Années 1984/85 - 1985/86

\title{
THEORIE DES NOMBRES
}

\section{(fascicule 1)}
J.-F. Jaulent : L'arithmétique des $\ell$-extensions (thèse de Doctorat d'Etat en Mathématiques, soutenue le 24 janvier 1986 à l'u niversité de Besançon)


Ce n'est pas sans une certaine émotion que j'exprime ici ma reconnaissance

à Georges GRAS, qui a dirigé mes recherches avec chaleur, humour et infinie patience; ses conseils, sa culture m'ont été extrèmement précieux ;

à Georges POITOU, qui m'a constamment manifesté sa bienveillance depuis mes débuts à l'Ecole Normale, et me fait l'honneur aujourd'hui de présider le jury de soutenance ;

à John COATES, qui a manifesté l'intérêt qu'il porte à ce travail en acceptant si volontiers de venir à Besançon participer au jury;

à Jean COUGNARD, dont l'amicale compréhension et la constante disponibilité ne m'ont jamais fait défaut ;

à Jacques MARTINET, qui m'a accueilli si cordialement à Bordeaux, entouré de ses conseils, fortifié de son énergie ;

à Michel WALDSCHMIDT, dont le cours d'arithmétique à Ulm a éveillé ma vocation de chercheur, et que je remercie tout particulièrement de participer au jury;

à Alain YGER, qui m'a proposé un second sujetpassionnant, et m'a initié avec patience aux secrets de la déconvolution.

Je tiens aussi à adresser mes plus vifs remerciements à Thérèse PARIS, qui, des mois durant, avec compétence et dévouement a assuré la dactylographie de la majeure partie de cette thèse;

à Catherine PAGANI, qui a poursuivi si heur eusement ce tr avail, avec l'énergie et l'efficacité que nous lui connaissons; à Rodolphe VRANA, qui a procédé au tirage avec sa rapidité et sa précision habituelle ; à Mauricette JAUBERT et à Louis POUPIN qui m'ont aidé à réali ser l'édition préliminaire.

Enfin, je n'aurais garde d'oublier que cette rédaction a pu être menée à bien grâce à l'hospitalité du C.N.R.S. Jel'en remer cie très sincèrement ici . 


\section{1.- L'ARITHMÉTIQUE DES l-EXTENSIONS ABÉLIENNES}

1. - Préliminaires à la théorie du corps de classes .............. 14

a.- Définition des $\ell$-groupes fondamentaux ................. 14

b. - Présentation du $\ell$-groupe des classes diidèles ............. 18

c. - Valeurs absolues $\ell$-adiques principales et formule du produit ........................... 21

2. - Isomorphisme du corps de classes ....................... 24

a. - Enoncé des théorèmes fondamentaux.................... 24

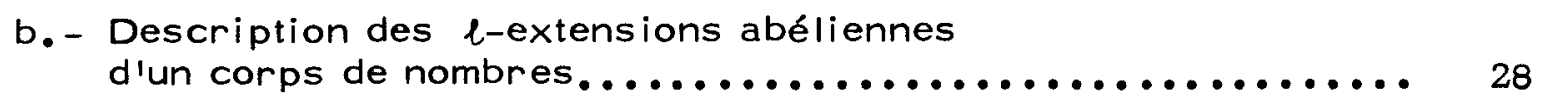

c.- Les conjectures de Leopoldt et de Gross................. 32

3.- Propriétés normiques des f-extensions abéliennes ........... 37

a. - La norme dans une extension quelconque de corps de nombres.................................. 37

b. - Applications aux $\ell$-genres cyclotomiques............... 40

c. - Récapitulatif des principaux groupes de normes ........... 43 


\section{2. - LA THÉORIE DE KUMMER \\ ET LE $K_{2}$ DES CORPS DE NOMBRES}

1. - Définition du radical universel $\Omega$

a.- Préliminaires: Théorie de Galois pour les radicaux universels

b. - Rappel de la description par Tate du groupe $K_{2}(K), \ldots \ldots$...

c. - Définition des groupes $\bar{K}_{2}(K)$, , et interprétation kummérienne.

2. - Introduction du noyau régulier $\Re \ldots \ldots \ldots \ldots \ldots \ldots \ldots \ldots \ldots \ldots \ldots$

a. - Interprétation symbolique : le groupe $R_{2}(\mathrm{~K})$............ 54

b. - Interprétation kummérienne : le groupe $\bar{R}_{2}\left(K_{\ell}\right)_{\ell} \ldots \ldots \ldots \ldots \ldots . . . .56$

c. - Interprétation régulière de la conjecture de Leopoldt....... 58

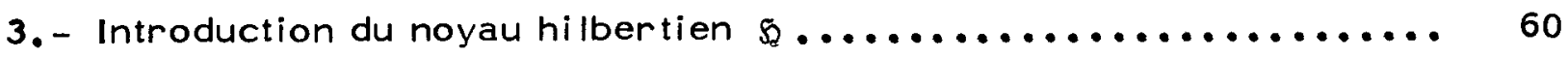

a. - Interprétation symbolique : le groupe $H_{2}(\mathrm{~K}) \ldots \ldots \ldots \ldots \ldots \ldots . \ldots 2$

b. - Interprétation kummérienne : le groupe $\bar{H}_{2}(\mathrm{~K})_{\ell} \ldots \ldots \ldots \ldots \ldots .65$

c. - Interprétation hilbertienne de la conjecture de Leopoldt...... 67

4. - Les résultats fondamentaux de la dualité.................. 70

a. - Comparaison des noyaux de la théorie de Kummer

et de la $K$-théorie............................ 70

b. - Dualité pour le noyau régulier et le noyau hilbertien ........ 71

c.- Isomorphisme du miroir......................... 75

\section{APPENDICE}

Les conjectures de Leopoldt et de Gross..................... 
CHAPITRE II

Sommaire.......................................

\section{1.- INDÉPENDANCE $\ell$ - ADIQUE DE NOMBRES ALgÉBRIQUES}

1.- Position du problème et énoncé de la conjecture............... 91

a. - Les deux complétions multiplicatives d'un corps de nombres.... 91

b. - Enoncé de la conjecture........................... 94

c. - Discussion de la conjecture énoncée.................. 98

2.-Minorations du rang $\mathfrak{f}$-adique et preuve de la conjecture dans le cas abélien.................................101

a.- Preuve de la conjecture dans le cas abélien...............101

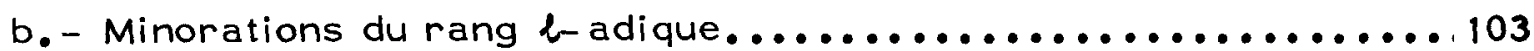

c. - Comparaison des divers résultats obtenus................ 108

3.- Application aux conjectures de Leopoldt et Gross...............111

a. - La conjecture de Leopoldt et le problème du rang $\ell$-adique des $S$-unités................................111

b. - La conjecture de Gross et le groupe des valeurs absolues $\ell$-adiques des $S$-unités.............................115

c. - Application aux problèmes normiques dans les

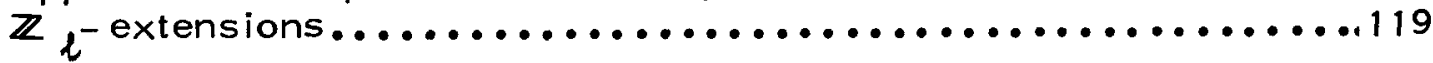

\section{2.- CALCUL INFINITÉSIMAL DANS UN CORPS DE NOMBRES ALGÉBRIQUES.}

1.-Eléments infinitésimaux d'un corps de nombres algébriques........126

a.- Définition des éléments infinitésimaux.................. 126

b. - Interprétation kummérienne des infinitésimaux.............128

c. - Définition des diviseurs infinitésimaux..................130

d. - Interprétation arithmétique du groupe des classes infinitésimales.................................133 
2.-Groupes de $S$-classes $T$-infinitésimales.................. 135

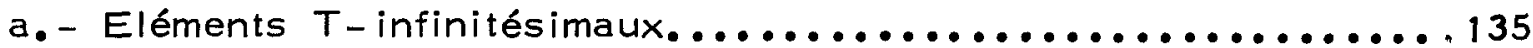

b. - S-classes $T$-infinitésimales...................... 137

c.- Interprétations arithmétiques : applications à la dualité........139

3.- La suite exacte des classes ambiges dans une extension

galoisienne.....................................142

a. - L'homomorphisme d'extension pour les groupes a ........... 142

b. - Application au sous-groupe de torsion 6 ................ 145

c.- Extension aux $\ell$-groupes de $S$-classes $T$-infinitésimales . . . . . . 148

4.- Genre infinitésimal d'une extension de corps de nombres..........150

a.- L'application norme pour les groupes a ................150

b. - S-genre T-infinitésimal d'une extension de corps

de nombres .......................................154

c.- Application au symbole de reste normique généralisé.........157

\section{CHAPITRE III}

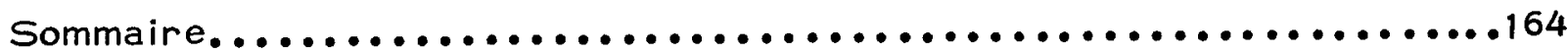

\section{1.- la formule des classes ambiges et ses GÉNÉRALISATIONS.}

1.- La formule de Chevalley pour les groupes de $\mathbf{S}$-classes

de diviseurs.......................................168

a.- Présentation du groupe des S-classes de diviseurs

d'un corps de nombres..............................168

b. - Démonstration de la formule des classes ambiges.............171

c. - Analyse de la formule dans le cas cyclique..................175

2.- Expression de la formule en termes de représentations dans le cas métabélien ................................180

a.- Préliminaires.................................180

b. - Enoncé des résultats............................182

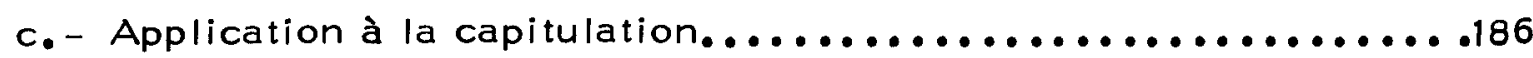


3.- Extension des résultats au cas procyclique.................. 189

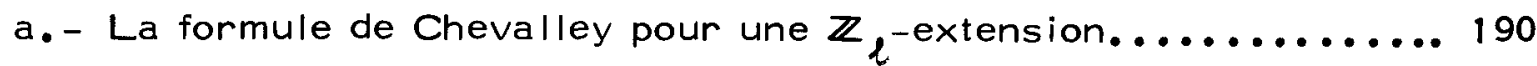

b. - Calcul du quotient de Herbrand dans le cas procyclique........ 193

c. - Analyse de la formule obtenue ; critères de trivialité pour les groupes $\mathrm{Cl}_{\mathrm{L}}^{\mathrm{S}}$

\section{2. - ÉLÉMENTS dE THÉORIE dES GENRES .}

1.- Présentation de la théorie des genres.

a.- Définition du corps des $\mathbf{S}$-genres relatif à une extension finie de corps de nombres................. 204

b. - La formule du produit pour le symbole de reste normique....... 209

c. - Comparaison du corps des genres et du corps des classes centrales................................ 214

2.- Expression de la formule des genres en termes de repré sentations..................................... 219

a.- Etude du cas métabélien.......................... 219

b. - Extension des résultats au cas procyclique :

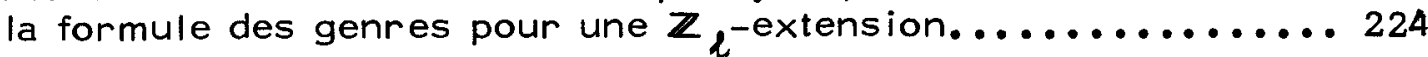

c. - Propriétés normiques des $S$ - unités dans une $\mathbb{Z}_{\ell}$-extension.... 229

\section{1.- STRUCTURE DES $\Lambda[\Delta]$-MODULES .}

1.-Présentation de l'algèbre d'Iwasawa généralisée.............. 244

a.- Position du problème.............................. 244

b. - Définition de l'algèbre d'lwasawa généralisée............. 246

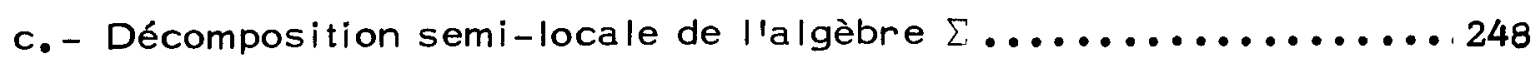


2.-Structure des $\Lambda[\Delta]$-modules dans le cas abélien.............251

a.- Paramètres attachés à un $\Lambda[\Delta]$-module noethérien..........251

b. - Etude de la filtration d'un $\Lambda[\Delta]$-module noethérien

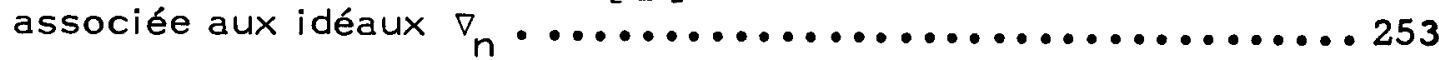

c. - Suites paramétrées de $\mathbb{Z}_{\ell}[\Delta]$-modules finis............. 257

3.- Structure des $\Lambda[\Delta]$-modules dans le cas métabélien............ 262

a. - Description des $\Sigma$-modules projectifs de type fini........... 263

b. - Classification des $\Sigma$-modules noethériens................ 267

c. - Paramètres attachés à un $\Sigma$-module noethérien............ 272

\section{2.- REPRÉSENTATIONS l-ADIQUES ASSOCIÉES AUX INVARIANTS CYCLOTOMIQUES.}

1.- Etude du groupe de Galois de la $\ell$-extension abélienne non ramifiée $l$-décomposée maximale de $K_{\infty} \ldots \ldots \ldots \ldots \ldots . \ldots \ldots$

a.- Définition du groupe C.......................... 281

b. - Comparaison avec le groupe des classes au sens ordinaire.... 285

c. - Capitulation et structure des groupes de classes............ 292

2.- Etude du groupe de Galois de la $\ell$-extension abélienne

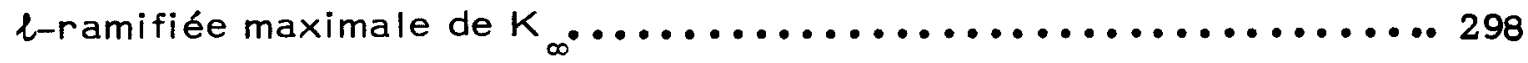

a.- Définition du groupe . ......................... 298

b. - Etude du sous-groupe de torsion $\tau$................. 302

c. - Capitulation pour les groupes $\varepsilon_{n}$, et application

à la $K$-théorie............................... 307

3.- Etude du groupe de Galois de la $l$-extension hilbertienne

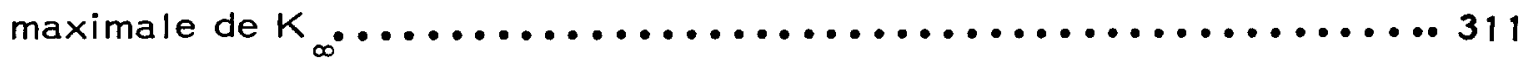

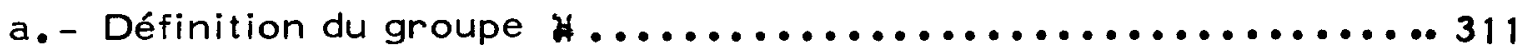

b. - Parallèle entre les conjectures de Leopoldt et de Gross....... 315

c. - Inégalités du miroir ( $l$ impair ) .................... 318

d. - Application à une conjecture de Coates.................. 321 
a. - Caractères associés aux suites paramétrées de $\mathbb{Z}_{\ell}[\Delta]$-modules finis........................... 328

b. - Caractères associés aux $\Lambda[\Delta]$-modules noethériens (avec conjugaison complexe) 
Les pages qui suivent traitent de l'arithmétique des l-extensions, et, plus précisément, de l'étude des $\ell$-groupes de classes (d'idèles, de radicaux, de symboles, de diviseurs,...) dans les $\ell$ extensions, finies ou non, de corps de nombres algébriques .

L'ensemble est organisé en quatre chapitres largement indépendants, eux-mêmes divisés en deux sections autonomes, qui exposent chacune un aspect de cette arithmétique, et restent directement accessibles au lecteur plus particulièrement intéressé par un résultat bien précis. Cette indépendance, cependant, n'est pas absolue, puisque nous utilisons, le cas échéant, dans chacune des huit sections qui constituent cette thèse, tel théorème essentiel établi plus avant, au même titre qu'un résultat classique.

L'unité de ce travail réside avant tout dans l'accent mis sur les méthodes f-adiques lors de la résolution des questions considérées. Nous entendons par là le développement systématique de techniques algébriques adaptées à l'étude des corps de nombres, de nature irréductiblement globales, mais où l'anneau $\mathbb{Z}_{\ell}$ des entiers $l-$ adiques tient un rôle central. Cet emploi des nombres $\ell$-adiques dans l'arithmétique algébrique, qui ne doit pas être confondu avec le recours aux méthodes locales, n'est d'ailleurs pas nouveau. Sans remonter aux origines, disons simplement ici que la présentatión par Serre des résultats d'iwasawa sur les groupes de classes des corps cyclotomiques - ce que nous appelons classiquement aujourd'hui la théorie d'lwasawa - en est une bonne illustration .

Nous résumons ci-dessous le contenu des différents chapitres. 
Le chapitre 1 est consacré aux $\ell$-extensions abéliennes.

La section 1 (L'ARITHMÉTIQUE DES l-EXTENSIONS ABÉLIENNES) expose les résultats fondamentaux de la théorie $\ell$-adique du corps de classes. Elle prend appui sur l'isomorphisme algébrique et topologique qu'elle établit entre le groupe de Galois $G_{K}$ de la $\ell$-extension abélienne maximale d'un corps de nombres donné, et le $\ell$-groupe des classes d'idèles de ce corps, défini comme le quotient $C_{K}=z_{K} / R_{K} d u$ l-groupe $g_{K}=\prod_{p \in P l_{K}}^{r e s} \frac{\lim }{K} K_{p}^{x} / K_{p}^{x l^{K}}$ des idèles généralisés par le sousgroupe $R_{K}=\mathbb{Z}_{l} \otimes_{\mathbb{Z}} K^{X}$ des idèles principaux. La description obtenue, qui élimine complètement le problème des normes universelles de la théorie de Chevalley, permet de rendre compte plus directement de l'arithmétique des $\ell$-extensions abéliennes, finies ou infinies. Elle ouvre enfin sur des interprétations simples des conjectures de Leopoldt et de Gross. Nous montrons ainsi que la conjecture de Leopoldt affirme exactement qulune unité généralisée $\epsilon \in \mathbb{Z}_{\ell} \otimes_{\mathbb{Z}} E_{K}$, qui est localement partout une racine de l'unité, doit être une racine globale de llunité; tandis que la conjecture de Gross postule qu'en un sens la formule du produit $\prod_{p \in P l_{K}} \nu_{p}=1$ est essentiellement la seule relation de liaison entre les

valeurs absolues $\ell$-adiques principales des éléments de $R_{K}$ •

La section 2 (LA THÉORIE DE KUMMER \& LE $K_{2}$ DES CORPS DE NOMBRES) peut être regardée comme une reconstruction de llarticle de Bertrandias et Payan sur les invariants cyclotomiques $\left({ }^{\star}\right)$ à la lumière des idées de Tate sur le $K_{2}$ des corps de nombres. Nous y établissons un parallèle entre la description kummérienne des $\ell$-genres dans une tour cyclotomique, et les résultats de Tate sur le $K_{2}\left(^{\star \star}\right)$. Plus précisément, à chaque corps de nombres $K$, nous associons un groupe universel $\bar{K}_{2}(K)$, ana-

$\left(^{\star}\right)$ T-extensions et invariants cyclotomiques - Ann. Sci. Éc. Norm. Sup. 5 (1972), 517-548.

$\left({ }^{\star}\right.$ ) Relations between $K_{2}$ and Galois cohomology - Inv. Math. 36 (1976), 255-274. 
logue au groupe symbolique $K_{2}(K)$, et deux groupes finis $\bar{R}_{2}(K)$ et $\bar{K}_{2}(K)$, qui correspondent aux noyaux régulier et hilbertien de la $K$-théorie, et explicitent les correspondances remarquables entre ces deux noyaux et certains groupes de Galois classiques. Dans le premier cas, qui fait intervenir le noyau régulier $R_{2}(K)$ et le sous-groupe de torsion $\sigma_{K} d u$ $\ell$-groupe des classes infinitésimales de $K$, la correspondance obtenue a déjà fait l'objet de travaux de Carroll $\left({ }^{\star}\right)$, Greenberg $\left({ }^{\star}{ }^{\star}\right)$, Kramer et Candiotti $\left({ }^{\star \star}\right)$; dans le second cas, qui concerne les noyaux hilber tiens $H_{2}(\mathrm{~K})$ et $\bar{H}_{2}(\mathrm{~K})$, elle est entièrement nouvelle.

En appendice, nous donnons plusieurs formulations équivalentes de la condition suffisante de la conjecture de Leopoldt introduite par Bertrandias et Payan $\left({ }^{\star \star \star}\right)$. Subsidiairement, nous montrons qu'elle entraîne aussi la conjecture de Gross.

Le chapitre 11 est consacré à l'arithmétique des infinitésimaux.

La section 1 (INDÉPENDANCE $\ell$-ADIQUE DE NOMBRES ALGÉBRIQUES) traite d'abord du problème de l'indépendance $\ell$-adique, qui se pose comme suit : Un nombre premier $l$ étant fixé, les logarithmes d'lwasawa, associés aux complétés de $K$ pour les places au-dessus

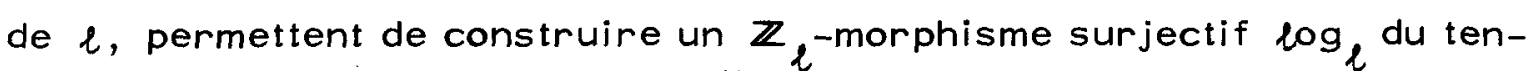
sorisé multiplicatif $\Omega_{K}=\mathbb{Z}_{\ell} \otimes_{\mathbb{Z}} K^{X}$ du corps $K$ sur un $\mathbb{Z}_{\ell}$-réseau

${ }^{\star}$ ) On the 2-primary part of $K_{2}(\theta)$ and on $\mathbb{Z}_{2}$-extensions for imaginary quadratic fields-Ph. D. Thesis, Cambridge, Mass. (1973). $\left({ }^{\star}\right)$ A note on $K_{2}$ and the theory of $\mathbb{Z}_{\mathrm{p}}$-extensions - Am. J. Math. 100 (1978), 1235-1245.

$\left({ }^{\star \star}\right)$ On $K_{2}$ and $\mathbb{Z}_{\ell}$-extensions of number fields - Am. J. Math. 100 (1978), 177-196.

$(\star \star \star \star)$-extensions et invariants cyclotomiques - Ann. Sci. Éc. Norm. Sup. 5 (1972), 517-548. 
$\log _{\ell} \Omega_{K}$ de son tensorisé additif $\mathbb{Z}_{\ell} \otimes_{\mathbb{Z}} K$. On se demande à quelle condition la restriction de cette application à un sous-module noethérien $m$ de $R_{K}$, engendré sur $\mathbb{Z}_{\ell}$ par des nombres algébriques, est une injection. Lorsque le corps $K$ est galoisien, et que le module $m$, stable par $G=G a l(K / Q)$, ne contient ni puissances de \& ni racines de l'unité, nous postulons que $\log _{\ell / m}$ est injective si et seulement si $\mathbb{Q}_{\ell} \otimes_{\mathbb{Z}}, m$ est contenu dans la représentation régulière de $\mathbb{Q}_{l}[G]$. Cette conjecture contient évidemment la conjecture de Leopoldt (pour laquelle $m=\mathbb{Z}_{\ell} \otimes_{\mathbb{Z}} E_{K}$ ', et, sous une restriction technique, celle de Gross. Nous montrons qu'elle est vérifiée dès que l'algèbre $\mathbb{Q}_{\ell}[G]$ est un produit direct de corps, c'est-à-dire dans tous les cas où les méthodes transcendantes établissent ces deux dernières conjectures. Nous proposons enfin des minorations du rang $l$-adique, directement dérivées d'un théorème de Waldschmidt $\left({ }^{\star}\right)$, qui conduit aux meilleures bornes connues et donne asymptotiquement le rang conjectural.

\section{La section 2 (CALCUL INFINITÉSIMAL DANS UN CORPS}

DE NOMBRES ALGÉBRIQUES) s'intéresse au groupe infinitésimal $\partial_{K}$ qu'on peut définir comme sous-module de $R_{K}$ formé des éléments (globaux) d'image locale 1 aux places divisant $l$ :

$\partial_{K}=\left\{x \in R_{K} \mid s_{l}(x)=1\right.$ dans $\left.K_{l}^{x}=\prod_{I \mid l} \frac{\lim }{K} K_{I}^{x} / K_{I}^{x \ell^{k}}\right\}$. Via la théorie de Kummer, le groupe $\jmath_{K}$ permet de décrire les $\ell$-extensions abéliennes $\ell$-décomposées du corps $K$ (i.e. celles pour lesquelles les places audessus de $\ell$ se décomposent complètement) ; dans l'isomorphisme du corps de classes, il correspond au contraire aux $\ell$-extensions labéliennes) de $K$ qui sont $\ell$-ramifiées (i.e. non ramifiées aux places étrangères à $\ell$ ). Comme il existe des relations précises entre radicaux (au sens ordinaire) et classes infinitésimales d'une part, radicaux au sens infinitésimal et classes (ordinaires) d'autre part, nous obtenons ainsi une double dualité entre les notions de $\ell$-ramification et de $\ell$-décomposition. Nous calculons ensuite la cohomologie des groupes infinitésimaux, ce qui généralise les résultats de Gras $\left(^{\star \star}\right)$ sur l'injectivité du transfert pour les

$\left(^{\star}\right)$ A lower bound for the p-adic rank of the units of an algebraic number field-Actes congrès Budapest (1981) .

$\left({ }^{\star \star}\right)$ Groupe de Galois de la p-extension abélienne p-ramifiée maximale d'un corps de nombres - J. reine angew. Math. 333 (1982), 86-132. 
groupes de Galois des $\ell$-extensions abéliennes maximales $\ell$-ramifiées, relativement à une $\ell$-extension de corps de nombres. Nous étudions enfin les propriétés normiques des infinitésimaux qui commandent les questions de déploiement de la ramification dans les $\ell$-extensions.

Le chapitre III est consacré à la théorie des genres.

\section{La section 1 (LA FORMULE DES CLASSES AMBIGES ET} SES GÉNÉRALISATIONS) commence par exposer la formule des classes ambiges de Chevalley dans le cadre des groupes de $\mathbf{S}$-classes de diviseurs. La suite exacte obtenue contient pour diverses spécifications de $S$ les formules données par Chevalley $\left({ }^{\star}\right)$, pour les groupes de classes d'idéaux au sens ordinaire, par Gras $\left({ }^{\star}\right.$ ) pour celles au sens restreint, ainsi que par d'autres auteurs. Elle s'appuie sur le formalisme des diviseurs qui est plus général que celui des idéaux. Dans un deuxième temps, nous reprenons l'ensemble des résultats obtenus pour les énoncer en termes de représentations $\ell$-adiques dans le cas semi-simple où l'extension considérée, cyclique de degré $l^{S}$, est métabélienne sur un sous-corps d'indice étranger à $\ell$. L'existence de cette structure supplémentaire permet de mettre en relief le rôle du quotient de Herbrand, qui peut alors ne pas se simplifier. Nous nous intéressons enfin au cas diune

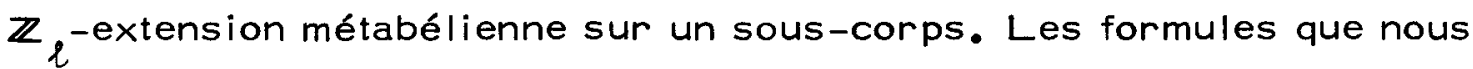
obtenons précisent et généralisent à la fois celles données indépendamment par Iwasawa $\left({ }^{\star \star \star}\right)$. Elles conduisent à des critères simples de trivialité ou de non trivialité des $\ell$-groupes de classes.

$\left(^{\star}\right)$ Sur la théorie du corps de classes dans les corps finis et les corps locaux - J. Fac. Sc. Tokyo 2 (1933), 402-405. $\left({ }^{\star}{ }^{\star}\right)$ Sur les $\ell$-classes d'idéaux dans les extensions cycliques relatives de degré premier $\ell$ - Ann. Sci. Inst. Fourier 23 (1973), 1-48. $\left({ }^{\star \star}\right)$ On cohomology groups of units for $\mathbb{Z}_{p}$-extensions - Am. J. Math. 105 (1983), 189-200. 
La section 2 (ÉLÉMENTS DE THÉORIE DES GENRES) définit puis calcule le nombre de $\mathbf{S}$-genres et le nombre de $\mathbf{S}$-classes centrales de diviseurs pour une extension finie quelconque de corps de nombres. Les formules obtenues contiennent, pour diverses spécifications de $S$, celles déjà connues pour les groupes de classes d'idéaux au sens ordinaire dans le cas galoisien, au sens restreint dans le cas abélien. Nous étendons pour cela aux extensions finies le symbole de reste normique défini par Hasse pour les extensions abéliennes, et nous établissons dans ce cadre une réciproque de la formule du produit. Nous reprenons ensuite l'ensemble de ces résultats pour les exprimer en termes de représentations dans le cas semi-simple, fini d'abord, infini ensuite, évoqué plus haut, où l'extension considérée est métabélienne sur un souscorps du corps de base. Le résultat essentiel est que les représentations obtenues, bien qulayant le même degré, ne coincident pas avec celles données par les groupes de classes ambiges. En particulier, dans le cas d'une $\mathbb{Z}_{\ell}$-extension métabélienne mais non abélienne sur un sous-corps, nos conclusions diffèrent totalement de celles de Greenberg $\left({ }^{\star}\right)$ pour la tour cyclotomique.

Le chapitre IV est consacré à la théorie d'Iwasawa.

La section I (STRUCTURE DES $\Lambda[\Delta]$-MODULES) établit les théorèmes généraux sur la structure des modules de type fini sur l'algèbre (éventuellement gauche) d'un groupe abélien $\Delta$ (d'ordre étr an ger à $\ell$ ) à coefficients dans un anneau d'lwasawa $\Lambda=\mathbb{Z}_{\ell}[[\gamma-1]]$.

$\left.{ }^{\star}\right)$ On a certain $\ell$-adic representation - Inv. Math. 21 (1973), 117-124. 
Nous introduisons ensuite la notion-clé de suite paramétrée de $\mathbb{Z}_{\ell}[\Delta]-$ modules finis, qui généralise la classique formule d'lwasawa sur le nombre de classes des corps cyclotomiques, et ramène la recherche des invariants d'un $\Lambda[\Delta]$-module noethérien, de torsion ou non, à l'étude systématique de certains de ses quotients finis : Lorsque $\Delta$ agit trivialement sur le groupe procyclique $\Gamma=\gamma^{\mathbb{Z}} \ell$, une suite $\left(x_{n}\right)_{n \in \mathbb{N}}$ est paramétrée par $\rho, \mu, \lambda$ et $\nu$ si, pour chaque caractère $l$-adique irréductible $\varphi$ du groupe $\Delta$, et tout $n$ assez grand, la $\&$-valuation $x_{n}^{\varphi}$ de llor dre de la $\varphi$-composante de $x_{n}$ est donné par la formule :

$$
x_{n}^{\varphi}=\left\langle\rho, \varphi>(n+1) \ell^{n}+\left\langle\mu, \varphi>\ell^{n}+\langle\lambda, \varphi>n+\langle\nu, \varphi>.\right.\right.
$$

Une formule semblable vaut dans le cas non abélien.

La section 2 (REPRÉSENTATIONS l-ADIQUES ASSOCIÉES AUX INVARIANTS CYCLOTOMIQUES) applique les résultats qui précèdent au cas de la $\mathbb{Z}$,extension cyclotomique d'un corps de nombres. Nous montrons, au moins sous les deux conjectures de Leopoldt et de Gross, que les paramètres associés aux divers modules classiques donnés par le corps de classes, la théorie du Kummer, ou la K-théorie (groupes de Galois respectifs des $\ell$-extensions abéliennes maximales l-ramifiée, hilbertienne, non ramifiée, non ramifiée et $\ell$-décomposée ; radicaux correspondant; noyaux régulier et hilbertien de la $K$-théorie) se déduisent tous de l'un quelconque d'entre eux par des formules explicites ne faisant intervenir que des invariants galoisiens simples du schéma d'extensions. Nous montrons également que les paramètres lambda vérifient un spiegelungssatz plus fort que celui de Leopoldt. Nous il lustrons enfin les résultats obtenus en calculant explicitement le ca ractère de défaut d'une conjecture de Coates $\left({ }^{\star}\right)$.

Deux tableaux, présentés en appendice, rassemblent les divers paramètres rencontrés au cours de cette étude.

$\left({ }^{\star}\right)$ On $K_{2}$ and some classical conjectures in algebraic number theory Ann. of Math. 95 (1972), 99-116. 
$-8-$ 
CHAPITRE I

L'ARITHMÉTIQUE DES $\ell$-EXTENSIONS ABÉLIENNES

LA THÉORIE DE KUMMER ET LE

$K_{2}$ DES CORPS DE NOMBRES

$-9-$ 


\section{1. - L'ARITHMÉTIQUE DES \&-EXTENSIONS ABÉLIENNES.}

1.- Préliminaires à la théorie du corps de classes................ 1.2

a.- Définition des $\ell$-groupes fondamentaux...................2

b. - Présentation du $\ell$-groupe des classes d'idèles.............. 1.6

c.- Valeurs absolues $\ell$-adiques principales et formule du produit............................... 9

2.- Isomorphisme du corps de classes........................12

a.- Enoncé des théorèmes fondamentaux......................12

b. - Description des h-extensions abéliennes d'un corps de nombres.................................16

c. - Les conjectures de Leopoldt et de Gross................... 1.20

3.-Propriétés normiques des $\ell$-extensions abéliennes.............. I.25

a. - La norme dans une extension quelconque de corps

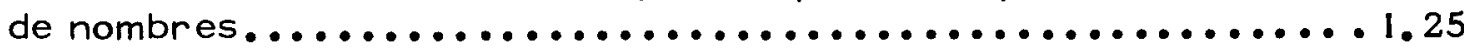

b. - Application aux $\ell$-genres cyclotomiques.................. 1.28

c. - Récapitulatif des principaux groupes de normes............. I.31

\section{2. - LA THÉORIE DE KUMMER ET LE $K_{2}$ DES CORPS DE NOMBRES.}

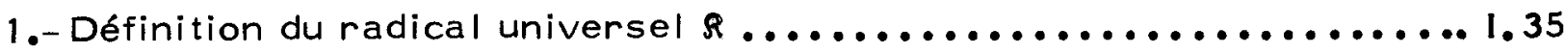

a.- Préliminaires : Théorie de Galois pour les radicaux universels...................................35

b. - Rappel de la description par Tate du groupe $K_{2}{ }^{(K)}{ }_{\ell} \ldots \ldots \ldots \ldots . .1 .37$

c. - Définition des groupes $\bar{K}_{2}{ }^{(K)} \ell$, et interprétation

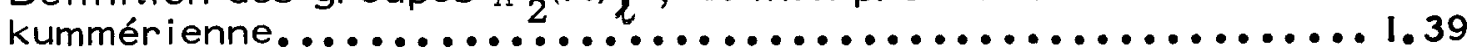

2.- Introduction du noyau régulier $\Re \ldots \ldots \ldots \ldots \ldots \ldots \ldots \ldots \ldots \ldots \ldots \ldots \ldots 1 . \ldots 1$

a.- Interprétation symbolique : le groupe $R_{2}(\mathrm{~K})_{2} \ldots \ldots \ldots \ldots \ldots \ldots \ldots .42$

b. - Interprétation kummérienne : le groupe $\bar{R}_{2}(\mathrm{~K})_{\ell} \ldots \ldots \ldots \ldots \ldots \ldots . . \ldots 44$

c.- Interprétation régulière de la conjecture de Leopoldt.......... 1.46 
3.- Introduction du noyau hilbertien $5 . \ldots \ldots \ldots \ldots \ldots \ldots \ldots \ldots \ldots \ldots \ldots \ldots$ a.- Interprétation symbolique : le groupe $H_{2}(\mathrm{~K})_{\ell} \ldots \ldots \ldots \ldots \ldots \ldots . . . . .50$

b. - Interprétation kummérienne : le groupe $\bar{H}_{2}(\mathrm{~K}), \ldots \ldots \ldots \ldots \ldots . . . . .53$ c. - Interprétation hilbertienne de la conjecture de Leopoldt......... I.55

4.- Les résultats fondamentaux de la dualité....................58

a. - Comparaison des noyaux de la théorie de Kummer et de la $K$-théorie ................................. 58

b. - Dualité pour le noyau régulier et le noyau hilbertien........... I.59

c. - Isomorphisme du miroir..........................1.63

\section{APPENDICE}

Les conjectures de Leopoldt et de Gross......................68 
$-12-$ 
La théorie du corps de classes global, telle qu'elle fut déve loppée par Takagi, Artin et Chevalley, a pour but la description de l'arithmétique des extensions abéliennes d'un corps de nombres donné, à l'aide des seuls éléments de ce corps. Dans sa formulation plus an cienne, celle de Takagi, elle décrit le groupe de Galois d'une extension abélienne finie $L / K$ de corpsde nombres comme groupe de congru ences attaché à un diviseur $\tau_{L} / K$ construit sur les places ramifiées dans cette extension. Ce point de vue, aujourd'hui encore le plus effi cace lorsquili s'agit d'apprécier numériquement une situation donnée, souffre cependant d'être limité au seul cas des extensions finies. La réinterprétation par Chevalley de l'ensemble des résultats de la théo rie à l'aide des groupes d'idèles permet de pallier agréablement cette difficulté. Mais deux autres problèmes surgissent alors: D'abord l'ap plication de réciprocité $\psi$ du groupe des classes diidèles $c_{K}=J_{K} / K X$ du corps $K$ dans le groupe de Galois $G a l\left(K^{a b} / K\right)$ de l'extension abélienne maximale de $K$ n'est jamais injective : Si $[K: \mathbb{Q}]=r_{K}+2 c_{K}$ est la décomposition canonique du degré de $K$ en ses contributions réelle et imaginaire, le noyau $C_{K}^{\circ}$ de $\psi$, qui est la composante connexe de l'élé ment unité dans $C_{k}$, est le produit dlune droite réelle $R$, de $\left(r_{k}+c_{k}-1\right)$ solénoïdes $(\boldsymbol{R} \oplus \hat{\mathbb{Z}}) / \mathbb{Z}$, et de $c_{k}$ tores $R / \mathbb{Z}(\star)$. L'existence de normes universelles dans la théorie est donc une première source de complications. Ensuite, la décomposition canonique du groupe de Galois $G_{K}=G a l\left(K{ }^{a b} / K\right)$ comme produit direct de ses $p$-composantes $q_{K}^{(p)}=\frac{\lim }{K} q_{K} / q_{K}^{p}$, pour tous les premiers $p$, ne se traduit pas commodément en termes de classes diidèles, puisque ni le numérateur $J_{K}$ ' ni le dénominateur $K^{X}$ ne sont des $\hat{\mathbb{Z}}$-modules.

$\left({ }^{\star}\right)$ Le groupe $\hat{\mathbb{Z}}$ est le complété profini de $\mathbb{Z}$ pour la topologie définie par ses sous-groupes dindice fini. 
Pour l'ensemble de ces raisons, nous proposons ici une pré sentation de la théorie du corps de classes, directement inspirée de celle de Chevalley, mais mieux adaptée, nous semble-t-il, à l'étude spécifique des $\ell$-extensions abéliennes d'un corps de nombres.

\section{1.- PRÉLIMINAIRES A LA THÉORIE DU CORPS DE CLASSES •}

\section{a.-Définition des $\ell$-groupes fondamentaux .}

$\ell$ étant un nombre premier fixé, nous désignons par $\mathbb{Z}_{\ell}=$ $\frac{\lim }{k} \mathbb{Z} / l^{k} \mathbb{Z}$ l'anneau des entiers $l$-adiques. A chaque corps de nom bres $K$ ( de degré fini sur $\mathbb{Q}$ ), nous allons associer deux $\mathbb{Z}_{\ell}$-modules : Le premier $\Omega_{K}$, global, défini à partir du groupe multiplicatif $K^{X}$ des éléments non nuls de $K$; le second $\partial_{K}$, semi-local, défini à partir des groupes multiplicatifs $K_{p}^{X}$ des complétés non complexes de $K$.

DÉFINITION 1,1,1.-Etant donné un corps de nombres $K$, nous appelons $\ell$-groupe des éléments généralisés de $K$, et nous notons $\Omega_{K}$, le tensorisé $\ell$-adique du groupe de ses éléments non nuls :

$$
R_{K}=\mathbb{Z} \ell^{\otimes} \mathbb{Z}^{X}
$$

Le groupe $R_{K}$ est la réunion des $l$-groupes de $S$-unités généralisées $\varepsilon_{K}^{S}=\mathbb{Z}_{l}{ }_{\mathbb{Z}} E_{K}^{S}$, lorsque $S$ parcourt les ensembles finis de places de $K$. Chacun des $\delta_{K}^{S}$ est compact pour sa topologie naturelle de $\mathbb{Z} l^{- \text {mo- }}$

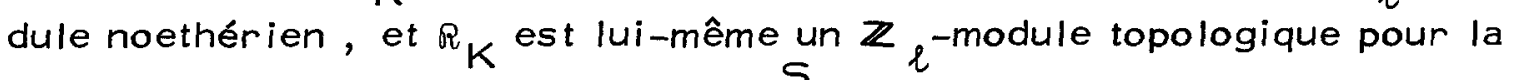
limite inductive des topologies des $\delta_{K}^{S}$.

Par définition de la topologie limite inductive, les sous-modules ouverts de $R_{K}$ sont exactement ceux qui intersectent chacun des $\delta_{K}^{S}$ suivant un sous-module relatif ouvert, clest-à-dire ( puisque les $\delta_{K}^{S}$ sont de type fini sur $\mathbb{Z}_{\ell}$ ) suivant un sous-module dindice fini . La topologie ainsi obtenue sur $Q_{K}$ est strictement plus fine que celle définie par ses sous-modules dindice fini.

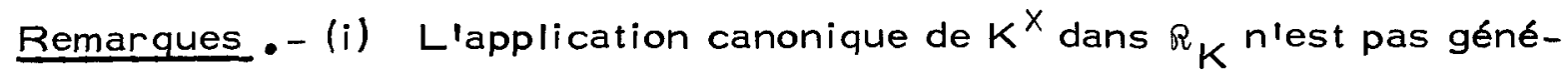
ralement injective. Plus précisément, son noyau est le sous-groupe $\mu_{k}^{\prime}$ des racines de llunité dans $K$ d'ordre étranger à $\ell$. Le quotient $K^{x} / \mu_{k}^{\prime}$, 
qui s'injecte dans $R_{K}$, s'identifie ainsi à un sous-groupe partout dense de $R_{K}$ •

(ii) Convenons diordonner les places de $K$ en posant $S_{n}=$ $\left\{p \in P I_{K} \mid N p \leq n\right\}$, avec la convention $N p= \pm 1$ pour les places archimédiennes. Nous avons évidemment $K^{X}=\bigcup_{n \in \mathbb{N}} E_{K}^{S}$, et chacun des $E_{K}^{S}=\left\{x \in K^{X} \mid v_{p}(x)=0, \forall p \in S_{n}\right\}$ est un $\mathbb{Z}$-module de type fini. En particulier $\mathfrak{R}_{K}=\bigcup_{n \in N} \delta_{K}^{S}$ est réunion dénombrable de $\mathbb{Z}_{l}$-modules compacts ${ }^{(\star)}$

(iii) Désignons par $k^{x}=\frac{\lim }{k} K^{x} / K^{x} l^{k}$ la limite projective des quotients d'exposant $\ell$-primaire du groupe $K^{x}$. Par passage à la limite à partir des surjections canoniques $\Omega_{K} \longrightarrow \Omega_{K} / R_{K} \ell^{k} \simeq K^{x} / K^{x} \ell^{k}$, le groupe $R_{K}$ slidentifie à un sous-module strict de $K_{K} \times$. Cependant, la topologie de $R_{K}$ n'est pas la restriction à $R_{K}$ de la topologie naturelle de $K^{x}$ définie par les sous-modules $K^{\times \ell^{k}}$.

DÉFINITION 1,1,2, - Pour chaque place $p$ du corps $K$, nous appelons $\ell-$ groupe des éléments généralisés du complété $K_{p}^{X}$, la limite projective $k{ }_{p}^{x}=\frac{\lim }{k} k_{p}^{x} / K l_{p}^{l^{k}}$

des quotients d'exposant $\ell$-primaire du groupe multiplicatif $K_{p}^{X}$. Le

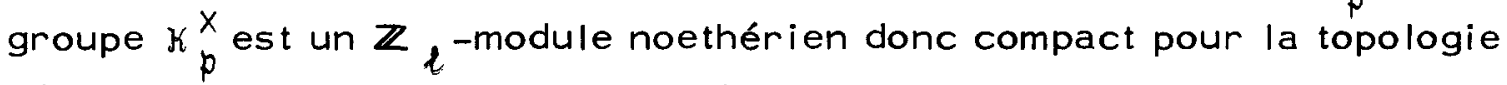
définie par ses sous-modules d'indice fini .

(i) Si p est une place archimédienne, deux cas se présentent: - ou bien $p$ est complexe, et le groupe $k_{p}^{x}$ est toujours nul. - ou bien pest réelle, et le groupe $k_{p}^{x}$ est isomorphe à $\mathbb{Z} / 2 \mathbb{Z}$ pour $\ell=2$, nul sinon.

(ii) Si $p$ est une place ultramétrique, le choix d'une unifor misante $\pi_{p}$ permet d'écrire formellement $\kappa_{p}^{x}=u_{p} \cdot \pi_{p}^{\mathbb{Z}} l$

en désignant par $u_{p}=\frac{\lim }{k} u_{p} / u_{p}^{l^{k}}$ la limite projective des quotients d'exposant $\ell$-primaire du groupe des unités du corps local $K_{p}$.

$\left({ }^{\star}\right)$ Pour une définition des valuations $v_{p}$ attachées aux places archimé diennes, voir $\mathrm{Ch} .111,1,1 \S 2$. 
- Lorsque $p$ divise $\ell$, le groupe $u_{p}$ n'est autre que le groupe des unités principales de $K_{p}$.

- Lorsque $p$ ne divise pas $l$, il s'identifie au $\ell$-sous groupe de Sylow $\mu_{p}$ du groupe des racines de l'unité dans $K_{p}$ • de $x_{p} \times\left(^{\star}\right)$

Dans les deux cas nous disons que $u_{p}$ est le groupe des unités

Démonstration :

(i) Si pest une place archimédienne, le groupe multiplicatif $K_{p}^{X}$ est soit divisible, lorsque $p$ est complexe, soit le produit de $\{ \pm 1\}$ par un groupe divisible, lorsque pest réelle. La limite projective $\kappa_{p}^{x}$ est donc nulle dans le premier cas, isomorphe à $\mathbb{Z}_{\ell} / 2 \mathbb{Z} l^{\text {dans le se- }}$ cond.

(ii) Si pest une place ultramétrique, la décomposition du groupe multiplicatif $K_{p}^{X}$

$$
K_{p}^{x}=\mu_{p}^{o} \cdot u_{p}^{1} \cdot \pi_{p}^{\mathbb{Z}_{l}}
$$

fait intervenir le groupe $\mu_{p}^{0}$ des racines de llunité d'ordre étranger à $p$, le sous-groupe $u_{p}^{1}$ des unités principales, et une uniformisante $\pi_{p}$ de $K_{p} \cdot$

- Si p est au-dessus de $\ell$, le groupe $\mu_{p}^{0}$ est $\ell$-divisible,

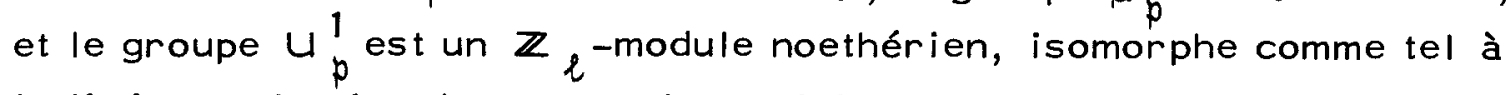
la limite projective de ses quotients finis. Il vient donc :

$$
u_{p}=\frac{\lim }{k} u_{p} / u_{p}^{l^{k}}=\frac{\lim }{k} u_{p}^{1} / u_{p}^{1} l^{k} \simeq u_{p}^{1} \text {, comme annoncé. }
$$

- Si p est étrangère à $l$, le groupe $\mu_{p}^{\circ}$ est le composé direct de son $\ell$-Sylow $\mu_{p}$ et de son sous-groupe $\ell$-divisible $\mu_{p}^{\prime}$. Enfin, le groupe $u_{p}^{1}$ est un $\mathbb{Z}_{p}$-module ( pour un $p \neq l$ ), donc $l$-divisible, et il vient

$$
u_{p}=\frac{\lim }{k} u_{p} / u_{p}^{l^{k}}=\mu_{p} \text {, comme annoncé . }
$$

Dans les deux cas, le groupe $k_{p}^{x}$ est le produit direct de $u_{p}$ et

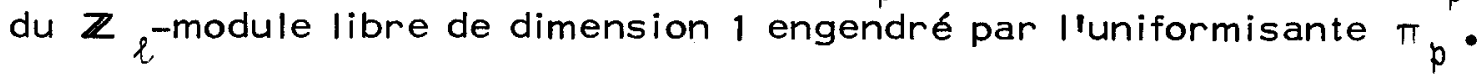

DÉFINITION 1.1.3.-Par $\ell$-groupe des idèles généralisés d'un corps de nombres $K$, nous entendons le produit :

$\left.{ }^{\star}\right)$ On a toujours $\mu_{p} \subset u_{p}$ lorsque $p$ est ultramétrique mais non lorsque $p$ est réelle. Dans ce dernier cas, en effet, $u_{p}$ est identiquement nul, et $\mu_{p}=\kappa_{p}^{x}$ égal à $\mathbb{Z}_{\ell} / 2 \mathbb{Z} \mathbb{Z}$. 


$$
\partial_{K}=\prod_{p \in P I_{K}^{*}}^{*} k_{p}^{x}
$$

des complétés profinis des groupes multiplicatifs des corps locaux $K_{\mathfrak{p}}$, restreint aux familles $\left(x_{p}\right)_{p} \in P I_{K}$ dont tous les éléments sont des unités, à l'exception d'un nombre fini d'entre eux.

Legroupe ${ }_{K}$ est la réuniondes $l$-groupes $u_{K}^{S}=\prod_{p \in S} k_{p}^{x} \prod_{p \notin S} u_{p}$,

lorsque $S$ parcourt les ensembles finis de places de $K$. Chacun des $U_{K}^{S}$ est compact pour sa topologie naturelle de $\mathbb{Z}_{\ell}$-module produit, et $g_{K}$

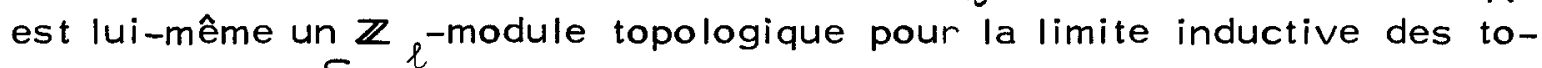
pologies des $U_{K}^{S}$.

Par définition de la topologie limite inductive, les sous-modules ouverts de $\partial_{K}$ sont ceux qui intersectent chacun des $U_{K}^{S}$ suivant un sous-module relatif ouvert. De fait, il est possible dlexhiber une base de sous-modules ouverts de $\partial_{K}$ en procédant comme suit : Pour chaque place ultramétrique $p$ de $K$, faisons choix d'une uniformisante $\pi_{p}$ dans $k_{p}^{x}$; si pest réelle, prenons $\pi_{p}=-1$; et, si p est complexe, $\pi_{p}=1$. Cela posé, le groupe $\partial_{K}$ s'écrit comme somme directe topologique du sous-module compact $u_{K}=\prod_{p \in P I} u_{p}$, formé des éléments unités, et du $\mathbb{Z}_{l}$-module $\underset{p \in P l}{\oplus} \pi_{p}^{\mathbb{Z}_{l}}$, engendré par les $\pi_{p}$, qui s'identifie au $\ell$-groupe $\theta_{K}$ des diviseurs de $K$. Les produits

$$
\left(\prod_{p \in P \mid K} u^{\prime} p\right) \cdot\left(\prod_{p \in P I_{K}}^{\oplus} \pi_{p}^{l^{n} p} \mathbb{z}_{l}\right)
$$

où, pour chaque place $p$ de $K, u_{p}^{\prime}$ est un sous-module d'indice fini de $u_{p}$ égal à $u_{p}$ pour presque tout $p$, et $n_{p}$ un entier naturel arbitraire, forment une base de sous-modules ouverts de $\partial_{K}$ •

Remarques - - (i) Llapplication canonique du groupe des idèles $J_{K}$ dans le $\ell$-groupe $\partial_{K}$ des idèles généralisés n'est jamais injective. Son noyau est le produit $\prod_{p \mid \infty} K_{p}^{\times 2} \cdot p \prod_{p} \mu_{p}^{0} \cdot p \prod_{\infty} \mu_{p}^{1} u_{p}^{1}$; son image est un sousgroupe partout dense de $\partial_{K}$.

(ii) Convenons d'ordonner les places de $K$ en posant $S_{n}=$ $\left\{p \in P I_{K} \mid N p \leq n\right\}$, avec la convention $N p= \pm 1$ pour les places archimédiennes. Nous avons évidemment $z_{K}=U_{n \in \mathbb{N}} U_{K}^{S_{n}}$, ce qui nous montre 
que $\partial_{K}$ est réunion dénombrable de $\mathbb{Z} \ell^{\text {-modules compacts }}$.

(iii) On peut regarder le groupe $\partial_{K}$ comme sous-module strict du produit $\prod_{p \in P I_{K}} k_{p}^{x}$. Cependant, la topologie de $\partial_{K}$ n'est pas la res triction du produit des topologies des $K_{p}^{X}$.

\section{b.-Présentation du $\ell$-groupe des classes d'idèles.}

THÉORËME \& DÉFINITION 1.1.4. - L'application naturelle du tensorisé $\ell$-adique $R_{K}=\mathbb{Z} \ell^{\otimes} \mathbb{Z} K^{X}$ du groupe multiplicatif du corps $K$ dans le $\ell$-groupe $\partial_{K}$ des idèles généralisés, induite par l'injection diagonale de $K^{X}$ dans $J_{K}$, est un monomorphisme continu. Le groupe quotient

$$
c_{K}=\partial_{K} / R_{K}
$$

est un $\mathbb{Z} l^{- \text {module compact }}$. Nous disons que c'est le $l$-groupe des classes diidèles du corps $K$.

Démonstration : Partons de I'injection diagonale $K^{x} \hookrightarrow \prod_{p \in P \mid K} K_{p}^{X}$ du groupe multiplicatif de $K$ dans le produit de ceux de ses complétés. Par passage au quotient, nous en déduisons, pour chaque entier $k$, un morphisme naturel

$$
K^{x} / K \times l \longrightarrow \prod_{p \in P I_{K}} \prod_{p}^{x} / K{ }_{p}^{* l},
$$

qui est injectif lorsque $\ell$ est impair, et dont le noyau est d'ordre 1 ou 2 dans tous les cas ( cf. [AT], Ch。 $\times, \S 1$, th. 1 ). Par passage à la li mite projective, nous obtenons par conséquent un morphisme injectif : $K^{x}=\frac{\lim }{k} K^{x} / K \times l \smile \prod_{p \in P I} \frac{\lim }{k} K_{p}^{x} / K x_{p}^{x l}=\prod_{p \in P I_{K}} K_{p}^{x}$. Sa restriction au départ à $\ell_{K}$, à l'arrivée à $\partial_{K}$ est le $\mathbb{Z} \ell^{- \text {morphisme }}$ cherché . Comme il est injectif d'après ce qui précède, nous identifie rons désormais $R_{K}$ avec son image canonique dans $\partial_{K}$. Cela étant, nous allons établir successivement :

(i) que l'application naturelle de $\Omega_{K}$ dans $\partial_{K}$ est continue ${ }^{(*)}$;

(ii) que $R_{K}$ est fermé dans $\partial_{K}$;

(iii) que le quotient $\partial_{K} / \Omega_{K}$ est un $\mathbb{Z}_{\ell}$-module compact .

(i) Le premier point résulte de la définition de la topologie limite inductive : Pour chaque ensemble fini $S$ de places de $K$, le groupe $\delta_{K}^{S}=R_{K} \cap U_{K}^{S}$ est un $\mathbb{Z} l^{-m o d u l e ~ d e ~ t y p e ~ f i n i, ~ d o n c ~ d i u n e ~ f a c ̧ o n ~ e t ~ d i u n e ~}$ 
seule un $\mathbb{Z} l^{\text {-module topologique }}$. En particulier la topologie de $\theta_{K}^{S}$ est induite par celle de $u_{K}^{S}$. Si donc $\theta_{K}$ est un sous-module ouvert de $g_{K}$, les sous-modules $\theta_{K} \cap u_{K}^{S}$ étant ouverts dans les $u_{K}^{S}$, il en est de même des sous-modules $\theta_{K} \cap \varepsilon_{K}^{S}$ dans les $\varepsilon_{K}^{S}$; ce qui veut dire que $\theta_{K} \cap \Omega_{K}$ est un sous-module ou vert de $\Omega_{K}$. En particulier linjection canonique de $R_{K}$ dans $\partial_{K}$ est bien continue.

(ii) Le second point s'établit comme suit : Etant donné unidèle généralisé $\mathfrak{r}$ de $\partial_{K}$ qui n'est pas dans $R_{K}$, fixons $S$ assez grand, contenant les places archimédiennes, pour avoir $\mathfrak{k} \in U_{K}^{S}$; puis, pour chaque place $p$ de $K$ n'appartenant pas à $S$, choisissons $n_{p}$ assez grand, de telle sorte que le diviseur $p^{\ell^{n_{p}}}$ soit principal (dans le $l$-groupe $\delta_{K}=\mathbb{Z} \ell^{\otimes} D_{K}$ des diviseurs de $\left.K\right)$. Cela étant, comme $\delta_{K}^{S}$ est fermé dans $U_{K}^{S}$, il existe un sous-module ouvert $\sigma^{S}$ de $U_{K}^{S}$ dont le translaté $k O^{S}$ ne rencontre pas $\delta_{K}^{S}$. Le $\mathbb{Z}_{l^{-m o d u l e}} \theta$ engendré dans $\partial_{K}$ par $\delta_{K}^{S}$ et les éléments $\pi_{p}^{\ell^{n_{p}}}$ pour $p \in S$ est alors un sous-module ou vert de $\partial_{K}$ dont le translaté $x \theta$ ne rencontre pas $\Omega_{K}$ •

(iii) Pour établir le troisième point, nous allons montrer que limage $u_{K}{ }^{R_{K} / R_{K}} \approx u_{K} / \varepsilon_{K}$ dans $c_{K}$, du sous-module compact $u_{K}$ de $\partial_{K}$ formé des idèles unités, en est un sous-module d'indice fini ( de sorte que $C_{K}$ sera compact comme réunion finie de compact). Or, cela résulte de la proposition :

PROPOSITION 1.1.5. - Le quotient $z_{K} / u_{K} \Omega_{K}$ du $\ell$-groupe des classes d'idèles du corps $K$ par le sous-groupe formé des classes des idèles unités s'identifie canoniquement au $\ell$-groupe fini des classes de diviseurs de $K$ :

$$
\partial_{K} / u_{K}{ }^{R_{K}} \simeq \mathrm{Cl}_{K}
$$

Démonstration: Le groupe des diviseurs dlun corps de nombres est le groupe abélien libre construit sur ses valuations : Comme la valuation $v_{p}$ associée à une place de $K$ est triviale si p est complexe, à valeurs dans $\mathbb{Z} / 2 \mathbb{Z}$ si $p$ est réelle, et dans $\mathbb{Z}$ si pest ultramétrique, le groupe $D_{K}$ est le produit direct de $r_{k}$ exemplaires de $\mathbb{Z} / 2 \mathbb{Z}$ et du groupe $I_{K}$ des idéaux de $K$. Son quotient $D_{K} / P_{K}$ par le sous-groupe des diviseurs principaux (i.e. par l'image canonique de $K^{X}$ dans $D_{K}$ ) est fini, d'après 
la géométrie des nombres: dans la terminologie des idéaux, c'est le groupe des classes au sens restreint ( cf. Ch.III.l, 1 a ). Son $\ell-S y l o w$ $C l_{K}=\mathbb{Z}_{\ell} \otimes_{\mathbb{Z}}\left(D_{K} / P_{K}\right)$ est donc canoniquement le quotient $D_{K} / p_{K} d u$ tensorisé $\ell$-adique $\theta_{K}=\mathbb{Z} \ell^{\otimes} D_{K}$ du groupe des diviseurs par celui $P_{K}=\mathbb{Z}_{\ell} \otimes_{\mathbb{Z}} P_{K}$ du sous-groupe principal $P_{K}$. Maintenant, $\theta_{K}$ s'identifie canoniquement à $\partial_{K} / u_{K}$, et $p_{K}$ est llimage canonique de $\Omega_{K}$ dans $\theta_{\mathrm{K}}$.

La correspondance entre $\ell$-groupes de classes d'idèles et de diviseurs peut ainsi se résumer par le diagramme commutatif exact (où

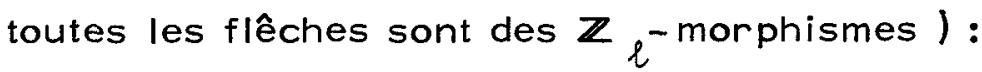

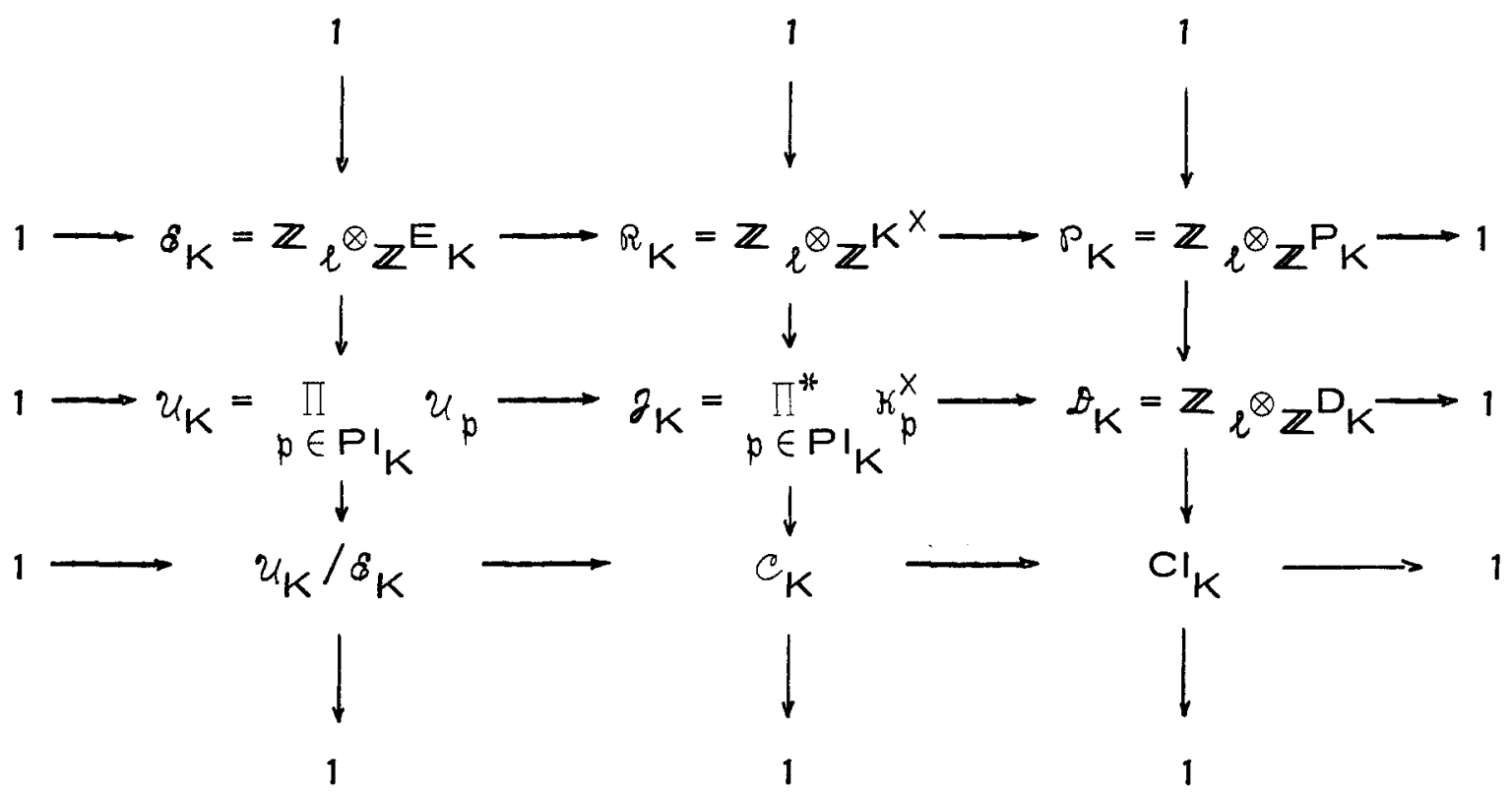

SCOLIE 1,1.6. - Pour tout ensemble fini $S$ de places de $K$, le quotient $C_{K}^{S}=z_{K} / \Omega_{K} U_{K}^{S}$ du $\ell$-groupe des classes diidèles $C_{K}$ par le sous groupe $c_{K}(S) \simeq u_{K}^{S} / \&_{K}^{S}$ des classes des idèles unités en dehors de $S$, s'identifie canoniquement au quotient $\mathrm{Cl}_{K} / \mathrm{Cl}_{K}(\mathrm{~S}) \mathrm{du} \ell$-groupe des classes de diviseurs du corps $K$ par le sous-groupe engendré par les classes des diviseurs construits sur les places de $S$. Nous disons que $l_{K}$ est le $\ell$-groupe des $S$-classes de diviseurs du corps $K$. 


\section{c.-Valeurs absolues 2 -adiques principales et formule du produit}

On pourrait craindre que la $\ell$-adification des groupes didèles fasse disparaitre la classique formule du produit pour les valeurs absolues. Il n'en est rien, à condition naturellement de remplacer les valeurs absolues habituelles par des valeurs absolues $\ell$-adiques conve nables.

Désignons pour cela par $\mathbb{Z}_{\ell}^{X}$ le groupe multiplicatif des élé ments inversibles de llanneau des nombres $\ell$-adiques, par $U_{\ell}=1+\ell \mathbb{Z} l$ le sous-groupe des unités principales de $\mathbb{Z}_{\ell}$, et par $u \mapsto\langle u\rangle$ la sur jection canonique de $\mathbb{Z}_{\ell}^{x}$ sur $u_{\ell}$ (de sorte que, pour $\ell$ impair, le quotient $u /\langle u\rangle$ est l'unique racine de l'unité dans $\mathbb{Z}_{\ell}$ qui est congrue à $u$ modulo $\ell$; tandis que si $\ell$ vaut $2,\langle u\rangle$ est toujours égal à $u$ ). Cela étant, nous avons:

DEFINITIONS 1.1.7.- Etant donnée une place $p$ d' un corps de nombres $K$, nous appelons valeur absolue $l$-adique principale dlun élément $x_{p}$ du groupe $\kappa_{p}^{x}$, et nous notons $\left|x_{p}\right|_{p}$, l'élément du $z_{\ell}$-module multi plicatif $u_{\ell}=1+\ell \mathbb{Z}_{\ell}$ défini par :

$\left|x_{p}\right|_{p}=1$, si $p$ est complexe;

$\left|x_{p}\right|_{p}=\left\langle s g\left(x_{p}\right)\right\rangle$, si p est réelle;

$\left|x_{p}\right|_{p}=\left\langle N p^{v_{p}(x)}\right\rangle$, si p estultramétrique et étrangère à $\ell$; $\left|x_{p}\right|_{p}=\left\langle N_{K_{p}} / \mathbb{Q}_{p}(x) \cdot N p^{-v_{q}(x)}\right\rangle$, si p est au-dessus de $\ell$.

Enfin, nous appelons valeur absolue $t$-adique principale d'un élément $x$ de $R_{K}$ relativement à la place $p$, et nous notons $|\times|_{p}$ la valeur absolue $\ell$-adique principale de l'élément $s_{p}(x)$, où $s_{p}$ est la surjection ca nonique de $\Omega_{K}$ dans $K_{p}^{x}$, induite par linjection naturelle de $K^{x}$ dans $K_{p}^{x}$.

Remarques.-(i) La définition donnée ici diffère de celle de Tate (cf. [TBS], Ch. VI, $\S 1$, déf. 1.1), qui ne s'étend pas aux $\mathbb{Z}_{\ell}$-mo dules. Plus précisément, pour chaque place $p$ de $K$, la quantité $|\times|_{p}$ est le crochet de la valeur absolue de Tate ; c'est pourquoi nous par lons de valeur absolue principale.

(ii) L'application $x_{p} \mapsto\left|x_{p}\right|_{p}$ est trivialement un $\mathbb{Z} \ell^{\text {-mor }}$ phisme de $\kappa_{p}^{x}$ dans $u_{\ell}$. En particulier, elle est continue et fermée 
pour la topologie naturelle de ces deux modules. Si p est ultramétrique, son image $\left|k_{p}^{x}\right|_{p}$ est, en outre, un sous-module dindice fini de $u_{l}$.

PROPOSITION 1.1.8. - (Formule du produit pour les valeurs absolues principales ) - L'application

$$
r=\left(x_{p}\right)_{p} \longrightarrow \prod_{\left.p \in P\right|_{K}}\left|x_{p}\right|_{p}=\|r\|
$$

est un $\mathbb{Z}_{\ell}$-morphisme continu du $\ell$-groupe $\delta_{K}$ des idèles généralisés du corps $K$ sur le groupe $U_{\ell}$ des unités principales de l'anneau $\mathbb{Z}_{\ell}$. Son noyau est un sous-module fermé de $\partial_{K}$, qui contient le tensorisé $\ell$-adique $R_{K}$ du groupe multiplicatif $K^{X}$.

Démonstration : Remarquons d'abord que la quantité $\Pi\left|\times_{p}\right|_{p}$ est bien définie puisque, pour chaque idèle $k$ de $\partial_{K}$, les composantes $x_{p}$ étant des unités pour presque tout $p$, le produit infini n'a en fait qu'un nom bre fini de facteurs distincts de 1 . Cela étant, pour montrer que l'ap plication $\mathfrak{k} \mapsto\|\mathfrak{k}\|$ est continue sur $\partial_{K}$, il nous suffit, d'après la pro priété universelle de la topologie limite inductive, de vérifier la conti nuité de sa restriction au sous-module $U_{K}^{S}$, pour chaque ensemblefini $S$ assez grand de places de $K$. Pour cela, nous pouvons supposer que $S$ contient les places au-dessus de $\ell$, auquel cas nous avons $\|\mathfrak{r}\|=$ $\prod_{p \in S}\left|x_{p}\right|_{p}$, pour chaque $x$ de $U_{K}^{S}$; et la continuité de $\|$. $\|$ résulte de celle des applications 1 . I sur chacun des facteurs $\kappa_{p}^{x}$.

Enfin, pour établir la formule du produit, il suffit de noter qu'elle est trivialement vérifiée sur le corps des rationnels, puis d'é crire que lion a, pour tout $x$ de $R_{K}$ :

$$
\begin{aligned}
\|x\|_{K}=\prod_{\left.p \in P\right|_{K}}|x|_{p} & =\prod_{p \in P I_{\mathbb{Q}}} \prod_{p}|x|_{p}=\prod_{p \in P I_{\mathbb{Q}}} \prod_{p \mid p}\left|N_{K_{p}} / \mathbb{Q}{ }_{p}^{(x)}\right|_{p}= \\
& =\prod_{p \in P I_{\mathbb{Q}}}\left|N_{K} / \mathbb{Q}(x)\right|=\left\|N_{K} / \mathbb{Q}(x)\right\|_{\mathbb{Q}}=1 .
\end{aligned}
$$

PROPOSITION 1.1.9. - Soit $p$ une place de $K$,et $\left.\right|_{p}$ la valeur abso lue $\ell$-adique principale associée.

(i) Si $p$ ne divise pas $\ell$, le noyau de llapplication $|\cdot|_{p}$ est le sous-module des unités de $k_{p}^{x}$ :

$$
\left|x_{p}\right|_{p}=1 \Leftrightarrow x_{p} \in u_{p} \text { 。 }
$$


(ii) Si p divise $l$, le noyau $k_{p}^{*}$ de l'application $\left.\left.\right|_{0}\right|_{p}$ est caractérisé par la condition :

$$
\left|x_{p}\right|_{p}=1 \Leftrightarrow \log _{l} N_{K_{p}} / Q_{l}\left(x_{p}\right)=\operatorname{Tr} K_{p} / Q_{l} \log _{p}\left(x_{p}\right)=0 \text {. }
$$

Le groupe $\kappa_{p}^{*}$ est le produit direct du sous-groupe $\mu_{p}$ des racines de I' unité dans $\kappa_{p}^{X}$, et d'un $\mathbb{Z}_{\ell}$-module libre de rang $\left[K_{p}: \mathbb{Q}_{\ell}\right]$.

Démonstration :

(i) Lorsque $p$ ne divise pas $\ell$, la condition $\left|x_{p}\right|_{p}=1$ s'écrit tout aussi bien $\vee_{p}\left(x_{p}\right)=0$; elle caractérise donc les unités de $\kappa_{p}^{x}$ ( On notera que, du fait des conventions utilisées, $(-1)$ n'est pas une unité dans $k \underset{p}{x}$, si p est réelle et $l$ égal à 2 l.

(ii) Lorsque $p$ divise $l$, la condition $\left|x_{p}\right|_{p}=1$ s'écrit en core $\left\langle N_{K_{p}} / Q_{\ell}\left(x_{p}\right) / N p^{V_{p}\left(x_{p}\right)}\right\rangle=1$. Elle affirme donc que la norme de $x_{p}$ s'écrit comme produit d'une puissance de $\ell$ (qui est nécessaire ment $N p_{p}\left(x_{p}\right)$ ), et dlune racine de llunité. Mais cette propriété caractérise précisément le noyau du logarithme d'lwasawa dans $\mathbb{Q}_{\ell}$, ce qui conduit à l'équivalence annoncée. Enfin, comme $\left|k_{p}^{x}\right|_{p}$ est d'indice fini dans $u_{l}$, il vient immédiatement ${ }^{\left({ }^{*}\right)}$ :

$$
\operatorname{dim}_{\mathbb{Z}_{\ell}} K_{p}^{K^{l}}=\operatorname{dim} \mathbb{Z}_{l} K_{p}^{x}-1=\operatorname{dim} \mathbb{Z}_{l} U_{l}=\left[K_{p}: Q_{l}\right] .
$$

COROLLAIRE 1.1.10. - Le noyau dans $g_{K}$ des valeurs absolues princi pales est le sous-module compact :

$$
\partial_{K}^{*}=\prod_{p \mid \ell_{\infty}} \mu_{p} \cdot \prod_{I \mid l} K_{1}^{*} \cdot
$$

Le groupe $\partial_{K}^{*}$ est composé direct du sous-groupe $\prod_{p} \mu_{p}$ des idèles unités qui sont localement des racines de llunité, et dlun $\mathbb{Z} \ell^{\text {-module }}$ libre de dimension $\sum_{l \mid l}\left[K_{l}: Q_{l}\right]=[K: \mathbb{Q}]$.

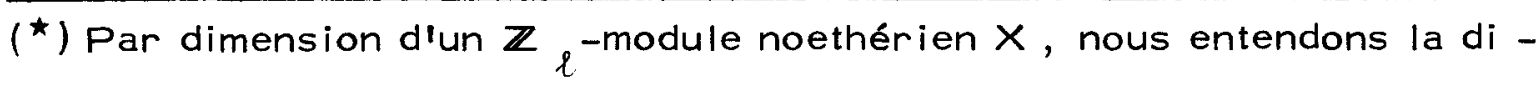
mension du $\mathbb{Q}_{\ell}$-espace vectoriel $\mathbb{Q}_{\ell} \otimes_{\mathbb{Z}} \times$. 


\section{2. - ISOMORPHISME DU CORPS DE CLASSES .}

\section{a.-Enoncé des théorèmes fondamentaux .}

Nous rappelons sans démonstration les résultats fondamentaux du corps de classes local pour les $\ell$-extensions, puisquilis sont es sentiellement bien connus sous cette forme (cf. par exemple, [La ], $\mathrm{Ch}$. VI , \$4). Nous donnons en revanche la preuve des résultats globaux .

THEOREME 1.1.11.- ( Corps de classes local) - Etant donné un corps local $K_{p}$, l'application de réciprocité induit un isomorphisme de $Z_{l}$ modules topologiques du complété profini $K^{\times}\left(K_{p}\right)=\frac{\lim }{K} K_{p}^{x} / K_{p}^{\times l}$ sur le groupe de Galois $D_{p}=\operatorname{Gal}\left(K_{p}^{a b} / K_{p}\right)$ de la $\ell$-extension abélienne maximale du corps $K_{p}$. Dans cet isomorphisme le groupe d'inertie $I_{p}=$ $\ln \left(K_{p}^{a b} / K_{p}\right)$ est l'image du sous-groupe $u\left(K_{p}\right)$ des unités de $K^{\times}\left(K_{p}^{p}\right)$. - Si $p$ ne divise pas $\ell$, la ramification est modérée, et le groupe $1_{p}$ est fini, isomorphe au $\ell$-Sylow $\mu_{p}$ du groupe des racines de l'unité dans $\mathrm{K}_{p}$.

- Si p divise $\ell$, la ramification est sauvage, et le groupe $I_{p}$ est infini. Dans ce cas, la suite des sous-groupes supérieurs de ramification est l'image dans $D_{p}$ de la filtration canonique $\left(u_{p}^{i}\right)_{i \geq 1}=$ $\left(1+p^{i}\right)_{i \geq 1}$ du groupe $u\left(K_{p}\right)^{p}$.

Remarques. - (i) L'application de réciprocité locale établit une cor respondance bijective entre les sous-modules fermés de $\kappa^{\times}{ }\left(K_{p}\right)$ et les $\ell$-extensions abéliennes de $K_{p}$. Plus précisément toute sous-extension $L_{P}$ de $K_{p}^{a b}$ est le corps des points fixes d'un unique sous-module fermé de $k^{X}\left(K_{p}^{p}\right)$. En particulier, si $H$ est un sous-groupe quelconque de $K^{x}\left(K_{p}\right)$, et $L_{P}$ son corps des invariants, la fermeture de $H$ dans $K^{X}\left(K_{p}\right)$ est le sous-module de $K^{X}\left(K_{p}\right)$ associé à $L_{B}$.

(ii) Dans la correspondance obtenue, les $\ell$-extensions abé liennes finies de $K_{p}$ sont associées aux sous-modules fermés d'indice fini de $k^{\times}\left(K_{p}\right)$, c'est-à-dire aux sous-modules ouverts de $k^{\times}\left(K_{p}\right)$. Ces sous-modules sont donc caractérisés comme groupes de normes associés aux $\ell$-extensions finies de $K_{p}$ : 
SCOLIE 1.1.12. - Si $L_{\mathcal{B}}$ est une $l$-extension finie quelconque de $K_{p}$, le sous-module ouvert de $\kappa^{X}\left(K_{p}\right)$ associé par le corps de classes local à la sous-extension maximale $L_{\mathcal{P}}^{a b}$ de $L_{\mathfrak{P}}$, qui est abélienne sur $K_{p}$, est l'image de $\kappa^{x}\left(L_{\mathcal{B}}\right)$ par la norme arithmétique. Il vient ainsi :

$$
\begin{aligned}
& D_{p}^{a b}\left(L_{\mathcal{B}} / K_{p}\right)=\operatorname{Gal}\left(L_{\mathcal{P}}^{a b} / K_{p}\right) \simeq K{ }^{\times}\left(K_{p}\right) / N_{L_{P}} / K_{p}\left(K^{X}\left(L_{P}\right)\right) \\
& I_{p}^{a b}\left(L_{P} / K_{p}\right)=\operatorname{In}\left(L_{P}^{a b} / K_{p}\right)=U\left(K_{p}\right) / N_{L_{P}} / K_{p}\left(U\left(L_{P}\right)\right) .
\end{aligned}
$$

Ce résultat s'étend sans difficulté aux extensions infinies, si l'on convient de dire qu'un élément de $k^{x}\left(K_{p}\right)$ est norme dans une telle extension lorsquil est norme dans chacune de ses sous-extensions finies. Dans ce cas, le théorème d'existence du corps de classes local affirme que tout sous-module fermé de $K^{X}\left(K_{p}\right)$ est le groupe des normes d'une unique $\ell$-extension abélienne de $K_{p}$.

THEOREME 1.1.13.- (Corps de classes global) - Etant donné un corps de nombres $\mathrm{K}$, l'application de réciprocité induit un épimorphisme con tinu du $\ell$-groupe $\partial_{K}$ des idèles généralisés de $K$ sur le groupe de Galois $G_{K}=G a l(K a b / K)$ de la $\ell$-extension abélienne maximale de $K$; le noyau de ce morphisme est le sous-groupe $R_{K}$ formé des idèles prin cipaux. Dans la correspondance obtenue, le sous-groupe de décompo sition $D_{p}$ dlune place $p$ de $K$ est llimage dans $G_{K}$ du sous-module $\kappa_{p}^{X}$ de $\partial_{K}$; et son sous-groupe d'inertie $I_{p}$ est celle du sous-groupe $u_{p}$ des unités de $K_{p}^{x}$.

Démonstration: Dlaprès Artin-Tate (cf. [AT], Ch. XIV, prop. 10), le morphisme surjectif du groupe des idèles de $K$ sur le groupe de Galois $G_{K}$ de la $\ell$-extension abélienne maximale de $K$ est nul sur le sous groupe $\ell$-divisible $J_{K}^{\text {div }}$ de $J_{K}$. Comme le $\ell$-groupe généralisé $\partial_{K}$ contient canoniquement le quotient $J_{K} / J_{K}^{\text {dir }}$ (cf. déf. 3 , rem. (i)), il slagit donc d'établir que le morphisme quotient $J_{K} / J_{K}^{\text {div }} \longrightarrow G_{K}$ se pro longe de façon unique en un $\mathbb{Z}_{l}$-morphisme $\psi$ de $\partial_{K}$ sur $G_{K}$, que ce prolongement est continu, et que son noyau est le groupe $\Omega_{K}$ des idèles principaux 。 
(i) existence du prolongement : Pour chaque place ultramé trique $p$ de $K$, faisons choix dlune uniformisante $\pi_{p}$; prenons $\pi_{p}=1$, si $p$ est complexe; $\pi_{p}=-1$, si p est réelle. Puisque le sous-groupe $u_{K}$ des unités de $y_{K}$ est contenu, par construction, dans celui $u_{K}$ de $J_{K}$, llapplication $\psi$ cherchée est bien définie sur le sous-groupe dense $u_{K} \cdot \underset{p \in P I_{K}}{\oplus} \pi_{p}^{\mathbb{Z}}$. Ecrivant alors $\psi\left(u \cdot \prod_{p} \pi_{p}^{\alpha}\right)=\psi(u) \prod_{p} \psi\left(\pi_{p}\right)^{\alpha}$, pour chaque famille $\left(\alpha_{p}\right)_{p}$ d'entiers $l$-adiques presque tous nuls, nous obtenons le prolongement cherché.

(ii) continuité de l'application obtenue : $\mathrm{Si} H$ est un sous groupe ouvert de $G_{K}$, le corps des points fixes qui lui correspond par la théorie de Galois est une $\ell$-extension abélienne finie de $K$; elle est ramifiée en un nombre fini de places. Par suite, si $S$ est la réunion des places ramifiées et de celles au-dessus de $l$, nous avons évidemment $\psi\left(u_{p}\right) \subset H$, pour chaque $p \in S$. De plus, le quotient $G_{K} / H$ étant un $l$-groupe fini, il existe un sous-module ouvert $u_{l}^{\prime}$ de $u_{l}=$ $\prod_{1} u_{I}$, et , pour chaque place $p$, un sous-module ouvert $\pi_{p} \ell^{n_{p}} \pi_{\ell} U_{l}$ de $\pi \mathbb{Z}_{l}$ dont l'image par $\psi$ est contenue dans $H$. Le produit $\prod_{p \in S} u_{p} \cdot u_{l}^{\prime} \cdot \prod_{p \in P I_{K}}^{\oplus} \pi_{p}^{l^{n_{p}} \mathbb{Z}_{l}}$ est alors un sous-module ouvert de $\partial_{K}$ contenu dans ${ }^{-1} \psi(H)$; ce qui prouve que $\psi$ est continue.

(iii) noyau : Nous savons déjà que le noyau de $\psi$ est un sous module fermé de $z_{K}$, qui contient l'image de $K^{X}$. Il contient donc la fermeture de cette image, qui est $R_{K}$. Pour voir qu'iln'a pas d'autres éléments, il suffit de remarquer que la construction des idèles généralisés a tué le sous-groupe des normes universelles, dont la structure est bien connue ( cf. [AT ], Ch. IX, $\$ 1$ ), et qui est le noyau dans $\mathrm{J}_{\mathrm{K}} / \mathrm{K}^{\mathrm{X}}$ de l'application de réciprocité.

Enfin, le lien entre le corps de classes global et le corps de classes local ne pose aucune difficulté particulière puisque, pour cha -

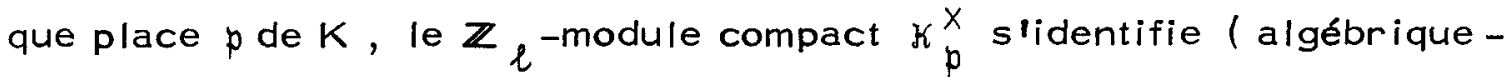
ment et topologiquement ( avec son image canonique dans le quotient $c_{K}=\delta_{K} / \alpha_{K}$. Plus généralement :

LEMME 1.1.14.- Si S est un ensemble fini de places de $K$, la surjection naturelle

$$
\stackrel{p}{p} \in S_{k}^{x} \longrightarrow\left(\underset{p \in S}{\oplus} k_{p}^{x}\right) R_{K} / R_{K} \subset c_{K}
$$


est un isomorphisme de $\mathbb{Z} e^{\text {-modules compacts }}{ }^{(*)}$.

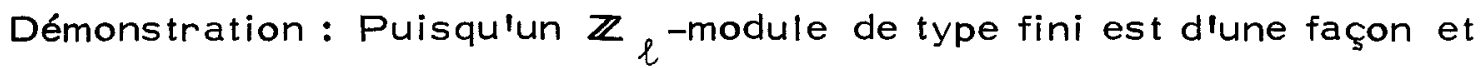

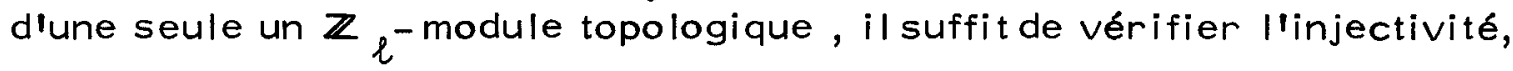
clest-à-dire le fait que $\Omega_{K}$ rencontre trivialement chaque somme finie $p \in S K_{p}^{X}$. Or cela résulte directement du fait que llunité est le seul élément de hauteur infinie dans $R$. En effet, si $\underset{x}{ }=\left(x_{p}\right)_{p}$ est un idèle principal contenu dans une somme finie $\underset{p \in S}{\oplus} K_{p}^{x}$, les conditions $x_{p}=1$ pour $p \in S$, montrent que $\mathfrak{x}$, qui est puissance $\ell^{k}$-ième locale en de hors de $S$, pour tout $k$, est puissance $l^{k}$-ième globale pour tout $k$ (cf. $[A T], C h . I X, \S 1$, th. 1 ) , i.e. de hauteur infinie dans $R_{K}$ •

Remarques - (i) L'application de réciprocité globale établit un iso morphisme de $\mathbb{Z}_{\ell}$-modules topologiques du $\ell$-groupe $C_{K}$ des idèles de $K$ sur le groupe de Galois $G_{K}$ de la $\&$-extension abélienne maximale de $K$. Elle met ainsi en bijection les sous-modules fermés de $\partial_{K}$ qui contiennent $R_{K}$ avec les $\ell$-extensions abéliennes de $K$, chaque sous-extension de $K^{a b}$ étant le corps des points fixes d'un unique sous-module fermé de $\partial_{K}$ contenant $R_{K}$ •

(ii) Dans la correspondance obtenue, les $\ell$-extensions abé liennes finies de $K$ sont associées aux sous-modules fermés d'indice fini de $\partial_{K}$ contenant $R_{K}$, c'est-à-dire aux sous-modules ouverts de $\partial_{K}$ qui contiennent $R_{K}$. Ces sous-modules sont donc caractérisés comme groupes de normes attachés aux 1 -extensions finies de $K$ :

SCOLIE 1.1.15. - Si $L$ est une $\ell$-extension finie quelconque de $K$, le sous-module ouvert de $C_{K}$ associé par le corps de classes global à la sous-extension maximale $L^{a b}$ de $L$ qui est abélienne sur $K$ est llimage de $C_{L}$ par la norme arithmétique :

$$
\operatorname{Gal}\left(L^{a b} / K\right) \simeq c_{K} / N_{L / K}\left(c_{L}\right)=\partial_{K} / N_{L / K}\left(z_{L}\right) R_{K} \cdot
$$

$\left({ }^{\star}\right)$ Ce résultat n'est pas vrai dans la théorie classique du corps de classes, puisque l'application $\underset{p \in S}{\oplus} K_{p}^{x} \longrightarrow\left(\underset{p \in S}{\oplus} K_{p}^{x}\right) K^{x} / K^{x}$ $\subset J_{K} / K^{X}$ n'est pas un homéomorphisme pour les topologies habituelles de ces deux groupes, dès que $S$ contient plus d'une place. 
Dans cette description, les sous-groupes de décomposition et d'inertie d'une place $p$ dans l'extension abélienne $L^{a b} / K$ sont donnés par les isomorphismes:

$D_{p}\left(L^{a b} / K\right) \simeq K x_{p}^{x} / K{ }_{p}^{x} \cap N_{L} / K\left(\partial_{L}\right) R_{K} \varepsilon_{1}\left(L^{a b} / K\right) \simeq u_{p} / u_{p} \cap N_{L} / K\left(\partial_{L}\right) R_{K}$

Remarque. - Lorsque $L$ est une extension abélienne de $K$, les groupes de décomposition et d'inertie d'une place $p$ dans $L / K$ s'identifient respectivement aux groupes de Galois et d'inertie de l'extension abélienne locale $L_{p} / K_{p}$. La correspondance entre le corps de classes local et le corps de classes global se traduit alors par les identités :

$$
\begin{aligned}
& N_{L_{p}} / K_{p}\left(K^{x}\left(L_{p}\right)\right)=K^{\times}\left(K_{p}\right) \cap N_{L / K}\left(z_{L}\right) R_{K} \\
& \& \quad N_{L_{p} / K_{p}}\left(u\left(L_{p}\right)\right)=u\left(K_{p}\right) \cap N_{L / K}\left(z_{L}\right) R_{K} \cdot \\
& \text { Dans le cas général, en revanche, il n'en est pas de même : }
\end{aligned}
$$

Si $L$ est une $\ell$-extension quelconque de $K$, l'extension galoisienne locale qui lui correspond est l'intersection $L_{p}=\bigcap_{P} L_{\mathfrak{P}}$ des complétés de $L$ pour les places au-dessus de $p$ ( pris dans une même clôture algébrique de $K_{p}$ ). D'après le corps de classes local, le groupe de Galois $D_{p}^{a b}(L / K)=\operatorname{Gal}\left(L_{p}^{a b} / K_{p}\right)$ de la sous-extension abélienne $L_{p}^{a b}$ de $L_{p}$ est donné par llisomorphisme :

$$
\begin{aligned}
D_{p}^{a b}(L / K) & =\operatorname{Gal}\left(L_{p}^{a b} / K\right) \simeq K^{x}\left(K_{p}\right) / N_{L_{p}} / K_{p}\left(K K^{\times}\left(L_{p}\right)\right) \\
& =K^{x}\left(K_{p}\right) \prod_{P \mid p} N_{L_{P}} / K_{p}\left(K \times\left(L_{P}\right)\right) .
\end{aligned}
$$

Mais il peut arriver que l'on ait $D_{p}^{a b}(L / K) \neq D_{p}\left(L^{a b} / K\right)$, puisque l'extension abélienne locale $L_{p}^{a b}$ peut contenir strictement la localisée de l'extension abélienne globale $L^{a b}$.

b.- Description des $\ell$-extensions abéliennes fondamentales d'un corps de nombres.

Nous définissons ici quelques unes des principales $\&$-exten sions abéliennes d'un corps de nombres étudiées dans cette thèse. Elles sont définies le plus souvent sous une condition maximale de plongement, de décomposition ou de ramification. Précisons d'abord ce que nous entendons par là : 
DÉFINITION 1.1.16. - Etant donnés deux ensembles finis (disjoints) $S$ et $T$ de places diun corps de nombres, nous disons qu'une extension $L$ de $K$ est $T$-ramifiée et $S$-décomposée lorsqulelle est non ramifiée aux places (finies) étrangères à $T$, et complètement décomposée aux places de $S$; autrement dit, lorsque pour chaque place $p$ de $K$ et chaque place $P$ de $L$ au-dessus de $p$, l'extension locale $L_{\beta} / K_{p}$ est non rami fiée si p nlappartient pas à $T$, et triviale si pappartient à $S$.

Convention: Lorsque pest une place réelle, le groupe multiplicatif $k_{p}^{x}$ est égal à $\mathbb{Z}_{\ell} / 2 \mathbb{Z}_{\ell}$, et son sous-groupe des unités $u_{p}$ est trivial d'après la définition 2 . La place p peut donc se décomposer dans une $\ell$-extension (abélienne) de $K$, mais non se ramifier, sans contredire le théorème 11 . Lorsqu'une place réelle n'est pas complètement dé composée dans une $l$-extension de $K$, nous disons qu'elle se complexifie. Nous parlons donc de complexification là où d'autres auteurs parlent de ramification $\dot{a} I^{\prime} i n f i n i$, et nous réservons le concept de ramifi cation aux seules places finies. En particulier, une extension est $\mathbf{S}$ ramifiée dès qulelle est non ramifiée aux places finies (=ultramétriques) n'appartenant pas à $S$, quel que soit le comportement des places à l'infini (= archimédiennes) .

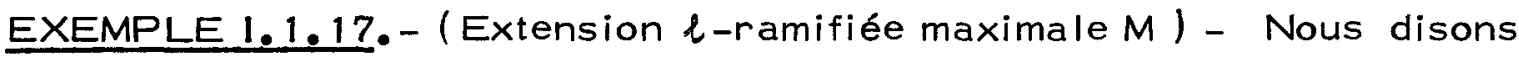
qu'une $\ell$-extension abélienne d'un corps de nombres $K$ est $\ell$-ramifiée, lorsqu'elle est non ramifiée aux places (finies) étrangères à $l$.

Dlaprès la théorie du corps de classes, le groupe de normes associé à la $\ell$-extension abélienne $\ell$-ramifiée maximale $M$ d'un corps $K$ est liimage dans $c_{K}$ du produit

$$
\partial_{K}^{M}=\prod_{p \nmid l} u_{p}=\prod_{p \nmid \ell_{\infty}} \mu_{p}
$$

des groupes dlunités locales attachés aux places étrangères à $\ell$. Le groupe de Galois $\mathrm{Gal}(\mathrm{M} / \mathrm{K})$ s'identifie par conséquent au quotient :

$$
\operatorname{Gal}(M / K) \approx z_{K} / R_{K} \prod_{p \not l} u_{p} \text {. }
$$

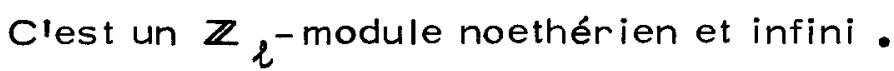

EXEMPLE 1.1.18. - (Extension hilbertienne maximale H) - Nous disons qu'une $\ell$-extension abélienne d'un corps de nombres $K$ est hilbertienne, lorsque chacune de ses sous-extensions cycliques peut se plonger dans 
une $l$-extension cyclique de degré arbitrairement grand.

Dlaprès Artin-Tate (cf. [AT], Ch. $X, \S 2$, th. 6), le groupe de normes associé à la $\ell$-extension hilbertienne maximale $H$ diun corps $K$ est limage dans $C_{K}$ du produit

$$
\partial_{K}^{H}=\prod_{p} \mu_{p}
$$

des $\ell$-groupes de racines de l'unité des complétés non complexes de $K$. L'inclusion $\partial_{K}^{M} \subset \partial_{K}^{H}$ montre que $H$ est contenue dans $M$. Plus préci sément, $M$ est une $\ell$-extension abélienne finie de $H$, de groupe de Galois :

$$
\operatorname{Gal}(M / H) \simeq \prod_{p \mid l \infty} \mu_{p} / \prod_{p \mid l \infty} \mu_{p} \cap R_{K} \mid=\prod_{p \mid l \infty} \mu_{p} / \mu .
$$

EXEMPLE 1.1.19. - (Corps de classes de valeurs absolues N) - Nous disons qulune $l$-extension abélienne d'un corps de nombres $K$ est un corps de classes de valeurs absolues, lorsqu'elle est fixée par le noyau

$$
g_{K}^{*}=\prod_{p \not l} u_{p} \cdot p \prod_{p} K_{p}^{*}
$$

dans $\partial_{K}$ des valeurs absolues $\ell$-adiques. Comme $\partial_{K}^{*}$ est un sous-module compact de $\partial_{K}$, son image $c_{K}^{*}$ dans $c_{K}$ est le groupe des normes associé à la $\ell$-extension abélienne maximale $N$ de $K$ qui est fixée par $\partial_{K}^{*}$. Nous disons que $N$ est le $\ell$-corps des classes de valeurs absolues de $K$. Son groupe de Galois

$$
\operatorname{Gal}(N / K) \simeq z_{K} / g_{K}^{*} R_{K} \simeq v_{\cdot} a_{\cdot}\left(z_{K}\right) / v_{0} a_{\cdot}\left(R_{K}\right)
$$

s'identifie, en effet, au quotient du groupe $v_{\bullet} \cdot a_{\bullet}\left(\partial_{K}\right)={\underset{p}{\oplus} \in I_{K}}_{k}\left|k_{p}^{x}\right|_{p}$ des valeurs absolues sur $K$ par le sous-groupe $v_{\bullet} a_{\bullet}\left(R_{K}\right)$ des valeurs absolues des éléments de $\mathfrak{R}_{K}$. C'est un $\mathbb{Z}_{\ell}$-module noethérien et infini .

EXEMPLE 1.1.20.- (Extension non ramifiée maximale C) - D'après la définition 16, une $\ell$-extension abélienne d'un corps de nombres est non ramifiée lorsqu'elle est non ramifiée aux places finies. Le groupe de normes associé à la $\ell$-extension abélienne maximale $C$ de $K$ qui estnon ramifiée est l'image dans $c_{K}$ du groupe $u_{K}=\prod_{p} u_{p}$ des idèles unités. D'après la proposition 5 , le groupe de Galois $\mathrm{Gal}(\mathrm{C} / \mathrm{K})$ 
s'identifie par conséquent au $\ell$-groupe fini des classes de diviseurs de K :

$$
\operatorname{Gal}(c / K) \simeq \theta_{K} / p_{K}=a_{K}
$$

EXEMPLE 1.1.21. - (Extension non ramifiée $l$-décomposée maximale $C^{\prime}$ ) Nous disons qulune $\ell$-extension abélienne de $K$ est $l$-décomposée lors que les places de $K$ au-dessus de $\ell$ s'y décomposent complètement. Le groupe de normes associé à la $\ell$-extension abélienne maximale de $K$ qui est non ramifiée et $\ell$-décomposée est donc le produit

$$
\partial_{K}^{C^{\prime}}=\prod_{p \not l} u_{p} \cdot p \mid l K_{p}^{x} \text {. }
$$

Enfin, le groupe de Galois $\mathrm{Gal}\left(\mathrm{C}^{\prime} / \mathrm{K}\right)$ s'identifie au quotient $C_{K}^{\prime}=$ $a_{K} / a_{K}(\ell)$ du $\ell$-groupe des classes de diviseurs de $K$ par le sous groupe engendré par les classes des diviseurs construits sur les pla ces au-dessus de $\ell$ :

$$
\operatorname{Gal}\left(C^{\prime} / K\right) \simeq \theta_{K}^{\prime} / P_{K}^{\prime}=C l_{K}^{\prime} \text {. }
$$

Le groupe $C l_{K}^{\prime}$ est le $\ell$-groupe des $\ell$-classes de diviseurs du corps $K$.

Les trois extensions $M, H$, et $N$ contiennent la $\mathbb{Z} \ell_{\ell}^{\text {-extension }}$ cyclotomique $K_{\infty}$ de $K$. En effet, d'un côté $H$ contient par définition la composée $Z$ des $\mathbb{Z}_{l}$-extensions de $K$, et en particulier $K_{\infty} ; i l$ en est donc de même pour $M$. Et d'un autre côté, nous avons trivialement $N_{K} \supset N_{F}$ pour chaque sous-corps $F$ de $K$ donc, en particulier $N=$ $N_{K} \supset N_{\mathbb{Q}}$. Maintenant, si $l$ est impair, $N_{\mathbb{Q}}$ est précisément la $\mathbb{Z}_{\ell}$ extension cyclotomique $\mathbb{Q}$ de $\mathbb{Q}$; et, si $\&$ vaut 2 , clest une extension quadratique de $Q_{\infty}$ $(\star)$

Lorsque $\ell$ est impair, nous obtenons ainsi le schéma de corps :

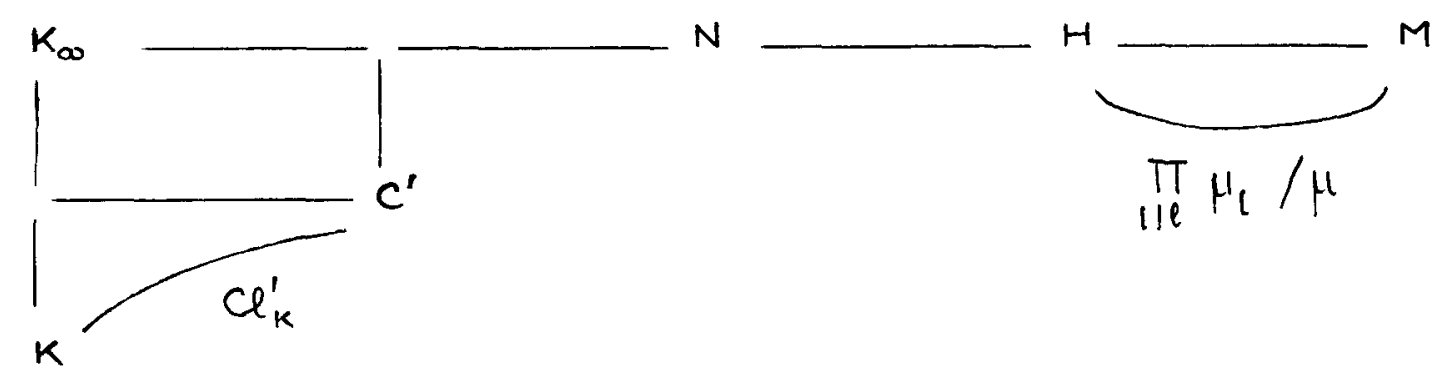

$\left({ }^{\star}\right)$ Plus généralement, le corps $N$ s'interprète comme $l$-corps des $\ell$ genres associé à l'extension procyclique $K_{\infty} / K$. 
En revanche, lorsque $\ell$ vaut 2 , il peut arriver que $H$ ne con tienne ni $N$ ni $C^{\prime}$ lorsque ces deux extensions se complexifient sur $K$. Pour conserver le même schéma de corps, il faut alors imposer une condition de décomposition à l'infini .

\section{c.- Les conjectures de Leopoldt et de Gross.}

Les dimensions respectives ${ }^{(*)}$ des groupes de Galois Gal(M/K), associé à la $\ell$-extension abélienne $\ell$-ramifiée maximale de $K$, et $\mathrm{Gal}(N / K)$, associé au $\ell$-corps des classes de valeurs absolues de $K$, sont gouvernées par les conjectures de Leopoldt et de Gross, ou plutôt par des généralisations convenables de ces conjectures excluant toute hypothèse restrictive sur le corps de base. Leopoldt a, en effet, énoncé sa conjecture sur la non nullité du régulateur $\ell$-adique dans le seul cas des corps de nombres totalement réels, et Gross dans celui des exten sions quadratiques totalement imaginaires dlun tel corps. Nous donnons ici les deux énoncés $\left.{ }^{(\star}\right)$ valables pour tout corps, que nous continuons par abus à appeler conjectures de Leopoldt et de Gross, l'important étant que le premier énoncé soit bien équivalent à celui de Leopoldt lorsque $K$ est totalement réel, et le second à celui de Gross lorsque $K$ est une extension quadratique totalement imaginaire diun corps $F$ totalement réel vérifiant la conjecture de Leopoldt.

THÉORĖME \& DÉFINITION 1.1.22. - Nous disons quiun corps de nom bres $K$ satisfait la conjecture de Leopoldt, lorsque les conditions équivalentes suivantes sont vérifiées:

(i) Le groupe de Galois $G a l(M / K)$ de la $l$-extension abé lienne $\ell$-ramifiée maximale de $K$ est un $\mathbb{Z}_{\ell}$-module de dimension $c_{k}+1$, où $c_{k}$ est le nombre de places complexes de $K$.

(ii) L'image $\varepsilon_{l}$ du groupe $\delta=\mathbb{Z}{ }_{l} \otimes_{\mathbb{Z}} E$ des unités généra lisées du corps $K$ dans le groupe $u_{\ell}=\prod_{1} \mid l u_{l}$ est un $\mathbb{Z} l^{- \text {module }}$ de dimension $r_{k}+c_{k}+1$, où $r_{k}$ est le nombre de places réelles et $c_{k}$ le nombre de places complexes de $K$.

$\left({ }^{\star}\right)$ c'est-à-dire les dimensions sur $\mathbb{Q}_{\ell}$ des espaces vectoriels produits associés: $\mathbb{Q}_{\ell} \mathbb{Z}_{\ell}$ Gal.

$\left({ }^{\star}\right)$ Ces deux énoncés sont discutés au chapitre II. 1 . 
(iii) L'application de semi-localisation $s_{\ell}=\left(s_{\mathfrak{l}}\right)_{\mathfrak{l} / \mathfrak{l}}$ induit un $\mathbb{Z}_{\ell}$-morphisme injectif du groupe $\delta=\mathbb{Z}_{\ell} \otimes_{\mathbb{Z}} E$ des unités généra lisées de $K$ dans le produit $u_{\ell}=\prod_{1} \mid \ell u_{l} \subset g$ des groupes diunités attachés aux places $\mathfrak{l}$ divisant $\ell$.

(iv) Le quotient $\prod_{p} \mu_{p} / \mu$ du produit des $\ell$-groupes de racines locales de l'unité dans 2 par le sous-groupe des racines globales s'injecte canoniquement dans le $\ell$-groupe des classes dijidèles $C$ du corps $K$.

(v) Pour qu'une unité généralisée $\varepsilon \in \delta$ du corps $K$ soit une racine de l'unité, il faut et il suffit qu'elle soit localement partout une racine de l'unité, ce qui s'écrit:

$$
\delta \cap \prod_{p} \mu_{p}=\delta \cap \mu \text {. }
$$

Remarque.- Lorsque le corps $K$ est totalement réel, la condition (i) s'écrit $\operatorname{dim} \mathbb{Z}_{\ell} \operatorname{Gal}(M / K)=1$. Elle exprime qu'il n'est d'autre $\mathbb{Z}_{\ell}$

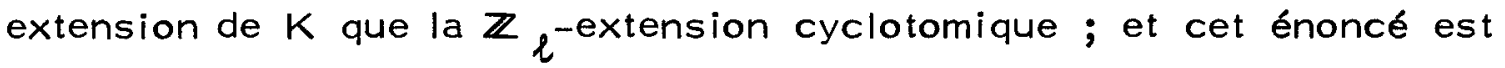
bien équivalent à celui de Leopoldt .

Démonstration des équivalences :

$(\mathrm{i}) \Leftrightarrow(\mathrm{ii}) \Leftrightarrow(\mathrm{iii})$ : Dlaprès l'exemple 17 , le groupe de Galois $\operatorname{Gal}(M / K)$ slidentifie au quotient $g / \Omega \prod_{p / l} u_{p}$. Nous obtenons donc

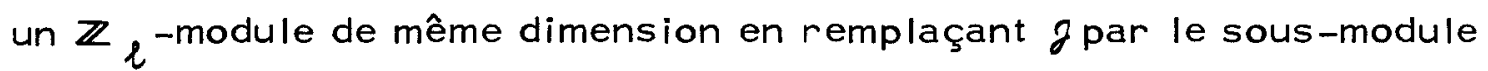
dindice fini $\prec \prod_{p} u_{p}$ (cf. proposition 5 ). Il vient alors :

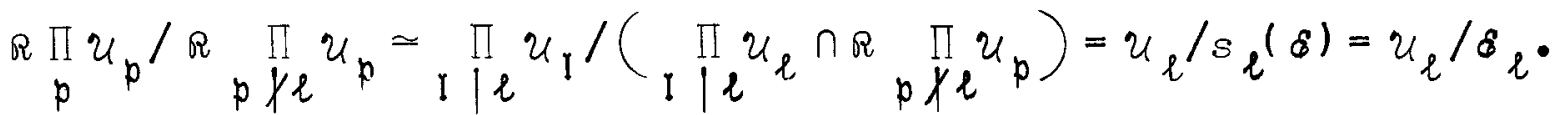
Maintenant, $U_{\ell}$ a pour dimension le degré $[K: Q]=r_{k}+2 c_{K}$ de $K$, et $\delta$ le nombre de Dirichlet $r_{k}+c_{k}-1$. Nous obtenons donc, comme annoncé : $\operatorname{dim}_{\mathbb{Z}} \operatorname{Gal}(M / K)=c_{k}+1 \Leftrightarrow \operatorname{dim} \mathbb{Z}_{\ell} \varepsilon_{\ell}=r_{k}+c_{k}-\left.1 \Leftrightarrow s_{\ell}\right|_{\mathscr{E}}$ est injectif.

$$
\text { (iii) } \Leftrightarrow(i v) \Leftrightarrow(v) \text { : Dans l'identification de } R=\mathbb{Z} \ell^{\otimes} \mathbb{Z} K^{x} \text { avec }
$$
son image canonique dans $\&$, l'application de $\&$ dans $u_{l}$ induite par l'homomorphisme de semi-localisation n'est autre que la restriction à $\mathscr{E}$ de 
la projection canonique de $u$ sur $U_{l}=\prod_{I} U_{l} \cdot \|$ vient done:

$$
\left.s \ell\right|_{\delta} \text { injectif } \Leftrightarrow \delta \cap \prod_{p \nmid \ell \ell} u_{p}=1 \text {. }
$$

Et la condition de droite peut encore s'écrire $\delta \cap \prod_{p} \mu_{p}=\delta \cap \mu$, puisque le groupe $\prod_{p \mid l_{\infty}} \mu_{p}$ est fini.

Bien entendu, si $l$ est impair, cette dernière identité s'écrit tout simplement : $\delta \prod_{p} \mu_{p}=\mu$. Cependant, si $\ell$ vaut 2 , la racine de l'unité $(-1)$ n'est pas une unité dès que $K$ possède des places réelles, de sorte que, dans ce cas, $\& \cap \prod_{p} \mu_{p}$ peut être strictement contenu dans $\mu$. Pour pallier cette difficulté, il est commode de reécrire la condi tion précédente sous la forme plus générale

$$
R \cap \prod_{p} \mu_{p}=\mu
$$

qui exprime que l'application naturelle du quotient $\prod_{p} \mu_{p} / \mu$ dans le $\ell$ groupe $C=\partial / R$ des classes diidèles de $K$ est un monomorphisme.

DÉFINITION 1.1.23.- Le noyau $\Delta_{\text {Leopeld }}$ de l'application naturelle du groupe $\delta \cap \mathbb{Z} l^{\otimes} \mathbb{Z} E$ des unités généralisées du corps $K$ dans le groupe $u_{\ell}=1 \prod_{1} U_{\ell}$ des unités semi-locales attaché aux places divisant $\ell$ est un $\mathbb{Z} \ell^{\text {-module libre de dimension finie } . ~ N o u s ~ d i s o n s ~ q u e ~} \Delta_{\text {Leopoldt }}$ est le groupe de défaut de la conjecture de Leopoldt dans le corps $K$, et que sa dimension $\delta_{\text {Leopoldt }}$ est le défaut dans $K$ de cette conjecture.

THÉORÈME \& DÉFINITION 1.1.24. - Nous disons qu'un corps de nom bres $K$ satisfait la conjecture de Gross, lorsque les conditions équi valentes suivantes sont vérifiées:

(i) Le groupe de Galois $\mathrm{Gal}(\mathrm{N} / \mathrm{K})$ du $\ell$-corps des classes de valeurs absolues de $K$ est un $\mathbb{Z}_{l}$-module de dimension 1 . Autre ment dit, $N$ est une $l$-extension finie de la $\mathbb{Z} l^{\text {-extension cyclotomique }}$ de $K$.

(ii) Le noyau $\varepsilon^{\prime *}$ des applications valeurs absolues $\mid$ I attachées aux places de $K$ au-dessus de $l$, restreintes au tensorisé 
$\ell$-adique $\delta^{\prime}=\mathbb{Z}_{\ell} \otimes_{\mathbb{Z}} E^{\prime}$ du groupe des $\ell$-unités de $K$, est un $\mathbb{Z}_{\ell}$ module de dimension $r_{k}+c_{k}$, où $r_{k}$ est le nombre de places réelles, et $c_{k}$ le nombre de places complexes du corps $K$.

(iii) L'image $\left|\varepsilon^{\prime}\right|_{\ell}$ du groupe $\delta^{\prime}=\mathbb{Z} \ell^{\otimes} \mathbb{Z} E^{\prime}$ des $\ell$-unités

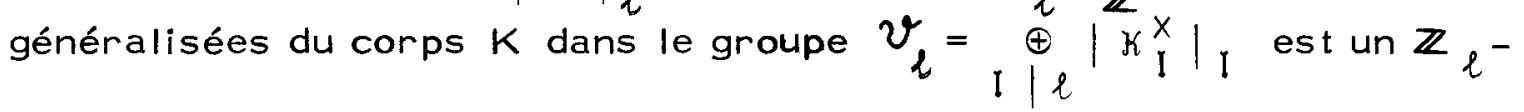
module de dimension $g-1$, où $g$ est le nombre de places de $K$ au dessus de $\ell$.

(iv) La famille ||$_{\ell}=\left(||_{1}\right)_{1} \mid \ell$ des valeurs absolues $\ell-$ adiques attachées aux places au-dessus de $l$ envoie le groupe $\delta^{\prime}=$ $\mathbb{Z}_{\ell} \otimes_{\mathbb{Z}} E^{\prime}$ des $\ell$-unités généralisées de $K$ sur un sous-groupe d'indice fini de la somme directe $\tilde{\vartheta}_{l}=\widetilde{\oplus}_{1}^{\oplus}\left|k_{l}^{x}\right|_{1}$ restreinte aux familles $\left(\sigma_{l}\right)_{1} \mid \ell$ qui vérifient la formule du produit.

(v) La famille $\left(||_{p}\right)_{p}$ des valeurs absolues $\ell$-adiques at tachées aux places de $K$ envoie le $l$-groupe $R=\mathbb{Z} l^{\otimes} \mathbb{Z} K K^{X}$ des idèles principaux sur un sous-module d'indice fini de la somme directe $\tilde{v}=$ $\underset{p}{\tilde{\oplus}}\left|K_{p}^{x}\right|_{p}$ restreinte aux familles $\left(\sigma_{p}\right)_{p}$ qui vérifient la formule du produit $\prod_{p} \sigma_{p}=1$.

Remarque.- Si $K$ est une extension quadratique totalement imaginaire d'un sous-corps $F$ totalement réel, le groupe de Galois $\Delta=G a l(K / F)$ est un groupe d'ordre 2 , engendré par la conjugaison complexe $\bar{T} \cdot I 1$

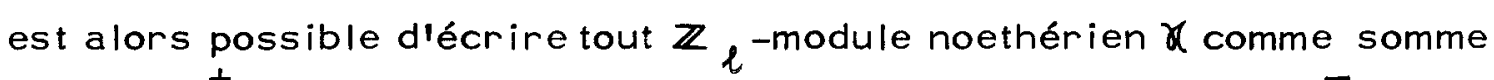
directe $x^{+} \oplus \mathcal{X}^{-}$de ses sous-modules propres pour llaction de $\bar{\tau}$, et ce, de façon exacte si $\ell$ est impair, à un fini près si $\ell$ vaut 2 . La conjec ture de Leopoldt pour $F$ permet ainsi d'affirmer que l'application de $\varepsilon^{+}$ dans $\tilde{v}^{+}$induite par les valeurs absolues est presque surjective, et la condition (iv) postule alors que le même résultat vaut de $\varepsilon^{\prime}$ dans $\tilde{v}^{-}$. Compte-tenu de l'égalité des dimensions, ceci revient à dire que la restriction des valeurs absolues $\left(||_{1}\right)|l| l$ à $\varepsilon^{\prime-}$ est injective, ce qui est précisément la conjecture de Gross .

Démonstration des équivalences :

(i) $\Leftrightarrow(\mathrm{ii}) \Leftrightarrow(\mathrm{iii})$ : Dlaprès l'exemple 19 , le groupe de Galois Gal $(N / K)$ slidentifie au quotient $g / R g^{*}=g / R \prod_{p \mid l} u_{p} \cdot 1 \mid l K_{1}^{*}$. 
Nous obtenons donc un $\mathbb{Z}_{\ell}$-module de même dimension en remplaçant $f$ par le sous-module d'indice fini $\Omega \prod_{p \not l} u_{p} \cdot I \prod_{l} x_{l}^{X} \cdot \|$ vient alors :

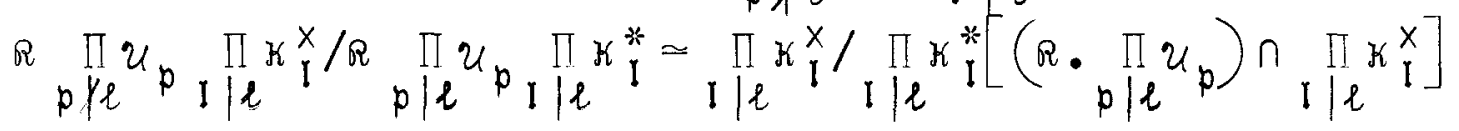
$=x_{l}^{x} / K_{l}^{*} \delta_{l}^{\prime} \simeq v_{l} /\left|\delta^{\prime}\right|_{l}$,

et $\delta_{l}^{\prime}$ désigne l'image dans $\kappa_{l}^{x}$ du groupe $\varepsilon^{\prime}=\mathbb{Z}_{\ell} \otimes_{\mathbb{Z}} E^{\prime}$ des $\ell$-unités généralisées, et ||$_{\ell}$ llapplication de $k_{\ell}^{x}$ dans $v_{\ell}$ composée des va leurs absolues $\ell$-adiques attachées aux places divisant $\ell$.

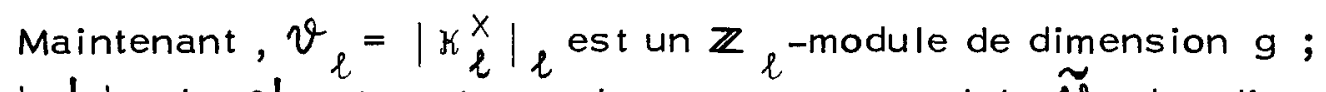
limage $\left|\delta^{\prime}\right|_{\ell}$ de $\varepsilon^{\prime}$ est contenue dans son sous-module $\tilde{v}_{\ell}$ de di mension $g-1$; et $\delta^{\prime}$ lui-même est un $\mathbb{Z} l$-module de dimension $r_{k}+c_{k}-1+g$. Nous avons donc, comme annoncé :

$\operatorname{dim}_{\mathbb{Z}_{l}} \operatorname{Gal}(N / K)=1 \Leftrightarrow \operatorname{dim} \mathbb{Z}_{\ell} \varepsilon^{\prime *}=r_{k}+c_{k} \Leftrightarrow \operatorname{dim} \mathbb{Z}_{\ell}\left|\varepsilon^{\prime}\right|_{\ell}=g-1$.

(iii) $\Leftrightarrow(i v) \Leftrightarrow(v)$ : L'assertion (iii) affirme que la dimension de $\left|\delta^{\prime}\right|_{\ell}$ est celle, g-1, du sous-module noethérien $\tilde{v}_{\ell}$ de $v v_{\ell}$ formé des familles $\left(\sigma_{l}\right)_{I} / \ell$ qui vérifient la formule du produit. D'après la proposition 8 , elle exprime donc que $\left|\delta^{\prime}\right|_{\ell}$ est d'indice fini dans $\tilde{v}_{\ell}$, ce qui est l'assertion (iv). Enfin, l'assertion ( $v$ ) affirme que le même résultat vaut pour $|R|$ dans $\tilde{v}$, ce qui est encore équivalent, puisque le quotient $v /|R| v_{\ell} \simeq g / \Omega \prod_{p \mid l} u_{p} \prod_{I \mid l} K_{l}^{x}$ est fini, qui s'identifie par l'isomorphisme du corps de classes au groupe de Galois Gal $\left(C^{\prime} / K\right)$ de la $\ell$-extension abélienne non ramifiée $\ell$-décomposée maximale de $K$.

DÉFINITION 1.1.25. - Le noyau $\Delta_{\text {Gross }}=\tilde{v}_{\ell} / \sqrt{\left|\varepsilon^{\prime}\right|_{l}}$ du sous groupe $\widetilde{v}_{l}$ de la somme directe $\oplus_{\mathfrak{l} \mid \ell}\left|k_{\mathfrak{l}}^{x}\right|_{\mathfrak{l}}$ formé des familles $\left(\sigma_{\mathfrak{l}}\right)_{\mathfrak{l}} \mid l$ qui vérifient la formule du produit, par le radical $\sqrt{\left|\mathscr{E}^{\prime}\right|}$ de l'image du groupe $\varepsilon_{\ell}^{\prime}$ des unités $l$-unités généralisées du corps $K$, est un $\mathbb{Z} \ell^{-}$ module libre de dimension finie. Nous disons que $\Delta_{\text {Gross }}$ est le groupe de défaut de la conjecture de Gross dans $K$, et que sa dimension $\delta_{G r o s s}$ est le défaut dans $K$ de cette conjecture . 


\section{3. - PROPRIÉTÉS NORMIQUES DES $\ell$-EXTENSIONS ABÉLIENNES .}

\section{a.- La norme dans une extension quelconque de corps de nombres .}

Considérons une extension finie quelconque de corps de nombres $\mathrm{K} / \mathrm{H}$. L'application norme $N_{K} / H$ est définie sur les groupes d'idèles généralisés par la formule:

$$
N_{K / H}\left(\left(x_{B}\right)_{P}\right)=\left(\prod_{\mathcal{P} \mid \mathcal{P}} N_{K_{\mathcal{P}}} / H_{p}\left(x_{\mathcal{B}}\right)\right)_{p}
$$

où, pour chaque place $p$ de $H$, et chaque place $\mathfrak{P}$ de $K$ au-dessus de $p$, la norme locale $N_{K_{\mathfrak{P}}} / H_{\mathfrak{p}}$ est le morphisme continu du groupe $\kappa_{\mathfrak{P}}^{x}=\underset{\mathrm{m}}{\lim } \mathrm{K}_{\mathfrak{P}}^{x} / K_{\mathfrak{P}}^{x \ell^{m}}$ dans le groupe $\psi_{p}^{X}=\underset{m}{i m} H_{p}^{X} / H_{p}^{X \ell^{m}}$ induite par la norme naturelle at tachée à l'extension locale $K_{\mathfrak{P}} / H_{\mathfrak{p}}$. Bien entendu, si $\mathfrak{k}=\left(x_{\mathfrak{B}}\right)_{\mathfrak{P}}$ est un idèle principal (i.e. si $\mathfrak{k}$ est contenu dans le tensorisé $R_{K}=\mathbb{Z}_{l} \otimes_{\mathbb{Z}} K^{X}$ ), sa norme $N_{K / H}(\mathfrak{k})$ est encore un idèle principal (i.e. un élément de $R_{H}=\mathbb{Z}_{\ell} \otimes_{\mathbb{Z}} H^{x}$ ): Plus précisément, la restriction aux sous-modules principaux $R$ de la norme généralisée $N_{K / H}$ n'est autre que l'application induite par la norme naturelle de $K^{X}$ dans $H^{X}$.

Par passage au quotient, nous obtenons une application continue et ouverte de $C_{K}$ dans $C_{H}$, que nous continuons par abus à noter $N_{K} / H$, et que nous désignons sous le nom de norme arithmétique ${ }^{(\star)}$. Dans l'isomorphisme du corps de classes, la norme arithmétique correspond à la restriction des automorphismes de Galois, ce qui se traduit par la commutativité du dia$\operatorname{gramme}^{(\star \star)}$

$\left(^{\star}\right)$ Ce qualificatif d'arithmétique doit être compris en concurrence de celui d'algébrique : $\mathrm{Si} \mathrm{K} / \mathrm{H}$ est galoisienne, la norme algébrique $\nu_{K / H}=\sum_{\sigma \in \operatorname{Gal}(K / H)} \sigma$, regardée comme opérateur sur $\mathcal{G}_{K}$, est composée de la norme arithmétique $N_{K / H}$ de $C_{K}$ dans $C_{H}$, et de l'homomorphisme d'extension $j_{K / H}$ de $\mathcal{G}_{H}$ dans $c_{K}$. $\left({ }^{\star}\right)$ cf. [AT ], ch. XIV, §5, th. $6 . \mathrm{b}$. 


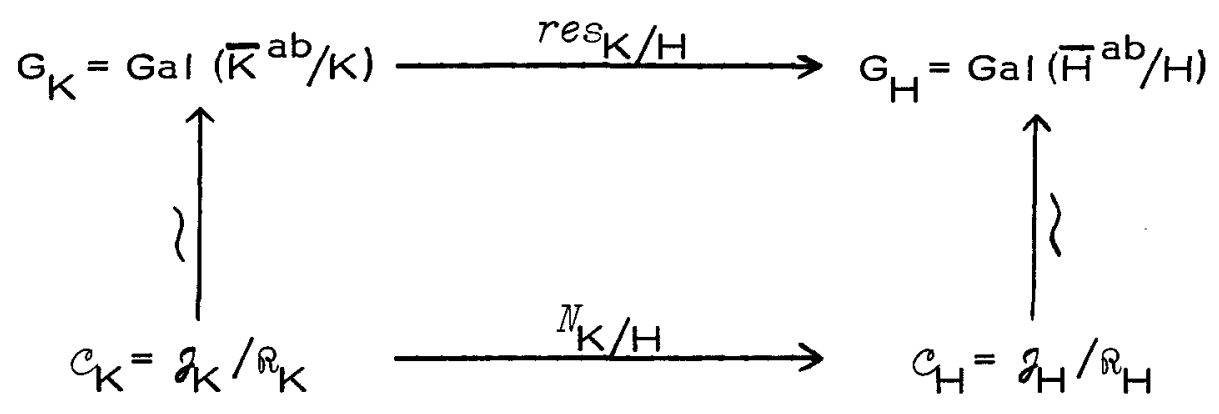

où $\overline{\mathrm{K}}^{\mathrm{ab}}$ (respectivement $\bar{H}^{\mathrm{ab}}$ ) est la $\ell$-extension abélienne maximale de

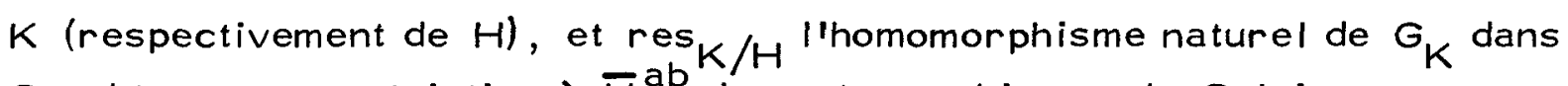
$G_{H}$ obtenu par restriction à $\bar{H}$ des automorphismes de Galois.

Une conséquence élémentaire mais essentielle de ce diagramme est le résultat suivant :

THÉORËME 1.1.26. - Soient $\mathrm{K} / \mathrm{H}$ une extension finie quelconque de corps de nombres, et $L$ une $\ell$-extension abélienne de $K$. Désignons par $c_{K}^{L}=\frac{1}{\partial_{K}} / R_{K}$ le sous-module fermé de $C_{K}$ qui lui correspond par le corps de classes. Alors :

(i) Le sous-module fermé de $G_{H}$, qui est associé à la $l$-extension abélienne maximale de $H$ contenue dans $L$, est liimage $C_{H}^{L}$ de $C_{K}^{L}$ par la norme arithmétique :

$$
C_{H}^{L}=\partial_{H}^{L} / R_{H} \text {, avec } \partial_{H}^{L}=N_{K / H}\left(\partial_{K}^{L}\right) R_{H} \text {. }
$$

(ii) Le corps $L$ provient, par composition avec $K$, dlune $l$-extension abélienne de $H$ si et seulement si le sous-module $C_{K}^{L}$ est saturé pour la norme arithmétique, ce qui s'écrit :

$$
\partial_{K}^{L}={ }^{-1} N_{K / H}\left(N_{K / H}\left(\partial_{K}^{L}\right) R_{H}\right) \text {. }
$$

Démonstration :

(i) Désignons par $G_{K}^{L}=G a l\left(\bar{K}^{a b} / L\right)$ le sous-groupe de $G_{K}$ associé à $L$ dans la correspondance de Galois. La l-extension abélienne maximale de $H$ qui est contenue dans $L$ est le corps des points fixes dans $\bar{H}^{a b}$ de la restriction à $\bar{H}^{a b}$ du groupe $G_{K}^{L}$. Dlaprès le diagramme, cette restriction est l'image dans $G_{H}$ du sous-groupe $N_{K / H}\left(C_{K}^{L}\right)$ de $C_{H}$. Ainsi, 
le sous-module de $q_{H}$ associé à $L \cap \bar{H}^{a b}$ par la correspondance du

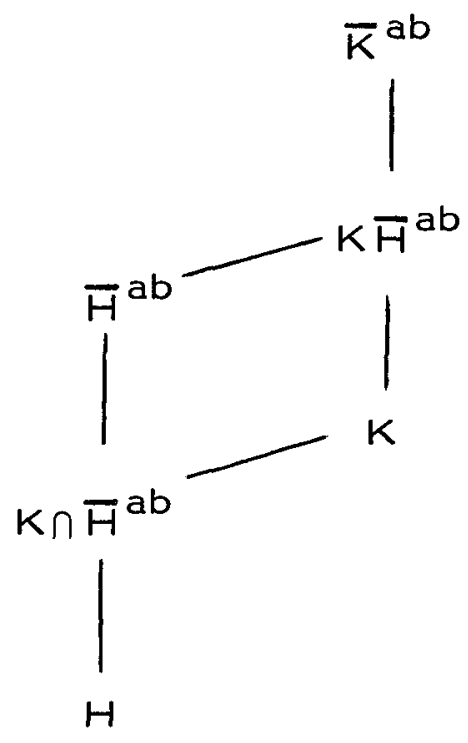
corps de classes est précisément limage de $C_{K}^{L}$ par la norme arithmétique, ce qui est bien l'assertion (i) du théorème.

(ii) Si maintenant $L^{\prime}$ est une autre $\ell$-extension abélienne de $K$, et $\mathcal{C}_{K}^{L}$ le sous-module de $c_{K}$ qui lui correspond, la condition $L^{\prime} \cap \bar{H}^{a b} \supset L \cap \bar{H}^{a b}$ s'écrit $N_{K / H}\left(C_{K}^{L}\right) \subset N_{K / H}\left(C_{K}^{L}\right)$, i.e. $\left.C_{K}^{L^{\prime}} c^{-1} N_{K / H}\left(N_{K} / H^{(} C_{K}^{L}\right)\right)$. La plus petite $\ell$-extension abélienne de $K$ qui contient $L \cap \bar{H}^{a b}$ est donc celle associée au saturé pour la norme du module $c_{K}^{L}$. C'est, bien entendu $K\left(L \cap \bar{H}^{\mathrm{ab}}\right)$. En particulier, l'égalité $L=K\left(L \cap \bar{H}^{a b}\right)$ a donc lieu si et seulement si $\mathcal{G}_{\mathrm{K}}^{\mathrm{L}}$ est saturé pour la norme arithmétique $N_{\mathrm{K} / \mathrm{H}}$.

L'étude des extensions (abéliennes) d'un corps de nombres donné $K$, qui proviennent par composition avec $K$ dlune extension abélienne sur un sous-corps donné $H$ de $K$, relève à proprement parler de la théorie des genres. Notre propos n'étant pas de la développer ici ${ }^{(\star)}$, donnons simplement la définition du $\ell$-corps des $S$-genres, avant dlappliquer sans plus attendre le théorème 26 au cas de la tour cyclotomique.

DÉFINITION 1.1.27. - Etant donnés une extension quelconque de corps de nombres $K / H$, et $S=S_{H}$ un ensemble fini de places de $H$, le $\ell$-corps des $S$-genres de l'extension $K / H$ est la $\ell$-extension maximale de $K$, non ramifiée et $S$ décomposée (i.e. non ramifiée aux places finies et complètement décomposée aux places de $K$ au-dessus de $S$ ), qui provient par composition avec $K$ d'une $\ell$-extension abélienne de $H$. Lorsque $S$ est l'ensemble des places de $H$ au-dessus de $\ell$, nous parlons simplement du $\ell$-corps des $\ell$-genres de l'extension $\mathrm{K} / \mathrm{H}$.

$\left.{ }^{\star}{ }^{\star}\right)$ Une approche de la théorie des genres fait l'objet de la section 2 du chapitre III. 


\section{b.- Application aux $\ell$-genres cyclotomiques.}

Précisons d'abord la différence, essentielle lorsque $\ell$ vaut 2 , entre la $\ell$-tour cyclotomique et la $\mathbb{Z} \ell^{\text {-extension cyclotomique d'un corps }}$ de nombres $K$ :

DÉFINITION \& PROPOSITION 1.1.28.- Nous appelons $\ell$-tour cyclotomique construite sur un corps de nombres $K$, et nous notons $T_{K}$ la réunion des $\ell$-sous-extensions finies de l'extension abélienne $K\left[\mu_{\ell}\right]$ engendrée sur $K$ par les racines d'ordre $\ell$-primaire de l'unité. Le sous-module fermé de $C_{K}$ associé au corps $T_{K}$ par la théorie du corps de classes est le noyau dans $\mathcal{C}_{K}$ de la formule du produit :

$$
c_{K}^{\top}=\tilde{\partial}_{K} / R_{K}, \quad \text { où } \tilde{\partial}_{K}=\left\{r \in \partial_{K} \mid\|\mathfrak{r}\|=1\right\} \text {. }
$$

- Si $\ell$ est impair, le groupe de Galois Gal $\left(T_{K} / K\right) \simeq 2_{K} / \widetilde{\partial}_{K}$ est isomorphe à $\mathbb{Z}_{\ell}$. Dans ce cas, $T_{K}$ n'est autre que la $\mathbb{Z}_{\ell}$-extension cyclotomique $K_{\infty}$ de $K$.

- Si $\ell$ vaut 2 , le groupe de Galois $G a l\left(T_{K} / K\right) \approx z_{K} / \tilde{z}_{K}$ peut contenir un sous-groupe de torsion d'ordre 2 . Lorsque cela est, la tour $T_{K}$ est une extension quadratique de la $\mathbb{Z}_{2}$-extension cyclotomique de $K$; dans le cas contraire, $T_{K}$ est égal à $K_{\infty}$. En toutes hypothèses cependant, il vient $T_{K}=K_{\infty}[i]$.

Démonstration : Lorsque $K$ est le corps $\mathbb{Q}$ des rationnels, la description du groupe $\tilde{\partial}_{K}$ est immédiate : Le groupe $\partial_{\mathbb{Q}}$ des idèles généralisés de $\mathbb{Q}$ admet, en effet, la décomposition directe :

$$
\partial_{\mathbb{Q}}=\prod_{p \in P \mid \mathbb{Q}} u_{p} \cdot \Omega_{\mathbb{Q}}
$$

de sorte que nous obtenons immédiatement :

$$
\tilde{\partial}_{\mathbb{Q}}=\prod_{p \neq l} u_{p} \cdot R_{\mathbb{Q}} \text {. }
$$

II vient donc :

$$
\partial_{Q} / \tilde{\partial}_{Q}=u_{l}=1+\ell \mathbb{Z}_{\ell}
$$


et deux cas se présentent :

- Si $\ell$ est impair, le groupe $u_{\ell}$ est isomorphe à $\mathbb{Z}_{\ell}$, et la $\ell$-extension abélienne $T_{\mathbb{Q}}$ associée à $\tilde{\partial}_{\mathbb{Q}}$ par la théorie du corps de

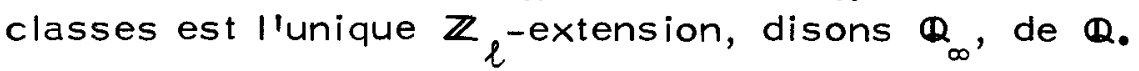

- Si $\ell$ vaut 2 , le groupe $u_{2}$ est isomorphe à $(\mathbb{Z} / 2 \mathbb{Z}) \times \mathbb{Z}_{2}$, et la 2-extension abélienne $T_{\mathbb{Q}}$ associée à $\widetilde{\partial}_{\mathbb{Q}}$ est une extension quadratique (totalement imaginaire) de la $\mathbb{Z}_{2}$-extension $\mathbb{Q}_{\infty}$ de $\mathbb{Q}$. Comme elle est non ramifiée en dehors de la place 2 , c'est, bien entendu, $\mathbb{Q}\left[\mu_{2} \infty\right]$.

La proposition est donc établie lorsque $K$ égale $Q$. Passons donc au cas général, et supposons maintenant que $K$ est un corps de nombres quelconque (de degré fini sur $\mathbb{Q}$ ). De l'identité $T_{K}=K . T_{\mathbb{Q}}$, nous déduisons, par le théorème 26 , que le sous-module de $\partial_{K}$ correspondant à $T_{K}$ est l'image réciproque par l'application norme $N_{K / \mathbb{Q}}$ de celui $\tilde{\partial}_{\mathbb{Q}}$ associé à $T_{\mathbb{Q}}$. C'est, comme annoncé $\tilde{\partial}_{K}$; ce qui achève la démonstration.

Cela posé, le lien entre la conjecture de Gross et la théorie cyclotomique des $\ell$-genres est donné par le résultat suivant :

THÉORĖME 1.1.29. - Le $l$-corps des classes de valeurs absolues $\left({ }^{\star}\right)$ d'un corps de nombres $K$ est la $\ell$-extension abélienne maximale de $K$, qui est non ramifiée et $l$-décomposée (i.e. non ramifiée aux places finies et complètement décomposée aux places au-dessus de $\ell$ ) sur la tour cyclotomique $T_{K}$. Autrement dit, $N_{K}$ est le $\ell$-corps des $\ell$-genres de l'extension infinie $T_{K} / K$.

Démonstration : II s'agit d'établir (i) que le corps $N_{K}$ contient la tour $T_{K}$; (ii) que llextension $N_{K} / T_{K}$ est non ramifiée et $\ell$-décomposée; (iii) qu'elle est maximale enfin (parmi les $\ell$-extensions abéliennes de $K$ ) sous ces deux conditions. Examinons successivement chacune des trois assertions :

(i) Nous savons déjà (cf. exemple 19) que le sous-module de $\gamma_{K}$ associé à $N_{K}$ est le produit $\gamma_{K}^{N}=\partial_{K}^{*} R_{K}$, où $\partial_{K}^{*}$ est le noyau dans $\partial_{K}$

$\left({ }^{\star}\right)$ Le corps $N_{K}$ est défini à l'exemple 19. 
des valeurs absolues $\ell$-adiques. Comme $\tilde{\sigma}_{K}^{N}$ est trivialement contenu dans $\tilde{\partial}_{K}$, nous en concluons que $N_{K}$ contient $T_{K}$.

(ii) Nous savons aussi (cf. exemple 19) que $N_{K}$ est $\ell-r a m i-$ fiée sur $K(i . e$. non ramifiée aux places finies étrangères à $\ell$ ). L'extension $N_{K} / T_{K}$ est donc $\ell$-ramifiée. Pour montrer qu'elle est en outre $\ell$-décomposée, il nous suffit de vérifier que les sous-groupes de décomposition $D_{l}\left(N_{K} / K\right)$ associés aux places de $K$ au-dessus de $\ell$ dans l'extension abélienne $N_{K} / K$ rencontrent trivialement le sous-groupe Gal $\left(N_{K} / T_{K}\right)$; autrement dit, que nous avons $\kappa_{l}^{x} \cap \tilde{\partial}_{K}=K_{I}^{x} \cap \partial_{K}^{*}{ }_{K}$, pour chaque place $I$ de $K$ au-dessus de $\ell$. Or, si $x=\left(x_{p}\right)$ est un élément de $K_{1}^{x} \cap \tilde{\partial}_{K}$, nous avons $x_{p}=1$, pour $p \neq l$, donc $|\mathfrak{k}|_{p}=1$ pour chaque $p$ distinct de $\mathfrak{l}$, et par suite $|\mathfrak{k}|_{1}=1$ en vertu de la formule du produit : $\|\mathfrak{k}\|=1$. $\|$ vient donc

$$
k_{l}^{x} \cap \tilde{\partial}_{K}=k_{l}^{*} \subset k_{j}^{x} \cap \partial_{K}^{x} \Omega_{K} ;
$$

ce qui est le résultat attendu.

(iii) Réciproquement, si $L$ est une $\ell$-extension abélienne de $K$, non ramifiée et $\ell$-décomposée sur $T_{K}$, les calculs qui précèdent montrent que le sous-module $\partial_{K}^{L}$ de $\partial_{K}$ associé à $L$ contient et les groupes $u_{p}$ pour $p \nmid \ell$ (en vertu de la condition de $\ell$-ramification), et les groupes $k_{l}^{*}$ pour $\mathfrak{I} \mid \ell$ (en vertu de la condition de $\ell$-décomposition sur $T_{K}$ ), et, bien entendu $\Omega_{K}$. Ainsi $\gamma_{K}^{L}$ contient $\gamma_{K}^{*}{ }^{*}{ }_{K}$; le corps $L$ est contenu dans $N_{K}$; et la maximalité de $\mathrm{N}_{K}$ est donc établie.

DÉFINITION 1.1.30.- Nous appelons groupe des classes de valeurs absolues diun corps de nombres le quotient

$$
z_{K}=\tilde{v}_{a_{K}} / v_{p_{K}} \simeq \tilde{z}_{K} / z_{K}^{*} r_{K}
$$

du groupe $\tilde{v}_{a_{K}}$ des valeurs absolues des idèles de masse 1 , par le sousgroupe des valeurs absolues des idèles principaux. Dans l'isomorphisme du corps de classes, le groupe ${ }^{3} K$ s'identifie au groupe de Galois relatif Gal $\left(N_{K} / T_{K}\right)$ du $\ell$-corps des classes de valeurs absolues $N_{K}$ sur la tour cyclotomique $T_{K}$. 
D'après l'assertion $(v)$ du théorème 24 , la conjecture de Gross pour le corps $K$ postule précisément la finitude du groupe ${ }^{K} K$ •

\section{c.-Récapitulatif des principaux groupes de normes •}

Nous présentons ci-dessous, sous forme de tableau, les sousmodules fermés du $\ell$-groupe des idèles généralisés attachés par la théorie du corps de classes aux principales $\ell$-extensions abéliennes d'un corps de nombres donné.

Le corps de base $K$ étant supposé fixé, nous omettons l'indice $K$.

\begin{tabular}{|c|c|c|c|}
\hline $\begin{array}{l}\text { l-extension } \\
\text { abélienne }\end{array}$ & $\begin{array}{l}\text { Définition de la } \ell \text {-extension } \\
\text { abélienne considérée }\end{array}$ & $\begin{array}{c}\text { Groupe de normes } \\
\text { associé }\end{array}$ & Commentaire \\
\hline K & corps de base & $g=\prod_{p}^{\text {res }} \kappa_{p}^{x}$ & $k_{p}^{x}=\underbrace{\lim }_{m} K_{p}^{x} / K_{p}^{x l^{m}}$ \\
\hline$c^{\prime}$ & $\begin{array}{c}\text { non ramifiée } \\
\ell \text {-décomposée maximale }\end{array}$ & $\prod_{I \mid l} k_{I}^{x} \cdot \prod_{p \not l e} u_{p} \cdot R$ & $u_{p}=\lim _{m} u_{p} / u_{p}^{l^{m}}$ \\
\hline C & non ramifiée maximale & $p^{\prod u_{p} \cdot R}$ & \\
\hline T & $\ell$-tour cyclotomique & $\tilde{g}$ & $\tilde{z}=\{x \in \partial\|x\|=1\}$ \\
\hline$N$ & $\begin{array}{l}\text { l-corps des classes } \\
\text { de valeurs absolues }\end{array}$ & $\prod_{1 \mid l} k_{l}^{*} \cdot \prod_{p X l} u_{p} \cdot R$ & $K_{1}^{*}=\left\{\left.x_{1} \in K_{1}|| x_{1}\right|_{1}=1\right\}$ \\
\hline Z & $\begin{array}{l}\text { composée des } \\
\mathbb{Z} l^{- \text {extensions }}\end{array}$ & $\sqrt{\prod_{p} \mu_{p} \cdot R}$ & $\mu_{p}=$ torsion de $k_{p}^{x}$ \\
\hline$c^{\prime} z$ & composée de $C^{\prime}$ et de $Z$ & $\sqrt{\varepsilon_{l}^{\prime}} \cdot \prod_{p \times l} \mu_{p} \cdot R$ & $\begin{array}{c}\sqrt{\varepsilon_{l}^{\top}} \text { radical dans } k_{\ell}^{x} \\
\text { de l'image de } \varepsilon^{\prime}\end{array}$ \\
\hline$C Z$ & composée de $C$ et de $Z$ & $\sqrt{\mathscr{E}_{\ell}} \cdot \prod_{p X l} \mu_{p} \cdot R$ & $\begin{array}{c}\sqrt{\xi_{\ell}} \text { radical dans } U_{\ell} \\
\text { de l'image de } \xi\end{array}$ \\
\hline$H$ & hilbertienne maximale & $p^{I \mu_{p} \cdot R}$ & \\
\hline$M$ & $\ell$-ramifiée maximale & $\prod_{p l e} u_{p} \cdot R$ & \\
\hline $\bar{K}^{a b}$ & maximale & $R$ & $R=\mathbb{Z}_{\ell} \otimes_{\mathbb{Z}} K^{x}$ \\
\hline
\end{tabular}


Nota. - Seuls les groupes de normes associés à $C^{\prime} Z$ et $C Z$ n'ont pas été calculés plus haut. Ils s'obtiennent comme intersections de ceux associés à $Z$ et à $C^{\prime}$ ou $C$. Nous avons noté $\varepsilon_{l}^{\prime}$ l'image dans $k_{l}^{x}=\prod_{I} k_{l}^{x} d u$ groupe des $\ell$-unités généralisées $\varepsilon^{\prime}=\mathbb{Z} l \otimes_{\mathbb{Z}} E^{\prime}$, et $\varepsilon_{\ell}$ celle, contenue dans $u_{l}=\prod_{l \mid \ell} U_{l}$, du groupe des unités généralisées $\varepsilon=\mathbb{Z}_{\ell} \otimes_{\mathbb{Z}} E$. 
Nous établissons dans cette section un parallèle entre la théorie de Tate pour le $K_{2}$ des corps de nombres (cf. [Ta 1 , [ $\left.\mathrm{Ta}_{2}\right]$, [ $\left.\mathrm{Ta}_{3}\right]$ ) et la description kummérienne des genres dans une tour cyclotomique. Nous sommes amenés, pour cela, à associer à chaque corps de nombres $K$ un groupe universel $\bar{K}_{2}(K)$, analogue au groupe symbolique $K_{2}(K)$, et deux sous-groupes finis $\bar{R}_{2}(K)$ et $\bar{K}_{2}(K)$, qui jouent, dans notre description, le même rôle que le noyau régulier $R_{2}(K)$ et le noyau hilbertien $H_{2}(K)$ dans la $K$-théorie. Ces divers groupes sont remarquables à plus d'un titre:

D'abord ils admettent une interprétation simple de type corps de classes, de sorte que leur arithmétique relève directement des méthodes, classiques ou non, de cette théorie.

Ensuite ils constituent une bonne approximation des noyaux de la $K$-théorie, en ce sens qu'ils possèdent le même rang ( $\mathrm{Si} K$ contient les racines $l^{n}$-ièmes de l'unité, les groupes $R_{2}(\mathrm{~K})$ et $\vec{R}_{2}(\mathrm{~K})$ d'une part, $H_{2}(\mathrm{~K})$ et $\bar{H}_{2}(K)$ d'autre part, ont même $\ell^{n}$-rang), et un ordre équivalent (Dans une tour cyclotomique, les ordres respectifs des groupes $\bar{R}_{2}\left(K_{n}\right)$ et $\vec{H}_{2}\left(K_{n}\right)$ sont donnés asymptotiquement, à un facteur borné près, par les mêmes formules que ceux des groupes $R_{2}\left(K_{n}\right)$ et $H_{2}\left(K_{n}\right)$ ).

Le parallèle obtenu explicite la correspondance, déjà remarquée par plusieurs auteurs (cf. $\left[\mathrm{Ca}_{2}\right],\left[\mathrm{Gb}_{3}\right],[\mathrm{KC}]$ ), entre le $l$-Sylow du noyau régulier $R_{2}(K)$ et le sous-groupe de torsion $\varepsilon_{K}$ du groupe de Galois de la $\ell$-extension abélienne $\ell$-ramifiée maximale de $K$, mais relie également le $\ell$-Sylow du noyau hilbertien $\mathrm{H}_{2}(\mathrm{~K})$ et le groupe de Galois de la $\ell$-extension abélienne non ramifiée $\ell$-décomposée maximale de $K$, et met en avant des interprétations nouvelles de la conjecture de Leopoldt, liées aux symboles donnés par le corps de classes. 


\section{0. - CONVENTIONS ET NOTATIONS .}

Dans toute cette section, $\ell$ est un nombre premier, et $K_{0} / F_{0}$ une extension abélienne de corps de nombres, de degré d étranger à $\ell$, supposée contenir les racines $\ell$-ièmes de l'unité si $\ell$ est impair, et les racines quatrièmes si $\ell$ vaut 2 ( par exemple $K_{0}=F_{0}[\zeta]$, pour une $r a-$ cine primitive $\ell$-ième de l'unité $\zeta$, et $\ell \neq 2) ; K_{\infty}={ }_{n \in N} K_{n}$ est la tour cyclotomique construite sur $K_{0}$, avec la conventiond'écriture $K_{n}=K_{0}\left[\mu_{n}\right]$, où $\mu_{n}$ désigne le groupe des racines $\ell^{n}$-ièmes de l'unité dans $\mathbb{C}$.

Nous notons $\mu_{\ell}=\stackrel{\lim }{\longrightarrow} \mu_{n}$ la réunion des $\mu_{n}$, puis $\sigma_{l}=\underline{\lim } \mu_{n}$ le module de Tate (que nous identifions à $\mathbb{Z}_{\ell}$ comme groupe abstrait, via l'exponentielle complexe $1 / \mathrm{m} \mapsto \exp (2 i \pi / m)$ ), et $\bar{\pi}_{\ell}$ le module opposé (c'est-à-dire le même groupe équipé de llaction opposée des groupes de Galois), qui n'est autre que le dual de Pontrjagin

$$
\sigma_{l}=\mu_{l}^{r}=\text { Hom } \mathbb{Z}_{l}\left(\mu_{l}, \mathbb{Q}_{l} / \mathbb{Z}_{l}\right)
$$

du groupe $\mu_{\ell}$. Le caractère w de l'action du groupe de Galois Gal $\left(K_{\infty} / F_{0}\right)$ sur le module de Tate $T_{l}$ est, par définition, le carac tère cyclotomique; le caractère opposé $\bar{\omega}$, défini par $\bar{\omega}(\tau)=\omega\left(\tau^{-1}\right)$, est le caractère anticyclotomique.

Si $r$ est le plus grand entier vérifiant $K_{r}=K_{0}$ ( de sorte que $K_{0}$ contient $\mu_{r}$ mais non $\mu_{r+1}$, nous supposons implicitement dans les cal culs qui suivent $n \geq r$ (et donc $\left.\left[K_{n+1}: K_{n}\right]=l\right)$. En particulier, par $\gamma_{n}$ nous entendons l'unique progénérateur du groupe $I_{n}=\mathrm{Gal}\left(K_{\infty} / K_{n}\right)$ dé fini par l'identité :

$$
\zeta^{Y_{n}}=\zeta^{1+e^{n}}
$$

pour tout $\zeta$ de $\mu_{\ell}$ (c'est-à-dire par la condition $\omega\left(\gamma_{n}\right)=1+\ell^{n}$ ).

Nous désignons enfin par $\Delta$ l'unique sous-groupe d'ordre $d$ de Gal $\left(K_{\infty} / F_{0}\right)$; nous notons $F_{\infty}=K_{\infty}^{\Delta}$ son corps des invariants, et, plus généralement, $F_{n}=K_{n}^{\Delta}$, de sorte que nous avons canoniquement $\Delta \approx$ $\operatorname{Gal}\left(K_{n} / F_{n}\right)$. L'algèbre $\mathbb{Z}_{\ell}[\Delta]$ est une algèbre semi-locale, composée directe d'extensions abéliennes finies, non ramifiées, de l'anneau $\mathbb{Z}_{\ell}$.

CONVENTION. - Etant donné un $\mathbb{Z}_{\ell}$-module multiplicatif $X$, nous disons qu'un élément $x$ de $\mathfrak{X}$ est : 
(i) de hauteur $m$, lorsque c'est une puissance $\ell^{m}$-ième dans $\mathfrak{X}$ (i.e. $\left.\mathbb{H} x_{m} \in \mathfrak{X}, x=x_{m}^{l}\right)$.

(ii) de hauteur $\infty$, lorsqu'il est de hauteur $m$ pour tout $m$ (i.e. $\forall m \in \mathbb{N}$ H $x_{m} \in \mathfrak{X}, x=x_{m}^{l}$ ).

(iii) divisible, lorsqu'il peut être indéfiniment divisé (i.e. $\exists\left(x_{m}\right)_{m \in \mathbb{N}} \in \mathfrak{x}^{\mathbb{N}}, x=x_{0}=x_{1}^{l}, x_{1}=x_{2}^{l}$, etc ... ). Nous notons $\mathfrak{X}_{\infty}=\prod_{\mathrm{m} \in \mathbb{N}} \mathfrak{X}^{l}$ le sous-module de $\mathfrak{X}$ formé des éléments de hauteur infinie, et $\mathfrak{X}_{\text {div }}=\mathfrak{X}_{\text {div }}^{\ell}$ le sous-module de $\mathfrak{X}_{\infty}$ constitué des élé ments divisibles de $\mathfrak{x}$.

Le module $\mathfrak{X}_{\text {div }}$ coïncide avec $\mathfrak{X}_{\infty}$ lorsque $\mathfrak{X}$ est de cotype fini (i.e. lorsque le sous-module $\ell^{\mathfrak{X}}$, annulé par $\ell$, est fini). Dans ce cas, le sous-module de torsion $t(x)$ s'écrit comme somme directe du sousmodule $t(x)_{\text {div }}$ et d'un $\mathbb{Z}_{\ell}$-module fini $\mathfrak{F}^{\mathfrak{x}}$ :

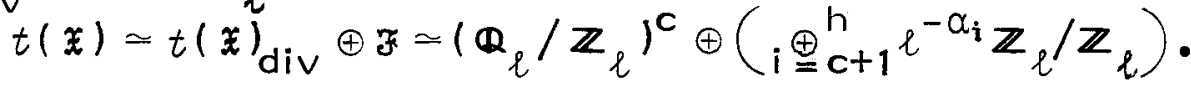

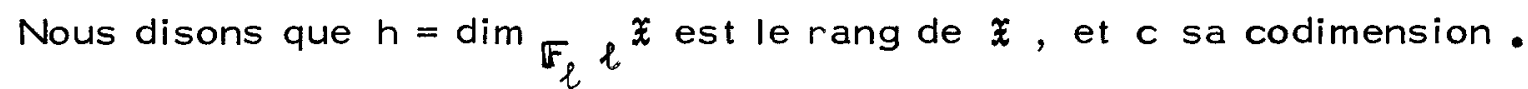

\section{1.- DÉFINITION DU RADICAL UNIVERSEL $\Omega$.}

DÉFINITION 1.2.1. - Par $\ell$-radical universel d'un corps de nombres $K$, nous entendons le tensorisé par $\mathbb{Q}_{\ell} / \mathbb{Z}_{\ell}$ de son groupe multiplicatif, et nous écrivons :

$$
\Omega_{K}=\left(Q_{l} / \mathbb{Z}_{l}\right) \otimes_{\mathbb{Z}^{K}}
$$

Le groupe $\Omega_{K}$ est un $\mathbb{Z}_{l}$-module de torsion dont tout élément admet une représentation de la forme $\ell^{-m} \otimes x$, avec $m \in N$ et $x \in K^{X}$. La condition $\ell^{-m} \otimes x=1$ signifie alors que l'élément $x$ est le produit $x=\zeta y^{l}$ d'une racine de l'unité et d'une puissance $l^{m}$-ième dans $k^{x}$.

a.-Préliminaires : théorie de Galois pour les radicaux universels

La tour cyclotomique $\mathrm{K}_{\infty}$ étant la réunion des sous-corps $\mathrm{K}_{\mathrm{n}}$, le radical universel $\Omega_{K_{\infty}}$, qui lui correspond, s'obtient naturellement comme limite inductive des radicaux $\Omega_{K_{n}}=\left(\mathbb{Q}_{l} / \mathbb{Z}_{l}\right) \otimes_{\mathbb{Z}} K_{n}^{x}$. Le point essentiel qui nous intéresse ici est que les groupes $\Omega_{K}$ vérifient la théorie de Galois : 
PROPOSITION 1.2.2. - L'application naturelle de $\Omega_{K_{n}}$ dans $\Omega_{K}$ est un morphisme injectif. Plus précisément, pour chaque naturel $n$, le radi cal $R_{K_{n}}$ associé au corps $K_{n}$ slidentifie canoniquement au sous-module de $\Omega_{K_{\infty}}$ fixé par le groupe de Galois $\Gamma_{n}=$ Gal $\left(K_{\infty} / K_{n}\right)$; ce qui s'écrit:

$$
s_{K_{n}}=s_{K_{\infty}}^{\Gamma_{n}} \text {. }
$$

Démonstration ${ }^{(*)}$ : Soit $x_{n}$ un élément de $K_{n}^{x}$. La relation $\ell^{-1} \otimes x_{n}=1$ dans $\Omega_{K}$ entraîne $K_{n}\left[\sqrt[l]{x_{n}}\right] \subset K\left[\sqrt[l]{x_{n}}\right]=K$, donc, d'après la théorie de Kummer, $x_{n} \equiv \zeta_{n}\left(\bmod x K_{n}^{x l}\right)$, pour un $\zeta_{n}$ de $\mu_{n}$, clest-à-dire finale ment $l^{-1} \otimes x_{n}=1$ dans $\Omega_{K_{n}}$. Autrement dit, tout élément diordre $l$ de $\Omega_{K_{n}}$ s'envoie sur un élément d'ordre $\ell$ de $\Omega_{K_{\infty}}$; et l'application natu relle de $\Omega_{K_{n}}$ dans $\Omega_{K_{\infty}}$ est donc injective.

Soit maintenant $\ell^{-p} \otimes x_{p}$ un élément de $\Omega_{K_{p}}$ fixé par $I_{n}$, pour un $n \leq p$. La condition $l^{-p} \otimes x_{p}^{Y_{n}-1}=1$ nous prouve ( puisque les racines de l'unité sont divisibles dans $K_{\infty} x_{\infty}$ ) que l'élément $x_{p}^{\gamma_{n}-1}$ est une puissance $\ell^{p}$-ième dans $K_{\infty}^{x}$. Ecrivons donc que $x_{p}^{\gamma_{n}-1}=y_{q}^{l^{p}}$, pour un $y_{q}$ dans $K_{q}^{x}$; et considérons l'élément $N_{\mathrm{q} / \mathrm{n}}\left(\mathrm{y}_{\mathrm{q}}\right)$. Nous avons $N_{\mathrm{q} / \mathrm{n}}\left(\mathrm{y}_{\mathrm{q}}\right)^{\ell^{p^{\mathrm{q}}}}=$ $N_{q / n}\left(x_{p}^{\gamma_{n}-1}\right)=x_{p}^{\gamma_{q}-1}=1$, de sorte que $N_{q} / n\left(y_{q}\right)^{q / n}$ est une racine de l'unité, disons $\zeta_{n}$, de $K_{n}$. Ecrivons $\zeta_{n}=N_{q} / n\left(\zeta_{q}\right)$, avec $\zeta_{q} \in \mu_{q}$; nous obtenons $N_{q} / n\left(y_{q} / \zeta_{q}\right)=1$, d'où (en vertu du théorème 90 de Hilbert appliqué à l'extension cyclique $\left.K_{q} / K_{n}\right): y_{q}=\zeta_{q} z_{q}^{\gamma_{n}-1}$, pour un $z_{q}$ de $K_{q}^{x}$. En particulier l'élément $\left(x_{p} z_{q}^{-l^{p}}\right) \gamma_{n}-1=\zeta_{q}^{l^{p}}$ est une racine de llunité. Posant alors $x_{n}=x_{p} z_{q}^{-l^{p}} \zeta_{q}^{-\ell^{p-n}}$, nous pouvons reécrire l'identité précédente sous la forme $x_{n} \gamma_{n}-1=1$, ce qui nous prouve que $x_{p}=x_{n} \zeta_{q}^{l^{p-n}} \quad z_{q}^{l^{p}}$ est le produit d'un élément de $k_{n}^{x}$, d'une racine de l'unité, et d'une puissance $\ell^{p}$-ième ; de sorte que nous avons bien $\ell^{-p} \otimes x_{p}=\ell^{-p} \otimes x_{n} \in A_{K}$, comme annoncé .

$\left({ }^{\star}\right)$ cf. $\left[\mathrm{Gi}_{2}\right]$, section 2 , où est établie l'identité $\ell^{\Omega_{K}}=\left(\ell^{\Omega_{K}}\right)_{\infty}^{\Gamma}$. 
Dans les sections qui suivent, nous serons amenés à considé rer les produits tensoriels $T_{\ell}^{\otimes} \mathbb{Z}_{l} \Re_{K}$ et $\bar{T}_{\ell} \otimes_{Z_{l}}{ }^{A_{K}}{ }_{\infty}$. Comme nous avons convenu didentifier $\mathbb{Z}_{\ell}, \pi_{\ell}$, et $\bar{\pi}_{\ell}$ en tant que groupes abstraits, ces deux produits ne sont rien d'autre que le radical ${ }_{K} K_{\infty}$ équipé d'une action différente des groupes de Galois. Le résultat sui vant nous sera utile :

PROPOSITION 1.2.3. - Supposons $\ell \neq 2$. Alors l'intersection des sous modules respectifs de $\pi_{\ell} \otimes_{\mathbb{Z}_{l}} \Omega_{K}$ et $\bar{\pi}_{\ell} \otimes_{Z_{l}}{ }^{\Omega_{K}}$ fixés par $\Gamma_{n}$ est le sous-module de $\ell^{n}$-torsion de $R_{K}$ :

$$
\left(\pi_{\ell} \otimes_{\mathbb{Z}}{ }^{\Omega_{K}}\right)^{\Gamma_{n}} \cap\left(\bar{\pi}_{\ell} \otimes_{\mathbb{Z}}{ }^{\Omega_{K}}\right)^{\Gamma_{n}}=l^{n}{ }^{\Omega_{K}} \text {. }
$$

Démonstration : Soit $\ell^{-p} \otimes x_{p}$ un élément de lintersection . L'identité $\ell^{-p} \otimes x_{p}^{\gamma_{n}-\omega}\left(\gamma_{n}\right)=1=\ell^{-p} \otimes x_{p}^{\gamma_{n}}-\bar{w}\left(\gamma_{n}\right)$ entraîne $e^{-1} \otimes x_{p}^{\gamma_{n}-\gamma_{n}^{-1}}=1$. L'élément $l^{-p} \otimes x_{p}$, annulé par $\left(\gamma_{n}-\gamma_{n}^{-1}\right)$ est fixé par $\gamma_{n}^{2}$, donc (sous la condition $\ell \neq 2$ ) par $\Gamma_{n}$. D'après la proposition 2 nous pouvons donc supposer $x_{p} \in K_{n}^{X}$, auquel cas nous obtenons immédiatement : $\left(l^{-p} \otimes x_{p}\right) \gamma_{n}-\omega\left(\gamma_{n}\right)=\left(l^{-p} \otimes x_{p}\right)^{l^{n}}=e^{-p+n} \otimes x_{p} ;$ ce qui établit le ré sultat.

\section{b.- Rappel de la description de Tate du groupe $K_{2}\left(K_{n}\right)_{\ell}$.}

Il est bien connu, depuis le résultat de finitude de Garland, que le $K_{2}$ dlun corps de nombres est un groupe de torsion, produit di rect comme tel de ses groupes de Sylow. Plus précisément, Tate a montré, dans le cas qui nous intéresse ici dlune tour cyclotomique, que les éléments d'ordre $l$-primaire de $K_{2}\left(K_{n}\right)$ sont ceux provenant du groupe $\left(\mu_{\ell} \otimes_{\mathbb{Z}} K_{\infty}^{x}\right)^{\Gamma}=\left(\pi_{\ell} \otimes_{\mathbb{Z}}{ }_{\ell}^{\Omega_{K}}\right)_{\infty}^{\Gamma}$ par les symboles universels $(\star)$ :

$(\star)$ cf. $\left[\mathrm{Ta}_{3}\right]$ 
THÉORÈME 1.2.4. - Le $\ell$-Sylow $K_{2}\left(K_{n}\right)_{\ell}$ du groupe $K_{2}\left(K_{n}\right)_{\ell}$ est donné, pour chaque naturel $n$, par la suite exacte courte canonique de $\mathbb{Z}_{\ell}$-mo dules:

(i) $1 \longrightarrow\left(\pi_{l}^{\otimes} \mathbb{Z}_{l}^{\beta_{K}}\right)_{\infty}^{\Gamma_{n}} \longrightarrow\left(\pi_{l i v}^{\otimes} \mathbb{Z}_{l}{ }^{\Omega_{K}}\right)_{\infty}^{\Gamma_{n}} \longrightarrow K_{2}\left(K_{n}\right)_{l} \longrightarrow 1$

La détermination de la codimension du sous-groupe divisible $\left(\pi_{\ell} \otimes_{\mathbb{Z}_{\ell}}{R_{K}}_{\infty}\right)^{\Gamma}$ est un des théorèmes essentiels de Tate sur le $K_{2}$ des corps de nombres ${ }^{(*)}$. On peut même dire que ce résultat est l'équiva lent, pour la $K$-théorie, de la conjecture de Leopoldt pour la $\ell$-ramification. Avant de développer ce point de vue, disons tout de suite que le résultat de Tate peut s'énoncer de façon sensiblement plus précise en termes de représentations. Pour chaque place à l'infini $p_{n}^{\infty}$ du corps $F_{n}$, désignons par $\Delta_{p^{\infty}}$ son sous-groupe de décomposition dans l'extension abélienne $K_{n} / F_{n}$ ( qui ne dépend que de la place $p^{\infty}$ de $F_{0}$ au-des sous de $p_{n}^{\infty}$ ); notons $x_{p^{\infty}}=$ Ind $\Delta_{\Delta_{p}^{\infty}}{ }^{1}{ }_{\Delta_{p}}$ l'induit à $\Delta$ du caractère de la représentation unité de $\Delta_{\text {po }}$; et formons la somme :

$$
x_{n}^{\infty}=p_{n}^{\infty} \sum_{\infty} x_{p^{\infty}}=\left.\left[K_{n}: K_{o}\right] p_{p}^{\infty}\right|_{\infty} x_{p^{\infty}}=\left[K_{n}: K_{o}\right] x^{\infty} .
$$

Les résultats du chapitreIV.2 (cf. $2 \xi c$ ) assurent l'existence d'un isomorphisme de $\mathbb{Z}_{\ell}[\Delta]$-modules :

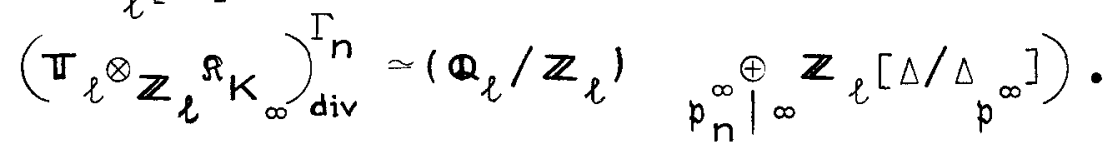

Autrement dit :

SCOLIE 1.2.5. - Le sous-groupe divisible maximal $\left(\pi_{l} \otimes_{\mathbb{Z}_{l}} \Omega_{K_{\infty}}\right)^{\Gamma}$ div du groupe $\left(\pi_{\ell} \otimes_{\mathbb{Z}_{l}}{ }^{\Omega_{K}}\right)_{\infty}^{\Gamma}$ est un $\mathbb{Z}_{l^{-m o d u l e} \text { divisible de cotype fini } . ~ S a}$ codimension est égale au nombre de places complexes $c_{n}$ du corps $K_{n}$ :

$$
\left(\Pi_{\ell} \otimes_{\mathbb{Z}_{l}}{ }^{\Omega_{K}}\right)_{\text {div }}^{\Gamma_{n}} \simeq\left(\mathbb{Q}_{\ell} / \mathbb{Z}_{\ell}\right)^{C_{n}} \text {. }
$$

(*) II s'agit de la conjecture principale énoncée dans $\left[\mathrm{Ta}_{1}\right]$. Une dé monstration cyclotomique de cette conjecture fait l'objet de $\left[\mathrm{Ta}_{2}\right]$. Ultérieurement, Tate en a produit une preuve cohomologique $\left[\mathrm{Ta}_{3}\right]$. 
Plus précisément, son dual de Pontrjagin $\left[\left(\pi_{\ell} \otimes_{\mathbb{Z}} \Omega_{K_{\infty}}\right)_{\text {dir }}^{I_{n}}\right]^{\nu}=$ $\operatorname{Hom}\left(\left(\mathbb{T}_{\ell} \otimes_{\mathbb{Z}_{\ell}}{\Omega_{K}}_{\infty}\right)_{\text {div }}^{\Gamma_{n}}, \mathbb{Q}_{\ell} / \mathbb{Z}_{\ell}\right)$ est un $\mathbb{Z}_{\ell}[\Delta]$-module projectif, qui a pour caractère la somme $\chi_{n}^{\infty}=\left[K_{n}: K_{0}\right] \chi^{\infty}$ des induits à $\Delta$ des carac tères des représentations unités des sous-groupes de décomposition des places à liinfini du corps $F_{n}$ dans l'extension $K_{n} / F_{n}$.

On notera que le caractère $\chi^{\infty}$, somme d'induits de caractères unités, est égal à son pseudo-inverse $\bar{X}^{\infty}$ (défini par $\bar{X}^{\infty}(\tau)=X^{\infty}\left(\tau^{-1}\right)$ ).

\section{c.- Définition des groupes $\bar{K}_{2}\left(K_{n}\right)$, et interprétation kummérienne.}

THÉORËME \& DÉFINITION 1.2.6.- Par $\bar{K}_{2}\left(K_{n}\right)$ nous entendons le quotient du groupe $\left(\bar{\pi}_{\ell} \otimes_{\mathbb{Z}} \Omega_{K_{\infty}}\right)^{\Gamma_{n}}$ par son sous-module divisible maximal $\left(\bar{\pi}_{\ell} \otimes_{\mathbb{Z}}{ }_{\ell}^{\Omega_{K}}\right)_{\infty}^{\Gamma_{n}}$ div , clest-à-dire le $\ell$-groupe de torsion défini par l'exac titude de la suite courte:

$$
1 \longrightarrow\left(\bar{\pi}_{\ell}^{\otimes} \mathbb{Z}_{\ell}{ }^{\Omega_{K}}\right)_{\infty}^{\Gamma_{\text {div }}} \longrightarrow\left(\bar{\pi}_{\ell}^{\otimes} \mathbb{Z}_{\ell}{ }^{\Omega_{K}}\right)^{\Gamma_{n}} \longrightarrow \bar{K}_{2}\left(K_{n}\right) \longrightarrow 1 .
$$

Le groupe $\bar{K}_{2}\left(K_{n}\right)$ décrit, via la théorie de Kummer, l'ensem ble des $l$-extensions de $K_{\infty}$ qui proviennent par composition diune exten sion abélienne de $K_{n}$, modulo celles qui proviennent par composition diune

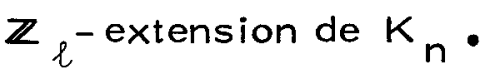

Démonstration : Désignons par $\mathcal{K}_{K_{\infty}}$ le groupe de Galois de la $\ell$-extension abélienne maximale de $K_{\infty}$, puis par $\Gamma_{n} X_{K_{\infty}}$ son quotient des genres re latif à l'extension procyclique $K_{\infty} / K_{n}$ (i.e.le plus grand quotient de $\mathcal{K}_{K_{\infty}}$ sur lequel $\Gamma_{n}$ opère trivialement). Nous avons $I_{n} \chi_{K}=\chi_{K} / \gamma_{K_{\infty}}^{\gamma_{n}-1}$, d'où :

$$
\begin{aligned}
\left(\bar{\pi}_{l} \otimes_{\mathbb{Z}_{l}}{ }^{K_{K}}\right)^{\Gamma n}=\left(\bar{\pi}_{l} \otimes_{\mathbb{Z}_{l}} \operatorname{Hom}\left(x_{K_{\infty}}, \mu_{l}\right)\right)^{\Gamma n} & =\operatorname{Hom}_{\Gamma_{n}}\left(x_{K_{\infty}}, \mathbb{Q}_{l} / \mathbb{Z}_{l}\right) \\
& =\operatorname{Hom}\left({ }^{\Gamma_{n}} x_{K_{\infty}}, \mathbb{Q}_{l} / \mathbb{Z}_{l}\right) .
\end{aligned}
$$

Et comme ${ }^{\Gamma}{ }^{\Gamma} K_{K_{\infty}}$ est le groupe de Galois de la plus grande $\ell$-extension 
de $K_{\infty}$ qui est abélienne sur $K_{n}$, son dual de Pontrjagin $\left(\bar{\pi}_{\ell} \otimes_{Z_{l}}{ }^{R_{K}}\right)_{\infty}^{\Gamma_{n}}=$ $\left(\bar{\mu}_{\ell} \otimes_{\mathbb{Z}} K_{\ell}^{X}\right)^{\Gamma_{n}}$ est bien le radical kummérien associé . En particulier son sous-groupe divisible maximal $\left(\bar{\pi}_{\ell} \otimes_{\mathbb{Z}_{l}}{ }^{I_{K}}\right)_{\infty}^{\Gamma_{n}}$ div est bien le radical sur $K_{\infty}$ de la composée $Z_{n}$ des $\mathbb{Z}_{\ell}$-extensions de $K_{n}$.

La conjecture de Leopoldt pour le corps $K_{n}$ ( et le nombre pre mier $\ell$ ) affirme l'existence d'exactement $\left(c_{n}+1\right) \mathbb{Z}_{\ell}$-extensions linéai rement indépendantes sur $K_{n}$, c'est-à-dire précisément l'égalité entre la codimension du groupe divisible $\left(\bar{\pi}_{\ell} \ell_{Z} l^{\Omega_{K}}\right)_{\infty}^{\Gamma_{n}}$ et celle $c_{n} d u$ groupe $\left(\pi_{\ell} \otimes_{\mathbb{Z}}{ }_{\ell}^{\Omega_{K}}\right)_{\infty}^{\Gamma_{n}}$ div . On sait, de façon générale, que la codimen sion du radical $\left(\bar{\pi}_{\ell} \otimes_{\mathbb{Z}}{ }_{\ell} K_{K_{\infty}}\right)_{\text {div }}^{\Gamma_{n}}$ n'excède $c_{n}$ que d'une quantité finie $d_{n}$, qui mesure donc le défaut de la conjecture de Leopoldt pour $K_{n}$, et qui reste d'ailleurs bornée lorsquion monte la tour cyclotomique $K_{\infty} / K_{0}$. Comme plus haut, ces résultats s'expriment en outre de façon plus précise en termes de représentations, la quantité $d_{n}$ s'interprétant comme le degré du caractère de défaut pour la conjecture de Leopoldt dans le corps $K_{n}$ (cf. Ch. IV.2, $2 \S \mathrm{b}$ ), le point remarquable étant que, sous la condition $d_{n}=0$, les groupes $\left(\bar{\pi}_{l} \otimes_{Z_{l}} \Omega_{K}\right)_{\infty}^{\Gamma_{n}}$ et $\left(\pi_{l i v}^{\otimes} \mathbb{Z}_{l} \Omega_{K}\right)_{\infty}^{\Gamma_{n}}$ définissent des représentations isomorphes (cf. Ch. IV.2, $2 \S c$ ):

SCOLIE 1.2.7. - Le sous-groupe divisible maximal $\left(\bar{\pi}_{\ell} \otimes_{Z}{ }_{\ell}^{\Omega_{K}}\right)_{\infty}^{\Gamma_{n}} d u$ groupe $\left(\overline{\mathbb{T}}_{\ell} \otimes_{\mathbb{Z}}{ }_{\ell}^{\Omega_{K}}\right)_{\infty}^{\Gamma_{n}}$ est un $\mathbb{Z}_{\ell}$-module de cotype fini, qui a pour codimension le nombre $c_{n}$ de places complexes du corps $K_{n}$ augmenté $d u$ défaut $d_{n}$ de la conjecture de Leopoldt :

$$
\left(\bar{\pi}_{\ell} \mathbb{Z}_{\ell}{ }^{\Omega_{K}}\right)_{\text {div }}^{\Gamma_{n}} \simeq\left(\mathbb{Q}_{\ell} / \mathbb{Z}_{\ell}\right)^{c_{n}+d_{n}} .
$$

Plus précisément, lorsque le défaut $d_{n}$ est nul, i.e. lorsque le corps $K_{n}$ vérifie la conjecture de Leopoldt, les groupes $\left(\bar{\pi} l^{\otimes} \mathbb{Z} l^{R_{K}}\right)_{\infty}^{\Gamma_{n}}$ et $\left(T_{l} \otimes_{\mathbb{Z}} \Omega_{K}\right)_{\infty}^{\Gamma_{n}}$ sont isomorphes comme $\mathbb{Z}_{\ell}[\Delta]$-modules . 


\section{2. - INTRODUCTION DU NOYAU RÉGULIER $\Re$.}

Le groupe $\Omega_{K_{\infty}}$ étant très gros, il est commode de faire inter venir un sous-groupe canonique plus petit, le noyau régulier $\Re_{K_{\infty}}$, que I'on peut définir comme suit :

DÉFINITION 1.2.8. - Etant donné un corps de nombres $K$, désignons par $D_{K}^{\prime}$ le groupe des diviseurs ${ }^{(*)}$ du localise $\theta_{K}^{\prime}=\theta_{K}[1 / \ell]$ en-dehors de $l$ de l'anneau des entiers de $K$. L'application canonique $x \rightarrow(x)^{\prime}$ de $K^{X}$ dans $I_{K}^{\prime}$ induit une surjection naturelle du radical universel $\Omega_{K}=\left(Q_{l} / \mathbb{Z}_{\ell}\right) \otimes_{\mathbb{Z}} K^{X}$ sur le produit tensoriel $\left(Q_{\ell} / \mathbb{Z}_{\ell}\right) \otimes \mathbb{Z}_{K} D_{K}^{\prime}$. Nous disons que le noyau de cette application est le noyau régulier du radical universel, et nous notons :

$$
\Re_{K}=\left\{l^{-k} \otimes x \in R_{K} \mid(x)^{\prime} \in D_{K}^{\prime l^{k}}\right\}
$$

Avant de justifier l'appelation de noyau régulier donnée au grou pe $\Re_{K}$, démontrons rapidement les analogues des propositions 2 et 3 pour les groupes $\Re_{K_{n}}$.

PROPOSITION 1.2.9.- Dans une tour cyclotomique, les noyaux régu liers vérifient la théorie de Galois; ce qui s'écrit :

$$
\Re_{K_{n}}=\Re_{K_{\infty}}^{\Gamma_{n}}
$$

Démonstration : Les places étrangères à $\ell$ étant non ramifiées dans une

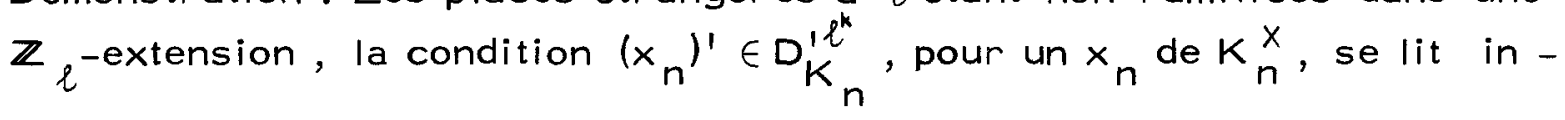
différemment dans un $K_{n}$ ou dans $K_{\infty}$; dioù le résultat annoncé, en vertu de la proposition 2 .

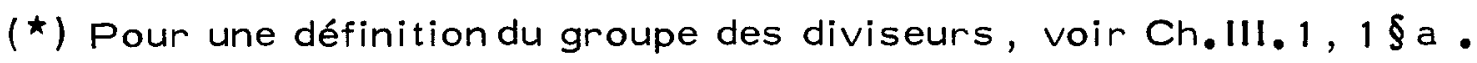


PROPOSITION 1.2.10.- Supposons $l \neq 2$. Alors l'intersection des sousmodules respectifs de $\bar{T}_{\ell} \mathbb{Z}_{\ell} \Re_{K_{\infty}}$ et $\overline{\mathrm{T}}_{\ell}{ }^{\otimes} \mathbb{Z}_{\ell}{ }^{R_{K}}$ fixés par $\Gamma_{n}$ est le sous-module de $\ell^{\mathrm{n}}$-torsion de $\Re_{K}$ :

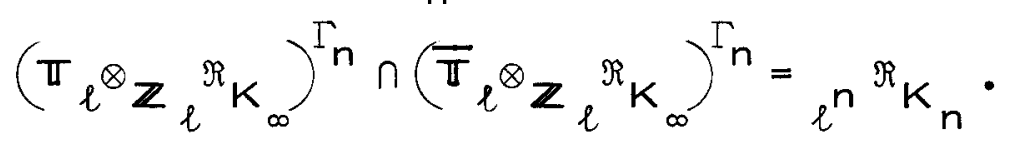

Démonstration : Cela résulte immédiatement de la proposition 3 , et de la proposition $9 \mathrm{ci-dessus.}$

a.- Interprétation symbolique: le groupe $R_{2}\left(\mathrm{~K}_{\mathrm{n}}\right)_{\ell} \cdot$

Rappelons d'abord la définition des symboles réguliers attachés à un corps de nombres $K$ : Pour chaque place non complexe $p$ de $K$, dé signons par $v_{p}$ la valuation qui lui correspond, et par $\langle,\rangle_{p}$ le carac tère crochet associé :

- si $p$ est une place finie, le crochet $\langle x\rangle_{p}$ dlune $p$-unité $x$ de $K^{X}$ est llunique racine de l'unité, d'ordre étranger à $p$, du complété $K_{p}$, qui vérifie la congruence $\langle x\rangle_{p} \equiv x(\bmod p)$;

- si p est une place réelle, le crochet $\langle x\rangle_{p}$ dlun élément $x$ de $K^{X}$ n'est autre que son signe dans le complété réel $K_{p}$.

L'application bilinéaire :

$$
(x, y) \mapsto(x, y)_{p}=\left\langle(-1)^{v_{p}(x) v_{p}(y)} x^{v_{p}(y)} / y_{p}^{v_{p}(x)}\right\rangle_{p}
$$

est alors un symbole sur $K$, à valeurs dans le groupe des racines de l'unité d'ordre étranger à $p$ du complété de $K$ pour la place $p$, appelé symbole régulier associé à $p$. En fait, les groupes étudiés ici étant tous des $\mathbb{Z}_{\ell} \ell^{\text {-modules }}$, il est plus commode de travailler non pas sur le sym bole régulier lui-même, mais sur sa $\ell$-partie, que nous continuons, par abus, à noter $(,)_{p}$. Cela étant,

- si p est une place de $K$ au-dessus de $\ell$, le $\ell$-symbole régulier $(,)_{p}$ est le symbole trivial;

- si p en revanche est étrangère à $\ell$, le $\ell$-symbole régulier $(,)_{p}$ prend ses valeurs dans le $\ell$-Sylow tout entier $\mu_{p}$ du groupe des racines de l'unité du complété $K_{\mathfrak{p}}$ de $K$ pour la place non complexe $p$. 
Considérons maintenant la suite exacte courte qui définit le groupe $\Re_{K_{\infty}}$ :

$$
1 \longrightarrow \Re_{K_{\infty}} \longrightarrow R_{K_{\infty}} \longrightarrow\left(\mathbb{Q}_{\ell} / \mathbb{Z}_{\ell}\right) \otimes_{\mathbb{Z}} D_{K_{\infty}}^{\prime} \longrightarrow 1
$$

Tensorisant par $\pi_{\ell}$, puis prenant les points fixes par $\Gamma_{n}$, nous obtenons la suite exacte de cohomologie :

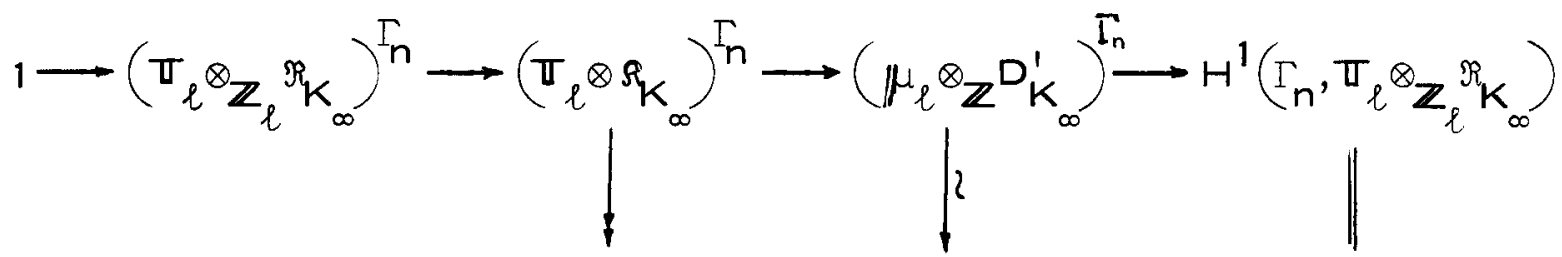

$$
\begin{aligned}
& K_{2}\left(K_{n}{ }^{\prime} \ell \stackrel{r}{\longrightarrow} \underset{p_{n} \mid \ell}{\oplus} \mu_{p_{n}}\right.
\end{aligned}
$$

Dans celle-ci, le terme de droite $H^{1}\left(\Gamma_{n}, T_{\ell} \otimes_{\mathbb{Z}_{\ell}} \Re_{K}\right)$ est nul en vertu d'un résultat de Tate ${ }^{(*)}$; le groupe $\left(\mu_{\ell} \otimes_{\mathbb{Z}} D_{K}^{\prime}\right)_{\infty}^{\Gamma_{n}}$ s'identifie à la somme $p_{n} \mid \ell \mu_{p_{n}}$ des $\ell$-Sylow des groupes de racines de l'unité des complétés respectifs de $K_{n}$ pour les places finies étrangères à $\ell$ (II suffit, pour s'en convaincre, de regarder la somme $\underset{p_{n} \mid \ell}{\oplus} \mu_{p_{n}}$, correspondant au corps $K_{\infty}$, comme étant plongée diagonalement dans celle $p_{\infty} \stackrel{\oplus}{\mu} \mathcal{L}_{\infty}$, correspondant à $K_{\infty}$, et d'avoir à l'esprit l'isomorphisme canonique $\left.\mu_{\ell}{ }^{\otimes} \mathbb{Z}_{K} \simeq p_{\infty}\right|^{\oplus} l^{\mu} p_{\infty}$ '; enfin l'application $r$ est induite par les sym boles réguliers, via la propriété universelle du $K_{2}$.

II vient donc :

PROPOSITION 1.2.11.-Le $l$-Sylow $R_{2}\left(K_{n}\right)_{l}$ du noyau dans $K_{2}\left(K_{n}\right)$ des symboles réguliers est l'image dans $K_{2}\left(K_{n}\right)$ du noyau régulier $\left(T_{\ell} \mathbb{Z}_{\ell} \Re_{K}\right)^{\Gamma_{n}}$ du groupe $\left(T_{\ell} \mathbb{Z}_{\ell}{ }^{\Omega_{K}}\right)_{\infty}^{\Gamma_{n}} ;$ ce qui se traduit par l'exactitude de la suite courte canonique :

(ii) $1 \longrightarrow\left(\mathrm{T}_{\ell} \otimes_{\mathbb{Z}_{\ell}}{ }^{R_{\mathrm{K}}}\right)_{\infty}^{\Gamma_{\mathrm{div}}} \longrightarrow\left(\mathrm{T}_{\ell} \otimes_{\mathbb{Z}_{\ell}}{ }^{R_{\mathrm{K}}}\right)^{\Gamma_{\mathrm{n}}} \rightarrow R_{2}\left(\mathrm{~K}_{\mathrm{n}}\right)_{\ell} \longrightarrow 1$.

( ) II s'agit de la conjecture principale, qui est démontrée dans $\left[\mathrm{Ta}_{2}\right]$. 
Le théorème de Garland ${ }^{\left({ }^{\star}\right)}$ sur le $K_{2}$ des corps de nombres montre que le groupe $R_{2}\left(K_{n}\right)$ est fini. Ainsi, puisque le groupe $\left(T_{\ell} \otimes_{Z}{ }^{\Omega_{K}}\right)^{\Gamma_{n}}$ est de cotype fini, il en est de même du groupe $\left(T_{\ell} \otimes_{Z_{\ell}} \Re_{K}\right)$. En particulier, les éléments divisibles de $\left(T_{\ell} \otimes_{Z_{l}} \Re_{K}\right)^{\Gamma_{n}}$ sont exactement ceux de hauteur infinie.

Autrement dit :

SCOLIE 1.2.12. - Pour chaque naturel n, les éléments divisibles du groupe $\left(\mathbb{T}_{\ell} \mathbb{Z}_{\ell}{ }^{\Omega_{K}}\right)^{\Gamma_{n}}$ sont ceux de hauteur infinie dans le sous-groupe de cotype fini $\left(\pi_{\ell} \otimes_{\mathbb{Z}_{\ell}}{ }^{\Re} K_{\infty}\right)^{\Gamma_{n}}$; ce qui s'écrit :

$$
\left(\pi_{l}^{\otimes} \mathbb{Z}_{l}{ }^{R_{K}}\right)_{\infty}^{\Gamma_{\text {div }}}=\left(\pi_{l}^{\otimes_{\mathbb{Z}_{l}}}{ }^{R_{K}}\right)_{\infty}^{\Gamma_{\text {div }}}=\left(\pi_{l} \otimes_{\mathbb{Z}_{l}} \Re_{K_{\infty}}\right)_{\infty}^{\Gamma_{n}} .
$$

\section{b.- Interprétation kummérienne : le groupe $\bar{R}_{2}\left(\mathrm{~K}_{\mathrm{n}^{\prime} l}{ }_{l} \cdot\right.$}

D'après la théorie de Kummer, la condition de puissance $\ell^{k}$ ième $(x)^{\prime} \in D_{K}^{\prime \ell^{k}}$, qui définit les éléments $\ell^{-k} \otimes x$ du noyau régulier $\Re_{K}$, caractérise les extensions $K_{\infty}\left[\sqrt[l^{k}]{x}\right] / K_{\infty}$ qui sont $\ell$-ramifiées (clest-àdire non ramifiées aux places finies étrangères à $\ell)^{(\star \star)}$. Le groupe $\overline{\mathrm{T}}_{\ell} \otimes_{\mathbb{Z}_{\ell}} \Re_{K_{\infty}}$ est donc le radical kummérien de la $\ell$-extension abélienne maximale $\ell$-ramifiée $M_{\infty}$ de $K_{\infty}$. En particulier, son sous-groupe in variant $\left(\mathbb{T}_{\ell} \otimes_{\mathbb{Z}_{\ell}} \Re_{K}\right)_{\infty} \Gamma_{n}$ décrit les $\ell$-extensions de $K$ qui proviennent par composition dlune extension abélienne $\ell$-ramifiée de $K_{n}$ (la condition de $l$-ramification se lisant indifféremment sur $K_{n}$ ou sur $K_{\infty}{ }^{\prime}$. Plus précisément, si $a$ est le groupe de Galois Gal $\left(\mathrm{M}_{\infty} / \mathrm{K}_{\infty}\right)$ et $\Gamma_{n} a=a / a^{Y_{n}-1}$ son quotient des genres relativement à l'extension pro cyclique $\mathrm{K}_{\infty} / \mathrm{K}_{\mathrm{n}}$, nous avons :

(*) Il s'agit en fait d'un théorème d'analyse harmonique, cf. [Ba] pour plus de détails.

$\left({ }^{\star}\right)$ Les corps $K_{n}$ étant complètement imaginaires, il n'y a pas lieu de se préoccuper des places à l'infini . 


$$
\left(\overline{\mathbb{U}}_{\ell} \otimes_{\mathbb{Z}_{\ell}} \mathfrak{R}_{K_{\infty}}\right)^{\Gamma_{n}}=\operatorname{Hom}_{\Gamma_{n}}\left(a, \mathbb{Q}_{\ell} / \mathbb{Z}_{\ell}\right)=\operatorname{Hom}\left({ }^{\Gamma_{n}} a, \mathbb{Q}_{l} / \mathbb{Z}_{l}\right) \text {. }
$$

DÉFINITION \& THÉORËME 1.2.13.- Par $\bar{R}_{2}\left(\mathrm{~K}_{\mathrm{n}}\right)_{l}$ nous entendons le quotient du groupe $\left(\bar{\pi}_{\ell} \otimes_{\mathbb{Z}_{l}} \Re_{K}\right)^{\Gamma_{n}}$ par le sous-groupe divisible $\left(\bar{\pi}_{\ell} \otimes_{\mathbb{Z}} R_{K}\right)_{\infty}^{\Gamma_{n}}$, c'est-à-dire le $\ell$-groupe de torsion défini par l'exactitude de la suite courte:

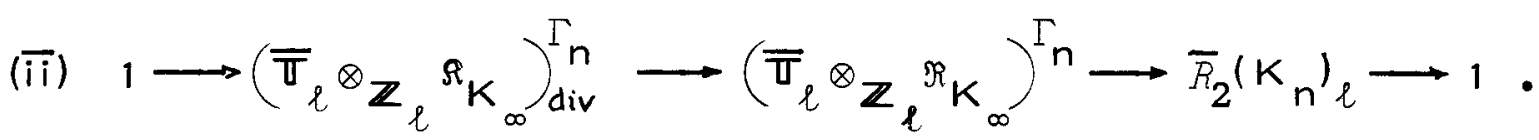

Le groupe $\bar{R}_{2}\left(K_{n}\right)$ est un $\ell$-groupe fini, qui s'identifie au dual de Pontrjagin $\sigma_{n}^{v}$ du sous-groupe de torsion $\sigma_{n}=a_{n}^{\text {tor }}$ du groupe de Galois $a_{n}=\operatorname{Gal}\left(M_{n} / K_{n}\right)$ de la $l$-extension abélienne maximale $l$-ra mifiée du corps $K_{n}$.

Démonstration : La tour cyclotomique $K_{\infty} / K_{n}$ étant une $\mathbb{Z}_{l}$-extension, les groupes $\Gamma_{n} a=\operatorname{Gal}\left(M_{n} / K_{\infty}\right)$ et $a_{n}=\operatorname{Gal}\left(M_{n} / K_{n}\right)$ ont le même sous-groupe de torsion $\boldsymbol{G}_{n}$, conformément au schéma de corps :

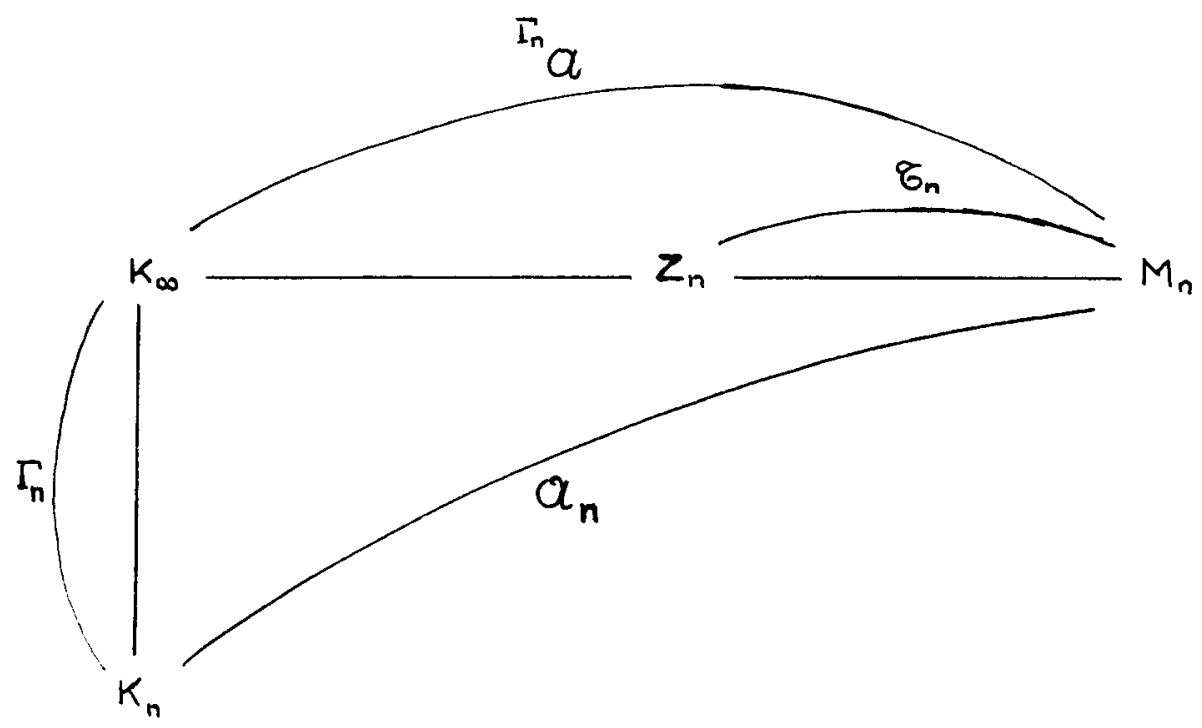

Cela étant, le groupe $\left(\bar{\pi}_{\ell} \otimes_{\mathbb{Z}_{\ell}} \Re_{K_{\infty}}\right)^{\Gamma_{n}}$, qui est le dual de Pontrjagin de $\Gamma_{n} a$, contient le groupe divisible $\left(\bar{\pi}_{\ell} \otimes_{\mathbb{Z}_{\ell}} \Omega_{K}\right)_{\infty}^{\Gamma_{n}}$, qui est le dual de Pontrjagin du quotient $\Gamma_{n} a / \sigma_{n}=\operatorname{Gal}\left(z_{n} / K_{n}\right)$; et la suite $(\overline{i i})$ a bien 
un sens. Cela étant, la théorie du corps de classes montre que le groupe $a_{n}$ est un $\mathbb{Z}_{\ell}$-module de type fini . Le sous-groupe de torsion $\sigma_{n}$ est donc fini, et le même résultat vaut pour son dual $\bar{R}_{2}\left(\mathrm{~K}_{n}\right)_{\ell}$, ce qui achève la démonstration .

SCOLIE 1.2.14.- Pour chaque naturel $n$, les éléments divisibles du groupe $\left(\bar{\pi}_{\ell} \otimes_{Z}{ }_{\ell}{ }_{K}\right)^{\Gamma_{n}}$ sont ceux de hauteurinfinie dans le sous-groupe de cotype fini $\left(\mathbb{U}_{\ell} \otimes_{\mathbb{Z}_{\ell}}{ }^{\Re_{K}}\right)_{\infty}^{\Gamma_{n}}$; ce qui s'écrit :

$$
\left(\bar{\pi}_{\ell} \otimes_{\mathbb{Z}_{\ell}}^{\Omega_{K}}\right)_{\infty}^{\Gamma_{\text {div }}}=\left(\bar{\pi}_{\ell} \otimes_{\mathbb{Z}_{l}}{ }^{R_{K}}\right)_{\infty}^{\Gamma_{\text {div }}}=\left(\bar{\pi}_{\ell} \otimes_{\mathbb{Z}_{\ell}} \Re_{K}\right)_{\infty}^{\Gamma_{n}} .
$$

\section{c.- Interprétation régulière de la conjecture de Leopoldt.}

Pour chaque naturel $n$, désignons par $\Lambda_{n}=\mathbb{Z}_{l}\left[\left[\gamma_{n}-1\right]\right]$ l'al gèbre d'Iwasawa construite sur le groupe $\Gamma_{n}$, puis par $\zeta$ le sous-groupe de $\Lambda_{n}$-torsion du groupe de Galois $a=G a l\left(M_{\infty} / K_{\infty}\right)$ de la $\ell$-extension abélienne maximale $\ell$-ramifiée du corps $K_{\infty}{ }^{(\star)}$. Nous savons, par un théorème d'Iwasawa ( $\mathrm{cf}$. $[\mathrm{Iw}] \S 8$, th. 18), que le groupe $a$ n'a pas de $\Lambda_{\mathrm{n}}$-sous-module fini non nul, et que son quotient $a / G$ est $\Lambda_{\mathrm{n}}$-pseudoisomorphe au produit de $c_{n}$ copies de l'algèbre $\Lambda_{n}$. En particulier, le groupe de Galois $a / \sigma a^{Y_{n}-1}$ est donc le produit direct de $c_{n}$ copies de $\mathbb{Z}_{\ell}$, et la conjecture de Leopoldt, qui affirme que le même résultat vaut pour le quotient des genres $\Gamma_{n} a=a / a^{\gamma_{n}-1}$, postule que le polynôme caractéristique du $\Lambda_{\mathrm{n}}$-module de torsion $\mathcal{G}$ est étranger au po lynôme cyclotomique $\omega_{n}=\gamma_{n}-1$, autrement dit, que $\sigma$ ne possède pas de sous-module infini fixé par $\Gamma_{n}$. En résumé, la conjecture de Leopoldt pour le corps $K_{n}$ s'écrit tout simplement :

$$
a^{\Gamma_{n}}=1
$$

D'un autre côté, la théorie de Kummer nous donne les iso morphismes:

(*) Comme on peut le vérifier sans peine, la définition de $\mathcal{G}$ est, en fait, indépendante de $n$. 


$$
\begin{aligned}
& a^{\Gamma_{n}}=\operatorname{Hom}_{\Gamma_{n}}\left(\Re_{K_{\infty}}, \mu_{l}\right)=\operatorname{Hom}_{\Gamma_{n}}\left(\bar{\pi}_{l} \otimes_{\mathbb{Z}_{l}} \Re_{K_{\infty}}, \mathbb{Q}_{l} / \mathbb{Z}_{l}\right) \\
& =\operatorname{Hom}\left({ }^{\Gamma_{n}}\left(\bar{\pi}_{\ell} \otimes_{\mathbb{Z}_{l}} \Re_{K_{\infty}}\right), \mathbb{Q}_{\ell} / \mathbb{Z}_{\ell}\right), \mathrm{i}_{\bullet} \text { e. } \\
& a^{\Gamma_{n}}=\operatorname{Hom}\left(H^{1}\left(\Gamma_{n}, \bar{T}_{\ell} \otimes_{\mathbb{Z}_{l}} \Re_{K_{\infty}}\right), \mathbb{Q}_{\ell} / \mathbb{Z}_{\ell}\right)=\stackrel{v}{H}^{1}\left(\Gamma_{n}, \bar{T}_{l} \otimes_{\mathbb{Z}_{l}} \Re_{K_{\infty}}\right) ;
\end{aligned}
$$

de sorte que la conjecture de Leopoldt pour $K_{n}$ s'écrit encore :

$$
H^{1}\left(\Gamma_{n}, \vec{T}_{\ell}^{\otimes} \mathbb{Z}_{\ell} \Re_{\infty}\right)=1
$$

Revenons maintenant sur la suite exacte courte qui définit le noyau régulier $\Re_{K_{\infty}}$ :

$$
1 \longrightarrow R_{K_{\infty}} \longrightarrow R_{K_{\infty}} \longrightarrow\left(Q_{l} / \mathbb{Z}_{\ell}\right) \otimes_{\mathbb{Z}} D_{K_{\infty}}^{\prime} \longrightarrow 1 \text {. }
$$

Tensorisant par $\overline{\mathrm{U}}_{\ell}$, puis prenant les points fixes par $\Gamma_{n}$, nous obte nons la suite exacte de cohomologie:

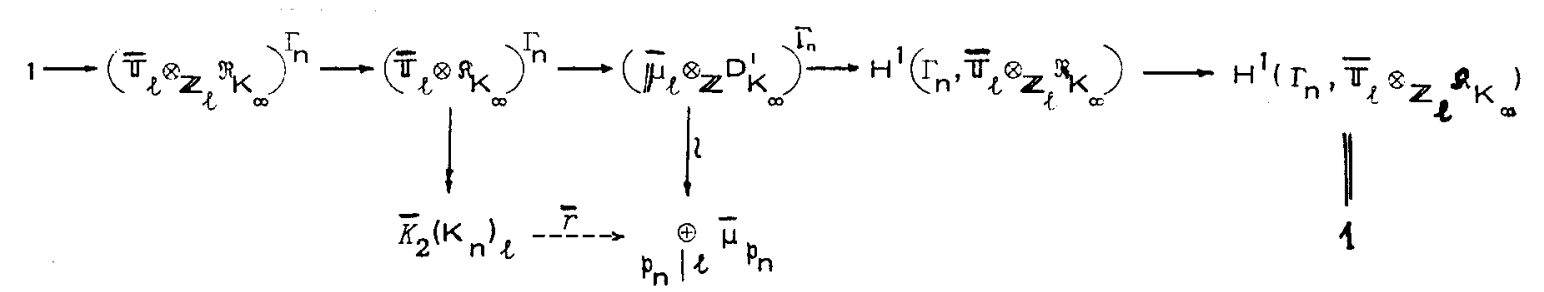

Dans celle-ci, le groupe $H^{1}\left(I_{n}, \overline{\mathbb{U}}_{\ell} \otimes_{\mathbb{Z}_{\ell}}{ }^{\Omega_{K}}\right)=\left(\mu_{\ell} \otimes_{\mathbb{Z}^{K}} K_{\infty}^{x}\right) /\left(\mu_{\ell} \otimes_{\mathbb{Z}^{K}} K_{\infty}^{X} \gamma_{n}-1\right.$ est nul, par un argument de Tate ${ }^{(\star)} ;$ le groupe $\left(\mu_{\ell} \otimes_{\mathbb{Z}} D_{K}^{\prime}\right)_{\infty}^{\Gamma_{n}}$ s'identifie, par un argument déjà utilisé, à la somme directe des opposés des l-Sylow des groupes de racines de l'unité des complétés respectifs de $K_{n}$ pour les places finies étrangères à $\ell$ (via l'isomorphisme canonique $\bar{\mu}_{\ell} \otimes_{\mathbb{Z}} D_{K}^{\prime} \simeq \underset{p_{\infty} \backslash l}{\oplus} \bar{\mu}_{p_{\infty}}$, et l'injection diagonale de $\underset{p_{n}{ }_{Y l}}{\oplus} \bar{\mu}_{p_{n}}$ dans $\left.p_{\infty} \stackrel{\oplus}{X \ell}^{\oplus} \bar{\mu}_{p_{\infty}}\right)$; enfin, l'application $\bar{r}$ est induite par les symboles réguliers.

$\left.{ }^{*}\right)$ Les valeurs spectrales de llaction de $\gamma_{n}$ sur $K_{n}{ }_{n}$ étant des racines de l'unité, leur produit par $w\left(\gamma_{n}\right)$ n'est jamais 1 , de sorte que $\left(\gamma_{n}-1\right)$ opère sur le produit $\mathbb{H}_{\ell} \otimes \mathbb{Z}^{K^{X}}$ surjectivement . 
Autrement dit :

THÉORËME 1.2.15. - Les quatre conditions suivantes sont équivalentes:

(i) La conjecture de Leopoldt est vérifiée dans le corps $K_{n}$ (pour le nombre premier $\ell$ ).

(ii) Le groupe de Galois $a=\operatorname{Gal}\left(M_{\infty} / K_{\infty}\right)$ de la $l$-extension abélienne maximale $\ell$-ramifiée sur la tour cyclotomique $K_{\infty}$ n'a pas de sous-module non nul fixé par $I_{n}$, i.e. $a^{I_{n}}=1$.

(iii) Le groupe de cohomologie $H^{1}\left(I_{n}, T_{\ell} \otimes_{\mathbb{Z}_{l}} \Re_{K_{\infty}}\right)$ est nul.

(iv) Les symboles réguliers induisent un épimorphisme du groupe $\bar{K}_{2}\left(K_{n}\right)_{l}$ sur la somme directe $\underset{p_{n} \chi_{\ell}}{\oplus} \bar{\mu}_{p}$ des opposés respectifs

des $\ell$-Sylow des groupes de racines de l'unité des complétés de $K_{n}$ pour les places non complexes étrangères à $\ell$.

SCOLIE 1.2.16.-Pour chaque naturel $n$, les symboles réguliers attachés aux places non complexes du corps $K_{n}$ donnent naissance à la suite exacte courte de $K$-théorie :

$$
1 \longrightarrow R_{2}\left(K_{n}\right)_{l} \longrightarrow K_{2}\left(K_{n}\right)_{l} \stackrel{r}{\longrightarrow} \underset{p_{n}}{\stackrel{\oplus}{X} \mu^{\mu}} \mu_{p} \longrightarrow 1 \text {. }
$$

Et la conjecture de Leopoldt dans $K_{n}$ postule l'existence diune suite ana logue pour les modules opposés :

$(\overline{\mathrm{iii}}) \quad 1 \longrightarrow \bar{R}_{2}\left(\mathrm{~K}_{\mathrm{n}}\right)_{\ell} \longrightarrow \bar{K}_{2}\left(\mathrm{~K}_{\mathrm{n}}\right)_{\ell} \stackrel{\bar{r}}{\longrightarrow} \underset{p_{n} \mathrm{X}_{l}}{\oplus} \mu_{p}-1$.

Ce dernier résultat sera précisé dans la section qui suit .

\section{3. - INTRODUCTION DU NOYAU HILBERTIEN $\mathscr{B}$.}

La définition du noyau régulier ne faisant intervenir que les places étrangères au nombre premier $\ell$, il estnécessaire, pour prendre en compte les places au-dessus de $\ell$, de faire intervenir un groupe plus petit, le noyau hilbertien, que l'on peut définir comme suit :

DÉFINITION 1.2.17.- Pour chaque naturel $n$, nous appelons noyau hil bertien attaché au corps $K_{n}$, et nous notons $\mathfrak{S}_{K_{n}}$ le sous-groupe du radical universel $A_{K_{n}}=\left(\mathbb{Q}_{\ell} / \mathbb{Z}_{\ell}\right) \otimes_{\mathbb{Z}} K_{n}^{X}$, qui est engendré par les nor mes cyclotomiques : 
$\mathscr{B}_{K_{n}}=\left\{\mathfrak{x} \in \mathbb{R}_{K_{n}} \mid \mathbb{H} k \in \mathbb{N}, \forall m \geq n\right.$, 岛 $\left.x_{m} \in K_{m}^{x}, \quad \mathfrak{E}=l^{-K} \otimes N_{m / n}\left(x_{m}\right)\right\}$. La réunion $\mathscr{S}_{K^{\prime}}=\frac{\lim }{\mathrm{n}} \mathfrak{S}_{K_{\mathrm{n}}}$ est, par définition, le noyau hilbertien de la tour cyclotomique $\mathrm{K}_{\infty}$.

Bien entendu, les groupes $\mathfrak{S}_{3} K_{n}$ vérifient les deux propriétés attendues :

PROPOSITION 1.2.18.- Dans une tour cyclotomique, les noyaux hilbertiens vérifient la théorie de Galois; ce qui s'écrit :

$$
\mathfrak{S}_{\mathrm{K}}=\mathfrak{S}_{\mathrm{K}}^{\Gamma_{\mathrm{n}}}
$$

Démonstration : II s'agit de slassurer de l'égalité $\mathfrak{S}_{K_{\mathrm{m}}} \cap \mathscr{R}_{\mathrm{K}}=\mathfrak{B}_{\mathrm{K}}{ }_{\mathrm{n}}$, pour chaque $m \geq n$. Soit donc $x$ un élément de $\mathfrak{S}_{2} K_{m}$. Pour chaque $m^{\prime} \geq m$, nous avons par définition $k=e^{-k} \otimes N_{m} / m^{\prime}\left(x_{m}\right)$, pour un $x_{m}$, de $K_{m^{\prime}}^{x}$ et un entier $k$ indépendant de $m^{\prime}$. Si maintenant $r$ est dans $\mathcal{S}_{\mathrm{K}}$, la norme arithmétique $N_{\mathrm{m} / \mathrm{n}}$ opère sur ç par élévation à la puissance $\left[K_{m}: K_{n}\right]=\ell^{m-n}$, et nous pouvons écrire:

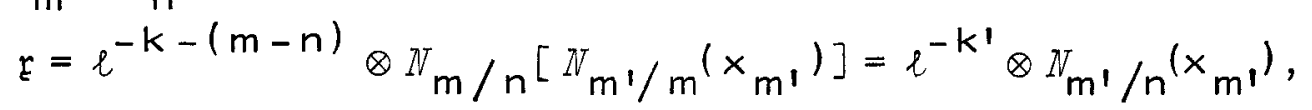

avec $k^{\prime}=k+(m-n)$ indépendant de $m^{\prime}$; ce qui nous prouve que $k$ est dans $\$_{K_{\mathrm{n}}}$.

PROPOSITION 1.2.19.- Supposons $\ell \neq 2$. Alors, lintersection des

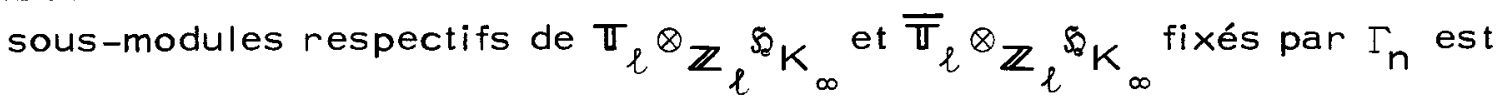
le sous-module de $\ell^{n}$-torsion de $\mathscr{B}_{K_{\mathrm{n}}}$ :

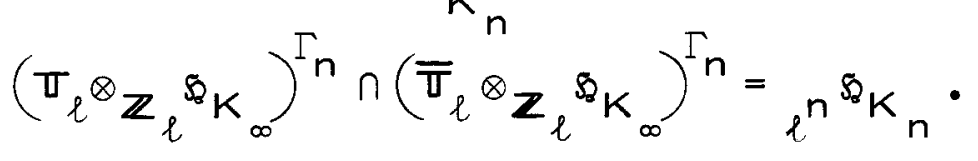

Démonstration: Cela résulte directement de la proposition 5 , et de la proposition $18 \mathrm{ci}$-dessus. 
a.- Interprétation symbolique: le groupe $H_{2}\left(\mathrm{~K}_{\mathrm{n}^{\prime} \ell} \cdot\right.$

Pour chaque place non complexe $p_{n} d u$ corps $K_{n}$, désignons $\operatorname{par}\left(\frac{1}{p_{n}}\right)$ le symbole de Hilbert associé à $p_{n}$ par le corps de clas ses local, ou plutôt la $\ell$-partie de ce symbole, qui prend ses valeurs dans le $\ell$-Sylow $\mu_{p}$ du groupe des racines de l'unité du complété de $K_{n}$ pour la place $p_{n}$. Le $\ell$-symbole $\left(\frac{1}{p_{n}}\right)$ envoie $K_{n}^{x} \times K_{n}^{x}$ sur le groupe $\mu_{p}$ tout entier, et coincide avec le symbole réguliersip $n$ ne divise pas $\ell$. Plus précisément, le théorème de Moore sur le $K_{2}$ des corps de nombres (cf. $\left.\left[\mathrm{Ta}_{1}\right], \S 8\right)$ affirme que la famille $\left(\left(\frac{1}{p_{n}}\right)\right)_{p_{n}}$ des symboles de Hilbert induit un morphisme surjectif du groupe $K_{2}\left(\mathrm{~K}_{\mathrm{n}}\right)_{\ell}$ sur le sous-groupe $\widetilde{\oplus} \mu_{p_{n}}$ de la somme directe des $\mu_{p_{n}}$, formé des familles ( $\left.\zeta_{p_{n}}\right)_{p_{n}}$ qui vérifient la formule du produit. Comme ce résultat est vrai pour $n$, par passage à la limite inductive, nous obtenons ainsi un morphisme surjectif du groupe $K_{2}\left(K_{n}{ }^{\prime}=\stackrel{\lim }{\longrightarrow} K_{2}\left(K_{n}\right)_{\ell}\right.$ sur le sous groupe $\widetilde{\oplus} \mu_{p_{\infty}}$ de la somme directe des $\ell$-Sylow des groupes de racines de l'unité des complétés non complexes de $K_{\infty}$, formé des familles $\left(\zeta_{P_{\infty}}\right)_{P_{\infty}}$ qui vérifient la formule du produit $\left.{ }^{\star}{ }^{\star}\right)$. Une autre façon d'ex primer ce résultat consiste à dire que les symboles de Hilbert $\left(\frac{1}{\mathfrak{p}_{\infty}}\right)$ attachés aux places non complexes du corps $K_{\infty}$ définissent un mor phisme surjectif du groupe $\bar{T}_{\ell} \otimes_{\mathbb{Z}_{l}}{ }^{R_{K}}=\mu_{l} \otimes_{\mathbb{Z}} K_{\infty}^{X}$ sur la somme di recte restreinte $\widetilde{\oplus} \mu_{p}$. Cela étant :

${ }^{\star}$ ) On prendra garde que, du fait de lidentification de chaque $\mu_{p_{n}}$ avec son image diagonale dans la somme $P_{\infty} \mid P_{n} \mu_{P_{\infty}}$, la formule du produit, qui se lit $\prod \zeta_{p_{\infty}}=1$ dans $K_{\infty}$, se lit $\prod \zeta_{p_{n}}^{g_{p_{n}}}=1$ dans $K_{n}$, si $g_{p}$ désigne l'indice de décomposition de la place $p_{n}$ dans l'extension $K_{\infty} / K_{n}$. 
LEMME 1.2.20. - Le noyau de l'épimorphisme du groupe $T_{\ell} \otimes_{\mathbb{Z}_{l}} \Re_{K}=$ $\mu_{\ell} \otimes \mathbb{Z}_{\infty} K_{\infty}^{x}$ sur la somme directe restreinte $\widetilde{\oplus} \mu_{p_{\infty}}$, donné par les sym boles de Hilbert $\left(\frac{l}{p}\right)$ est le groupe $\pi_{\ell} \mathbb{Z}_{\ell}{ }^{\mathfrak{S}_{K}} \mathrm{~K}_{\infty}$.

Démonstration : Le noyau de cet épimorphisme est constitué par les éléments $\zeta \otimes x$ de $\mu_{\ell} \otimes \mathbb{Z}_{\infty} K_{\infty}^{x}$ qui vérifient les conditions locales $\left(\frac{\zeta_{2} x}{p_{m}}\right)=1$, pour chaque $m$ assez grand, clest-à-dire, d'après la définition normique du symbole de Hilbert, pour lesquels le nombre $x$ est norme locale partout dans chacune des extensions cycliques $k_{m+k} / k_{m}$, si $\zeta$ est une racine primitive $\ell^{k}-i e ̀ m e$ de llunité. Prenons donc un tel élé ment $\zeta \otimes x$, supposons par exemple $x \in K_{n}^{x}$, notons $m^{\prime}=m+k$, et utilisons le principe des normes de Hasse pour écrire $x=I_{\mathrm{m}} / \mathrm{m}\left(x_{\mathrm{m}}\right)$ avec $x_{m^{\prime}} \in K_{m^{\prime}}^{x}$, dès que $m$ est assez grand. La condition $N_{m^{1} / m^{\prime}}\left(x_{m^{\prime}}^{\gamma_{n}}{ }^{-1}\right)$ $=x^{\gamma_{n}-1}=1$, et le théor ème 90 de Hilbert appliqué à l'extension cyclique $K_{m^{\prime}} / K_{m}$ nous permettent d'écrire $x_{m^{\prime}}^{\gamma_{n}-1}=y_{m^{\prime}}^{\gamma_{m}-1}$, pour un $y_{m}$, de $K_{m}^{x}$, c'est-à-dire $x_{m^{\prime}}^{\gamma_{n}-1}=N_{m} / n\left(y_{m^{\prime}}^{\gamma_{n}}-1\right)$ (l'opérateur norme étant pris algé briquement $)$, donc finalement $x_{m^{\prime}}=N_{m / n}\left(y_{m^{\prime}}\right) \cdot z_{m^{\prime}}$, avec $z_{m}, \in K_{n}^{X}$.

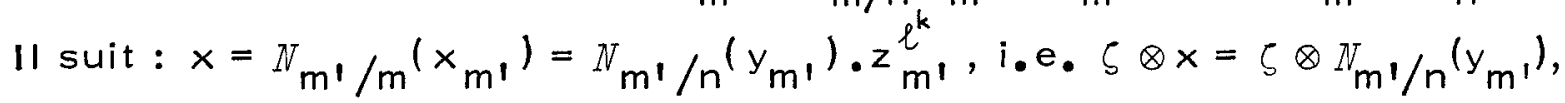
ce qui nous prouve que $\zeta \otimes x$ est bien dans $\pi_{l} \otimes \mathbb{Z}_{l} \mathfrak{S}_{K}$.

Réciproquement, la définition normique du noyau hilbertien montre de façon évidente que le groupe $\pi_{\ell} \otimes_{\mathbb{Z}_{\ell}}{ }^{\mathfrak{S}_{K}} \mathrm{~K}_{\infty}$ est contenu dans le noyau des symboles $\left(\frac{1}{p}\right)$; d'où, bien entendu, son nom .

Considérons maintenant la suite exacte courte donnée par le lemme 20:

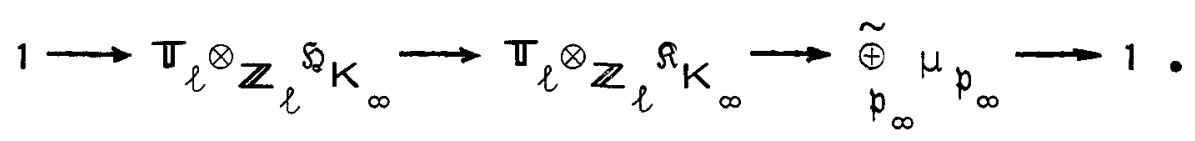

Prenant les points fixes par $\Gamma_{n}$, nous obtenons la suite exacte de cohomologie ( où le groupe $H^{1}\left(\Gamma_{n}, \sigma_{l} \otimes_{\mathbb{Z}_{l}} \Omega_{K}\right)$ est nul, par un argument déjà utilisé ) : 


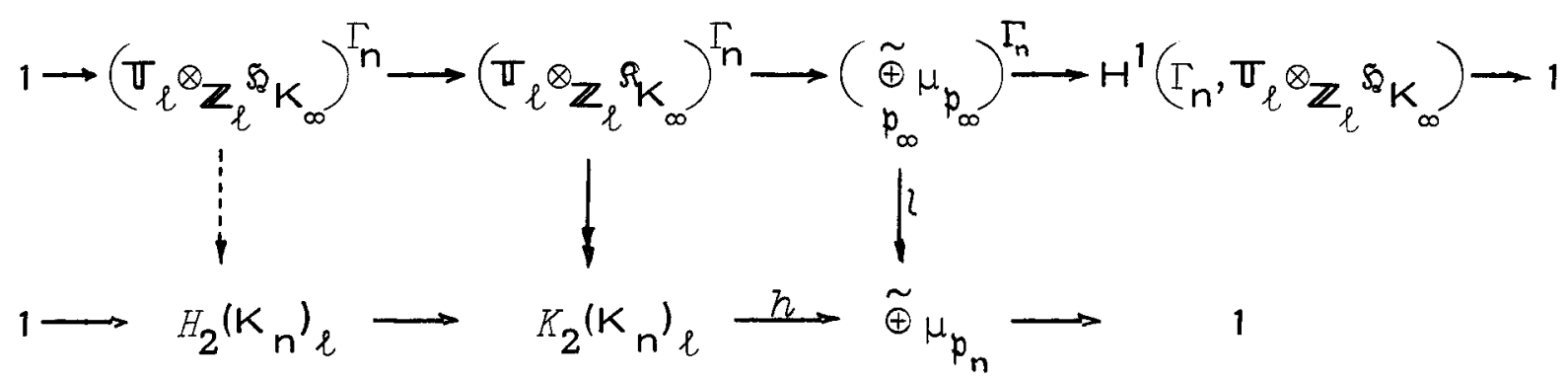

Puis, par rapprochement avec la suite exacte de Moore pour le groupe $K_{2}\left(K_{n}\right)_{l}$, nous concluons à la trivialité du groupe de cohomologie $H^{1}\left(\Gamma_{n}, T_{l} \otimes_{\mathbb{Z}_{l}} \mathscr{S}_{K}\right)$ ainsi qu'à l'identité :

THÉORĖME 1.2.21.- Le $\ell$-Sylow $H_{2}\left(K_{n}\right)_{\ell}$ du noyau dans $K_{2}\left(K_{n}\right)$ des symboles de Hilbert est limage dans $K_{2}\left(\mathrm{~K}_{n}\right)_{\ell}$ du noyau hilbertien $\left(\pi_{l} \otimes_{\mathbb{Z}_{l}} \mathscr{B}_{K}\right)^{\Gamma_{n}}$ du groupe $\left(T_{l} \otimes_{\mathbb{Z}_{l}} \Omega_{K}\right)^{\Gamma_{n}}$; ce qui se traduit par l'exactitude de la suite courte canonique :

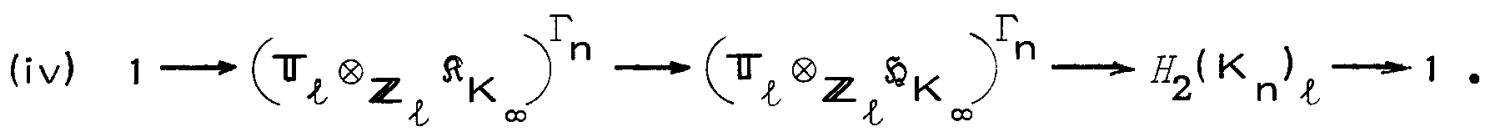

SCOLIE 1.2.22. - Pour chaque naturel $n$, le groupe $\left(T_{\ell} \otimes_{\mathbb{Z}_{\ell}}{ }^{\mathscr{S}_{K}}\right)_{\infty}^{\Gamma_{n}}$ est l'ensemble des éléments de hauteur infinie du groupe $\left(\pi_{\ell}^{\otimes_{\mathbb{Z}}}{ }_{\ell}{ }_{K}\right){ }_{\infty}{ }^{n}$, ce que nous écrivons :

$$
\left(\mathbb{T}_{\ell} \mathbb{Z}_{\ell}{ }^{\mathfrak{S}_{K}}\right)_{\infty}^{\Gamma_{n}}=\left(\pi_{\ell} \mathbb{Z}_{\ell}{ }^{R_{K}}\right)_{\infty}^{\Gamma_{n}} .
$$

En particulier, le groupe $H_{2}\left(K_{n}\right)$ mesure donc l'écart entre le sous groupe divisible maximal et le sous-groupe des éléments de hauteur in finie du groupe $\left(\pi_{\ell} \otimes_{\mathbb{Z}_{l}}{ }^{\Omega_{K}}\right)_{\infty}^{\Gamma_{n}}$.

Démonstration : Les résultats de Tate (cf. $\left[\mathrm{Ta}_{1}\right], \S 5$ ) montrent que le groupe $H_{2}\left(K_{n}\right)_{l}$ est le sous-groupe $\bigcap_{k \in \mathbb{N}} K_{2}\left(K_{n}\right)_{l}^{l^{k}}$ des éléments de hauteur infinie dans $K_{2}\left(K_{n}\right)$. Comme tout élément divisible est trivialement de hauteur infinie, cette caractérisation du noyau hilbertien se relève dans $\left(\pi_{\ell} \mathbb{Z}_{\ell}{ }^{\Omega_{K}}\right)^{\Gamma_{n}}$; ce qui donne le résultat annoncé . 


\section{b.- Interprétation kummérienne : Le groupe $\bar{H}_{2}\left(\mathrm{~K}_{\mathrm{n}}\right)_{\ell}$ •}

L'introduction du noyau hilbertien $S_{3}$ (sous une forme sensiblement moins générale) est due à Bertrandias et Payan, qui avaient en vue l'étude d'une condition suffisante de la conjecture de Leopoldt, liée à un problème de plongement (cf. [BP ]). D'après Artin-Tate, en effet (cf. [AT] , $10 \S 3$, th. 6), une condition nécessaire et suffisante pour qu'une extension cyclique $L_{m}=K_{m}\left[\sqrt[m]{x_{m}}\right]$, définie par un élément $\ell^{-m} \otimes x_{m}$ de $\mathscr{S}_{K_{m}}$, se plonge dans une $\ell$-extension cyclique de $K_{m}$ de degré arbitrairement grand est précisément que, pour chaque place non complexe $\mathfrak{P}_{m}$ de $K_{m}$, l'une quelconque $S_{p_{m}}$ des racines primitives de $\mu_{p_{m}}$ soit norme dans l'extension locale $K_{m, p}\left[\sqrt[\ell]{x_{m}}\right] / K_{m, p}$, ce qui se lit sur le symbole de Hilbert $\left(\frac{S_{m}, x_{m}}{p_{m}}\right)$, pour une racine pri mitive $\ell^{m}$-ième de l'unité $\zeta_{m}$. Plus généralement, si l'extension $L_{m}$ provient par composition d'une $\ell$-extension cyclique $L_{n}$ de degré $\ell^{m}$ sur un sous-corps $K_{n}$ de $K_{m}$ (i.e. si l'élément $\zeta_{m} \otimes x_{m}$ tombe dans $\left.\left(\overline{\mathrm{T}}_{\ell} \otimes \mathbb{Z}_{\ell}{A_{K}}_{\mathrm{m}}\right) \Gamma_{n}\right)$, le même critère appliqué à l'extension globale $L_{n} / K_{n}$ et l'équivalence, donnée par le corps de classes local: $\left(\frac{\zeta_{p_{m}}, L_{m, P_{m}} / K_{m}, P_{m}}{p_{m}}\right)=1 \Leftrightarrow\left(\frac{\zeta_{p_{n}}, L_{n, P_{n}} / K_{n, p_{n}}}{p_{n}}\right)=\left(\frac{N\left(\zeta_{p_{m}}\right), L_{n, P_{n}} / K_{n, p_{n}}}{p_{n}}\right)=1$ montrent que la trivialité des symboles $\left(\frac{\zeta_{m}, x_{m}}{p_{m}}\right)$ est encore la condition pour que l'extension $L_{n}$ se plonge dans une $\ell$-extension cyclique de $K_{n}$, de degré arbitrairement grand. Autrement dit :

PROPOSITION 1.2.23. - Pour chaque naturel $n$, le groupe $\left.\left(\bar{\pi}_{\ell} \otimes_{Z}{ }_{l}^{\mathscr{S}_{K}}\right)_{\infty}\right)^{\Gamma_{n}}$ est l'ensemble des éléments de hauteur infinie dans $\left(\bar{\pi}_{\ell} \otimes_{\mathbb{Z}_{l}}{ }_{\ell} K_{K_{\infty}}\right)^{\Gamma_{n}}$; ce que nous écrivons :

$$
\left(\bar{\pi}_{\ell} \otimes_{\mathbb{Z}_{l}}{ }_{S_{K}}\right)^{\Gamma_{n}}=\left(\bar{\pi}_{\ell} \otimes_{\mathbb{Z}_{l}}{ }^{\Omega_{K}}\right)_{\infty}^{\Gamma_{n}} .
$$


DÉFINITION \& THÉORĖME 1.2.24. - Par $\bar{H}_{2}\left(K_{n}\right)_{\ell}$ nous entendons le quotient du groupe $\left(\overline{\mathbb{U}}_{\ell} \otimes_{Z_{\ell}} \mathscr{S}_{K}\right)_{\infty}^{\Gamma_{n}}$ des éléments de hauteur infinie dans $\left(\bar{\pi}_{\ell} \otimes_{\mathbb{Z}_{\ell}} \Omega_{K}\right)^{\Gamma_{n}}$ par son sous-groupe divisible maximal $\left(\bar{\pi}_{\ell} \otimes_{\mathbb{Z}_{\ell}}{ }^{\Omega_{K}}\right)_{\infty}^{\Gamma_{n}}$, clest-à-dire le $\ell$-groupe de torsion défini par l'exactitude de la suite courte:

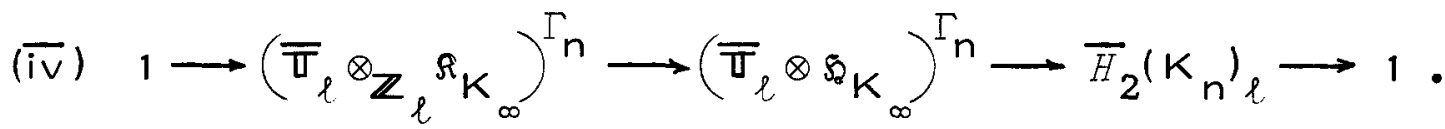

Le groupe $\bar{H}_{2}\left(K_{n}\right)$ est le sous-groupe des éléments de hauteur infinie dans $\bar{K}_{2}\left(K_{n}\right)_{\ell}$. Clest un $\ell$-groupe fini, qui s'identifie au dual de Pontrjagin du sous-groupe de torsion $\varepsilon_{n}^{\prime}=\not_{n}^{\text {tor }}$ du groupe de Galois $H_{n}=\operatorname{Gal}\left(H_{n} / K_{n}\right)$ de la composée des $l$-extensions cycliques de $K_{n}$ qui se plongent dans une $\ell$-extension cyclique de $K_{n}$ de degré arbitrairement grand. Nous disons que $H_{n}$ est la $\ell$-extension hilbertienne maximale du corps $K_{n}$.

Démonstration: Nous avons vu que le groupe divisible $\left(\overline{\bar{T}}_{\ell} \otimes_{\mathbb{Z}}{ }_{\ell}{ }^{\Omega_{K}}\right)^{\Gamma_{n}}$ est le dual de Pontrjagin du groupe de Galois $G a l\left(Z_{n} / K_{\infty}\right)$ associé à la composée $Z_{n}$ des $\mathbb{Z}_{\ell}$-extensions de $K_{n}$. Le sous-groupe des élé ments de hauteur infinie $\left(\overline{\mathrm{U}}_{\ell} \otimes \mathbb{Z}_{\ell} \Omega_{K}\right)_{\infty}^{\Gamma_{n}}$ correspond lui au groupe de

Galois Gal $\left(H_{n} / K_{\infty}\right)$ associé à la composée $H_{n}$ des $\ell$-extensions cy cliques de $K_{n}$, qui se plongent dans une $\ell$-extension cyclique de degré arbitrairement grand . Leur quotient $\bar{H}_{2}\left(K_{n}\right)$ est donc, comme annoncé, le dual de Pontrjagin du groupe de torsion $\sigma_{n}^{1}=G a l\left(H_{n} / Z_{n}\right)$. Le fait qu'il soit fini résulte de l'inclusion immédiate $Z_{n} \subset H_{n} \subset M_{n}$, et de la finitude du groupe $\sigma_{n}=\operatorname{Gal}\left(M_{n} / Z_{n}\right)$.

PROPOSITION 1.2.25. - Pour chaque naturel n, les symboles de Hilbert

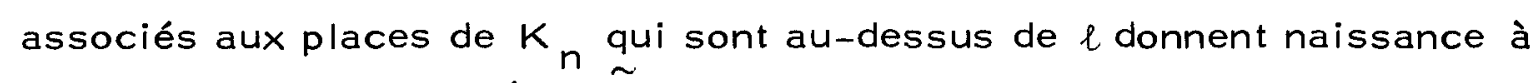
la suite exacte courte (où $\oplus$ désigne la somme directe restreinte aux familles qui vérifient la formule du produit) :

(v) $1 \longrightarrow H_{2}\left(\mathrm{~K}_{\mathrm{n}}\right)_{\ell} \longrightarrow R_{2}\left(\mathrm{~K}_{\mathrm{n}}\right)_{\ell} \longrightarrow \tilde{I}_{\mathrm{n}} \tilde{\sim}_{\ell} \mu_{1} \longrightarrow 1$. 
Et la même suite vaut pour les modules opposés si la conjecture de Leopoldt est vérifiée dans le corps $K_{n}$ :

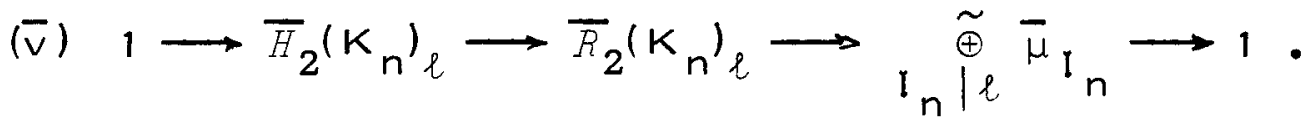

Démonstration : La première suite provient directement du théorème de Moore sur le $K_{2}$ des corps de nombres ( $\mathrm{cf} .\left[\mathrm{Ta}{ }_{1}\right], \S 2$ ), par restriction au noyau régulier. Elle conduit à la formule bien connue :

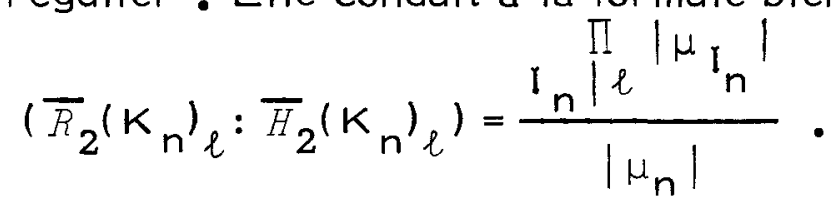

Pour démontrer la seconde suite, il suffit d'en vérifier l'exactitude à droite qui seule pose problème, clest-à-dire, en fait, de montrer que la même formule :

$$
\left(\bar{R}_{2}\left(\mathrm{~K}_{\mathrm{n}}\right)_{\ell}: \bar{H}_{2}\left(\mathrm{~K}_{\mathrm{n})_{\ell}}\right)=\frac{\mathrm{I}_{\mathrm{n}}\left|e^{\mid}\right| \mu_{\mathrm{l}} \mid}{\left|\mu_{\mathrm{n}}\right|}\right.
$$

vaut encore sous la conjecture de Leopoldt. Mais, comme le quotient $\bar{R}_{2}\left(K_{n}\right)_{\ell} / \bar{H}_{2}\left(K_{n^{\prime}}\right)$ est le dual de Pontrjagin du groupe de Galois Gal $\left(M_{n} / H_{n}\right)$, cette dernière égalité résulte de l'isomorphisme déjà connu, toujours sous la conjecture de Leopoldt ${ }^{(\star)}$ :

$$
\operatorname{Gal}\left(M_{n} / H_{n}\right) \simeq\left(l_{n} \mid e^{\mu} l_{n}\right) / \mu_{n} \text {. }
$$

\section{c.- Interprétation hilbertienne de la conjecture de Leopoldt .}

Rappelons que nous avons désigné par $\Lambda_{n}=\left[\left[\gamma_{n}-1\right]\right]$ l'al gèbre dilwasawa construite sur le groupe $\Gamma_{n}$, par $\forall$ le sous-groupe de $\Lambda_{\mathrm{n}}$-torsion du groupe de Galois $a=\operatorname{Gal}\left(\mathrm{M}_{\infty} / \mathrm{K}_{\infty}\right)$ de la $\ell$-extension abélienne maximale $\ell$-ramifiée de $K_{\infty}$, et par $Z_{\infty}=M_{\infty}^{G}$ le corps des invariants de $G$. Introduisons maintenant la sous-extension $H_{\infty}$ de $M_{\infty}$, réunion des extensions hilbertiennes $H_{n}$ associées aux étages finis $K_{n}$ de la tour cyclotomique, et notons $\not=\underline{1 \mathrm{im}} \not_{\mathrm{n}}$ son groupe de Galois .

$\left.{ }^{\star}\right) \mathrm{cf}$. Ch. I.I. 
Linclusion évidente $Z_{\infty} \subset H_{\infty} \subset M_{\infty}$ nous montre que le groupe $\operatorname{Gal}\left(M_{\infty} / H_{\infty}\right.$ ) est un $\Lambda_{n}$-module de torsion, autrement dit, que les groupes de Galois $a$ et $\not$ sont des $\Lambda_{n}$-modules de même dimension $c_{n}$. En particulier, la conjecture de Leopoldt pour le corps $K_{n}$, que nous avons écrite $a^{\Gamma_{n}}=1$ dans la section $2 \S \mathrm{c}$, se lit tout aussi bien sur le groupe $\boldsymbol{\not}$, et exprime la finitude du sous-groupe invariant $\boldsymbol{\not}^{\Gamma_{n}}$. Tout comme pour $a^{\Gamma_{n}}$, cette condition est, en fait, équivalente à la nullité du groupe $\sharp^{\Gamma_{n}}$. Admettons provisoirement ce résultat, et retenons que la conjecture de Leopoldt dans $K_{n}$ (pour le nombre premier $\ell$ ) s'écrit:

$$
{ } \Gamma_{n}=1 \text {. }
$$

D'un autre côté, la théorie de Kummer nous donne les isomor phismes :

$$
\begin{aligned}
& \mathcal{H}^{\Gamma_{n}}=\operatorname{Hom}_{\Gamma_{n}}\left(\mathscr{B}_{K_{\infty}}, \mu_{\ell}\right)=\operatorname{Hom}_{\Gamma_{n}}\left(\overline{\mathbb{T}}_{\ell} \otimes_{\mathbb{Z}_{\ell}} \mathfrak{S}_{K_{\infty}}, \mathbb{Q}_{\ell} / \mathbb{Z}_{\ell}\right) \\
& =\operatorname{Hom}\left({ }^{\Gamma_{n}}\left(\overline{\boldsymbol{T}}_{\ell} \mathbb{Z}_{\ell} \mathbb{S}_{K_{\infty}}, \mathbb{Q}_{\ell} / \mathbf{Z}_{\ell}\right)\right) \text {, i.e. }
\end{aligned}
$$

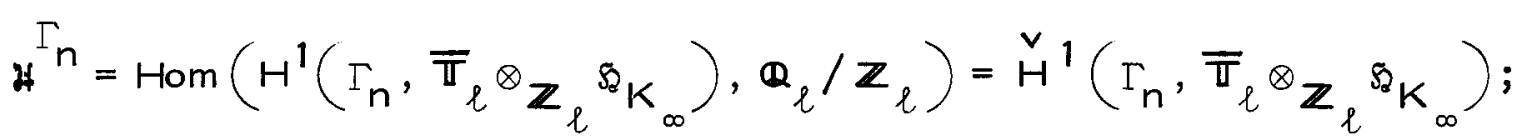

de sorte que la conjecture de Leopoldt pour $K_{n}$ s'écrit encore :

$$
H^{1}\left(\Gamma_{n}, \bar{\nabla}_{l} \otimes \mathbb{Z}_{\ell} \mathbb{K}_{\infty}\right)=1 \text {. }
$$

Reprenons maintenant, sous une forme légèrement différente, la suite exacte courte donnée par le lemme 20 , qui caractérise le noyau hilbertien $\mathfrak{S}_{K_{\infty}}$ :

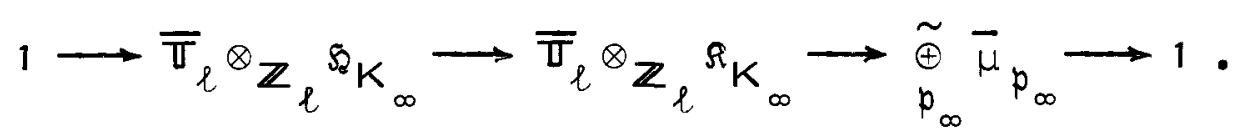

Prenant les points fixes par $I_{n}$, et notant que le groupe $H^{1}\left(I_{n}, \bar{\pi}_{\ell} \otimes_{\mathbb{Z}_{l}} \Omega_{K}{ }_{\infty}\right)$ est nul, nous obtenons la suite exacte de cohomologie :

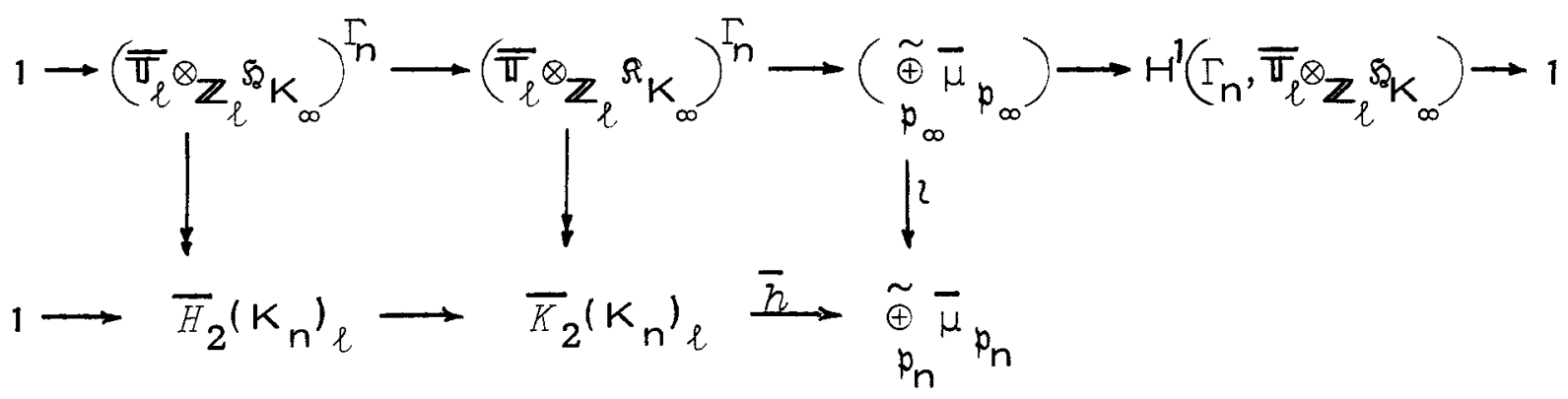


Nous pouvons donc énoncer l'analogue du théorème 15:

THÉORÈME 1.2.26.- Les qua tre conditions suivantes sont équivalentes :

(i) La conjecture de Leopoldt est vérifiée dans le corps $K_{n}$ ( pour le nombre premier $\ell$ ).

(ii) Le groupe de Galois $\not H=\operatorname{Gal}\left(H_{\infty} / K_{\infty}\right)$ de la réunion $H_{\infty}$ des extensions hilbertiennes maximales associées aux corps $K_{n} n^{\prime}$ a pas de sous-module non nul fixé par $\Gamma_{n}$, i.e. H $\Gamma_{n}=1$.

(iii) Le groupe de cohomologie $H^{1}\left(I_{n}, T_{l} \otimes_{\mathbb{Z}} \mathbb{B}_{K}\right)$ est nul.

(iv) Les symboles de Hilbert induisent un épimorphisme du groupe $\bar{K}_{2}\left(K_{n}\right)$ sur la somme restreinte $\underset{p_{n}}{\oplus} \bar{\mu}_{p_{n}}$ des opposés respectifs des $\ell$-Sylow des groupes de racines de l'unité des complétés non complexes du corps $K_{\mathrm{n}}$.

SCOLIE 1.2.27.- Pour chaque naturel $n$, les symboles de Hilbert attachés aux places non complexes du corps $K_{n}$ donnent naissance à la suite exacte courte de $K$-théorie (de Calvin Moore) :

$$
1 \longrightarrow H_{2}\left(K _ { n _ { l } } { } ^ { \prime } K _ { 2 } \left(K_{n_{l}}{ }^{h} \underset{p_{n}}{\oplus} \mu_{p_{n}} \longrightarrow 1\right.\right.
$$

Et la conjecture de Leopoldt postule l'existence d'une suite analogue pour les modules opposés :

$$
1 \longrightarrow \bar{H}_{2}\left(\mathrm{~K}_{n}\right)_{l} \longrightarrow \bar{K}_{2}\left(\mathrm{~K}_{\mathrm{n}}\right)_{l} \stackrel{\bar{\hbar}}{\longrightarrow} \underset{p_{n}}{\widetilde{\oplus}_{\mathrm{p}}} \bar{\mu}_{p_{n}} \longrightarrow 1 .
$$

Démonstration : Seulreste à vérifier que le groupe $\aleph^{\Gamma_{n}}$ ne peut être fini sans être nul. Or, si la conjecture de Leopoldt était en défaut dans $K_{n}$, ce corps admettrait des $\mathbf{Z}_{\ell}$-extensions en excès ; le polynôme carac téristique du sous-module de $\Lambda_{n}$-torsion de $\not$ serait divisible par $\left(\gamma_{n}-1\right)$; et le groupe $\psi^{\Gamma_{n}}$ serait infini. Sa finitude suppose donc vérifiée la conjecture de Leopoldt. Mais, dans cette hypothèse, la surjectivité de l'appli cation $\bar{r}$, donnée par le scolie 16 , et la proposition 25 assurent la sur jectivité de l'application $\bar{h}$, qui équivaut bien à lidentité $\not \Gamma_{n}=1$. 


\section{4. - LES RÉSULTATS FONDAMENTAUX DE LA DUALITÉ.}

a.- Comparaison des noyaux de la théorie de Kummer et de ceux de la $K$ théorie : préliminaires .

$$
\text { Considérons d'abord les groupes } K_{2}\left(K_{n}\right)_{l}^{\text {et }} \bar{K}_{2}\left(K_{n}\right) \text {. }
$$

Nous avons :

THEOREME 1.2.28.- Pour chaque naturel $m \leq n$, les symboles de Hilbert induisent un isomorphisme :

$$
\bar{K}_{2}\left(K_{n}\right)_{l} / \bar{K}_{2}\left(K_{n}\right)^{l^{2}} \simeq \underset{p_{n}}{\oplus} \bar{\mu}_{p_{n}} / \bar{\mu}_{p_{n}}^{l^{m}},
$$

du groupe $\ell^{m} \bar{K}_{2}\left(K_{n}\right)$ sur la somme directe des quotients d'exposant $\ell^{m}$ des opposés respectifs des groupes de racines de l'unité des complétés non complexes de $\mathrm{K}_{\mathrm{n}}$, restreinte aux familles qui véri fient la formule du produit ( prise dans $\mu_{n} / \mu_{n}^{l^{m}}$ ). Cet isomorphisme est le pendant exact de celui donné par Tate pour la $K$-théorie :

$$
K_{2}\left(K_{n}\right)_{l} / K_{2}\left(K_{n}\right)^{l^{m}} \simeq \underset{p_{n}}{\widetilde{\oplus}} \mu_{p_{n}} / \mu_{p_{n}}^{l^{m}} .
$$

Le même résultat vaut d'ailleurs pour $m>n$, en-dehors du cas spécial $\left.{ }^{(}\right)$, donc en particulier, dès que $l$ est impair.

Démonstration : Comme $\bar{K}_{2}\left(K_{n}\right)$ est le dual de Pontrjagin du groupe de Galois Gal $\left(A_{n} / K_{\infty}\right)$ attaché à la $\ell$-extension abélienne maximale $A_{n}$ de $K_{n}$, le théorème sera établi si nous montrons que le sous -

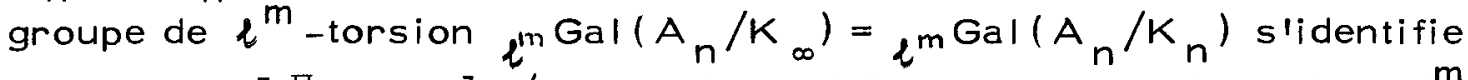
au quotient $\left[\prod_{p} l^{m \mu} \mu_{p} / l^{m \mu}\right.$ du produit des groupes de racines $l^{m}$. ièmes locales de l'unité par l'image diagonale du sous-groupe des

${ }^{\star}$ ) Le cas spécial a lieu lorsque, $\ell$ valant 2 , et les nombres -1 , $\zeta_{n}+2, \zeta_{n}^{-1},-\left(\zeta_{n}+2+\zeta_{n}^{-1}\right)$ n'étant pas des carrés dans $k_{n}$, cette dernière propriété est localement en défaut aux places divisant 2 (cf. $[\mathrm{AT}], \mathrm{Ch} . \times, \S 1$ ). 
racines $l^{m}$-ièmes globales. Transportée par l'isomorphisme ducorps de classes (cf. théorème 1.1 .13 ), cette condition signifie que les élé ments $l^{m}$ du $l$-groupe des classes diidèles $\partial_{n} / R_{n}$ du corps $K_{n}$ sont les images canoniques des racines $l^{m}$ - ièmes de l'unité dans $\partial_{n}$.

- Sous l'hypothèse $m \leq n$, il est facile de voir directement qu'elle est trivialement satisfaite, au moyen de la théorie de Kummer. $\mathrm{Si}$, en effet, $\vartheta_{\mathrm{n}}$ est un idèle généralisé représentant une classe d'ordre $l^{m}$ dans $\partial_{n} / R_{n}$, l'élément global $\mathfrak{r}_{n}=\mathfrak{g}_{n}^{\ell^{m}}$ est localement partout une puissance $\ell^{m}$-ième, et l'extension kummérienne $K_{n}\left[\sqrt[l]{\mathfrak{x}_{n}}\right]$ est donc triviale, puisque complètement décomposée en chaque place $p_{n}$ de $K_{n}$, ce qui montre que $\mathfrak{E}_{n}$ est la puissance $l^{m}$-ième d'un élément $z_{n}$ de $\beta_{n}$. En particulier la classe de $z_{n}$ dans le quotient $\partial_{n} / \Omega_{n}$ est représentée par le quotient $\vartheta_{n} / z_{n}$, qui est bien une ra -

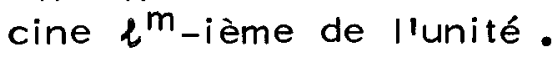

- Lorsque $m$ est strictement plus grand que $n$, cet argument ne s'applique pas. Dans ce cas, il peut arriver que la propriété a vancée soit en défaut: c'est le cas spécial recensé par Artin-Tate . II ne se produit que lorsque $l$ vaut 2 ( cf. [AT], Ch. $\times, \S 1$ ).

Du rapprochement de l'isomorphisme obtenu et de celui donné par Tate (cf. $\left[\mathrm{Ta}_{1}\right], \S 5$, th. 2 ), nous déduisons immédiatement :

COROLLAIRE 1.2.29. - Pour chaque naturel $m \leq n$, il existe un isomorphisme canonique, compatible avec l'action de Galois :

$$
\mu_{m} \otimes_{\mathbb{Z}} \bar{K}_{2}\left(K_{n}\right)_{l} \simeq \bar{\mu}_{m} \otimes_{\mathbb{Z}} K_{2}\left(K_{n}\right)_{l} \cdot
$$

Et le même résultat vaut pour $m>n$, en-dehors du cas spécial.

Bien entendu, ce résultat n'apporte aucune information di recte sur les noyaux hilbertiens, puisque ceux-ci ne font intervenir que les éléments de hauteur infinie.

b.- Dualité pour le noyau régulier et le noyau hilbertien.

Revenons sur la suite exacte $(\overline{i i})$ induite par les symboles réguliers, donnée par le scolie 16 :

$$
1 \longrightarrow \bar{R}_{2}\left(K_{n}\right) \longrightarrow \bar{K}_{2}\left(K_{n}\right)_{l} \stackrel{\vec{r}}{\longrightarrow} p_{n}|l| \bar{\mu}_{p_{n}} \longrightarrow 1 \text {. }
$$


Prenant les puissances $l^{m}$-ièmes, pour un $m \leq n$, puis formant le diagramme du serpent, nous obtenons la suite exacte longue:

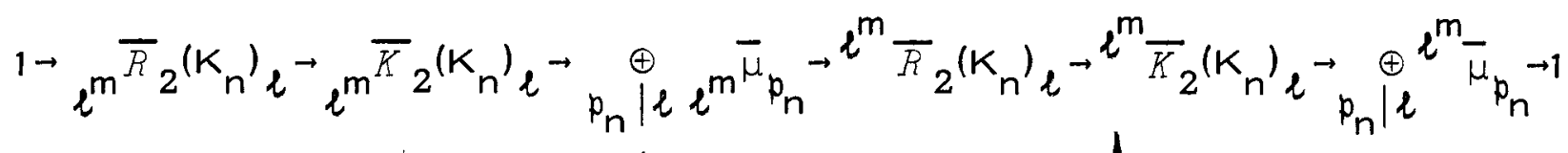

$$
\begin{aligned}
& \left.\vec{\mu}_{m} \otimes_{\mathbb{Z}^{K}}\right|_{n} ^{x} \rightarrow \bar{\mu}_{m}^{\otimes_{\mathbb{Z}} D_{n}^{\prime}} \\
& 12 \\
& \widetilde{\oplus}_{n} l^{m} \bar{\mu}_{p_{n}}
\end{aligned}
$$

Dans celle-ci, le quotient $\ell^{m} \bar{R}_{2}\left(\mathrm{~K}_{\mathrm{n}}\right)_{\ell}$ s'identifie à la somme restreinte $\underset{p_{n}}{\oplus} l^{m} \bar{\mu}_{p_{n}}$, en vertu du théorème $28 ;$ le groupe $l^{m} \bar{K}_{2}\left(K_{n}\right) l$ provient de $\bar{\mu}_{m} \otimes \mathbb{Z}_{n} K_{n}^{x}$, par construction de $\bar{K}_{2}\left(K_{n}\right)$; et la somme directe $\stackrel{\oplus}{\oplus} p_{n} \mid \ell \ell^{m} \bar{\mu}_{p_{n}}$ s'identifie canoniquement au produit tensoriel $\bar{\mu}_{m} \otimes_{\mathbb{Z}} D_{n}^{\prime}$, où $D_{n}^{\prime}$ désigne le groupe des diviseurs du corps $K_{n}$ qui sont étrangers à $l$. Rassemblant ces résultats, nous obtenons la suite exacte courte :

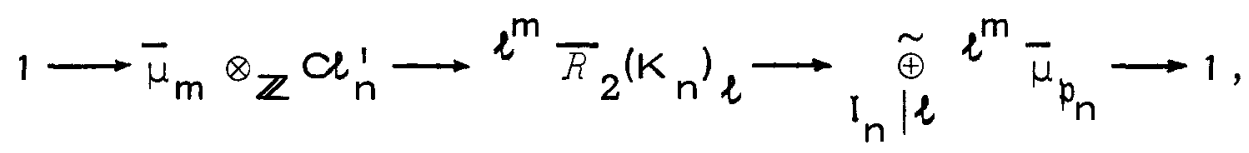

où $C_{n}^{\prime}$ désigne le $\ell$-groupe des $\ell$-classes de diviseurs du corps $K_{n}$ (i.e. le $\ell$-Sylow du quotient $D_{n}^{\prime} / P_{n}^{\prime}$ du groupe des diviseurs de $K_{n}$ étrangers à \& par l'image canonique de $K_{n}^{x}$ dans $D_{n}^{\prime}$ ). En fait, le corps $K_{n}$ étant supposé complexe, le groupe $C_{n}^{\prime}$ s'identifie cano niquement au quotient du $l$-groupe des classes dilidéaux au sens ordinaire par le sous-groupe engendré par les images des places au dessus de $\ell$. Nous disons, pour abréger, que $C \ell_{n}^{\prime}$ est le $\ell$-groupe des $\ell$-classes de $K_{n}$.

Bien entendu, la même méthode appliquée à partir de la suite exacte (iii):

$$
1 \longrightarrow R_{2}{ }^{\left(K_{n}\right)_{l} \longrightarrow K_{2}\left(K_{n}\right)_{l} \longrightarrow} p_{n} \mid \ell \mu^{\mu} \longrightarrow 1
$$

conduit à un résultat ana logue pour le quotient $\ell^{m} R_{2}\left(\mathrm{~K}_{\mathrm{n}}\right)_{\ell}$. C'est le résultat de Tate (cf. $\left[\mathrm{Ta}_{3}\right]$, th. 6.2). 
Le point remarquable est que la suite obtenue est scindée :

THÉOREME 1.2.30.-Pour chaque naturel $m \leq n$, les symboles de Hilbert attachés aux places de $K_{n}$ qui sont au-dessus de $\ell$ donnent nais sance à la suite exacte courte scindée :

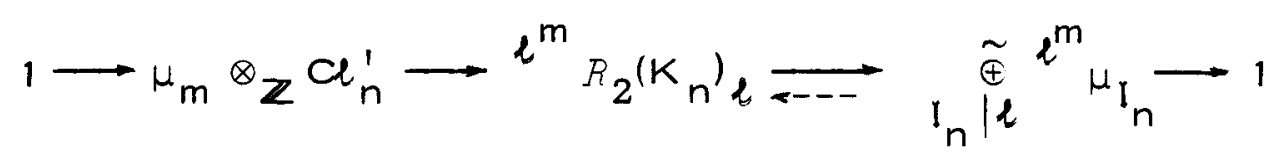

Dans celle-ci, $C l_{n}^{\prime}$ est le $l$-groupe des $l$-classes du corps $K_{n}$, et le signe $\widetilde{\oplus}$ signifie que la somme est restreinte aux familles qui véri fient la formule du produit (prise dans $e_{\mu_{n}}^{m}$ ).

Lorsque le corps $K_{n}$ vérifie la conjecture de Leopoldt, la même suite vaut pour les modules opposés:

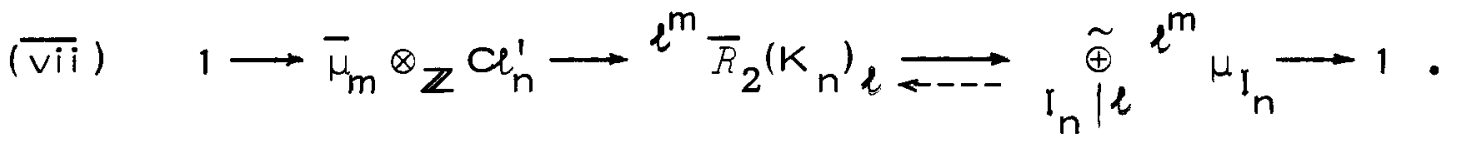

Démonstration: D'après ce qui précède, seul reste à vérifier le fait que les deux suites sont bien scindées.

Ecrivons les, pour simplifier, sous la forme légèrement différente :

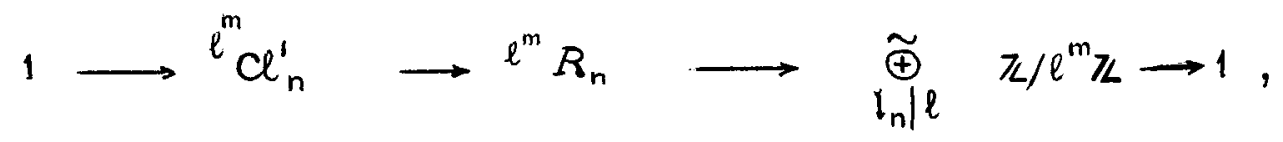

en désignant par $R_{\mathrm{n}}$ l'un quelconque des deux modules $\bar{\mu}_{\mathrm{n}} \otimes R_{2}\left(\mathrm{~K}_{\mathrm{n}}\right)_{\ell}$ et $\mu_{\mathrm{n}} \otimes \bar{R}_{2}\left(\mathrm{~K}_{\mathrm{n}}\right)_{\ell}$. Cela étant, l'injectivité de l'application naturelle $\ell^{m} \mathrm{Cl}_{\mathrm{n}}^{\prime} \longrightarrow \ell^{m} R_{\mathrm{n}}$, pour chaque $\mathrm{m} \leq \mathrm{n}$, prouve que le quotient $l^{m} C_{n}^{\prime}=C l_{n}^{\prime} / C l_{n}^{\prime} l^{m}$ s'identifie à un sous-module pur de $R_{n}$. Comme $R_{n}$ est fini, cela suffit à montrer que la suite exacte précédente est scindée.

COROLLAIRE 1.2.31.- Sous la conjecture de Leopoldt dans $K_{n}$, il existe un isomorphisme de $\mathbb{Z} l[\Delta]$-modules :

$$
\mu_{\mathrm{n}} \otimes \mathbb{Z}_{2} \bar{R}_{\mathrm{n} l \ell}=\overline{\mathrm{K}}_{\mathrm{n}} \otimes_{\mathbb{Z}} R_{2}\left(\mathrm{~K}_{\mathrm{n}}\right)_{\ell} \text {. }
$$

L'existence de cet isomorphisme résulte naturellement du fait que les suites exactes du théorème 30 sont scindées. Comme $\bar{R}_{2}\left(K_{n}\right)$ 
est canoniquement le dual de Pontrjagin du groupe de torsion $b_{n}$ (cf. théorème 13), le corollaire 31 établit une dualité entre le sous-groupe de $l^{n}$-torsion $\ell^{n} \sigma_{n}$ de $\sigma_{n}$ (qui est donc le sous-groupe de $\ell^{n}$-tor sion du groupe de Galois $a_{n}=\operatorname{Gal}\left(M_{n} / K_{n}\right)$ attaché à la $\ell$-extension abélienne $\ell$-ramifiée maximale de $\mathrm{K}_{\mathrm{n}}$ ) et le quotient $\ell^{n} R_{2}\left(\mathrm{~K}_{\mathrm{n}}\right) \ell$ d'exposant $\ell^{\mathrm{n}}$ du noyau modéré $R_{2}\left(\mathrm{~K}_{\mathrm{n}}\right)_{\ell}$ de la $K$-théorie. Le résultat établi contient donc la dualité entre les groupes $l^{G} \mathrm{~K}$ et $R_{2}(\mathrm{~K}) / R_{2}(\mathrm{~K})^{l}$, relevée par Cramer et Candiotti, qui correspond au cas $n=1$ ( cf. [CK]). Mais $\mathrm{il}$ apporte surtout une information plus précise : Alors que les dualités établies par Carroll ( pour $\ell=2$ ), par Cramer et Candiotti (pour $l$ impair) ne concernent que les structures de groupe, le corollaire 31 affirme l'existence ( sous la conjecture de Leopoldt) d'un isomorphisme de modules galoisiens du tensorisé $\bar{\mu}_{n} \otimes R_{2}\left(K_{n}\right)$ sur le dual de Pontrjagin du groupe $\bar{\mu}_{n} \otimes \mathbb{Z} l^{n} \sigma_{n}$.

Remarque 1. - II n'y a pas lieu d'attendre d'isomorphisme canonique du tensorisé $\mu_{\mathrm{n}} \otimes_{\mathbb{Z}} \mathrm{l}^{\mathrm{n} \bar{R}_{2}}\left(\mathrm{~K}_{\mathrm{n}}\right)$ sur le tensorisé $\bar{\mu}_{\mathrm{n}} \otimes_{\mathbb{Z}} \mathrm{ln}^{R} 2^{\left(\mathrm{K}_{\mathrm{n}}\right)}$, quoique ces deux groupes soient effectivement isomorphes en vertu du corollaire 31 . L'un et l'autre sont, en effet, des quotients du sousgroupe de $l^{n}$-torsion du noyau régulier $\Re_{n}$. Cependant, le contre exemple de Greenberg ( cf. $\left[\mathrm{Gb}_{2}\right]$ ) montre que les sous-groupes de $l^{n} \Re_{n}$ qui leur correspondent ( à savoir le radical initial des $\mathbb{Z}_{l^{-e x-}}$ tensions $l^{n}\left(\bar{\pi}_{l} \otimes_{\mathbb{Z}} \Omega_{\infty}\right)_{\text {div }}^{\Gamma_{n}}$, diune part, et le noyau universel $\ell^{n}\left(\bar{\pi}_{\ell} \otimes \mathbb{Z}_{\infty} \Re_{\text {div }}^{\Gamma_{n}}\right.$ d'autre part $)$ ne coincident pas toujours.

Remarque 2. - Les suites exactes (vii) et ( $\overline{\mathrm{vi}})$ étant scindées, le théorème 30 permet d'exprimer les $\ell^{\mathrm{m}}$-rangs des groupes $R_{2}\left(\mathrm{~K}_{\mathrm{n}}\right)_{\ell}$ et $\bar{R}_{2}\left(\mathrm{~K}_{\mathrm{n}}\right)_{\ell}$, pour chaque $\mathrm{m} \leq \mathrm{n}$, à partir de ceux correspondant au $l-$ groupe des $l$-classes $C_{n}^{\prime}$. Il donne également les caractères des $\mathbb{F}_{\ell}[\Delta]$-modules $R_{2}\left(\mathrm{~K}_{\mathrm{n}}\right) / R_{2}\left(\mathrm{~K}_{\mathrm{n}}\right)$ et $\bar{R}_{2}\left(\mathrm{~K}_{\mathrm{n}}\right) / \bar{R}_{2}\left(\mathrm{~K}_{\mathrm{n}}\right)^{l}$ en fonction de celui du quotient $\mathrm{Cl}_{\mathrm{n}} / \mathrm{Cl}_{\mathrm{n}} l\left(\mathrm{cf} \cdot\left[\mathrm{Ja}_{7}\right]\right.$, pour plus de détails).

Partons maintenant des deux suites (vii) et ( $\overline{\mathrm{vii}}$ ), induites par les symboles de Hilbert, et données par le scolie 27 : Appliquant le même procédé que plus haut, nous obtenons les suites exactes : 


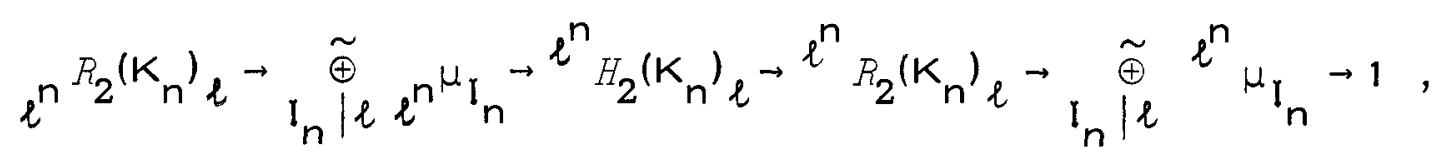

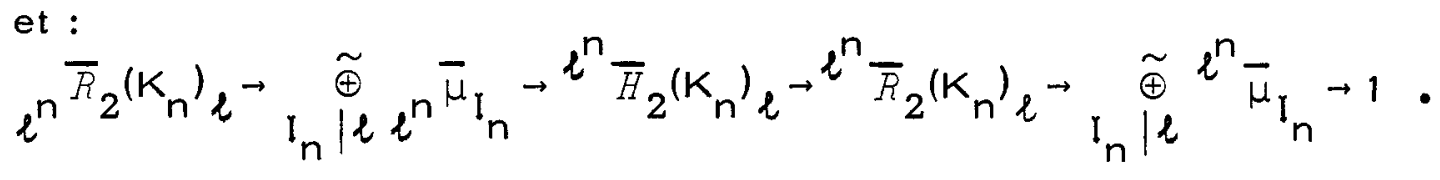

Désignons par $H_{\mathrm{n}}$ l'un quelconque des deux groupes $\bar{\mu}_{\mathrm{n}} \otimes_{\mathbb{Z}}{ }_{2}{ }^{(\mathrm{K}} \mathrm{n}^{\prime} \mathrm{l}$ ou $\mu_{n} \otimes \mathbb{Z}_{2} \bar{H}_{2}\left(K_{n}\right)$; et appuyons-nous sur l'isomorphisme précédent $\bar{\mu}_{n} \otimes_{Z} R_{2}\left(K_{n}\right)_{l} \simeq \mu_{n} \otimes_{\mathbb{Z}} \bar{R}_{2}\left(\mathrm{~K}_{\mathrm{n}}\right)_{l}$, donné par le corollaire 31 , pour noter $R_{\mathrm{n}}$ ce dernier groupe. Nous pouvons alors écrire les deux suites précédentes sous la forme légèrement modifiée :

$$
\Re_{\mathrm{n}} \longrightarrow \underset{\mathrm{I} \mid l}{\tilde{\oplus}} \mathbb{Z} / l^{\mathrm{n}} \mathbb{Z} \longrightarrow H_{\mathrm{n}} \longrightarrow R_{\mathrm{n}} \longrightarrow \underset{I_{\mathrm{n}} \mid l}{\oplus} \mathbb{Z} / l^{\mathrm{n}} \mathbb{Z} \longrightarrow 1
$$

où la flèche de gauche est induite par les symboles de Hilbert. II vient donc :

COROLLAIRE 1.2.32. - Sous la conjecture de Leopoldt dans $K_{n}$, il existe un isomorphisme de $\mathbb{Z}_{\ell}[\Delta]$-modules :

$$
\mu_{n} \otimes_{\mathbb{Z}} \bar{H}_{2}\left(K_{n}\right)_{\ell} \simeq \bar{\mu}_{n} \otimes_{\mathbb{Z}} H_{2}\left(K_{n}\right)_{\ell} .
$$

D'après le théorème 24 , l'isomorphisme obtenu établit une dualité entre le sous-groupe de $\ell^{n}$-torsion $\ell^{n} \sigma_{n}^{\prime}=\ell^{n} H_{n}$ du groupe de Galois $\mu_{n}=\operatorname{Gal}\left(H_{n} / K_{n}\right)$ de la $\ell$-extension hilbertienne maximale de $K_{n}$ et le quotient $\ell^{n} H_{2}\left(K_{n}\right)$ du noyau hilbertien $H_{2}\left(K_{n}\right)_{\ell}$ de la $K$ - théorie.

\section{c.- Isomorphisme du miroir.}

Rappelons brièvement la définition des éléments infinitési maux d'un corps de nombres, relativement à un premier $\ell$ fixé ( cf. chapitre II, pour plus de détails ) : Le corps $K$ étant donné, l'injection canonique du groupe multiplicatif $K$ ' des éléments de $K^{x}$ étran gers à $l$ dans le groupe des unités de l'algèbre semi-locale $\mathbb{Z} \ell^{\otimes} \mathbb{Z} K$ induit une surjection naturelle du tensorisé $\Omega_{K}^{\prime}=\mathbb{Z}_{l} \otimes_{\mathbb{Z}} K^{\prime}$ sur le sous-groupe des unités principales $U_{K}^{1}=\prod_{I \mid l} U_{I}^{1}$ semi-locales de $K$. 
Nous appelons infinitésimaux les éléments du noyau $\Im_{K}$ de cette application.

DÉFINITION 1.2.33. - Nous appelons noyau infinitésimal d'un corps de nombres $K$, et nous notons $\mathfrak{F}_{K}^{\prime}$, le sous-groupe du noyau régu lier $\Re_{K}$ engendré par les images des infinitésimaux :

$\mathbb{S}_{K}^{\prime}=\left\{\mathfrak{x} \in \Re_{K} \mid \mathbb{E} \in \mathbb{N}\right.$, $\left.\mathbb{H} x^{\infty} \in \widetilde{S}_{K}, \mathfrak{x}=l^{-m} \otimes x^{\infty}\right\}$

Comme les infinitésimaux sont précisément les éléments glo baux d'image locale 1 aux places $I$ de $K$ divisant $\&$, les symboles de Hilbert sauvages sont triviaux sur les éléments de $\mathbb{\complement}_{K}^{\prime}$, et $\mathbb{\Xi}_{K}^{\prime}$, qui est contenu par définition dans le noyau régulier $\Re_{K}$, est en fait dans le noyau hilbertien $S_{2} K$.

Cela posé, écrivons $\mathfrak{S}_{\infty}^{\prime}={ }_{n} \in \mathbb{N}^{\cup} \mathbb{E}_{n}^{\prime}$ le radical infinitésimal associé à la $\mathbb{Z} l^{\text {-extension cyclotomique }} K_{\infty}=\underset{n \in \mathbb{N}}{U} K_{n}$ construite sur $K$. Nous avons:

THÉORÈME 1.2.34. - Le noyau infinitésimal $\mathbb{S}_{\infty}^{\prime}$ est le radical kummérien associé à la $\ell$-extension abélienne maximale non ramifiée et $\ell$-décomposée ( $i . e$. non ramifiée aux places finies et complètement décomposée à celles divisant $\& \mid C_{\infty}^{\prime}$ du corps $\left.K_{\infty}\right)$. Il vient donc :

$$
\widetilde{c}_{\infty}^{\prime} \simeq \operatorname{Hom}\left(c_{\infty}^{\prime}, \mu_{\ell}\right)
$$

puisque le groupe $C_{\infty}^{\prime}=\frac{\varliminf \mathrm{im}}{\mathrm{n}} C_{n}^{\prime}$ correspond à $\mathrm{Gal}\left(C_{\infty}^{\prime} / K_{\infty}\right)$ dans l'isomorphisme du corps de classes.

Démonstration: D'après la théorie de Kummer, pour que l'extension $K_{\infty}\left[\sqrt[\ell^{k}]{x}\right] / K_{\infty}$, définie par un élément $z=l^{-k} \otimes x$ de $\Re_{\infty}$ soit $l$-dé composée, il faut et il suffit que l'élément $x$ soit une puissance $l^{k}-$ ième dans l'algèbre semi-locale $\hat{k}_{n}=\mathbb{Z}_{l^{\otimes}} \mathbb{Z}_{n}$, pour chaque $n$ assez grand. Désignons par $R_{n}$ le tensorisé $\ell$-adique $\mathbb{Z} \ell^{\otimes} \mathbb{Z}_{n} K_{n}^{X} d u$ groupe multiplicatif de $K_{n}$. Par surjectivité de l'application de semilocalisation $s_{n}$, la condition requise, qui s'écrit $s_{n}\left(x_{n}\right)=u_{n}^{l}$ dans $\hat{\mathrm{K}}_{\mathrm{n}}$, affirme l'existence d'un $y_{\mathrm{n}}$ de $\Omega_{\mathrm{n}}$ vérifiant : 
$s_{n}(x)=s_{n}(y)^{l}$, i.e. $s_{n}\left(x / y^{l}\right)=1$. II vient donc $x=l^{-k} \otimes\left(x / y^{l}\right)$, avec $x / y^{l} \in \Im_{n}$, comme annoncé .

PROPOSITION 1.2.35. - Dans une tour cyclotomique, les noyaux infinitésimaux vérifient la théorie de Galois :

$$
\mathfrak{s}_{\infty}^{1}=\mathfrak{s}_{\infty}^{1} \Gamma
$$

En particulier, liisomorphisme fondamental de la théorie de Kummer pour les noyaux infinitésimaux s'écrit :

$$
\mathfrak{s}_{n}^{\prime} \cong \operatorname{Hom}_{\Gamma_{n}}\left(\mathfrak{s}_{\infty}^{\prime}, \mu_{\ell}\right)
$$

Démonstration : II s'agit simplement de vérifier l'égalité $\mathbb{E}_{m}^{\prime} \cap \Re_{n}=\mathbb{E}_{n}^{\prime}$, pour $m \geq n$, et plus précisément linclusion $\mathfrak{S}_{m}^{\prime} \cap \Re_{n} \subset \mathbb{E}_{n}^{\prime}$. Soit donc $r=l^{-k} \otimes x_{m}^{\infty}=l^{-k} \otimes x_{n}$ un élément de lintersection, avec $x_{m}^{\infty} \in \Im_{m}$ et $x_{n} \in R_{n}$. Nous avons alors $x_{n}=\zeta_{m} x_{m}^{\infty} y_{m}^{l^{k}}$ pour une racine $l^{m}$ ième de l'unité $\zeta_{m} \in \mu_{m}$ et un y $y_{m}$ convenable dans $R_{m}$, ce qui nous prouve que l'extension semi-locale $\hat{k}_{n}\left[\sqrt[\ell]{u_{n}}\right] / \hat{k}_{n}$ ( où $u_{n}=s_{n}\left(x_{n}\right.$ ) est l'image semi-locale de $x_{n}$ ) est cyclotomique. Par la théorie de Kummer, nous en concluons que $u_{n}$ est le produit $\xi_{n} v_{n}^{l^{k}}$ d'une racine de l'unité semi-locale et d'une puissance $l^{k}$-ième dans $\hat{k}_{n}{ }_{n}$. Ecrivant $v_{n}=s_{n}\left(y_{n}\right)$, puis choisissant $h$ assez grand pour avoir $\xi_{n}^{l^{h}}=1$, nous obtenons donc $s_{n}\left(x_{n} / y_{n}^{l^{k}}\right)^{l^{h}}=\left(u_{n} / v_{n}^{l^{k}}\right)^{l^{h}}=1$, d'où :

$x=l^{-k} \otimes x_{n}=l^{-k} \otimes\left(x_{n} / y_{n}^{l^{k}}\right)=l^{-k-h} \otimes\left(x_{n} / y_{n} l^{k}\right)^{l^{h}}, \quad$ et $\left(x_{n} / y_{n}^{l^{k}}\right)^{l^{h}} \in \Im_{n}$;

ce qui est le résultat attendu. L'isomorphisme annoncé résulte alors du théorème 34 .

L'importance des groupes $\mathfrak{S}_{n}^{\prime}$ tient au théorème fondamental suivant : 
THÉORÈME 1.2.36. - Isomorphisme du miroir - Lorsque le corps $K$ vérifie la conjecture de Leopoldt, le noyau infinitésimal $\mathfrak{S}_{K}^{\prime}$ s'identifie canoniquement au sous-groupe de torsion $\sigma_{K}^{\prime}=y_{K}^{t o r} d u$ groupe de Galois H $_{K}=\mathrm{Gal}\left(H_{K} / K\right)$ de la composée des $\ell$-extensions cy cliques de $K$ qui se plongent dans une $l$-extension cyclique de degré arbitrairement grand. Autrement dit, $\mathfrak{S}_{K}^{\prime}$ est le dual de Pontrjagin du groupe $\bar{H}_{2}(\mathrm{~K}){ }_{\ell}$.

Démonstration: Pour établir ce résultat, nous nous appuyerons sur la description des infinitésimaux donnée au chapitre 1 ( section 2 , $\S 1)^{(*)}$. Partons de la suite exacte $(\overline{\mathrm{vi}})$ de la proposition 25 :

$$
1 \longrightarrow \bar{H}_{2}(\mathrm{~K}) \longrightarrow \bar{R}_{2}(\mathrm{~K}) \longrightarrow \underset{\mathrm{I} / \mathrm{l}}{\tilde{e}_{l}} \bar{\mu}_{\mathrm{I}} \longrightarrow 1 \text {. }
$$

La suite duale s'écrivant précisément :

$$
1 \longrightarrow\left({ }_{I_{\mathrm{n}} \mid l}^{\oplus} \mu_{I_{\mathrm{n}}}\right) / \mu_{\mathrm{K}} \longrightarrow \sigma_{\mathrm{K}} \longrightarrow \sigma_{\mathrm{K}}^{\prime} \longrightarrow 1
$$

nous allons démontrer le théorème 36 en la retrouvant directement, à partir de la description infinitésimale du groupe $\sigma_{\mathrm{K}}$ comme quo tient $\theta_{K, \infty} / P_{K, \infty}$ du groupe des diviseurs quasi-infinitésimaux par le sous-groupe des diviseurs principaux infinitésimaux. Considérons pour cela un diviseur quasi-infinitésimal a . Par définition du groupe $D_{K, \infty}$ nous pouvons trouver un naturel $k$ tel que sa puissance $k^{k}$ ième soit principale infinitésimale, c'est-à-dire de la forme $a^{l^{k}}=\left(x^{\infty}\right)$ pour un $x^{\infty}$ de $\Im_{K}$ défini à une unité infinitésimale près. Sous la conjecture de Leopoldt dans $K$, l'élément $x^{\infty}$ est unique, et l'application naturelle :

$$
a\left(\bmod P_{K, \infty}\right) \mapsto l^{-k} \otimes x^{\infty}
$$

est ainsi un épimorphisme de $\sigma_{K}$ sur $\overleftarrow{E}_{K}^{\prime}$. Pour déterminer son noyau, considérons un diviseur a d'image triviale, c'est-à-direvérifiant $a^{l^{k}}=\left(x^{\infty}\right)$, avec $l^{-k} \otimes x^{\infty}=1$. Cette dernière condition s'écrit $x^{\infty}=\zeta_{S} \ell^{\ell^{k}}$, avec $\zeta \in \mu_{K}$ et y convenable dans $R_{K}$. Elle signifie donc que le diviseur a est principal, et qulil est engendré par un élé-

(*) Récemment, Nguyen Quang Do a proposé une démonstration cohomologique de ce résultat ( $\mathrm{cf} \cdot\left[\mathrm{Ng}_{3}\right]$ ). 
ment $y \in R_{K}$ dont l'image semi-locale est une racine de l'unité . Comme y est défini à une racine globale de l'unité près, l'application de semi-localisation induit un isomorphisme :

$$
\text { (y) }\left(\bmod p_{K, \infty}\right) \longrightarrow s_{K}(y)\left(\bmod \mu_{K}\right)
$$

du noyau de l'application précédente sur le quotient $\hat{\mu}_{k} / \mu_{k}=\underset{I_{k} / \ell}{\oplus} \mu_{I_{k}} / \mu$, ce qui conduit à la suite exacte attendue :

$$
1 \longrightarrow\left(\underset{I_{K} \mid l}{\oplus} \mu_{I_{K}}\right) / \mu_{K} \longrightarrow \sigma_{K} \longrightarrow \varepsilon_{K}^{\prime} \longrightarrow 1 \text {. }
$$


$-80-$ 
Nous présentons ci-dessous une condition suffisante de la conjecture de Leopoldt, introduite par Bertrandias et Payan [BP] et déjà étudiée par Gillard $\left[\mathrm{Gi}_{1}\right]$ et Miki $\left[\mathrm{Mi}_{1}\right]$. Nous en donnons des formulations équivalentes via la théorie $\ell$-adique du corps de classes, ou au moyen de la $K$-théorie des corps de nombres. Nous montrons en outre qu'elle implique également la conjecture de Gross.

Les notations sont celles des sections 1 et 2 .

THEOREME 1.A.1. - Soient $\ell$ un nombre premier, et $K$ un corps de nombres algébriques contenant les racines $2 \ell$-ièmes de l'unité. Les six conditions suivantes sont équivalentes :

(i) Le $\ell$-Sylow $H_{2}(\mathrm{~K})$ du noyau dans $K_{2}(\mathrm{~K})$ des symboles de Hilbert est nul.

(ii) Le corps $K$ possède exactement $c_{k}+1$ extensions cycliques de degré $\ell$ linéairement indépendantes qui sont cycliquement plongeables (i.e. plongeables dans une $\ell$-extension cyclique de degré arbitrairement grand) $\left({ }^{\star}\right)$.

(iii) Le noyau infinitésimal $\mathfrak{F}_{K}^{\prime}$ attaché au corps $K$ est nul.

(iv) Le noyau infinitésimal $\mathfrak{S}_{K}^{\prime}$ attaché à la $\mathbb{Z}_{l^{-e x t e n s i o n ~ c y c l o-~}}$ tomique $K_{\infty}$ de $K$ est nul.

(v) La $\mathbb{Z}_{\ell}$-extension cyclotomique $K_{\infty}$ de $K$ ne possède pas de $\ell$-extension abélienne ramifiée $\ell$-décomposée non triviale. du corps $K$ est nul.

(vi) Le groupe $\sigma_{K}=\tilde{v}_{a_{K}} / v_{p_{K}}$ des classes de valeurs absolues

$\left({ }^{\star}\right)$ Le corps $K$ étant supposé ici totalement imaginaire, l'entier $c_{K}$ vaut donc $\frac{1}{2}[K: \mathbb{Q}]$. 
Lorsqu'elles sont satisfaites, les conjectures de Leopoldt et de Gross sont vérifiées à chaque étage fini de la tour cyclotomique $K_{\infty} / K$.

La condition ( $i$ ) est celle introduite par Bertrandias et Payan. Elle implique en particulier que le corps $K$ possède au plus (et donc exac-

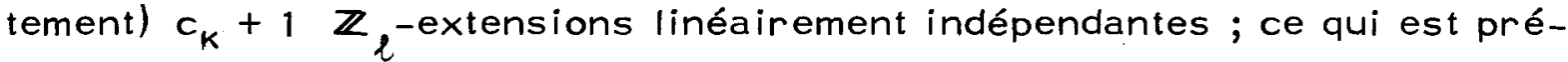
cisément la conjecture de Leopoldt pour $K$ (cf. th.1.1.22, assertion (i)). La condition $(v)$ est celle considérée par Gillard, qui note qu'elle résulte de (ii). Pour voir qu'elle entraîne en fait la conjecture de Leopoldt dans la totalité de la tour $K_{\infty} / K$, il suffit de remarquer que, ci celle-ci était en défaut à un étage donné, disons $K_{n}$, le tensorisé $\ell$-adique $\mathbb{Z}_{\ell} \otimes_{\mathbb{Z}} K_{n}^{x}$ contiendrait une unité infinitésimale non triviale $\epsilon_{n}$ de sorte que l'extension abélienne $K_{\infty}\left[\sqrt[l]{\epsilon_{n}}\right] / K_{\infty}$ serait infinie non ramifiée et $l$-décomposée, contrairement à $(v)$. L'équivalence de $(i i)$ et de $(v)$ a été établie par Gillard, et indépendamment par Miki, sous la condition subsidiaire que les étages initiaux de la tour $K_{\infty} / K$ ne soient pas $\ell$-décomposés. Comme nous allons le voir, cette restriction n'est nullement nécessaire.

Auparavant, notons que la condition (i) implique la conjecture de Leopoldt, et la condition ( $v i$ ) celle de Gross. Le dernier point résulte trivialement du théorème 1.1.26, puisqulune façon d'énoncer la conjecture de Gross consiste précisément à affirmer la finitude du groupe $\widetilde{J}_{K}$ • Le premier s'établit comme suit : Sous la condition $H_{2}(K) l=1$, le noyau dans $K X / K^{X \ell}$ des symboles universels $\left\{\zeta_{l},.\right\}$ coincide avec celui des symboles de Hilbert. Or le théorème fondamental de la $K$-théorie des corps de nombres montre que ce noyau universel est un $\mathbb{F}_{\ell}$-espace vectoriel de dimension $c_{k}+1$. Comme le noyau hilbertien contient évidem-

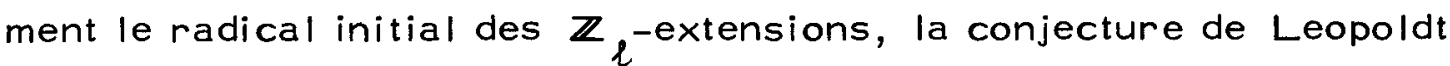
pour $K$ en résulte.

Démonstration : Nous avons les équivalences :

(i) $\Leftrightarrow(i i)$, sous Leopoldt; d'après le corollaire 1.2.32 qui donne $H_{2}(\mathrm{~K})_{\ell}=1 \Leftrightarrow \bar{H}_{2}(\mathrm{~K})_{\ell}=1$, et puisque, sous Leopoldt, la condition (ii) s'écrit encore $\bar{H}_{2}(\mathrm{~K})_{\ell}=1$.

(ii) $\Leftrightarrow(i i i)$, sous Leopoldt; en vertu du théorème 1.2.36 qui montre que $\mathfrak{s}_{K}^{\prime}$ est le dual de Pontrjagin de $\bar{K}_{2}(K)_{\ell}$. 
(iii) $\Leftrightarrow(i v)$ car $\mathbb{E}_{K}^{\prime}$ étant le sous-groupe de $\mathfrak{S}_{K_{\infty}}^{\prime}$ fixé par $\Gamma=\operatorname{Gal}\left(K_{\infty} / K\right)$, l'identité $\mathbb{S}_{K}^{\prime}=1$ entraîne $\widetilde{E}_{K_{n}}^{\prime}=1$, pour tout $n$, i.e. $\mathfrak{V}_{K_{\infty}}^{\prime}=1$ (cf. proposition 1.2 .35 ).

(iv) $\Leftrightarrow(v)$ car $\mathfrak{S}_{K_{\infty}}^{\prime}$ est le radical kummérien de la $\ell$-extension abélienne non ramifiée $\ell$-décomposée maximale $C_{\infty}^{\prime}$ de $K_{\infty}$.

$(v) \Leftrightarrow(v i)$ car $\xi_{K}$ est le quotient des $\ell$-genres $\Gamma_{G a l}\left(C_{\infty}^{\prime} / K_{\infty}\right)$ de l'extension procyclique $K_{\infty} / K$ (cf. théorème 1.1 .29 ).

Comme nous savons déjà que les conditions (i), (ii) et (v) entraînent la conjecture de Leopoldt, le théorème en résulte. 
CHAPITRE II

INDÉPENDANCE l-ADIQUE DE NOMBRES ALGÉBRIQUES

CALCUL INFINITÉSIMAL DANS UN CORPS DE NOMBRES ALGÉBRIQUES 


\section{1. - INDÉPENDANCE l-ADIQUE dE NOMBRES ALgÉBRIQUES .}

1.-Position du problème du problème et énoncé de la conjecture....... II.3

a.- Les deux complétions multiplicatives d'un corps de nombres....................................

b. - Enoncé de la conjecture......................... 11.6

c. - Discussion de la conjecture énoncée.................... II. 10

2.-Minorations du rang $\ell$-adique et preuve de la conjecture

dans le cas abélien....................................13

a. - Preuve de la conjecture dans le cas abélien............... II.13

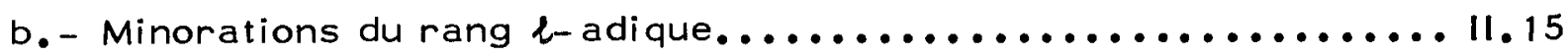

c. - Comparaison des divers résultats obtenus................ II.20

3.- Application aux conjectures de Leopoldt et Gross............... II.23

a. - La conjecture de Leopoldt et le problème du rang

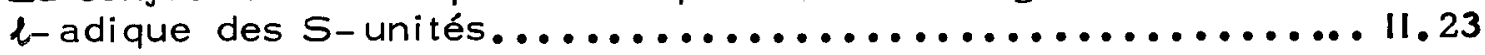

b. - La conjecture de Gross et le groupe des valeurs

absolues $\ell$ - adiques des $s$-unités.................... 11.27

c. - Application aux problèmes normiques dans les

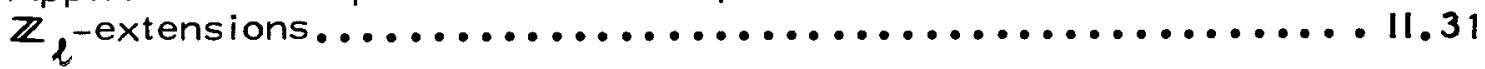

\section{2.- CALCUL INFINITÉSIMAL DANS UN CORPS DE NOMBRES ALGÉBRIQUES.}

1.-Eléments infinitésimaux d'un corps de nombres algébriques........ II.37

a.- Définition des éléments infinitésimaux................. 11.37

b. - Interprétation kummérienne des infinitésimaux............. II.39

c. - Définition des diviseurs infinitésimaux................. II.41

d. - Interprétation arithmétique du groupe des classes infinitésimales.................................11.44 
2.-Groupes de $S$-classes $T$-infinitésimales................. 11.46

a.- Eléments $T$-infinitésimaux......................... 11.46

b. - S-classes T-infinitésimales........................ 11.48

c.- Interprétations arithmétiques : applications à la dualité....... 11.50

3.- La suite exacte des classes ambiges dans une extension

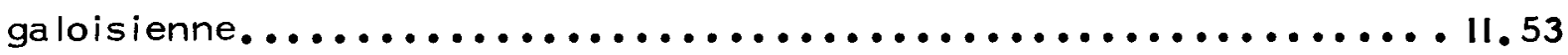

a.- L'homomorphisme d'extension pour les groupes a .......... 11.53

b. - Application au sous-groupe de torsion $\tau$...................

c. - Extension aux $\ell$-groupes de $S$-classes T-infinitésimales....... 11.59

4.- Genre infinitésimal d'une extension de corps de nombres.......... II.61

a.- L'application norme pour les groupes . ................ II.62

b. - S-genre T-infinitésimal d'une extension de corps

de nombres................................... 11.65

c.- Application au symbole de reste normique généralisé......... II.68 
$-88-$

$8-$ 
Le problème de l'indépendance $l$-adique de nombres algé briques slest posé historiquement à l'occasion d'un calcul analytique : On sait depuis longtemps que la fonction zêta complexe d'un corps de nombres se prolonge en une fonction méromorphe sur le plan complexe avec un unique pôle, simple, au point 1 , et que l'expression du résidu en ce point fait intervenir le produit du nombre de classes de ce corps par le régulateur complexe du groupe de ses unités. De façon tout à fait semblable, on sait aujourd'hui que la fonction zêta $\ell$-adique, qui est méromorphe sur le disque unité fermé du corps $\mathbb{C}_{\ell}$, admet un unique pôle éventuel au point 1 , avec pour résidu, outre un certain nombre de facteurs canoniques, le produit du nombre de classes par le régu lateur $\ell$-adique des unités, la non nullité de ce régulateur étant la condition de la présence effective du pôle (cf. $\left[\mathrm{Se}_{2}\right]$ ).

En fait, clest en 1962 que Leopoldt, dans son étude sur l'arithmétique des corps abéliens (cf. $\left[L_{2}\right]$ ), introduit le régulateur $\ell$-adique des unités, et suggéra sa fameuse conjecture sur l'égalité du rang $\ell-$ adique des unités et du nombre de Dirichlet $r_{k}+c_{k}-1$. En 1965 , Ax (cf. $[\mathrm{Ax}]$ ), s'appuyant sur un analogue $\ell$-adique dû à Mahler du théo rème de Guel'fond-Schneider ${ }^{(\star)}$, démontra cette conjecture dans un certain nombre de cas particuliers ; mais c'est Brumer, en 1967 , en généralisant au cadre $\ell$-adique le récent résultat de Baker sur l'indépendance $\ell$-adique de nombres algébriques (cf. $[\mathrm{Br}]$ ), qui établit finalement le résultat pour tous les corps abéliens. Dans les autres cas, cependant, la conjecture n'a guère avancé depuis : les méthodes algébriques, illustrées au Chapitre IV (cf. Ch.IV.2, $3 \S b$ ), donnent des résultats précis, mais qui ne permettent de conclure que pour des corps particuliers; les méthodes transcendantes donnent seulement

${ }^{\star}$ ) Il slagit de la version $\ell$-adique du septième problème de Hilbert . 
des minorations du rang $\ell$-adique : le résultat actuellement le plus fin , da à $W$ aldschmidt (cf. $\left[W_{a}\right]$ ), et sur lequel nous reviendrons plus loin, permet ainsi dlaffirmer que le rang $\ell$-adique des unités est du moins la moitié du nombre de Dirichlet ${ }^{\star}$. De ce fait, une démonstration de la conjecture de Schanuel $\ell$-adique résoudrait complètement le problème .

D'un autre côté, la conjecture de Leopoldt sur la non nullité du régulateur $\ell$-adique des unités et les relations attendues entre ce régulateur et le résidu au point 1 de la fonction zêta $\ell$-adique ont été généralisées par Serre aux fonctions $L$ d'Artin $\ell$-adiques attachées à certaines représentations galoisiennes. Etudiant le comportement de ces mêmes fonctions au voisinage de 0 , Gross énonça une conjecture analogue à celle de Leopoldt $\left.{ }^{(\star}\right)$, mais faisant intervenir cette fois le régulateur $\ell$-adique construit sur les $\ell$-unités imaginaires d'un corps à conjugaison complexe, conjecture qu'il démontra dans le cas abélien, à l'aide du théorème de Baker - Brumer .

Le parallèle extrêmement intéressant entre ces deux conjec tures appelle plusieurs commentaires : Dlabord la différence, soulignée par plusieurs auteurs $(\star \star \star)$, entre les régulateurs qui leur corres pondent, est en grande partie factice : Leopoldt a introduit sa conjec ture pour un corps totalement réel; Gross pour un corps à conjugaison complexe (i.e. pour une extension quadratique totalement imaginaire diun corps totalement réel); dans les deux cas, pour un type de corps dans lequel le comportement des places à l'infini est très particulier. En llabsence de conditions du même ordre sur les places au-dessus de $l$, il n'y a pas lieu de s'étonner si réapparait dans le résultat la dissymétrie introduite dans les hypothèses. De fait, l'étude synoptique du chapitre IV (cf.Ch. IV.2, $3 \S \mathrm{c}$ ) fait apparaitre une parfaite dualité entre les interprétations arithmétiques de ces deux conjectures, et fournit un même critère algébrique de leur validité. Enfin, et c'est ce qui

(*) On a même une conclusion sensiblement plus précise (cf.th.15).

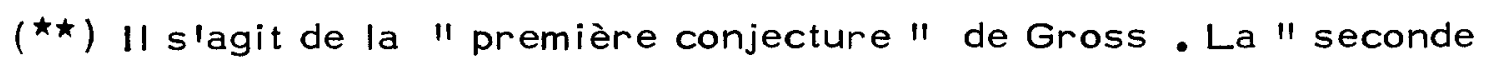
conjecture " concerne les relations entre le régulateur des $\ell$-unités et le coefficient dominant du développement Taylorien de la fonction $L$ d'Artin au voisinage de l'origine. Pour plus de détails sur les conjec tures de Gross, on pourra consulter le livre de Koblitz [Ko].

$\left({ }^{\star \star}\right)$ Ainsi $[\mathrm{Ko}],\left[\mathrm{Fe}_{1}\right], \ldots$ : les deux régulateurs n'ont pas la même dimension. 
nous intéresse plus particulièrement ici, les arguments de transcen dance invoqués pour justifier llune ou l'autre de ces conjectures ne prennent jamais en compte la nature arithmétique des éléments algé briques manipulés.

C'est pourquoi nous avançons ici une conjecture générale, contenant celles dûes à Leopoldt et à Gross, qui décrit le comporte ment $l$-adique de n'importe quel sous-module noethérien du groupe multiplicatif des éléments non nuls d'un corps de nombres algébriques. Plus précisément, nous postulons que le rang $\ell$-adique d'un tel mo dule se lit sur le caractère de la représentation galoisienne qui lui est associée.

Avant d'énoncer cette conjecture, traçons d'abord le cadre général du problème :

\section{1.- POSITION DU PROBLËME ET ÉNONCÉ DE LA CONJECTURE .}

\section{a.-Les deux complétions multiplicatives d'un corps de nombres.}

Un corps de nombres $K$ étant donné, il existe deux façons

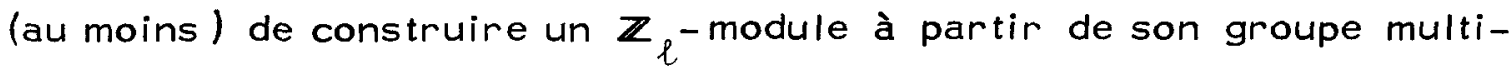
plicatif :

- La plus simple consiste à former directement le produit ten soriel $\ell=\mathbb{Z}_{\ell} \otimes \mathbb{Z} K^{X}$. Le groupe obtenu est un $\mathbb{Z}_{\ell}$-module qui n'est ni de type fini, ni même compact pour la topologie des sous-modules d'indice fini, mais cependant réunion dénombrable de sous-modules com pacts : $S i\left(S_{n}\right)_{n} \in N$ est une suite croissante de parties qui recouvre l'ensemble $P I_{K}$ des places non complexes de $K$, le groupe $R$ est la réunion croissante, lorsque $n$ décrit $\mathbf{N}$, de ses sous-modules noethériens $\delta^{S}$, où $\delta^{S}=\mathbb{Z}_{\ell} \otimes_{\mathbb{Z}}$, $E^{S}$ désigne le tensorisé $\ell$-adique du groupe des $S$ unités de $K$ (i.e. la fermeture de llimage de $E^{S}$ dans $R$ ).

- Une autre façon de procéder consiste à introduire dlabord le complété semi-local de $K$ pour les places au-dessus de $l$, et à considérer ensuite le groupe multiplicatif $K_{l}^{x}=\left(\mathbb{Z}_{l} \otimes_{\mathbb{Z}} K\right)^{x}$ de l'algèbre obte nue. Comme $\mathrm{K}_{\ell}$ est la somme directe des complétés de $\mathrm{K}$ pour les pla ces au-dessus de $\ell$, le groupe $K_{\ell}^{X}$ est le produit des groupes multi plicatifs des corps locaux $\mathrm{K}_{\mathfrak{l}}$ : 


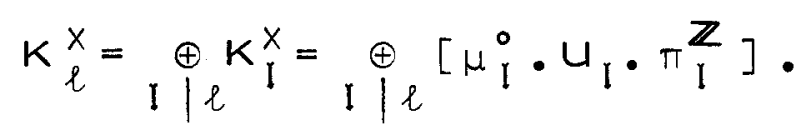

Sa décomposition directe fait donc intervenir les groupes $\mu_{j}^{0}$ de racines locales de l'unité d'ordre étranger à $l$, les groupes locaux d'unités principales $u_{1}=(1+1)$, et autant de $\mathbb{Z}$-modules libres de rang 1 associés à une uniformisante locale $\pi_{\mathfrak{l}}$ qu'ily a dans $K$ de places au-des sus de $\ell$. Le sous-groupe $u_{l}=1 \stackrel{\oplus}{\oplus} u_{l}$ étant déjà un $\mathbb{Z}_{l}$-module , la manière la plus économique de fabriquer un $\mathbb{Z}_{\ell}$-module à partir de $k_{l}^{x}$ est de former le complété profini :

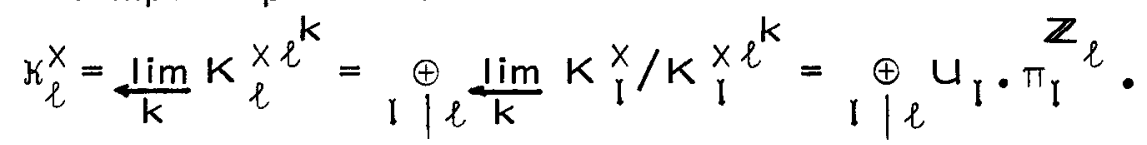

Le groupe $\kappa_{\ell}^{x}$ ainsi construit, produit direct des complétés profinis $\varkappa_{\mathfrak{l}}^{x}$ des groupes multiplicatifs des corps locaux $K_{l}$ est un $\mathbb{Z}_{\ell}$-module com pact, et même noethérien .

Cela posé, llinjection naturelle de $k^{x}$ dans $k_{\ell}^{x}(i . e$. l'applica tion diagonale de $K^{X}$ dans le produit $\left(\underset{l}{\oplus} K^{X}\left(\begin{array}{l}x \\ I\end{array}\right)\right.$ se prolonge de façon unique en un morphisme continu du tensorisé $R=\mathbb{Z}_{\ell} \mathbb{Z}_{\mathbb{Z}} K^{x}$ dans le complété profini $k_{l}^{x}=\frac{\lim }{k} K_{\ell}^{x} / k_{l}^{x l}$. Nous allons voir que cet homomorphisme est surjectif.

THÉORĖME \& DÉFINITION 11.1.1.- Etant donné un corps de nombres $K$, l'application naturelle $s$ du tensorisé $\ell$-adique $\Omega=\mathbb{Z}_{\ell} \mathbb{Z}^{K} K^{X}$ du groupe multiplicatif de $k$ dans le complété profini $k_{\ell}^{x}=\frac{\varliminf_{k}}{k} K_{\ell}^{x} / K_{\ell}^{x}{ }_{\ell}$ du groupe des éléments inversibles de l'algèbre semi-locale $K_{\ell}=\mathbb{Z}_{\ell} \otimes_{\mathbb{Z}} K$, est un épimorphisme continu appelé application de semi-localisation.

Démonstration : La surjectivité de llapplication s repose sur deux argu ments :

- Dlune part, chaque classe d'idéaux d'un corps de nombres contient un idéal étranger à un nombre fini de places données. En par ticulier chacun des idéaux premiers $\mathfrak{l}$ de $K$ au-dessus de $\ell$ est associé à un idéal $a_{\mathfrak{l}}$ étranger à $l$. Ecrivant $\mathfrak{l}=x_{\mathfrak{l}} a_{\mathfrak{l}}$, nous voyons qulilest donc 
toujours possible de trouver dans $K^{X}$ une famille d'éléments $\left(x_{1}\right)$ I $\mid \ell$ vérifiant les conditions de valuation :

(i) $x_{l}$ est une uniformisante locale pour la place $l$ (i.e. $\left.v_{1}\left(x_{1}\right)=1\right)$

(ii) $x_{l}$ est une unité locale pour les places $I I \mid I$ autres que $\mathfrak{l}\left(i . e . v_{\mathfrak{l}}\left(x_{\mathfrak{l}}\right)=0\right)$.

- D'autre part, le corps $K$ est dense dans le produit $K_{\ell}=\underset{l \mid l}{\oplus} K_{l}$ de ses complétés pour les places au-dessus de $l$. Si donc $\left(u_{i}\right)_{i}=1, \ldots, n$ est une famille génératrice du $\mathbb{Z}_{l}$-module $u_{l}=1 \stackrel{\oplus}{e} u_{l}$, et $m$ un entier naturel, il est encore possible de résoudre dans $K^{X}$ les $n$ systèmes de congruences:

$$
\text { (iii) } s\left(y_{i}\right) \equiv u_{i}\left(\bmod I^{m}\right), \quad \forall I \mid \ell \text {. }
$$

Cela étant, si $\mathrm{m}$ est choisi plus grand que le module d'hyper -

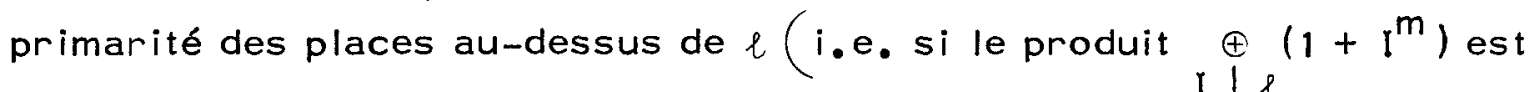
contenu dans $\left.u_{l}^{l}\right)$, le lemme de Nakayama montre que les éléments $\left(s\left(x_{i}\right)\right)_{i \mid l}$ et $\left(s\left(y_{i}\right)\right)_{i=1, \ldots, n}$ engendrent conjointement le $\mathbb{Z}_{l}$-module $k_{l}^{x}$, ce qui établit le théorème.

Il est bien clair, en revanche, que l'application de semi-loca lisation $s$ n'est jamais injective (comme sa "restriction "à $K^{x}$ ), le groupe $k_{l}^{X}$ étant un $\mathbb{Z}_{\ell}$-module de type fini, mais non le tensorisé $R$. Le premier problème de l'indépendance $\ell$-adique est donc le suivant :

Problème 1. - Etant donnés $x_{1}, \ldots, x_{n}$ dans $K^{x}$, à quelle condition la restriction de $s$ au sous-module $\mathbb{Z}_{l}$-engendré par les images de ces élé ments dans $R$ est-elle injective ?

Posée sous cette forme, la question n'est cependant pas totalement satisfaisante, le rôle dans $\kappa_{l}^{x}$ des places au-dessus de $l$ étant par trop particulier : il est clair, par exemple, que les images $s\left(x_{i}\right)$, pour $i=1, \ldots, n$, dlun nombre fini d'uniformisantes locales associées à des places distinctes au-dessus du premier $\ell$ sont trivialement indépen dantes; mais ce résultat est en défaut pour tout autre premier $p \neq l$. Pour lisser la situation, il est naturel de faire appel au logarithme $\ell-$ adique : L'application $\log _{\ell}$, qui est définie sur le sous-groupe princi pal $u_{\ell}$ de $k_{\ell}^{X}$ par son développement en série entière: 


$$
\log _{\ell} u=\sum_{k=1}^{\infty}(-1)^{k-1} \frac{(u-1)^{k}}{k},
$$

se prolonge de façon unique à $k_{\ell}^{x}$ grâce à son équation fonctionnelle

$$
\log _{\ell} x y=\log _{\ell} x+\log _{\ell} y \text {, }
$$

si llon convient de poser $\log _{\ell} \ell_{1}=0$, pour chaque place de I de $\mathrm{K}$ audessus de $\ell$, en désignant par $\ell$ l limage dans $k_{\mathfrak{l}}^{x}$ du nombre premier $\ell$. II vient alors :

LEMME 11.1.2. - Le logarithme d'Iwasawa Log $_{\ell}$ est un morphisme continu du groupe multiplicatif $\kappa_{\ell}^{X}$ dans le groupe additif $K_{\ell}=I \stackrel{\oplus}{e} K_{I}$. Son image $\log _{\ell} k_{\ell}^{x}$ est un $\mathbb{Z}_{\ell}$-réseau de $K_{\ell}$. Son noyau est le radical dans $\kappa_{\ell}^{x}$ du $\mathbb{Z}_{\ell}$-module libre engendré par les images $\ell_{l}$ du nombre premier $l$ dans chacun des facteurs locaux $\kappa_{\mathfrak{l}}^{X}$; en particulier, il contient le groupe $\mu_{\ell}=1 \stackrel{\oplus}{1 \mid \ell} \mu_{I}$ des racines de l'unité dans $k_{\ell}^{x}$.

DÉFINITION 11, 1.3. - Nous notons $\log _{\ell}$ et nous appelons logarithme $\ell-$ adique l'homomorphisme naturel $\log _{\ell} \circ s$ du tensorisé multiplicatif $R=\mathbb{Z}_{\ell} \otimes K^{X}$ dans le tensorisé additif $K_{\ell}=\mathbb{Z}_{\ell} \otimes \mathbb{Z} K$ composé du lo garithme d'lwasawa et de l'application de semi-localisation .

Cela posé, le problème fondamental de l'indépendance $\ell$-adique peut s'énoncer comme suit :

Problème 2 - Etant donnés $x_{1}, \ldots, x_{n}$ dans $k^{x}$, à quelle condition la restriction du logarithme $l$-adique $\log _{\ell}$ au sous-module de $R$ qui est $\mathbb{Z}_{l}$ engendré par les images de ces éléments est-elle injective?

\section{b.- Enoncé de la conjecture.}

La notion-clé de cette conjecture étant celle de représentation monogène, commençons par énoncer les principaux résultats sur les représentations qui nous seront utiles :

Etant donnés un groupe fini $G$ et un corps $F$ de caractéristique nulle, écrivons :

$$
F[G]=\underset{i=1}{\oplus} F[G] e_{i}=\underset{i=1}{\oplus} A_{i}
$$


la décomposition semi-simple de l'algèbre $F[G]$ associée auxidempotents centraux minimaux $e_{i}$ de $F[G]$.

Chacune des algèbres simples $A_{i}=F[G] e_{i}$ s'écrit comme somme di recte dlidéaux à gauche minimaux deux à deux isomorphes :

$$
A_{i}=\oplus_{j=1}^{r_{i}} F[G] e_{i j}=\bigoplus_{j=1}^{r_{i}} V_{i j}
$$

où les $\left(e_{i j}\right)_{j=1, \ldots, r_{i}}$ sont un système complet d'idempotents primitifs ( non centraux pour $r_{i}>1$ ) au-dessus de $e_{i}$.

Le caractère régulier de $F[G]$ se décompose alors comme somme :

$$
x_{\text {rég }}=\sum_{i=1}^{r} r_{i} x_{i}
$$

des caractères $X_{i}$ attachés aux modules indécomposables $V_{i j}=F[G] e_{i j}$, avec les multiplicités $r_{\mathbf{i}}$.

Considérons maintenant un $F[G]$-module $M$, et écrivons $X=\sum_{i=1}^{r} m_{i} X_{i}$ la décomposition irréductible du caractère associé. Si $N=\mathrm{F}[\mathrm{G}]$. $\mathrm{x}$ est un sous-module monogène de $M$, le caractère $\chi_{N}$ qui lui correspond est contenu à la fois dans $\chi_{M}$ (puisque $N$ est contenu dans $M$ ) et dans $X_{\text {rég }}$ (puisque $N$ s'identifie à un quotient de $F[G]$ ). Autrement dit, nous avons simultanément :

$$
X_{N \prime} \leq x_{M} \quad \text { et } \quad X_{N} \leq x_{\text {rég }} \text {, }
$$

ce que nous pouvons encore écrire :

$$
x_{N} \leq x_{M} \wedge x_{\text {rég }} \text {, }
$$

en convenant de noter $x_{M} \wedge x_{\text {rég }}=\sum_{i=1}^{r} \min \left\{m_{i}, r_{i}\right\} x_{i}$ le plus grand caractère contenu à la fois dans $X_{M}$ et dans $X_{\text {rég }}$. Nous allons voir que cette dernière inégalité caractérise les sous-modules monogènes de $M:$

THÉORÈME 11.1.4.- Pour qu'un $F[G]$-module noethérien soit mono gène, il faut et il suffit que son caractère soit contenu dans le caractère régulier.

Démonstration : Soit $N$ un $F[G]$-module noethérien dont le caractère $X_{N}=\sum_{i=1}^{r} n_{i} X_{i}$ est contenu dans le caractère régulier. Lidentité 
$n_{i} \leq r_{i}$ pour $i=1, \ldots, r$, se traduit par llexistence dlun isomorphisme $\varphi$ de $I$ pour la somme directe :

$$
N \simeq \underset{i=1}{\oplus} \oplus_{j=1}^{n_{i}} v_{i j}
$$

Posons alors $x_{i j}=\varphi^{-1}\left(e_{i j}\right)$, puis $x=\sum_{i=1}^{r} \sum_{j=1}^{n_{i}} x_{i j}$; et considérons le $F[G]$-module engendré par $x$. Nous obtenons sans peine, par or thogonalité des idempotents $e_{i j}$, la décomposition :

$$
F[G] x=\underset{i=1}{\oplus} \oplus_{j=1}^{n_{i}} F[G] x_{i j} \simeq \underset{i=1}{\oplus} \oplus_{j=1}^{n_{i}} V_{i j}
$$

Et $F[G] \times$, sous-module de $N$ de même caractère, ne peut être que $N$.

COROLLAIRE 11.1.5. - Dans un $F[G]$-module noethérien $M$, les sous modules monogènes maximaux sont ceux de caractère $X_{M} \wedge X_{\text {rég }}$. En particulier, ils sont deux à deux isomorphes .

Démonstration: D'après ce qui précède, il s'agit de vérifier que les sous-modules monogènes de caractère maximal sont exactement les sous modules monogènes maximaux. Or, d'un côté, tout sous-module de ca ractère $X_{M} \wedge X_{\text {rég }}$ est monogène maximal, puisqulil est monogène d'après le théorème, mais qulaucun sous-module de $M$ strictement plus grand ne peut l'être; et, d'un autre côté, si $N$ est un sous-module de $M$, de caractère $x_{N}<\left(x_{M} \wedge x_{\text {rég }}\right)$, et $x_{i}$ un facteur irréductible de $\left(\chi_{M} \wedge x_{\text {rég }}\right)-\chi_{N}$, le quotient $M / N$ contient un sous-module de caractère $\chi_{i}$, dont l'image réciproque dans $M$ est un sous-module contenant strictement $N$, de caractère $x_{N}+x_{i} \leq\left(x_{M} \wedge x_{\text {rég }}\right)$, donc monogène.

Venons-en maintenant à notre sujet : Soit $K$ une extension galoisienne finie du corps de rationnels; notons $G$ songroupe de Galois, et $\ell$ un nombre premier. Le corps $K$, regar dé comme $\mathbb{Q}[G]$-module , étant libre et de rang 1 , son localisé $K_{\ell}=\mathbb{Q}_{\ell} \otimes_{\mathbb{Q}} K$ est donc un $\mathbb{Q}_{\ell}[G]-$ module libre et monogène, clest-à-dire un $\mathbb{Q}_{\ell}[G]$-module de caractère $\chi_{\text {rég }}$ - Considérons un sous-module noethérien $M$ du groupe multiplicatif $\mathrm{K}^{\mathrm{X}}$, et notons $M_{\ell}=\log _{\ell}\left(\mathbb{Z}_{\ell} \mathbb{Z}_{\mathbb{Z}} M\right)$ le sous-module de $\mathrm{K}_{\ell} \mathbb{Z}_{\ell}$-engendré par les logarithmes des éléments de $M$. Si $M$ est stable par $G$, il 
en est de même de $M_{\ell}$ ( puisque le logarithme commute à l'action de Galois), et le caractère $\chi_{M_{\ell}}$ du $Q_{\ell}[G]$-module $Q_{\ell} \otimes_{\mathbb{Z}}{ }^{M} l=Q_{\ell} \log _{\ell} M$ est contenu à la fois dans celui $\chi_{\text {rég }}$ du $\mathbb{Q}_{\ell}[G]$-module $K_{\ell}$ (puisque $\mathbb{Q}_{\ell} \log _{\ell} M$ est un sous-module de $\left.K_{\ell}\right)$, et dans celui $\chi_{M}$ du $\mathbb{Q}[G]-$ mo -

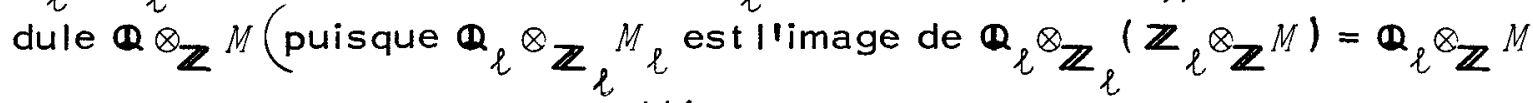
par le logarithme $\ell$-adique $)^{(*)}$. En particulier, l'égalité $x_{M_{l}}=x_{M}$ ne peut avoir lieu que si $X_{M}$ est contenu dans $X_{\text {rég }}$, i.e., d'après le théorème 4 ci-dessus, si le $\mathbb{Q}[G]$-module $\mathbb{Q} \otimes \mathbb{Z} M$ est monogène 。

Ce résultat nous amène à poser la conjecture suivante :

CONJECTURE 11.1.6.- Soient $K$ une extension galoisienne finie du corps des rationnels, $G$ son groupe de Galois, et $l$ un nombre premier. Etant donné un sous-module noethérien $M$ du groupe multiplicatif $\mathrm{K}^{X}$, stable pour llaction de $G$, la restriction du logarithme $\ell$-adique $\log _{\ell}$ au $\mathbb{Z}_{\ell}[G]$-module $m=\mathbb{Z}_{\ell} \mathbb{Z}^{M}$, engendré par llimage de $M$ dans le tensorisé $\mathbb{Z} \ell^{\otimes} \mathbb{Z}^{\mathrm{K}}$, est injective si et seulement si les trois conditions suivantes sont réunies :

(i) Le groupe $M$ ne contient pas les racines $l^{\text {ièmes }}$ de llunité. (ii) Le radical de $M$ dans $K^{X}$ ne contient pas $\ell$, i.e. $\ell \notin \sqrt{M}$. (iii) Le $\mathbb{Q}[G]$-module $\mathbb{Q} \otimes_{\mathbb{Z}} M$ est monogène, i.e. $x_{M} \leq x_{\text {rég }}$.

Les deux premières conditions sont évidemment nécessaires puisque les racines $\ell$-primaires de l'unité ${ }^{(\star \star)}$ et le nombre premier $\ell$ sont contenus dans le noyau du logarithme $\ell$-adique ; la troisième d'après la discussion qui précède. La conjecture énoncée postule donc que ces conditions sont également suffisantes. Avant de la discuter, tironsen immédiatement une conséquence :

( $\left.{ }^{\star}\right)$ Nous notons indifféremment $\chi_{M}$ le caractère du $\mathbb{Q}[G]$-module $\mathbb{Q} \otimes_{\mathbb{Z}} M$ et celui du $\mathbb{Q}_{\ell}[G]$-module $\mathbb{Q}_{\ell} \otimes \mathbb{Z} M$.

$(\star \star)$ Le produit tensoriel par $\mathbb{Z}_{\ell}$ a tué les racines de l'unité d'ordre étranger à $\ell$. 
THÉORĖME 11.1.7.- Sous la conjecture précédente, le caractère $\chi_{M_{l}}$ du $\mathbb{Q}_{\ell}[G]$-module $\mathbb{Q}_{\ell} \log _{\ell} M$, engendré dans $K_{\ell}$ par liimage $M_{\ell}=$ $\log _{\ell}\left(Z_{\ell} \mathbb{Z}_{\mathbb{Z}} M\right)$ du tensorisé $\ell$-adique de $M$, est donné, en fonction du caractère $\chi_{M}$ du $\mathbb{Q}[G]$-module $\mathbb{Q} \otimes \mathbb{Z} M$, par la formule :

(i) $\quad X_{M_{l}}=X_{M} \wedge X_{\text {rég }}$, si $\ell$ n'appartient pas au radical de $M$.

(ii) $X_{M_{l}}=\left(X_{M}-1\right) \wedge X_{\text {rég }}$, dans le cas contraire.

Démonstration : Quittes à remplacer $M$ par l'une de ses puissances $M^{\text {n }}$, nous pouvons toujours supposer que $M$ ne contient dlautre racine de l'unité que 1 . Cela étant, distinguons les deux éventualités :

(i) $\quad \ell \notin \sqrt{M}$. Dans ce cas, nous avons évidemment $\chi_{M_{\ell}} \leq \chi_{M}$ et $X_{M_{l}} \leq X_{\text {rég }}$, comme vu plus haut, donc $X_{M_{l}} \leq\left(X_{M} \wedge X_{\text {rég }}\right)$. Réci proquement, le corollaire 5 nous donne linégalité opposée $X_{M_{e}} \geq$ $\left(X_{M} \wedge X_{\text {rég }}\right)$, puisque, si $\bar{N}$ est un sous-module monogène maximal de $\mathbb{Q} \otimes \otimes_{\mathbb{Z}} M$, et $x \in M$ un générateur de $\bar{N}^{(\star)}$, la restriction du logarithme $\ell$-adique du $\mathbb{Z}_{\ell}[G]$-module $x^{\mathbb{Z}_{\ell}[G]}$ est injective, en vertu de la conjecture.

(ii) $l \in \sqrt{M}$. Dans ce cas, il existe un naturel $m \geq 1$, et un sous-module $N$ de $M$, de caractère $\chi_{M}=\chi_{M}-1$, tel que la somme $N \oplus \ell^{m \mathbb{Z}}$ soit directe et d'indice fini dans $M$. L'argument précédent appliqué à $N$ permet alors de conclure.

\section{c.- Discussion de la conjecture énoncée.}

DÉFINITION 11.1.8.- Convenons de dire qu'un $\mathbb{Z}[G]$-module noethérien $M$ est quasi-monogène lorsque le $\mathbb{Q}[G]$-module associé $\mathbb{Q} \otimes \mathbb{Z} M$ est monogène. Un $\mathbb{Z}[G]$-module quasi-monogène est donc un sur-module d'indice fini dlun $\mathbb{Z}[G]$-module monogène.

PROPOSITION 11.1.9. - La conjecture précédente est vraie pour les modules $M$ dont tous les éléments sont étrangers à $\ell$, sous la conjecture de Schanuel $\ell$-adique $(\star \star)$

(*) Le groupe $M$ étant pris sans torsion, il est canoniquement un sous module de son tensorisé $\mathbb{Q} \otimes_{\mathbb{Z}} M$.

$\left({ }^{\star}\right)$ Sous la forme faible, la conjecture de Schanuel $\mathfrak{b}$-adique postule que les logarithmes $\ell$-adiques de nombres algébriques $\mathbb{Q}$-multiplicativement indépendants et étrangers à $\ell$ sont $\mathbb{Q}$-algébriquement indépendants . 
Pour établir cette proposition, nous nous appuyerons sur le lemme :

LEMME $11,1,10 .-$ Tout sous-module quasi-monogène $M$ du $\mathbb{Z}$ [G]-module $K^{X}$, dont tous les éléments sont étrangers à $l$, est contenu dans un sous-module quasi-monogène $N$ de $\mathrm{K}^{X}$ qui possède les trois propriétés suivantes :

(i) Les éléments de $N$ sont étrangers à $\ell$.

(ii) Le $\mathbb{Q}[G]$-module $\mathbb{Q} \otimes \mathbb{Z} N$ est libre et monogène .

(iii) $\mathbb{Q} \otimes \mathbb{Z} M$ est facteur direct de $\mathbb{Q} \otimes \mathbb{Z} N$.

Démonstration du lemme : Puisque $M$ est de type fini, nous pouvons trouver un nombre premier $\mathrm{p}$, étranger à $\ell$ et à tous les éléments de $M$, qui se décompose complètement dans l'extension galoisienne $K / \mathbb{Q}$. Notons $x$ un générateur d'une puissance principale d'un quelconque des idéaux premiers $p$ de $K$ au-dessus de $p$. Par hypothèse, le groupe de Galois $G$ opère fidèlement sur les places de $K$ au-dessus de $p$; le sousmodule $x^{\mathbb{Z}}[G]$, engendré dans $K^{x}$ par $x$ et ses conjugués, est donc isomorphe à $\mathbb{Z}[G]$. Il est linéairement disjoint de $N$, puisque $\mathrm{p}$ est étranger à tous les éléments de $M$. Considérons le $\mathbb{Q}[G]$-module $\bar{P}=Q \otimes \mathbb{Z}\left(M \oplus x^{\mathbb{Z}[G]}\right)$ engendré par $M$ et $x:$ II contient la représenta tion régulière; et le corollaire 5 nous assure donc que ses sous-mo dules monogènes maximaux sont tous isomorphes à $Q[G]$. Choisissons en un $\bar{N}$, qui contienne $\bar{M}=\mathbb{Q} \otimes \mathbb{Z} M$; le sous-groupe $N$ formé des éléments de $M \oplus \mathrm{x}^{\mathbb{Z}}[\mathrm{G}]$ qui tombent dans $\bar{N}$ convient.

Démonstration de la proposition: Il s'agit d'établir que, pour tout sousmodule quasi-monogène et sans $\mathbb{Z}$-torsion $M$ du $\mathbb{Z}[G]$-module $\mathrm{K}^{\mathrm{x}}$, le rang $\ell$-adique $\mathrm{rg}_{\mathbb{Z}} \log _{\ell}\left(\mathbb{Z}_{\ell} \mathbb{Z}_{\mathbb{Z}} M\right)$ du $\mathbb{Z}_{\ell}$-module libre engendré par l'image logarithmique de $M$ dans $\mathrm{K}_{\ell}$ est égal au rang rg $\mathbb{Z} M$ du $\mathbb{Z}$-module libre $M$, dès lors que tous les éléments de $M$ sont étrangers à $l$. D'après le lemme, il suffit de faire la démonstration lorsque $\mathbb{Q} \otimes \mathbb{Z} M$ est la représentation régulière de $\mathbb{Q}[G]$. Cela étant, notons $x \in M$ un générateur du $\mathbb{Q}[G]$-module $\mathbb{Q} \otimes_{\mathbb{Z}} M$, et considérons la matrice $\ell-$ adique : 


$$
x_{\ell}=\left[\log _{\ell} x^{\sigma \tau}\right]_{(\sigma, \tau) \in G \times G} \text {. }
$$

Son déterminant est un polynôme en les logarithmes $\ell$-adiques des conjugués de $x$, qui n'est pas le polynôme nul : En effet, les conjugués de $x$ étant par hypothèse $\mathbb{Q}$-multiplicativement indépendants, la matrice réelle:

$$
X=\left[\log \left|x^{\sigma \tau}\right|\right](\sigma, \tau) \in G \times G
$$

est régulière, puisque liimage de $M$ dans $R^{[K: \mathbb{Q}]}$ par le plongement logarithmique :

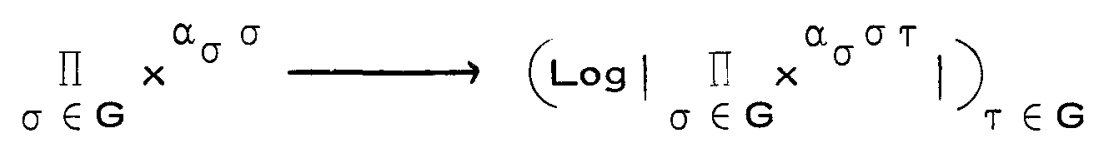

est un réseau de $R^{[K: \Phi]}$, en vertu d'un argument classique de géo métrie des nombres (cf. [Sa $\left.{ }_{1}\right], C h . I V, \$ 4$ ). La conjecture de Schanuel permet alors d'affirmer que la matrice $x_{\ell}$ est également régulière, ce qui signifie que llimage de $M$ dans $K_{\ell}[K: \mathbb{Q}]$ par le plongement logarith mique :

$$
\prod_{\sigma \in G} x^{\alpha \sigma^{\sigma}} \longrightarrow\left(\log _{\ell}\left(\prod_{\sigma \in G} x^{\alpha \sigma^{\sigma \tau}}\right)\right)_{T \in G}
$$

est un réseau de $K_{l}[K: \mathbb{Q}]$, et implique en particulier que les nombres $\left(\log _{\ell} x^{\sigma}\right)_{\sigma \in G}$ sont $K_{\ell}$-linéairement indépendants .

Pour pouvoir appliquer au module $M$ la conjecture de Schanuel, nous avons supposé que tous les éléments de $M$ étaient étrangers à $l$. De fait, la conjecture énoncée plus haut requiert seulement que $M$ ne contienne aucune puissance de $\ell$. Il parait donc raisonnable d'énoncer la conjecture de Schanuel $\ell$-adique sous la forme faible plus générale suivante:

Conjecture de Schanuel modifiée - Si les logarithmes $l$-adiques de nombres algébriques sont $\mathbb{Q}$ - linéairement indépendants, ils sont $\mathbb{Q}$ - al gébriquement indépendants.

Cela posé, la proposition 9 se généralise immédiatement sous la forme: 
PROPOSITION 11.1.11. - La conjecture énoncée est vraie sous la con jecture de Schanuel modifiée.

Ce dernier résultat est l'une des présomptions les plus fortes que nous ayons en faveur de la conjecture énoncée.

\section{2. - MINORATIONS DU RANG $\ell$-ADIQUE ET PREUVE DE LA CONJECTURE DANS LE CAS ABÉLIEN.}

\section{a.-Preuve de la conjecture dans le cas abélien .}

Considérons un sous-module noethérien et sans torsion $M$ du groupe multiplicatif $\mathrm{K}^{\mathrm{x}}$; notons $r_{M}=r g_{\mathbb{Z}} M$ son rang, et $r_{M_{\ell}}=r g_{\mathbb{Z}} M_{\ell}$ celui du $\mathbb{Z}_{\ell}$-module libre $\log _{\ell}\left(\mathbb{Z}_{\ell} \otimes \mathbb{Z} M\right)$. Si $M$ est stable pour l'action de $G$, les entiers $r_{M}$ et $r_{M_{\ell}}$ sont les degrés respectifs des représenta tions associées à $M$ et à $M_{\ell}$ :

$$
r_{M}=\operatorname{deg} x_{M} \quad \text { et } \quad r_{M_{l}}=\operatorname{deg} x_{M_{l}} \text {. }
$$

En particulier, le rang conjectural du module $M_{\ell}$ est donné par les for mules:

$$
r_{\mu_{l}}=\left\{\begin{array}{l}
\operatorname{deg}\left[\left(x_{M}-1\right) \wedge x_{\text {rég }}\right], \text { si } \ell \in \sqrt{M}, \\
\operatorname{deg}\left[X_{M} \wedge x_{\text {rég }}\right], \text { sinon, }
\end{array}\right.
$$

ce quilil est commode d'écrire de façon indifférenciée :

$$
r_{M_{l}}=\operatorname{deg}\left[X_{M}^{\prime} \wedge x_{r e ́ g}\right] \text {, en posant } X_{M}^{\prime}=\left\{\begin{array}{l}
X_{M}-1 \text {, si } l \in \sqrt{M} \\
X_{M} \text {, dans le cas contraire. }
\end{array}\right.
$$

Nous allons voir que la conjecture énoncée est vraie dès que le groupe $G$ est abélien, ce qui nous donnera au passage une première mi noration du rang $\ell$-adique $r_{M}$.

THÉORÈME 11.1.12. - Soient $M$ un sous-module noethérien du groupe multiplicatif $K^{X}$, stable pour l'action de $G=G a l(K / \mathbb{Q})$, puis $X_{M}$ le caractère du $\mathbb{Q}[G]$ - module $\mathbb{Q} \otimes_{\mathbb{Z}} M$, et $X_{M_{l}}$ celui du $\mathbb{Q}_{\ell}[G]$-module $\mathbb{Q}_{\ell} \otimes_{\mathbb{Z}_{\ell}} M_{\ell}$, engendré dans $K_{\ell}$ par liimage $M_{\ell}=\log _{\ell}\left(\mathbb{Z}_{\ell} \otimes_{\mathbb{Z}} M\right) \mathrm{du}$ 
tensorisé $\ell$-adique de $M$. Alors le caractère $\chi_{M_{l}}$ contient chaque caractère $\ell$-adique irréductible $\psi$ représenté dans le caractère $\chi_{M}^{\prime}$ défini par $X_{M}^{\prime}=X_{M}-1$, si $\ell=\sqrt{M} ; X_{M}^{\prime}=X_{M}$ sinon:

$$
x_{M_{\ell}} \geq \sum_{\psi \leqslant X^{\prime}} \psi
$$

En particulier, la conjecture énoncée est vraie dès que le groupe $G$ est abélien, et, plus généralement, dès que l'algèbre $\mathbb{Q}_{\ell}[G]$ est un produit direct de corps.

Démonstration : Supposons d'abord que $M$ ne contienne pas de puissance de $\ell$. Pour chaque caractère rationnel irréductible $X$ représenté dans $X_{M}$, nous pouvons trouver un $x_{X}$ dans $M$ qui engendre un $\mathbb{Q}[G]$-module irréductible de caractère $X$.

- Si $x$ est irréductible sur $Q_{\ell}$, le $\mathbb{Q}_{\ell}[G]$-module engendré par $\log _{\ell} \times{ }_{\chi}$ (qui est non nul, puisque $M$ ne contient pas de puissance de $\ell$ ) est encore irréductible de caractère $\chi$; et il n'y a rien à démontrer.

- Sinon $X$ s'écrit comme somme de caractères $\psi$, irréductibles $\operatorname{sur} \mathbb{Q}_{\ell}$, à valeurs dans une extension cyclotomique de $\mathbb{Q}$. Faisons choix d'une base $\left(x^{\sigma}\right)_{\sigma \in H}$ du $\mathbb{Q}$-espace $x^{\mathbb{Q}[G]}$, formé de conjugués de $x$. Si llun des caractères au-dessus de $X$, disons $\psi$, n'était pas repré senté dans le $\mathbb{Q}_{\ell}[G]$-module $\mathbb{Q}_{\ell} \otimes_{\mathbb{Z}_{\ell}} M_{\ell}$, nous aurions simultanément :

$$
\sum_{\sigma \in G} \psi\left(\sigma^{-1}\right) \log _{\ell} x^{\sigma}=0
$$

dans $K_{\ell}$, mais :

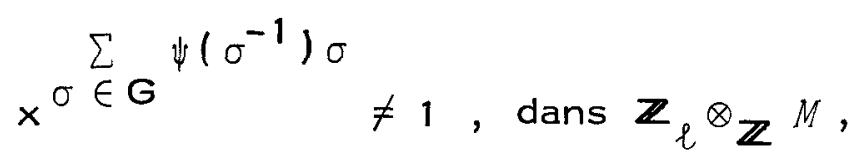

donc, en exprimant les conjugués de $x$ en fonction des seuls $\left(x^{\sigma}\right)_{\sigma} \in H$, une relation de dépendance linéaire non triviale, à coefficients algé briques, entre les logarithmes $\ell$-adiques des $\left(x^{\sigma}\right)_{\sigma \in H}$, contrairement au théorème d'indépendance de Baker-Brumer ( $\mathrm{cf} \cdot[\mathrm{Br}]$ ).

(*) Cette inégalité peut être regardée comme une généralisation du théo rème fondamental de $[E K W]$ pour le module des unités. 
Enfin, si $M$ contient des puissances de $\ell$, il suffit de remarquer que $M$ contient un sous-module dindice fini de la forme $\ell^{m \mathbb{Z}} \oplus N$, où $N$ est un $\mathbb{Z}[G]$-module de caractère $X_{N}=X_{M}-1=X_{M}^{\prime}$, et de raisonner sur $N$ pour se ramener au cas précédent .

En résumé, la conjecture est ainsi établie dans tous les cas où les caractères $\ell$-adiques irréductibles sont représentés une fois et une seule dans le caractère régulier, c'est-à-dire (cf. [My], prop.3) lorsque l'algèbre de groupe $\mathbb{Q}_{\ell}[G]$ est un produit direct de corps .

COROLLAIRE 11.1.13. - Le rang $\ell$-adique diun $\mathbb{Z}[G]$-module multiplicatif $M$ est au moins la racine carrée de son rang conjectural $r_{M_{l}}^{c o n j}=$ $\operatorname{deg}\left(x_{M}^{\prime} \wedge x_{\text {rég }}\right):$

$$
r_{M_{l}} \geq \sqrt{r_{M_{e}}^{\text {conj }}}
$$

Démonstration : Dans la décomposition $\chi_{\text {rég }}=\sum d_{\varphi} \varphi$ du caractère ré gulier comme somme de caractères absolument irréductibles (à valeurs dans une extension cyclotomique de $Q$ ), les indices $d \varphi$ sont les degrés respectifs des caractères $\varphi$ (cf. $\left[\mathrm{Se}_{1}\right], \mathrm{Ch} .1, \S 2.4$ ). Comme, d'après le théorème 12 ci-dessus, chaque caractère absolument irréductible $\varphi$ représenté dans $X_{M}^{\prime}$ l'est encore dans $X_{M_{l}}$, il vient donc:

$$
\begin{aligned}
r_{M_{l}} \geq \sum_{\varphi \mid X_{M}^{\prime}} \operatorname{deg} \varphi=\sum_{\varphi \mid X_{M}^{\prime}} \sqrt{\mathrm{d} \varphi \operatorname{deg} \varphi} & \leq \sqrt{\sum_{\varphi \mid X_{M}^{\prime}{ }^{d} \varphi} \operatorname{deg} \varphi}=\sqrt{\operatorname{deg}\left(\sum_{\left.\varphi \mid X_{M}^{\prime} \varphi_{\varphi} \varphi\right)}\right.} \\
& \geq \sqrt{\operatorname{deg} X_{M_{l}}^{\text {conj }}}=\sqrt{r_{M_{l}}^{\text {conj }}} .
\end{aligned}
$$

Clest le résultat démontré par Emsalem, Kisilevsky, et Wales ( c f. $[E K W]$ ), lorsque $M$ est le groupe des unités.

\section{b.- Minoration du rang $\ell$-adique.}

La minoration du rang $\ell$-adique donnée par le corollaire 13 peut être sensiblement améliorée à l'aide d'un résultat de transcendance, dû à Waldschmidt, sur les matrices à coefficients logarithmes de nom bres algébriques. Il vient, en effet : 
PROPOSITION $11,1.14$ - - Le rang $\ell$-adique $r_{M_{l}}=$ deg $X_{M_{l}}$ d'un sous module noethérien $M$ du $\mathbb{Z}[G]$-module $K^{X}$ est au moins la moitié du rang conjectural $r_{M_{l}}^{\text {conj }}=\operatorname{deg}\left(\chi_{M}^{\prime} \wedge \chi_{\text {rég }}\right)$ :

$$
r_{M_{\ell}} \geq \frac{1}{2} r_{M_{\ell}}^{\text {conj }}
$$

Démonstration : II suffit naturellement d'établir ce résultat lorsque $M$ est un $\mathbb{Z}[G]$-module sans $\mathbb{Z}$-torsion ne contenant pas de puissances de $\ell$. Cela étant, prenons un $x$ dans $M$ engendrant un sous-module monogène maximal du produit $\bar{M}=\mathbb{Q} \otimes \mathbb{Z} M$; et posons $N=x^{\mathbb{Z}}[\mathrm{G}]$. La proposition sera démontrée si nous prouvons liinégalité $r_{N_{\ell}} \geq \frac{1}{2} r_{N}$. Considérons pour cela la matrice en les logarithmes réels des modules des conjugués de $x$ :

$$
X=\left[\log \left|x^{\sigma \tau}\right|\right]_{(\sigma, \tau)} \in G \times G \cdot
$$

Par un argument de géométrie des nombres déjà invoqué (cf. [Sa, ${ }_{1}$, Ch.IV, \$4), cette matrice a pour rang $r_{N}$. Le théorème de Waldschmidt (cf. $\left[W_{a_{2}}\right], \S 1$, th. 1.1 ) permet alors dlaffirmer que la matrice en les logarithmes $\ell$-adiques :

$$
x_{\ell}=\left[\log _{l}\left(x^{\sigma \tau}\right)\right]_{(\sigma, \tau)} \in G \times G
$$

a pour rang au moins $\frac{1}{2} r_{N}{ }^{(*)}$. L'inégalité annoncée en résulte.

Enoncé en termes de représentations, le théorème de Waldschmidt conduit même à un résultat sensiblement plus précis : Ecrivons $X_{\text {rég }}=$ $\sum r_{X} \times$ la décomposition du caractère régulier comme somme de caractères rationnels irréductibles. Nous avons :

${ }^{\star}$ ) En fait, le théor ème énoncé dans $\left[\mathrm{Wa}_{2}\right]$ suppose que les $x^{\sigma \top}$ soient des unités $\ell$-adiques. Cependant, cette condition n'est nullement indispensable : Dans les démonstrations techniques, en effet, le changement de variables $x=\exp \left(\ell^{m} \log _{\ell} x\right)$, avec $m$ grand, permet toujours de se ramener à des unités principales. Outre l'algébricité de $x$, seule est donc requise la validité de limplication $\sum_{\sigma \in G} a_{\sigma} \log _{\ell} x^{\sigma}=0 \Rightarrow$ $\sum_{\sigma \in G} a_{\sigma} \log \left|x^{\sigma}\right|=0$ ( pour des $\left(a_{\sigma}\right)$ dans $\mathbb{Z}$ ), c'est-à-dire le fait que le $\mathbb{Z}[G]$-module multiplicatif engendré par $\times$ ne contienne pas de puissance de $\ell$. 
THÉOREMME 11.1.15. - Soient $M$ un sous-module galoisien isotypique du groupe multiplicatif $K^{X}$, ne contenant pas de puissances de $l$, de caractère $X_{M}=m X$, et $X_{M \ell}$ le caractère du $\mathbb{Z}_{\ell}[G]$-module $\log _{\ell}\left(\mathbb{Z}_{\ell} \mathbb{Z}^{M}\right)$.

- Si le caractère rationnel irréductible $\chi$ ne se décompose pas sur $Q_{\ell}(i . e$. si $\chi$ est un multiple $s \psi$ diun caractère $\ell$-adique irréductible $\psi)$, le caractère $\chi_{M_{l}}$ vérifie les minorations :

$$
\begin{aligned}
x_{M_{l}} \geq & \frac{m}{2} x=\frac{1}{2} x_{M}, \text { si } x_{M} \text { est contenu dans } x_{\text {rég }} \\
x_{M_{l}} & \geq \frac{k}{k+1} r_{X} x=\frac{k}{k+1}\left(x_{M} \wedge x_{\text {rég }}\right), \\
& \text { avec } k=\left[\frac{m}{r_{X}}\right], \text { dans tous les autres cas. }
\end{aligned}
$$

- Si le caractère rationnel irréductible $x$ se décompose sur $\mathbb{Q}_{\ell}$ (i.e. s'il existe au moins deux caractères $\ell$-adiques irréductibles dis tincts représentés dans $\chi$ ), les formules précédentes restent valables pour les degrés :

$$
\begin{aligned}
& r_{M_{\ell}} \geq \frac{1}{2} r_{M_{\ell}}^{\text {conj }}=\frac{1}{2} r_{M}, \text { si } x_{M} \text { est contenu dans } x_{\text {rég }} \cdot \\
& r_{M_{\ell}} \geq \frac{k}{k+1} r_{M_{\ell}}^{\text {conj }} \text {, avec } k=\left[\frac{m}{r_{X}}\right] \text {, dans tous les autres cas. }
\end{aligned}
$$

Démonstration : Lorsque $\chi_{M}$ est contenu dans $x_{\text {rég }}$, linégalité $r_{m_{\ell}} z$ $\frac{1}{2} r_{M}$ est donnée par la proposition 14 . Si le caractère $\chi$ est un multiple $s \psi$ d'un caractère $\ell$-adique irréductible $\psi$, le caractère $X_{M_{\ell}}$ est luimême un multiple de $\psi$ ( tout comme $x_{M}$ ) et l'inégalité $r_{M_{l}} \geq \frac{1}{2} r_{M}$ s'écrit donc:

$$
X_{M_{l}} \geq \frac{1}{2} X_{M} \text {, comme annoncé. }
$$

On notera que cette inégalité, qui s'écrit encore $X_{M_{l}} \geq \frac{s m}{2} \psi$, donne en fait $X_{M_{l}} \geq\left[\frac{s m+1}{2}\right] \psi$, puisque $\psi$ est contenu un nombre entier de fois dans $X_{M_{l}}$ (mais ce résultat pourrait être en défaut pour $X$ ). Supposons donc désormais $m \geq r_{X}$, et posons $k=\left[\frac{m}{r_{X}}\right]$. Par hypothèse, nous pouvons trouver dans $M$ un sous-module $N$, somme di recte de $k$ sous-modules monogènes $x_{i} \mathbb{Z}[G]$ ( pour $i=1, \ldots, k$ ), isotypiques de caractère $r_{X} \chi$; et le théorème sera établi si nous prouvons l'inégalité $r_{M_{l}} \geq \frac{k}{k+1} r_{x}$ deg $x$. Pour cela, introduisons l'idempotent 
central $e_{X}$ de l'algèbre $\mathbb{Q}_{\ell}[G]$ attaché au caractère $X$; faisons choix d'une base $H \subset G$ du $\mathbb{Q}$-espace vectoriel $\mathbb{Q}[G] e_{\chi}$; et considérons le $\mathbb{Z}$-module $X$, de rang $k r_{X}$ deg $\chi$, engendré dans $K_{l}^{H}$ par les images $\left(\log _{l} \times_{i}^{\sigma \tau}\right)_{\sigma \in H}$ des conjugués des $x_{i}$ dans le plongement logarithmique de $M$ dans $\mathrm{K}_{\ell}^{\mathrm{H}}$. Nous pouvons naturellement l'écrire comme somme di recte $X=\underset{i=1}{\oplus} X_{i}$ des $\mathbb{Z}$-modules associés à chacun des $x_{i}$, de sorte qu'en vertu d'un lemme d'Emsalem (cf. [E], lemme 4), le coefficient $\theta\left(X, K_{\ell}^{H}\right)$ défini par :

$$
\theta\left(x, K_{l}^{H}\right)=\min _{V} \frac{\operatorname{corg}_{\mathbb{Z}}(V \cap x)}{\operatorname{codim}_{K_{l}} V}=\min _{V} \frac{\operatorname{rg}_{\mathbb{Z}} \times-r g_{\mathbb{Z}}(V \cap x)}{\operatorname{dim}_{K_{l}} K_{l}^{H}-\operatorname{dim}_{K_{l}} V}
$$

(lorsque $V$ parcourt les sous-espaces stricts de $K_{l}^{H}$ qui sont rationnels sur Q), vérifie la minoration:

$$
\theta\left(X, K_{l}^{H}\right) \geq \sum_{i=1}^{k} \theta\left(X_{i}, K_{l}^{H}\right)=\sum_{i=1}^{k} 1=k \text {. }
$$

Le théorème principal de Waldschmidt ( $c f .\left[W a_{2}\right], c h .4$, th. 4.1) conduit alors à la minoration :

$$
\mathrm{rg}_{N_{l}} \geq \frac{\mathrm{k}}{\mathrm{k}+1}\left(r_{x} \operatorname{deg} x\right)
$$

ce qui est le résultat attendu.

Remarque : Nous avons supposé, dans l'énoncé du théorème 15 , que le module $M$ ne contenait pas de puissances de $\ell$. De fait, lorsque $M$ est isotypique, la condition $\ell \in \sqrt{M}$ ne peut avoir lieu que si $\chi_{M}$ est un multiple $m 1$ du caractère unité. Mais comme celui-ci n'est représenté qu'une fois dans le caractère régulier, le caractère $X_{M_{l}}=\left(X_{M}-1\right) \wedge X_{\text {rég }}$ est alors égal à 0 ou à 1 suivant que $X$ contient une ou plusieurs fois le caractère unité. Dans le premier cas, $X_{M_{l}}$ est trivialement nul ; dans le second, le module $M$ contient un nombre algébrique $x \in \mathrm{K}^{X}$ qui n'est pas dans le noyau du logarithme $\log _{l}$, et le caractère $X_{M_{l}}$ n'est pas nul. Dans les deux cas, il vient donc directement $\chi_{M_{l}}=\chi_{M_{l}}^{\text {conj }_{M_{l}}}=$ $\left(X_{M}-1\right) \wedge X_{\text {rég }}$, sans quill soit nécessaire de faire appel à un quelconque argument transcendant . 
COROLLAIRE $11.1 .16^{\left({ }^{*}\right)}$.- Le rang $\ell$-adique $r_{M_{l}}$ d'un sous-module galoisien $M$ de $\mathrm{K}^{X}$ est égal à son rang conjectural $r_{M_{l}}^{\text {conj }}$ dès que $M$ est assez grand en un sens indépendant de $\ell$. Plus précisément, si chaque caractère rationnel irréductible $\chi$ représenté dans $\chi_{M}$ l'est au moins $\frac{r_{\chi}}{s_{\chi}}$ fois, le caractère $X_{M_{l}}$ a sa valeur conjecturale $\chi_{M_{l}}^{\text {conj }}=X_{M}^{\prime} \wedge X_{\text {rég }}$. Les entiers $r_{X}$ et $s_{X}$ sont définis par la représentation matricielle $\mathbb{Q}[G] \mathrm{e}=$ ${ }^{M} r_{X}\left(D_{\chi}\right)$ du facteur simple de l'algèbre $\mathbb{Q}[G]$ associé au caractère $\chi$ :

le premier $r_{\chi}$ est le nombre de fois où $\chi$ est représenté dans le carac tère régulier ; le second $s_{X}$, égal au degré sur $Q$ du centre du corps gauche $D_{X}$, est l'indice de Schur du caractère $x$ •

Démonstration : Il suffit évidemment de faire la démonstration lorsque $M$ est isotypique, i.e. quand $X_{M}$ est de la forme $m X$, pour un caractère rationnel irréductible $X$. Si les caractères absolument irréductibles $\varphi$ au-dessus de $X$ sont de degré 1 , ils sont représentés une seule fois dans le caractère régulier, et le théorème 12 donne immédiatement $X_{1 T_{l}}=X_{M_{l}}^{c o n j}$. C'est le cas en particulier lorsque $X$ est le caractère unité 1 . Dans tout ce qui suit, nous pouvons donc supposer $\chi \neq 1$, auquel cas $M$ ne contient pas de puissances de $l$. Cela étant, d'après le théorème 15 , pour $m \geq r_{\chi}$ nous avons :

$$
r_{M_{l}} \geq \frac{k}{k+1} r_{\chi} \operatorname{deg} x>r_{\chi} \operatorname{deg} \chi-\frac{r_{\chi} \operatorname{deg} x}{k}, \text { avec } k=\left[\frac{m}{r_{\chi}}\right] \text {. }
$$

Ecrivons alors $X=s \chi_{\varphi \mid X} \varphi$ la décomposition absolument irréductible du caractère $\chi$ (de sorte que nous avons $r_{\chi} s_{X}=$ deg $\varphi$ ). Si le caractère $X$ était strictement inférieur à sa valeur conjecturale $X_{M_{l}}^{\text {conj }}=r_{X} X$, nous aurions : $\quad r_{M_{l}} \leq r_{X} \operatorname{deg} X-\operatorname{deg} \varphi$.

L'égalité $X_{M_{l}}=X_{M_{c}}^{\text {conj }}$ a donc lieu dès que la quantité $\frac{r_{X} \text { deg } X}{k}$ est infé rieure à deg $\varphi$, clest-à-dire pour

$$
k \geq r_{\chi} \frac{\operatorname{deg} x}{\operatorname{deg} \varphi}, \text { i.e. } m \geq r_{x}^{2} \frac{\operatorname{deg} x}{\operatorname{deg} \varphi}=\frac{r_{x}}{s_{x}} \operatorname{deg} x \text {. }
$$

(*) L'égalité $r_{M_{e}}=r_{M_{e}}^{\text {conj }}$ dès que $M$ est assez grand, en un sens indépendant de $l$, est dûe à Emsalem : C'est le résultat principal de [E]. La borne proposée ici est plus fine que celle de [E]. 


\section{c.- Comparaison des résultats obtenus .}

Donnons d'abord quelques exemples, qui illustrent les limites des résultats précédents :

Exemple 1 - Si $G$ est le groupe quaternonien $H_{8}$, l'algèbre $\mathbb{Q}[G] \approx$ $\mathbb{Q} \oplus \mathbb{Q} \oplus \mathbb{Q} \oplus \mathbb{Q} \oplus \mathbb{H}$ est composée de quatre exemplaires de $\mathbb{Q}$, et d'un du corps des quaternions sur $\mathbb{Q}$.

- Si $\ell$ vaut 2 , le facteur simple $\mathbb{Q}_{2} \otimes_{Q} \mathbf{H}$ est le corps $\mathbf{H}_{2}$ des quaternions sur $\mathbb{Q}_{2} ;$ l'algèbre $\mathbb{Q}_{2}$ [G] est un produit direct de corps, et la conjecture est vraie en vertu du théorème 12 .

- Dans tous les autres cas, le produit tensoriel $\mathbb{Q}_{\ell} \otimes_{\mathbb{Q}} H$ est isomorphe à $M_{2}(\mathbb{Q})$; le caractère rationnel $p$ correspondant à $H$ s'écrit $\rho=2 \pi$, pour un caractère $\ell$-adique ( absolument) irréductible $\pi$. Si donc $M$ est un sous-module galoisien de $\mathrm{K}^{\mathrm{X}}$, ne contenant pas de puis sances de $\ell$, ayant pour caractère le caractère régulier

$$
x_{M}=1+x_{1}+x_{2}+x_{3}+\rho,
$$

le théorème 12 donne seulement :

$$
x_{M_{l}} \geq 1+x_{1}+x_{2}+x_{3}+\pi \text {. }
$$

D'après le corollaire 16, en revanche, l'égalité $\chi_{M_{l}}=\chi_{M_{l}}^{\text {conj }}$ est assu rée dès que $\chi_{M}$ contient deux fois le caractère $\rho$ :

$$
x_{M} \geq 1+x_{1}+x_{2}+x_{3}+2 \rho \Rightarrow x_{M l}=1+x_{1}+x_{2}+x_{3}+\rho \text {. }
$$

Exemple 2. - Si $G$ est le groupe métacyclique $M_{21}$, la décomposition semi-simple de l'algèbre $\mathbb{Q}[G]$ s'écrit :

$$
\mathbb{Q}[\mathbf{G}] \simeq \mathbb{Q} \oplus \mathbb{Q}[\sqrt{-3}] \oplus \mathbf{3}^{(\mathbb{Q}[\sqrt{-7}]) .}
$$

L'écriture rationnelle irréductible $\chi_{\text {réy }}=1+\chi+3 p$ du caractère ré gulier fait donc intervenir un caractère $\chi$ de degré 2 ( qui se décompose sur $\mathbb{Q}_{\ell}$ comme somme de deux caractères absolument irréductibles, lorsque $(-3)$ est un carré dans $\left.Q_{\ell}\right)$ et un caractère $\rho$ de degré 6 . - Si $(-7)$ n'est pas un carré dans $\mathbb{Q}_{\ell}$, le caractère $\rho$ est irré ductible sur $\mathbb{Q}_{\ell}$. Par suite, si $M$ est un sous-module galoisien de $K^{X}$, ne contenant pas de puissances de $l$, ayant pour caractère le caractère régulier $X_{M}=X_{\text {rég }}$, le théorème 12 donne $X_{M_{\ell}} \geq 1+\chi+\rho$, et le théor ème 15: $\chi_{M_{l}} \geq 1+X+2 \rho$. Enfin, d'après le corollaire 16 , l'égalité $X_{M_{l}}=X_{H_{e}}^{c o n j}$ a lieu sous la condition suffisante $X_{M} \geq 1+X+18 \rho$. 
- Si (-7) est un carré dans $\mathbb{Q}_{\ell}$, le caractère $\rho$ se décompose comme somme $\rho_{1}+\rho_{2}$ de deux caractères $\ell$-adiques (absolument) ir réductibles, de degré 3 . Si donc, comme plus haut, $M$ est un sous module galoisien de $K^{X}$, ne contenant pas de puissances de $\ell$, ayant pour caractère le caractère régulier $X_{M}=X_{\text {iég }}$, le théorème 12 donne toujours $X_{M_{l}} \geq 1+X+P$, mais le théorème 15 permet seulement d'af firmer que l'inégalité est stricte, sans préciser celui des caractères $\rho_{i}$ au-dessus de $\rho$ qui figure au moins deux fois dans $\chi$. Enfin, le co rollaire 16 donne encore $X_{M_{l}}=X_{M_{l}}^{\text {conj }}$, sous la condition suffisante $x \geq 1+x+18 \rho$.

Exemple 3 - Si $G$ est le groupe symétrique $\Xi_{4}$, la décomposition semi-simple de l'algèbre $\mathbb{Q}[\mathbf{G}]$ s'écrit :

$$
\mathbb{Q}[G] \simeq \mathbb{Q} \oplus \mathbb{Q} \oplus M_{2}(Q) \oplus M_{3}(\mathbb{Q}) \oplus M_{3}(\mathbb{Q}),
$$

et la décomposition du caractère régulier comme somme de caractères rationnels irréductibles

$$
X_{\text {reg }}=1+\varepsilon+2 \theta+3 \psi+3 \epsilon \psi,
$$

fait donc intervenir deux caractères de degré 1 , un caractère de degré 2 , et deux caractères de degré 3 . Tous sont absolument irréducti bles $\left.{ }^{\star}\right)^{*}$. En particulier, si $M$ est un sous-module galoisien de $\mathrm{K}^{x}$, ne contenant pas de puissances de $\ell$, ayant pour caractère le carac tère régulier $X_{M}=X_{\text {rég }}$, le théorème 12 donne l'inégalité $X_{M_{\ell}} \geq 1+\epsilon+\theta+\psi+\varepsilon \psi$, et le théorème 15 l'inégalité plus forte $X_{M_{l}} \geq 1+\epsilon+\theta+2 \psi+2 \epsilon \psi$. Enfin, le corollaire 16 assure l'égalité $X_{M_{l}}=X_{M_{l}}^{\text {conj }}$, sous la condition suffisante $X_{M} \geq 1+\varepsilon+4 \theta+9 \psi+9 \varepsilon \psi$.

On voit sur ces exemples que le théorème 15 , s'il ne permet en aucun cas d'étendre le domaine de validité de la conjecture énoncée, conduit toujours néanmoins à des estimations du rang $\ell$-adique sensi blement meilleures que celles résultant du théorème 12 . La différence

$\left({ }^{\star}\right)$ Plus généralement, toutes les représentations de $\epsilon_{n}$ étant réali sables sur $\mathbb{Q}$, les caractères d'un tel groupe sont à valeurs rationnelles, et le théorème 12 vaut donc trivialement pour les groupes symétriques en l'abscence de tout argument transcendant. 
entre les minorations obtenues est d'ailleurs d'autant plus nette que les caractères irréductibles ont un indice élevé : Par exemple, si $M$ est un sous-module isotypique de $K^{X}$ de caractère $x_{M}=m x($ avec $\chi \neq 1)$, contenu dans le caractère régulier, le rang $\ell$-adique $r_{M_{l}}$ est donné en fonction du rang $r_{M}$ par le tableau suivant :

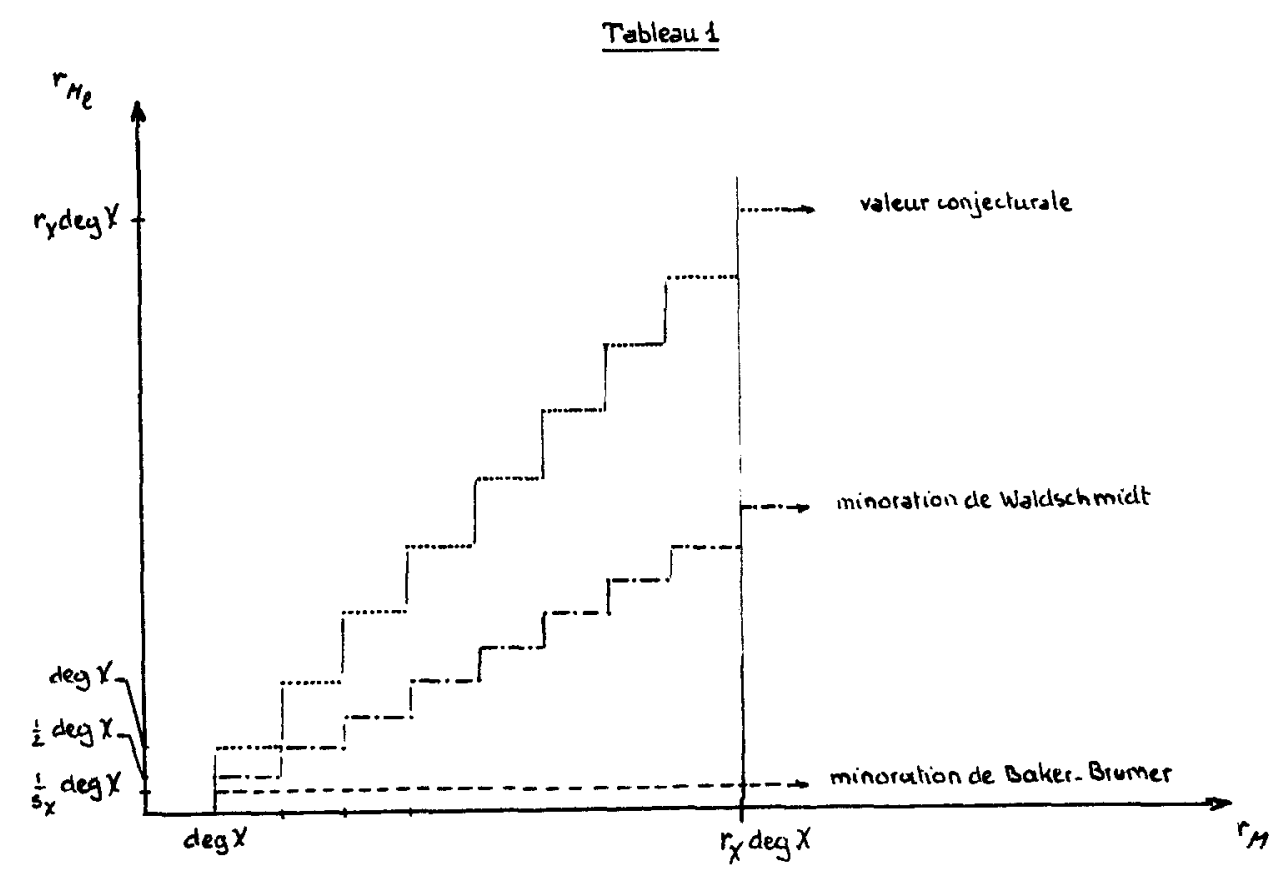

Pour $m \geqslant r_{x}$, il vient de même:

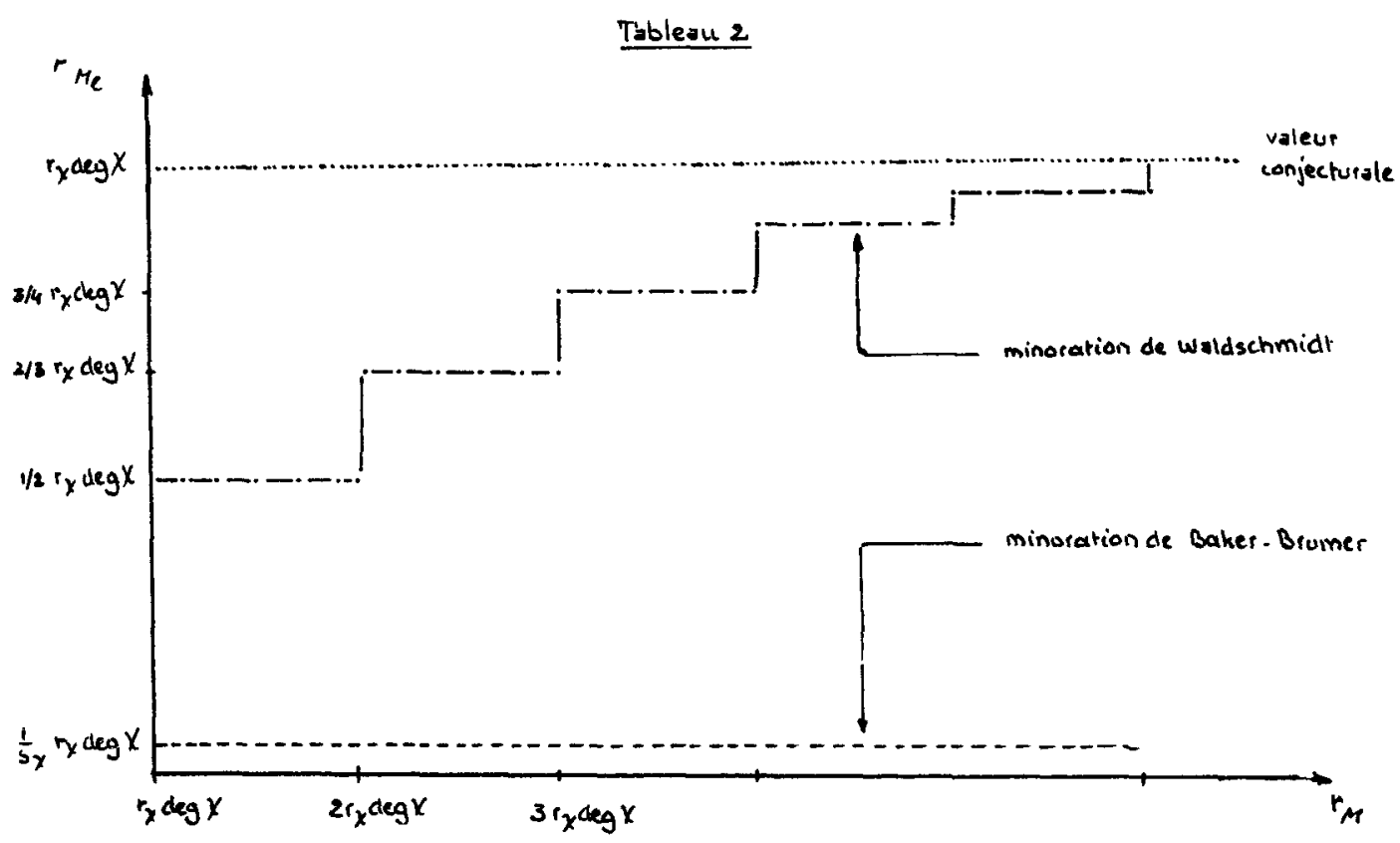




\section{3. - APPLICATION AUX CONJECTURES DE LEOPOLDT ET DE GROSS .}

\section{a.-La conjecture de Leopoldt et le problème du rang $\ell$-adique des S-unités .}

Les sous-groupes de type fini de $K^{\times}$les plus intéressants du point de vue de l'arithmétique sont naturellement les groupes de S-unités. Rappelons que, si $S$ désigne un ensemble fini de places du corps des rationnels, contenant la place à l'infini, et $S_{K}$ l'ensemble des places de $K$ au-dessus de $S$, les $S$-unités du corps $K$ sont les éléments inversibles de llanneau $\sigma_{K}^{S}$ des $S$-entiers de $K$ :

$$
\sigma_{K}^{S}=\left\{x \in K \mid v_{p}(x) \geq 0, \quad \forall p \notin S_{K}\right\} \text {. }
$$

Plus généralement :

DÉFINITION 11.1.17.- Etant donné un ensemble fini quelconque $S=S_{F}$ de places diun sous-corps $F$ de $K$, nous appelons groupe des $S$-unités du corps de nombres $K$, et nous notons $E_{K}^{S}$, le noyau, dans le groupe multiplicatif $K^{x}$, des valuations associées aux places de $K$ au-dessus de $S\left({ }^{\star}\right)$ :

$$
E_{K}^{S}=\left\{x \in K^{x} \mid v_{p}(x)=0, \quad \forall p \notin S_{K}\right\}
$$

Les S-unités sont donc les $\mathbf{S}$-unités au sens ordinaire qui sont positives en chaque place $p$ de $K$ n'appartenant pas à $S_{K}$ •

Comme les places complexes ne jouent aucun rôle dans la définition des groupes $E_{K}^{S}$, et que les carrés vérifient trivialement la condition de positivité aux places réelles, les groupes de S-unités ont la même dimension $\left({ }^{\star}\right.$ ) qulils soient pris au sens ordinaire ou au sens de la définition précédente. Nous supposerons donc pour simplifier, dans tout ce qui suit, que l'ensemble $S_{K}$ contient les places archimédiennes du corps $K$, auquel cas les deux définitions coïncident. Cela posé, lorsque le

${ }^{\star}$ ) Pour une définition des valuations associées aux places d'un corps de nombres, voir Ch. III.1, $\$ 1, a$. $\left({ }^{\star \star}\right)$ Nous appelons dimension d'un $\mathbb{Z}$-module $M$, la dimension sur $\mathbb{Q}$ de l'espace vectoriel $\mathbb{Q} \otimes_{\mathbb{Z}} M$. 
corps $K$ est galoisien sur $\mathbb{Q}$, le groupe $\mathbb{Q} \otimes_{\mathbb{Z}} E_{K}^{S}$ est un module noethérien sur l'algèbre de Galois dont le caractère $\chi^{S}$ est connu depuis Herbrand :

PROPOSITION 11.1.18.- Soient K/F une extension galoisienne de corps de nombres, $G=G a l(K / F)$ son groupe de Galois, et $S$ un ensemble fini de places de $F$, contenant les places archimédiennes. Pour chaque $p$ de $S$, désignons par $X_{p}$ linnduit à $G$ du caractère de la représentation unité du sous-groupe de décomposition $D_{\mathfrak{P}}$ de l'une quelconque des places de $K$ au-dessus de $p$; notons enfin $X_{S}$ la somme des $X_{p}$, lorsque $p$ décrit $S$. Le caractère du $\mathbb{Q}[G]$-module $\mathbb{Q} \otimes_{\mathbb{Z}} E_{K}^{S}$ construit sur les $S$-unités de $K$ est donné par la formule :

$$
x^{S}=x_{S}-1=\sum_{p \in S} x_{p}-1
$$

Nous disons que $\chi^{S}$ est le caractère du groupe des $S$-unités dans l'extension $K / F$.

Démonstration : Le cas des unités, où $S$ se réduit aux places archimédiennes, constitue le théorème de Herbrand proprement dit (cf. [He]). La proposition s'en déduit facilement, puisque, si $S^{\prime}$ est réunion de $S$ et d'une place $p$, le quotient $E_{K}^{S^{\prime}} / E_{K}^{S}$ s'identifie au sous-groupe principal du groupe $I_{K}(p)$ des idéaux de $K$ construits sur les premiers au-dessus de $p$. II vient donc :

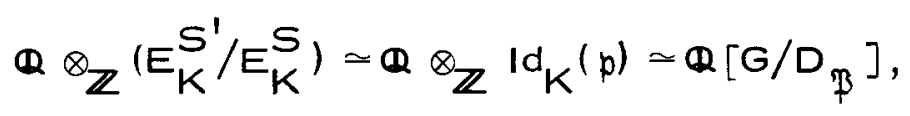

puisque $G$ opère transitivement sur les places de $K$ au-dessus de $p$; d'où, comme attendu :

$$
x^{s^{\prime}}=x^{s}+x_{p}
$$

Introduisons maintenant le tensorisé $\ell$-adique $\varepsilon_{K}^{S}=\mathbb{Z}_{\ell} \otimes_{\mathbb{Z}} E_{K}^{S}$ du groupe des $S$-unités. D'après le théorème 7 , nous pouvons énoncer directement la proposition :

PROPOSITION 11.1.19.- Soient $K / Q$ une extension galoisienne du corps des rationnels, et $S$ un ensemble fini de places de $\mathbb{Q}$ contenant la place 
à l'infini. Sous la conjecture énoncée plus haut, le caractère du $\mathbb{Z}_{l^{-}}$ module $\log _{\ell} \varepsilon_{K}^{S}$ construit sur les $S$-unités du corps $K$ (i.e. le caractère de l'action du groupe de Galois $G a l(K / Q)$ sur le $\mathbb{Q}$,-espace vectoriel $\mathbb{Q}_{l} \otimes_{\mathbb{Z}_{l}} \log _{l} \varepsilon_{K}^{S}$ ) est donné par la formule :

$$
\begin{aligned}
& x_{l}^{S}=x^{S} \wedge x_{\text {rég }}, \quad \text { si } l \notin S ; \\
& x_{l}^{S}=\left(x^{S}-1\right) \wedge x_{\text {rég }}, \quad \text { si } l \in S .
\end{aligned}
$$

COROLLAIRE 11.1.20.- Lorsque l'ensemble $S$ ne contient pas $l$, le noyau $\delta_{K, \infty}^{S}$ de llapplication naturelle du groupe $\delta_{K}^{S}=\mathbb{Z}_{l} \otimes_{\mathbb{Z}} E_{K}^{S}$ des $S$-unités généralisées dans le groupe $u_{l}=u_{K}, l$ des unités principales semi-locales du corps $K$, est un $\mathbb{Z}_{l}[G]$-module $\left({ }^{\star}\right)$ dont le caractère $\chi_{\infty}^{S}$ est donné, sous la conjecture précédente, par la formule:

$$
x_{\infty}^{S}=x^{S}-\left(x^{S} \wedge x_{\text {rég }}\right)
$$

Remarque.- Le corollaire 20 et la proposition précédente valent, indépendamment de la conjecture énoncée, dans deux cas particuliers importants :

(i) Si les sous-groupes de décomposition des places de $S$ sont distingués et à quotient abélien : Dans ce cas, les caractères absolument irréductibles représentés dans $\chi^{S}$ le sont une fois et une seule dans le caractère régulier, et le théorème 12 assure la conclusion. De fait la condition requise sur les groupes de décomposition s'interprète comme suit : Il existe un sous-corps $F$ de $K$, abélien sur $\mathbb{Q}$, tel que les places de $S$ ne se décomposent pas dans l'extension relative $\mathrm{K} / F$. Comme $S$ contient par hypothèse les places archimédiennes, cela implique en particulier, que $K$ est soit une extension abélienne de $Q$, soit une extension quadratique totalement imaginaire d'un corps abélien réel.

(ii) Si l'ensemble $S$ est assez grand; ce qui peut s'entendre dans deux sens :

- En un sens arithmétique : Soit $u \in U_{l}$ une unité semi-locale engendrant un $\mathbb{Z}_{\ell}[G]$-module $U^{\prime}$ d'indice fini dans $U_{l}$; choisissons $m$ $(\star)$ Nous disons que $\delta_{K}^{S}, \infty$
corps $K$. le groupe des S-unités infinitésimales du 
supérieur au module d'hyperprimarité pour avoir $\underset{l \mid l}{\oplus}\left(1+I^{m}\right) \subset U_{l}^{l}$; et invoquons le théorème d'approximation simultanée pour exhiber un $x$ de $\mathrm{K}^{\mathrm{X}}$ qui vérifie la congruence

$$
s_{\ell}(x) \equiv u\left(\bmod \underset{I \mid l}{\oplus}\left(1+I^{m}\right)\right)
$$

Le lemme de Nakayama nous assure alors que les images dans $u_{l}$ des conjugués de $x$ engendrent le $\mathbb{Z} \ell^{\text {-module }} u^{\prime}$, et donc que le logarithme $\ell$-adique $\log _{\ell} \times$ de l'élément $x$ est une base normale de l'algèbre $K_{l}=\mathbb{Z}_{l} \otimes_{\mathbb{Z}} K$ sur le corps $\mathbb{Q}_{l}$. En particulier, il suit immédiatement :

$$
x_{l}^{S}=x^{S} \wedge x_{r e ́ g}=x_{r e ́ g} \text {, }
$$

dès que l'ensemble $S_{K}$ contient les places de $K$ qui divisent $x$.

- En un sens algébrique indépendant de $\ell$ : D'après le résultat d'Emsalem, en effet, l'égalité entre $\chi_{l}^{S}$ et sa valeur conjecturale a lieu dès que le caractère $\chi^{S}$ est assez gros, par exemple si dans la famille $\left(\theta_{p}\right)_{p \in S}$ des classes de conjugaison dans $G$ des sous-groupes de décomposition attachés aux places de $S_{K}$, chaque élément représenté apparait au moins $[K: \mathbb{Q}]$ fois (cf. corollaire 16 supra). Dans linterprétation du corps de classes (cf. Ch. I.1), ce dernier résultat a la conséquence suivante : 11 existe un ensemble fini $S_{K}$ de places de $K$ tel que l'extension abélienne maximale $S_{K}$-décomposée du corps $K$ ne contienne de $\mathbb{Z}_{\ell}$-extension pour aucun premier $\ell$.

COROLLAIRE 11.1.22. - La conjecture énoncée plus haut contient la conjecture de Leopoldt.

Démonstration: Sous sa forme for te (cf. théorème 1.1.22), la conjecture de Leopoldt affirme que la dimension $\ell$-adique du groupe des unités d'un corps de nombres est égale au nombre de Dirichlet, autrement dit que l'application de semi-localisation $S_{\ell}$ envoie injectivement le tensorisé l-adique $\varepsilon_{K}=\mathbb{Z}_{l} \otimes_{\mathbb{Z}} E_{K}$ du groupe des unités dans le groupe $u_{l}={ }_{I}^{\oplus} u_{!}^{1}$ des unités principales semi-locales de ce corps. Naturellement, si la conjecture de Leopoldt était fausse dans $K$, elle le serait a fortiori dans tout corps de nombres plus grand. Aussi n'est-ce point restreindre la géné- 
ralité que supposer $K$ galoisien sur $\mathbb{Q}$. Cela étant, lorsque $S$ se réduit à la place à l'infini, nous avons

$$
x^{S}=x_{\infty}-1 \leq x_{r e ́ g} \text {, en vertu du théorème de Herbrand, }
$$

donc, comme attendu :

$$
x_{l}^{S}=x^{S}=x_{\infty}-1 \text {, par la proposition } 19 \text {, sous la conjecture }
$$

énoncée. En d'autres termes, le tensorisé $\varepsilon_{K}=\mathbb{Z}_{l} \otimes_{\mathbb{Z}} E_{K}$ étant $\mathbb{Z}_{l}[G]-$ quasi monogène, la restriction à $\delta_{K}$ de l'homomorphisme de semi-localisation est conjecturalement injective.

\section{b.- La conjecture de Gross et le groupe des valeurs absolues $l$-adiques des}

\section{S-unités.}

Soient $K$ une extension finie quelconque du corps des rationnels, $n=[K: \mathbb{Q}]$ son degré, et $g$ le nombre de places de $K$ qui sont au-dessus de $\ell$. La trace semi-locale $\operatorname{Tr}_{\ell}$ est une application continue de l'algèbre artinienne $K_{l}=\mathbb{Z}_{l} \otimes_{\mathbb{Z}} K=\oplus_{1 \mid l} K_{l}$, de dimension $n$ sur $\mathbb{Q}_{l}$, sur la sousalgèbre $K_{l}^{\text {déc }}=\underset{l \mid l}{\oplus} Q_{\ell}$ de dimension $g$. Ses composantes $\left(T_{r_{l}}\right)_{l} \mid l$ sont les traces locales $\operatorname{Tr}_{1}=\operatorname{Tr}_{K_{I}} / Q_{\ell}$ associées aux complétions de $K$ pour les places au-dessus de $l$; et la trace globale $\operatorname{Tr}=\operatorname{Tr}_{K_{l}} / \mathbb{Q} l_{l}$, qui est induite par la trace arithmétique de $K$ sur $Q$, est simplement la composée de la trace semi-locale $\operatorname{Tr}_{\ell}$ et de la surjection canonique $\left(x_{1}\right)_{I} \mid l \mapsto \sum_{l \mid l} x_{l}$ de $\stackrel{\oplus}{\oplus} \mathbb{Q}_{\ell}$ sur $\mathbb{Q}$.

Cela dit, le problème de l'indépendance $\ell$-adique des traces locales de logarithmes de nombres algébriques se pose comme suit:

Problème 3.- Etant donnés $x_{1}, \ldots . x_{n}$ dans $K^{x}$, à quelle condition la res triction de l'application $\operatorname{Tr}_{\ell} \circ \log _{\ell}$ au sous-module $m$ du produit tensoriel $Q=\mathbb{Z} \ell \mathbb{Z}_{\mathbb{Z}} K^{X} \mathbb{Z}_{\ell}$-engendré par ces éléments est-elle injective ?

La première conjecture de Gross postule cette injectivité, aux racines de l'unité près, dans la situation suivante : $K$ est une extension quadratique totalement imaginaire d'un sous-corps totalement réel, et $m$ 
est le tensorisé $\ell$-adique $\mathbb{Z}_{\ell} \mathbb{Z}_{\mathbb{Z}} E^{1-}$ du groupe $E^{1-}$ des $\ell$-unités imaginaires de $K\left({ }^{\star}\right)$.

Pour examiner cette conjecture à la lumière de la conjecture générale sur llindépendance $l$-adique énoncée plus haut, nous pouvons, sans restreindre la généralité, nous ramener au cas où le corps $K$ est galoisien sur $\mathbb{Q}$. Cela étant, la représentation galoisienne de l'algèbre

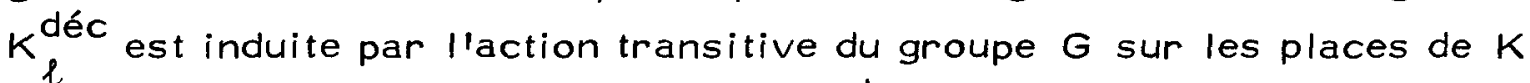
au-dessus de $l$. Son caractère $x_{l}=\operatorname{Ind}_{D_{l}}{ }^{1} D_{D_{l}}$ est donc l'induit au groupe $G=G a l(K / Q)$ du caractère de la représentation unité du sous-groupe de décomposition de l'une quelconque des places $I$ de $K$ au-dessus de $\ell$.

Si donc $M$ est un sous-groupe noethérien du groupe multiplicatif $K^{X}$, stable par Galois, et $\chi_{M}$ le caractère de la représentation associée, le caractère $x_{T}$ du module image $T_{l}=\operatorname{Tr}_{l} \circ \log _{l}\left(\mathbb{Z}_{l} \otimes_{\mathbb{Z}} M\right)$ vérifie les deux inégalités:

$$
x_{T_{l}} \leq x_{M} \quad \& \quad x_{T} \leq x_{l} \quad ;
$$

ce que nous pouvons résumer par la formule:

$$
x_{T} \leq x_{M} \wedge x_{l}
$$

Plus précisément, si $M$ contient des puissances de $l$, nous avons même $\chi_{T} \leq X_{M}-1$ (d'après les propriétés du logarithme l-adique rappelées en 1.b), d'où finalement :

$$
x_{T} \leq x_{M}^{\prime} \wedge x_{l}
$$

en posant, comme plus haut :

$$
X_{M}^{\prime}=\left\{\begin{array}{l}
X_{M}, \text { si } M \text { ne contient pas de puissance de } l \\
X_{M}-1, \text { dans le cas contraire. }
\end{array}\right.
$$

II vient donc:

\footnotetext{
$\left({ }^{\star}\right)$ Si T est la conjugaison complexe, les $\ell$-unités imaginaires sont celles qui vérifient l'identité $x^{\tau}=x^{-1}$.
} 
PROPOSITION $11.1 .23 .-$ Soient $K$ une extension galoisienne finie du corps des rationnels, $G$ son groupe de Galois, $l$ un nombre premier, et $X_{l}$ l'induit à $G$ du caractère de la représentation unité du sous-groupe de décomposition de l'une quelconque des places de $K$ au-dessus de $l$. Etant donné un sous-module noethérien $M$ du groupe multiplicatif $K^{x}$, stable pour l'action de $G$, la restriction de l'application $T r l_{\ell} \log _{\ell}$ composée de la trace semi-locale et du logarithme $l$-adique au $\mathbb{Z} l^{\text {-module }}$ $m=\mathbb{Z}_{\ell} \otimes_{\mathbb{Z}} M$, engendré par l'image de $M$ dans le tensorisé $\mathbb{Z}_{\ell} \otimes_{\mathbb{Z}} K^{X}$, n'est injective que si les trois conditions nécessaires suivantes sont réunies :

(i) Le groupe $M$ ne contient pas les racines $\ell$-ièmes de l'unité.

(ii) Le radical de $M$ dans $K^{X}$ ne contient pas $\ell$, i.e. $\ell \notin \sqrt{M}$.

(iii) Le caractère $X_{M}$ associé au module $M$ est contenu dans $X_{l}$.

Comme plus haut, il s'agit évidemment de savoir si les conditions avancées sont également suffisantes. Nous avons ainsi :

THÉORĖME 11.1.24.- Conservons les notations de la proposition précédente, et notons $\bar{x}_{l}=x_{\text {rég }}-x_{l}$ le supplémentaire du caractère $x_{l}$. Si les deux caractères $x_{M} \wedge x_{l}$ et $x_{M} \wedge \bar{x}_{l}$ sont étrangers (autrement dit, si nous avons $\left.x_{M} \wedge x_{l} \wedge \bar{x}_{l}=0\right)$, alors, sous la conjecture générale d'indépendance $\ell$-adique énoncée plus haut, les trois conditions nécessaires de la proposition 23 sont également suffisantes.

Démonstration: Supposons réunies les trois conditions de la proposition 23. D'après la conjecture 6 , nous avons alors $X_{M}=X_{M}$. D'un autre côté, le noyau $\mathrm{Ker}^{\mathrm{Tr}} \mathrm{r}_{\ell}$ dans l'algèbre $K_{\ell}$ de la trace semi-locale $\operatorname{Tr} l$ est évidemment un $Q_{l}[G]$-module de caractère $x_{r e ́ g}-x_{l}=\bar{x}_{l}$. Son intersection avec le $\mathbb{Z}_{l}[G]$-module noethérien $M_{l}=\log _{l}\left(\mathbb{Z}_{l} \otimes_{\mathbb{Z}} M\right)$ est donc un sous-module de celui-ci dont le caractère associé est au plus $X_{M} \wedge \bar{\chi}_{l}$. Il suit donc :

$$
x_{T} \geq x_{M}-\left(x_{M} \wedge \bar{x}_{l}\right)=x_{M} \wedge x_{l} \text {, ce qui est précisément le }
$$

résultat attendu. 
COROLLAIRE 11.1.25.- Soit $K$ une extension galoisienne du corps des rationnels, pour laquelle le caractère $x_{l}$ est saturé (i.e. vérifie l'identité $\left.x_{l} \wedge \bar{x}_{l}=0\right)$. Alors, la conjecture générale sur l'indépendance $l$-adique énoncée plus haut entraîne la conjecture de Gross pour le corps $K$.

Démonstration: D'après le théorème 1.1 .24 , une façon d'énoncer la conjecture de Gross généralisée pour le corps $K$ consiste à dire que les valeurs absolues $\ell$-adiques attachées aux places de $K$ au-dessus de $\ell$ envoient le tensorisé $\ell$-adique $\delta_{K}^{\prime}=\mathbb{Z}_{\ell} \otimes_{\mathbb{Z}} E_{K}^{\prime}$ du groupe des $\ell$-unités de $K$ sur un sous-module d'indice fini de la somme directe $\widetilde{\oplus}_{\mathfrak{l} / \ell}(1+\ell \mathbb{Z} l)$ restreinte aux familles $\left(\nu_{I}\right)_{I} \mid \ell$ qui vérifient la formule du produit $\prod_{l \mid l} \nu_{l}=1$

Transposons ce résultat à l'aide du diagramme commutatif :

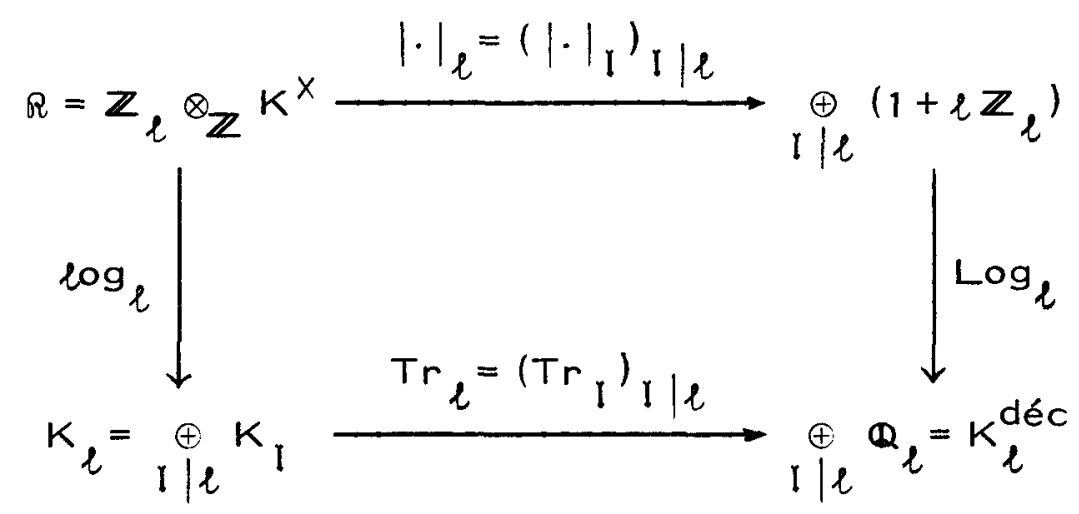

L'image du module $\underset{l \mid l}{\oplus}\left(1+\ell \mathbb{Z}_{\ell}\right)$ par le logarithme $\log _{\ell}$ étant un $\mathbb{Z}_{\ell}$ réseau de $\mathrm{K}_{\ell}^{\text {déc }}, \mathfrak{l} \mid \ell$ une autre façon d'énoncer la conjecture de Gross généralisée consiste à dire que l'image $\operatorname{Tr}_{\ell} \circ \log _{\ell}\left(\varepsilon_{K}^{\prime}\right)$ de $\delta_{K}^{\prime}$ dans $K{ }_{l}^{\text {déc est }}$

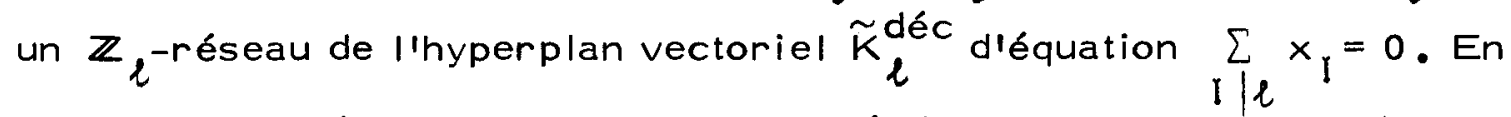
termes de représentations, cela revient à dire que le caractère de la

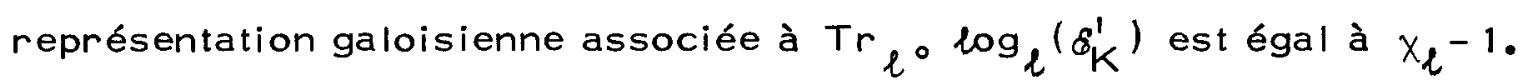

Maintenant, nous connaissons le caractère associé au groupe des l-unités $E_{K}^{\prime}$. D'après la proposition 18 , il est égal à $x_{\infty}+x_{l}-1$. En particulier $E_{K}^{\prime}$ contient donc un $\mathbb{Z}[G]$-module libre, disons $M$, de caractère $\chi_{l}-1$. Et le théorème 24 nous montre alors que, sous la condition $x_{l} \wedge \bar{x}_{l}=0$, la restriction au tensorisé $\mathbb{Z}_{l} \otimes_{\mathbb{Z}} M$ de l'application $\operatorname{Tr}_{l} \log _{l}$ est injective. Le groupe $\operatorname{Tr}_{\ell} \circ \log _{\ell}\left(\varepsilon_{K}^{\prime}\right)$ contient donc un $\mathbb{Z}_{\ell}[G]$-module 
libre de caractère $x_{l}-1$, ce qui est bien le résultat annoncé.

\section{Remarques. -}

(i) La condition subsidiaire invoquée $\chi_{l} \wedge \bar{X}_{l}=0$ est trivialement vérifiée lorsque le groupe $G$ est abélien, et, plus généralement, dès que l'algèbre $\mathbb{Q}[G]$ est un produit direct de corps, donc dans tous les cas où la conjecture 6 est établie par des arguments de transcendance.

(ii) Lorsque le corps $K$ est une extension quadratique totalement imaginaire d'un sous-corps totalement réel, on peut la remplacer par la condition plus faible $\bar{\chi}_{l}^{-} \wedge \bar{x}_{l}^{-}=0$ (où $\chi^{-}$désigne la partie imaginaire d'un caractère $\chi$ ), puisque la partie réelle de la conjecture de Gross généralisée est contenue dans la conjecture de Leopoldt.

(iii) Dans le cas général, cependant, lorsque la décomposition de l'algèbre $Q[G]$ fait intervenir une ou plusieurs algèbres simples non intègres, cette condition peut n'être pas vérifiée, et il n'y a plus alors d'évidence algébrique en faveur de la conjecture de Gross.

\section{c.- Application aux problèmes normiques dans les $\mathbb{Z}_{\ell}$-extensions}

Le problème du rang $\ell$-adique des groupes de $S$-unités gouverne

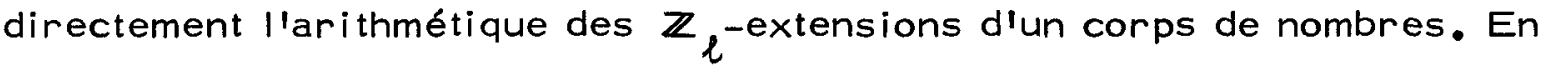
particulier il intervient de façon essentielle dans l'étude des propriétés normiques des sous-groupes de type fini de $K^{X}$ dans une $\mathbb{Z}_{l}$-extension donnée $K_{\infty} / K$ : Si $K_{\infty}=\underset{n \in \mathbb{N}}{U} K_{n}$ est une $\mathbb{Z}_{\ell}$-extension de $K$, les éléments de $K^{X}$ qui sont normes dans l'extension procyclique $K_{\infty} / K$ sont, par définition, ceux qui sont normes dans chacune des $\ell$-extensions finies $K_{n} / K$, clest-à-dire, d'après le principe de Hasse, ceux qui sont normes locales partout dans chacune des $\ell$-extensions cycliques $K_{n} / K$. Plus généralement, nous disons qu'un élément $x$ du tensorisé $\ell=\mathbb{Z} \otimes_{\mathbb{Z}} K^{X}$ est une norme dans l'extension procyclique $K_{\infty} / K$, lorsquilil est contenu dans le tensorisé du groupe des normes $\mathbb{Z}_{\ell} \otimes_{\mathbb{Z}}\left(\bigcap_{n \in \mathbb{N}} N_{K_{n}} / K\left(K_{n}^{x}\right)\right)$.

En une place $p$ de $K$ complètement décomposée dans $K_{\infty} / K$, les extensions locales $K_{n}, p_{n} / K_{p}$ étant triviales, tous les éléments sont des normes locales et le problème normique ne se pose donc pas. 
- En une place $p$ de $K$ presque totalement inerte dans $K_{\infty} / K$, les diverses extensions locales $K_{\infty}, p_{\infty} / K_{p}$ (correspondant aux places de $K_{\infty}$ au-dessus de $p$ ) étant infinies et non ramifiées, les éléments de $Q$ qui sont normes locales sont ceux étrangers à $p$.

Le problème des normes locales dans une $\mathbf{Z}_{\mathfrak{l}}$-extension ne se pose donc en fait qu'aux places ramifiées. Or, pour celles-ci, il est toujours possible d'en restreindre l'étude aux seuls groupes d'inertie $I_{\mathfrak{l}}\left(K_{\infty} / K\right)$ : Si $\mathfrak{l}$ est une telle place, en effet, le groupe $I_{\mathfrak{l}}\left(K_{\infty} / K\right)$, qui est isomorphe à $\mathbb{Z}_{\ell}$, est d'indice fini, disons $f_{l}$ dans le groupe de décomposition $D_{l}\left(K_{\infty} / K\right)$, également isomorphe à $\mathbb{Z}_{l}$. Ainsi, pour décider si un élément $x$ de $R$ est norme locale en $I$ dans l'extension $K_{\infty} / K$, il suffit de déterminer si sa puissance $f_{l}$-ième (ou toute autre puissance plus grande au sens de la divisibilité) a une image triviale ou non dans le groupe $I_{l}\left(K_{\infty} / K\right)$, via liisomorphisme du corps de classes local, qui identifie $I_{\mathfrak{l}}\left(K_{\infty} / K\right)$ à un quotient du groupe $U_{\mathfrak{l}}^{1}$ des unités principales du corps $K_{I}$. En résumé, lorsque l'élément $\times$ est donné, la procédure se présente comme suit :

(i) Vérifier d'abord que $x$ est étranger aux places de $K$ qui sont presque totalement inertes dans $K_{\infty} / K$;

(ii) puis, pour chaque place $I$ de $K$ ramifiée dans $K_{\infty} / K$, faire choix d'un élément $\pi_{\mathfrak{l}}$ de $K_{l}^{X}$, non unité, qui soit norme dans l'extension locale $K_{\infty}, I_{\infty} / K_{\mathfrak{l}}$, et envoyer $x$ dans $U_{1}^{1}$ par llapplication de localisation $s_{\mathfrak{l}}$ en tordant par une puissance convenable de $\pi_{\mathfrak{l}}\left({ }^{\star}\right)$,

$$
x \longmapsto s_{l}(x) \cdot \pi_{l}^{-v_{l}(x) / v_{l}\left(\pi_{l}\right)} \text {. }
$$

(iii) Regarder enfin l'image dans $l_{l}\left(K_{\infty} / K\right)$ de l'élément obtenu. La première étape étant sans malice, nous allons nous intéresser ici à la seconde; la troisième, qui relève en fait de la théorie des genres, est étudiée au chapitre III.2.

Supposons, pour simplifier, que $\mathrm{K}$ et $\mathrm{K}_{\infty}$ soient tous deux galoisiens sur $\mathbb{Q}$. Dans ce cas, les places ramifiées dans $K_{\infty} / K$ sont exacte-

$\left({ }^{\star}\right)$ Comme expliqué plus haut, quitte à remplacer $\times$ par l'une de ses puissances, on peut toujours supposer que $v_{l}\left(\pi_{l}\right)$ divise $v_{l}(x)$. 
ment les places de $K$ au-dessus de $\ell$, et il est possible de choisir les éléments $\left(\pi_{l}\right)$ l le de façon compatible avec l'action de Galois en imposant l'identité de conjugaison :

$$
\pi_{\mathfrak{l} T}=\pi_{\mathfrak{l}}^{T} \quad, \quad \forall T \in G=\operatorname{Gal}(\mathrm{K} / Q)
$$

Cela étant, le premier problème qui se pose est le suivant :

Problème 4. - Soient $K$ une extension galoisienne finie du corps des rationnels, et $G$ son groupe de Galois. Supposons choisi, pour chaque place $\mathfrak{l}$ de $K$ au-dessus de $l$, un élément $\pi_{\mathfrak{l}}$ de $K_{l}^{X}$, non unité, de telle sorte que nous ayons:

$$
\pi_{l T}=\pi_{I}^{\tau} \quad, \quad \forall T \in G \quad \text { (dans l'algèbre } K_{l}=\underset{I \mid l}{\oplus} K_{l} \text { ). }
$$

Etant donné un sous-module noethérien $M$ du groupe multiplicatif $\mathrm{K}^{X}$, stable pour l'action de $G$, et satisfaisant la condition $\nu_{l}\left(\pi_{l}\right) / \nu_{l}(x)$ pour tout $\times$ de $M$ distinct de 1 , et tout $I$ divisant $l$, que peut-on dire de limage $m_{l}$ de son tensorisé $\mathbb{Z}_{l} \otimes_{\mathbb{Z}} M$ dans le groupe $u_{l}$ des unités principales de $K_{\ell}$ donnée par l'application

$$
x \longmapsto\left(s_{1}(x) \cdot \pi_{1}^{-v_{1}}(x) / v_{1}\left(\pi_{1}\right)\right)_{\mathfrak{l} \mid l} ?
$$

Plus précisément, peut-on exprimer le caractère $\chi_{m_{l}}$ de la représentation galoisienne associée à $m_{l}$ en fonction de celui $X_{M}$ de la représentation galoisienne associée à $M$ ?

La proposition suivante apporte un élément de réponse :

PROPOSITION 11.1.26. - Soient $S$ un ensemble fini de places de $\mathbb{Q}$, et $M$ un sous-groupe diindice fini du groupe des $\mathrm{S}$-unités de $\mathrm{K}$. Sous les hypothèses du problème 4 , tous les caractères $\ell$-adiques irréductibles représentés dans $X_{M}$, sauf un peut-être, le sont encore dans $X_{M}$.

Démonstration: $S i S$ ne contient pas $l$, les éléments $\pi_{l}$ n'interviennent pas, et $M_{l}$ n'est autre que l'image canonique dans $u_{l}$ du tensorisé $\mathbb{Z}_{\ell} \otimes_{\mathbf{Z}} M$. Dans ce cas, la proposition résulte directement du théorème 12. 
Si $S$ contient $l$, les choses sont plus complexes. Faisons choix d'une place $I$ de $K$ au-dessus de $l$, et d'une I-unité non triviale $x$ dans $M$; puis considérons le sous-module $N=x^{\mathbb{Z}[G]}$ de $M$ engendré par l'élément $x$ et ses conjugués. D'après ce qui précède, la proposition sera établie si nous montrons que tous les caractères $l$-adiques irréductibles représentés dans $X_{N}$ le sont encore dans $X_{\eta_{l}}$. Raisonnons par l'absurde, en supposant qu'il existe deux caractères $\ell$-adiques irréductibles distincts $\varphi$ et $\psi$ représentés dans $\chi_{M}$ mais non dans $X_{\eta_{\ell}}$. Les deux éléments

$$
\prod_{\tau \in G} x^{\varphi\left(\tau^{-1}\right) \tau} \quad \varepsilon \quad \prod_{\tau \in G} x^{\psi\left(\tau^{-1}\right) \tau}
$$

sont alors $\mathbb{Z}_{\ell}$-linéairement indépendants dans $\mathbb{Z}_{\ell} \otimes_{\mathbb{Z}} M$, mais d'image triviale dans $u_{l}$. II vient donc:

$$
\begin{aligned}
& \sum_{\tau \in G} \varphi\left(\tau^{-1}\right)\left[\log _{\mathfrak{l}} x^{T}-v_{\mathfrak{l}}\left(x^{T}\right) / v_{\mathfrak{l}}\left(\pi_{\mathfrak{l}}\right) \log _{\mathfrak{l}} \pi_{\mathfrak{l}}\right]=0= \\
& =\sum_{\tau \in G} \psi\left(\tau^{-1}\right)\left[\log _{\mathfrak{l}} x^{\tau}-v_{\mathfrak{l}}\left(x^{T}\right) / v_{\mathfrak{l}}\left(\pi_{\mathfrak{l}}\right) \log _{\mathfrak{l}} \pi_{\mathfrak{l}}\right] .
\end{aligned}
$$

Dans la formule obtenue, la quantité $v_{I}\left(x^{\top}\right)$ est nulle dès que $T$ n'appartient pas au sous-groupe de décomposition $D_{\mathfrak{l}}$ de la place $l$ dans $K / \mathbb{Q}$. En particulier, les caractères $\varphi$ et $\psi$ doivent agir trivialement sur $D_{I}$ (c'est-à-dire qu'ils sont contenus dans le caractère $x_{l}=\operatorname{lnd}_{D_{l}}^{G}{ }^{1} D_{l}$ défini plus haut) sans quoi nous aurions $\sum_{\tau \in D_{l}} \varphi\left(\tau^{-1}\right)=0$ ou $\sum_{\tau \in D_{l}} \psi\left(\tau^{-1}\right)=0$, et, par conséquent

$$
\sum_{\tau \in G} \varphi\left(\tau^{-1}\right) \log _{\mathfrak{l}} x^{\top}=0 \quad \text { ou } \quad \sum_{\tau \in G} \psi\left(\tau^{-1}\right) \log _{\mathfrak{l}} x^{\tau}=0
$$

en contradiction avec le théorème d'indépendance de Baker-Brumer. Mais si $\varphi$ comme $\psi$ agissent trivialement sur le sous-groupe $D_{I}$, nous obtenons par différence l'identité :

$$
\sum_{\tau \in G}\left[\varphi\left(\tau^{-1}\right) \psi(1)-\psi\left(\tau^{-1}\right) \varphi(1)\right] \log _{\downarrow} x^{\tau}=0
$$

qui contredit à nouveau le théorème de Baker-Brumer. D'où le résultat. 
COROLLAIRE 11.1.27.- Lorsque le groupe $G$ est abélien (et, plus généralement, dès que l'algèbre $Q_{\ell}[G]$ est un produit direct de corps), le caractère $X_{M_{l}}$ s'écrit

$$
x_{M_{l}}=x_{M}-\psi
$$

où $\psi$ est soit le caractère nul, soit llun des facteurs irréductibles du caractère $x_{l}$.

Les conséquences arithmétiques de ce résultat étant développées dans la deuxième section du chapitre III, limitons-nous ici à quelques remarques :

Remarque 1.- Il peut effectivement arriver que l'un des caractères $l$ adiques irréductibles représentés dans $\chi_{M}$ ne le soit plus dans $M_{\ell}$. Prenons par exemple pour $\pi_{l}$ l'image du nombre premier $\ell$ dans le complété $K_{\mathfrak{l}}$. Dans ce cas, le logarithme $\mathfrak{l}$-adique $\log _{\mathfrak{l}} \pi_{\mathfrak{l}}$ étant nul, le groupe $\log _{\ell} M_{l}$ n'est autre que $\log _{l}\left(\mathbb{Z}_{\ell} \otimes_{\mathbb{Z}} M\right)$. Et la conjecture 6 postule que son caractère, qui est encore $\chi_{M_{\ell}}$, est égal à $\chi_{M}^{\prime} \wedge X_{\text {rég }}$. En particulier, si $M$ contient des puissances de $l$, mais une seule fois la représentation unité, le caractère unité est représenté dans $\chi_{M}$ mais non dans $\chi_{M_{\ell}}$.

Remarque 2. - Il est facile de voir, sous la conjecture générale diindépendance $l$-adique énoncée, que le caractère $\chi_{M_{l}}$ vérifie la minoration :

$$
x_{M_{l}} \geq\left(x_{M} \wedge x_{r e ́ g}\right)-\left(x_{M} \wedge x_{l}\right)
$$

Il est clair cependant qu'en général cette inégalité est stricte. De fait, il n'est pas évident de prédire la valeur du caractère $\chi_{M}$ dès qu'un ou plusieurs caractères $\ell$-adiques représentés dans $X_{M}$ interviennent dans $\chi_{\text {rég }}$ avec une multiplicité.

Disons pour finir que, lorsque $K_{\infty}$ est la $\mathbb{Z}_{\ell}$-extension cyclotomique de $K$, l'application naturelle du groupe $u_{\ell}$ dans la somme directe $\oplus I_{\mathfrak{l}}\left(K_{\infty} / K\right)$ des groupes d'inertie associés aux places au-dessus de $l$, $1 \mid \ell$ se factorise par la trace semi-locale $\mathrm{Tr}_{\ell}$. Dans ce cas le problème normique est exactement "résolu" par la conjecture de Gross généralisée. 
$-124-$ 
Nous exposons dans cette section les résultats fondamentaux de l'arithmétique des infinitésimaux. Notre propos est de montrer comment les groupes introduits slintégrent dans un formalisme très souple, par ticulièrement adapté à l'étude des $\ell$-extensions abéliennes de corps de nombres.

La notion-clé de cette section est celle d'élément infinitésimal d'un corps de nombres algébriques: Les infinitésimaux sont les limites (en un sens très précis) des suites convergentes d'éléments globaux qui sont ultimement arbitrairement près de 1 , en chaque place au dessus de $l$, et, plus généralement, en chaque $p$ dlun ensemble fini $T$ donné; nous parlons alors d'éléments T-infinitésimaux. Par les procé dés usuels, ces éléments permettent de construire des groupes de classes infinitésimales ( un diviseur principal infinitésimal étant un diviseur principal engendré par un élément infinitésimal) et des radicaux infinitésimaux ( un radical au sens de Kummer étant infinitésimal s'il peut être représenté par des infinitésimaux). D'un côté, la théorie du corps de classes interpréte le groupe des classes T-infinitésimales de divi seurs comme groupe de Galois de la $l$-extension abélienne T-ramifiée maximale du corps considéré, et permet donc de donner dans le cadre de la T-ramification des énoncés tout à fait analogues à ceux que l'on connait dans le cadre de la non-ramification : calculs de classes inva riantes, problèmes de norme, de théorie des genres, de capitula tion, etc ... D'un autre côté, la théorie de Kummer interpréte le ra dical T-infinitésimal comme radicalkummérien associé à la $\ell$-extension abélienne $T$-décomposée maximale du corps considéré. Enfin, ces deux interprétations ( l'une galoisienne, l'autre kummérienne) ouvrent sur une approche nouvelle de la dualité : Les résultats présentés, qui font intervenir deux ensembles finis de places $S$ et $T$, mettent doublement en relation la $\ell$-extension abélienne maximale $S$-décomposée et $T$-ra mifiée du corps considéré, et celle qui est $T$-décomposée et $S$-ramifiée; 
ils sont obtenus de façon extrèmement simple. Bien entendu, du fait des limites propres à la théorie de Kummer, cette dualité ne trouve sa pleine expression qu'en présence des racines de l'unité, clest-à-dire dans la perspective de la théorie cyclotomique, exposée plus haut.

\section{1. - ÉLÉMENTS INFINITESIMAUX DIUN CORPS DE NOMBRES ALGÉBRIQUES.}

\section{a.- Définition des éléments infinitésimaux.}

Un nombre premier $l$ étant fixé, nous avons associé à chaque corps de nombres algébriques $K$ deux $\mathbb{Z}_{\ell}$-modules multiplicatifs (cf. section $1,1 \S \mathrm{a}$ ) :

tif $\mathrm{K}^{\mathrm{X}}$;

- le tensorisé $\ell$-adique $R=\mathbb{Z}_{\ell} \mathbb{Z} K^{X}$ du groupe multiplica - le complété profini $k_{l}^{x}=\frac{\lim }{m} k_{l}^{x} / k_{l}^{x} l^{m}$ du groupe des élé ments inversibles de l'algèbre semi-locale $K_{l}=\mathbb{Z}_{l} \otimes_{\mathbb{Z}} K$.

Nous avons $v u$, en outre, que l'injection naturelle de $K^{x}$ dans $K_{l}^{X}$ ( induite par l'injection diagonale de $K$ dans le produit $K_{l}=l \stackrel{\oplus}{\oplus} K_{l}$ de ses complétés pour les places au-dessus de $\ell$ ) se prolonge de façon unique en un épimorphisme continu $s_{\ell}$ de $R \operatorname{sur} k_{l}^{x}(\mathrm{cf.ll.1.1})$, que nous avons appelé application de semi-localisation. Le groupe qui nous intéresse ici est le noyau de cet épimorphisme :

DÉFINITION 11.2.1 - Etant donné un corps de nombres $K$, nous appe lons groupe des éléments infinitésimaux du corps $K$, et nous notons $R_{\infty}$, le noyau dans $R$ de l'application de semi-localisation, i.e. le sous groupe de $R$ défini par l'exactitude de la suite courte canonique de $\mathbb{Z}_{l^{-}}$ modules :

$$
1 \longrightarrow R_{\infty} \longrightarrow R \stackrel{s_{\ell}}{\longrightarrow} K_{\ell}^{X} \longrightarrow 1
$$

L'appellation d'infinitésimal peut se comprendre comme suit : les infinitésimaux sont les limites dans $R$ des suites convergentes d'éléments de $\mathrm{K}^{X}$ dont les images dans $\mathrm{K}_{\ell}^{X}$ convergent vers 1 ; ce sont donc les éléments globaux généralisés, qui sont localement arbitrairement près de 1 ( la théorie est multiplicative), pour les places au-dessus de $l$. 
Remarque 1.- Le groupe $R_{\infty}$ peut se définir à partir de la filtration canonique du sous-groupe principal $u_{l}$ de $k_{l}^{x}$ : Pour chaque naturel $n \geq 1$, désignons par $R_{n}$ le sous-groupe des éléments de $k$ dont llimage semi locale tombe dans $\oplus\left(1+l^{n}\right)$, ce que nous pouvons écrire : $1 \mid \ell$

$$
R_{n}=\left\{x \in R\left|s_{\ell}(x) \equiv 1\left(\bmod ^{x} I^{n}\right), \forall \backslash\right| l\right\} .
$$

Posons de même $R_{0}={ }^{-1} S_{l}\left(U_{l}\right)$ (le groupe $R_{0}$ est le tensorisé $\mathbb{Z}_{l} \mathbb{Z} K_{0}^{x}$ du groupe des éléments de $K^{X}$ qui sont étrangers à $\ell$ ). Nous obtenons immédiatement :

$$
R_{\infty}=\bigcap_{n \in \mathbb{N}} R_{n}
$$

En particulier, le groupe $R_{\infty}$ est sans $\mathbb{Z} l^{\text {torsion }}$.

Remarque 2. - Si nous restreignons l'application de semi-localisation au groupe des $S$-unités généralisées $\delta^{S}=Z_{l} \otimes E^{S}$, pour un ensemble fini quelconque $S$ de places non complexes de $K$, le noyau obtenu $\delta_{\infty}^{S}$ est un

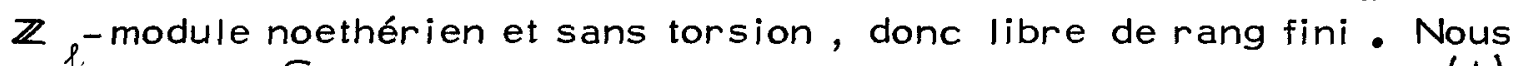
disons que $\delta_{\infty}^{S}$ est le groupe des $\underline{S}$-unités infinitésimales du corps $K^{(*)}$. Lorsque $S$ est vide, $\varepsilon_{\infty}^{S}$ est le groupe $\delta_{\infty}$ des unités infinitésimales ; il mesure le défaut de la conjecture de Leopoldt dans le corps $K$.

ll est intéressant de comparer le groupe $\Omega_{\infty}$ au noyau du loga rithme $\ell$-adique dans $R_{0}$. Nous savons que le noyau dans $k_{\ell}^{x} d u$ logarithme d'lwasawa $\log _{\ell}$ est engendré par les racines de llunité et les images respectives $\ell_{j}$ du nombre $\ell$ dans chacun des facteurs locaux $K^{X}$. Comme $R_{0}$ est précisément le sous-groupe des éléments de $R$ qui sont des unités locales, le noyau dans $\ell_{0}$ du logarithme $l$-adique est liimage réciproque par l'application de semi-localisation du groupe $\mu_{\ell}$ des racines de l'unité dans $\kappa_{l}^{X}$ : c'est donc le radical du sous-groupe infinitésimal $\Omega_{\infty^{*}}$

PROPOSITION 11.2 .2 - Le noyau dans ${ }_{0}$ du logarithme $l$-adique est le radical $\sqrt{\Omega}_{\infty}$ du sous-groupe infinitésimal de $R$. Nous disons que $\sqrt{R}_{\infty}$ est le groupe des éléments quasi-infinitésimaux du corps $K$. Le quotient $\sqrt{\Omega_{\infty}} / \Omega_{\infty}$ slidentifie au sous-groupe $\mu_{\ell}$ des racines de l'unité dans $k x$, i.e. au $\ell$-Sylow du groupe des racines de l'unité de l'algèbre semi locale $K_{\ell}=\mathbb{Z}_{\ell} \otimes_{\mathbb{Z}} K$.

${ }^{\star}$ ) Le caractère de la représentation galoisienne associée au groupe $\delta_{\infty}^{S}$ a été calculé dans la section $1 \S 5$ (cor.11.1.20), sous la conjecture diindépendance $\ell$-adique formulée dans cette section. 


\section{b.- Interprétation kummerienne des infinitésimaux.}

Si $\mu$ désigne le $\ell$-Sylow du groupe des racines de l'unité dans $K$, et $\ell^{m}$ l'ordre de $\mu$, la théorie de Kummer établit un isomorphisme entre le groupe de Galois de l'extension abélienne maximale d'exposant $\ell^{m}$ de $K$, et le groupe $\operatorname{Hom}\left(K^{x}, \mu\right) \approx \operatorname{Hom}(\Omega, \mu)$. Cet isomorphisme s'obtient en associant à l'élément $\sigma$ du groupe de Galois, I'homomorphisme $f_{\sigma}$ défini par la formule :

$$
f_{\sigma}(x)=\sqrt[\ell^{m}]{x}(\sigma-1)
$$

Nous disons que le quotient $\left(l^{-m} \mathbb{Z} / \mathbb{Z}\right) \otimes_{\mathbb{Z}} K^{x} \simeq\left(l^{-m} \mathbb{Z}_{l} / \mathbb{Z}_{l} l^{\otimes} \mathbb{Z} l_{l}\right.$ est le radical kummérien de l'extension considérée. Plus généralement à chaque $\ell$-extension abélienne de $K$ correspond un sous-groupe de ce radical, défini par llidentité :

$$
\operatorname{Rad}(L / K)=\left\{l^{-k} \otimes x \in\left(l^{-m} \mathbb{Z} / \mathbb{Z}\right) \otimes K^{x} \mid K\left[\sqrt[l^{k}]{x}\right] \subset L\right\},
$$

et caractérisé par liisomorphisme de dualité : $\operatorname{Rad}(L / K) \simeq H o m(G a l(L / K), \mu)$. Nous disons que $L$ est kummérienne lorsque $\operatorname{Rad}(L / K)$ et $G a l(L / K)$ ont le même ordre, c'est-à-dire lorsque $L / K$ est d'exposant $l^{m}$. Cela posé, nous avons :

THÉORĖME 11,2.3. - Une extension Kummérienne $L / K$ est $\ell$-décomposée (en ce sens que les places de $K$ au-dessus de $\ell$ se décomposent complètement dans $L / K)$, si et seulement si les éléments de son radical peuvent être représentés par des infinitésimaux.

Démonstration : Considérons une extension kummérienne $L / K$, et notons $\ell^{-k} \otimes \times$ un élément de son radical . Si l'extension $L / K$ est $l$-décomposée, alors pour chaque place $l$ de $K$ au-dessus de $\ell$, l'extension locale $K_{I}[\sqrt[\ell]{x}] / K_{l}$ est triviale, et l'élément $x$ est donc une puissance $\ell^{k}-i e ̀ m e$ dans l'algèbre semi-locale $K_{\ell}=\underset{l \mid \ell}{\oplus} K_{I}$. Ecrivons alors $s_{\ell}(x)=u^{\ell^{k}}$, pour un $u$ de $k^{x}$, et choisissons y dans $R$ au-dessus de $u$. Nous obte nons $s_{\ell}(x)=u^{\ell^{k}}=s(y)^{\ell^{k}}$, et l'élément infinitésimal $x y^{-l^{k}}$ a même classe que $\times$ modulo $\Omega^{l^{k}}$. Réciproquement, si chaque élément $l^{-k} \& \times$ du radical de $L$ peut être représenté par un infinitésimal, i.e. si $\times$ s'écrit $x_{\infty} y^{l^{k}}$ avec $x_{\infty} \in R_{\infty}$, l'identité $s_{l}(x)=s_{l}(y)^{l}$ montre que les éléments $x$ sont des puissances $\ell^{k}$-ièmes locales. Toutes les sous-extensions cycliques 
$K[\sqrt[\ell]{x}] / K$ sont alors $\ell$-décomposées, ce qui prouve que $L / K$ elle même est $\ell$-décomposée.

Parmi les $\ell$-extensions abéliennes de $K$, celles qui sont $\ell-$ ramifiées nous intéressent plus particulièrement :

PROPOSITION II.2.4. - Nous disons quiune extension de $K$ est $l$-rami fiée lorsqu'elle est non ramifiée aux places (finies)étrangères à $\ell$. Le radical $\operatorname{Rad}(M / K)$ de la $\ell$-extension abélienne $\ell$-ramifiée maximale de $K$ est le noyau $R$, dans $\left(e^{-m} \mathbb{Z} / \mathbb{Z}\right) \otimes \mathbb{Z}^{x}$, des valuations asso ciées aux places finies et étrangères à $\ell$ du corps $K$ :

$\operatorname{Rad}(M / K)=\left\{l^{-k} \otimes x \in\left(l^{-m} \mathbb{Z} / \mathbb{Z}\right) \otimes \mathbb{Z}^{K^{x}} \mid v_{p}(x) \equiv 0\left(\bmod \ell^{k}\right), \forall p \not l \infty\right\}$

Démonstration : C'est un résultat classique de la théorie de Kummer.

COROLLAIRE $11.2 .5_{0}$ - Le radical $\operatorname{Rad}\left(C^{\prime} / K\right)$ de la $l$-extension abé lienne non ramifiée $\ell$-décomposée maximale de $K$ est le sous-groupe de $\operatorname{Rad}(M / K)$ engendré par les classes des infinitésimaux :

$\operatorname{Rad}\left(C^{\prime} / K\right)=\left\{l^{-k} \otimes x \in\left(l^{-m} \mathbb{Z}_{l} / \mathbb{Z}_{l}\right) \otimes_{\mathbb{Z}_{l}} \Omega \mid x \in R_{\infty} \& v_{p}(x) \equiv 0\left(\bmod l^{k}\right), \forall p \times l_{\infty}\right\}$

Démonstration : Dlaprès la proposition 4 , la condition sur les valua tions signifie que l'extension est $\ell$-ramifiée; et dlaprès le théorème 3 , celle sur la représentation par des infinitésimaux, qu'elle est en outre l-décomposée.

Il est d'ailleurs extrêmement intéressant de comparer le groupe $\operatorname{Rad}\left(C^{\prime} / K\right)$ au noyau dans $\left(l^{-m} \mathbb{Z}_{l} / \mathbb{Z}_{\ell}\right) \otimes_{\mathbb{Z}_{l}}{ }^{R_{\infty}}$ des valuations :

PROPOSITION 11.2.6.- Désignons par : $\Re_{\infty}=\left\{l^{-k} \otimes x \in\left(l^{-m} \mathbb{Z}_{l} / \mathbb{Z}_{l}\right) \otimes_{\mathbb{Z}_{l}{ }^{R}} \mid v_{p}(x) \equiv 0\left(\bmod l^{k}\right), \forall p \nmid l \infty\right\}$, le noyau, dans le groupe $\left(\ell^{-m} \mathbb{Z}_{\ell} / \mathbb{Z}_{\ell}\right) \otimes_{\mathbb{Z}_{\ell}}{ }^{\ell_{\infty}}$, des valuations atta chées aux places finies et étrangères à $l$. Il existe une suite exacte courte canonique loù $\ell^{m} \mu_{\ell}$ est le sous-groupe de $l^{m}$-torsion du groupe multiplicatif $\left.K_{l}^{X}\right)$ :

$$
1 \longrightarrow \ell^{m} \mu_{l} / \mu \rightarrow \Re_{\infty} \longrightarrow \operatorname{Rad}\left(C^{\prime} / K\right) \longrightarrow 1
$$


Démonstration : D'après le corollaire 5 , llapplication naturelle de $\Re_{\infty}$ dans $\operatorname{Rad}\left(C^{\prime} / K\right)$ est une surjection. Le problème est donc de déter miner son noyau . Or, si $\ell^{-m} \otimes x_{\infty}$ est un élément de $\Re_{\infty}$ d'image 1 dans $\operatorname{Rad}\left(C^{\prime} / K\right)$, l'infinitésimal $x_{\infty}$ est une puissance $l^{m}$-ième dans $k^{x}$. De l'égalité $x_{\infty}=y^{l^{m}}$, nous tirons $s(y)^{l^{m}}=1$, ce qui montre que $s(y)$ est une racine $\ell^{m}$-ième de l'unité dans $k_{l}^{x}$. Comme y est connu à une racine globale de l'unité près, nous définissons un homomorphisme du noyau étudié dans le quotient $\ell^{m} \mu_{l} / \mu$ en associant à l'élément $\ell^{-m} \otimes x_{\infty}$ du noyau la classe de $s(y)$ dans $\ell^{m} \mu_{l} / \mu$. Reste à vérifier que ce morphisme est bijectif. D'un côté liidentité $s(y) \in \mu$ implique que y soit le produit d'une racine globale de l'unité et dlun infinitésimal $y_{\infty}$; dans ce cas,$x_{\infty}=y^{l^{m}}=y_{\infty}^{l^{m}}$ est la puissance $l^{m}$-ième d'un infinitésimal, et $\ell^{-m} \otimes x_{\infty}$ vaut $1 ;$ ce qui prouve linjectivité. D'un autre côté, si $\zeta$ est une racine $l^{m}$-ième de llunité dans $k_{l}^{x}$, et y un élé ment de $\kappa^{x}$ au-dessus de $\zeta$, l'élément $x_{\infty}=y^{l^{m}}$ est un infinitésimal, et le produit $\ell^{-m} \otimes x_{\infty}$ est dans $\Re_{\infty}$; ce qui achève la démonstration.

\section{c.-Définition des diviseurs infinitésimaux.}

Rappelons que le groupe des diviseurs d'un corps de nombres est le groupe abélien $D$ construit sur les valuations attachées aux places non complexes de ce corps. Comme les valuations ultramé triques, attachées aux idéaux premiers non nuls, sont à valeurs dans $\mathbb{Z}$, et les valuations réelles à valeurs dans $\mathbb{Z} / 2 \mathbb{Z}$, le groupe $D$ est le produit direct du groupe ld des idéaux de $K$, et d'un $\mathbb{F}_{2}$-espace vectoriel de dimension égale au nombre de plongements réels de $K$ (cf. IIl. $1, \S 1$, pour plus de détails). Son sous-groupe principal $P$, image canonique de $K^{x}$ dans $D$, est, par définition, le groupe des diviseurs principaux du corps $K$.

DÉFINITION 11.2.7. - Par groupe des diviseurs généralisés du corps $K$, nous entendons le tensorisé $l$-adique $\theta=\mathbb{Z}_{l} \otimes_{\mathbb{Z}} D$ du groupe des di viseurs de $K$; par groupe des diviseurs principaux généralisés, le tensorisé $\ell$-adique $P=\mathbb{Z}_{\ell} \mathbb{Z}^{P}$ du sous-groupe principal $P$. Le groupe $P$ est limage canonique dans $\theta$ du groupe multiplicatif $R^{X}$; et le quotient fini $C \ell=D / P$ est le $\ell$-groupe des classes de diviseurs du corps $K$. 
PROPOSITION 11.2.8.-Pour chaque naturel $n$, écrivons $p_{n}$ l'image dans le groupe des diviseurs $\theta$ du sous-groupe $R_{n}$ de $R$. Lintersection $P_{\infty}=\bigcap_{n \in N} P_{n}$ est l'image dans $A$ du groupe infinitésimal $Q_{\infty}^{X}$. Nous disons que $P_{\infty}$ est le groupe des idéaux principaux-infinitésimaux du corps $K$.

Démonstration : Soit $x$ un élément de $R$ engendrant un diviseur princi pal généralisé $a \in n \in \mathbb{N} P_{n}$. Pour chaque naturel $n$, il existe alors une unité $\epsilon_{n}$ dans $\delta$, et un élément $x_{n}$ dans $Q_{n}$, qui vérifient $x=\epsilon_{n} \times{ }_{n}$. Cela étant, puisque le groupe \& est compact, la suite $\left(\epsilon_{n}{ }^{\prime} n \in \mathbb{N}^{\text {pos - }}\right.$ sède une valeur d'adhérence $\epsilon_{\infty}$. L'élément $x_{\infty}=x / \varepsilon_{\infty}$, qui s'écrit $x_{\infty}=x_{n} \epsilon_{n} / \epsilon_{\infty}$ pour $n$ arbitrairement grand est infinitésimal; d'où le résultat annoncé : $a=(x)=\left(x_{\infty}\right)$. Les idéaux principaux infinitési maux sont donc les idéaux principaux engendrés par les éléments in finitésimaux.

DÉFINITION 11.2.9. - Nous disons qu'un diviseur généralisé a est quasi-infinitésimal $^{(\star)}$ lorsqulune de ses puissances $a^{l^{k}}$ est princi pale infinitésimale. Le groupe $D_{\infty}$ des diviseurs quasi-infinitésimaux est donc le radical $\sqrt{P_{\infty}}$ dans $\mathcal{A}$ du sous-groupe des diviseurs principaux-infinitésimaux .

Remarque. - Les diviseurs principaux qui sont quasi-infinitésimaux ne sont pas, en général, infinitésimaux. En fait, la condition $(x) \in \theta_{\infty}$ s'écrit $s(x) \in \sqrt{s(\theta)}$; tandis que la condition $(x) \in p_{\infty}$ s'écrit $s(x) \in s(\delta)$. Le groupe quotient $\left(P \cap D_{\infty}\right) / P_{\infty}$ est donc isomorphe au sous-groupe de torsion du quotient $u_{\ell} / s(\varepsilon)$.

PROPOSITION 11.2.10. - Le quotient $a=\delta_{0} / p_{\infty}$ du groupe des diviseurs généralisés étrangers à $l$ par le sous-groupe des diviseurs princi-

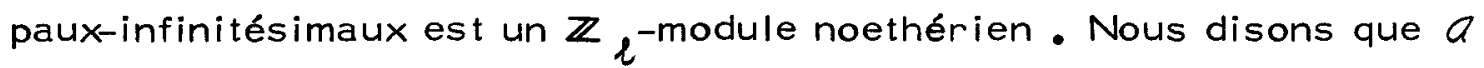
est le $\ell$-groupe des classes infinitésimales du corps $K$. Son sous groupe de torsion $\zeta$, qui est fini, s'identifie au quotient $D_{\infty} / p_{\infty} d u$ groupe des diviseurs quasi-infinitésimaux par le sous-groupe principalinfinitésimal.

$\left({ }^{\star}\right)$ Ces idéaux sont dits infinitésimaux dans [ Ja ]. La remarque qui suit justifie le changement de vocabulaire. 
Démonstration : II s'agit d'établir que $a$ est de type fini . Introduisons pour cela le sous-groupe $p_{0}=\delta_{0} \cap p$ des diviseurs principaux étran gers à $\ell$, et considérons la suite exacte courte canonique :

$$
1 \longrightarrow p_{0} / p_{\infty} \longrightarrow \theta_{0} / p_{\infty} \longrightarrow \theta_{0} / p_{0} \longrightarrow 1 \text {. }
$$

Le terme de gauche $p_{0} / p_{\infty}$ s'identifie au quotient $u_{l} / s(\varepsilon) ;$ il est donc de type fini, comme quotient de $u_{l}$. Le terme médian est le groupe $a$. Enfin, le terme de droite $\delta_{0} / p_{0}$ s'identifie au $\ell$-groupe $C \ell$ des classes de diviseurs du corps $K$; il est donc fini.

Nombre de propriétés usuelles du $\ell$-groupe des classes $C l$ se transportent sans difficulté au groupe des classes infinitésimales $a$. Par exemple :

PROPOSITION 11.2.11.- Toute classe de a peut être représentée par un diviseur étranger à un ensemble fini $S$ de places donné.

Démonstration : Un ensemble fini $S$ de places de $K$ étrangères à $\ell$ étant donné, il en existe un autre $S^{\prime}$, ne rencontrant pas $S$, tel que la restriction aux $S^{\prime}$ - unités généralisées de l'homomorphisme de semi-loca lisation recouvre $u_{\ell}$ : En effet, si $x_{1}, \ldots, x_{n}$ est une famille finie d'éléments de $K^{X}$, étrangers à $l$, dont les images par $s$ engendrent $l$-adiquement $u_{\ell}$, nous pouvons résoudre pour chaque $i=1, \ldots, n$, le sys tème de congruences (où $\nu$ est le degré de $\ell$-hyperprimarité) :

$$
y_{i} \equiv x_{i}\left(\bmod l^{\nu}\right), \forall l \mid l \quad \varepsilon \quad y_{i} \equiv 1(\bmod p), \forall p \in S \text {. }
$$

L'ensemble des places intervenant dans les $\left(y_{i}\right)$ convient donc.

Cela étant, considérons un diviseur $a \in D_{0}$. La proposition 11 étant vraie dans le groupe $C l$, il existe un $x$ dans $R_{0}$ tel que le diviseur $x$ a soit étranger à $S$; et, d'après ce qui précède, il existe aussi un y, étranger à $S$, tel que le produit $x y$ soit infinitésimal. Le diviseur $(x y)$ a est donc associé à $a$ dans $C l$, et étranger à $S$.

Le groupe $a$ n'étant pas dénombrable, il est clair en revanche que toute classe de $a$ ne peut pas être représentée par un diviseur premier. On peut toutefois vérifier que les classes des diviseurs premiers sont denses dans $a$. 


\section{d.- Interprétation arithmétique du groupe des classes infinitésimales .}

Pour chaque naturel $n$, la théorie du corps de classes interprète le $\ell$-groupe des classes de rayon $\theta_{0} / p_{n}$ comme groupe de Galois de la $l$-extension abélienne maximale de $K$ de conducteur $i_{n}=\left(\prod_{1 \mid l} l^{n}\right)$. Dans cet isomorphisme, le groupe de la $\ell$-extension abélienne maxi -

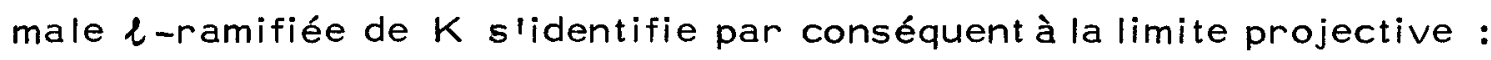

$$
\operatorname{Gal}(M / K) \simeq \frac{\lim }{n} D_{0} / P_{n}=D_{0} / P_{\infty}=a \text {, }
$$

c'est-à-dire au $\ell$-groupe des classes infinitésimales de $K$. II vient donc :

THÉOREMME 11.2.12. - Dans l'isomorphisme du corps de classes, le $l$ groupe des classes infinitésimales $a$ du corps $K$ s'identifie au groupe de Galois $\mathrm{Gal}(\mathrm{M} / \mathrm{K})$ de la $\ell$-extension abélienne $\ell$-ramifiée maximale de $K$.

Remarque.- Désignons par $C$ la $l$-extension abélienne non ramifiée (i.e. non ramifiée aux places finies) maximale du corps $K$. Dans l'iso morphisme du corps de classes, la suite exacte courte de la propo sition 10 :

$$
1 \longrightarrow p_{0} / p_{\infty} \longrightarrow D_{0} / p_{\infty} \longrightarrow D_{0} / p_{0} \longrightarrow 1
$$

prend la forme bien connue :

$$
1 \longrightarrow \operatorname{Gal}(\mathrm{M} / \mathrm{C}) \longrightarrow \operatorname{Gal}(\mathrm{M} / \mathrm{K}) \longrightarrow \operatorname{Gal}(\mathrm{C} / \mathrm{K}) \longrightarrow 1 \text {. }
$$

Cette dernière suite apporte malheureusement assez peu d'in formations sur le groupe $a$; en particulier elle ne donne ni son rang, ni son sous-module de torsion 6 . La dualité, en revanche, conduit à un résultat beaucoup plus précis : Comme première application des infinitésimaux, nous allons démontrer deux suites exactes très sim ples, qui traduisent précisément cette dualité entre les notions de $\ell-$ ramification et de $\ell$-décomposition .

Considérons d'abord la $\ell$-extension abélienne ramifiée maxi male $M$ du corps $K$. Dlaprès le théorème 12 , son groupe de Galois s'identifie au $\ell$-groupe des classes infinitésimales $a$; et, d'après la proposition 4 , son radical de Kummer est le noyau $\Re$ des valuations attachées aux places finies étrangères à $l$. 
Considérons ensuite la $\ell$-extension abélienne non ramifiée $\ell$-décomposée maximale $C^{\prime}$ de $K$. D'après la théorie du corps de classes, son groupe de Galois s'identifie au $\ell$-groupe $C \ell^{\prime}$ des $\ell$-classes de divi seurs du corps $K(i . e$. au quotient du $\ell$-groupe $C \ell$ des classes de di viseurs par le sous-groupe $C \ell(\ell)$, engendré par les classes des di viseurs premiers au-desus de $\ell$ ) ; et, dlaprès le corollaire 5 , son radical de Kummer est l'image dans $\Re$ du radical infinitésimal $\Re_{\infty}$. Nous avons :

THÉORÈME $11,2.13$ - Soient $M$ la $\ell$-extension abélienne $\ell$-ramifiée maximale d'un corps de nombres $K$, et $C^{\prime}$ la sous-extension maximale de $M$ qui est $\ell$-décomposée. Il existe deux suites exactes courtes de

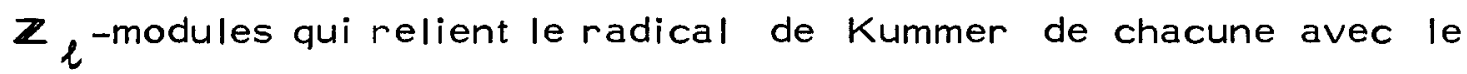
groupe de Galois de l'autre :

- La première fait intervenir limage $\mathbb{S}^{\prime}=\left(l^{-m} \mathbb{Z} / \mathbb{Z}\right) \otimes_{\mathbb{Z}} E^{\prime}$, dans le radical $\Re$,du groupe $E^{\prime}$ des $\ell$-unités de $K$. Elle s'écrit :

$$
1 \longrightarrow \mathbb{E}^{\prime} \longrightarrow \mathfrak{R} \ell^{m} \mathrm{C} \ell^{\prime} \longrightarrow 1 \text {. }
$$

- La seconde fait intervenir l'image $\mathbb{S}_{\infty}=\left(l^{-m} \mathbb{Z}_{\ell} / \mathbb{Z}_{l}\right) \otimes_{\mathbb{Z}_{l}} \varepsilon_{\infty}$, dans le radical $\Re_{\infty}$, du groupe $\AA_{\infty}$ des unités infinitésimales de $K$. Elle s'écrit :

$$
1 \longrightarrow \xi_{\infty} \longrightarrow \Re_{\infty} \longrightarrow \ell^{m} a \longrightarrow 1
$$

Dans les deux cas , $\ell^{m}$ représente l'ordre du $\ell$-groupe $\mu$ des racines de l'unité dans $K$.

Remarque : La suite (i) est bien connue. Elle permet dans tous les cas d'exprimer le $\ell^{m}-$ rang du groupe $\Re=\operatorname{Hom}(a, \mu)$ en fonction de celui du groupe $C \ell^{\prime}$. Comme elle est compatible avec l'action de Galois, on peut toujours se ramener, en effet, au cas où le corps $K$ contient les ra cines $\ell$-ièmes de l'unité; les méthodes semi-simples exposées dans la section IV. 2 conduisent alors au résultat (cf. [Gr]).

La suite (ii), en revanche, utilise de façon essentielle les infinitésimaux. Lorsque le corps $K$ vérifie la conjecture de Leopoldt, elle montre que le radical $\Re_{\infty}$ slidentifie au sous-groupe de $l^{m}$-torsion $\ell^{m} \tau$ de $a$. Dans tous les cas, elle montre que $\Re_{\infty}$ contient $\ell^{m} \tau$ comme facteur direct; et comme $\Re_{\infty}$ est lié au radical $\operatorname{Rad}\left(C^{\prime} / K\right)$ par la suite 
exacte scindée de la proposition 6 , elle permet d'obtenir directement le $l^{m}$-rang du sous-groupe de torsion de $a$ à partir de celui du groupe $C \ell^{\prime}(\star)$.

Démonstration du théorème : La première suite exacte s'obtient très simplement en associant à l'élément $l^{-K} \otimes x$ de $\Re$ la classe $c l^{\prime}(a) d u$ diviseur $a=\sqrt[e^{k}]{(x)^{\prime}}$ dans $C l^{\prime}$; la seconde en associant à l'élément $l^{-k} \otimes x_{\infty}$ de $\Re_{\infty}$ la classe cl $(a)$ du diviseur $a=\sqrt[l]{(x)}$ dans $a$. Dans les deux cas le noyau de l'homomorphisme obtenu est engendré par l'image des unités: des $\ell$-unités dans le premier cas, des unités infinitésimales dans le second. Dans les deux cas enfin, l'image est formée des éléments de $l^{m}$-torsion du groupe d'arrivée.

\section{2.- GROUPES DE S-CLASSES T-INFINITÉSIMALES.}

\section{a.-Eléments T-infinitésimaux .}

Pour chaque place $p$ du corps $K$, non complexe et étrangère à $\ell$, désignons par $k_{p}^{x}$ le complété profini $\frac{\lim }{n} k_{p}^{x} / K_{p}^{x}{ }_{p}$ du groupe multiplicatif du corps local $K_{p}$ : Si p est ultramétrique, le choix d'une uniformisante $\pi_{p}$ de $K_{p}$ permet diidentifier le groupe $K_{p}^{x}$ au produit di rect $\mu_{p} \cdot \pi_{p}^{z_{l}}$ du $\ell$-Sylow $\mu_{p}$ du groupe des racines de liunité du corps

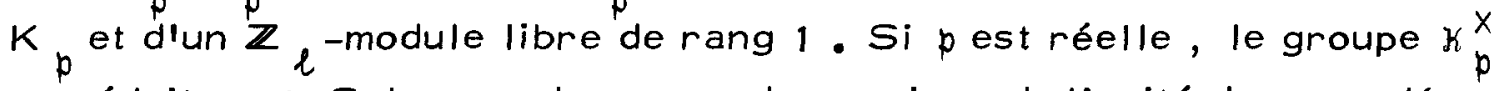
se réduit au $\ell$-Sylow $\mu_{p}$ du groupe des racines de l'unité du corps $K_{p}$ : il est nul si $l$ est différent de 2 , égal à $\{ \pm 1\}$ sinon .

Fixons maintenant un ensemble fini $T$ de places non complexes de $K$ : et considérons le groupe semi-local :

$$
k_{T}^{x}=\underset{p \in T}{\oplus} k_{p}^{x}
$$

L'injection diagonale du groupe $K^{X}$ dans le produit $\underset{p \in T}{\oplus} K_{p}^{X}$ se prolonge de façon unique en un $\mathbb{Z} l^{- \text {morphisme du tensorisé } \ell=\mathbb{Z}} l^{\otimes} \mathbb{Z}^{K X}$ dans

(*) Tout récemment, Nguyen Quang Do a obtenu une version cohomologique de la suite exacte correspondante. Sa démonstration utilise les résultats de dualité globale de Poitou-Tate. 
le complété profini $\kappa_{T}^{X}$. L'application obtenue est l'homomorphisme de semi-localisation $s$ associé à l'ensemble $T$. Il est surjectif en vertu du lemme de Nakagama : En effet, d'une part le groupe $k_{T}^{x}$ est bien un

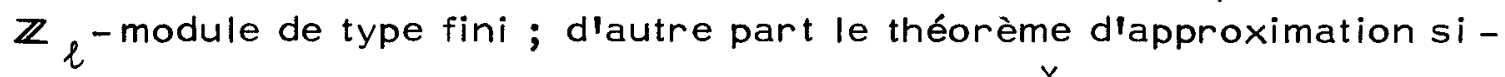
multanée montre que l'application naturelle de $K^{x}$ dans le quotient $k \frac{X}{T} / K_{T}^{\times l} \simeq \underset{p \in T}{\oplus} K_{p}^{X} / K{ }_{p}^{X l}$ est une surjection. Cela étant nous avons :

DEFINITION 11.2.14.- Etant donnés un corps de nombres $K$, et $T$ un ensemble fini de places non complexes de $K$, nous appelons groupe des éléments $T$-infinitésimaux du corps $K$, et nous notons $R_{T}$, le noyau dans le tensorisé $R=\mathbb{Z} l^{\otimes} \mathbb{Z}^{X}$ de l'homomorphisme de semi-locali sation associé à l'ensemble $T$, autrement dit le sous-groupe de $R$ défini par l'exactitude de la suite courte de $\mathbb{Z}_{\ell}$-modules :

$$
1 \longrightarrow \kappa_{T} \longrightarrow \curvearrowleft \stackrel{s_{T}}{\longrightarrow} \kappa_{T}^{x}=\underset{p \in T}{\oplus} k_{p}^{x} \longrightarrow 1 \text {. }
$$

Arrêtons-nous un instant sur les contraintes imposées par les conditions d'infinitésimalité :

- Si $p$ est une place réelle, la condition $s_{p}(x)=1$ signifie simplement que $s_{p}(x)$ est un carré; si $l$ est différent de 2 , elle est donc toujours vérifiée.

- Si $p$ est une place finie étrangère à $l$, la condition $s_{p}(x)=1$ signifie que $x$ est étranger à $p$ (ce qui se lit sur la factorisation du di viseur principal associé), et que $s_{p}(x)$ est une puissance $l^{m}$-ième ( si $\ell^{m}$ est l'ordre du groupe $\mu_{p}$ ). Cette dernière condition est, en fait, une congruence multiplicative : Elle s'écrit $x=y z^{l^{m}}$, pour un $z$ de $R$, et un $y$ de $K^{X}$ vérifiant $y \equiv 1(\bmod p)$.

- Si $p$ est une place au-dessus de $l$, la condition $s_{p}(x)=1$, analogue quant à la forme aux précédentes, est en revanche plus exi -

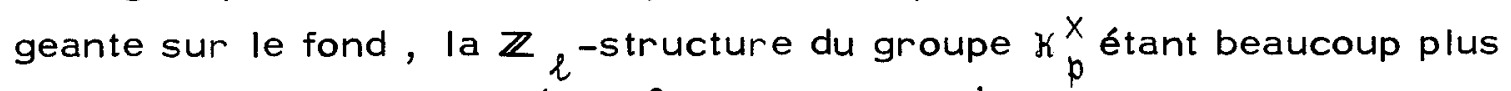
riche. Comme plus haut (cf. $1 \S$ a, remarque 1 ), on peut la regarder comme une infinité de congruences multiplicatives : Par exemple, si T est un ensemble fini de places non complexes étrangères à $l$, et $T^{*}=$ $T$ TPI ( $\ell$ ) la réunion de $T$ et des places au-dessus de $\ell$, le groupe $R_{T}$ des éléments $T^{*}$-infinitésimaux s'obtient comme intersection de groupes de congruences :

$$
R_{T}=\bigcap_{n \in \mathbb{N}}\left(R_{T} \cap R_{n}\right)
$$




\section{b.-S-classes T-infinitésimales}

Regardons maintenant les groupes de diviseurs. Si $S$ est un ensemble fini de places non complexes de $K$, le groupe des $\mathbf{S}$-diviseurs généralisés du corps $K$ est le quotient $\theta^{S}=\theta / \theta(S)$ du groupe des diviseurs généralisés $\theta=\mathbb{Z} l^{\otimes} \mathbb{Z}$ par le sous-groupe $\theta(S) \mathbb{Z} l^{\text {-engendré }}$ par les diviseurs construits sur les places de $S$; ils'identifie par con séquent au sous-groupe des diviseurs de $D$ qui sont étrangers à $S$.

Le groupe des $S$-diviseurs principaux $p^{S}$ est llimage canonique de $R$ dans $\delta^{S}$. Un S-diviseur principal est donc représenté dans $\mathscr{D}$ par le produit $x a$ dlun diviseur principal $(x)$ et d'un diviseur a appartenant à $D(S)$. Le groupe quotient $C l^{S}=\delta^{S} / p^{S}$, qui s'identifie canonique ment au quotient $\mathrm{Cl} / \mathrm{Cl}(\mathrm{S}) \mathrm{du} \ell$-groupe des classes $\mathrm{Cl}$ par son sous groupe $C \ell(S)$ engendré par les classes des diviseurs construits sur les places de $S$, est le $\ell$-groupe des $S$-classes de diviseurs $d u$ corps $K$.

DÉFINITION II.2.15. - Soient $S$ et $T$ deux ensembles finis disjoints de places non complexes du corps $K$. Par groupe des $S$-diviseurs principaux $T$-infinitésimaux de $K$, nous entendons l'image canonique $P_{T}^{S} d u$ sous-groupe $T$-infinitésimal $R_{T}$ de $R$ dans le groupe $\theta^{S}$ des S-divi seurs de $K$. Les $S$-diviseurs principaux $T$-infinitésimaux sont donc les S-diviseurs principaux engendrés par les éléments T-infinitési maux .

PROPOSITION 11,2,16.- Si les places de $S U T$ sont étrangères à $l$, désignons par $T^{*}=T \cup P I(\ell)$ la réunion de $T$ et de l'ensemble des places au-dessus de $\ell$. Pour chaque naturel $n$, désignons par p $S, n$ le sous-groupe de $p_{T}^{S}$ formé des idéaux $S$-principaux engendrés par les éléments du groupe de congruences $R_{T, n}=R_{T} \cap R_{n}$. II vient :

$$
P_{T}^{*}=n_{n \in \mathbb{N}} P_{T, n}^{S} \text {. }
$$

Démonstration: Elle est en tous points analogue à celle de la proposition 8 . Soit $\times a$, avec $\times \in R_{T}$ et $a \in D(S)$, un diviseur de $K$ dont la classe modulo $\mathscr{D}(S)$ tombe dans lintersection $\bigcap_{n \in \mathbb{N}} P^{S}, n$. Pour chaque naturel $n$, nous pouvons alors écrire $x a=x_{n} a_{n}$, pour un $x_{n}$ 
de $R_{T, n}$ et $a_{n}$ dans $D(S)$. Le quotient $\times / x_{n}$ est ainsi une $S$-unité $T$ infinitésimale $\epsilon_{n}$. Maintenant, le groupe $\varepsilon_{T}^{S}=\varepsilon^{S} \cap R_{T}$ des $S$-unités $T$ infinitésimales, qui est un $\mathbb{Z}_{\ell}$-module de type fini , est compact. La suite $\left(\epsilon_{n}\right)_{n} \in \mathbb{N}$ possède donc une valeur d'adhérence $\epsilon_{\infty}$. Posant $x_{\infty}=$ $x / \epsilon_{\infty}$, nous obtenons immédiatement $x \mathfrak{a}=x_{\infty} a_{\infty}$, avec $x_{\infty} \in \mathbb{R} T^{*}$, et $a_{\infty} \in \hat{\theta}(S)$, comme attendu.

DÉFINITION I1.2.17. - Nous disons qu'un S-diviseur généralisé a est $T$-quasi-infinitésimal, lorsqulune de ses puissances a ${ }^{l^{k}}$ est $S$-principale T-infinitésimale . Le groupe des S-diviseurs T-quasi-infinitésimaux est donc le radical $\sqrt{p_{T}^{S}}$ dans $\theta^{S}$ du groupe des S-diviseurs prin cipaux T-infinitésimaux.

PROPOSITION 11.2.18. - Le quotient $C^{S} l_{T}^{S}=D_{T}^{S} / p T_{T}^{S}$ du groupe $D_{T}^{S}$ des S-diviseurs généralisés étrangers à $T$ par le sous-groupe $p \frac{S}{T}$ des $S$ diviseurs principaux $T$-infinitésimaux est un $\mathbb{Z} \ell^{- \text {module noethérien }}$. Nous disons que $\mathrm{Cl}_{T}^{S}$ est le $\ell$-groupe des $S$-classes $T$-infinitésimales du corps $K$. Son sous-groupe de torsion $\sigma_{T}^{S}$, qui est fini, s'identifie au quotient $\sqrt{ } P_{T}^{S} / P_{T}^{S}$ du groupe des $S$-diviseurs $T$-quasi-infinitési maux par le sous-groupe T-infinitésimal.

Démonstration: II s'agit de vérifier que $C_{S}^{S}$ est de type fini . Intro duisons pour cela le sous-groupe $\theta_{T}^{S} \cap p^{S}$ des $S$-diviseurs principaux étrangers à $T$, et considérons la suite exacte courte canonique :

$$
1 \longrightarrow\left(D_{T}^{S} \cap p^{S}\right) / p_{T}^{S} \longrightarrow D_{T}^{S} / p_{T}^{S} \longrightarrow D_{T}^{S} /\left(D_{T}^{S} \cap p^{S}\right) \rightarrow 1 \text {. }
$$

Par l'homomorphisme de semi-localisation $S_{T}$, le terme de gauche $\left(\theta_{T}^{S} \cap P^{S}\right) / p_{T}^{S} s^{\prime}$ identifie au quotient $\left[\left(\underset{p \in T, P I(l)}{\oplus} \mu_{P}\right) \oplus\left(\underset{P \in T \cap P I(l)}{\oplus} U_{P}\right)\right] / s_{T}\left(E^{S}\right)$

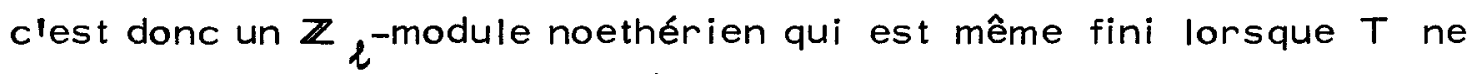
rencontre pas l'ensemble $P l(l)$ des places de $K$ au-dessus de $l$. Le terme médian $\theta_{T}^{S} / P_{T}^{S}$ est le groupe $C_{l}^{S}$ étudié. Enfin, le terme de gauche $\hat{\theta}_{T}^{S} /\left(\theta_{T}^{S} \cap p^{S}\right)$ s'identifie canoniquement au $\ell$-groupe $C^{S}$ des $S$-classes de diviseurs de $K$; il est donc toujours fini.

Les groupes $\mathrm{Cl}_{T}^{S}$ ainsi définis contiennent comme cas particu liers les divers groupes de classes rencontrés plus haut. En particu lier, le $\ell$-groupe des $\ell$-classes de diviseurs $C \ell^{\prime} s^{\prime}$ écrit $C \ell^{\prime}=C \ell^{P I}(\ell)$, 
tandis que le $\ell$-groupe des classes infinitésimales $a$ prend la forme $a=C \ell_{P l}(l)$. Nous allons voir que les suites exactes de dualité qui les relient se généralisent sans peine aux groupes $\mathrm{Cl}_{\mathrm{T}}^{\mathrm{S}}$.

c.- Interprétations arithmétiques; applications à la dualité.

Dans la section $11.1 . \S \mathrm{c}$, nous avons associé à chaque $\ell-e x-$ tension $L$ de $K$ (kummérienne ou non) son radical de Kummer $\operatorname{Rad}(L / K)=\left\{l^{-k} \otimes x \in\left(e^{-m} \mathbb{Z} / \mathbb{Z}\right) \otimes \mathbb{Z}^{x} \mid K\left[\sqrt[k^{k}]{x}\right] \subset L\right\}$, qui est caractérisé par l'isomorphisme de dualité :

$$
\operatorname{Rad}(L / K) \simeq \operatorname{Hom}(\mathrm{Gal}(L / K), \mu) \text {. }
$$

DÉFINITION 11.2.19. - Etant donnés deux ensembles finis disjoints $S$ et $T$ de places non complexes du corps $K$, nous appelons $T$-radical $S$-infinitésimal de $K$, et nous notons $\Re_{S}^{\top}$ le sous-groupe du tensorisé $\left(\ell^{-m} \mathbb{Z}_{\ell} / \mathbb{Z}_{\ell}\right) \otimes_{\mathbb{Z}_{l}}{ }^{R} s$ du groupe des éléments S-infinitésimaux défi ni par l'identité :

$\Re_{S}^{T}=\left\{l^{-k} \otimes x \in\left(l^{-m} \mathbb{Z}_{l} / \mathbb{Z}_{l}\right) \otimes_{\mathbb{Z}_{l}} R_{S} \mid v_{p}(x) \equiv 0\left(\bmod l^{k}\right), \forall p \in T\right\}$. Le groupe $\Re_{S}^{\top} n^{\prime}$ 'est pas un radical au sens de Kummer : Plus précisément :

PROPOSITION 11.2.20. - Désignons par $\operatorname{Rad}_{S}^{\top}$ l'image canonique du groupe $\Re_{S}^{T}$ dans le radical de Kummer $\left(e^{-m} \mathbb{Z} / \mathbb{Z}\right) \otimes \mathbb{Z} \mathrm{K}^{\mathrm{X}}$. Nous ob tenons la suite exacte courte de $\mathbb{Z} \ell^{\text {-modules : }}$

$$
1 \longrightarrow \ell^{m} \mu_{S} / \mu \longrightarrow R_{S}^{\top} \longrightarrow \operatorname{Rad}_{S}^{\top} \longrightarrow 1
$$

où $\mu_{S}=\underset{p \in S}{\oplus} \mu_{p}$ est le $\ell$-Sylow du groupe des racines de llunité du complété semi-local $K_{S}=\underset{p \in S}{\oplus} K_{p}$.

Démonstration: Un élément $\ell^{-m} \otimes x$ de $\Re_{S}^{\top}$ a une image triviale dans $\operatorname{Rad}_{S}^{T}$ si $x$ est la puissance $\ell^{m}$-ième dlun élément y de $R$. En associant à un tel élément l'image $s_{S}(y) . \mu$ de y dans $\mu_{T} / \mu$, nous définissons un morphisme du noyau cherché dans le sous-groupe $\ell^{m} \mu_{S} / \mu$ de $\mu_{S} / \mu$. 
Reste à vérifier que ce morphisme est bijectif. Or, d'un côté liidentité $s_{S}(y) \in \mu$ implique que y soit le produit d'une racine globale de l'unité et d'un élément S-infinitésimal $y_{\infty}$; dans ce cas $x=y^{l^{m}}=y_{\infty}^{l^{m}}$ est la puissance $l^{m}$-ième d'un S-infinitésimal, et $l^{-m} \otimes x$ vaut 1 ; ce qui prouve linjectivité. D'un autre côté, si $\zeta$ est une racine $\ell^{m}-i e ̀ m e d e$ l'unité dans $k_{S}^{x}$, et y un élément de $R$ au-dessus de $\zeta$, l'élément $x=y^{l^{m}}$ est $S$-infinitésimal, et le produit $\ell^{-m} \otimes x$ est dans $\Re_{S}^{\top}$; ce qui achève la démonstration.

THÉORÈME 11.2.21.- Soient $S$ et $T$ deux ensembles finis disjoints de places du corps $K$, non complexes et étrangères à $l$, puis $S^{*}=S \cup P I(l)$ et $T^{*}=T \cup P I(\ell)$ leurs réunions respectives avec l'ensemble $P I(\ell)$ des places de $K$ au-dessus de $\ell$.

(i) Désignons par $\mathrm{C}_{T}^{\mathrm{S}}$ * la $\ell$-extension abélienne maximale de $K$ qui est $T^{*}$-ramifiée et $S$-décomposée.

1) Le groupe de Galois Gal $\left(C_{T}^{*} / K\right)$ s'identifie au $l$-groupe des $S$ classes $T^{*}$-infinitésimales $\mathrm{Cl}_{\mathrm{T}^{*}}^{\mathrm{S}}$.

2) Le radical de Kummer $\operatorname{Rad}\left(C_{T^{*}} T^{*} / K\right)$ est l'image Rad $T_{S}^{*} d u T^{*}$-radical S-infinitésimal $\Re_{S}^{T^{*}}$.

(ii) Désignons par $C_{T}^{S^{*}}$ la $\ell$-extension abélienne maximale de $K$ qui est $T$-ramifiée et $S^{*}$-décomposée.

1) Le groupe de Galois Gal $\left(C_{S^{*}}^{*} / K\right)$ s'identifie au $\ell$-groupe des $S^{*}$ classes $\mathrm{T}$-infinitésimales $\mathrm{Cl}_{\mathrm{T}}^{\mathrm{S}^{*}}$.

2) Le radical de Kummer $\operatorname{Rad}\left(C_{T}^{S^{*}} / K\right)$ est l'image $\operatorname{Rad}_{T}^{T} S^{*}$ du T-radical $S^{*}$-infinitésimal $R^{\top} S^{*}$.

Démonstration :

(i) Le corps $C_{T^{*}}^{S^{*}}$ est, par définition, la $\ell$-extension abé lienne de $K$, qui est non ramifiée aux places finies étrangères à $T^{*}$, et complètement décomposée aux places de $S$. Clest donc la réunion, lorsque $n$ décrit $\mathbb{N}$, des $\ell$-extensions abéliennes $S$-décomposées ma ximales de conducteurs respectifs $\tilde{i}_{n}=\prod_{p \in T} p \cdot \prod_{l \in P I(\ell)^{1}} \mathfrak{l}^{n}$. Son groupe de Galois Gal $\left(C_{T^{*}}^{S^{*}} / K\right)$ s'identifie par conséquent à la limite projective des groupes de classes de rayons : $\operatorname{Gal}\left(C_{T^{*}}^{S} / K\right)=\frac{\lim }{n} \theta_{T^{*}} / P_{T, n} \theta_{T^{*}}(S)=\frac{\lim }{n} \theta_{T^{*}}^{S} / P_{T, n}^{S}=\theta_{T^{*}}^{S} / P_{T^{*}}^{S}=C l_{T^{*}}^{S}$ 
Le radical kummérien $\operatorname{Rad}\left(\mathrm{C}_{T^{*}}^{S} / K\right)$ est l'ensemble des éléments $l^{-k} \otimes x$ du groupe $\left(l^{-m} \mathbb{Z}_{l} / \mathbb{Z}_{l}\right) \otimes_{\mathbb{Z}}{ }_{l}$, pour lesquels l'ex tension cyclique $K[\sqrt[\ell]{x}] / K$ est $T^{*}$-ramifiée et $S$-décomposée. Cela étant, comme $T^{*}$ contient les places au-dessus de $l$, la condition de $T^{*}$-ramification se lit sur les valuations $: v_{p}(x) \equiv 0\left(\bmod \ell^{k}\right), \forall p \in T^{*}$. La condition de $S$-décomposition exprime que $s_{s}(x)$ est une puissance $\ell^{k}$-ième dans $k \frac{x}{s}$; autrement dit, que $x$ est le produit d'une puis sance $l^{k}$-ième de $R$ et d'un $S$-infinitésimal $x_{\infty} \in R_{S}$. D'où le résultat annoncé .

(ii) Le corps $\mathrm{C}_{\mathrm{T}}^{S^{*}}$ est, par définition, la $\ell$-extension abélienne maximale de $K$, qui est non ramifiée aux places finies étran gères à $l$, et complètement décomposée aux places de $S^{*}$. En particulier, elle est de degré fini sur $K$. La théorie du corps de classes $i-$ dentifie donc directement son groupe de Galois Gal $\left(C_{T}^{S^{*}} / K\right)$ au $\ell-$ groupe de classes de rayons : $D_{T} / P_{T} D_{T}\left(S^{*}\right)=\mathscr{D}_{T}^{*} / P_{T}^{*}=C \ell_{T}^{*}$. Enfin, le radical kummérien $\operatorname{Rad}\left(C_{T}^{*} / K\right)$ se calcule comme plus haut. Cependant, la condition sur les valuations $v_{p}(x) \equiv 0$ $\left(\bmod \ell^{k}\right), \forall p \in T$ ne caractérise pas les extensions $K\left[\sqrt[\ell^{k}]{x}\right] / K$ qui sont T-ramifiées : elle n'exclut pas la ramification au-dessus de $l$. Ce fait est sans importance dans ce cas, puisque la condition de $S^{*}$-décompo sition impose aux places au-dessus de $l$ de se décomposer complète ment .

Remarque : Même en l'absence de toute hypothèse sur les places au dessus de $\ell$, le groupe de Galois $\operatorname{Gal}\left(\mathrm{C}_{T}^{S} / K\right)$ de la $\ell$-extension abélienne T-ramifiée $S$-décomposée maximale s'identifie toujours au $\ell$ groupe $\mathrm{Cl}_{T}^{S}$ des $S$-classes T-infinitésimales. En revanche, s'il existe des places au-dessus de $l$ qui ne sont représentées ni dans $S$ ni dans $T$, le groupe $R_{S}^{T}$ peut contenir strictement le radical $\operatorname{Rad}\left(C_{T}^{S} / K\right)$.

THËOREME 11,2.22. - Etant donnés deux ensembles finis disjoints $S$ et $T$ de places non complexes du corps $K$, il existe une suite exacte canonique de $\mathbb{Z} \ell^{\text {-modules : }}$

$$
1 \longrightarrow \mathbb{F}_{T}^{S} \longrightarrow R_{T}^{S} \longrightarrow \ell^{m} \mathrm{Cl}_{T}^{S} \longrightarrow 1 .
$$


Dans celle-ci , $\mathbb{E}_{T}^{S}=\left(\ell^{-m} \mathbb{Z}_{l} / \mathbb{Z}_{l}\right) \otimes_{\mathbb{Z}} \varepsilon_{l}^{\delta} \frac{S}{T}$ est le tensorisé du groupe des S-unités $T$-infinitésimales du corps $K ; \Re \frac{S}{T}$ est le $S$-radical T-infinitésimal; $\ell^{m} C l_{T}^{S}$ est le sous-groupe de $\ell^{m}$-torsion du $\ell-$ groupe des $S$-classes $T$-infinitésimales de $K$; et $\ell^{m}$ est l'ordre du $\ell-$ Sylow $\mu$ du groupe des racines de llunité de $K$.

Démonstration : La suite exacte s'obtient tout simplement en associant à l'élément $l^{-m} \otimes x$ de $R_{T}^{S}$ la classe $c l \underset{T}{S}(a)$ dans le groupe $C_{m} \ell_{T}^{S} d u d i-$ viseur a défini par la décomposition $(x)=a^{\ell^{m}}$. b du diviseur principal $(x)$ comme produit d'une puissance $l^{m}$-ième et d'un diviseur $b \in \theta_{T}(S)$. Comme $x$ est est défini à la puissance $\ell^{m}$-ième près d'un T-infinitési mal, nous obtenons ainsi un épimorphisme du groupe $\Re_{T}^{S}$ sur le sousgroupe de $l^{m}$-torsion de $\mathrm{Cl}_{T}^{\mathrm{S}}$, dont le noyau est formé des classes de $\Re_{T}^{S}$ qui sont représentées par des $S$-unités $T$-infinitésimales.

Compte-tenu des interprétations arithmétiques données par le théorème 21 , le théorème 22 établit une double dualité entre la $\ell$-extension abélienne maximale $C_{T}^{S}$ qui est $T^{*}$-ramifiée et $S$-décomposée, et celle $C_{S}^{T}$ qui est $S$-ramifiée et $T^{*}$-décomposée.

\section{3. - LA SUITE EXACTE DES CLASSES AMBIGES DANS UNE EXTENSION GALOISIENNE.}

\section{a.- L'homomorphisme d'extension pour les groupes $a$.}

Etant donnée une extension quelconque de corps de nombres $L / K$, I'homomorphisme $d_{L / K}$ d'extension des diviseurs induit, par passage au quotient, une application naturelle du $\ell$-groupe des classes infinitésimales $a(K)$ du corps $K$ dans celui $a(L)$ de $L$ : Clest l'homo morphisme diextension $j=j_{L / K}$, qui correspond au transfert dans linterprétation, par la théorie du corps de classes, des groupes $a$. Lorsque $L$ est une extension galoisienne de $K$, de groupe de Galois $G$, il est naturel de comparer le groupe étendu $j_{L / K}(a(K))$ au sous groupe ambige $a^{G}(L)$ de $a(L)$. Nous avons besoin pour cela d'un lemme de cohomologie : 
LEMME 11.2.23. - Soient L/K une extension galoisienne de corps de nombres, et $G$ son groupe de Galois.

(i) Le groupe de cohomologie $H^{1}\left(G, D_{0}(L)\right)$ est nul.

(ii) Le groupe de cohomologie $H^{1}\left(G, R_{\infty}(L)\right)$ est nul .

(iii) Le quotient $P_{\infty}(L)^{G} / d_{L / K}(P(K))$ s'identifie au groupe de cohomologie $H^{1}\left(G, \varepsilon_{\infty}(L)\right)$.

(iv) Le groupe $H^{1}\left(G, P_{\infty}(L)\right)$ est isomorphe au noyau $\operatorname{Ker}\left[H^{2}\left(G, \delta_{\infty}(L)\right) \longrightarrow H^{2}(G, R(L))\right]$.

Démonstration : Elle est en tous points analogue à celle du lemme III.1.4.

(i) n'est rien d'autre que le théorème 90 de Hilbert pour les diviseurs généralisés.

(ii) s'obtient en écrivant la suite exacte de cohomologie as sociée à la suite courte

$$
1 \longrightarrow R_{\infty}(L) \longrightarrow R(L) \longrightarrow K_{l}^{X}(L) \longrightarrow 1
$$

qui définit le sous-groupe infinitésimal (cf. définition 1 ). Dans la suite obtenue

$1 \longrightarrow R_{\infty}(K) \longrightarrow R(K) \longrightarrow K_{l}^{X}(K) \longrightarrow H^{1}(G, R(L)) \longrightarrow H^{1}(G, R(L))$ le terme de droite $H^{1}(G, R(L))$ est nul en vertu du théorème 90 de Hilbert, et llapplication $R(K) \longrightarrow K_{\ell}^{X}(K)$ est surjective ; ce qui donne le résultat attendu: $H^{1}\left(G, R_{\infty}(L)\right)=1$.

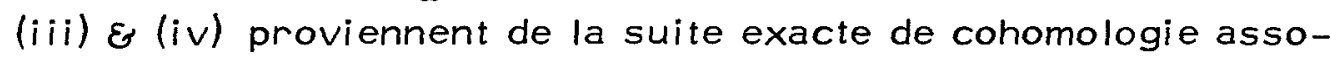
ciée à la suite courte qui définit $R_{\infty}$ : $1 \longrightarrow \varepsilon_{\infty}(L) \longrightarrow R_{\infty}(L) \longrightarrow P_{\infty}(L) \longrightarrow 1$ (cf.pproposition 8 ) . 11 vient, en effet : $\begin{aligned} 1 \rightarrow \delta_{\infty}(K) \rightarrow R_{\infty}(K) \rightarrow P_{\infty}^{G}(L) \rightarrow H^{1}\left(G, \delta_{\infty}(L)\right) \rightarrow H^{l}\left(G, R_{\infty}(L)\right) \rightarrow H^{\top}\left(G, P_{\infty}(L)\right) & \rightarrow H^{2}\left(G, \varepsilon_{\infty}(L)\right) \\ & \rightarrow H^{2}(G, \Omega(L))\end{aligned}$ et le groupe $H^{1}\left(G, \Omega_{\infty}(L)\right)$ est nul, en vertu de l'assertion (ii).

THÉORËME 11.2.24. - Dans une extension galoisienne finie $L / K$ de corps de nombres, de groupe de Galois $G$, le sous-groupe ambige $a(L)^{G}$ du $\ell$-groupe des classes infinitésimales du corps $L$ est donné par la suite exacte de modules finis (où $j_{L / K}$ est l'homomorphisme de $Q(K)$ dans $a(L)$ induit par l'extension $d_{L / K}$ des diviseurs ) :

$$
\begin{aligned}
1 \rightarrow \delta_{\infty}(L)^{G} / \delta_{\infty}(K) \rightarrow \operatorname{Ker} d_{L / K} \rightarrow \operatorname{Ker} j_{L / K} & \rightarrow H^{\prime}\left(G, \delta_{\infty}(L)\right) \rightarrow \theta_{0}(L)^{G} / d_{L} / K\left(D_{0}(K)\right) \\
& \rightarrow a(L)^{G} / j_{L / K}(a(K)) \rightarrow H^{1}\left(G, P_{\infty}(L)\right) \rightarrow 1 .
\end{aligned}
$$


Démonstration : Partons de la suite exacte courte qui définit le groupe $a(L)$ :

$$
1 \longrightarrow P_{\infty}(L) \longrightarrow \theta_{0}(L) \longrightarrow a(L) \longrightarrow 1
$$

La suite exacte de cohomologie associée :

$1 \longrightarrow P_{\infty}(L)^{G} \longrightarrow D_{0}(L)^{G} \longrightarrow a(L)^{G} \longrightarrow H^{1}\left(G, P_{\infty}(L)\right) \longrightarrow H^{1}\left(G, D_{0}(L)\right)$ s'arrête en vertu du lemme 23 (i). L'homomorphisme d'extension donne lieu par conséquent au diagramme commutatif :

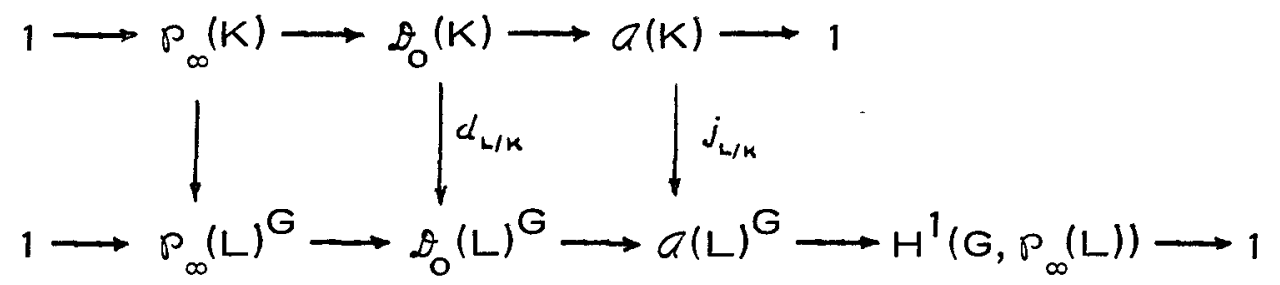

qui conduit, via le lemme du serpent, à la suite exacte annoncée. Seul reste donc à vérifier que tous les termes de la suite exacte obtenue sont finis. Or, les termes $\mathrm{Ker} d_{L / K}$ et $\theta_{0}(L)^{G} / \phi_{0}(K)$ sont bien connus : Le premier mesure la complexification żl'infini ; le second la ra mification aux places(finies) étrangères à $\ell$ (cf. scolie III.1.6). Enfin, les deux groupes de cohomologie $H^{1}\left(G, \&_{\infty}(L)\right)$ et $H^{1}\left(G, P_{\infty}(L)\right) \simeq$ $\operatorname{Ker}\left[H^{2}\left(G, \varepsilon_{\infty}(L)\right) \longrightarrow H^{2}(G, R(L))\right]$, qui sont annulés par l'exposant de $G$, sont llun et l'autre finis puisque le groupe de défaut $\delta_{\infty}(L)$ est un $\mathbb{Z} \ell^{\text {-module de type fini } . ~}$

COROLLAIRE 11,2,25, - Formule des classes ambiges infinitésimales Etant donnée une extension galoisienne finie de corps de nombres $L / K$, l'homomorphisme d'extension des diviseurs induit un pseudo-isomor phisme $j_{L / K}$ du $\ell$-groupe des classes infinitésimales $a(K)$ dans le sous-groupe ambige $Q(L)^{G}$ de $a(L)$. Noyau et Conoyau de ce pseudoisomorphisme sont liés par liidentité :

$\frac{\mid \text { Coker } j_{L / K} \mid}{\left|\operatorname{Ker} j_{L / K}\right|}=\frac{p \not l}{\prod_{p \mid \infty} e_{p}(L / K)} \frac{\left|H^{1}\left(G, p_{\infty}(L)\right)\right|}{\left|H^{1}\left(G, \varepsilon_{\infty}(L)\right)\right|}\left(\delta_{\infty}(L)^{G}: \delta_{\infty}(K)\right)$.

En particulier, lorsque l'extension $L / K$ est absolument $l$-ramifiée (i.e. non ramifiée aux places finies ètrangères à $l$, et non complexifiée aux places réelles ), il vient exactement :

$$
\left\{\begin{array}{c}
\operatorname{Ker} j_{L / K} \simeq H^{1}\left(G, \delta_{\infty}(L)\right) \quad \& \\
\text { Coker } \dot{j}_{L / K} \simeq H^{1}\left(G, P_{\infty}(L)\right) \simeq \operatorname{Ker}\left[H^{2}\left(G, \delta_{\infty}(L)\right) \longrightarrow H^{2}(G, R(L))\right] .
\end{array}\right.
$$


COROLLAIRE 11.2.26. - Supposons réunies les deux conditions sui vantes :

(i) L'extension $L / K$ est absolument $\ell$-ramifiée.

(ii) Le corps $L$ vérifie la conjecture de Leopoldt .

Alors I Thomomorphisme d'extension $j_{L / K}$ est un isomorphisme de $a(K)$ sur $a(L)^{G}$.

COROLLAIRE 11.2.27.- Plus généralement, supposons réunies les deux conditions plus faibles suivantes:

(i) L'extension $L / K$ est non complexifiée.

(ii) Le défaut de la conjecture de Leopoldt est constant dans $L / K\left(\right.$ i.e. $\left.r g_{\mathbb{Z}} \delta_{\infty}(L)=r g_{\mathbb{Z}} \varepsilon_{l}(K)\right)$.

Alors I'homomorphisme d'extension $j_{L / K}$ envoie injectivement $a(K)$ dans $a(L)^{G(\star)}$.

Démonstration: II slagit de vérifier que, sous la condition (ii), le groupe de cohomologie $H^{1}\left(G, \delta_{\infty}(L)\right)$ est nul or l'hypothèse faite entraîne que $\delta_{\infty}(K)$ est d'indice fini dans $\delta_{\infty}(L)$. Et comme le groupe

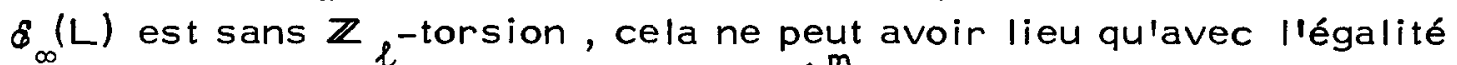
$\delta_{\infty}(K)=\delta_{\infty}(L)$. En effet, liidentité $\varepsilon^{\ell^{m}} \in \delta_{\infty}(K)$, pour un $\epsilon$ de $\delta_{\infty}(K)$ implique $e^{\ell^{m}(\sigma-1)}=1, \forall \sigma \in G$, donc $\epsilon^{\sigma-1}=1, \forall \sigma \in G$, clest-àdire finalement $\epsilon \in \delta_{\infty}(K)$, comme attendu .

\section{b.- Application au sous-groupe de torsion $\ell$.}

La capitulation Ker $j_{L / K}$ étant finie d'après le théorème 2 , elle ne concerne que les éléments de torsion du groupe $a(K)$. Comme l'homomorphisme d'extension envoie naturellement le sous-groupe de torsion $\zeta(K)$ de $a(K)$ dans le sous-groupe ambige $G(L)^{G}$ de celui de $a(L)$, nous pouvons reprendre, pour le groupe de torsion $\zeta$ la construction de la section précédente, à partir cette fois de la suite exacte courte donnée par la proposition 10 :

$\left({ }^{\star}\right)$ Ce résultat est dû à Gras $\left(\left[G r_{s}\right]\right.$, prop.ll.3) . La condition (ii) est toujours vérifiée en particulier dans une tour cyclotomique, au moins à partir dlun certain rang (cf. $\left[G_{2}\right]$ ) 


$$
1 \longrightarrow P_{\infty}(L) \longrightarrow \theta_{\infty}(L) \longrightarrow \sigma(L) \longrightarrow 1 \text {. }
$$

Une difficulté, toutefois, se présente: Le groupe de cohomologie $H^{1}\left(G, \phi_{\infty}(L)\right)$ pouvant ne pas être trivial, la suite exacte de cohomologie associée :

$1 \longrightarrow P_{\infty}(L)^{G} \longrightarrow D_{\infty}(L)^{G} \longrightarrow G(L)^{G} \longrightarrow H^{1}\left(G, P_{\infty}(L)\right) \longrightarrow H^{1}\left(G, D_{\infty}(L)\right)$ ne slarrête pas, non plus par conséquent que celle déduite, via le lemme du serpent, du diagramme commutatif donné par l'homomorphisme d'extension :

$1 \rightarrow \delta_{\infty}(L)^{G} / \delta_{\infty}(K) \rightarrow \operatorname{Ker} d_{L / K} \rightarrow \operatorname{Ker} j_{L / K} \rightarrow H^{1}\left(G, \delta_{\infty}(L)\right) \rightarrow D_{\infty}(L)^{G} / d_{L} / K\left(D_{\infty}(K)\right) \rightarrow$ $\rightarrow \tau(L)^{G} / \dot{j}_{L / K}(\zeta(K)) \rightarrow H^{1}\left(G, p_{\infty}(L)\right) \rightarrow \ldots$

D'après le lemme 23 (iv), cependant, le groupe de cohomo logie $H^{1}\left(G, P_{\infty}(L)\right)$ s'injecte dans celui $H^{2}\left(G, \varepsilon_{\infty}(L)\right)$, qui est, a priori, mieux connu : Par exemple, lorsque $G$ est cyclique, $H^{2}\left(G, \varepsilon_{\infty}(L)\right)$ est nul dès que $\varepsilon_{\infty}(K)$ l'est, clest-à-dire lorsque le corps de base $K$ vérifie la conjecture de Leopoldt .

II vient donc :

THÉORËME 11.2.28. - Soit L/K une extension galoisienne de corps de nombres vérifiant la condition $H^{2}\left(G, \varepsilon_{\infty}(L)\right)=1$. Si $j_{L / K}$ désigne I'ho momorphisme du $\ell$-groupe des classes infinitésimales $a(K)$ du corps $K$ dans celui $a(L)$ de $L$, induit par l'extension des diviseurs $\alpha_{L / K}$, le sous-groupe ambige $\zeta(L)^{G}$ du $l$-groupe de torsion de $a(L)$ est donné par la suite exacte :

$$
\begin{aligned}
1 \rightarrow \delta_{\infty}(L)^{G} / \delta_{\infty}(K) \rightarrow \operatorname{Ker} d_{L / K} \rightarrow \operatorname{Ker} j_{L / K} & \rightarrow H^{1}\left(G, \delta_{\infty}(L)\right) \rightarrow D_{\infty}(L)^{G} / d_{L} / K\left(D_{\infty}(K)\right) \rightarrow \\
& \rightarrow G(L)^{G} / j_{L} / K(G(K)) \rightarrow 1 .
\end{aligned}
$$

COROLLAIRE 11.2.29. - Sous la conjecture de Leopoldt, l'ordre du sous-groupe ambige $\boldsymbol{G}(L)^{G}$ est donné par la formule :

$$
\left|\sigma(L)^{G}\right|=|\sigma(K)|\left(\theta_{\infty}(L)^{G}: d_{L / K}\left(\theta_{\infty}(K)\right)\right)=|\sigma(K)| \frac{\left(D_{0}(L)^{G}: d_{L} / K\left(\theta_{0}(K)\right)\right)}{\left(\theta_{0}(L)^{G}: d_{L / K}\left(\delta_{0}(K)\right) \delta_{\infty}(L)^{G}\right)}
$$

Démonstration : L'égalité de gauche provient directement du théorème 28 , sous la condition $\mathscr{E}_{\infty}(\mathrm{L})=1$. Cela étant, puisque la capitulation $\mathrm{Ker} j_{\mathrm{L}} / \mathrm{K}$ est finie, l'intersection $\theta_{\infty}(L) \cap a_{L / K}\left(\theta_{0}(K)\right)$ se réduit à $d_{L / K}\left(\theta_{0}(K)\right)$, 
et le quotient $\theta_{\infty}(L)^{G} / \alpha_{L / K}\left(\mathcal{L}_{\infty}(K)\right)$ s'écrit encore $\begin{aligned}\left(\theta_{\infty}(L) G\right. & d_{L / K}\left(\theta_{0}(K)\right) / d_{L} / K\left(\theta_{0}(K)\right) ; \text { d'où le résultat annoncé . } \\ & \text { Le numérateur }\left(\theta_{0}(L)^{G}: d_{L} / K\left(\delta_{0}(K)\right)\right) \text { étant bien connu (d'a- }\end{aligned}$ près le scolie III.1.6, il est égal au produit $\prod_{p \nmid \ell_{\infty}} e_{p}(L / K)$ des indices de ramification des places finies étrangères à $\ell)$, disons un mot sur le dénominateur $\left(\theta_{0}(L)^{G}: d_{L / K}\left(\theta_{0}(K)\right) \theta_{\infty}(L)^{G}\right)$. D'après Gras $\left[\mathrm{Gr}_{5}\right]$, il vient, en effet:

PROPOSITION 11,2,30.- Etant donné un corps de nombres $K$, désignons par log l'unique prolongement fonctionnel du logarithme $l$-adique au groupe $\mathcal{D}$ des diviseurs généralisés, à valeurs dans le quotient $K_{\ell} / \mathbb{Q} \ell^{\cdot \log _{\ell} E}$, défini sur le sous-groupe principal p par la formule: $\log (x)=\log _{\ell} x \cdot\left(\mathbb{Q}_{\ell} \cdot \log _{\ell} E\right)$.

Le groupe $D_{\infty}$ des diviseurs quasi-infinitésimaux est le noyau dans $D_{0}$ du logarithme généralisé.

Démonstration: Un diviseur a étant donné, faisons choix d'une puis sance $a^{l^{k}}=(x)$. D'après l'équation fonctionnelle du logarithme, nous avons, par définition :

$\log a=l^{-k} \log a^{l^{k}}=l^{-k} \log (x)=l^{-k} \log _{\ell} \times \cdot\left(Q_{\ell} \log _{\ell} E\right) \in K_{l} / \mathbb{Q}_{\ell} \cdot \log _{\ell} E$. Supposons maintenant a étranger à $\ell$. L'égalité $\log a=0$ a lieu si et seulement si $\log _{\ell} \times$ tombe dans le $Q_{l}$-espace vectoriel engendré par $\log _{\ell} E$, clest-à-dire si et seulement si un multiple non nul de $\log _{\ell} x$ tombe dans le sous-module $\mathbb{Z}_{\ell} \cdot \log _{\ell} E=\log _{\ell} \&$ Quitte à grossir $k$, nous pouvons donc écrire cette condition $\log _{\ell} x=\log _{\ell} \varepsilon$, pour un $\epsilon$ de $\delta$, ce qui revient à dire, d'après la proposition 2 , que $x$ est le produit d'une unité et d'un quasi-infinitésimal . Les diviseurs étrangers à $\ell$, de logarithme log $a=0$ sont donc exactement ceux dont une puissance finie $a^{\ell^{k}}=(x)$ est principale-infinitésimale, ce qui est précisément la définition des diviseurs quasi-infinitésimaux .

COROLLAIRE 11,2,31. - Sous les hypothèses du corollaire 29 , l'ordre du sous-groupe ambige $\sigma(L)^{G}$ est donné par la formule :

$$
\left|\sigma(L)^{G}\right|=|\sigma(K)| \frac{\left(\theta_{0}(L)^{G}: d_{L} / K\left(\theta_{0}(K)\right)\right)}{\left(\log \left[\theta_{0}(L)^{G}\right]: \log \left[\theta_{0}(K)\right]\right)} \text {. }
$$


Remarque 1.- L'ordre du sous-groupe ambige $G(L)^{G}$ dépend donc non seulement de la ramification proprement dite (que mesure le groupe $\left.\theta_{0}(L)^{G} / a_{L / K}\left(\theta_{0}(K)\right)\right)$, mais encore des propriétés $\ell$-adiques des di viseurs ramifiés. Cependant, la contribution du dénominateur $\log \left[\theta_{0}(L)^{G}\right] / \log \left[\theta_{0}(K)\right]$ reste bornée indépendamment de la ramification : En effet d'une part son $\ell$-rang est majoré par la dimension $\left[L_{\ell}: \mathbb{Q}_{\ell}\right]=[L: \mathbb{Q}]$ du $\mathbb{Q}_{\ell}$-espace vectoriel $L_{l}=\mathbb{Z} \mathbb{Q}_{\ell} \mathbb{Z} L$, d'autre part son exposant divise celui du groupe $G$. En particulier, llordre du groupe $\sigma(L)^{G}$ est donc arbitrairement grand avec le nombre de places ramifiées dans $L / K$.

Remarque 2. - L'extension aux diviseurs de l'application logarithme permet de donner une interprétation simple du groupe $H^{1}\left(G, \theta_{\infty}(L)\right)$. D'après le lemme 23 (i) en effet, la suite exacte de cohomologie associée à la suite courte $1 \rightarrow D_{\infty}(L) \rightarrow D_{0}(L) \rightarrow \log \left[D_{0}(L)\right] \rightarrow 1$ conduit à |isomorphisme: $H^{1}\left(G, \theta_{\infty}(L)\right) \simeq\left[\log \left[D_{0}(L)\right]^{G} / \log \left[\theta_{0}(L)^{G}\right]\right]$.

\section{c.-Extension aux $\ell$-groupes de S-classes T-infinitésimales.}

$L / K$ désignant toujours une extension galoisienne de corps de nombres, supposons donnés deux ensembles finis disjoints $S_{K}=S$ et $T_{K}=T$ de places non complexes de $K$. Notons $S_{L}$ (respectivement $T_{L}$ ) l'ensemble des places non complexes de $L$ au-dessus de $S_{K}$ (respectivement $T_{K}$ ) et convenons d'écrire $C l S_{T}^{S}(L)$ pour $C l_{T_{L}}^{S_{L}}(L)$. Les calculs de classes ambiges exposés plus haut se généralisent sans difficulté aux groupes $\mathrm{Cl}_{\mathrm{T}}^{\mathrm{S}}$ de $\mathrm{S}$-classes T-infinitésimales.

Enonçons sans démonstration les principaux résultats :

LEMME 11.2.32. - Soient L/K une extension galoisienne de corps de nombres, $G$ son groupe de Galois, $S$ et $T$ deux ensembles finis dis joints de places non complexes de $K$, aucune place réelle de $T$ ne se ramifiant dans $L / K$.

(i) Le groupe de cohomologie $H^{1}\left(G, D_{T}^{S}(L)\right)$ est nul.

(ii) Le groupe de cohomologie $H^{1}\left(G, R_{T}(L)\right)$ est nul •

(iii) Le quotient $P_{T}^{S}(L)^{G} / d_{L / K}^{S}\left[P_{T}^{S}(K)\right]$ s'identifie au groupe de cohomologie $H^{1}\left(G, \varepsilon_{T}^{S}(L)\right)$.

(iv) Le groupe $H^{1}\left(G, P_{T}^{S}(L)\right)$ est isomorphe au noyau $\operatorname{Ker}\left[H^{2}\left(G, \delta_{T}^{S}(L)\right) \longrightarrow H^{2}\left(G, R_{T}(L)\right)\right]$. 
L'application $d_{L / K}^{S}$, obtenue par passage au quotient à partir de $d_{L / K}$, est I'homomorphisme d'extension des S-diviseurs. La restriction sur T assure lidentité $R_{T}(L)^{G}=\Omega_{T}(K)$, dans la démonstration de (ii) .

THÉORËME 11,2.33. - Suite exacte des classes ambiges - Dans une extension galoisienne finie $L / K$ de corps de nombres, de groupe de Galois $G$, le sous-groupe ambige $C_{l} \ell_{T}(L)^{G}$ du $\ell$-groupe des $S$-classes T-infinitésimales du corps $L$ est donné par la suite exacte de modules finis ( où $j S_{T, L / K}$ est l'homomorphisme de $C \ell_{T}^{S}(K)$ dans $C \ell_{T}^{S}(L)$ induit par l'extension $a_{T, L / K}^{S}$ des $S$-diviseurs étrangers à $T$ ) :

$$
\begin{aligned}
1 \rightarrow \delta_{T}^{S}(L)^{G} / \delta_{T}^{S}(K) \rightarrow \operatorname{Ker} d_{T}^{S} \rightarrow \operatorname{Ker} j_{T}^{S} & \rightarrow H^{1}\left(G, \delta_{T}^{S}(L)\right) \rightarrow D_{T}^{S}(L)^{G} / d \frac{S}{T}\left[D_{T}^{S}(K)\right] \\
& \rightarrow C l_{T}^{S}(L)^{G} / j_{T}^{S}\left[C l_{T}^{S}(K)\right] \rightarrow H^{1}\left(G, P_{T}^{S}(L)\right) \rightarrow 1 .
\end{aligned}
$$

COROLLAIRE 11.2.34.- Formule des classes ambiges - Etant donnés $L / K$ une extension galoisienne de corps de nombres, $G$ son groupe de Galois, $S$ et $T$ deux ensembles finis disjoints de places non complexes de $K$ tels qulaucune place réelle de $T$ ne se ramifie dans $L / K$, I'homomorphisme d'extension des $S$-diviseurs $a_{L}^{S} / K$ induit un pseudo-isomorphisme $j S_{T, L / K}$ du $\ell$-groupe des $S$-classes infinitésimales $C_{\ell} S(K)$ dans le $\ell$-groupe ambige $C \ell \underset{T}{S}(L)^{G}$. Noyau et conoyau de ce pseudo isomorphisme sont liés par liidentité :

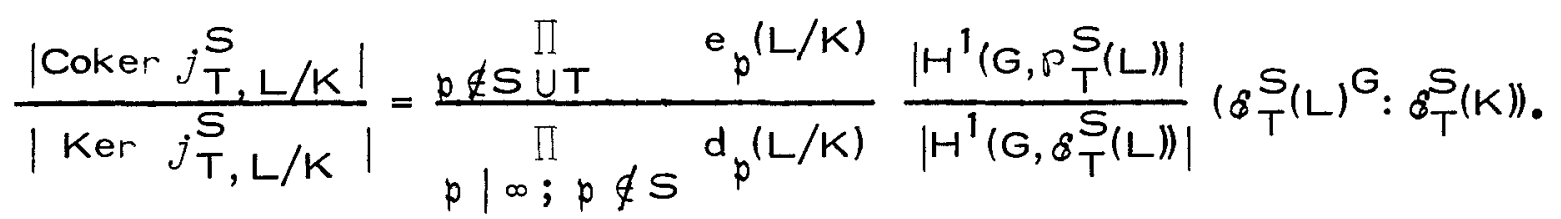

En particulier, lorsque l'extension L/K est absolument $S T$ ramifiée (i.e. ni complexifiée, ni ramifiée aux places (finies) nlappartenant pas à $S$ ou à $T$ ), il vient exactement :

$$
\text { Ker } j_{T, L / K}^{S} \simeq H^{1}\left(G, \varepsilon_{T}^{S}(L)\right) \quad \varepsilon
$$

Coker $j_{T, L / K}^{S} \simeq H^{1}\left(G, P_{T}^{S}(L)\right) \simeq \operatorname{Ker}\left[H^{2}\left(G, \delta_{T}^{S}(L)\right) \longrightarrow H^{2}\left(G, R_{T}(L)\right)\right]$.

COROLLAIRE 11,2.35,- Lorsque l'extension $L / K$ est cyclique, la formule des classes ambiges prend la forme:

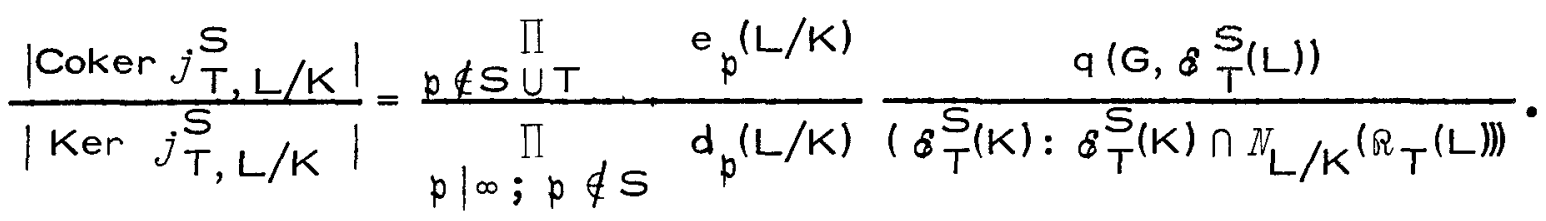


Et le quotient de Herbrand $q\left(G, \delta_{T}^{S}(L)\right)$ ne dépend que de $S$ et des places de $T$ qui divisent $l$.

Dans le cas cyclique, en effet, l'image du groupe $H^{2}\left(G, \varepsilon_{T}^{S}(L)\right)$ dans le groupe de cohomologie $H^{2}\left(G, R_{T}(L)\right)$ sidentifie au quotient $\left.\varepsilon_{T}^{S}(L){ }^{G} /\left[\varepsilon_{T}^{S}(L) \cap N_{L} / K^{\left(R_{T}\right.}(L)\right)\right]=\varepsilon_{T}^{S}(L){ }^{G} /\left[\varepsilon_{T}^{S}(K) \cap N_{L} / K^{(R}{ }_{T}^{(L))}\right] ;$ ce qui conduit bien au résultat annoncé. Dans la formule obtenue, le dénominateur ( $\left.\delta_{\mathrm{T}}^{S}(\mathrm{~K}): \delta_{\mathrm{T}}^{\mathrm{S}}(\mathrm{K}) \cap N_{\mathrm{L}} / \mathrm{K}^{(R} \mathrm{T}(\mathrm{L})\right)$ ) est un terme local qui peut se calculer à llaide des symboles locaux de reste normique lors que le groupe $\delta_{T}^{S}(K)$ est connu numériquement. Enfin, le quotient de Herbrand $q\left(G, \delta_{T}^{S}(L)\right)$, qui est inchangé lorsqu'on remplace le groupe $\varepsilon_{T}^{S}(L)$ par un sous-groupe d'indice fini, ne dépend donc que de la représentation $\ell$-adique de $G$ associée au $\mathbb{Q}_{l}$-espace vectoriel $\mathbb{Q}_{\ell} \mathbb{Z}_{l}{ }_{l}^{S}(L)$. II est donc indépendant des places de $T$ qui ne divisent pas $\ell$. Lorsque $T$ ne contient pas de places au-dessus de $l$, il est donné par la proposition 111.1 .8 . Lorsque $T$ contient les places au-dessus de $l$, il peut être estimé grâce à la conjecture d'indépendance $l$-adique avancée dans la section II, 1 . En particulier, il est tout à fait explicite si L est abélienne sur $\mathbb{Q}$.

\section{4. - GENRE INFINITÉSIMAL DIUNE EXTENSION DE CORPS DE NOMBRES •}

a.-L'application norme pour les groupes $a$.

Une extension finie $L / K$ de corps de nombres étant donnée , le prolongement de la norme arithmétique $N_{L / K}$ aux groupes de diviseurs généralisés induit, par passage au quotient, un morphisme na turel du $\ell$-groupe des classes infinitésimales $a(L)$ du corps $L$ sur ce lui $a(K)$ du corps $K$, que nous continuons, par abus, à noter $N_{L / K}$ (ou encore $N$ en l'absence d'ambiguité). Dans linterprétation des groupes $a$ donnée par la théorie du corps de classes, llapplication obtenue correspond simplement à la restriction des isomorphismes de Galois. En particulier, nous avons immédiatement : 
THÉORËME 11.2.35. - Soient L/K une $\ell$-extension finie quelconque de corps de nombres, $M_{K}$ la $\ell$-extension abélienne maximale $\ell$-ramifiée de $K$, et $Z_{K}$ la sous-extension de $M_{K}$ composée de toutes les $\mathbb{Z}_{\ell^{-}}$ extensions du corps $K$. Alors :

(i) Le quotient $a(K) / N_{L / K}(a(L))$ s'identifie au groupe de Galois $\mathrm{Gal}\left(\mathrm{L} \cap \mathrm{M}_{K} / K\right.$ ) de la sous-extension abélienne maximale de $L$ qui est $\ell$-ramifiée sur $K$.

(ii) Le quotient $\log (a(K)) / N_{L / K}[\log (a(L))]$ s'identifie au groupe de Galois $G a l\left(L \cap Z_{K} / K\right)$ de la sous-extension maximale de $L$ qui est contenue dans la composée $Z_{K}$ des $\mathbb{Z}_{\ell}$-extensions de $K$. En particulier, si $L$ est abélienne sur $K$ et $\ell-r a m i f i e ́ e$, on a l'équivalence :

$$
L \subset Z_{K} \Leftrightarrow N_{L / K}(a(L)) \subset G(K)
$$

Démonstration: L'assertion (i) résulte directement de la théorie du corps de classes, compte-tenu de l'isomorphisme $a(K) \simeq \mathrm{Gal}\left(\mathrm{M}_{K} / K\right)$ donné par le théorème 12 . L'assertion (ii) s'obtient de même , puisque, d'après la proposition 30 , la suite exacte courte canonique

$$
1 \longrightarrow \tau(K) \longrightarrow a(K) \longrightarrow \log a(K) \longrightarrow 1
$$

permet d'interpréter l'image du groupe $a(K)$ par le logarithme généralisé comme groupe de Galois de la sous-extension $Z_{K}$ de $M_{K}$ fixée par $\tau(K)$, c'est-à-dire comme groupe de Galois de la composée des $\mathbb{Z}_{\ell^{-}}$ extensions de $K$. L'équivalence $L \subset Z_{K} \Leftrightarrow N_{L / K}(a(L)) \subset q(K)$, lorsque $L$ est abélienne et $\ell$-ramifiée sur $K$ résulte directement des inter prétations précédentes: le groupe $N_{L / K}(a(L))$ siidentifie au sousgroupe de Gal $\left(M_{K} / K\right)$ qui fixe $L$; et le groupe de torsion $G(K)$ à celui qui fixe $Z_{K}$.

Si linterprétation des conoyaux $a(K) / N_{L / K}(a(L))$ et $\log a(K) / N_{L / K}(\log a(L))$ est claire, celle du groupe $\mathcal{G}(K) / N_{L / K}(\tau(L))$ soulève des problèmes plus délicats : D'après les résultats de la section $2 \S \mathrm{d}$, le sous-groupe de torsion $\zeta$ du $\ell$-groupe des classes infini tésimales a se comporte comme le dual du $\ell$-groupe des $\ell$-classes $C \ell^{\prime}$. Le problème de la surjectivité de la norme pour les groupes $\zeta$ est analogue à celui de la capitulation pour les groupes $C \ell^{\prime}$; c'est donc une question difficile. Du point de vue du corps de classes, il correspond d'ailleurs à une capitulation: 
SCOLIE II,2.36. - Etant donnée une $\ell$-extension finie quelconque de corps de nombres, convenons de dire qulune $l$-extension $F$ de $K$ capitule dans $L$, lorsque l'extension composée $F L$ est contenue dans la

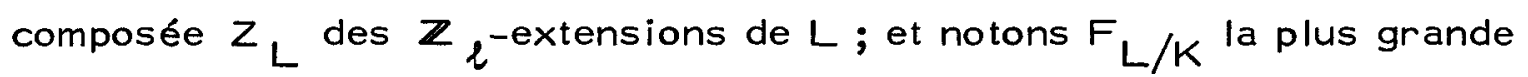
$\ell$-extension abélienne $\ell$-ramifiée de $K$, qui capitule dans $L$. Le quo tient $G(K) / N_{L / K}(G(L))$ s'identifie au groupe de Galois $G a l\left(F_{L / K} / Z_{K}\right)$.

Dans un cas particulier au moins llindice $\left(\zeta(\mathrm{K}): N_{L / K}(\sigma(L))\right.$ peut se calculer facilement : Lorsque les deux groupes $a(K)$ et $a(L)$ ont même rang essentiel, l'application induite par la norme sur le $\mathbb{Z} \ell^{- \text {mo - }}$ dule libre $\log a(L)$ est injective, et la suite exacte du serpent associée au diagramme commutatif

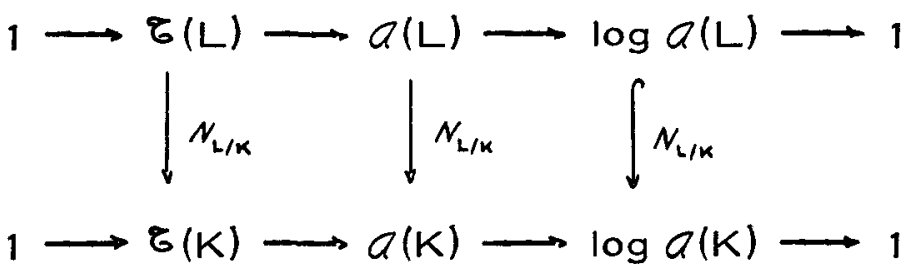

s'écrit :

$1 \rightarrow{ }_{N} \zeta(L) \rightarrow{ }_{N} a(L) \rightarrow 1 \rightarrow \sigma(K) / N_{L} / K^{(Z(L)) \rightarrow a(K) / N_{L / K}}(a(L)) \rightarrow \log a(K) / N_{L} / K(a(L)) \rightarrow 1$. 11 vient alors :

$$
\begin{aligned}
& { }_{N} G(L)={ }_{N} a(L) \quad \text { et } \quad\left(\sigma(K): N_{L / K}(G(L))\right)=\frac{\left(a(K): N_{L} / K^{(a(L)))}\right.}{\left(\log a(K): N_{L} / K^{(\log a(L)))}\right.} \\
& \text { Plus précisément : }
\end{aligned}
$$

PROPOSITION 11,2,36. - Dans une $\ell$-extension finie de corps de nom bres totalement réels, qui vérifient la conjecture de Leopoldt, noyau et conoyau de l'application induite par la norme arithmétique entre les groupes $\sigma$ sont donnés par les formules:

$$
\begin{aligned}
& \left(\sigma(K): N_{L / K}(\sigma(L))\right)=\frac{\left(a(K): N_{L / K}(a(L))\right)}{\left(\log a(K): N_{L / K}(\log a(L))\right)}
\end{aligned}
$$

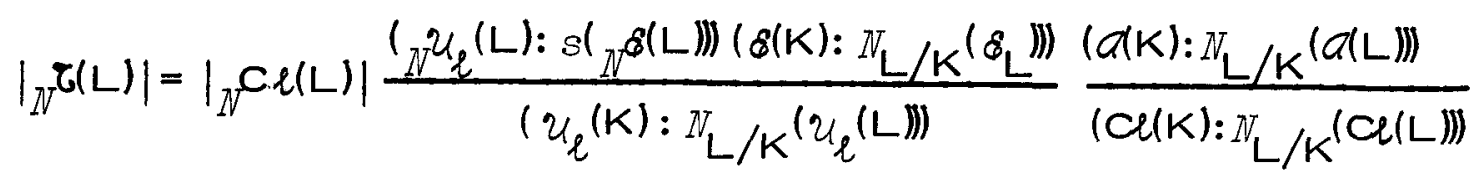


Démonstration: L'assertion (i) est établie ci-dessus, en même temps que l'égalité ${ }_{N} G(L)={ }_{N} Q(L)$.

Pour établir (ii), partons de la suite exacte courte de la proposition 10:

$$
1 \longrightarrow u_{\ell} / s(\xi) \longrightarrow a \longrightarrow c l \longrightarrow 1 \text {. }
$$

Formant le diagramme commutatif associé à l'application norme $N_{\mathrm{L}} / \mathrm{K}$, et appliquant le lemme du serpent, nous obtenons la suite longue :

$$
\begin{aligned}
& 1 \rightarrow{ }_{N}\left[u_{l}(L) / s\left(\delta^{\prime}(L)\right)\right] \rightarrow{ }_{N} Q(L) \rightarrow{ }_{N} C_{l}^{\prime}(L) \rightarrow u_{l}(K) / s\left(\delta^{\prime}(K)\right) N_{L} / K\left(U_{l}(L)\right) \\
& \rightarrow a(K) / N_{L} / K(G(L)) \rightarrow C l(K) / N_{L} / K(C l(L)) \rightarrow 1 . \\
& \text { D'un autre côté, la suite courte canonique (exacte sous la }
\end{aligned}
$$
conjecture de Leopoldt) :

$$
1 \longrightarrow \varepsilon \stackrel{s}{\longrightarrow} u_{l} \longrightarrow u_{l} / s(\varepsilon) \longrightarrow 1 \text {. }
$$

conduit, par le même procédé à la suite longue :

$$
\begin{aligned}
& 1 \rightarrow{ }_{N} \delta(L) \rightarrow{ }_{N} u_{\ell}^{X}(L) \rightarrow{ }_{N}\left[u_{\ell}(L) / s(\delta(L))\right] \rightarrow \delta(K) / N_{L} / K\left(\delta_{L}\right) \rightarrow u_{l}(K) / N_{L} / K\left(u_{l}(L)\right) \\
& \rightarrow u_{l}(K) / s(\&(K)) N_{L} / K\left(u_{l}(L)\right) \rightarrow 1 \text {. }
\end{aligned}
$$

Rassemblant ces résultats, nous obtenons :

$\left|{ }_{N} G(L)\right|=\left.\right|_{N} a(L) \mid=$

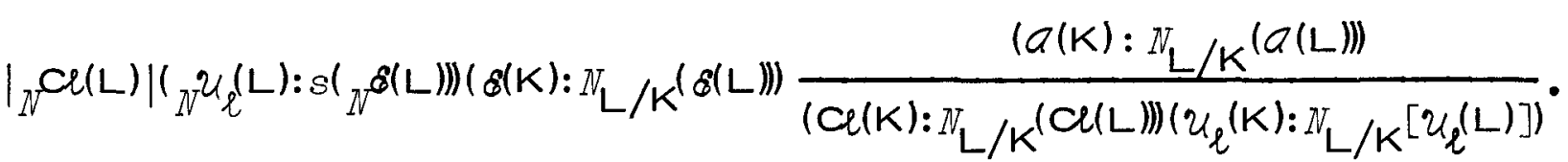

Dans la formule obtenue, l'indice $\left(a(K): N_{L / K}(a(L))\right)$ est égal au degré $\left[L \cap M_{K}: K\right]$ de la sous-extension abélienne maximale $\ell$-ramifiée de $L / K$; I'indice $\left(C \ell(K): N_{L / K}(C \ell(L)\right.$ ) est le degré $\left[L \cap C_{K}: K\right]$ de la sous-extension abélienne maximale non ramifiée de $L / K$; et l'indice $\left(u_{\ell}(K): N_{L / K}\left(u_{\ell}(L)\right)\right)$ est le produit des indices de ramification e $\left(L^{a b} / K\right)$ des extensions abéliennes locales associées aux places au-dessus de $l^{(\star)}$.

Naturellement, clest l'hypothèse $L$ totalement réel qui assure l'égalité des rangs essentiels rg.ess. $a(L)=$ rg.ess. $a(K)=1$, sous la conjecture de Leopoldt, et donc la finitude du noyau de la norme ${ }_{N} a(L)$. Dans le cas général, l'étude du groupe ${ }_{N} Q(L)$ relève de la théorie des genres.

(*) Lorsque l'extension $L / K$ est abélienne on retrouve ainsi la formule donnée par Gras dans ce cas ( $\left.c f_{0}\left[G r_{4}\right]\right)$. 


\section{b.-S-genre T-infinitésimal d'une extension de corps de nombres.}

Les formules normiques de la théorie des genres slexprimant de façon sensiblement plus simple en termes de classes diidèles plutôt que de rayons, commençons par donner une interprétation idèlique du $\ell$-groupe $\mathrm{Cl}_{\mathrm{T}}^{\mathrm{S}}$ des $S$-classes $\mathrm{T}$-infinitésimales.

Désignons par $z=\frac{\lim }{n} \mathrm{~J} / \mathrm{J}^{l^{n}}$ le $l$-groupe des idèles d'un corps de nombres, i.e. le produit des complétés profinis $k_{p}^{x}=\frac{\lim }{n} k_{p}^{x} / k_{p}^{x} l^{n}$ attachés aux places non complexes de ce corps, restreint aux familles $\left(x_{p}\right)_{p}$ dont presque tous les éléments sont des unités ( $D^{\prime}$ laprès les ré sultats de la section $2 \S a$, le groupe $u_{p}=\frac{\lim }{n} u_{p} / u_{p}^{l^{n}}$ des unités de $x_{p}^{x}$ slidentifie au groupe $u_{p}^{\prime}=(1+p)$ des unités principales du corps local $K_{p}$, lorsque $p$ est au-dessus de $\ell$; au $\ell$-Sylow $\mu_{p}$ du groupe des racines de llunité dans $K_{p}$, sinon). Linjection diagonale du groupe multiplicatif $K^{X}$ dans le groupe des idèles $J$ se prolonge canoni-

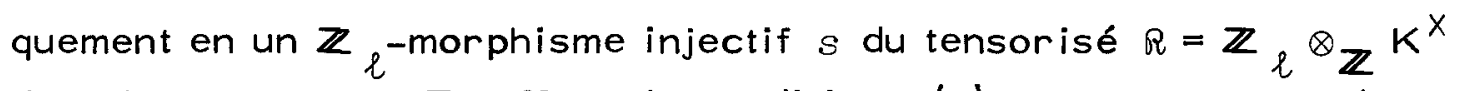
dans le groupe $g$ : En effet, la condition $s(x)=1$, pour un $x$ de $R$, permet d'écrire $x=x_{n} z_{n}^{l}$ pour chaque naturel $n$, avec $z_{n}$ dans $R$ et $x_{n}$ dans $K^{x}$, puissance $\ell^{n}$-ième locale partout, donc ( cf. [AT], $I X \S 1$, th. 1 ) puissance $\ell^{n-1}$-ième d'un élément global $y_{n}$ (en fait , puissance $\ell^{n}$-ième, sauf dans le cas spécial, pour $\ell=2$ ); ce qui donne immédiatement $x \in n_{n \geq 1} R^{l^{n}}=\{1\}$. Nous $n$ 'hésitons donc pas à confondre $R$ avec son image naturelle dans $\partial$.

DÉFINITION I1.2.37.- Par $\ell$-groupe des classes d'idèles d'un corps de nombres $K$, nous entendons le quotient $g / R$ du complété $g=\frac{1 \mathrm{im}}{n} \mathrm{~J} / \mathrm{J} l^{n}$ du groupe des idèles de $K$ par le tensorisé $\ell$-adique $R=\mathbb{Z}_{\ell}^{\otimes} \mathbb{Z}^{X}$ de son groupe multiplicatif.

Si $S$ et $T$ sont deux ensembles finis disjoints de places non complexes de $K$, nous notons $\partial_{T}(S)=p \notin S \cup T u_{p} \cdot \prod_{p \in S} K_{p}^{x}$ le $l-$ groupe des $S$-idèles étrangers à $T$, et $R S_{T}$ l'image canonique de $R$ dans le quotient $g_{T}^{S}=g / g_{T}(S)$. 
PROPOSITION 11.2.38, - Le quotient $\partial_{T}^{S} / R T_{T}^{S}$ s'identifie au $l$-groupe $c l_{T}^{S}=\theta_{T}^{S} / P_{T}^{S}$ des $S$-classes $T$-infinitésimales du corps $K$.

Démonstration : Le théorème d'approximation simultanée montre que chaque classe de $g / \Omega$ peut être représentée par un idèle étranger à $T$, qui est défini modulo $g_{T}(S) \cdot R_{T}$. En associant à cet idèle $\times$ la classe $\operatorname{cl}_{T}^{S}(a)$ du diviseur $a=\prod_{p} p^{\mathcal{L}_{p}}$, nous définissons par passage au quo tient, un isomorphisme naturel du quotient $\partial_{T}^{S} / R_{T}^{S}$ sur le groupe $\delta_{T}^{S} / p_{T}^{S}$ des $S$-classes $T$-infinitésimales du corps $K$.

Cela posé, considérons une extension finie quelconque de corps de nombres $\mathrm{L} / \mathrm{K}$. La définition 111.2 .1 se généralise comme suit :

DÉFINITION 11.2.39.- Etant donnés deux ensembles finis disjoints $S$ et $T$ de places non complexes diun corps $K$, le $\ell$-corps des $S$-genres $T$-infinitésimaux associé à une extension finie quelconque $L$ de $K$, est la plus grande $\ell$-extension $\bar{C}_{T, L / K}^{S} S$-décomposée et $T$-ramifiée sur $L$, qui provient par composition avec $L$ dlune $\ell$-extension abélienne de $K$. Le sous-groupe $\bar{d}_{T}^{S}(L / K)$ de $C_{T}^{S}(L)$ associe à $\bar{C}_{T, L / K}^{S}$ par la thérie du corps de classes est le S-genre principal T-infinitésimal . Le quo tient $G_{T}^{S}(L / K) \approx G a l\left(\bar{C}_{T}^{S} L / K / L\right)$ est le quotient des genres.

THÉORÈME 11.2.40,- Formule des genres - Soient $M$ la $\ell$-extension abélienne maximale de $K$, contenue dans le $\ell$-corps $\bar{C} S, L / K$ des $S$ genres $T$-infinitésimaux de l'extension $L / K$, et $K^{\prime}=C_{T}^{S}, K$ la $l$-exten sion abélienne maximale de $K$, qui est $S$-décomposée et $T$-ramifiée . Le degré de l'extension $M / K^{\prime}$ est donné par la formule :

$$
\left[M: K^{\prime}\right]=\frac{p \in S d_{p}^{a b}(L / K) \cdot p \notin S \cup T e_{p}^{a b}(L / K)}{\left(\delta_{T}^{S}(K): \delta_{T}^{S}(K) \cap \eta_{L / K}^{10 c}\right)}
$$

Dans celle-ci, $e_{p}^{a b}(L / K)$ est la $\ell$-partie de llindice de ramification de la sous-extension maximale $L_{p}^{a b}$, abélienne sur $K_{p}$, du corps $L_{p}=$ $\mathfrak{P} \cap_{p} L_{P} ;$ et $d_{p}^{a b}(L / K)$ est la $l$-partie du degré de cette extension; $\delta \frac{S}{S}(K)$ est le groupe des $S$-unités T-infinitésimales $\delta S(K) \cap \Omega(K) d u$ 
corps $K$; et $\eta_{L / K}$ est le sous-groupe des éléments de $R(K)$ qui sont normes dans chacune des extensions galoisiennes locales $L_{p} / K_{p}$ as sociées à $L / K$.

Démonstration: La situation peut être décrite par le schéma (où sont représentés les corps et les groupes de Galois ) :

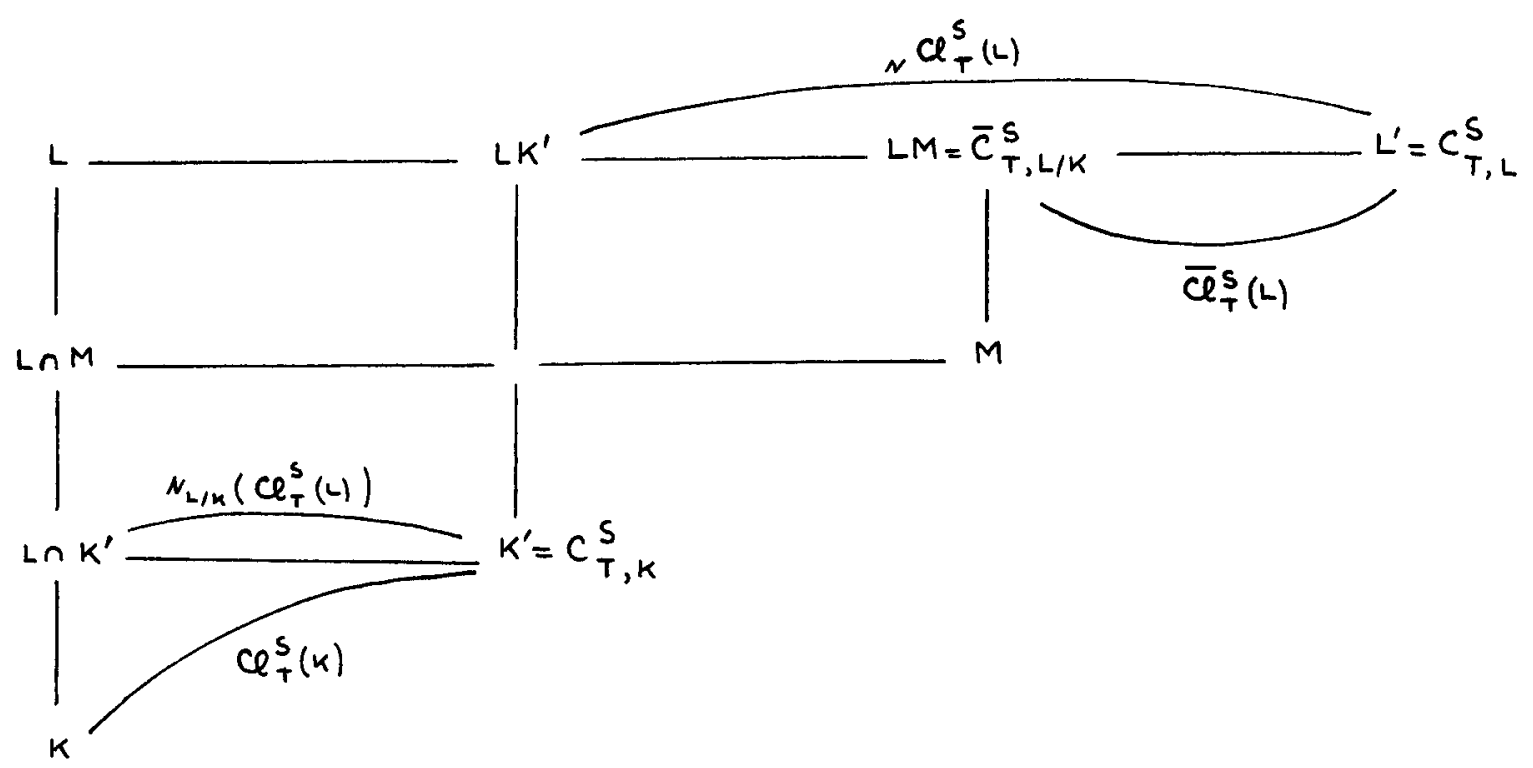

Ecrivons $L^{\prime}$ pour $C \stackrel{S}{T}, L$. Le corps $M$ étant la plus grande $\ell$-extension abélienne de $K$ contenue dans $L^{\prime}$, la théorie du corps de classes nous donne immédiatement :

$\operatorname{Gal}(M / K)=z_{K} / N_{L^{\prime} / K}\left(z_{L^{\prime}}\right) \Omega_{K}=z_{K} / N_{L / K}\left[N_{L^{\prime} / L^{\prime}}\left(z_{L^{\prime}}\right)\right] \Omega_{K}$ 。

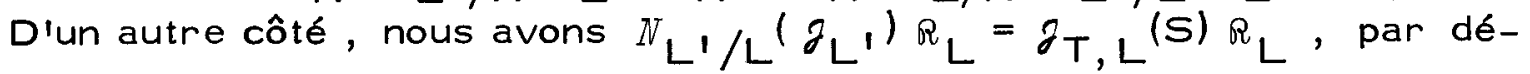
finition du corps $L^{\prime}$, et, par suite:

II vient donc :

$$
\operatorname{Gal}(M / K) \simeq \partial_{K} / N_{L / K}\left(\partial_{T, L}(S)\right) R_{K} \text {. }
$$

$$
\begin{aligned}
{\left[M: K^{\prime}\right] } & =\left(\partial_{T, K}(S) R_{K}: N_{L / K}\left[\partial_{T, L}(S)\right] R_{K}\right) \\
& =\frac{\left(\partial_{T}, K(S): N_{L} / K\left[\partial_{T, L}(S)\right]\right)}{\left(R_{K} \cap \partial_{T, K}(S): R_{K} \cap N_{L / K}\left[\partial_{T, L}(S)\right]\right)},
\end{aligned}
$$

i.e.:

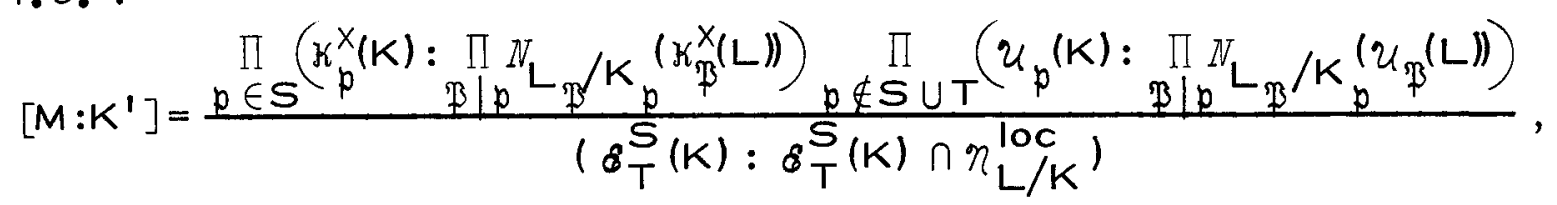

ce qui est bien le résultat annoncé. 
COROLLAIRE $11.2 .41 .-$ Soient $L / K$ une extension finie quelconque de corps de nombres, $M$ la $\ell$-extension abélienne maximale de $K$ telle que l'extension composée $L M / L$ soit absolument $\ell$-ramifiée, et $K$ ' la $\ell-$ extension abélienne, absolument $\ell$-ramifiée, maximale de $K$. Le degré de l'extension $M / K^{\prime}$ est donné par la formule :

$$
\left[M: K^{\prime}\right]=\frac{\prod_{p l \infty} d_{p}^{a b}(L / K) \cdot \prod_{p x e} e_{p}^{a b}(L / K)}{\left(\delta_{\infty}^{\text {ord }}(K): \delta_{\infty}^{\text {ord }}(K) \cap \eta_{L / K}^{\text {loc }}\right)},
$$

où $\varepsilon_{\infty}^{\text {ord }}(K)$ est le groupe des unités (au sens ordinaire) infinitési males du corps $K$ 。

11 est donc égal au produit des indices de ramification $e_{p}^{a b}(L / K)$, pour $p$ ne divisant pas $\ell$, dès que le corps $K$ vérifie la conjecture de Leopoldt.

Démonstration: II suffit de prendre $S=P \mid(\infty)$ et $T=P \mid(\ell)$, dans le théorème. Sous la conjecture de Leopoldt, le résultat obtenu corres pond à un déploiement de la ramification sur lequel nous allons reve nir.

\section{c.- Application au symbole de reste normique généralisé .}

Dans la section $111.2 \S 2$, nous avons défini le symbole de reste normique généralisé $\left(\frac{, L / K}{p}\right)$ at taché à une place non complexe $p$ d'un corps de nombres $K$, dans une extension finie quelconque $L$ de $K$, comme composé de linjection naturelle du groupe multiplicatif de $K$ dans celui de son complété $K_{p}$, et de l'isomorphisme de réciprocité

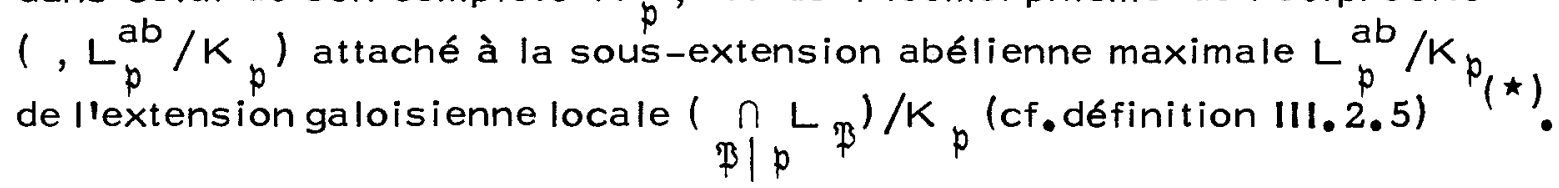
Ce symbole prend donc ses valeurs dans le groupe de décomposition abélianisé $D_{p}^{a b}(L / K)=$ Gal $\left(L_{p}^{a b} / K_{p}\right)$, défini comme groupe de Galois de l'extension abélienne locale $L_{p}^{\text {ab }} / K_{p}$. Cela étant, comme tout groupe abélien est composé direct de ses sous-groupes de Sylow, nous pou vons définir un $\ell$-symbole de reste normique généralisé en composant le précédent avec la projection canonique du groupe $D_{p}^{a b}(L / K)$ sur son l-Sylow. Plus précisément :

$\left.{ }^{\star}{ }^{\star}\right)$ La définition du symbole de reste no rmique dans le cas abélien est due à Hasse [ Ha 1 . 
DÉFINITION II.2.42. - Etant données une extension finie quelconque de corps de nombres $L / K$, et une place non complexe $p$ de $K$, nous ap pelons $l$-symbole de reste normique généralisé associé à la place $p$ dans l'extension $L / K$, et nous continuons par abus à noter $\left(\frac{L / K}{p}\right)$, la surjection naturelle du tensorisé $\ell_{K}=\mathbb{Z}_{\ell} \otimes_{\mathbb{Z}} K^{X}$ dans le groupe de Galois $D_{p}^{a b}(L / K)=\operatorname{Gal}\left(L_{p}^{a b} / K_{p}\right)$ de la $\ell$-sous-extension abélienne maximale de l'extension galoisienne locale $\left(\cap_{\mathcal{P} / P} L_{\mathcal{P}}\right) / K_{\mathcal{P}}$.

Cette définition s'étend sans difficulté au cas où l'extension $L / K$ est infinie: En effet, comme la restriction du symbole de Hasse à une sous-extension est le symbole de Hasse pris dans cette sous extension, le symbole $\left(\frac{, L / K}{p}\right)$ peut se définir comme limite projective des symboles $\left(\frac{, F / K}{p}\right)$ lorsque $F$ parcourt les sous-extensions finies de $L / K$. Cela posé, nous avons :

THÉOREME 11,2.43, - Suite exacte des genres - Soient $S$ et $T$ deux ensembles finis disjoints de places non complexes d'un corps de nombres $K$, et $L$ une extension finie quelconque de $K$. Si $K$ ' désigne la $\ell$-extension abélienne maximale de $K$ qui est $S$-décomposée et $T$-ramifiée, $L$ 'la $\ell$-extension abélienne maximale de $L$ qui est $S$-décomposée et T-ramifiée, et $M$ la sous-extension maximale de $L^{\prime}$ qui est une $l-$ extension abélienne de $K$, il existe une suite exacte courte canonique:

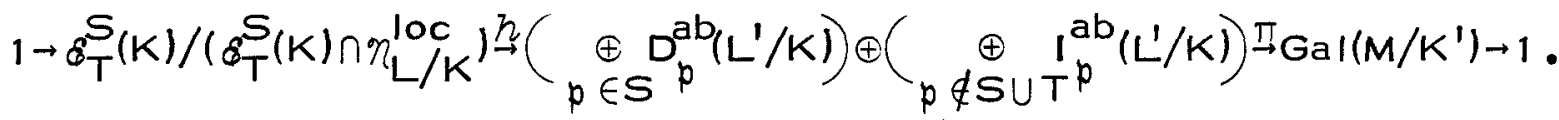
Dans celle-ci, $h$ est la composée des symboles généralisés $\left(\frac{, L 1 / K}{p}\right)$, à valeurs dans la somme directe $\underset{p}{\oplus D_{p}}\left(L^{\prime} / K\right)$ des groupes de décomposi tion abélianisés associés à $L ' / K$, et $\pi$ est la projection canonique $\left(\sigma_{p}\right)_{p} \longrightarrow \prod_{p}\left(\left.\sigma_{p}\right|_{M}\right)$

Démonstration: L'extension L'/L étant $S$-décomposée et T-ramifiée, les extensions $L / K$ et $L ' / K$ ont mêmes groupes de décomposition abé lianisés $D_{p}^{a b}$ aux places de $S$, et mêmes groupes d'inertie abélianisés 
$\mathrm{I}_{p}^{a b}$ aux places finies étrangères à $T$. Le terme médian de la suite slécrit donc tout aussi bien :

$$
\left[\underset{p \in S}{\oplus} D_{p}^{a b}(L / K)\right] \oplus\left[\underset{p \notin S \cup T}{\oplus} 1_{p}^{a b}(L / K)\right] \text {. }
$$

Cela étant :

(i) L'application $\hbar$ est injective : Les S-unités T-infinitésimales sont en effet normes locales en-dehors de $S$ et des places ramifiées qui ne sont pas contenues dans $T$. La condition $h(\epsilon)=1$ caractérise donc celles qui sont normes locales partout dans l'extension $L / K$.

(ii) L'application composée $\pi \circ \hbar$ est nulle, d'après la for mule du produit pour le symbole de Hasse appliquée à l'extension abélienne $M / K$.

(iii) L'application $\pi$ est surjective, par maximalité du corps $K^{\prime}$ sous les deux conditions de $S$-décomposition et de T-ramification. L'exactitude de la suite résulte donc de la formule des genres donnée par le théorème 40 .

COROLLAIRE 11.2.44.- Réciproque de la formule du produit - Etant donnés un ensemble fini $T$ de places non complexes d'un corps de nom bres $K$, et $L$ une extension finie de $K$, les éléments $\left(\sigma_{p}\right)_{p}$ de la somme directe $\oplus_{p} D_{p}^{a b}(L / K)$ des $l$-groupes de décomposition abéliani sés associés aux places non complexes de $K$ dans $L / K$, qui vérifient la formule du produit $\left.\prod_{p} \sigma_{p}\right|_{L^{a b}}$ (prise dans la $\ell$-sous-extension abé lienne maximale de $L / K)$, et les conditions $\sigma_{p}=1$, pour $p \in T$, sont les images par les $\ell$-symboles de reste normique généralisés $\left(\frac{, L / K}{p}\right)$ des éléments T-infinitésimaux. Autrement dit il existe une suite exacte courte canonique :

$1 \longrightarrow R_{T}(K) / R_{T}(K) \cap \eta_{L / K}^{l o c} \longrightarrow D_{p}^{\oplus} D_{p}^{a b} \longrightarrow G^{a b}(L / K) \longrightarrow 1$.

Démonstration : Une famille $\left(\sigma_{p}\right)_{p}$ étant donnée, choisissons $S$ assez grand, étranger à $T$, contenant les places p pour qui $\sigma_{p}$ est non nul, et tel qu'on ait $L^{\prime}=L$ et $K^{\prime}=K$, avec les notations du théorème. La suite exacte des genres nous donne alors une S-unité T-infinitési male $€$ qui vérifie les conditions requises : 


$$
\left(\frac{\epsilon, L / K}{p}\right)=\sigma_{p}, \quad \forall p
$$

COROLLAIRE 11.2.45. - Déploiement de la ramification - Soient L/K une $\ell$-extension abélienne de corps de nombres, $M$ la $\ell$-extension abélienne maximale de $K$ qui est absolument $\ell$-ramifiée sur $L$, et $K$ ' celle qui est absolument $\ell$-ramifiée sur $K$. Si le corps $K$ vérifie la conjec ture de Leopoldt, le groupe de Galois $\mathrm{Gal}\left(\mathrm{M} / \mathrm{KI}^{\prime}\right)$ sidentifie à la somme directe des $\ell$-sous-groupes d'inertie des places de $K$ étrangères à $\ell$ : $\operatorname{Gal}(M / K I) \simeq\left[\underset{p / \infty}{\oplus} D_{p}(L / K)\right] \oplus\left[\underset{\text { D pll }}{\oplus} I_{p}(L / K)\right]$

Nous disons que la ramification est déployée dans le corps des genres infinitésimaux $\mathrm{M} / \mathrm{K}$.

Démonstration: II suffit d'appliquer le théorème 43 avec $S=P \mid(\infty)$ et $T=F I(\ell)$.

Remarque. - Le corollaire vaut encore, si L/K n'est pas une l-extension. 
$-161-$ 
$-162-$ 
CHAPITRE III

LA FORMULE DES CLASSES AMBIGES ET SES GÉNÉRALISATIONS ÉLÉMENTS DE THÉORIE DES GENRES 


\section{1.- La formule des classes ambiges et ses GÉNÉRALISATIONS.}

1.- La formule de Chevalley pour les groupes de S-classes

de diviseurs ................................... III.2

a.- Présentation du groupe des $\mathrm{S}$-classes de diviseurs

d'un corps de nombres............................ III.2

b. - Démonstration de la formule des classes ambiges............. III.5

c. - Analyse de la formule dans le cas cyclique................ III.9

2.- Expression de la formule en termes de représentations

dans le cas métabélien............................... III.14

a.- Préliminaires................................... 111.14

b. - Enoncé des résultats........................... III.16

c. - Application à la capitulation............................ 20

3.- Extension des résultats au cas procyclique.................. III.23

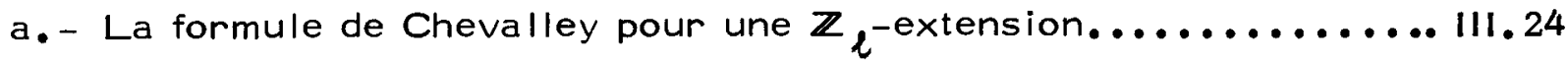

b. - Calcul du quotient de Herbrand dans le cas procyclique......... III.27

c.- Analyse de la formule obtenue ; critères de trivialité pour les groupes $\mathrm{Cl}_{L}^{S}$

\section{2. - ÉLÉMENTS DE THÉORIE DES GENRES .}

1.- Présentation de la théorie des genres..................... III.37

a. - Définition du corps des S-genres relatif à une extension finie de corps de nombres.................111.37

b. - La formule du produit pour le symbole de reste normique........ III.42

c. - Comparaison du corps des genres et du corps des classes centrales. 
2.- Expression de la formule des genres en termes de repré sentations..................................... III.52

a. - Etude du cas métabélien.......................... III.52

b. - Extension des résultats au cas procyclique :

la formule des genres pour une $\mathbb{Z}$, extension.............. III.57

c. - Propriétés normiques des $S$-unités dans une $\mathbb{Z}_{\mathfrak{l}}$-extension...... 111.62 


$$
-166-
$$


Les calculs de classes invariantes remontent aux débuts mêmes de la théorie du corps de classes, et plus précisément aux travaux es sentiels de Takagi sur cette question (cf., par exemple [TK]). Toutefois, ils n'y apparaissent d'abord que comme une étape technique dans la démonstration des inégalités fondamentales de la théorie. Aussi, n'est-ce que dans la thèse de Chevalley, parue en 1933, que furent présentées pour la première fois l'ensemble des suites exactes qui conduisent à la formule des classes ambiges que nous connaissons au jourd'hui (cf. [Ch], p. 402-405), l'utilisation du tout récent théorème de Herbrand sur les unités ( cf. $\left[\mathrm{He}_{1}\right]$ ) ayant permis de passer du cas cyclique d'ordre premier au cas cyclique général. En fait, les idées essentielles de ce calcul se trouvaient déjà dans le Zahlbericht de Hilbert, qui les met en oeuvre dans la démonstration de son théorème 94 sur lequel nous reviendrons plus loin. Le fait qu'elles aient été si souvent redécouvertes depuis, jusque dans les périodes les plus récentes, à l'occasion de différents problèmes, tient sans aucun doute à la qualité des informations sur le comportement des groupes de classes d'idéaux que la suite exacte des classes ambiges apporte directement . En 1956 , Iwasawa, dans une courte note sur les unités ( cf. $\left[\mathrm{Iw}_{1}\right]$ ) donna une interprétation cohomologique de plusieurs morphismes intervenant dans la démonstration de Chevalley, permettant ainsi d'en produire une démonstration valable pour toute extension galoisienne, et non plus seulement dans le cas cyclique. C'est ce point de vue que nous adoptons ici, dans le cadre plus général des groupes de $\mathbf{S}$-clas ses de diviseurs. 


\section{1. - LA FORMULE DE CHEVALLEY POUR LES GROUPES DE S- CLASSES DE DIVISEURS .}

a.- Présentation du groupe des $S$-classes de diviseurs d'un corps de

A chaque place $p$ d'un corps de nombres est associée canoni quement une valuation $v_{p}$ qu'on peut définir comme suit :

- Pour une place ultramétrique, correspondant à un idéal premier $p$ de l'anneau des entiers de $K$, la $p$-valuation $v p(x)$ d'un élé ment $x$ de $K^{X}$ est l'exposant de $p$ dans la décomposition primaire de l'idéal principal engendré par cet élément. L'ensemble des valeurs de la valuation $v_{p}$ est donc le groupe $\mathbb{Z}$.

- Pour une place archimédienne, il y a lieu de distinguer deux cas : Si p est réelle, la p-valuation d'un élément $\times$ de $K^{X}$ est dé terminée par son signe sg $p(x)$ dans le complété $K_{p} \approx R, i . e . v_{p}(x)=0$ si $x>0$ dans $K_{p}$, et $v_{p}(x)=1$ si $x<0$ dans $K_{p}$; llapplication $v_{p}$ prend alors ses valeurs dans $\mathbb{Z} / 2 \mathbb{Z}$. Si p est complexe, $v_{p}$ est la valuation triviale.

DÉFINITION III.1.1.- Par groupe des diviseurs d'un corps de nombres $K$, nous entendons le groupe abélien libre $D_{K}$ engendré par les valua tions attachées aux places de $K$. Le groupe $D_{K}$ s'identifie à la somme directe du groupe $I d_{K}$ des idéaux fractionnaires de $K$ et du produit de $r_{K}$ copies de $\mathbb{Z} / 2 \mathbb{Z}$, où $r_{k}$ est le nombre de places réelles de $K$.

Cela posé, si l'on identifie le groupe $\mathbb{Z} / 2 \mathbb{Z}$ au groupe multi plicatif $\{-1,+1\}$, l'application de $K^{X}$ dans le groupe des diviseurs $D_{K}$, donnée par les valuations, prend la forme:

$$
x \mapsto(x)=\left(x \theta_{K}, \operatorname{sgn}(x)\right),
$$

où $x \theta_{K}$ est l'idéal principal engendré par $x$, et $\operatorname{sgn}(x)$ la signature de $x$, i.e. le $r_{k}$-uplet des signes de ses plongements réels. Nous disons que $(x)$ est le diviseur principal engendré par l'élément $x$. Comme il existe dans $K^{X}$ des éléments de toutes signatures ( $f$, par exeimple, $[c h]$ ), chaque classe du quotient $C_{K}=D_{K} / P_{K}$ du groupe des diviseurs $D_{K}$ 
par le sous-groupe $P_{K}$ des diviseurs principaux peut être représentée par un idéal, et il existe donc un isomorphisme naturel

$$
c \ell_{K} \simeq 1 d_{K} / P r_{K}^{+}
$$

du groupe $C_{K}$ sur le quotient $I_{K} / P_{K}+\underset{K}{d u}$ groupe des idéaux de $K$ par le sous-groupe formé des idéaux principaux engendré par les éléments de $\mathrm{K}^{\mathrm{X}}$ de signature unité. Autrement dit :

PROPOSITION III.1.2. - Le quotient $d_{K}=D_{K} / P_{K}$ du groupe des di viseurs du corps $K$ par le sous-groupe des diviseurs principaux est un groupe fini, appelé groupe des classes de diviseurs, qui slidentifie au groupe des classes d'idéaux au sens restreint, c'est-à-dire au quotient $I_{K} / P_{K}^{+}$du groupe des idéaux de $K$ par le sous-groupe des idéaux principaux engendrés par les éléments de $K^{X}$ totalement positifs ( $i$.e. de signature unité ). Son ordre diffère donc de celuidu groupe des classes diidéaux au sens ordinaire d'un facteur 2 -primaire, égal à lindice dans $\{-1,+1\}^{r_{k}}$ de la signature $\operatorname{sgn}\left(E_{K}^{o r d}\right.$ ) du groupe des unités au sens ordinaire de $K$.

Ce dernier point résulte directement du diagramme commutatif exact, où nous avons repéré par un + , le noyau de la signature :

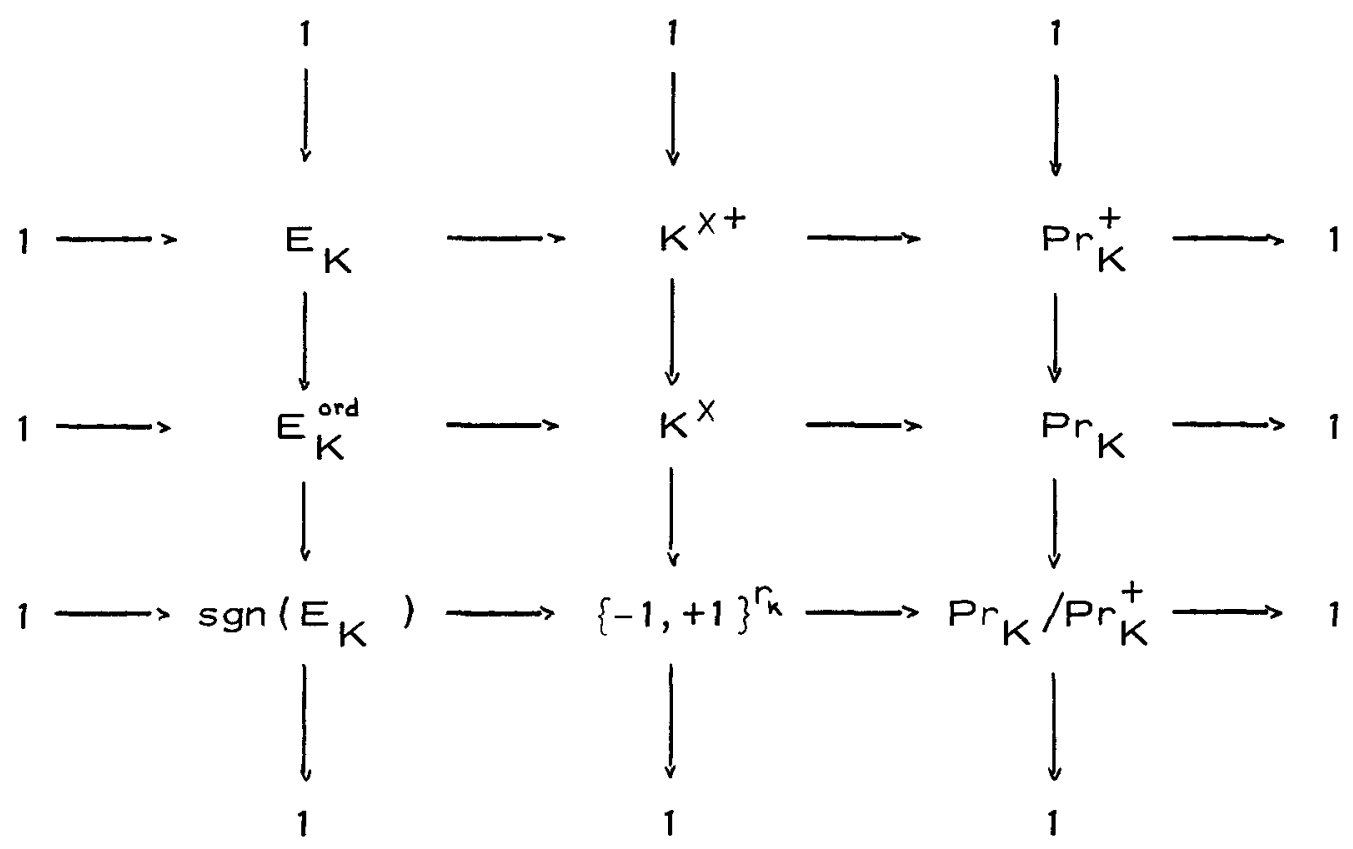


Convention.- Dans tout ce qui suit, nous appelons groupe des unités du corps $K$, et nous notons $E_{K}$, le groupe

$$
E_{K}=\left\{x \in K^{x} \mid v_{p}(x)=0, \forall p \in P I_{K}\right\} \text {, }
$$

c'est-à-dire le noyau de la surjection canonique de $K^{X}$ dans $P_{K}$. Nous appelons groupe des unités au sens ordinaire, et nous notons $E_{K}^{\text {ord }}$ le noyau de la surjection canonique de $\mathrm{K}^{{ }^{X}}$ dans $\mathrm{Pr}_{\mathrm{K}}$. Les unités de $\mathrm{K}$ ( au sens des diviseurs) sont donc les unités au sens ordinaire ( i.e. au sens des idéaux) qui sont totalement positives (i.e. de signature unité ).

Bien entendu, lorsqu'on s'intéresse exclusivement au $\ell$-Sylow du groupe des classes, pour un nombre premier $l$, la distinction entre

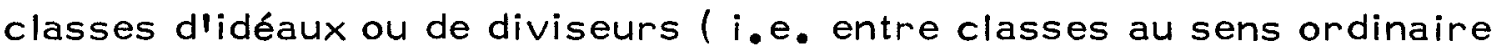
ou au sens restreint ) ne se pose que pour $\ell=2$. Dans ce cas, il n'est pas indifférent de raisonner sur l'un ou l'autre groupe dès que la signature des unités (au sens ordinaire) n'épuise pas le groupe $\{-1,+1\}^{r_{k}}$. Nous allons voir que le point de vue des classes de diviseurs est préférable car plus fin :

DÉFINITION III,1.3.- Etant donné un ensemble fini $S$ de places non complexes de $\mathrm{K}$, nous appelons groupe des $\mathbf{S}$-classes de diviseurs du corps $K$, et nous notons $a_{K}^{S}$, le quotient $a_{K} / a_{K}(s)$ du groupe des classes de diviseurs de $K$ par le sous-groupe de $a_{K}$ engendré par les classes des places de $K$ appartenant à $S$.

Lorsque llensemble $S$ contient les places réelles de $K$, le groupe $\alpha_{K}^{S}$ sidentifie au groupe des $S^{0}$-classes didéaux du corps $K$, i.e. au groupe des classes diidéaux du localisé en-dehors de $S^{*}$ de l'anneau des entiers de $K$, où $S^{\circ}$ est l'ensemble des places finies con -

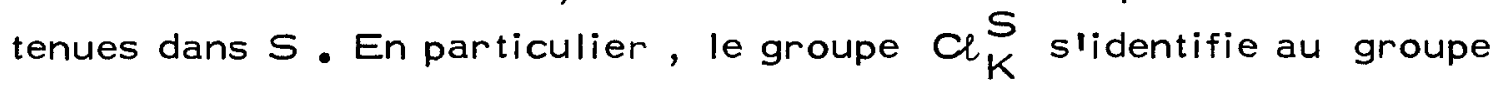
des classes dijiéaux au sens ordinaire lorsque $S$ est exactement l'en semble des places réelles de $K$.

Du point de vue de la théorie du corps de classes, le groupe $a_{K}$ des classes de diviseurs du corps $K$ correspond au groupe de Galois de l'extension abélienne maximale de ce corps, qui est non ra mifiée aux places finies $(=u l t r a m e ́ t r i q u e s)$. Son quotient $d_{K}^{S}$ 
correspond ainsi au groupe de Galois de l'extension abélienne maximale de $K$, qui est non ramifiée aux places finies et complètement décompo sée aux places de $S$. En particulier, si $S$ est l'ensemble des places réelles de $K$, le groupe $C_{K}^{S}$ (qui slidentifie alors au groupe des classes d'idéaux au sens ordinaire ) correspond au groupe de Galois de l'extension abélienne maximale de $K$ qui est non ramifiée et décomposée à l'infini ( $i$ e. non ramifiée aux places finies et non complexifiée aux places réelles).

Cela dit, pour étudier les quotients $C_{K}^{S}$, il est commodedintroduire les deux groupes :

le groupe des $S$-diviseurs $D_{K}^{S}=D_{K} / D_{K}(S)$, quotient du groupe des diviseurs par le sous-groupe $D_{K}(S)$ engendré par les places de $S$;

- le groupe des $S$-diviseurs principaux $P_{K}^{S}=P_{K} D_{K}(S) / D_{K}(S)$ image canonique de $P_{K}$ dans $D_{K}^{S}$.

Enfin, conformément aux conventions précédentes, nous no tons $E_{K}^{S}$ le groupe des $S$-unités de $K$ :

$$
E_{K}^{S}=\left\{x \in K^{X} \mid v_{p}(x)=0, \forall p \notin S\right\} \text {. }
$$

Nous obtenons alors les deux suites exactes canoniques (qui sont le point de départ de la démonstration de la formule des classes ambiges ) :
(a)

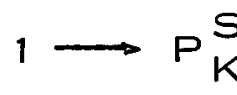
$P \mathrm{~K}$
$D_{K}^{S}$
$a_{K}^{s}$

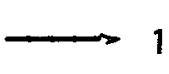
(b)

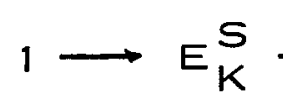
$\longrightarrow$
$\mathrm{K}^{\mathrm{x}} \longrightarrow \mathrm{P}_{\mathrm{K}}^{\mathrm{S}}$

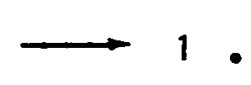

b.- Démonstration de la formule des classes ambiges -

Une extension finie $L / K$ de corps de nombres étant donnée, le prolongement des valeurs absolues définit un morphisme naturel du groupe des diviseurs $D_{K}$ vers le groupe $D_{L}$, qui envoie le sous groupe principal $P_{K}$ sur celui $P_{L}$ de $D_{L}$ : Dans la décomposition ca nonique $D=$ Id $\oplus\{-1,+1\}^{r_{k}}$ du groupe des diviseurs, la restriction de cet homomorphisme au groupe Id correspond à l'extension des idéaux : 


$$
a_{K} \longrightarrow a_{K} \theta_{L} ;
$$

c'est pourquoi nous l'appelons homomorphisme d'extension, et nous le notons $a_{L / K}$. Contrairement à sa restriction aux idéaux, il n'est pas injectif dès lors qu'une au moins des places réelles de $K$ se complexifie dans $L$. Si, maintenant, $S_{K}$ est un ensemble fini de places non com plexes de $K$, et $S_{L}$ l'ensemble des places non complexes de $L$ au-dessus des précédentes, l'homomorphisme d'extension envoie $D_{K}^{S}$ dans $D_{L}^{S}$ et $\mathrm{P}_{\mathrm{K}}^{\mathrm{S}}$ dans $\mathrm{P}_{\mathrm{L}}^{\mathrm{S}}$, d'où, par passage au quotient, $\mathrm{Cl}_{\mathrm{K}}^{S}$ dans $\mathrm{Cl}_{\mathrm{L}}^{S}$. Nous notons $j_{L / K}^{S}$ cette dernière application, et $\operatorname{Cap}_{L / K}^{S}$ son noyau, qui représente donc la $S$-capitulation dans l'extension $L / K$.

Lorsque, de plus, l'extension L/K est galoisienne, de groupe de Galois $G$, I'homomorphisme d'extension $j_{L / K}^{S}$ envoie le groupe des $S$-classes de diviseurs de $K$ dans le sous-groupe ambige $C_{L}^{S G}$ de ce lui de $L$; et le problème se pose d'évaluer l'indice correspondant $\left(a_{L}^{S G}: j_{L / K}^{S}\left(d_{K}^{S}\right)\right)$, ainsi que llordre $\left|C_{L}^{S} S / K\right|$ de la $S-c a-$ pitulation. Nous avons besoin pour cela d'un résultat préliminaire :

LEMME 111.1.4.- Dans une extension galoisienne $L / K$ de corps de nombres, on a les isomorphismes, où $G$ désigne le groupe $G a l(L / K)$ :

$$
\begin{aligned}
& \text { (i) } P_{L}^{S G} / d_{L / K}^{S}\left(P_{K}^{S}\right) \simeq H^{1}\left(G, E_{L}^{S}\right) \text {. } \\
& \text { (ii) } H^{1}\left(G, P_{L}^{S}\right) \simeq \operatorname{Ker}\left[H^{2}\left(G, E_{L}^{S}\right) \longrightarrow H^{2}\left(G, L^{x}\right)\right] . \\
& \text { (iii) } H^{1}\left(G, D_{L}^{S}\right)=1 .
\end{aligned}
$$

Démonstration: Partons de la suite exacte (b) appliquée au groupe $P_{L}^{S}$, et formons la suite exacte de cohomologie correspondante :

$1 \rightarrow E_{L}^{S G} \rightarrow K^{x} \rightarrow P_{L}^{S G} \rightarrow H^{1}\left(G, E_{L}^{S}\right) \rightarrow H^{1}\left(G, L^{x}\right) \rightarrow H^{1}\left(G, P_{L}^{S}\right) \rightarrow H^{2}\left(G, E_{L}^{S}\right) \rightarrow H^{2}\left(G, L^{X}\right)$.

Le groupe $H^{1}\left(G, L^{X}\right)$ étant nul, en vertu du théorème 90 de Hilbert

généralisé ( cf. $[C F]$, ch. $V, \S 27$ ), nous obtenons les deux premiers isomorphismes :

$$
\begin{array}{ll} 
& P_{L}^{S G} / d_{L / K}\left(P_{K}^{S}\right) \simeq H^{1}\left(G, E_{L}^{S}\right) \\
\Leftrightarrow \quad & H^{1}\left(G, P_{L}^{S}\right) \simeq \operatorname{Ker}\left[H^{2}\left(G, E_{L}^{S}\right) \rightarrow H^{2}\left(G, L^{x}\right)\right] .
\end{array}
$$

Pour établir le troisième, remarquons que si $p_{L}$ est une place non complexe de $L$ et $G_{P_{L}}$ son groupe de décomposition dans $L / K$, le groupe de cohomologie $H^{1}\left(G_{p_{L}}, D\left(p_{L}\right)=\operatorname{Hom}\left(G_{p}, D\left(P_{L}\right)\right)\right.$ est nul en toutes hypothèses : 
- si $P_{L}$ est ultramétrique, le groupe $D\left(P_{L}\right)$ est isomorphe à $\mathbb{Z}$, et $H^{1}\left(G_{P_{L}}, D\left(P_{L}\right)\right)$ est donctrivial, puisque le groupe $G_{P_{L}}$ est fini .

- si $p_{L}$ est réelle, la place $p_{L}$ est complètement décom posée dans l'extension $L / K$, et $H^{1}\left(G_{p_{L}}, D\left(p_{L}\right)\right.$ est encore trivial, puisque le groupe $G p_{p_{L}}$ l'est.

Par le lemme de Shapiro (cf. [CF ], Ch. IV, § 4), il vient donc $H^{1}\left(G, \underset{p_{L} \mid p_{k}}{\oplus} D\left(p_{L}\right)\right)=H^{1}\left(G_{p_{L}}, D\left(p_{L}\right)\right)=1$, dès que la place $p_{k}$ n'a pas de prolongement complexe dans $L$; ce qui achève la démonstration .

Considérons maintenant la suite exacte (a) pour le corps L . La suite exacte de cohomologie associée

$1 \longrightarrow P_{L}^{S G} \longrightarrow D_{L}^{S G} \longrightarrow C_{L}^{S G} \longrightarrow H^{1}\left(G, P_{L}^{S}\right) \longrightarrow H^{1}\left(G, D_{L}^{S}\right)$

slarrête, en vertu de llassertion (iii) du lemme. En la comparant à la suite exacte (a) écrite pour le corps $K$

$1 \longrightarrow \mathrm{P}_{\mathrm{K}}^{\mathrm{S}} \longrightarrow \mathrm{D}_{\mathrm{K}}^{\mathrm{S}} \longrightarrow \mathrm{Cl}_{\mathrm{K}}^{\mathrm{S}} \longrightarrow 1$

à l'aide des homomorphismes d'extension, nous pouvons donc former le diagramme commutatif exact :

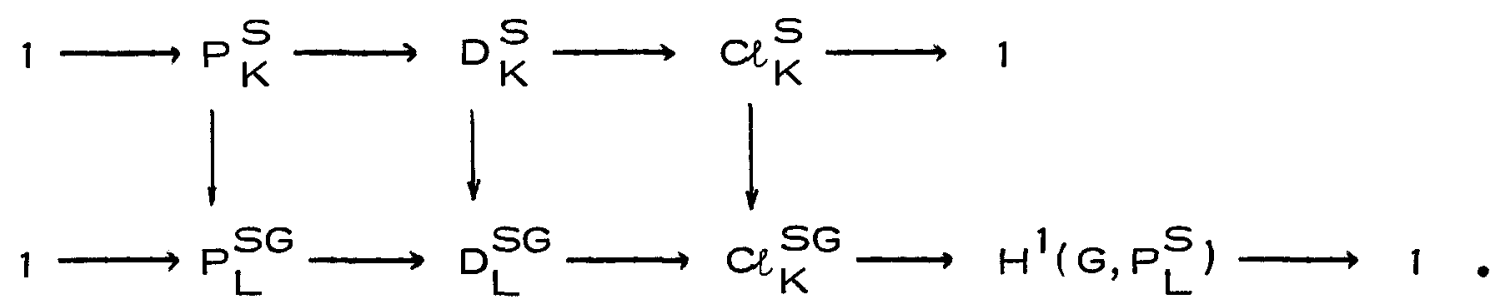

Par le lemme du serpent, et compte-tenu des isomorphismes donnés par le lemme 4 , nous obtenons la suite exacte annoncée :

THÉORÈME III,1.50- (Suite exacte des classes ambiges ) - Dans une extension galoisienne $L / K$ de corps de nombres, de groupe de Galois $G$, noyau et conoyau de l'homomorphisme d'extension $j_{L / K}^{S}$ du groupe $C_{K}^{S}$ des $S$-classes de diviseurs de $K$, dans le sous-groupe ambige $C_{L}^{S G}$ de delui de $L$, sont liés par la suite exacte longue : $1 \rightarrow E_{L}^{S G} / E_{K}^{S} \rightarrow \operatorname{Ker} d_{L / K}^{S} \rightarrow \operatorname{Ker} j_{L / K}^{S} \rightarrow H^{1}\left(G, E_{L}^{S}\right) \rightarrow$ Coker $a_{L / K}^{S} \rightarrow$ Coker $j_{L / K}^{S}$ $\rightarrow H^{2}\left(G, E_{L}^{S}\right) \rightarrow H^{2}\left(G, L^{X}\right)$. 
SCOLIE 111.1.6. - Dans la suite exacte des classes ambiges, le noyau Ker $a_{L / K}^{S}$ de l'extension des diviseurs mesure la $S$-complexification (i.e. la complexification dans $L / K$ des places (réelles) n'appartenant pas à $S$ ) et le conoyau Coker $\alpha_{L / K}^{S}$ la $S$-ramification (i.e. la rami fication dans $L / K$ des places (finies) nlappartenant pas à $S$ I. Plus précisément, il vient :

$\left|\operatorname{Ker} d_{L / K}^{S}\right|=\prod_{p \notin S ; p \mid \infty} \mathrm{d}_{p}(L / K)$ \& $\mid$ Coker $a_{L / K}^{S} \mid=\prod_{p \notin S} e_{p}(L / K)$, si, pour chaque place $p$ de $K, d_{p}(L / K)$ désigne le degré de llune quelconque des extensions locales $L_{p} / K_{p}$, et $e_{p}(L / K)$ son indice de ramification.

Démonstration: L'extension des idéaux étant injective, le noyau de $a_{L / K}^{S}$ est formé des seuls diviseurs de $K$ construits sur les places réelles n'appartenant pas à $S$ qui se complexifient dans $L / K$; d'où la première formule. Pour établir la seconde, associons à chaque place $p_{k}$ de $K$, qui nlappartient pas à $S$, et dont les prolongements à $L$ sont non complexes, le produit $P_{L}=\prod_{L} p_{L}$ des places de $L$ qui sont au-dessus. Le groupe étendu $a_{L / K} S_{L}\left(D_{K}^{S}\right)$ est engendré par les diviseurs de laforme $P_{L}$, et le groupe ambige $D_{L}^{S G}$ par les $P_{L}$; d'où la seconde formule.

COROLLAIRE 111.1.7.- Soient $L / K$ une extension galoisienne de corps de nombres, de groupe $G$, et $S$ un ensemble fini de places.

(i) Si L/K est S-ramifiée (i.e. non ramifiée en-dehors de $S$ ), le noyau de l'homomorphisme $j_{L / K}^{S}$, du groupe $C_{L}^{S}$ des $S$-classes de diviseurs de $K$ dans le sous-groupe ambige $C_{L}^{S G}$ de celuide $L$, contient le groupe $H^{1}\left(G, E_{L}^{S}\right)$; et son conoyau est un sous-groupe de $H^{2}\left(G, E_{L}^{S}\right)$.

(ii) En particulier si L/K est simultanément $S$-ramifiée et $S$-complexifiée (i.e. non ramifié aux places finies en-dehors de $S$, et non complexifiée aux places réelles en-dehors de $S$ ), le groupe $H^{1}\left(G, E_{L}^{S}\right)$ mesure exactement la $S$-capitulation :

$$
\operatorname{Cap}_{L / K}^{S}=\operatorname{Ker} j_{L / K}^{S} \simeq H^{1}\left(G, E_{L}^{S}\right) \text {. }
$$


Ce dernier résultat est l'une des informations les plus fortes que nous possédions sur la $\mathrm{S}$-capitulation. Cela dit, le groupe de co homologie $H^{1}\left(G, E_{L}^{S}\right.$ ) est, en général, très mal connu. C'est, par exemple un problème ouvert que de décider si son ordre est au moins égal au degré de l'extension $L / K$ lorsque celle-ciest non ramifiée partout et complètement décomposée aux places de $S$ comme aux places réelles. Dans le cas cyclique, la réponse est affirmative, ce qui gé néralise le théorème 94 de Hilbert ( cf. proposition 10, ci-dessous). A l'opposé, si L est l'extension abélienne maximale de $K$ qui est non ramifiée et $\mathrm{S}$-décomposée, une généralisation facile du théorème d'Artin-Fürtwangler (cf. [Fu]) permet d'affirmer que les S-classes de diviseurs de $K$ capitulent dans $L$; autrement dit que l'on a alors exactement :

$$
\left|H^{1}\left(G, E_{L}^{S}\right)\right|=\left|\operatorname{Cap}_{L / K}^{S}\right|=\left|C_{K}^{S}\right|=[L: K] \text {. }
$$

c.- Analyse de la formule dans le cas cyclique .

Supposons maintenant que $L / K$ soit une extension cyclique ; notons $\mathrm{g}$ un générateur de son groupe de Galois $\mathrm{G}$, et $N=\sum_{\mathrm{i}=1}^{[\mathrm{L}: \mathrm{K}]} \mathrm{g}^{\mathrm{i}}$ l'opérateur norme. Dans ce cas, le groupe de cohomologie $H^{2}\left(G, L^{X}\right)$ n'est autre que le quotient $K^{x} / N\left(L^{x}\right)$, et la suite exacte des S-clas ses ambiges prend la forme:

$$
\begin{array}{r}
1 \rightarrow E_{L}^{S G} / E_{K}^{S} \rightarrow \operatorname{Ker} d_{L / K}^{S} \rightarrow \operatorname{Ker} j_{L / K}^{S} \rightarrow H^{1}\left(G, E_{L}^{S}\right) \rightarrow \text { Coker } d_{L / K}^{S} \rightarrow \text { Coker } j_{L / K}^{S} \\
\rightarrow H^{2}\left(G, E_{L}^{S}\right) \rightarrow E_{L G}^{S G} / E_{L}^{S G} \cap N\left(L^{X}\right) \rightarrow 1 .
\end{array}
$$

Dans celle-ci, nous pouvons encore remplacer $E_{L}^{S G} \cap N\left(L^{x}\right)$ par $E_{K}^{S} \cap N\left(L^{X}\right)$. En effet, si le groupe $E_{K}^{S}$ des $S$-unités de $K$ est, en général, contenu strictement dans le sous-groupe invariant $E_{L} S$ de $E_{L}^{S}$ (tout simplement parce que la condition de positivité aux places réelles non contenues dans $S$, qui est requise dans $K$, n'existe pas dans $L$ s'il y a complexification), cette distinction n'intervient pas lorsquion travaille sur des normes : Si p est une place réelle de $K$ qui se complexifie dans $L$, lidentité $N_{\mathbb{C} / \mathbf{R}}\left(\mathbb{C}^{X}\right)=\mathbf{R}_{+}^{X}$ montre que les normes locales en $p$ ( et donc, a fortiori, en normes globales) sont posi tives dans $K_{p}$. II vient donc: 


$$
\frac{\left|C_{L}^{S G}\right|}{\left|a_{K}^{S}\right|}=\frac{\mid \text { Coker } j_{L / K}^{S} \mid}{\left|\operatorname{Ker} j_{L / K}^{S}\right|}=\frac{\mid \text { Coker } a_{L / K}^{S} \mid}{\left|\operatorname{Ker} a_{L / K}^{S}\right|} \frac{\left(E_{L}^{S G}: E_{K}^{S}\right)}{\left(E_{L}^{S G}: E_{K}^{S} \cap N\left(L^{X}\right)\right.} \frac{\left|H^{2}\left(G, E_{L}^{S}\right)\right|}{\left|H^{1}\left(G, E_{L}^{S}\right)\right|}
$$

Dans cette formule, les ordres du noyau et du conoyau de llapplication $d_{L}^{S} / K$ sont donnés par la proposition 6 , et llindice normique $\left(E_{K}^{S}: E_{K}^{S} \cap N\left(L^{X}\right)\right.$ peut se calculer à l'aide des symboles de Hasse, comme expliqué plus loin dans la section 2 . Venons en donc au quotient de Herbrand :

$$
q\left(G, E_{L}^{S}\right)=\frac{\left|H^{2}\left(G, E_{L}^{S}\right)\right|}{\left|H^{1}\left(G, E_{L}^{S}\right)\right|}
$$

Nous savons quili est multiplicatif, ce qui permet de le calculer par dévissage. Formons pour cela la suite exacte canonique (où $P_{L}(S$ ) est le groupe des diviseurs principaux de $L$ engendrés par les $S$-unités):

Nous obtenons immédiatement :

$$
1 \longrightarrow E_{L} \longrightarrow E_{L}^{S} \longrightarrow P_{L}(S) \longrightarrow 1 \text {. }
$$

$q\left(G, E_{L}^{S}\right)=q\left(G, E_{L}\right) \cdot q\left(G, P_{L}(S)\right)=q\left(G, E_{L}^{\text {ord }}\right) \cdot q\left(G, D_{L}(G)\right)$,

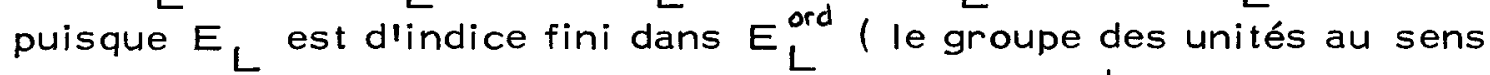
ordinaire) et $P_{L}(S)$ dans $D_{L}(S)$. Et comme $q\left(G, E_{L}^{\text {ord }}\right.$ ) est connu depuis Herbrand pour être égal au quotient $\left(\prod_{p \mid \infty} d_{p}(L / K)\right) /[L: K]$ de la complexification par le degré de l'extension ( résultat qui est le coeur même de la démonstration de Chevalley), seul reste à évaluer le se cond facteur $q\left(G, D_{L}(S)\right)$. Nous avons :

PROPOSITION 111.1.8. - Dans une extension cyclique de corps de nombres $L / K$, de groupe de Galois $G$, le quotient de Herbrand $q\left(G, D_{L}(S)\right)$ du groupe des diviseurs construits sur les places de $S$ est le produit des degrés locaux $d_{p}(L / K)=\left[L_{P} / K_{p}\right]$ attachés auxplaces non réelles de $S$ :

$$
q\left(G, D_{L}(S)\right)=\prod_{p \in S ; p \gamma_{\infty}} d_{p}(L / K)=p \in S ; p \prod_{\infty}\left[L_{p} ; K_{p}\right] .
$$

En particulier, le quotient de Herbrand du groupe des S-uni tés est donné par la formule:

$$
q\left(G, E_{L}^{S}\right)=\frac{p \in S_{i p \gamma_{\infty}}^{d} p^{(L / K)} p \prod_{\infty} d_{p}(L / K)}{[L: K]}
$$


Démonstration: Nous avons $H^{1}\left(G, D_{L}(S)\right)=1$, d'après le lemme 4 ; et, avec les notations de la proposition 6 :

$H^{2}\left(G, D_{L}(S)\right)=\left(D_{L}(S)^{G}: N\left(D_{L}(S)\right)\right)=\prod_{p \in S ; p \gamma_{\infty}}\left(P_{L}^{\mathbb{Z}}: P_{L}^{d}{ }^{(L / K) \mathbb{Z}}\right)$, comme attendu .

Récapitulant ce qui précède, nous pouvons donc énoncer :

THÉORÈME III.1.9.- (Formule des S-classes ambiges) - Dans une extension cyclique $L / K$ de corps de nombres, de groupe de Galois $G$, le nombre de $S$-classes ambiges de diviseurs est donné par la formule:

$$
\left|a_{L}^{S G}\right|=\left|C l_{K}^{S}\right| \frac{\prod_{S}{ }^{d} p^{(L / K)} \prod_{p \notin S} p^{(L / K)}}{[L: K]\left(E_{K}^{S}: E_{K}^{S} \cap N\left(L^{X}\right)\right.},
$$

où $E_{K}^{S}$ est le groupe des S-unités du corps $K ; d_{p}(L / K)$ le degré de l'extension locale $L_{p} / K_{p}$ associée à la place $p$, et e $p_{p}(L / K) \quad$ l'indice de ramification de cette extension (avec la convention e $p(L / K)=1$, lorsque $p$ est archimédienne).

Remarque.- Sur la formule obtenue, il est loin d'être évident que la quantité $\mid \mathrm{Cl}_{L}^{S G}$ | soit une fonction décroissante de $S$. De fait, nous avons $\left.\left|d_{L}^{S G}\right|_{L}^{L}\right|_{S^{\prime}} ^{G} d_{L}^{S} \mid$, puisque $G$ est cyclique, et, si $S^{\prime}$ contient $S$, le groupe ${ }^{G} C l_{L}^{S^{\prime}}$ est canoniquement un quotient de ${ }^{G} C_{L}^{S}$.

Applications - (i) Prenons $S$ vide, la formule des classes ambiges s'écrit alors :

$$
\left|C l_{L}^{G}\right|=\left|C l_{K}\right| \frac{\prod_{p} e_{p}(L / K)}{[L: K]\left(E_{K}: E_{K} \cap N\left(L^{X}\right)\right)} \text {. }
$$

Elle exprime le nombre de classes ambiges de diviseurs (i.e. le nom bre de classes ambiges d'idéaux au sens restreint) de $L$ en fonction du nombre de classes de diviseurs de $K$, de la ramification ( aux places finies ) dans $L / K$, du degré de l'extension, et de llindice normique associé aux unités ( au sens des diviseurs). Clest la formule donnée par Gras lorsque $[L: K]$ est premier ( cf. $\left[G r_{1}\right]$ ). 
(ii) Prenons pour $S$ l'ensemble des places réelles de $\mathrm{K}$; la formule s'écrit :

$$
\left|\alpha_{L}^{\text {ord }}\right|=\left|\alpha_{K}^{\text {ord }}\right| \frac{\prod_{p} e_{p}(L / K) \cdot \prod_{p} d_{p}(L / K)}{[L: K]\left(E_{K}^{\text {ord }}: E_{K}^{\text {ord }} \cap N\left(L^{x}\right)\right)} .
$$

Elle exprime le nombre de classes ambiges d'idéaux (au sens ordi naire) de $L$, en fonction du nombre de classes d'idéaux de $K$, de la ramification ( aux places finies) et de la complexification (aux places réelles ) dans $L / K$, du degré de l'extension, et de l'indice normique associé aux unités ordinaires (i.e. au sens des idéaux); c'est, aux notations près, la formule de Chevalley.

COROLLAIRE 111.1.10.- ( Théorème 94 de Hilbert généralisé ) - Soit $L / K$ une extension cyclique non triviale de corps de nombres. Sup posons $L / K$ non ramifiée (aux places finies) et $S$-décomposée. Alors :

(i) Le groupe $C_{K}^{S}$ des $S$-classes de diviseurs de $K$ n'est pas nul.

dans $\mathrm{Cl}_{\mathrm{L}}^{\mathrm{S}}$.

(ii) Il existe dans $d_{K}^{S}$ une classe non triviale qui capitule Plus précisément, il vient :

$$
\left|\operatorname{Cap}_{L / K}^{S}\right| \geq[L: K] \text {. }
$$

Démonstration : L'extension $L / K$ étant supposée sans ramification , donc S-ramifiée, l'assertion (i) du corollaire 7 nous donne l'inéga lité : $\left|\operatorname{Cap}_{L / K}^{S}\right| \geq\left|H^{1}\left(G, E_{L}^{S}\right)\right|$; et la proposition 8 nous dit que le quotient de Herbrand $q\left(G, E_{L}^{S}\right)=\left|H^{2}\left(G, E_{L}^{S}\right)\right| /\left|H^{1}\left(G, E_{L}^{S}\right)\right|$ est égal à $1 /[L: K]$; d'où le résultat annoncé .

Remarque 1.- Si $K$ possède une extension abélienne $L$, non ramifiée et $S$-décomposée, qui vérifie $C l_{L}^{S}=1$, pour un ensemble fini $S$ de places donné, clest nécessairement l'extension abélienne maximale non ramifiée et $S$-décomposée, disons $F$, de $K$. Dans le cas contraire, en effet, l'extension $F / L$ serait non triviale et, bien entendu, abélienne, non ramifiée, et S-décomposée, contrairement à l'hypothèse $C l_{L}^{S}=1$. En particulier, si $L$ est cyclique sur $K$, le groupe $C_{K}^{S} \simeq \mathrm{Gal}(L / K)$ est lui-même cyclique, d'ordre $[L: K]$. 
Plus généralement, pour chaque sous-extension $K^{\prime} / K$ de $L / K$, le même argument, appliqué à l'extension cyclique $L^{\prime} / K$, montre alors que le groupe $C_{K^{\prime}}^{S}$ est encore cyclique, d'ordre $\left[L: K^{\prime}\right]$. D'un autre côté, l'extension $L / K$ étant non ramifiée et $S$-décomposée, les $S-u n i t e ́ s$ de $K$ sont normes locales partout dans $L / K$, donc normes globales (en vertu du principe de Hasse, puisque $L / K$ est supposée cyclique), donc normes de S-unités de $L$ (d'après la suite exacte des classes ambiges, puisque $C_{L}^{S}$ est nul par hypothèse). A fortiori cette dernière condition est-elle vérifiée dans chacune des sousextensions $K^{\prime} / K$. II vient donc $H^{2}\left(G_{a l}\left(K^{\prime} / K\right), E_{K^{\prime}}^{S}\right)=1$, i.e. $\left|H^{\prime}\left(G a l\left(K^{\prime} / K\right), E_{K^{\prime}}^{S}\right)\right|=\left[K^{\prime}: K\right]$. Autrement dit, la S-capitula tion Cap $K_{K^{\prime} / K}$ dans $K^{\prime} / K$ est d'ordre $\left[K^{\prime}: K\right]$; et le groupe $C l_{K^{\prime}}$, qui a pour ordre $\left[L: K^{\prime}\right]=[L: K] /\left[L: K^{\prime}\right]=\left(C_{K}^{S}: \operatorname{Cap}_{K^{\prime} / K}\right)^{\prime}$, est llimage de $C_{K}^{S}$ par l'homomorphisme d'extension $j_{K 1 / K}$.

Remarque 2.- Lorsque le corps $K$, l'ensemble de places $S$, et le degré de l'extension cyclique $L / K$ sont donnés, le dénominateur [L:K] ( $\left.E_{K}^{S}: E_{K}^{S} \cap N\left(L^{X}\right)\right)$, dans la formule des classes ambiges, est majoré par la quantité $[L: K]\left(E_{K}^{S}: E_{K}^{S[L: K]} \leq(S[K: \mathbb{Q}])^{[L: K]}\right.$, où $S$ est le nombre de places de $\mathbb{Q}$ qui sont soit la place réelle, soit au-dessous dlune place de $S$. En particulier, l'ordre du groupe ambige $d_{L}^{S G}$ est arbitrairement grand avec la ramification. Nous retrouvons ainsi, en le généralisant, le résultat de [BR] .

Une première extension de la formule de Chevalley auxclasses de diviseurs (en fait aux classes d'idéaux prises au sens restreint), dans le cas des extensions cycliques de degré premier, figure dans la thèse de Gras ( $c f_{\bullet}\left[G r_{1}\right], C h . I V, A \& 1$ ), qui étudie également les noyaux d'ordre supérieur $\left(\mathrm{Cl}^{G} ;\left(\mathrm{Cl} / \mathrm{Cl}^{G}\right)^{G}\right.$; etc... $)$. Plus récem ment, Federer a proposé une démonstration de la formule des S-classes ambiges dans le cas cyclique, voisine de celle que nous donnons ici, mais l'idée de considérer des $S$-classes d'idéaux est antérieure puis qu'elle avait déjà été développée par Gillard à l'occasion d'une question de théorie d'lwasawa ( $c f_{\bullet}\left[\mathrm{Gi}_{1}\right]$, appendice), puis par nous-même dans un autre contexte (cf. $\left[\mathrm{Ja}_{1}\right]$ ), et, dans les deux cas, à l'instar de Gras (cf. $\left[\mathrm{Gr}_{2}\right], \S 1$ ), en abordant le problème en termes de repré sentations. 


\section{2. - EXPRESSION DE LA FORMULE EN TERMES DE REPRÉSENTATIONS DANS LE CAS MÉT ABELIEN.}

a.-Préliminaires.

Fixons un nombre premier $\ell$, et intéressons nous désormais au $\ell$-Sylow du groupe des S-classes de diviseurs; convenons, pour ne pas alourdir les notations, de continuer à le noter $\mathrm{Cl}^{S}$. Si L/K est une extension galoisienne de corps de nombres, de degré étranger à $l$, de groupe de Galois $G$, l'élément $e=\frac{1}{[L: K]} \sum_{g \in G} g$ est un idempo tent de l'algèbre $\mathbb{Z}_{\ell}[G]$, de sorte que l'homomorphisme d'extension identifie canoniquement le $\ell$-groupe des $S$-classes $d_{K}^{S}$ à un facteur direct de $C l_{L}^{S}$, et, plus précisément, à son sous-groupe ambige $C_{L}^{S G}$. Le problème de comparer les ordres de $c_{K}^{S}$ et $C_{L}^{S G}$ ne se pose donc que dans la mesure où celui de $G$ est divisible par $l$, c'est-à-dire, en dernière analyse, lorsque $G$ est un $\ell$-groupe .

Nous supposons, dans ce qui suit, que $G$ est cyclique, et nous nous intéressons à la situation suivante : $L$ est une $l$-extension cyclique de $K$, galoisienne sur un sous-corps $F$, et $K / F$ est abé lienne, de degré d étranger avec $\ell$. Sous ces hypothèses, le groupe de Galois Gal (L/F) s'écrit comme produit direct de son $\ell$-sous-groupe de Sylow $G=G a l(L / K)$ et du quotient associé $\Delta=G a l(K / F)$. De plus, le groupe $G$ étant supposé cyclique, l'homomorphisme de $\Delta$ dans Aut $G$, qui définit le produit, se factorise par un caractère $\ell$-adique de $\Delta$, ce qui permet d'écrire

$$
\text { (*) } \operatorname{Tg} \mathrm{T}^{-1}=\mathrm{g}^{X(\mathrm{~T})}, \forall \mathrm{T} \in \Delta \text { et } \mathrm{g} \text { générateur de } \mathrm{G} \text {, }
$$

en faisant choix une fois pour toutes d'un relèvement de $\Delta$ dans Gal (L/F). Ainsi, lorsque $X$ est le caractère unité, le groupe Gal (L/F) est abé lien (c'est la situation étudiée dans $\left[G i_{1}\right]$ et $\left[G r_{2}\right]$ ) . Dans tous les autres cas, le groupe Gal(L/F) est métabélien (clest la situation dé crite dans $\left[\mathrm{Ja}_{1}\right]$ ).

D'un autre côté, le degré d de la sous-extension abélienne $\mathrm{K} / \mathrm{F}$ étant supposé étranger à $\ell$, l'algèbre de Galois $\mathbb{Z}_{\ell}[\Delta]$ est semi-locale, en fait produit direct d'extensions abéliennes non ramifiées de $\mathbb{Z}_{\ell}$ : 
à chaque caractère $\ell$-adique irréductible $\varphi$ du groupe $\Delta$ correspond un idempotent primitif de l'algèbre $\mathbb{Z}_{\mathfrak{l}}[\Delta]$

$$
e_{\varphi}=\frac{1}{d} \sum_{\tau \in \Delta} \varphi(\tau) \tau^{-1}
$$

et la $\varphi$-composante $\mathbf{Z}_{\varphi}=\mathbb{Z}_{\ell}[\Delta] e_{\varphi}$ s'identifie à l'anneau local des entiers d'une extension cyclotomique non ramifiée de $\mathbb{Q}$, qui a pour de gré celui $d_{\varphi}=\left[Z_{\varphi}: \mathbb{Z}_{\ell}\right]$ du caractère $\varphi$. La décomposition obtenue s'étend, bien entendu, à l'algèbre $\mathbb{Z}_{\ell}[\mathrm{Gal}(L / F)]$ regardée comme module sur elle-même, chaque facteur $\mathbb{Z}_{\ell}[\mathrm{Gal}(\mathrm{L} / \mathrm{F})]$ e $\varphi$ étant pro jectif et indécomposable, mais il ne s'agit plus là d'un isomorphisme d'algèbres dès que $\chi$ est non trivial. Pour traduire les relations de commutation $(*)$, il est alors commode d'introduire la résolvante

$$
\theta=\frac{1}{d} \sum_{\tau \in \Delta} x\left(\tau^{-1}\right)\left[g^{X(\tau)}-1\right]
$$

construite sur un générateur g du groupe $G$, dont on vérifie facilement qu'elle engendre lidéal d'augmentation $1_{G}$ de l'algèbre $\mathbb{Z}_{\ell}[G]$. La relation (*) s'écrit alors:

$$
\text { (**) } \tau \theta \tau^{-1}=\chi(\tau) \theta, \quad \forall \tau \in \Delta ;
$$

ce qui se traduit, sur les idempotents primitifs de l'algèbre $\mathbb{Z}_{\ell}$ [G], par une translation des caractères :

$$
(* * *) \text { e } \varphi^{\theta=\theta \text { e }} \varphi x, \quad \forall \varphi \in R \mathbb{Z}_{l}(\Delta) \text {. }
$$

Revenons maintenant à notre sujet : Les divers groupes qui interviennent dans la suite exacte des classes ambiges n'étant pas tous des $\mathbb{Z}_{\ell}$-modules, introduisons les tensorisés respectifs

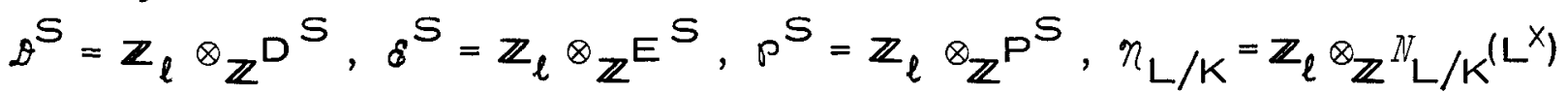

des groupes de $S$-diviseurs, de $S$-unités, de $S$-diviseurs principaux, et de normes. Par un argument de platitude, nous obtenons immédiatement la suite exacte de $\mathbb{Z}_{\ell}$-modules ( ou nous avons conservé par abus les notations $d_{L / K}^{S}$ et $j_{L / K}^{S}$ pour désigner, de ces applications, les restrictions aux $\ell$-Sylow :

$$
\begin{aligned}
& 1 \rightarrow \delta_{L}^{S G} / \delta_{K}^{S} \rightarrow \operatorname{Ker} d_{L / K}^{S} \rightarrow \operatorname{Ker} j_{L / K}^{S} \rightarrow H^{1}\left(G, \theta_{L}^{S}\right) \rightarrow \text { Coker } d_{L / K}^{S} \rightarrow \text { Coker } j_{L / K}^{S} \\
& \rightarrow H^{2}\left(G, \varepsilon_{L}^{S}\right) \rightarrow \delta_{L}^{S G} / \delta_{K}^{S} \cap \eta_{L / K} \rightarrow 1 \text {. }
\end{aligned}
$$

Dans celle-ci, les applications canoniques induites par la norme, 
l'extension, l'inclusion, etc ... , sont évidemment des $\mathbb{Z}_{\ell}[\Delta]-$ mor phismes ; mais il n'en est plus de même pour les isomorphismes d'ori gine cohomologique donnés par le lemme 4 . Convenons, en effet, de repérer par un indice $\varphi$ la composante d'un $\mathbb{Z}_{\ell}[\Delta]$-module attachée à un caractère irréductible $\varphi$ du groupe $\Delta$ (c'est-à-dire l'image de ce module par l'idempotent primitif e $\left.{ }_{\varphi}\right)$. Cela posé, nous avons :

LEMME 111.1.11.- Sous les hypothèses précédentes, il existe des isomorphismes de $\mathbf{Z}_{\ell}$-modules :

$$
\left[P_{L}^{S G} / d_{L / K}^{S}\left(P_{K}^{S}\right)\right]_{\varphi} \simeq H_{\varphi x}^{1}\left(G, \delta_{L}^{S}\right) \text {. }
$$

$$
\begin{aligned}
& {\left[C_{L}^{S G} / d^{S}\left(\delta_{L}^{S G}\right)\right]_{\varphi} \simeq H_{\varphi X}^{1}\left(G, P_{L}^{S}\right)} \\
& \simeq \operatorname{Ker}\left[H_{\varphi x}^{2}\left(G, 8_{L}^{S}\right) \rightarrow H_{\varphi x}^{2}\left(G, \mathbb{Z} \otimes_{\mathbb{Z}^{L}}{ }^{X}\right)\right] .
\end{aligned}
$$

Démonstration :

(i) Soit $(x)$ un S-diviseur principal et invariant par $G$. La résolvante $\theta$ engendrant l'idéal d'augmentation $I_{G}$, l'élément $x{ }^{\theta}$ est alors une $S$-unité qui est annulée par la norme algébrique $\nu=\sum_{g \in G} g$. Comme $x$ est défini à une $S$-unité près, nous réalisons ainsi l'isomor phisme de $p_{L}^{S G} / d_{L / K}^{S}\left(P_{K}^{S}\right)$ sur $H^{1}\left(G, \delta_{L}^{S}\right)$, induit par le lemme 4 . Et le résultat annoncé provient simplement de la translation des caractères donnée par liidentité $(* * *)$.

(ii) Soit a un $S$-diviseur appartenant à une classe ambige . Le diviseur $(x)=a^{\theta}$ est alors S-principal et annulé par la norme arithmétique. Comme a est défini à un $S$-diviseur principal près, nous réalisons ainsi liisomorphisme de $\alpha_{L}^{S G} / d^{S}\left(\theta_{L}^{S G}\right)$ sur $H^{1}\left(G, p_{L}^{S}\right)$ consi déré dans la démonstration de la suite exacte des classes ambiges. Comme plus haut, la factorisation par la résolvante $\theta$ se traduit par une translation des caractères. Cela dit, lisomorphisme de $H^{1}\left(G, P_{L}^{S}\right)$ sur $\operatorname{Ker}\left[H^{2}\left(G, \varepsilon_{L}^{S}\right) \rightarrow H^{2}\left(G, Z \otimes \otimes_{\mathbb{Z}} L^{X}\right)\right]$ s'obtient tout simplement en asso ciant à la classe du diviseur $(x)$ dans le premier groupe celle de la $\mathbf{S}$ unité $N_{L / K}(x)$ dans le second; c'est un isomorphisme de $\mathbb{Z}_{\ell}[\Delta]$ modules.

\section{b.- Enoncé des résultats.}

Distinguons deux cas, suivant la parité de $\ell$ : 
1) Si $\ell$ vaut 2 , le groupe Aut $G$ est un 2 -groupe, et les hypothèses faites impliquent que $L$ soitune extension abélienne de $F$. Il vient donc:

THÉORËME III.1.12. - Soient L une 2-extension cyclique d'un corps de nombres $K$, de groupe $G$, abélienne sur un sous-corps $F$ de $K$ d'indice $[F: K]$ impair, et $S$ un ensemble fini de places de $F$. Alors, pour chaque caractère 2 -adique irréductible $\varphi$ du groupe $\Delta=\mathrm{Gal}(\mathrm{K} / \mathrm{F})$, les $\varphi$-composantes du noyau et du conoyau de I'homomorphisme d'extension $j_{L / K}^{S}$ du 2 -groupe des $S$-classes de diviseurs $C e_{K}^{S} d u$ corps $K$, dans le sous-groupe ambige $C_{L}^{S G}$ de celui de $L$, sont liées par la suite exacte de $\mathbb{Z}_{2}$-modules :

$$
\begin{aligned}
1 \rightarrow\left(\delta_{L}^{S G} / \delta_{K}^{S}\right) \rightarrow \operatorname{Ker}{ }_{\varphi} d_{L / K}^{S} & \rightarrow \text { Ker }{ }_{\varphi}^{j} j_{L / K}^{S} \rightarrow H_{\varphi}^{1}\left(G, \delta_{L}^{S}\right) \rightarrow \text { Coker } d_{L / K}^{S} \\
& \rightarrow \text { Coker } \varphi_{L}^{j}{ }_{L}^{S} / K^{\rightarrow H^{2}}\left(G, \delta_{L}^{S}\right) \rightarrow\left(\delta_{L}^{S G} / \delta_{K}^{S} \cap \eta_{L} / K{ }_{\varphi}^{\prime} \rightarrow 1 .\right.
\end{aligned}
$$

En particulier, la $\varphi$-partie du 2 -nombre de $S$-classes am biges est donnée par la formule:

$$
\frac{\left|C e_{L, \varphi}^{S G}\right|}{\left|C e_{K, \varphi}^{S}\right|}=\frac{\mid \text { Coker } \varphi_{L / K}^{S} \mid}{\left|\operatorname{Ker}_{\varphi} j_{L / K}^{S}\right|}=\frac{\mid \text { Coker } d_{L / K}^{S} \mid}{\mid \text { Ker } d_{L / K}^{S} \mid} \frac{q_{\varphi}^{\left(G, \varepsilon_{L}^{S}\right)}}{\left(\delta_{K}^{S}: \varepsilon_{K}^{S} \cap \eta_{L / K}\right)_{\varphi}} \text {, }
$$

où $q_{\varphi}\left(G, \varepsilon_{L}^{S}\right)$ est la $\varphi$-partie du 2 -quotient de Herbrand du groupe des S-unités.

2) Si $\ell$ est impair, iln'y a plus lieu de distinguer entre classes dijdéaux et de diviseurs. Il vient donc :

THÉORĖME III.1.13.- Soient $L$ une $\ell$-extension cyclique diun corps $K$, de degré impair, de groupe $G$, métabélienne sur un sous-corps $F$ de $K$ d'indice relatif $[K: F]$ étranger à $\ell$, puis $X$ le caractère du groupe de Galois $\Delta=G a l(K / F)$ qui factorise le produit semi-direct $G \times \Delta$, et $S$ un ensemble fini de places de $F$. Alors, pour chaque caractère $\ell$-adique irréductible $\varphi$ du groupe $\Delta$, les $\varphi$-composantes du noyau et du conoyau et du conoyau de l'homomorphisme d'extension $j_{L}^{S} / K d u l-$ groupe des classes diidéaux $C_{K}^{S}$ de $K$ dans le sous-groupe ambige $C \ell_{L}^{S G}$ de celui de $L$, sont liés par la suite exacte courte de $\mathbb{Z}_{\ell}$-mo dules: 


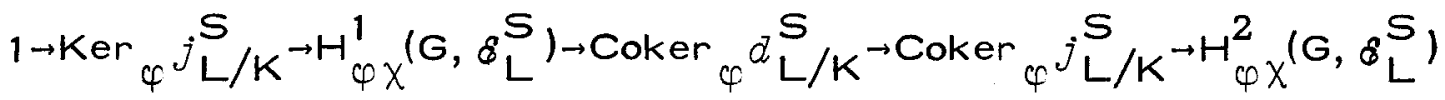

$$
\begin{aligned}
& \rightarrow\left(\varepsilon_{K}^{S} / \varepsilon_{K}^{S} \cap \eta_{L / K}\right)_{\varphi X} \rightarrow 1 \text {. }
\end{aligned}
$$

En particulier, la $\varphi$-partie du $\ell$-nombre de $S$-classes am bige est donnée par la formule:

$\frac{\left|C_{L}^{S G}, \varphi\right|}{\left|C_{K}^{S}, \varphi\right|}=\frac{\mid \text { Coker }_{\varphi} j_{L / K}^{S} \mid}{\left|\operatorname{Ker}_{\varphi} j_{L / K}^{S}\right|}=\frac{\mid \text { Coker } \varphi^{d}{ }_{L / K}^{S} \mid}{\left(\delta_{K}^{S}: \varepsilon_{K}^{S} \cap \eta_{L / K}{ }^{S} \varphi x\right.} q_{\varphi x}{ }^{\left(G, \varepsilon_{L}^{S}\right)}$,

où $q \varphi \chi^{\left(G, \delta_{L}^{S}\right)}$ est la $\varphi X$-partie du $\ell$-quotient de Herbrand du groupe des S-unités.

SCOLIE III.1.14. - Pour chaque place $p$ de $F$, désignons par $\chi_{p}$ l'induit à $\Delta$ du caractère de la représentation unité de son sous-groupe de décomposition $\Delta_{p}$ dans l'extension abélienne $K / F$, notons $e_{p}(L / K)$ l'indice de ramification associé à $p$ dans la $\ell$-extension cyclique $L / K$, et $d_{p}(L / K)$ le degré de l'extension locale correspondante. Alors, pour chaque caractère $\ell$-adique irréductible $\varphi$ de $\Delta$, les $\varphi$-parties du noyau et du conoyau de l'homomorphisme d'extension des diviseurs sont donnés par les formules:

$$
\begin{array}{ll} 
& \left|\operatorname{Ker}_{\varphi} d_{L / K}^{S}\right|=\prod_{p \mid \infty ; p \notin S}{ }^{S} d_{p}(L / K)^{\left\langle\varphi, x_{p}\right\rangle} \\
\varepsilon & \mid \text { Coker }{ }_{\varphi}{ }_{L / K}^{S} \mid=\prod_{p \notin S} e_{p}(L / K)
\end{array}
$$

Démonstration : Le quotient $\Delta / \Delta_{p}$ opérant facilement sur les places de $K$ au-dessus de $p$, le sous-module de $\theta_{K}$ engendrées par celles-ci est donc isomorphe à $\mathbb{Z}_{\ell}\left[\Delta / \Delta_{p}\right]$, si $p$ est ultramétrique, et à $\mathbb{Z}_{\ell} \otimes_{\mathbb{Z}} \mathbb{F}_{2}\left[\Delta / \Delta_{p}\right]$, sinon. Dans le premier cas, la $\varphi$-partie de ce module est un $\mathbb{Z}_{\ell}$-module libre de dimension $\left\langle\varphi, \chi_{p}\right\rangle$; dans le second cas, et si $l$ vaut 2 , clest un $\mathbb{F}_{2}$-espace vectoriel de dimension $\left\langle\varphi, x_{p}\right\rangle$; et, dans llun et l'autre, nous concluons comme dans le lemme 6 .

SCOLIE 111.1.15.- Conservons les notations précédentes. Alors, pour chaque caractère $\ell$-adique $\Phi$ du groupe $\Delta$, contenu dans le caractère régulier, et stable par translation de $\chi(i . e$. vérifiant $\Phi \chi=\Phi)$, la 
$\Phi$-composante du $\ell$-quotient de Herbrand du groupe des S-unités est donnée par la formule :

$q_{\Phi}\left(G, \delta_{L}^{S}\right)=[L: K]^{-\langle\Phi, 1\rangle} \cdot \prod_{p X_{\infty} ; p \in S^{d}} d_{p}(L / K)^{\left\langle\Phi, X_{p}\right\rangle} \cdot \prod_{p \mid \infty} d_{p}(L / K)^{\left\langle\Phi, X_{p}\right\rangle}$.

Démonstration: D'après $(* * *)$, la condition de stabilité sur le carac tère $\Phi$ signifie que l'idempotent associé e ${ }_{\Phi}=\frac{1}{\mathrm{~d}} \sum_{T \in \Delta} \Phi\left(\tau^{-1}\right)_{T}=\sum_{\varphi \mid \Phi}$ e $\varphi$ est central dans l'algèbre de Galois $\mathbb{Z}_{\ell}[\mathrm{Gal}(\mathrm{L} / \mathrm{F})]$. Lorsque c'est le cas, la $\Phi$-partie du $\ell$-quotient de Herbrand des $S$-unités n'est autre que le $\ell$-quotient de Herbrand de la $\Phi$-composante du groupe des $S$ unités, qui se calcule par la méthode de la proposition. Il vient donc :

puis

$$
q_{\Phi}\left(G, \varepsilon_{L}^{S}\right)=q\left(G, \varepsilon_{L, \Phi}^{S}\right)=q\left(G, \varepsilon_{L}, \Phi\right) \cdot q\left(G, \theta_{L}, \Phi(S)\right) \text {, }
$$

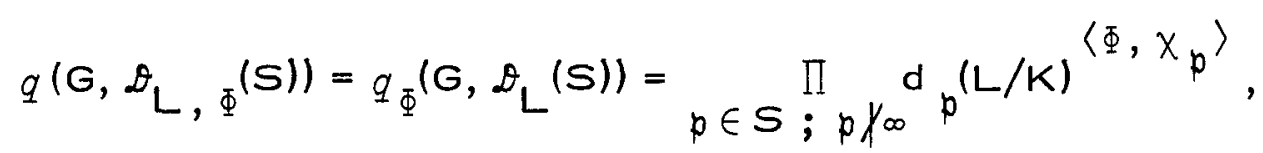

par une généralisation immédiate des résultats de la proposition 8 . D'un autre côté, le lemme de Herbrand sur les unités d'un corps de nombres algébriques affirmant l'existence d'une suite exacte de $\mathbb{Z}_{\ell}[G a l(L / F)]$-modules ( où $M$ est un module fini, et $G_{p} \times \Delta_{p}$ le sous groupe de décomposition dans $L / F$ de l'une quelconque des places de $L$ au-dessus de $p$ ) :

$$
1 \longrightarrow \mathbb{Z}_{\ell} \longrightarrow \underset{p \mid \infty}{\oplus} \mathbb{Z}_{\ell}\left[G \times \Delta / G_{p} \times \Delta_{p}\right] \longrightarrow \varepsilon_{L} \longrightarrow M \longrightarrow 1
$$

nous en déduisons par localisation l'existence d'une suite exacte de $\mathbb{Z}[G]$-modules :

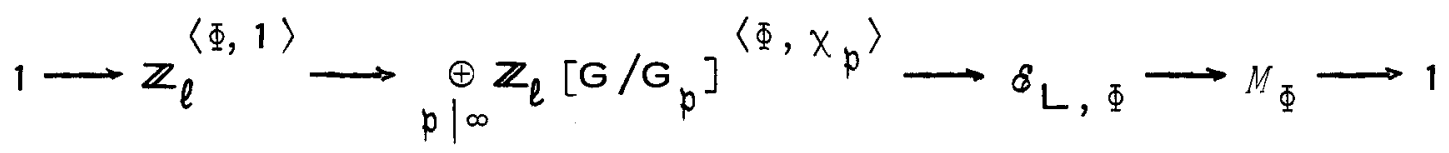

où $M_{\Phi}$ est encore un module fini, ce qui nous donne, comme attendu :

$$
\begin{aligned}
& q\left(G, \varepsilon_{L, \Phi}\right)=\frac{\prod_{\infty} q\left(G, \mathbb{Z}_{\ell}[G / G)_{p}\right]\left\langle\Phi, \chi_{p}\right\rangle}{q(G, \mathbb{Z})^{\langle\Phi, 1\rangle}} \\
& =\left\{\begin{array}{c}
1 /|G|^{\langle\Phi, 1\rangle}, \text { si } l \text { est impair } \\
\frac{\prod_{\infty} d_{p}(L / K)^{\left\langle\Phi, \chi_{p}\right\rangle}}{|G|^{\langle\Phi, 1\rangle}} \text {, si \& vaut } 2 . \\
-185-
\end{array}\right. \text {. }
\end{aligned}
$$


Remarque. - On prendra garde que le calcul qui précède suppose effectivement que le caractère $\Phi$ est associé à un idempotent central de l'al gèbre de Galois . Lorsque ce n'est pas le cas, le groupe $\varepsilon_{L}^{S}$, $\Phi^{\text {n'est }}$ plus généralement un G-module, et l'étude numérique de cas particu liers laisse augurer que le comportement du quotient $q_{\Phi}\left(G, \varepsilon_{L}^{S}\right.$ ) est imprévisible, car il met en jeu de façon extrêmement diophantienne l'arithmétique des classes et des unités.

COROLLAIRE 111,1.16.- Dans une $\ell$-extension cyclique de corps de nombres $L / K$, métabélienne sur un sous-corps $F$, l'ordre de la $\Phi-$ composante du $\ell$-groupe des $S$-classes ambiges est donné, avec les notations du scolie 14 , et pour chaque caractère central $\Phi$ de l'algè bre de Galois, par la formule :

$$
\left|C e_{L, \Phi}^{S G}\right|=\left|C e_{K, \Phi}^{S}\right| \frac{\prod_{K} S_{p}(L / K)^{\left\langle\Phi, x_{p}\right\rangle} \prod_{p \in S} d_{p}(L / K)^{\left\langle\Phi, X_{p}\right\rangle}}{[L: K]^{\langle\Phi, 1\rangle}\left(\varepsilon_{K, \Phi}^{S}, \delta_{K, \Phi}^{S} \cap \eta_{L / K}\right)} .
$$

c.- Application à la capitulation .

Supposons maintenant que l'ensemble $S$ contienne les places réelles de $F$ qui se complexifient dans $L / K$. Le groupe $H_{\Phi}^{1}\left(G, \varepsilon_{L}^{S}\right.$ ) gouverne alors la $\Phi$-partie de la capitulation ( puisque $C_{a p} S_{L / K}$ s'iden tifie dans ce cas au noyau de l'application $\left.H_{\Phi}^{1}\left(G, \delta_{L}^{S}\right) \longrightarrow \operatorname{Ker} d_{L}^{S} / K\right)$. Comme le quotient de Herbrand $q_{\Phi}\left(G, \varepsilon_{L}^{S}\right)$ est parfaitement explicite, le calcul de l'ordre $\left|H_{\Phi}^{l}\left(G, \delta_{L}^{S}\right)\right|$ se ramène à celui de l'ordre $\left|H_{\Phi}^{2}\left(G, \delta_{L}^{S}\right)\right|$, que I'on peut encadrer à partir de linclusion immé diate :

$$
E_{K}^{S}[L: K] \subset N_{L / K}\left(E_{L}^{S}\right) \subset E_{K}^{S} \cap N_{L / K}\left(L^{x}\right) \text {. }
$$

Désignons, en effet, par $\bar{S}$ la réunion de $S$ et des places à l'infini du corps $F$. D'un côté, le groupe $\varepsilon_{K}^{S}$, $\Phi$ est composé direct d'un $\mathbb{Z}_{\ell}$-module libre de dimension $\left\langle\sum_{p \in S} X_{p}-1, \Phi\right\rangle$, et de la $\Phi$-composante $\mu_{K, \Phi}$ du $\ell$-Sylow $\mu$ du groupe des racines de l'unité dans $K$; lin $\operatorname{dice}\left(\varepsilon_{K, \Phi}^{S}: \varepsilon_{K, \Phi}^{S}[L: K]\right)$ est donc parfaitement explicite. D'un autre 
côté, la détermination du groupe $\delta_{K}^{S}, \Phi \cap \eta_{L / K}$ relève de méthodes locales ( le fait d'être norme dans une extension cyclique se lisant loca lement), et l'indice ( $\left.\delta_{K, \Phi}^{S}: \delta_{K, \Phi}^{S} \cap n_{L / K}\right)$ est donc toujours calculable dans la pratique, dès que $\hat{\delta}_{K}$ est connu numériquement. En uti lisant le théorème de Grumwald-Wang ( cf. [AT], Ch. X, \&2 ), il est même possible de construire des extensions cycliques $L / K$, de degré premier, qui sont non ramifiées, et où l'indice $\left(\varepsilon_{K}: \varepsilon_{K} \cap \eta_{L / K}\right)$ est arbitrairement grand, ce qui revient à dire, compte-tenu des en cadrements précédents, que la capitulation y est arbitrairement grande $\left.{ }^{(}\right)$.

Inversement, si le caractère $\Phi$ n'est pas représenté dans le groupe $\delta_{K}^{S}\left(i . e . s i \delta_{K}^{S}, \Phi\right.$ est sans torsion, et si la quantité $\sum_{p \in \bar{S}} X_{p}-1, \Phi>$ est nulle) les calculs qui précèdent montrent que le groupe $\operatorname{Cap}_{L / K}^{S}, \Phi$ est trivial, c'est-à-dire que la restriction aux $\Phi-$

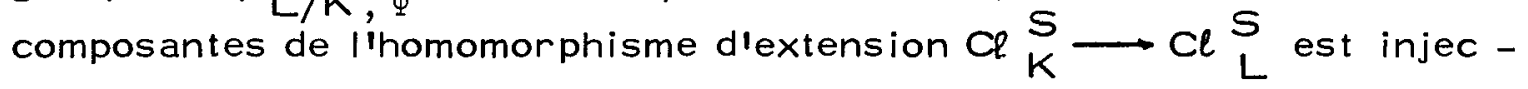
tive. Ce dernier résultat est essentiellement connu dans la situation suivante : $l$ est impair ; $K$ est une extension quadratique totalement imaginaire d'un sur-corps totalement réel de $F$; l'ensemble $S$ est vide ( ou réduit aux places à l'infini ; ce point étant ici sans importance du fait de l'imparité de $\ell$ ) ; et $\Phi$ est un caractère imaginaire de $\Delta$ (i.e. un ca ractère $\ell$-adique de $\Delta$ dont les facteurs irréductibles prennent des valeurs négatives sur la conjugaison complexe), ne contenant pas le caractère de l'action de $\Delta$ sur $\mu_{k}$, lorsque $\mu_{k}$ est non trivial. Plus précisément :

PROPOSITION III.1.17.- Soient $L$ une $\ell$-extension cyclique d'un corps de nombres $K$, de degré impair, métabélienne sur un sous-corps $F$, et $\chi$ le caractère $\ell$-adique du groupe de Galois $\Delta=\mathrm{Gal}(\mathrm{K} / \mathrm{F})$ qui défi nit le produit semi-direct $G \times \Delta$. Supposons que $K$ soit une extension quadratique totalement imaginaire d'un sur-corps totalement réel de $F$, et convenons de dire qu'un caractère $\ell$-adique irréductible $\varphi$ de $G$ est réel ou imaginaire suivant qu'il prend une valeur positive ou négative sur la conjugaison complexe. Supposons, en outre, que le caractère $X$ soit réel. Alors:

$\left({ }^{\star}\right)$ On trouvera les détails de la construction de telles extensions dans llarticle de Schipper [Sc]. 
(i) Si le corps $K$ ne contient pas les racines $l$-ièmes de l'unité, la restriction de l'homomorphisme d'extension $j_{L / K}$ à la com posante imaginaire $C_{\ell_{K}}$ du $\ell$-groupe des classes diidéaux de $K$ est une injection .

(ii) Si le $\ell$-Sylow $\mu_{k}$ du groupe des racines de llunité dans $K$ est non trivial, la même conclusion subsiste sous la condition suffisante $H^{1}\left(G, \mu_{L}\right)=1$.

Démonstration : Désignons par $\Phi=$ Ind $\stackrel{\Delta}{\infty}_{\Delta_{\infty}}{ }^{1} \Delta_{\infty}$ l'induit à $\Delta$ du caractère de la représentation unité du sous-groupe de décomposition commun des places réelles de $F$ dans l'extension $K / F$; et notons $\bar{\Phi}=X_{\text {rég }}-\Phi$ le ca ractère supplémentaire. L'entier $\ell$ étant supposé impair, nous avons : $\left|\operatorname{Cap}_{L / K}^{-}\right|=\left|\operatorname{Cap}_{L / K}, \bar{\Phi}\right| \leq\left|H \frac{1}{\Phi}\left(G, \delta_{L}\right)\right|=\left|H^{1}\left(G, \delta_{L}^{-}\right)\right|=\left|H^{1}\left(G, \mu_{L}\right)\right|$, puisque, $\chi$ étant réel, le caractère $\bar{\Phi}$ est invariant par $\chi$. D'où la conclusion.

Le résultat obtenu peut être généralisé grâce à la proposition suivante :

PROPOSITION III.1.18.- Soient, comme plus haut, $L$ une $l$-exten sion cyclique d'un corps de nombres $K$, métabélienne sur un souscorps $F$, et $\Phi$ le caractère $l$-adique du groupe $\Delta=G a l(K / F)$ attaché à un idempotent central $e_{\Phi}$ de l'algèbre de Galois. Supposons donnés un ensemble fini $S$ de places de F ( contenant les places réelles complexifiées dans $L / K$, si $l$ vaut 2 ), et $S^{\prime}$ réunion de $S$ et d'un ensemble fini de places de $F$ ne présentant pas de $\Phi$-décomposition dans $L / K$ (i.e. pour lesquelles la $\Phi$-partie $g_{p}(L / K)^{\left\langle\Phi, X_{p}\right\rangle}$ de l'indice de décomposi tion $g_{p}(L / K)=[L: K] / d_{p}(L / K)$ est égale à 1$)$. Alors l'ordre $d u$ groupe de cohomologie $H_{\Phi}^{1}\left(G, \varepsilon_{L}^{S^{\prime}}\right)$ divise celui du groupe $H_{\Phi}^{1}\left(G, \varepsilon_{L}^{S}\right)$. En particulier, si $H_{\Phi}^{1}\left(G, \varepsilon_{L}^{S}\right)$ est nul, $H_{\Phi}^{1}\left(G, \varepsilon_{L}^{S^{\prime}}\right)$ l'est aussi, et la restriction aux $\Phi$-composantes de l'homomorphisme d'extension $\mathrm{Cl} \mathrm{S}_{\mathrm{K}}^{\mathrm{S}^{\prime}} \rightarrow \mathrm{Cl} \mathrm{S}_{\mathrm{L}}^{\prime}$ est injective. 
Démonstration : Formons le quotient

$$
\frac{\left|H_{\Phi}^{1}\left(G, \delta_{L}^{S}\right)\right|}{\left|H_{\Phi}^{1}\left(G, \varepsilon_{L}^{S^{\prime}}\right)\right|}=\frac{\left|H_{\Phi}^{2}\left(G, \delta_{L}^{S}\right)\right|}{\left|H_{\Phi}^{2}\left(G, \varepsilon_{L}^{S^{\prime}}\right)\right|} \frac{q_{\Phi}\left(G, \delta_{L}^{S^{\prime}}\right)}{q_{\Phi}\left(G, \delta_{L}^{S}\right)} \text {. }
$$

Le premier facteur s'écrit encore

$$
\frac{\left|H_{\Phi}^{2}\left(G, \delta_{L}^{S}\right)\right|}{\left|H_{\Phi}^{2}\left(G, \varepsilon_{L}^{S^{\prime}}\right)\right|}=\frac{\left(\varepsilon_{\mathrm{K}}^{S}: N\left(\varepsilon_{L}^{S}\right)\right)_{\Phi}}{\left(\varepsilon_{\mathrm{K}}^{S^{\prime}}: N\left(\varepsilon_{\mathrm{L}}^{S^{\prime}}\right)\right)_{\Phi}}=\frac{\left(\varepsilon_{\mathrm{K}}^{S} \cap N\left(\varepsilon_{\mathrm{L}}^{S^{\prime}}\right): N\left(\varepsilon_{\mathrm{L}}^{S}\right)\right)_{\Phi}}{\left(\varepsilon_{\mathrm{K}}^{S^{\prime}}: \varepsilon_{\mathrm{K}}^{S} N\left(\delta_{\mathrm{K}}^{S^{\prime}}\right)\right)_{\Phi}} ;
$$

c'est donc un multiple entier de la quantité fractionnaire

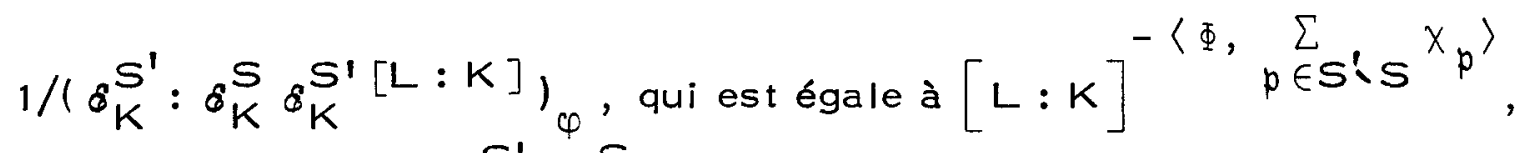
puisque le quotient $\delta_{K}^{S^{\prime}} / \delta_{K}^{S}$ est un $\mathbb{Z}_{\ell}$-module libre de dimension $\left\langle\Phi, \sum_{p \in S^{\prime} S} X_{p}\right\rangle$. Enfin, le second facteur est donné par le scolie 15 ; et il vaut précisément $[L: K]^{\left\langle\Phi, \sum_{p} \in S^{\prime} S^{\left.X_{p}\right\rangle}\right.}$, ce qui donne bien le résultat attendu.

\section{3. - EXTENSION DES RÉSULTATS DU CAS PROCYCLIQUE.}

Nous conservons les hypothèses de la section précédente, à ceci près que nous supposons désormais que $L / K$ est, non plus une extension cyclique de corps de nombres, mais une $\mathbb{Z}_{\ell}$-extension $\left.{ }^{(}\right)$. Suivant l'usage, nous notons $\Gamma$ son groupe de Galois, que le choix d'un générateur topologique $y$ identifie au groupe additif des entiers $\ell$ adiques:

$$
\Gamma=\gamma^{\mathbb{Z}_{l}}
$$

Nous imposons toujours à L d'être métabélienne sur un sous corps $F$ de $K$, de degré relatif $d=[L: F]$ étranger à $l$, et nous

${ }^{\star}$ ) Clest dans $\left[\mathrm{Ja}_{4}\right]$ que nous avons développé pour la première fois l'idée d'écrire la formule des classes ambiges dans une $\mathbf{Z}_{\ell}$-extension. Ultérieurement, Iwasawa $\left[\mathrm{IW}_{6}\right]$ a proposé une autre approche de ce résultat 
notons $x$ le caractère $\ell$-adique du groupe abélien $\Delta=$ Gal (K/F) qui définit le produit semi-direct $\Gamma \times \Delta$; c'est-à-dire que nous écrivons :

$$
\tau \gamma \tau^{-1}=\gamma^{X\left({ }^{\tau}\right)}, \quad \forall \tau \in \Delta \text {, }
$$

en faisant choix une fois pour toutes d'un relèvement de $\Delta$ dans Gal (L/F) . La résolvante

$$
\theta=\frac{1}{\mathrm{~d}} \sum_{\tau \in \Delta} X\left(\tau^{-1}\right)\left[\gamma^{X(\tau)}-1\right]
$$

engendre alors l'idéal d'augmentation de l'algèbre d'lwasawa $\Lambda=\mathbb{Z}_{\ell}[[\gamma-1]]$, ce qui permet d'écrire directement $\Lambda=\mathbb{Z}_{\ell}[[\theta]]$, tout en conservant les relations de commutation:

$$
\theta_{\varphi}=e_{\varphi x}^{\theta}
$$

pour chaque idempotent primitif e $\varphi_{\varphi}$ de llalgèbre semi-locale $\mathbb{Z}_{\ell}[\Delta]$.

a.-La formule de Chevalley pour une $\mathbb{Z}_{\ell}$-extension

Pour chaque naturel $n$, désignons par $\Gamma_{n}$ l'unique sous-groupe fermé dindice $l^{n}$ dans $r$, et notons $K_{n}$ son corps des invariants. Comme $L$ est la réunion des $K_{n}$, le groupe de Galois $\Gamma=G a l(L / K)$ est la limite projective des quotients $\Gamma / \Gamma_{n}$, et les groupes de cohomologie $H^{i}\left(\Gamma, E_{L}^{S}\right)$ associés aux $S$ - unités de $L$ sidentifient aux limites inductives des groupes finis $H^{i}\left(\Gamma / \Gamma_{n}, E_{K_{n}}^{S}\right)$ (cf. $\left.[C F], C h_{\bullet} V, \S 2.4\right)$. Le $\ell$-groupe des $S$-classes de diviseurs $C \ell S$ étant lui aussi la limite inductive des $\ell$-groupes $c \ell_{K_{n}}^{S}$ attachés aux sous-corps $K_{n}$ de degré fini, nous pouvons déduire la suite exacte des $S$-classes ambiges pour l'extension procyclique $L / K$ des mêmes suites écrites pour ses sous extensions cycliques $K_{n} / K$. Une simplification apparait alors : Les places ramifiées dans $L / K$ l'étant totalement dans $L / K_{n}$ pour $n$ assez grand, il ne peut y avoir ramification qulaux places au-dessus de $\ell$, llindice de ramification d'une place modérée étant nécessairement fini car borné par la $\ell$-partie du degré résiduel du corps de base. En outre, le même argument montre que les places réelles ne peuvent se complexifier dans une $\mathbf{Z}_{\ell}$-extension. L'extension des diviseurs y est donc toujours injective, ce qui dispense de distinguer suivant la parité de $\ell$.

En résumé, pour chaque caractère $\ell$-adique irréductible $\varphi$ 
du groupe $\Delta$, et indépendamment de la parité de $\ell$, la suite exacte des $S$-classes ambiges (théorèmes 12 \& 13 ) prend la forme limite :

$$
\begin{aligned}
& 1 \rightarrow \operatorname{Cap}_{L / K}^{S}, \varphi^{S} H_{\varphi x}^{1}\left(\Gamma, \varepsilon_{L}^{S}\right) \rightarrow \operatorname{Ram} \underset{L / K, \varphi}{S} \rightarrow\left[C l_{L}^{S} / j_{L / K}^{S} \text { Cl } \underset{K}{S}\right]_{\varphi} \\
& \rightarrow H_{\varphi x}^{2}\left(\Gamma, \delta_{L}^{S}\right) \rightarrow \underline{\lim }\left(\delta_{K}^{S} / \delta_{K}^{S} \cap \eta_{n}\right){ }_{\varphi} \rightarrow 1 \text {, }
\end{aligned}
$$

où le groupe

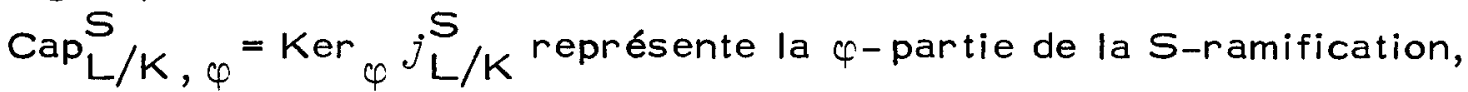
$\operatorname{Ram} S_{L / K, \varphi}^{S}=$ Coker $d_{L / K}^{S}$ est la $\varphi$-partie de la $S$-ramification . et où la limite inductive à droite est prise pour le système inductif don né par les applications normes $N_{n+1 / n}$ •

Cela étant :

PROPOSITION III.1.19.- Dans une $\mathbb{Z}_{\ell}$-extension $L$ d'un corps de nombres $K$, métabélienne sur un sous-corps $F$, le quotient $R a m s / K=$ $\delta_{L}^{S T} / \phi_{K}^{S} d u l$-groupe des $S$-diviseurs ambiges du corps $L$, par le sous-module des $S$-diviseurs de $K$, est un $Z_{\ell}[\Delta]$-module divisible de corang fini ${ }^{(\star)}$ :

$$
\sum_{p \in R \backslash S} x_{p}
$$

où $p$ parcourt l'ensemble des places de $F$, nlappartenant pas à $S$ et (sauvagement) ramifiées dans $\mathrm{L} / \mathrm{K}$. Autrement dit, nous avons

$$
\operatorname{Ram}_{L / K}^{S} \simeq \underset{p \in R 、 S}{\oplus}\left(\left(\mathbb{Q}_{\ell} / \mathbb{Z}_{l}\right) \otimes_{\mathbb{Z}_{l}} \mathbb{Z}_{l}[\Delta] e_{\chi_{p}}\right),
$$

si e $x_{p}$ désigne l'idempotent de l'algèbre $\mathbb{Z}[\Delta]$ associé à l'induit $x_{p}=$ Ind $\Delta_{p}{ }^{1} \Delta_{p}$ à $\Delta$ du caractère de la représentation unité du sous-groupe de décomposition $\Delta_{p}$ de la place $p$ dans l'extension abélienne $K / F$.

En particulier, pour chaque caractère $\ell$-adique irréductible $\varphi$ du groupe $\Delta$, le corang de la $\varphi$-composante du module $R^{2} S / K$ est donné par l'identité :

${ }^{\star}$ ) Nous appelons corang d'un $\mathrm{Z}_{\ell}$-module $M$ le rang de son dual de Pontrjagin Hom $\mathbb{Z}_{\ell}\left(M, \mathbf{Q}_{\ell} / \mathbb{Z}_{\ell}\right)$. 


$$
\operatorname{crg} \operatorname{Ram}_{L / K, \varphi}^{S}=\left\langle\varphi, \sum_{p \in R \backslash s} \chi_{p}\right\rangle .
$$

Démonstration: L'extension $L / K$ étant non complexifiée aux places réelles, il n'y a pas d'inconvénient à identifier les diviseurs de $K$ avec leurs images dans $\mathscr{D}_{\mathrm{L}}$; ce qui justifie la notation $\mathscr{D}_{\mathrm{L}}^{S \Gamma} / \mathscr{D}_{\mathrm{K}}^{\mathrm{S}}$. Cela posé, le $\ell$-groupe des diviseurs ambiges $D{ }_{L}$ est engendré par les diviseurs étendus de $K$, et ceux construits sur les produits ambiges de places ramifiées, lesquels sont divisibles dans $2{ }_{L}^{T}$ : Si pest une place de $F$ qui se ramifie dans $L / K$, le sous-module $\mathscr{D}_{L}$ engendré par les places de $L$ au-dessus de ps'écrit, comme $\mathbb{Z}_{\ell}[\Delta]$-module :

$$
\mathscr{Q}_{L}(p)=\mathbb{Q}_{\ell}\left[\Delta / \Delta_{p}\right]^{\left[\Gamma: \Gamma_{p}\right]},
$$

si $\Gamma_{p}$ est le sous-groupe de décomposition de $p$ dans $L / K$. Son sous groupe ambige est donc:

$$
\mathscr{Q}_{L}(p)^{\Gamma}=\mathbb{Q}_{\ell}\left[\Delta / \Delta_{p}\right] \simeq \mathbb{Q}_{\ell}[\Delta] e_{\chi_{p}}
$$

d'où le résultat annoncé, le sous-groupe étendu de $K$ s'écrivant :

$$
\mathscr{F}_{K}(p)=\mathbb{Z}_{\ell}\left[\Delta / \Delta_{p}\right] \approx \mathbb{Z}_{\ell}[\Delta] e_{\chi_{p}} \text {. }
$$

Revenons maintenant sur la suite exacte des classes ambiges : Le groupe $\operatorname{Cap}_{L / K}^{S}$ est fini $S_{S}$ puisque contenu dans $d \underset{K}{S}$; nous venons de voir que le quotient $\operatorname{Ram}_{L / K}$ est divisible et de corang fini ; et le même résultat vaut pour les deux groupes de droite $H^{2}\left(\Gamma, \sigma_{L}^{S}\right)$ et $\underset{n}{\lim }\left(\delta_{K}^{S} / \varepsilon_{K}^{S} \cap \eta_{n}\right)$, qui sont des quotients du produit tensoriel $\mathbb{S}_{K}^{S}=\left(Q_{l} / \mathbb{Z}_{l}\right) \otimes_{\mathbb{Z}} E_{K}^{S}\left(^{(*)}:\right.$

(i) $H^{2}\left(\Gamma, \delta_{L}^{S}\right)=\circledast_{K}^{S} / N \mathbb{E}_{L}^{S}$,

où $\quad N \mathbb{S}_{\mathrm{L}}^{\mathrm{S}}=\left\{\ell^{-\mathrm{n}} \otimes x \in\left(\mathbb{Q}_{\ell} / \mathbb{Z}_{\ell}\right) \otimes_{\mathbb{Z}} \mathrm{K}^{\mathrm{x}} \mid \mathrm{x} \in N_{\mathrm{n}}\left(\mathrm{E}_{\mathrm{K}_{\mathrm{n}}}^{\mathrm{S}}\right)\right\}$;

(ii) $\quad \frac{\lim }{n}\left(\varepsilon_{K}^{S} / \varepsilon_{K}^{S} \cap n_{n}\right)=\varepsilon_{K}^{S} / \varepsilon_{K}^{S} \cap n_{L / K}$,

où

$$
n_{L / K}=\left\{l^{-n} \otimes x \in\left(\mathbb{Q}_{l} / \mathbb{Z}_{l}\right) \otimes_{\mathbb{Z}} K^{x} \mid x \in N_{n}\left(K_{n}^{x}\right)\right\} .
$$

$\left({ }^{\star}\right)$ On notera que le sous-groupe de torsion de $E_{K}^{S}$ (i.e. le groupe des racines de l'unité de $K$ ) est tué dans le produit tensoriel par le groupe divisible $\mathbb{Q}_{\ell} / \mathbb{Z}_{l}$. 
Les deux groupes $H^{1}\left(\Gamma, \varepsilon_{L}^{S}\right)$ et $d_{L}^{S \Gamma}$ sont donc de corang fini. Par suite :

THÉORĖME $111,1.20$. - Dans une $\mathbb{Z}_{\ell}$-extension $L$ d'un corps de nombres $K$, métabélienne sur un sous-corps $F$, la $\varphi$-composante du $\ell_{-}$ groupe des $S$-classes ambiges est donnée, pour chaque caractère $\ell$ adique irréductible $\varphi$ du groupe abélien $\Delta=G a l(K / F)$, par la suite exacte de $\mathbb{Z}_{l}$-modules de cotype fini :

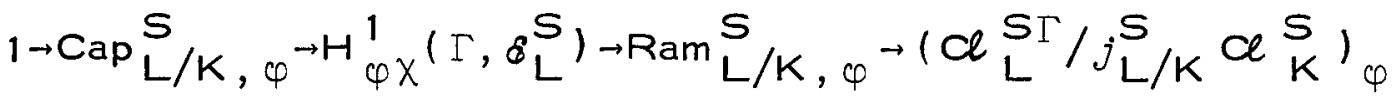

$$
\begin{aligned}
& \rightarrow H_{\varphi x}^{2}\left(\Gamma, \&_{L}^{S}\right) \rightarrow\left(\mathbb{E}_{K}^{S} / \mathbb{E}_{K}^{S} \cap \mathfrak{N}_{L / K}\right)_{\varphi} \rightarrow 1 \text {. }
\end{aligned}
$$

En particulier, la $\varphi$-partie de la codimension ${ }^{\left({ }^{\star}\right)}$ du $l$-groupe des $S$-classes ambiges est donnée par la formule :

$$
\operatorname{codim} c_{L, \varphi}^{S \Gamma}=\left\langle\varphi, \sum_{p \in R \backslash S} x_{p}\right\rangle-n_{L / K}^{S}, \varphi x^{+} q_{L / K, \varphi x}^{S}
$$

où $R$ est l'ensemble des places (sauvagement) ramifiées dans l'extension procyclique $\mathrm{L} / \mathrm{K} ; n_{\mathrm{L} / \mathrm{K}, \varphi x}$ est la $\varphi$ X-partie de la codimension du $\ell$-groupe divisible $\mathbb{E}_{K}^{S} / \mathbb{E}_{K}^{S} \cap \mathfrak{N}_{L / K}$; et $q_{L / K, \varphi X}^{S}$ est donné par l'identité :

$$
q_{L / K, \varphi X}^{S}=\operatorname{codim} H_{\varphi X}^{2}\left(\Gamma, \delta_{L}^{S}\right)-\operatorname{codim} H_{\varphi x}^{1}\left(\Gamma, \delta_{L}^{S}\right) \text {. }
$$

DÉFINITION III.1.21.- Nous disons que la quantite $q_{\mathrm{L} / \mathrm{K}, \varphi x}^{\mathrm{S}}=$ codim $H_{\varphi \chi}^{2}\left(\Gamma, \varepsilon_{L}^{S}\right)-\operatorname{codim} H_{\varphi X}^{1}\left(\Gamma, \varepsilon_{L}^{S}\right)$ est la $\varphi X$-partie du quotient de Herbrand généralisé attaché aux $\mathrm{S}$ - unités dans l'extension procy clique $L / K$.

b.- Calcul du quotient de Herbrand dans le cas procyclique $\left.{ }^{(\star \star}\right)$.

II repose tout entier sur le lemme suivant:

(*) Nous appelons codimension d'un $\mathbb{Z}_{\ell}$-module $M$ la dimension de son dual de Pontrjagin : codim $\mathbb{Z}_{\ell} M=\operatorname{dim} \mathbb{Q}_{\ell}\left(\mathbb{Q}_{\ell} \otimes_{\mathbb{Z}_{\ell}} \operatorname{Hom}_{\mathbb{Z}_{\ell}}\left(M, \mathbb{Q}_{\ell} / \mathbb{Z}_{\ell}\right)\right.$. $\left({ }^{\star}\right)$ Le calcul du quotient de Herbrand des $S$-unités, dans un cadre moins général, a été effectué par Iwasawa dans $\left[\mathrm{Iw}_{6}\right]$ par une méthode complètement différente, qu'on peut regarder comme duale de celle dé veloppée dans $\left[\mathrm{Ja}_{4}\right]$, qui fait intervenir la théorie des genres . 
LEMME 111.1 .22 - - Soient $1 \longrightarrow \mathrm{A} \longrightarrow \mathrm{B} \longrightarrow \mathrm{C} \longrightarrow 1$

une suite exacte courte de $\mathbb{Z}[\Gamma]$-modules discrets, et

$1 \longrightarrow \mathrm{A} \longrightarrow \mathrm{B} \longrightarrow \mathrm{C}$

la suite exacte obtenue après produit tensoriel par $\mathbb{Z}_{\ell}$.

Si les conditions suivantes sont réunies:

(i) Le groupe $C$ est un $\mathbb{Z}_{l}$-module de torsion, dont le sousgroupe invariant $C^{\Gamma}$ est de cotype fini ;

(ii) Le sous-groupe $a^{\Gamma}$ est d'indice fini dans $\beta^{\Gamma}$;

alors les quotients de Herbrand généralisés $q(\Gamma, a)$ et $q(\Gamma, \beta)$ existent simultanément et sont égaux.

Démonstration : Considérons la suite exacte de cohomologie :

$$
\begin{aligned}
1 \rightarrow a^{\Gamma} \rightarrow B^{\Gamma} \rightarrow C^{\Gamma} \rightarrow H^{1}(\Gamma, a) \rightarrow H^{1}(\Gamma, \beta) \rightarrow H^{1}(\Gamma, c) \rightarrow H^{2}(\Gamma, a) & \rightarrow H^{2}(\Gamma, \beta) \\
& \rightarrow H^{2}(\Gamma, c) .
\end{aligned}
$$

Dans celle-ci, le quotient $B^{\Gamma} / Q^{\Gamma}$ est fini par hypothèse; et le groupe $H^{2}(\Gamma, C)$ est nul, puisque $C$ est de torsion. Enfin, le dual de Pontrjagin $C^{\nu}=$ Hom $\mathbb{Z}_{l}\left(c, \mathbb{Q}_{l} / \mathbb{Z}_{\ell}\right)$ du groupe $C$ est un $\Lambda$-module compact, dont le quotient invariant $\Gamma C^{\nu}=$ Hom $\mathbb{Z}_{\ell}\left(c^{\Gamma}, \mathbb{Q}_{\ell} / \mathbb{Z}_{\ell}\right)$ est de type fini sur $\mathbb{Z}_{\ell}$. Clest donc un $\Lambda$-module de type fini, ce qui entraîne en particu lier que son quotient $\Gamma C^{\nu}$ et son sous-groupe invariant $C^{v} \Gamma$ ont même dimension, ou, ce qui revient au même, que leurs duaux respectifs $C^{\Gamma}$ et ${ }^{\Gamma} C=H^{1}(\Gamma, C)$ ont même codimension finie.

Ainsi dans la suite longue obtenue, les groupes $H^{1}(\Gamma, a)$ et $H^{1}(\Gamma, \beta)$ d'une part, $H^{2}(\Gamma, a)$ et $H^{2}(\Gamma, \beta)$ diautre part, sont-ils simultanément de cotype fini. Lorsque clest le cas, l'égalité des quo tients de Herbrand slobtient tout simplement en écrivant que la somme alternée des codimensions des termes de la suite est nulle.

THÉORÈME $111,1.23$. - Lorsque $\Phi$ est le caractère associé à un idem potent central de l'algèbre de Galois $\Lambda[\Delta]$ ( i.e. lorsque $\Phi$ est un ca ractère $l$-adique du groupe $\Delta=G a l(K / F)$, contenu dans le caractère régulier, et stable par translation de $\chi$ ), la $\Phi$-partie du quotient de Herbrand généralisé attaché aux $S$-unités dans l'extension procy clique $L / K$ est donné par la formule : 


$$
q_{L / K, \Phi}^{S}=\left\langle\Phi, \sum_{p \in S} \delta_{p} X_{p}-1\right\rangle \text {. }
$$

Dans celle-ci, $\chi_{p}$ désigne comme plus haut l'induit $\Delta$ du caractère de la représentation unité du sous-groupe de décomposition de la place $p$ dans l'extension abélienne $\mathrm{K} / \mathrm{F}$; et l'entier $\delta_{\mathrm{p}}$ est égal à 0 si p est complètement décomposée dans l'extension procyclique $L / K$, et à 1 sinon .

Démonstration : Le caractère $\Phi$ étant associé à un idempotent central, la $\Phi$-partie du quotient de Herbrand des $S$-unités n'est autre que le quotient de Herbrand de la $\Phi$-partie du groupe $\varepsilon_{L}^{S}$. Il peut donc se calculer à l'aide du lemme, par passage à la limite inductive à partir du théorème de représentation de Herbrand, qui nous assure l'exis tence d'une suite exacte courte de $\mathbb{Z}[\Gamma]$-modules discrets :

$$
1 \longrightarrow M \longrightarrow \delta_{\mathrm{L}}^{\mathrm{S}} \longrightarrow \mathrm{T} \longrightarrow 1
$$

dans laquelle $T$ est un $\mathbb{Z}$-module de torsion, et $M$ est donné par l'isomorphisme :

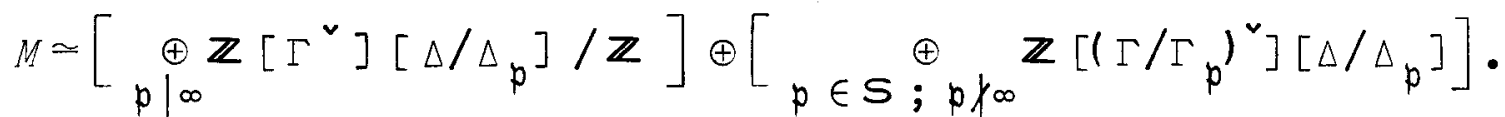
où $\Gamma^{v}=\underset{n}{\lim } \Gamma / \Gamma_{n}$ est le dual de Pontrjagin du groupe $\Gamma$, et $\Gamma_{p}$ comme $\Delta_{p}$ sont les sous-groupes de décomposition de la place p respective ment dans l'extension procyclique $L / K$ et dans l'extension abélienne $K / F$. Après produit tensoriel par $\mathbb{Z}_{\ell}$ et localisation, nous obtenons donc la suite exacte courte :

$$
1 \longrightarrow m_{\Phi} \longrightarrow \delta_{L, \Phi}^{S} \longrightarrow \sigma_{\Phi} \longrightarrow 1
$$

où $\sigma_{\Phi}$ est un $\mathbb{Z}_{l}$-module fini, et $m_{\Phi}$ est donné par llisomorphisme :

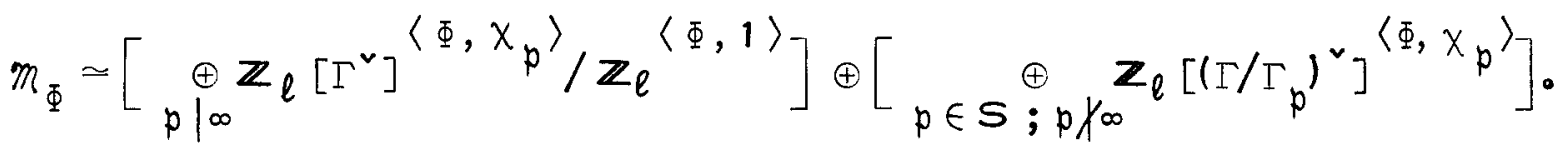
Et, dlaprès les calculs du lemme 15 , nous avons directement :

$$
H_{\Phi}^{1}(\Gamma, m)=\underset{n}{\lim } H_{\Phi}^{1}\left(\Gamma / \Gamma_{n}, m^{\Gamma_{n}}\right) \simeq\left(\mathbb{Q}_{\ell} / \mathbb{Z}_{\ell}\right)^{\langle\Phi, 1\rangle}
$$

\& $\quad H_{\Phi}^{2}(\Gamma, m)=\underset{n}{\lim } H_{\Phi}^{2}\left(\Gamma / \Gamma_{n}, m^{\Gamma_{n}}\right) \simeq\left(\mathbb{Q}_{l} / \mathbb{Z}_{l}\right) p \in \sum_{i p \mid \infty} \delta_{p}\left\langle\Phi, x_{p}\right\rangle$. Cela étant, comme $m_{\Phi}^{\Gamma}$ a même rang que $\delta_{K}^{S}, \Phi$, et comme les deux 
groupes de cohomologie $H_{\Phi}^{1}\left(\Gamma, \varepsilon_{L}^{S}\right)$ et $H_{\Phi}^{2}\left(\Gamma, \varepsilon_{L}^{S}\right)$ sont de cotype fini ( en vertu du théorème 20 ), les conditions du lemme sont bien véri fiées, et le quotient de Herbrand cherché est donné par la formule :

$$
\begin{aligned}
& q_{L / K, \Phi}^{S}=q\left(\Gamma, \delta_{L, \Phi}^{S}\right)=q\left(\Gamma, m_{\Phi}\right)=\left\langle\Phi, \sum_{p \in S ; p X_{\infty}} \delta_{p} X_{p}-1\right\rangle \\
& =\left\langle\Phi, \sum_{p \in S} \delta_{p} x_{p}-1\right\rangle \text {. }
\end{aligned}
$$

Le théorème 23 ci-dessus conduit à dlintéressants critères de non trivialité pour les groupes $c \ell_{L}^{S}$. Ainsi, lorsque l'ensemble $S$ contient les places du corps $F$ qui se ramifient dans la tour $L / K$, le groupe Ram ${ }_{L / K}^{S}$ est nul, et la $S$-capitulation Cap ${ }_{L / K}^{S}$ s'identifie au $^{S}$ groupe de cohomologie $H^{1}\left(\Gamma, \varepsilon_{L}^{S}\right)$. Dans ce cas, ce dernier groupe étant nécessairement fini, le deuxième groupe de cohomologie $H^{2}\left(\Gamma, \delta_{L}^{S}\right)$ est parfaitement connu : clest un $\mathbb{Z}_{\ell}$-module divisible de cotype fini, dont la codimension est alors égale au quotient de Herbrand:

$$
q_{L / K}^{S}=\left(\sum_{p \in S} \delta_{p}\left[\Delta: \Delta_{p}\right]\right)-1 \text {. }
$$

Plus généralement, lorsque $S$ ne contient pas nécessairement les places de F ramifiées dans la tour $L / K$, la $S$-capitulation Cap $S / K$ est seulement un sous-groupe de $H^{1}\left(\Gamma, \delta_{L}^{S}\right)$. Mais, même dans ce cas, la finitude du groupe $H^{1}\left(\Gamma, \delta_{L}^{S}\right)$, où de llune de ses composantes, apporte une information intéressante :

PROPOSITION III.1.24.- Conservons les notations du théorème 20 . Alors :

(i) Si le groupe $H_{\varphi x}^{1}\left(\Gamma, \delta_{L}^{S}\right)$ est nul, pour un caractère adique irréductible $\varphi$ de $\Delta$, il en est de même du groupe $C_{a p} S / K, \varphi$; autrement dit, la restriction aux $\varphi$-composantes de l'homomorphisme d'extension $\mathrm{Cl} \underset{\mathrm{K}}{\mathrm{S}} \longrightarrow \mathrm{Cl}_{\mathrm{L}}^{\mathrm{S}}$ est injective.

(ii) Si le groupe $H_{\varphi x}^{1}\left(\Gamma, \delta_{L}^{S}\right)$ est fini, pour un caractère $\ell-$ adique irréductible $\varphi$ de $\Delta$, la $\varphi$-composante du $\ell$-groupe $c \ell S_{L}^{S}$ des $S$ classes de diviseurs du corps $L$ contient un facteur direct isomorphe au groupe $\operatorname{Ram} S / K, \varphi$, qui est un $\mathbb{Z}_{\ell}$-module divisible de codimension $\left\langle\varphi, \sum_{p \in R \backslash S} x_{p}\right\rangle$. 
Démonstration: La première assertion est immédiate, le groupe Cap ${ }_{L / K}^{S}, \varphi$ étant contenu dans $H_{\varphi X}^{1}\left(\Gamma, \varepsilon_{L}^{S}\right)$ en vertu de la suite exacte des classes ambiges. Pour établir la seconde, il suffit de remarquer que, sous la condition $H_{\varphi x}^{1}\left(\Gamma, \delta_{L}^{S}\right)$ fini, limage $\left(\delta_{L}^{S} \Gamma / \delta_{K}^{S} P_{L}^{S} \Gamma\right)$ du groupe divisible $\operatorname{Ram}_{L / K}^{S}$ dans le quotient ( $C e_{L}^{S}{ }_{S} / j_{L / K}^{S}$ Ce ${ }_{K}^{S}$ ) est un groupe divisible isomorphe à $\operatorname{Ram} L / K, \varphi$. En particulier, le sous-groupe de $C_{L} S \Gamma, \varphi$, engendré par les classes des $S$-diviseurs ambiges, est alors un $\mathbb{Z}_{\ell}$-module divisible de codimension codim $\operatorname{Ram}_{L / K, \varphi}^{S}=\left\langle\varphi, \sum_{p \in R \backslash S} x_{p}\right\rangle$.

La proposition obtenue donne directement des critères simples de non trivialité du $\ell$-groupe des $S$-classes. Ainsi :

SCOLIE III.1.25. - Supposons que le corps $K$ soit une extension qua dratique totalement imaginaire d'un sur-corps totalement réel de $F$, et que le caractère $X$ agisse trivialement sur la conjugaison complexe. Alors :

(i) Si L est la $\mathbb{Z}_{\ell}$-extension cyclotomique de $K$, le groupe $H_{\varphi X}^{1}\left(\Gamma, \delta_{L}\right)=H_{\varphi}^{1}\left(\Gamma, \delta_{L}\right)$ est nul pour chaque caractère $\ell$-adique irréductible imaginaire $\varphi$ du groupe $\Delta$.

(ii) Si $L$ est une $\mathbb{Z}_{\ell}$-extension non cyclotomique de $K$, le groupe $H_{\varphi X}^{1}\left(\Gamma, \delta_{L}\right)$ est nul pour chaque caractère $l$-adique irré ductible imaginaire $\varphi$ du groupe $\Delta$, à l'exception éventuelle d'un caractère de dimension 1 lorsque $K$ contient les racines $l$-ièmes de l'unité, auquel cas il reste cependant fini.

Démonstration: Lorsque $\mathrm{K} / \mathrm{F}$ admet une conjugaison complexe, contenue dans le noyau de $X$, la composante imaginaire du tensorisé $\delta_{L}=\mathbb{Z}_{\ell} \otimes_{\mathbb{Z}} E_{L}$ du groupe des unités est stable pour llaction de $\Gamma$, et se réduit à son sous-module de torsion, $\mu_{L}$. Cela étant :

- Si $L$ est la $\mathbb{Z}_{\ell}$-extension cyclotomique de $K$, nous avons trivialement $H^{1}\left(\Gamma, \mu_{L}\right)=H^{2}\left(\Gamma, \mu_{L}\right)=1$.

- Si L n'est pas la $\mathbb{Z}_{\ell}$-extension cyclotomique de $K$ ( ce qui ne peut advenir qu'en contradiction avec la conjecture de Leopoldt), 
le groupe $\mu_{L}$ est un $\mathbb{Z}_{\ell}$-module fini. Il vient donc $H_{\varphi x}^{1}\left(\Gamma, \mu_{L}\right)=1$, sauf, peut-être, pour $\varphi X=\omega$, où $\omega$ est le caractère dellaction de $\Delta$ sur $\mu_{L}$, lorsque $\mu_{L}$ est non nul. Dans ce cas encore, $H_{\omega}^{1}\left(\Gamma, \mu_{L}\right)$ est fini, comme quotient de $\mu_{L}$.

Application. - Soient $K$ une extension quadratique totalement imaginaire d'un corps $F$ totalement réel, $\ell$ un nombre premier impair, et $L$ la $\mathbb{Z}_{\ell}$-extension cyclotomique de $\mathrm{K}$. Alors la composante imaginaire $C_{\ell}-$ du $\ell$-groupe des classes d'idéaux de $L$ contient un $\mathbb{Z}_{\ell}$-module divi sible isomorphe à $\left(Q_{\ell} / \mathbb{Z}_{\ell}\right)^{g_{\ell}^{-}}$, où $g_{\ell}^{-}$est le nombre de places de $k$ au-dessus de décomposées dans $K / F(\star)$.

Remarque.- II n'est pas nécessaire de supposer que le caractère $\varphi X$ n'est pas représenté dans le groupe $\delta_{L}^{S}$ pour conclure à la trivialité du groupe $H_{\varphi X}^{1}\left(\Gamma, \delta_{L}^{S}\right)$. La proposition 18 en fournit une excellente il lustration. Reprenons l'exemple ci-dessus, et supposons totalement ramifiées dans la tour cyclotomique $L / K$ les places au-dessus de $\ell$ décomposées dans l'extension quadratique $\mathrm{K} / \mathrm{F}$. Dans ce cas nous avons encore $H_{\varphi}^{1}\left(\Gamma, \delta_{L}^{S}\right)=1$, pour chaque caractère imaginaire $\varphi \mathrm{du}$ groupe $\triangle$ et chaque partie $S$ de l'ensemble des places de $F$ au-dessus de $\ell$. En particulier, la composante imaginaire Cap $\frac{S}{L} / K$ de la $S-$
capitulation est alors réduite à $1^{(\star \star)}$.

c.- Analyse de la formule obtenue; critères de trivialité pour les groupes Ce ${ }_{\mathrm{L}}^{\mathrm{S}}$.

Réunissant les théorèmes 20 et 23 , nous obtenons :

THEOREME III.1.26. - Sous les hypothèses du théor ème 23 , la codi mension de la $\Phi$-partie du $\ell$-groupe des $S$-classes ambiges de diviseurs est donnée par la formule :

$$
\operatorname{codim} c e_{L, \Phi}^{S T}=\left\langle\Phi,\left(\sum_{p \in S \cup R} \delta_{p} x_{p}\right)-1\right\rangle-n_{L / K, \Phi}^{S} \cdot
$$

Dans celle-ci, $R$ est l'ensemble des places ramifiées dans l'extension procyclique $L / K$; l'entier $\delta_{p}$ est égal à 0 ou 1 suivant que la place $p$

${ }^{\star}$ ) Ce résultat est dû à Gold $\left[\mathrm{Go}_{2}\right]$

$\left({ }^{\star}\right)$ Autre résultat de Gold (op. cit.). 
est complètement décomposée ou non dans $L / K$; le caractère $X_{p}$ est l'induit à $\triangle$ de celui de la représentation unité du sous-groupe de dé composition de $p$ dans $\mathrm{K} / \mathrm{F}$; et l'indice $n_{\mathrm{L} / \mathrm{K}}^{\mathrm{S}} \Phi$ est la codimension de la $\Phi$-partie du $\ell$-groupe divisible $\mathbb{E}_{K}^{S} / \mathbb{E}_{K}^{S} \cap n_{L} / K$. En particulier, le $\ell$-groupe $C \ell \underset{L, \Phi}{S} \Gamma_{\text {, }}$ est fini si et seulement si
est réalisée l'égalité $\left({ }^{\prime}\right)$ :

$$
n_{L / K, \Phi}^{S}=\left\langle\Phi,\left(\sum_{p \in S \cup R} \delta_{p} X_{p}\right)-1\right\rangle \text {. }
$$

Démonstration: D'après le théorème 20 , nous avons codim $C l S S_{L}^{S}, \Phi=$ $\left\langle\Phi, \sum_{p \in R \backslash S} \delta_{p} X_{p}\right\rangle+q_{L / K, \Phi}^{S}-n_{L / K, \Phi}^{S} ;$ et, dlaprès le théorème $23: q_{L / K, \Phi}^{S}=\left\langle\Phi, \sum_{p \in S} \delta_{p} X_{p}-1\right\rangle$; d'où le résultat .

Le critère ainsiobtenu (la finitude du $\ell$-groupe ambige $C_{\ell} S \Gamma$, ${ }^{S}$, qui se lit sur l'indice normique $n_{L / K}^{S}, \Phi$ ) est évidemment une condition nécessaire pour que le groupe $C \ell_{L, \Phi}$ soit lui-même fini . Cependant cette condition n'est nullement suffisante, le groupe cl ${ }^{S} L_{\text {, }} \Phi$ pouvant être fini sans être nul, mais non le groupe $C e \stackrel{S}{L}, \Phi$. Plus précisément :

PROPOSITION III.1.27.- Soient, comme plus haut, $L$ une $\mathbb{Z}_{l}$-ex tension d'un corps de nombres $K$, métabélienne sur un sous-corps $F$, et $\Phi$ le caractère $l$-adique du groupe abélien $\Delta=G a l(K / F)$ attaché à un idempotent central de l'algèbre $\Lambda[\Delta]$. Les assertions suivantes sont équivalentes :

(i) La $\Phi$-composante $C l \stackrel{S}{L}$, $\Phi$ du $\ell$-groupe des $S$-classes de diviseurs du corps $L$ est finie.

(ii) La $\Phi$-composante $C l \underset{L}{S}$, $\Phi$ du $l$-groupe des $S$-classes de diviseurs du corps $L$ est triviale. est triviale.

(iii) La $\Phi$-composante $d \int_{L, \Phi}^{S}$ du sous-groupe ambige de $C S$

Démonstration: L'équivalence des deux premières assertions est bien

$\left.{ }^{\star}{ }^{*}\right)$ L'indice $n_{L / K}^{S}, \Phi$, qui mesure le comportement normique des unités, peut se calculer, dans certains cas, sous la conjecture d'indépendance $\ell$-adique exposée au chapitre II. Ce point est développé dans la section 2 qui suit. 
connue pour les $\ell$-groupes de classes didéaux au sens or dinaire ( $c f_{.}$, par exemple, [Go]). Elle provient simplement du fait, donné par le corps de classes, que, pour $n$ assez grand, la norme arithmétique $N_{\mathrm{n}+1} / \mathrm{n}$ induit une surjection naturelle de la $\Phi$-composante $\mathrm{Cl} \mathrm{K}_{\mathrm{n}+1}{ }^{\mathrm{S}}$, $\Phi$ du $\ell$-groupe des $S$-classes de diviseurs de $K_{n+1}$ dans celle $C \ell K_{n}^{S}$, $\Phi$ de $K_{n}$ : Si donc $C l L_{L, \Phi}^{S} \underset{n}{\lim } C e_{K_{n}}^{S}$, $\Phi$ est fini, I'homomorphismedlextension $j_{L / K_{n}}^{S}$ identifie $C \ell_{K_{n}}^{S}$, $\Phi$ avec $C e_{L}^{S}$, pour chaque $n$ assez grand; dans cette identification l'opérateur norme $N_{n+1 / n}$ correspond à l'élévation à la puissance $\ell$-ième; et sa surjectivité prouve que le groupe $c e_{L, \Phi}^{S}$ est divisible donc trivial, puisqulil est fini . Inversement, si le groupe $C e_{L}^{S}$, $\Phi$ 'est pas nul, le quotient ce $K_{n}^{S}$, $\Phi$ / Cap $L_{L / K_{n}}$, $\Phi$ est non nul pour $n$ assez grand; comme il est fini, il contient au moins une classe non nulle $c_{n}$ invariante par $\Gamma$ ( $c f$. $\left.\left[\mathrm{Se}_{2}\right], \mathrm{Ch} . \mathbf{I X}, \S 1\right)$. Cela étant, si $a_{n}$ est un diviseur de $\mathrm{K}_{n}$ re présentant cette classe, son étendu à $L$ ne capitule pas dans $C l{ }_{S} L$, et engendre une classe ambige de $c e \stackrel{S}{S}, \Phi$. D'où l'équivalence des deux dernières assertions .

COROLLAIRE III.1.28.- Conservons les mêmes notations, et suppo sons réunies les trois conditions :

(i) $\operatorname{Ram}_{L / K, \Phi}^{S}=1$, i.e. $\left\langle\Phi, \sum_{p \in R \backslash S} X_{p}\right\rangle=0$;

(ii) $\mathrm{Cl} \underset{\mathrm{K}, \Phi}{\mathrm{S}}=1$;

(iii) $\mathbb{S}_{K, \Phi}^{S}=1$, i.e. $\left\langle\Phi, \sum_{p \in \bar{S}} x_{p}-1\right\rangle=0 \quad$ (où $\bar{S}$ est la réunion de $S$ et des places à l'infini $)^{(\star)}$.

Alors le $\ell$-groupe des $S$-classes de diviseurs de $L$ a une $\Phi$-composante triviale : $C_{L}^{S}{ }_{L}^{S}=1$.

Démonstration: $\|$ slagit évidemment d'établir la trivialité du $\ell$-groupe

${ }^{\star}$ ) La condition requise $\mathbb{E}_{K, \Phi}^{S}=1$ ( où $\otimes_{K}^{S}=\left(Q_{\ell} / \mathbb{Z}_{l}\right) \otimes_{\mathbb{Z}_{S}}^{E_{K}^{S}}$ ) est légèrement plus faible que celle $\delta_{K, \Phi}^{S}=1$ ( où $\delta_{K}^{S}=\mathbb{Z}_{\ell} \otimes_{\mathbb{Z}} E_{K}{ }_{K}$ ), qui fait intervenir les racines de l'unité contenues dans $K$. 
ambige $C e \underset{L}{S} \Gamma_{\Phi}$ sous les trois conditions énoncées. Or cela résulte directement de la suite exacte des $\mathbf{S}$-classes ambiges donnée par le théorème 20 . En effet, le groupe $\operatorname{Ram} S / K, \Phi$ est nul par hypothèse ; le groupe $H_{\Phi}^{2}\left(\Gamma, \delta_{L}^{S}\right)$ comme quotient de ${ }_{K}, \Phi$; et le groupe $j_{L / K}^{S} \propto_{K, \Phi}^{S}$ comme image de $C l \underset{K}{S}, \Phi$. D'où la conclusion annoncée, la traductión en termes de caractères des conditions (i) et (ii) résultant des calculs qui précèdent ( cf. proposition 19 et théorème 23 ).

Ce résultat est essentiellement bien connu dans le cas parti culier suivant ( $\mathrm{cf}$. [Gi $\left.\mathrm{G}_{3}\right]$ ): $\mathrm{F}$ est le corps des rationnels; $\mathrm{K}$ un corps abélien imaginaire de degré étranger à $l ; L$ est la $\mathbb{Z}_{l}$-extension cyclotomique de $K$; et, si $K_{+}$est le sous-corps réel maximal de $K$, les places de $K_{+}$au-dessus de $l$ ne se décomposent pas dans $\mathrm{K} / \mathrm{K}_{+}$. Plus généralement :

PROPOSITION III.1.29.- Soient $\ell$ un nombre premier impair, et $L$ une $\mathbb{Z}_{\ell}$-extension dlun corps de nombres $K$, métabélienne sur un sous corps $F$. Supposons que $K$ soit une extension quadratique totalement imaginaire dlun sur-corps totalement réel $K_{+}$de $F$, et que le carac tère $\chi$ agisse trivialement sur la conjugaison complexe. Alors, pour chaque caractère $\ell$-adique imaginaire $\Phi$ du groupe $\Delta=\mathrm{Gal}(\mathrm{K} / F)$, la condition $d_{K, \Phi}=1$ entraîne $d l_{L, \Phi}=1$; et le même résultat vaut encore pour les $\ell$-groupes de $S$-classes, si $S$ est contenu dans l'en semble $S(\ell)$ des places de $F$ au-dessus de $\ell$ :

$$
\mathrm{Cl} \mathrm{K}, \Phi_{\mathrm{K}, \Phi}^{\mathrm{S}}=1 \Rightarrow \mathrm{C} \ell_{\mathrm{L}, \Phi}^{\mathrm{S}}=1 \text {. }
$$


$-202-$ 


\section{ÉLÉMENTS DE THÉORIE DES GENRES}

La théorie des genres pour les corps de nombres a une ori gine très ancienne puisqulelle remonte en fait aux Disquisitiones Arithmeticae de Gauss . Bien entendu, Gauss étudiait les formes quadratiques, tandis que le point de vue actuel met l'accent sur l'arithmétique des extensions abéliennes. Ce n'est qu'en 1951 , pourtant, que Hasse donna une interprétation du genre des corps quadratiques basée sur la théorie du corps de classes, ouvrant ainsi la voie aux développements ultérieurs ( $\left.\mathrm{cf}_{\circ}\left[\mathrm{Ha}_{2}\right]\right)$. Peu après, lyanaga et Tamagawa s'intéressèrent au cas absolument cyclique (cf. [IT]), puis Leopoldt au cas absolument abélien général ( $\mathrm{cf}_{\circ}\left[\mathrm{Le}_{2}\right]$, et $\left[\mathrm{Ha}_{4}\right]$ ). En 1959 , Fröhlich donna une définition du corps des genres attaché à un corps de nombres arbitraire ( $\mathrm{cf} .\left[\mathrm{Fr}_{1}\right]$ ), qui est tout à fait générale, puisqu'elle s'étend sans grande modification aux extensions relatives quelconques $L / K$ de corps de nombres $\left.{ }^{\star}\right)$.

Clest précisément par llexposé de cette généralisation que nous ouvrons cette section, en nous plaçant d'emblée dans le cadre des groupes de $\mathrm{S}$-classes de diviseurs. Auparavant, rappelons que le calcul du nombre de genres pour les extensions relatives galoi siennes est dû à Furuta $\left(\mathrm{cf} \cdot\left[\mathrm{Fu}_{1}\right]\right.$ ). Son résultat, qui vaut pour les genres au sens ordinaire, a été précisé par Goldstein dans le cas restreint, mais sous réserve d'abélianité de l'extension. Plus récemment d'ailleurs, Gurak a étendu à ce cas les résultats de Fröhlich sur la caractérisation du genre principal à partir des caractères diArtin ( cf. $\left[\mathrm{Gu}_{2}\right]$ ).

$\left({ }^{\star}\right)$ Pour plus de détails sur le genre des extensions finies du corps des rationnels, on pourra se reporter au livre d'Ishida consacré à cette question . 


\section{1. - PRÉSENTATION DE LA THÉORIE DES GENRES.}

\section{a.-Définition du corps des S-genres, relatif à une extension finie de corps de nombres.}

Nous conservons les conventions de la section précédente. En particulier, pour chaque corps de nombres $K$, nous désignons par Cl $\mathrm{K}$ son groupe des classes de diviseurs (qui s'identifie canonique ment au groupe des classes d'idéaux de ce corps, prises au sens res treint). Enfin, si $S$ est un ensemble fini de places non complexes d'un sous-corps $K$, nous notons $S_{K}$ l'ensemble des places non complexes de $K$ au-dessus de $S$, et $C \ell_{K}^{S}$ le quotient de $c e_{K}$ par le sous-groupe $C l_{K}(S)$ formé des classes des diviseurs construits sur les places de $S_{K}$. Cela dit :

DÉFINITION 111.2.1.-Etant donnés une extension finie quelconque de corps de nombres $L / K$, et un ensemble fini $S$ de places non complexes du corps $K$, le corps des $S$-genres de l'extension $L / K$ est la plus grande extension $\bar{C}_{L / K}$ de $L$, qui est non ramifiée et $S$-décomposée (i.e. non ramifiée aux places finies et complètement décomposée aux places au-dessus de S), et qui provient par composition dlune extension abélienne de $\mathrm{K}$.

Le corps des $S$-genres $\bar{C} L_{S} / K$ est donc la sous-extension maximale du corps des $S$-classes $C_{L}^{S}$ qui provient par composition d'une extension abélienne de $K$. Le sous-groupe de $C_{L} S$, qui lui correspond par la théorie du corps de classes, est le $\mathbf{S}$-genre princi-

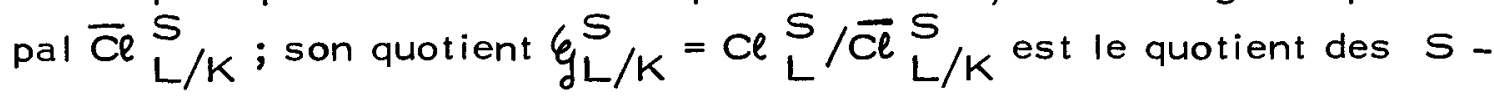
genres.

Remarques.-(i) Lorsque l'ensemble de places $S$ est vide, le corps $\bar{C}_{L / K}$ est l'extension maximale de $L$, non ramifiée (aux places finies), qui provient par composition d'une extension abélienne de $K$. Dans le vocabulaire des classes d'idéaux, $\bar{C}_{L / K}$ est le corps des genres au sens restreint. Nous disons que $\bar{C}_{L / K}$ est le corps des genres de l'extension $L / K$, sans précision . 
(ii) Lorsque $S$ est l'ensemble des places réelles de $K$, le corps $\bar{C}_{L / K}^{S}$ est l'extension maximale de $L$, non ramifiée (aux places finies) et non complexifiée (aux places réelles), qui provient par com position d'une extension abélienne de $K$. Nous disons que $\bar{C}_{L / K}^{S}$ est le corps des genres au sens ordinaire de l'extension $L / K$.

Considérons donc une extension finie de corps de nombres $L / K$, et notons $\bar{C} L / K$ son corps des S-genres. D'après la définition ci-dessus, le corps $\overline{\mathrm{C}} \mathrm{L} / \mathrm{K}$ est le composé de $L$ et de la sous-exten sion maximale $C_{L}^{S} a b$ de son corps des $S$-classes $C_{L}^{S}$, qui est abé lienne sur $K$. En particulier , $\vec{C}_{L / K}^{S}$ contient le corps des $S$-classes $C_{K}^{S}$ de $K$. Si donc nous désignons par $L^{a b}$ la sous-extension maxi male de $L$ qui est abélienne sur $K$, nous obtenons le schéma de corps :

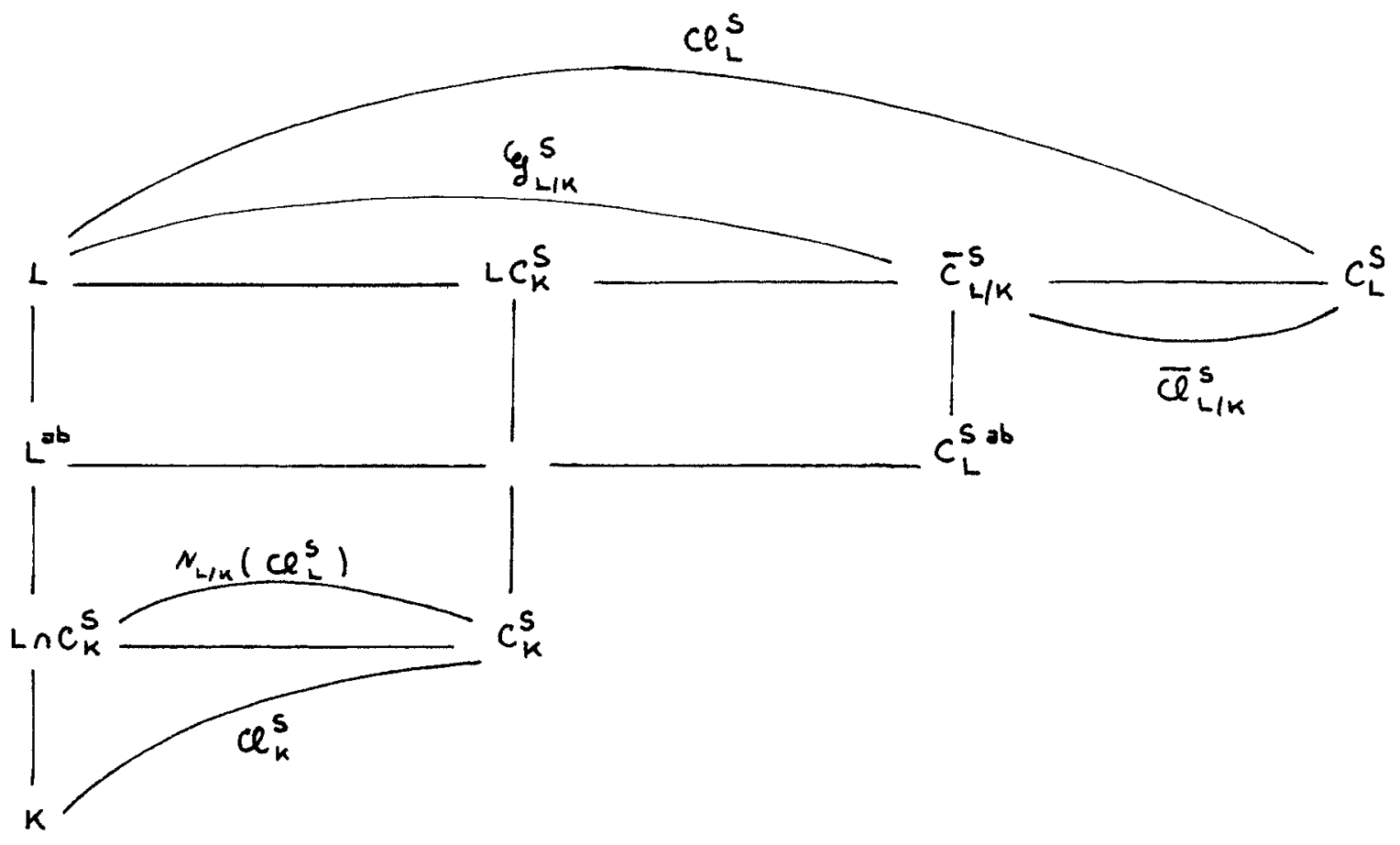

Pour chaque corps de nombres $F$, désignons par $J_{F}$ le groupe des idèles de $F$, par $J_{F}(S)$ le sous-groupe de $J_{F}$ formé des idèles unités en-dehors de $S$, et par $U_{F}$ le groupe des idèles unités. Cela posé, puisque $C_{L}^{S}$ ab est la sous-extension maximale de $C_{L}^{S}$ qui est abélienne sur $K$, la théorie du corps de classes nous donne directe ment l'isomorphisme : 
$\operatorname{Gal}\left(C_{L}^{S a b} / K\right) \approx J_{K} / N C_{L}^{S}$ ab $/ K\left(J_{L}^{S a b}\right) K^{x}=J_{K} / N C_{L}^{S} / K\left(C_{L}^{S}\right) K^{x}$. D'un autre côté, nous avons ${ }_{C_{L} / L}\left(J_{C_{L}}\right) L^{x}=J_{L}(S) L^{x}$, par définition du corps des $S$-classes $C_{L}^{S}$. Il vient donc :

$$
\operatorname{Gal}\left(C_{L}^{S} a b / K\right) \simeq J_{K} / N_{L / K}\left(J_{L}(S)\right) K^{x} \text {. }
$$

Cela étant, nous obtenons :

$$
\left[C_{L}^{S a b}: C_{K}^{S}\right]=\left(J_{K}(S) K^{x}: N_{L / K}\left(J_{L}(S)\right) K^{x}\right)=\frac{\left(J_{K}(S): N_{L} / K^{\left.\left(J_{L}(S)\right)\right)}\right.}{\left(K^{x} \cap J_{K}(S): K^{x} \cap N_{L / K}\left(J_{L}(S)\right)\right)} ;
$$

c'est-à-dire, finalement :

$$
\left[C_{L}^{S a b}: C_{K}^{S}\right]=\frac{p \in S\left(K_{p}^{x}: \prod_{P \mid p} N_{L_{P}} / K_{p}\left(L_{P}^{x}\right)\right) \cdot \prod_{p \notin S}\left(u_{p}: \prod_{P \mid p} N_{L_{P}} / K_{p}\left(u_{P}\right)\right)}{\left(E_{K}^{S}: E_{K}^{S} \cap N_{L / K}\right)}
$$

en notant $K_{p}^{X}$ le groupe multiplicatif du complété de $K$ pour la place $p$, et $U_{p}$ le sous-groupe des unités; $L \underset{P}{X}$ le groupe multiplicatif du complété de $L$ pour la place $P$, et $U_{\mathcal{B}}$ le sous-groupe des unités; $E_{K}^{S}$ le groupe des $S$-unités du corps $K$ (au sens des diviseurs), et $N_{L / K}$ le sous groupe de $K^{X}$ formé des éléments dont liimage diagonale dans $J_{K}$ tombe dans le sous-groupe normique $N_{L / K}\left(J_{L}\right)$.

Pour interpréter les différents indices intervenant dans la formule obtenue, introduisons, pour chaque place non complexe $p$ de $K$, le corps $L_{p}=\prod_{\mathfrak{P} \mid \mathfrak{p}} L_{\mathfrak{P}}$ intersection des complétés de $L$ au-dessus de $p$ dans une même clôture algébrique de $K_{p}$, et notons $L_{p}^{a b}$ la sous-ex tension maximale de $L_{p}$ qui est abélienne sur $K_{p}$.

DÉFINITION III.2.2.- Etant données une extension finie $L / K$ de corps de nombres, et une place $p$ de $K$, nous disons que le groupe de Galois $D_{p}^{a b}(L / K)=G_{a l}\left(L_{p}^{a b} / K\right)$ de la sous-extension abélienne maximale $L_{p}^{a b}$ de l'extension galoisienne locale $L_{p}=\prod_{\mathfrak{P} \mid p} L_{\mathfrak{P}}$ du complété $K_{p}$ est le groupe de décomposition abélianisé de la place $p$ dans l'extension $L / K$; 
et nous appelons groupe d'inertie abélianisé le sous-groupe d'inertie $I_{p}^{a b}(L / K)$ de $\operatorname{Gal}\left(L_{p}^{a b} / K_{p}\right)$.

L'ordre du premier groupe $a_{p}^{a b}(L / K)=\left|D_{p}^{a b}(L / K)\right|$ est le degré de l'extension abélienne locale $L_{p}^{a b} / K_{p}$; et celui du second $e_{p}^{a b}(L / K)=\left|1_{p}^{a b}(L / K)\right|$ est son indice de ramification.

Cela posé, la théorie du corps de classes local nous donne les isomorphismes :

$D_{p}^{a b}(L / K)=\operatorname{Gal}\left(L_{p}^{a b} / K_{p}\right) \simeq K K_{p}^{x} / N_{L_{p}} / K_{p}\left(L_{p}^{x}\right)=K_{p}^{x} / \prod_{P / p} N_{L_{p}} / K_{p}\left(L_{p}^{X}\right)$

et $\quad i_{p}^{a b}(L / K)=u_{p} / \prod_{p \mid p} N_{L_{p}} / K_{p}\left(u_{B}\right)$.

En particulier, le groupe $N_{L / K}=K^{x} \cap N_{L / K}\left(J_{L}\right)$ s'interprète comme l'ensemble des éléments de $K^{X}$ qui sont normes dans chacune des exten sions abéliennes locales $L_{p}^{a b} / K_{p}$ ( ou, ce qui revient au même, dans chacune des extensions galoisiennes locales $L_{p} / K_{p}$ '. Nous disons que $N_{L / K}$ est le sous-groupe des éléments de $K X$ qui sont normes lo cales partout dans l'extension $L / K$.

Récapitulant ce qui précède, nous obtenons :

THEOREME 111.2.3.- ( Formule des S-genres) - Soit L/K une exten sion finie quelconque de corps de nombres. Pour tout ensemble fini $S$ de places non complexes du corps $K$, l'ordre $g_{L / K}^{S}=\left[\bar{C}_{L / K}^{S}: L\right] d u$ quotient des $S$-genres de l'extension $L / K$ est donné par la formule :

$$
g_{L / K}^{S}=\frac{h_{K}^{S}}{\left[L^{a b}: K\right]} \frac{\prod_{L} d_{p}^{a b}(L / K) \prod_{p \notin S} e_{p}^{a b}(L / K)}{\left(E_{K}^{S}: E_{K}^{S} \cap N_{L / K}\right)}
$$

Dans celle-ci, $h_{K}^{S}=\left[C_{K}^{S}: K\right]$ est le nombre de $S$-classes de diviseurs du corps $K$; $L^{\text {ab }}$ est la sous-extension maximale de $L$ qui est abélienne sur $K ; e_{p}^{a b}(L / K)$ est llindice de ramification ${ }^{(\star)}$ de la sous-extension

$\left.{ }^{\star}{ }^{\star}\right)$ Conformément aux conventions de la section 1 , la ramification n'intéresse que les places finies. Pour les places réelles, nous par lons, s'il y a lieu, de complexification. 
maximale $L_{p}^{a b}$, abélienne sur $K_{p}$, du corps $L_{p}=\underset{\mathfrak{P} \mid p}{\cap L_{P}}$; et $d_{p}^{a b}(L / K)$ est le degré de cette extension; enfin $E_{K}^{S}$ est le groupe des $S$ - unités de $K^{(*)}$, et $N_{L / K}=K^{x} \cap N_{L / K}\left(J_{L}\right)$ est le groupe des éléments de $K^{x}$ qui sont normes locales partout dans l'extension $L / K$.

Applications - - (i) Lorsque l'extension $S$ est vide, l'indice $g_{L / K}$ est le nombre de genres. Il est donné par la formule :

$$
g_{L / K}=\frac{n_{K}}{\left[L^{a b}: K\right]} \frac{\prod_{p} e_{p}^{a b}(L / K)}{\left(E_{K}: E_{K} \cap N_{L / K}\right)} ;
$$

$h_{K}$ est le nombre de classes de diviseurs, et $E_{K}$ le groupe des unités du corps $K$

(ii) Lorsque $S$ est l'ensemble des places réelles de $K$, l'in dice correspondant $g_{L / K}$ est le nombre de genres au sens ordinaire; il s'écrit :

$$
g_{L / K}^{\text {ord }}=\frac{h_{K}^{\text {ord }}}{\left[L^{a b}: K\right]} \frac{\left.\prod_{p} e_{p}^{a b}(L / K) \prod_{p}\right|_{\infty} ^{a b}(L / K)}{\left(E_{K}^{\text {ord }}: E_{K}^{\text {ord }} \cap N_{L / K}\right)}
$$

$h_{K}$ est le nombre de classes dijidéaux, et $E_{K}$ le groupe des unités au sens ordinaire du corps $K^{(\star \star \star)}$.

Remarque. - La définition du corps des S-genres, que nous avons donnée plus haut, s'applique sans changement aux extensions infinies de corps de nombres. Dans ce cas cependant, le quotient des $\mathbf{S}$-genres peut être infini, et les calculs précédents ne sont pas fondés. De fait, la formule des $S$-genres reste valable sous réserve que soient vérifiées les deux conditions suivantes:

(*) II slagit des $\mathbf{S}$-unités au sens des diviseurs, i.e. des S-unités au sens des idéaux qui sont en outre positives aux places réelles n'appar tenant pas à $S$.

$\left({ }^{\star}\right)$ Lorsque $L / K$ est abélienne, nous retrouvons ainsi la formule de Goldstein (cf. [Gd], th.2.1).

$(\star \star \star)$ Lorsque L/K est galoisienne, la formule obtenue est due à Furuta (cf. $\left.\left[F u_{1}\right]\right)$. 
(i) Iln'y a qu'un nombre fini de places de $K$ qui se ramifient dans L.

(ii) Pour chaque place non complexe $p$ de $K$, l'extension abé lienne locale $L_{p}^{a b} / K_{p}$ est de degré fini .

\section{b.- La formule du produit pour le symbole de reste normique.}

Soit $L / K$ une extension abélienne (finie) de corps de nombres . Si $p$ est une place non complexe de $K$, le symbole de reste normique $\left(\frac{, L / K}{p}\right)$, introduit par Hasse dans $\left[\mathrm{Ha}_{1}\right]$, est composé de l'injection du groupe multiplicatif de $K$ dans celui de son complété $K_{p}$, de l'application de réciprocité locale $\left(\cdot, L_{\beta} / K_{p}\right)$ associé à l'extension locale $L_{\mathfrak{P}} / K_{\mathfrak{p}}$ au-dessus de $L / K$, et de llisomorphisme canonique $d u$ groupe de Galois Gal( $\left.L_{p} / K_{p}\right)$ sur le groupe de décomposition $D_{p}(L / K)$ de la place $p$ dans $L / K$. On sait que les symboles de Hasse $\left(\frac{, L / K}{p}\right)$ sont directement reliés au symbole d'Artin global (l'élément $\left(\frac{x, L / K}{p}\right)$ étant le symbole d'Artin $\left(\frac{L / K}{a}\right)$ de l'idéal $a=y p^{-V_{p}(y)}$ construit sur un $p$-associé convenable $y$ de $x$ ), et que la formule du produit :

$$
\prod_{p}\left(\frac{\cdot, L / K}{p}\right)=1
$$

est essentiellement la seule relation entre les divers symboles $\left(\frac{\cdot, L / K}{p}\right)$ attachés à une même extension (en ce sens que toute autre relation de dépendance multiplicative lui est proportionnelle).

Supposons maintenant que $\mathrm{L} / \mathrm{K}$ soit une extension finie quel conque de corps de nombres. Dans ce cas, il est encore possible de définir un symbole de reste normique pour chaque place non complexe $p$ de $K$, en introduisant les extensions abéliennes locales $L_{p}^{a b} / K_{p}$ :

DÉFINITION III.2.4.- Etant données une extension finie quelconque de corps de nombres $L / K$, et une place non complexe $p$ du corps $K$, nous appelons symbole de reste normique généralisé, associé à la place p 
dans l'extension $L / K$, I'application $\left(\frac{\cdot, L / K}{p}\right)$ du groupe multiplicatif $K^{x}$ sur le groupe de décomposition abélianisé $D_{p}^{a b}(L / K)$, compo sée de linjection naturelle du groupe multiplicatif de $K$ dans celui de son complété $K_{p}$, et de l'isomorphisme de réciprocité $\left(\cdot, L_{p}^{a b} / K_{p}\right)$ attaché à la sous-extension abélienne maximale $L_{p}^{a b} / K_{p}$ del'extension galoisienne locale $\left(\bigcap_{\mathcal{P} \mid p} L_{\mathcal{P}}\right) K_{\mathcal{P}}$.

Bien entendu, le symbole généralisé induit le symbole usuel lorsque l'extension est abélienne, de sorte que, si $L^{a b}$ désigne la sous-extension maximale de $L$ abélienne sur $K$, les restrictions à $L^{a b}$ des symboles de reste normique généralisés pris dans $L / K$ vérifient la formule du produit :

$$
\left.\prod_{p}\left(\frac{\dot{L}, L / K}{p}\right)\right|_{L^{a b}}=1 \text {. }
$$

Le but de cette section est d'établir la réciproque de ce der nier résultat :

THÉORËME 111.2.5.- ( Réciproque de la formule du produit) - Étant donnée une extension finie quelconque $L / K$ de corps de nombres, les familles $\left(\sigma_{p}\right)_{p}$ appartenant à la somme directe $\oplus_{p} D_{p}^{a b}(L / K)$ des groupes de Galois attachés aux extensions abéliennes locales associées à $L / K$, dont les restrictions à la sous-extension abélienne globale $L^{a b}$ vérifient la formule du produit $\left.\prod_{p} \sigma_{p}\right|_{L} a b=1$, sont celles provenant par les symboles de reste normique généralisé d'un même élément de $K^{x}$. En d'autres termes, les symboles généralisés $\left(\frac{\dot{L} L / K}{p}\right)_{p \in P I_{K}}$ induisent un isomorphisme canonique du quotient $K^{x} / N_{L / K}$ sur la somme directe restreinte $\underset{p}{\tilde{\oplus}} \mathrm{D}_{p}^{\mathrm{ab}}(\mathrm{L} / K)$.

LEMME III.2.6. - Soient $S$ un ensemble fini de places non complexes de $K$. Les $S$-unités de $K$ qui sont normes locales partout dans $L / K$ sont exactement celles qui sont normes locales partout dans l'extension 
$\bar{C}_{L / K}^{S} / K$. De plus, les indices de décomposition $a_{p}^{a b}(L / K)$ et $e_{p}^{a b}(L / K)$, attachés aux places non complexes $p$ du corps $K$ dans l'extension $L / K$, s'interprètent encore comme étant les ordres respectifs des groupes de décomposition et d'inertie abélianisés $D_{p}^{a b}\left(C_{L / K}^{S} / K\right)$ et $I_{p}^{a b}\left(C_{L / K}^{S} / K\right)$ attachés à ces mêmes places dans l'extension $C_{L / K}^{S} / K$.

Démonstration : Cela résulte directement de l'égalité des groupes d'idèles $J_{L}(S)=N_{\bar{C}_{L / K}^{S}} / L\left(J_{L / K}^{S}(L)\right)$, l'extension abélienne $\bar{C}_{L / K}^{S} / L$ étant par hypothèse non ramifiée et $\mathrm{S}$ - décomposée .

THÉORËME 111.2.7.- ( Suite exacte des $S$-genres ) - Soient $L / K$ une extension finie quelconque de corps de nombres, $S$ un ensemble fini de places non complexes de $K$, puis $\bar{C}_{L / K}^{S}$ le corps des $S$-genres de l'extension, $C_{K}^{S}$ le corps des $S$-classes de $K, E_{K}^{S}$ le groupe des $S$ unités, et $N_{L / K}$ le sous-groupe de $K^{X}$ formé des éléments qui sont normes locales partout dans l'extension $L / K$. Il existe une suite exacte courte canonique :

$$
\begin{aligned}
& 1 \longrightarrow E_{K}^{S} / E_{K}^{S} \cap N_{L / K} \stackrel{\eta}{\longrightarrow}\left(\underset{p \in S}{\oplus} D_{p}^{a b}\left(\bar{C}_{L / K}^{S} / K\right)\right) \oplus\left(\underset{p \notin S}{\oplus} I_{p}^{a b}\left(\bar{C}_{L}^{S} / K\right)\right) \\
& \stackrel{\pi}{\rightarrow} \operatorname{Gal}\left(\mathrm{C}_{\mathrm{L} / \mathrm{K}}^{\mathrm{S} a b} / \mathrm{C}_{\mathrm{K}}^{\mathrm{S}}\right) \rightarrow 1
\end{aligned}
$$

où l'application $\eta$ est induite par les symboles généralisés $\left(\frac{\cdot, C_{L}^{S} / K / K}{p}\right)$, et $\pi$ est la projection canonique $\left.\left(\sigma_{p}\right)_{p} \mapsto \prod_{p} \sigma_{p}\right|_{C_{L / K}^{S} \text { ab }}$ de la somme

directe des groupes de décomposition et d'inertie abélianisés sur le groupe de Galois de l'extension abélienne $C_{L / K}^{S} / C_{K}^{S}$.

Démonstration : L'application $\pi$ est surjective, par maximalité du corps des $S$-classes $C_{K}^{S}$; et $\eta$ est injective, en vertu du lemme 6 ; linclu sion Im $\eta \subset$ Ker $\pi n$ 'est autre que la formule du produit; et l'exactitude de la suite, qui constitue donc une réciproque partielle de cette for mule, résulte du calcul du nombre de $S$-genres effectué plus haut (cf. théorème 3 ). 
Démonstration du théorème 5 : Prenons $S$ assez grand, i.e. contenant les places ramifiées dans l'extension $L / K$, et suffisamment de places pour engendrer les groupes de classes de diviseurs de $L$ comme de $K$. Dans ce cas, le corps des $S$-classes $C_{L}^{S}$ coincide avec $L$, et son sous-corps $C_{K}^{S}$ avec $K$. La suite exacte du théorème 7 prend donc la forme :

$1 \longrightarrow E_{K}^{S} / E_{K}^{S} \cap N_{L / K} \longrightarrow \underset{p \in S}{\oplus} D_{p}^{a b}(L / K) \longrightarrow G a l\left(L^{a b} / K\right) \longrightarrow 1$ et les $\mathbf{S}$ - unités sont bien normes locales en-dehors de $\mathbf{S}$ dans l'extension $L / K$. Passant à la limite avec $S$, nous obtenons donc la suite exacte courte attendue :

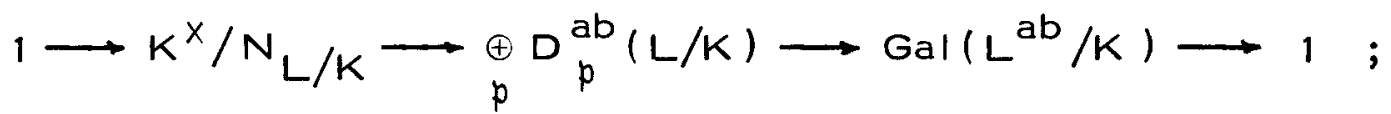

ce qui établit le théorème 5 .

SCOLIE III.2.8. - Dans une extension finie $L / K$ de corps de nombres, les symboles de reste normique généralisés donnent naissance à la suite exacte courte canonique :

$1 \longrightarrow K^{x} / N_{L / K} \longrightarrow \underset{p}{\oplus} D_{p}^{a b}(L / K) \longrightarrow G a l\left(L^{a b} / K\right) \longrightarrow 1$

Lien avec le théorème de Moore - $K$ étant un corps de nombres ( de degré fini), supposons donnée, pour chaque place non complexe d'un ensemble fini $S$, une racine de l'unité $\zeta_{p}$ dans le complété $K_{p}$. Notons $\mu_{k}$ le groupe des racines de llunité du corps $K$, puis $m$ son ordre, et $m_{p}$ celui du groupe $\mu_{K_{p}}$ des racines de l'unité dans $K_{p}$, $n$ enfin un multiple commun non nul des $m_{p}$ associés aux places de $S$, et, plus généralement, $n \wedge m_{p}$ le plus grand diviseur commun de $n$ et de $m_{p}$.

Par le théorème d'approximation simultanée, nous pouvons choisir un a dans $K$ dont limage dans le quotient $K{ }_{p}^{x} / \mu_{K_{k}} K_{p}^{x m}$ soit exactement d'ordre $m_{p}$ (par exemple, si $p$ est ultramétrique, en imposant à a d'être une uniformisante locale), et ce, pour chaque $p$ de l'ensemble fini $S$. Prenons un tel a, et considérons l'extension $L=K[\sqrt[n]{a}]$ : sa sous-extension maximale $L^{a b}$ a pour degré $m$, et, pour tout $p$ de $S$, la sous-extension maximale $L_{p}^{a b}$ de $K_{p}[\sqrt[n]{a}]$, abélienne sur $K_{p}$, a pour degré $m_{p}$. Si donc $\sigma_{p}$ désigne l'élément du 
groupe de décomposition $D_{p}^{a b}(L / K)$ défini par:

$\sqrt{a}^{\left(\sigma_{p}-1\right)}=\zeta_{p}$, pour $p \in S \quad \& \quad \sigma_{p}=1$, pour $p \notin S$,

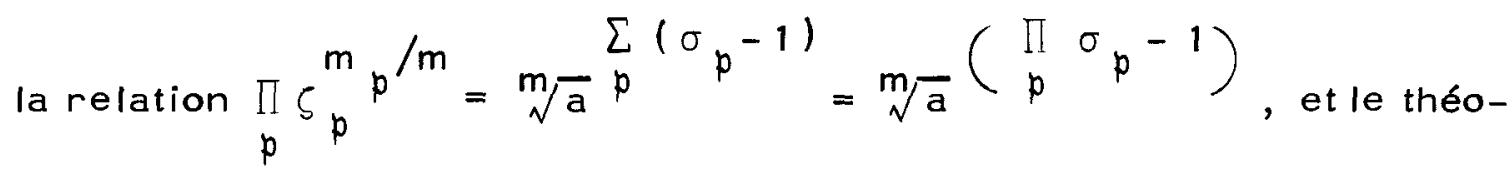
rème 5 nous montrent que la formule du produit

$$
\prod_{p} \zeta_{p}^{m} p^{/ m}=1
$$

est la condition pour que les $\sigma_{p}$ soient les symboles de reste normique généralisés, dans l'extension $L / K$, d'un élément $b$ de $K^{X}$ qui est nor me locale en-dehors de $S$. Lorsqu'elle est vérifiée, nous avons ainsi :

$$
\sigma_{p}=\left(\frac{b, K[\sqrt[n]{a}] / K}{p}\right) \text {, pour chaque place } p ;
$$

donc, en particulier :

$\left(\frac{a, b}{p}\right)_{m}=S_{p}$, pour $p \in S$; et $\left(\frac{a, b}{p}\right)_{m}^{m} m_{p}^{/ m} p^{\wedge n}=1$, pour $p \notin S$; ce qui établit l'exactitude de la suite induite par les symboles de Hilbert $\left(\frac{1}{p}\right)=\left(\frac{1}{p}\right)_{m p}:$

$$
K^{x} \otimes{ }_{\mathbb{Z}} K^{x} \longrightarrow \oplus_{p} \mu_{K} / \mu_{K}^{n} \longrightarrow \mu_{k} / \mu_{K}^{n} \longrightarrow 1
$$

Le résultat obtenu vaut pour tout $n$ assez grand donc, finalement, pour tout $\mathrm{n}$ non nul. Il est plus faible que le théorème de Moore (cf. [CW]), qui affirme l'exactitude de la suite pour $n=0$, mais dont la démonstration fait intervenir un argument analytique de densité. Il suffit cepen dant à montrer qu'il n'est d'autre loi de réciprocité pour le symbole de Hilbert que la formule du produit $\prod_{p}\left(\frac{,}{p}\right)^{m} p^{/ m}=1$. 
c.- Comparaison du corps des genres et du corps des classes centrales.

L'idée d'étudier les extensions centrales des corps de nombres abéliens est due à Fröhlich, qui signala le premier le rôle joué dans ces questions par les multiplicateurs de Schur associés aux grou pes de Galois ( $\mathrm{cf}_{\bullet}\left[\mathrm{Fr}_{2}\right]$ ). En fait, la notion d'extension centrale peut se généraliser en l'absence de toute hypothèse galoisienne, de par l'existence de la norme arithmétique :

DÉFINITION III.2.9.- Etant donnés une extension finie quelconque $L / K$ de corps de nombres, et un ensemble fini $S$ de places non complexes de $K$, nous appelons corps des $S$-classes centrales de l'extension $L / K$, et nous notons $C_{L / K}^{S}$, le sous-corps du corps des $S$-classes de $L$ fixé par le groupe diidèles

$$
J_{N / K}^{S}={ }_{N} J_{L} \cdot J_{L}(S) \cdot L^{X}
$$

où ${ }_{N} \mathrm{~J}_{L}$ est le noyau dans $\mathrm{J}_{\mathrm{L}}$ de la norme arithmétique $N_{\mathrm{L}} / \mathrm{K}$ •

Cette définition trouve sa justification dans la proposition sui -

vante :

PROPOSITION III,2.10.- Supposons l'extension L/K galoisienne. Alors le corps des $S$-classes centrales $C_{L / K}^{S}$ de $L / K$ est la plus grande sousextension galoisienne du corps des $S$-classes $C_{L}^{S}$ pour laquelle le groupe de Galois Gal( $\left.C_{L / K}^{S} / L\right)$ est contenu dans le centre de Gal ( $\left.C_{L / K}^{S} / K\right)$. Autrement dit,$C_{L / K}^{S}$ est la sous-extension de $C_{L}^{S}$ fixée par le sous-groupe $C \underset{L}{S} / K$ de $C S$, image du groupe des $S$ classes de diviseurs par lijéal d'augmentation de l'algèbre de Galois $\mathbb{Z}[\operatorname{Gal}(L / K)]$.

Démonstration : Ilsuffit naturellement de vérifier l'identité des grou pes d'idèles ${ }_{N} J_{L} \cdot U_{L}$ et $J_{L}^{I} G \cdot U_{L}$, où $I_{G}$ est l'idéal d'augmenta tion de llalgèbre de Galois $\mathbb{Z}[G a l(L / K)]$. Or le quotient $J_{L} / U_{L} d u$ groupe des idèles par le sous-groupe des idèles unités s'identifie au groupe des diviseurs $D_{L}$, et les diviseurs de norme 1 sont précisé ment ceux contenus dans le sous-groupe $D_{L}{ }_{L}{ }^{\prime}$. 
SCOLIE III.2.11.- Soient L/K une extension finie quelconque de corps de nombres, et $S$ un ensemble fini de places non complexes de $K$. Les groupes dijièles attachés par la théorie du corps de classes au composé avec $L$ du corps des $S$-classes de $K$, au corps des $S$-genres de l'extension $L / K$, et au corps des $S$-classes centrales de $L / K$ sont respectivement :

(i) pour le composé $L C_{K}^{S}$, le groupe :

$J_{L / K}^{S *}=\left\{\mathfrak{r} \in J_{L} \mid N_{L / K}(\mathfrak{r}) \in J_{K}(S) \cdot K^{X}\right\} ;$

(ii) pour le corps $\bar{C}_{L / K}^{S}$, le groupe :

$$
J_{L / K}^{S}=\left\{\mathfrak{r} \in J_{L} \mid N_{L / K}(\mathfrak{r}) \in N_{L / K}\left(J_{L}(S)\right) \cdot K^{x}\right\} ;
$$

(iii) pour le corps $C_{L / K}^{S}$, le groupe :

$$
J_{L / K}^{S}=\left\{\mathfrak{x} \in J_{L} \mid N_{L / K}(\mathfrak{x}) \in N_{L / K}\left(J_{L}(S) \cdot L^{x}\right)\right\} \text {. }
$$

THÉORËME 111.2.12.- (Formule des S-classes centrales ) - Soit L/K une extension finie quelconque de corps de nombres. Pour tout ensemble fini $S$ de places non complexes du corps $K$, le nombre $h_{L / K}^{S}=$ $\left[C_{L / K}^{S}: L\right]$ de $S$-classes centrales de l'extension $L / K$ est donné par la formule :

$n_{L / K}^{S}=\frac{h_{K}^{S}}{\left[L^{a b}: K\right]} \cdot \frac{\prod_{K} S^{d_{p}^{a b}(L / K)} \cdot \prod_{p \notin S} e_{p}^{a b}(L / K)}{\left(E_{K}^{S}: E_{K}^{S} \cap N_{L / K}\left(L^{x}\right)\right)} \cdot k_{L / K} \cdot$ Dans celle-ci, $h_{K}^{S}=\left[C_{K}^{S}: K\right]$ est le nombre de $S$-classes de divi seurs du corps $K ; L^{a b}$ est la sous-extension maximale de $L$ qui es $t$ abélienne sur $K ; e_{p}^{a b}(L / K)$ est l'indice de ramification de la sous extension maximale $L_{p}^{a b}$, abélienne sur $K_{p}$, du corps $L_{p}=\bigcap_{\mathcal{P} / p} L_{\mathfrak{P}}$; et $d_{p}^{a b}(L / K)$ est le degré de cette extension; enfin $E_{K}^{S}$ est le groupe des $\mathrm{S}$-unités de $K$, et $k_{L / K}=\left(N_{L / K}: N_{L / K}\left(L^{x}\right)\right)$ est le nombre de noeuds de l'extension $L / K$.

Démonstration : Considérons le quotient $J_{L / K}^{S} / J_{L / K}^{S}$. En associan $t$ à la classe de liidèle $\mathfrak{r} \in \bar{J}_{L / K}^{S}$ celle de l'élément $x \in N_{L / K}$, défini par $x^{-1} N_{L / K}(\mathfrak{x}) \in N_{L / K}\left(J_{L}(S)\right)$, nous définissons un isomorphisme : 


$$
\bar{J}_{L / K}^{S} / J_{L / K}^{S} \simeq N_{L / K} / N_{L / K}\left(L^{x}\right)\left(E_{K}^{S} \cap N_{L / K}\right) \text {. }
$$

II vient donc :

$$
\begin{aligned}
{\left[C_{L / K}^{S}: \bar{C}_{L / K}^{S}\right]=\left(\bar{J}_{L / K}^{S}: J_{L / K}^{S}\right) } & =\left(N_{L / K}: N_{L / K}\left(L^{x}\right) .\left(E_{K}^{S} \cap N_{L / K}\right)\right) \\
& =\frac{\left(N_{L / K}: N_{L / K}\left(L^{x}\right)\right)}{\left(E_{K}^{S} \cap N_{L / K}: E_{K}^{S} \cap N_{L / K}\left(L^{x}\right)\right)} .
\end{aligned}
$$

Et l'expression du nombre de $S$-classes centrales résulte de celle du nombre de $S$-genres donnée par le théorème 5 .

Applications - - (i) Lorsque l'ensemble $S$ est vide, l'indice $h_{\mathrm{L} / \mathrm{K}}$ est le nombre de classes centrales. Il est donné par la formule :

$$
h_{L / K}=\frac{h_{K}}{\left[L^{a b}: K\right]} \cdot \frac{\prod_{p}^{a b}(L / K)}{\left(E_{K}: E_{K} \cap N_{L / K}\left(L^{x}\right)\right)} \cdot k_{L / K}
$$

$h_{K}$ est le nombre de classes de diviseurs de $K, E_{K}$ est le groupe des unités ( au sens des diviseurs), et $\kappa_{L / K}$ est le nombre de noeuds de l'extension $\mathrm{L} / \mathrm{K}$.

(ii) Lorsque $S$ est l'ensemble des places réelles de $K$, liin dice correspondant $h_{L / K}^{\text {ord }}$ est le nombre de classes centrales diidéaux;

$$
h_{L / K}^{\text {ord }}=\frac{h_{K}^{\text {ord }}}{\left[L^{a b}: K\right]} \frac{\left.\prod e_{p}^{a b}(L / K) \cdot \prod_{p}\right|_{p} ^{a b}(L / K)}{\left(E_{K}^{\text {ord }}: E_{K}^{\text {ord }} \cap N_{L} / K^{\left(L^{X}\right)}\right.} \cdot \kappa_{L} / K \cdot
$$

$h_{K}^{\text {ord }}$ est le nombre de classes d'idéaux, et $E_{K}^{\text {ord }}$ le groupe des unités au sens ordinaire du corps $K$.

COROLLAIRE 111.2.13.- Soient L une extension finie quelconque du corps des rationnels, $\bar{C}=\bar{C}_{L / \mathbb{Q}}$ le corps des genres correspondant, et $C=C C_{L / Q}$ le corps des classes centrales de $L / Q$. Alors le groupe de Galois $\mathrm{Gal}\left(\mathrm{C} / \overline{C_{1}}\right)$ est isomorphe au groupe des noeuds $\mathrm{K}_{\mathrm{L} / \mathbb{Q}}=$ $N_{L / Q} / N_{L / Q}{ }^{\left(L^{X}\right)^{(*)}}$.

${ }^{\star}$ ) Cerésultat a été établi par Garbanati dans le cas oì L est une exten sion galoisienne du corps des rationnels ( $\mathrm{cf}$. $\left[\mathrm{Ga}_{3}\right]$ ). 
Démonstration: C'est une conséquence immédiate de lisomorphisme établi ci-dessus :

$\operatorname{Gal}(\overline{\mathrm{C}} / \mathrm{C}) \simeq \bar{J}_{L / Q} / J_{L / Q} \simeq N_{L / Q} / N_{L / Q}\left(L^{x}\right)\left(E_{Q} \cap N_{L / Q}\right)$, et du fait que la seule unité ( au sens des diviseurs) du corps des rationnels est le nombre 1 .

COROLLAIRE 111.2,14.- Soient $L$ une extension finie quelconque du corps des rationnels, et $C=C \mathbb{L}_{\mathbb{Q}}$ le corps des classes centrales as socié. Pour quiun rationnel non nul soit une norme globale dans l'ex tension $L / Q$, il faut et il suffit quil soit norme locale partout dans l'extension $C / Q$.

Démonstration: En l'absence d'unité rationnelle autre que 1 , nous avons en effet :

$x \in N_{L / \mathbb{Q}}\left(L^{x}\right) \Leftrightarrow x \in N_{L / \mathbb{Q}}\left(U_{L} \cdot L^{x}\right) \Leftrightarrow x \in N_{C / \mathbb{Q}}\left(J_{C}\right)$, comme annoncé.

Appendice : le groupe des noeuds - Le nombre de noeuds $k_{L / K}$ fut in troduit par Scholz ( $\mathrm{cf} .[\mathrm{Sz}]$ ) à l'occasion de l'étude de corps biqua dratiques $^{(\star)}$. Ces corps sont, en effet, les plus petites extensions galoisiennes de $\mathbb{Q}$ dans lesquelles le principe des normes de Hasse (i.e. le fait que les normes locales $N_{L / K}$ soient exactement les normes glo bales $N_{L / K}\left(L^{X}\right)$ ) peut se trouver en défaut : Pour voir cela, suppo sons donnée une extension finie quelconque $L / K$ de corps de nombres, et formons le diagramme commutatif associé à la norme arithmétique :

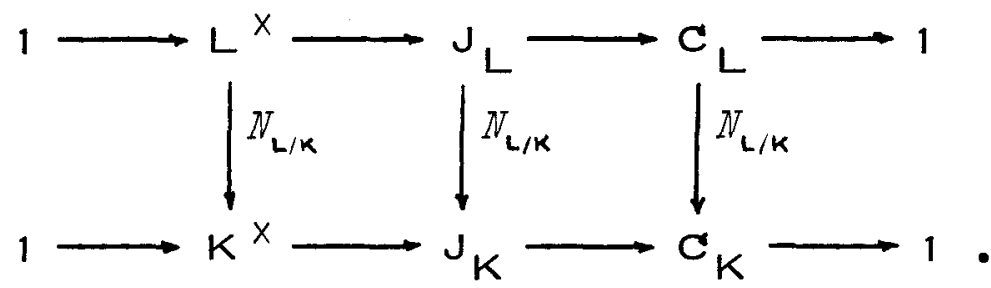

Par le lemme du serpent, nous obtenons la suite exacte longue :

${ }^{\star}$ ) En fait Scholz définit plusieurs groupes de noeuds associés respectivement au groupe multiplicatif du corps, au groupe des unités, et au groupe des classes d'idéaux. Il est d'ailleurs possible d'en définir bien d'autres. On pourra consulter sur ce sujet l'article très complet de Jehne [Je]. 
${ }_{1 \rightarrow{ }_{N}} \mathrm{~L}^{\mathrm{X}} \rightarrow{ }_{N} \mathrm{~J}_{\mathrm{L}} \rightarrow{ }_{N} \mathrm{C}_{\mathrm{L}} \rightarrow \mathrm{K}^{\mathrm{x}} / N_{\mathrm{L} / \mathrm{K}}\left(\mathrm{L}^{\mathrm{x}}\right) \rightarrow \mathrm{J}_{\mathrm{K}} / N_{\mathrm{L} / \mathrm{K}}\left(\mathrm{J}_{\mathrm{L}}\right) \rightarrow \mathrm{C}_{\mathrm{K}} / N_{\mathrm{L} / \mathrm{K}}\left(\mathrm{C}_{\mathrm{L}}\right) \rightarrow 1$. Cela étant, la théorie du corps de classes global nous dit que le terme de droite $C_{K} / N_{L / K}\left(C_{L}\right)$ s'identifie au groupe de Galois $G^{\text {ab }}(L / K)=$ $\mathrm{Gal}\left(L^{a b} / K\right.$ ) de la sous-extension abélienne maximale $L^{a b} / K$ de $L / K$; et la théorie du corps de classes local interprète le quotient $J_{K} / N_{L} / K\left(J_{L}\right)$ comme somme directe $\oplus_{p}^{a b}(L / K)$ des groupes de Galois respectifs $D_{p}^{a b}(L / K)=\operatorname{Gal}\left(L_{p}^{a b} / K_{p}\right)$ des extensions abéliennes locales asso ciées à $L / K$. La partie droite de la suite prend donc de la forme :

$1 \rightarrow N_{L / K} / N_{L / K}\left(L^{x}\right) \rightarrow K^{x} / N_{L / K}\left(L^{x}\right) \rightarrow \underset{p}{\oplus} D_{p}^{a b}(L / K) \rightarrow G^{a b}(L / K) \rightarrow 1 ;$ ce qui permet de retrouver la suite exacte courte du scolie 8 . Quant à la partie gauche, elle s'écrit encore:

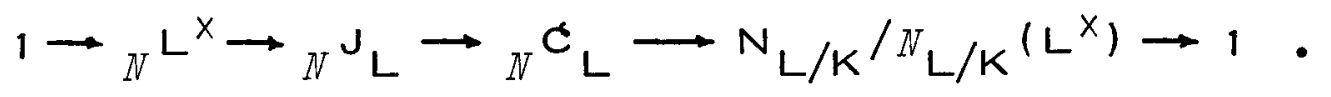

Elle interprète donc le groupe des noeuds $K_{L / K}=N_{L / K} / N_{L / K}\left(L^{x}\right)$ comme quotient du groupe ${ }_{N} C_{L}$ des classes dididèles de norme 1 par le sous-groupe des classes des idèles de norme 1 .

(i) Lorsque l'extension $L / K$ est galoisienne, de groupe $G$, le noyau ${ }_{N} C_{L}$ s'écrit aussi $H^{-1}\left(G, C_{L}\right)$, et le noyau ${ }_{N} J_{L}$ s'écrit de même $H^{-1}\left(G, J_{L}\right) \simeq \underset{p}{\oplus} H^{-1}\left(D_{p}, L_{p}^{x}\right)$, si $D_{p}=G a l\left(L_{p} / K_{p}\right)$ est le groupe de Galois local associé à la place $p$. Il vient donc :

$$
\begin{aligned}
K_{L / K} & \simeq \operatorname{Coker}\left[\underset{p}{\oplus} H^{-1}\left(D_{p}, L_{p}^{x}\right) \longrightarrow H^{-1}\left(G, C_{L}\right)\right] \\
& \simeq \operatorname{Coker}\left[\underset{p}{\oplus} H^{-3}\left(D_{p}, \mathbb{Z}\right) \longrightarrow H^{-3}(G, \mathbb{Z})\right],
\end{aligned}
$$

via les isomorphismes cohomologiques du corps de classe.

$$
\text { Le dual de Pontrjagin } \check{K}_{L / K}=\operatorname{Hom}\left(K_{L / K}, Q / \mathbb{Z}\right) \text { est donc }
$$
le noyau de l'application naturelle de $H^{3}(G, \mathbb{Z})$ dans la somme directe $\underset{p}{\oplus} H^{3}\left(D_{p}, \mathbb{Z}\right)$. C'est l'interprétation de Tate, qui montre en particulier que $K_{L} / K$ est nul lorsque $G$ est cyclique et, plus généralement, dès que $D_{p}$ estégal à $G$ pour au moins un $p(c f .[C F], C h . V I I I, \S 11.4$ ) • 
(ii) Lorsque l'extension $L / K$ est abélienne, le groupe $H^{3}(G, \mathbb{Z}) \simeq H^{2}(G, Q / \mathbb{Z})$ s'identifie au carré alterné $G \wedge G$ du groupe $G$. De même $H^{3}\left(D_{p}, \mathbb{Z}\right) \simeq H^{2}\left(D_{p}, Q / \mathbb{Z}\right)$ est isomorphe à $D_{p} \wedge D_{p} \cdot \|$ vient donc:

$$
\check{K}_{L / K} \simeq \operatorname{Ker}\left[G \wedge G \longrightarrow \underset{p}{\oplus}\left(D_{p} \wedge D_{p}\right)\right] \text {. }
$$

C'est l'interprétation de Razar (cf. $[R z]$ ). Par exemple, si G est le groupe de $k$ lein $\mathbb{Z} / 2 \mathbb{Z} \times \mathbb{Z} / 2 \mathbb{Z}$, le principe des normes de Hasse vaut si $D_{p}$ est égal à $G$ pour au moins un $G$; il est en défaut sinon. Ainsi pour $L=\mathbb{Q}[\sqrt{p}, \sqrt{q}], p$ et $q$ premiers congrus à 1 modulo 4 , vérifiant $\left(\frac{p}{q}\right)=\left(\frac{q}{p}\right)=1$, et $K=\mathbb{Q}$.

\section{2. - EXPRESSION DE LA FORMULE DES GENRES EN TERMES DE REPRÉ- SENTATIONS.}

\section{a.-Etude du cas métabélien.}

Nous reprenons dans cette section les hypothèses de la sec tion $1, \S 2$. En particulier, $\ell$ est un nombre premier, $L$ une $\ell$-ex tension cyclique de $K$, galoisienne sur un sous-corps $F$, et $K / F$ est abélienne de degré d étranger à $\ell$; le groupe de Galois Gal(L/F) s'écrit comme produit semi-direct du $\ell$-Sylow $G=G a l(L / F)$ et $d u$ quotient abélien $\Delta=G a l(F / K)$; l'homomorphisme de $\Delta$ dans Aut $G$, qui définit le produit se factorise par un caractère $\ell$-adique $\chi$ du groupe $\Delta$, conformément à l'identité :

(*) $\quad \operatorname{Tg}^{-1}=g^{X(\tau)}$, quels que soient $\tau$ dans $\Delta$ et g générateur de $G$; et cette relation se traduit, dans l'algèbre du groupe $\mathbb{Z}$ [Gal(L/F)], par l'identité de translation des caractères:

(**) $\quad \mathrm{e}_{\varphi x} \theta=\theta \mathrm{e}_{\varphi}, \quad \forall \varphi \in \mathrm{R}_{\mathbb{Z}_{\ell}}(\Delta)$,

où e $\mathrm{e}_{\varphi}=\frac{1}{\mathrm{~d}} \sum_{\tau \in \Delta} \varphi\left(\tau^{-1}\right) \tau$ est l'idempotent primitif de l'algèbre $\mathbb{Z}_{\ell}[\Delta]$ associé à un caractère $\ell$-adique irréductible $\varphi$ du groupe $\Delta$, et $\theta=$ $\frac{1}{d} \sum_{\tau \in \Delta} X\left(\tau^{-1}\right)\left[g^{X(\tau)}-1\right]$ est la résolvante de Lagrange construite sur un générateur arbitraire $g$ du groupe $G$. 
Cela posé, fixons un ensemble fini $S$ de places non complexes de $F$, désignons par $\alpha \underset{L}{S}$ le $\ell$-groupe des $S$-classes de diviseurs du corps $L$, et intéressons-nous à son quotient des genres $Q_{L}^{S} / K$. Une simplification intervient ici : L'extension $L / K$ étant supposée cyclique, le $\ell$-corps des $S$-genres $\bar{C}_{L}^{S} / K$ coincide avec le $\ell$-corps des $S$-classes centrales $C_{L / K}^{S}$, ce qui permet d'écrire directement :

$$
G_{L / K}^{S}=C L_{L}^{S} / C e_{L}^{S I} G=C e_{L}^{S} / C e_{L}^{S \theta}
$$

puisque la résolvante $\theta$ engendre l'idéal d'augmentation de l'algèbre $\mathbb{Z}_{\ell}[G]$. Compte-tenu de l'identité de translation des caractères rap pelée plus haut, il vient donc:

THÉOREME 111.2.15. - (Formule de translation des S-classes ) - Soient $L$ une $\ell$-extension cyclique diun corps de nombres $K$, de groupe $G$, métabélienne sur un sous-corps $F$ de $K$, d'indice relatif $[K: F]$ étranger à $\ell$, puis $x$ le caractère du groupe de Galois $\Delta=G a l(K / F)$ qui factorise le produit semi-direct $G \times \Delta$, et $S$ un ensemble fini de places non complexes de $F$. La résolvante de Lagrange $\theta$, construite sur un générateur $\mathrm{g}$ du groupe $G$, donne naissance à la suite exacte :

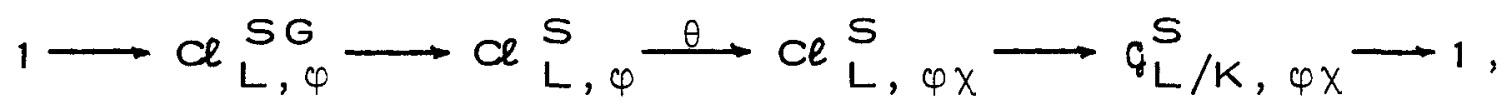
qui relie le $\ell$-quotient des $S$-genres $C_{L}^{S} / K$ avec le sous-groupe ambige ${ } S_{L} G$ du $l$-groupe des $S$-classes de diviseurs du corps $L$. En particulier, les ordres respectifs $h_{L, \varphi}^{S}, g_{L / K, \varphi}^{S}$, et $a_{L / K, \varphi}^{S}$ des $\varphi$ composantes respectives du $\ell$-groupe des $S$-classes de diviseurs de $L$, du $\ell$-quotient des $S$-genres, et du $\ell$-sous-groupe ambige sont liés par la formule de translation des $S$-classes :

$$
\frac{n_{L, \varphi}^{S}}{n_{L, \varphi x}^{S}}=\frac{a_{L / K}^{S}, \varphi}{g_{L / K}^{S}, \varphi x} \text {. }
$$

Lorsque $\Phi$ est le caractère d'un idempotent central de l'algèbre de Galois (i.e. lorsque $\Phi$ est un caractère $\ell$-adique de $\Delta$, contenu dans le caractère régulier, et stable par translation de $\chi$ ), la formule des S-classes conduit à lididentité : 


$$
g_{L / K, \Phi}^{S}=a_{L / K, \Phi}^{S}
$$

qui exprime l'égalité entre la $\Phi$-partie du $\ell$-nombre de $S$-genres et celle du $\ell$-nombre de classes ambiges. En particulier, lorsque $L$ est abélienne sur $F$, l'expression du $\ell$-nombre de $S$-genres, pour cha que caractère $\ell$-adique $\varphi$, résulte directement des calculs effectués dans la section III $\$ 2$.

A l'opposé, lorsque $\varphi$ est un caractère $\ell$-adique irréductible du groupe $\Delta$, qui n'est pas stable par translation de $\chi$, la formule de translation des $S$-classes permet d'exprimer la $\Phi$-partie du nombre de $S$-classes de diviseurs du corps $L$, pour chaque caractère $\psi$ de l'or bite de $\varphi$, à partir de la seule $\varphi$-partie de ce nombre et des nombres de $\boldsymbol{S}$-genres et de $\boldsymbol{S}$-classes ambiges pour les divers caractères de l'orbite. Par exemple, si L est métacyclique sur F (i.e. si Xest le caractère d'une représentation finie de $\Delta$, nous disposons ainsi d'un procédé permettant de calculer le nombre de $\mathbf{S}$-classes de diviseurs

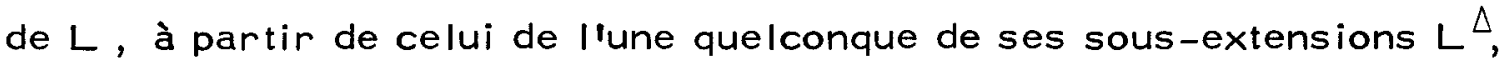
fixée par un relèvement de $\Delta$.

II faut naturellement pour cela évaluer directement la $\varphi$ partie du $\ell$-nombre de $S$-genres pour chaque caractère $\ell$-adique $\varphi$ du groupe $\Delta$. Partons donc de la suite exacte des $S$-genres, telle qu'elle est donnée par le théorème 7 . Formant le produit tensoriel terme à terme avec l'anneau $\mathbb{Z}_{\ell}$, nous obtenons la suite exacte de $\mathbb{Z}_{\ell}$ modules:

$$
\begin{aligned}
1 \rightarrow \delta_{K}^{S} / \delta_{K}^{S} \cap n_{L / K} \rightarrow\left[\underset{p \in S}{\oplus D}\left(C_{L / K}^{S} / K\right)\right] & \oplus\left[\underset{p \notin S}{\oplus 1}\left(C_{L / K}^{S} / K\right)\right] \\
& \rightarrow G a l\left(C_{L / K}^{S} / C_{K}^{S}\right) \rightarrow 1 .
\end{aligned}
$$

Dans celle-ci $\varepsilon_{K}^{S}=\mathbb{Z}_{\ell} \otimes_{\mathbb{Z}} E_{K}^{S}$ est le tensorisé du groupe des $S$ - uni tés, et $n_{L / K}=\mathbb{Z}_{\ell} \otimes_{\mathbb{Z}} N_{L / K}$ celui du groupe des normes ( au sens global comme au sens local); $D_{p}\left(C_{L / K}^{S} / K\right)$ est le groupe de décomposition de la place $p$ dans la $\ell$-extension abélienne $C_{L / K}^{S} / K$, et $I_{p}\left(C_{L / K}^{S} / K\right)$ son sous-groupe diinertie; $C_{L / K}$ est le $l_{\text {-corps des }}$ $S$-genres de $L / K(i . e$. la $\ell$-extension abélienne maximale de $K$ qui est non ramifiée et $S$-décomposée sur $L$ ) et $C_{K}^{S}$ le $\ell$-corps des $S$-classes de $K(i . e$. la $\ell$-extension abélienne maximale de $K$ qui est non ramifiée et $S$-décomposée ). 
Cela étant, les propriétés galoisiennes du symbole de Hasse

montrent que la suite obtenue est même une suite exacte de $\mathbb{Z}_{\ell}[\Delta]$ modules pour l'action naturelle du groupe $\Delta$ sur le terme médian donnée par la formule:

$$
\left(\sigma_{p}\right)_{p}^{T}=\left(\sigma_{p}^{\chi(\tau)}\right)_{p}, \quad \forall \tau \in \Delta .
$$

II vient donc :

THÉORËME 111.2.16. - Soient $L$ une $\ell$-extension cyclique d'un corps de nombres $K$, de groupe $G$, métabélienne sur un sous-corps $F$ de $K$ d'indice relatif $[K: F]$ étranger à $\ell$, puis $X$ le caractère du groupe de Galois $\Delta=$ Gal $(K / F)$ qui factorise le produit semi-direct $G \times \Delta$, et $S$ un ensemble fini de places non complexes de $F$. La suite exacte courte de $\mathbb{Z}_{\ell}[\Delta]$-modules

$$
\begin{aligned}
1 \rightarrow \delta_{K}^{S} / \delta_{K}^{S} \cap n_{L / K} \rightarrow\left[{ \underset { p \in S } { \oplus } D _ { p } ( C _ { L / K } ^ { S } / K ) ] } ^ { \oplus } \left[{\left.\underset{p \notin S}{\oplus} I_{p}\left(C_{L / K}^{S} / K\right)\right]}\right.\right. & \rightarrow G a l\left(C_{L / K}^{S} / C_{K}^{S}\right) \rightarrow 1
\end{aligned}
$$

conduit à la formule des $\mathrm{S}$-genres :

$$
g_{L / K, \varphi}^{S}=h_{K, \varphi}^{S} \frac{\prod_{K} \in S_{p}(L / K)^{\left\langle\varphi, x x_{p}\right\rangle} \prod_{p \notin S} e_{p}(L / K)^{\left\langle\varphi, x_{p}\right\rangle}}{[L: K]^{\langle\varphi, x\rangle} \cdot\left(\delta_{K}^{S}: \delta_{K}^{S} \cap n_{L / K}\right)_{\varphi}} .
$$

Dans celle-ci, $g_{\mathrm{L} / K, \varphi}^{\mathrm{S}}$ est la $\varphi$-partie du $\ell$-nombre de $S$-genres dans l'extension cyclique $L / K, h_{K}^{S}, \varphi$ est celle du $l$-nombre de $S$ classes de diviseurs du corps $K ; d_{p}(L / K)=\mid\left(D_{p}\left(C_{L / K}^{S} / K\right) \mid\right.$ est l'ordre du groupe de décomposition de la place $p$ dans $L / K$, et $e_{p}(L / K)=\left|I_{p}\left(C_{L / K}^{S} / K\right)\right|$ est celui du sous-groupe diinertie; $X_{p}$ est l'induit à $\Delta$ du caractère de la représentation unité du sous-groupe de décomposition de $p$ dans l'extension abélienne $\mathrm{K} / \mathrm{F}$; enfin $\delta_{\mathrm{K}}^{\mathrm{S}}=$ $\mathbb{Z}_{\ell} \otimes_{\mathbb{Z}} E_{K}^{S}$ est le tensorisé $\ell$-adique du groupe des $S$-unités $($ au sens des diviseurs / du corps $K$; et $\eta_{L / K}=\mathbb{Z}_{\ell} \otimes_{Z} N_{L / K}$ celui $d u$ groupe des normes.

(*) II s'agit en fait de lidentite $\left(\frac{x^{\top}, L / K}{p}\right)=\tau\left(\frac{x_{2} L / K}{p}\right) \tau^{-1}$ dont on trouvera par exemple une démonstration dans $\left[\mathrm{Gr}{ }_{10}\right]$. 
Démonstration : La suite exacte courte obtenue donne directement :

$$
\left.|G a|\left(C_{L / K}^{S} / C_{K}^{S}\right)\right|_{\varphi}=\frac{\prod_{\varphi} d_{p}(L / K)^{\left\langle\varphi, x x_{p}\right\rangle} \prod_{p \notin S} e_{p}^{(L / K)^{\left\langle\varphi, x_{p}\right\rangle}}}{\left(\delta_{K}^{S}: \delta_{K}^{S} \cap n_{L / K}\right)_{\varphi}} \text {, }
$$

pour chaque caractère $\ell$-adique irréductible $\varphi$ du groupe $\Delta$, compte tenu de l'action de $\Delta$ sur le terme médian rappelée plus haut . La for mule annoncée en résulte, en vertu de l'identité :

$$
\begin{aligned}
\left.g_{L / K, \varphi}^{S}=\mid \operatorname{Gal}_{L / K}^{S} / L\right)\left.\right|_{\varphi} & =\left|\operatorname{Gal}\left(C_{L / K}^{S} / C_{K}^{S}\right)\right|_{\varphi} \frac{\left|G^{S}\left(C_{K}^{S} / K\right)\right|_{\varphi}}{|G a l(L / K)|_{\varphi}} \\
& =\left.|G a|\left(C_{L / K}^{S} / C_{K}^{S}\right)\right|_{\varphi} \frac{h_{K}^{S}}{[L: K]^{\langle L, X\rangle}} \cdot
\end{aligned}
$$

II est instructif de comparer ce dernier résultat avec l'expression du $\ell$-nombre de $S$-classes ambiges donnée par le théorème III.1.13. D'après le théorème $15 \mathrm{ci}$-dessus, il vient ainsi :

COROLLAIRE III.2.17. - Sous les hypothèses du théorème 16, la for mule de translation des $S$-classes prend la forme:

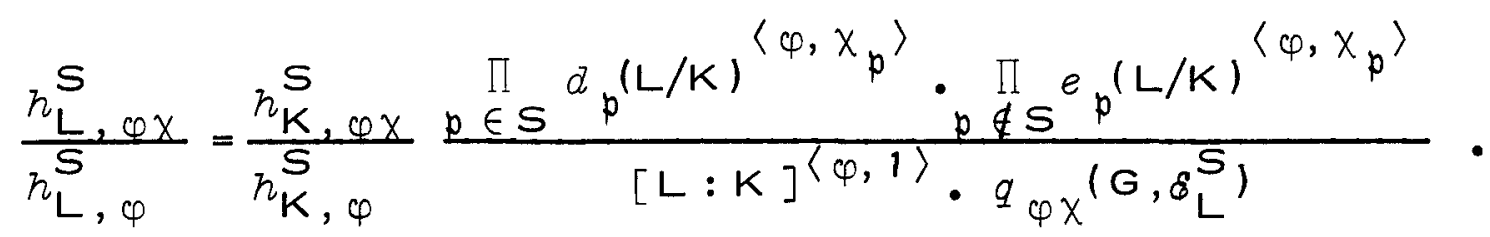

Remarques.- (i) Prenons pour $\Phi$ le caractère d'un idempotent central de l'algèbre de Galois. La formule de translation des $S$-classes nous re donne alors l'expression du quotient de Herbrand des S-unités calculé dans la section 1 ( scolie III.1.15):

$q_{\Phi}\left(G, \varepsilon_{L}^{S}\right)=\prod_{p \in S} d_{p}(L / K)^{\left\langle\Phi, X_{p}\right\rangle} \cdot \prod_{p \notin S} e_{p}(L / K)^{\left\langle\Phi, X_{p}\right\rangle} /[L: K]^{\langle\Phi, 1\rangle}$.

(ii) Prenons $\varphi$ quelconque, mais fixons $S$ assez grand pour annuler $c e \underset{L}{S}$ comme $C S_{K}^{S}$. Dans ce cas, la formule du corollaire donne directement l'expression du quotient de Herbrand $q \varphi \chi^{\left(G, \delta_{L}^{S}\right)}$. La structure galoisienne du groupe des $S$-unités se régularise donc pour $S$ assez grand. 
b.- Extension des résultats au cas procyclique: La formule des genres pour une $\mathbb{Z}_{\ell}$-extension.

Nous reprenons ici les hypothèses de la section $1, \S 3,2$ : Le corps $L$ est donc désormais une $\mathbb{Z}_{\ell}$-extension d'un corps de nombres $K$, de groupe de Galois $\Gamma=\gamma^{\mathbb{Z}_{\ell}}$, métabélienne sur un sous-corps $F$ de $K$, d'indice relatif $d=[K: F]$ étranger à $\ell$; et nous continuons à noter $x$ le caractère $\ell$-adique du groupe $\Delta=\mathrm{Gal}(\mathrm{K} / \mathrm{F})$ qui factorise le produit semi-direct $\Gamma \times \Delta$, clest-à-dire que nous écrivons

$$
\tau \gamma T^{-1}=\gamma^{X(\tau)}, \quad \forall T \in \Delta,
$$

en faisant choix une fois pour toutes d'un relèvement de $\Delta$ dans Gal(L/F). Comme plus haut, nous notons

$$
\theta=\frac{1}{d} \sum_{\tau \in \Delta} X\left(\tau^{-1}\right)\left[\gamma^{\chi(\tau)}-1\right]
$$

la résolvante de Lagrange construite sur un progénérateur $\gamma$ de $\Gamma$, ce qui nous permet d'écrire

$$
\Lambda=\mathbb{Z}_{\ell}[[\gamma-1]]=\mathbb{Z}_{\ell}[[\theta]]
$$

l'algèbre d'lwasawa du groupe $\Gamma$, tout en conservant les raltions de commutation

$$
{ }^{\theta} \mathrm{e}_{\varphi}=\mathrm{e}_{\varphi x^{\theta}, \quad \forall \varphi \in \mathrm{R}} \mathbb{Z}_{\ell}(\Delta)
$$

pour chaque idempotent primitif de l'algèbre semi-locale $\mathbb{Z}_{\ell}[\Delta]$.

Les considérations de théorie des genres développées dans le paragraphe précédent s'étendent alors sans difficulté au cas procy clique considéré ici. L'introduction du $\ell$-corps des $S$-genres n'appelle pas de remarque particulière: c'est toujours l'extension maximale de $L$, qui est non ramifiée ( aux places finies ), complètement décomposée aux places au-dessus de $S$, et provient par composition avec $L$ dlune $\ell$-extension abélienne de $K$. La définition des symboles de Hasse pour une extension infinie peut se faire comme suit : Si $K \subset M \subset L$ est une tour de corps de nombres (de degrés finis), les restrictions à $M$ des symboles de Hasse $\left(\frac{\cdot, L / K}{P}\right)$ associés à $L / K$ ne sont autres que les symboles de Hasse $\left(\frac{\cdot, M / K}{P}\right)$ associés à $M / K$. Par suite, si $L / K$ est une extension infinie de corps de nombres, $L$ est la limite 
inductive de ses sous-extensions $M$ de degré fini sur $K$, on peut donc définir les symboles de Hasse associés à L/K comme limites projec tives des symboles finis $\left(\frac{\cdot, M / K}{P}\right)$ :

$$
\left(\frac{\cdot, L / K}{P}\right)=\frac{\lim }{M}\left(\frac{\cdot, M / K}{P}\right)
$$

Cela posé, nous avons :

THÉORÈME III.2.18.- Etant donnés $S$ un ensemble fini de places non complexes diun corps de nombres $K$, et $L$ une $\mathbb{Z}_{l}$-extension de $K$, métabélienne sur un sous-corps $F$ de $K$, d'indice relatif $d=[K: F]$ étranger à $\ell$, les symboles de Hasse associés à l'extension infinie $C_{L / K}^{S} / K$ induisent une suite exacte courte de $\mathbb{Z}_{\ell}[\Delta]$-modules :

$$
\begin{aligned}
1 \rightarrow \delta_{K}^{S} / \delta_{K}^{S} \cap n_{L / K} \rightarrow\left[{ }_{p \in S}^{\oplus} D_{p}\left(C_{L / K}^{S} / K\right)\right] & \oplus\left[\underset{p \notin S}{\oplus} I_{p}\left(C_{L / K}^{S} / K\right)\right] \\
& \rightarrow G a l\left(C_{L / K}^{S} / C_{K}^{S}\right) \rightarrow 1 .
\end{aligned}
$$

Dans celle-ci, $\delta_{K}^{S}=\mathbb{Z}_{\ell} \otimes_{\mathbb{Z}} E_{K}^{S}$ est le tensorisé du groupe des $S$ unités du corps $K$, et $\eta_{L / K}=\mathbb{Z}_{\ell} \otimes_{\mathbb{Z}}\left(\cap_{n \in \mathbb{N}} N_{K} / K\right)$ celui du groupe $N_{L / K}$ des éléments de $K^{x}$ qui sont normes locales partout (et donc normes globales) dans chacune des sous-extensions cycliques $K_{n} / K$ de $L / K ; C_{L / K}^{S}$ est le $l$-corps des $S$-genres de l'extension procyclique $L / K$, et $C_{K}^{S}$ le $\ell$-corps des $S$-classes de $K ; D_{p}\left(C_{L / K}^{S} / K\right)$ est le groupe de décomposition de la place $p$ dans l'extension abélienne $C_{L / K}^{S} / K$, et $I_{P}\left(C_{L / K}^{S} / K\right)$ le sous-groupe diinertie correspondant .

COROLLAIRE 111.2.19.- Sous les hypothèses du théorème 18 , la 4 partie de la dimension du $\ell$-quotient des $S$-genres de l'extension procyclique $L / K$ est donnée, pour chaque caractère $l$-adique irréduc tible $\varphi$ du groupe $\Delta$, par la formule :

$$
\operatorname{dim}_{\mathbb{Z}_{\ell}} q_{L / K, \varphi}^{S}=\left\langle\varphi, \sum_{p \in R \cup S}{ }^{\delta} p \times x_{p}-\nu_{L / K}^{S}-x\right\rangle \text {. }
$$

$R$ est l'ensemble des places de $F$ qui se ramifient (sauvagement) dans $L / K ; X_{p}$ est l'induit à $\Delta=G a l(K / F)$ du caractère de la représentation 
unité du sous-groupe de décomposition de $p$ dans $L / K ; \delta_{p}$ est égal à 0 ou à 1 suivant que $p$ est complètement décomposée ou non dans l'extension procyclique $L / K ; \nu_{L / K}^{S}$ est le caractère du $Z_{\ell}[\Delta]$-module projectif $\varepsilon_{K}^{S} / \varepsilon_{K}^{S} \cap \eta_{L / K}$, et $x$ celui du $\mathbb{Z}_{\ell}[\Delta]$-module $\Gamma=G a l(L / K)$. Autrement dit, le groupe $\mathcal{q}_{L / K}^{S}$ est somme directe diun $\mathbb{Z}_{\ell}[\Delta]$ module fini, et d'un $\mathbb{Z}_{\ell}[\Delta]$-module projectif de caractère :

$$
x\left[\sum_{p \in S \cup R} \delta_{p} x_{p}-1\right]-\nu_{L / K}^{S} \text {. }
$$

Applications.-(i) Lorsque l'ensemble $S$ est vide, la suite exacte des $S$-genres prend la forme:

$1 \longrightarrow \varepsilon_{K} / \delta_{K} \cap n_{L / K} \longrightarrow \operatorname{P\in R}_{p}^{\oplus} I_{p}\left(C_{L / K} / K\right) \longrightarrow G a l_{L}\left(C_{L / K} / K\right) \longrightarrow 1$. Dans celle-ci, $\delta_{K}$ est le tensorisé du groupe des unités (au sens strict) du corps $K$, et le terme médian est un $\mathbb{Z}_{\ell}$-module libre dont la dimen sion est égale au nombre $t$ de places de $K$ qui se ramifient dans $L / K$.

(ii) Lorsque $S$ est l'ensemble $R$ des places de $F$ qui se ramifient dans $L / K$, il vient :

$1 \longrightarrow \varepsilon_{K} / \varepsilon_{K} \cap n_{L / K} \longrightarrow \underset{P \in R}{\oplus} D_{P}\left(C_{L / K}^{R} / K\right) \longrightarrow \operatorname{Gal}\left(C_{L / K}^{R} / C_{K}^{R}\right)-1$; et le terme médian est encore un $\mathbb{Z}_{\ell}$-module libre de dimension $t$.

(iii) Lorsque $S$ est l'ensemble des places réelles de $F, C_{L}^{S} / K$ est le $\ell$-corps des genres au sens ordinaire, et la suite exacte fait intervenir alors le tensorisé du groupe des unités, au sens ordinaire, du corps $K$ :

$1 \longrightarrow \delta_{K}^{\text {add }} / \delta_{K}^{\text {ord }} \cap n_{L / K} \longrightarrow{\underset{p \in R}{P}}_{P}\left(C_{L / K} / K\right) \longrightarrow$ Gall $\left._{L / K} / C_{K}\right) \rightarrow 1$. Ici encore, le terme médian est un $\mathbb{Z}_{\ell}$-module libre de dimension $t{ }^{(\star)}$.

Démonstration du théorème : L'exactitude à droite comme à gauche de la suite courte proposée est immédiate : A droite, la surjectivité de l'ap plication $\pi$ traduit la maximalité de la $\ell$-extension abélienne $C_{K}^{S}$ sous les deux conditions de non ramification et de S-décomposition; à gauche,

$\left({ }^{\star}\right)$ On notera que les places réelles ne peuvent se ramifier ni dans la tour $L / K$, ni dans l'extension $S$-décomposée $C_{L / K}^{S} / L$. 
l'injectivité de $\eta$ résulte de la caractérisation des symboles profinis et de la définition du groupe $n_{L / K}$. Seule reste à montrer l'exactitude au centre, i.e. que chaque famille $\left(\sigma_{p}\right)_{p}$ de la somme directe

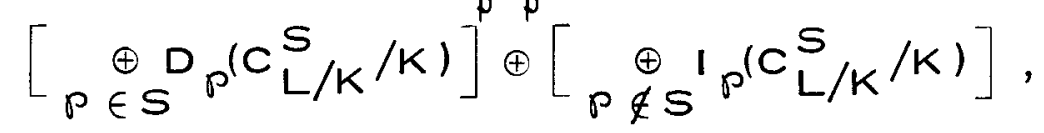

qui vérifie la formule du produit $\prod_{p} \sigma_{p}=1$, provient, par les symboles de Hasse profinis, d'une $S$-unité généralisée $\epsilon \in \delta_{K}^{S}$. Or, nous savons déjà, par le théorème 16 , que ce résultat est vrai à chaque étage fini de la tour $L / K$, autrement dit, qu'une telle famille étant donnée, nous pouvons trouver, pour chaque naturel $n$, une $S$-unité généralisée $\epsilon_{\mathrm{n}} \in \varepsilon_{\mathrm{K}}^{\mathrm{S}}$, qui vérifie :

$$
\left(\frac{{ }^{\epsilon}, C_{K_{n}}^{S} / K}{p}\right)=\left.\left(\frac{\epsilon_{n}, C_{L}^{S} / K}{p}\right)\right|_{C_{K_{n} / K}^{S}}=\left.\sigma\right|_{C_{K_{n} / K}^{S}} .
$$

Maintenant, le groupe $\delta_{K}^{S}$ étant compact, la suite $\left(\epsilon_{n}\right)_{n} \in \mathbb{N}$ possède une valeur d'adhérence $\varepsilon_{\infty} \in \varepsilon_{K}^{S}$, qui est manifestement solution du problème posé .

L'exactitude étant établie, considérons les différents termes de la suite : Chacun des groupes $D_{p}\left(C_{L / K}^{S} / K\right)$ et $I_{p}\left(C_{L / K}^{S} / K\right)$ est soit nul, soit isomorphe à $\mathbb{Z}_{\ell} ;$ le terme médian est donc un $\mathbb{Z}_{\ell}[\Delta]$ module projectif de caractère

$$
\sum_{p \in S} \delta_{p} x x_{p}+\sum_{p \in R \backslash S} x_{p}=x_{p \in R \cup S} \sum_{p} x_{p}
$$

Son sous-groupe $\delta_{K}^{S} / \delta_{K}^{S} \cap \eta_{L / K}$ est donc également $\mathbb{Z}_{\ell}[\Delta]$-pro jectif. Et l'expression de la dimension du $\ell$-quotient des $S$-genres s'obtient alors en remarquant que dans la suite exacte courte scindée

$$
1 \longrightarrow C_{L / K}^{S} \longrightarrow \operatorname{Gal}\left(\mathrm{C}_{L / K}^{S} / K\right) \longrightarrow \Gamma \longrightarrow 1
$$

le groupe $\Gamma=$ Gal(L/K) est un $\mathbb{Z}_{\ell}[\Delta]$-module projectif de caractère $X$, et le terme médian est pseudo-isomorphe à $G a l\left(C_{L / K}^{S} / C_{K}^{S}\right.$ ).

SCOLIE III.2.20.- Conservons les notations des théorèmes 111.1 .19 et 111.2 .18 . L'isomorphisme naturel du tensorisé $\left(\mathbb{Q}_{\ell} / \mathbb{Z}_{\ell}\right) \otimes_{\mathbb{Z}_{\ell}} \&_{K}^{S}$ sur le groupe $\mathbb{S}_{K}^{S}=\left(\mathbb{Q}_{\ell} / \mathbb{Z}_{\ell}\right) \otimes_{\mathbb{Z}} E_{K}^{S}$ induit un isomorphisme de $\mathbb{Z}_{\ell}[\Delta]-$ modules : 


$$
\left(\Phi_{\ell} / \mathbb{Z}_{l}\right) \otimes_{\mathbb{Z}_{l}}\left(\varepsilon_{K}^{S} / \delta_{K}^{S} \cap \eta_{L} / K\right) \simeq \mathbb{E}_{K}^{S} / \mathbb{E}_{K}^{S} \cap N_{L} / K
$$

En particulier, pour chaque caractère $\ell$-adique irréductible $\varphi$ du groupe $\Delta$, la $\varphi$-partie $\left\langle\varphi, \nu_{L / K}^{S}\right\rangle$ de la dimension du quotient $\varepsilon_{K}^{S} / \varepsilon_{K}^{S} \cap \eta_{L / K}$ est égale à celle $n_{L / K, \varphi}^{S}$ de la codimension du groupe $\mathbb{E}_{K}^{S} / \mathbb{E}_{K}^{S} \cap \mathbb{N}_{L / K}$.

Démonstration : Le groupe $\delta_{K}^{S} / \varepsilon_{K}^{S} \cap \eta_{L / K}$ étant sans $\mathbb{Z}_{l}$-torsion, nous avons bien :

$$
\begin{aligned}
\left(\Phi_{l} / \mathbb{Z}_{l}\right) \otimes_{\mathbb{Z}_{l}}\left(\varepsilon_{K}^{S} \cap \eta_{L / K}\right) & \simeq\left(Q_{l} / \mathbb{Z}_{\ell}\right) \otimes_{\mathbb{Z}_{l}} \varepsilon_{K}^{S} /\left(Q_{l} / \mathbb{Z}_{l}\right) \otimes_{\mathbb{Z}_{l}}\left(\varepsilon_{K}^{S} \cap \eta_{L / K}\right) \\
& \simeq \varepsilon_{K}^{S} /\left(Q_{l} / \mathbb{Z}_{l}\right) \otimes_{\mathbb{Z}_{l}}\left(\varepsilon_{K}^{S} \cap \eta_{L / K}\right) .
\end{aligned}
$$

Il s'agit donc de vérifier que dans la description obtenue le groupe $\mathbb{E}_{K}^{S} \cap \eta_{L / K}$ correspond bien au produit $\left(Q_{l} / \mathbb{Z}_{l}\right) \otimes_{\mathbb{Z}_{l}}\left(\varepsilon_{K}^{S} \cap \eta_{L / K}\right)$, ce qui est sans malice.

La formule obtenue souligne la parenté entre l'expression de la codimension du $\ell$-groupe des $S$-classes ambiges donnée dans la section 1 ( théorème 111.1 .20 ), et celle de la dimension du $l$-quo tient des $S$-genres obtenue plus haut (corollaire III.2.19) :

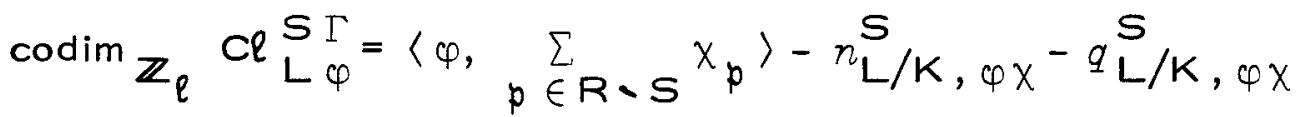

$$
\begin{aligned}
& \operatorname{dim}_{\mathbb{Z}_{l}} \mathcal{G}_{L / K, \varphi X}^{S}=\left\langle\varphi, \sum_{p \in R \cup S} x_{p}\right\rangle-n_{L / K}^{S} \varphi X^{-\langle\varphi, 1\rangle} .
\end{aligned}
$$

Lorsque $\Phi$ est le caractère associé à un idempotent central de l'algèbre $\Lambda[\Delta]$, lidentité codim $\mathbb{Z}_{l} C_{L}^{S T}=\operatorname{dim} \mathbb{Z}_{l} G_{L / K}^{S}, \Phi$ permet ainsi de retrouver l'expression du quotient de Herbrand profini $q_{\Phi}\left(\Gamma, \varepsilon_{L}^{S}\right)$ donné dans la section 1 ( théorème 111.1 .23$)^{(\star)}$. En revanche, lors que $\Phi$ n'est pas central, l'écart entre les expressions obtenues se traduit sur les invariants lambda d'Iwasawa du groupe $C_{L}^{S}=\stackrel{l i m}{L} d_{K}^{S}$ par une formule analogue à la formule de translation des S-classes énoncée plus haut (corollaire 17$)^{(\star \star)}$.

$\left({ }^{\star}\right)$ C'est la méthode employée dans $\left[\mathrm{Ja}_{4}\right]$.

$\left.{ }^{\star \star}\right)$ La formule de translation pour les invariants $\lambda$ est exposée à la fin du chapitre IV. 


\section{c.-Propriétés normiques des $S$-unités dans une $\mathbb{Z}_{\ell}$-extension}

Nous nous proposons de préciser ici le caractère $\nu_{L / K}^{S}$ du $\mathbb{Z}_{\ell}[\Delta]$-module projectif $\varepsilon_{K}^{S} / \varepsilon_{K}^{S} \cap \eta_{L / K}$. Dlaprès ce qui précède, en effet, nous disposons de deux majorations de $\nu_{L / K}^{S}$ :

la première, donnée par le théorème 19 , ci-dessus, provient des contraintes normiques décrites par les symboles de Hasse:

$$
\nu_{L / K}^{S} \leq x\left[\sum_{p \in S \cup R} \delta_{p} x_{p}-1\right] \text {, }
$$

- la seconde résulte directement du caractère du $\mathbb{Z}_{\ell}[\Delta]-$ module projectif $\delta_{K}^{S} / \mu_{K}$ ( cf. corollaire 111.1 .23 ), puisque le sous groupe de torsion $\mu_{K}$ de $\delta_{K}^{S}$ (i.e. le $\ell$-Sylow du groupe des racines de llunité dans $K$ ) est contenu dans le groupe des normes $\eta_{L / K}$. Si $\bar{S}$ est la réunion de $S$ et des places à liinfini, il vient ainsi :

$$
\nu_{L / K}^{S} \leq \sum_{p \in \bar{S}} x_{p}-1 \text {. }
$$

De fait, lorsque $F$ est le corps des rationnels, nous allons montrer que ces deux majorations déterminent complètement le caractère $\nu_{L / K}^{S}$.

THEOREME 111.2 .21 - Soient $L$ une $\mathbb{Z}_{\ell}$-extension d'un corps abélien $K$, métabélienne sur le corps des rationnels, et $S$ un ensemble fini de places de $\mathbb{Q}$. Notons o l'unique place à l'infini du corps $\mathbb{Q} ; \times$ le ca ractère $\ell$-adique du groupe de Galois $\Delta=G a l(K / Q)$ qui définit le produit semi-direct $G a l(L / \mathbb{Q})=\Gamma \times \Delta$; puis, pour chaque place $p$ de $\mathbb{Q}$, $X_{p}$ l'induit à $\Delta$ du caractère unité du sous-groupe de décomposition de $p$ dans l'extension $K / \mathbb{Q}$; posons enfin $\delta_{p}=0$ ou 1 selon que $p$ se dé compose complètement ou non dans l'extension procyclique $L / K$. Alors le caractère du $\mathbb{Z}_{\ell}[\Delta]$-module projectif $\varepsilon_{K}^{S} / \varepsilon_{K}^{S} \cap \eta_{L / K}$ des S-unités modulo les normes est donné, comme plus grand diviseur commun de deux caractères, par la formule :

$$
\nu_{L / K}^{S}=\left[\sum_{p \in S \cup\{\infty\}} x_{p}-1\right] \wedge\left[\sum_{p \in S \cup\{l\}} \delta_{p} x_{p}-1\right] \text {. }
$$

Avant de démontrer ce résultat, tirons en quelques enseigne ments : 
Le caractère $\nu_{L / K}^{S}$ étant ainsi connu explicitement ( du moins dans le cas absolument métabélien ), la dimension du quotient des $\mathbf{S}-$ genres $C_{L}^{S} / K$ est donnée par le théorème 19 . Si, de plus, le corps $K$ est connu numériquement, i.e. si l'on sait expliciter un système fon damental de $\mathbf{S}$-unités ainsi qulune pseudo-base du $\boldsymbol{l}$-groupe des $\boldsymbol{S}$ classes de diviseurs, il est même possible de préciser le sous-mo dule de torsion $q_{L / K}^{S}$ ( il suffit de calculer des symboles de Hasse). Le quotient $g_{L / K}^{S}$ est donc, de ce point de vue, entièrement effectif. II n'en est pas de même, en revanche, du sous-groupe ambige Ce $S I$ introduit dans la section 1 . Son étude, en effet, fait intervenir de

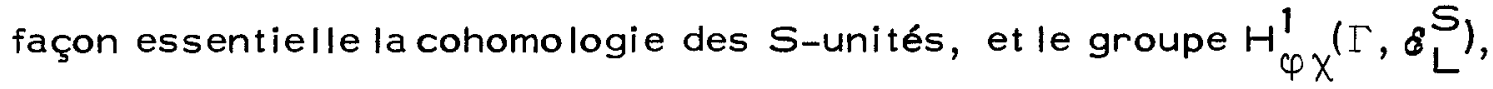
qui gouverne la $\varphi$-partie de la $S$-capitulation, ne se calcule pas dans $\mathrm{K}$.

Lorsque $L$ n'est pas absolument métabélienne, le théorème précédent est en défaut, par exemple parce que le plus grand diviseur comm un de la restriction de deux caractères à un sous-groupe n'est pas, en général, la restriction à ce sous-groupe du plus grand divi seur commun de ces deux caractères. De fait, la décomposition du groupe des 5 -unités $\delta_{K}^{S}$ par les idempotents primitifs de l'algèbre $\mathbb{Z}_{\ell}[\Delta]$ ne suffit pas, dans ce cas, à rendre compte des propriétés normiques des S-unités. Cependant, si L est absolument galoisienne, et $\mathrm{si}$ les caractères $\ell$-adiques irréductibles du groupe $\mathrm{Gal}(\mathrm{K} / \mathbb{Q})$ re présentés dans le quotient $\delta_{K}^{S} / \mu_{K}$ le sont une seule fois dans le caractère régulier, les résultats d'indépendance de la section $I I, 1$ conduisent à une expression explicite du caractère $\nu_{L / K}^{S}$, tout à fait ana logue à celle donnée ici, mais faisant intervenir cette fois les caractères $\ell$-adiques du groupe fini, éventuellement gauche, Gal(K/Q). Cela dit, commençons par établir un lemme préliminaire (qui ne dépend pas de |'hypothèse $F=Q$ ) :

LEMME III.2.22. - Soit $p$ une place de $S$ qui est presque complète ment inerte dans l'extension procyclique $L / K$, et $S^{\prime}=S \cup\{p\}$. Alors :

(i) Le caractère $x_{p}$ est stable par multiplication par $x$ (i.e. $\left.x_{p}(x-1)=0\right)$. Autrement dit, la restriction de $x$ au sous - 
groupe de décomposition $\Delta_{p}$ de $p$ dans l'extension abélienne $K / F$ est le caractère unité, ce qui s'écrit : $\chi\left(\Delta_{p}\right)=1$

(ii) Les caractères des $\mathbb{Z}_{\ell}[\Delta]$-modules $\delta_{K}^{S} / \varepsilon_{K}^{S} \cap \eta_{L / K}$ et $\varepsilon_{K}^{S^{\prime}} / \varepsilon_{K}^{S^{\prime}} \cap \eta_{L / K}$ sont liés par liidentité :

$$
v_{L / K}^{S}=v_{L / K}^{S \prime}+x_{p} \text {. }
$$

Démonstration : En une place non ramifiée $P$ de $K$, le sous-groupe du groupe procyclique $D_{p}\left(C_{L / K}^{S} / K\right)$, engendré par le symbole de Hasse $\left(\frac{x, C_{L}^{S} / K / K}{P}\right)$ d'un élément donné $x$ de $K^{x}$, ne dépend que de la $p$ valuation de cet élément . L'application induite

$$
\varepsilon_{K}^{S} / \varepsilon_{K}^{S I} \rightarrow p D_{p}^{\oplus}\left(C_{L / K}^{S} / K\right)
$$

est donc presque surjective, l'image des $S$ - unités étant d'indice fini dans le groupe $\hat{D}_{K}(S)$. Comme le caractère du $\mathbb{Z}_{\ell}[\Delta]$-module $D_{K}\left(\{p\}\right.$ ) est connu (c'est l'induit $\chi_{p}$ du caractère unité du sousgroupe de décomposition $\left.\Delta_{p}\right)$, ainsi que celui de la somme ${ }_{p / p}^{\oplus} D_{p}\left(C_{L / K}^{S} / K\right)$ (c'est le translaté $\chi_{p}$ du précédent), l'égalité des degrés entraîne celle des caractères et l'injectivité de l'application considérée. D'où les deux assertions du lemme.

Démonstration du théorème : Par application récurrente du lemme, nous pouvons nous ramener au cas où les places de $S$ sont soit com plètement décomposées, soit presque totalement ramifiées dans l'ex tension procyclique $L / K$. La formule à établir s'écrit alors :

$$
\nu_{L / K}^{S}=\left(\sum_{p \in S \cup\{\infty\}} x_{p}-1\right) \wedge x\left(x_{\ell}-1\right) \text {. }
$$

Distinguons deux cas :

$1^{\text {er }}$ cas $:$ Si $S$ ne contient pas $\ell$, l'application de semi-localisation s envoie le tensorisé $\delta_{K}^{S}=\mathbb{Z}_{\ell} \otimes_{\mathbb{Z}} E_{K}^{S}$ du groupe des $S$-unités

$\left.{ }^{\star}\right)$ L'assertion contraposée " $\chi\left(\Delta_{p}\right) \neq 1 \Rightarrow p$ complètement décomposée dans $L / K$, si $p$ ne divise pas $\ell p^{\prime \prime}$ a été établie indépendamment par Carroll et Kisilevsky ( cf. $\left[\mathrm{CK}_{2}\right]$ ) par une voie complètement diffé rente. 
dans le groupe $U_{\ell}=\underset{I \mid \ell}{\oplus} U_{\mathfrak{l}}$ des unités principales semi-locales du corps $K$. Le groupe $u_{\ell}$ ( ou plutôt son quotient $u_{\ell} / \mu_{\ell}$ par le sous groupe des racines semi-locales de l'unité ) est un $\mathbb{Z}_{\ell}[\Delta]$ - module de caractère régulier. Cela étant, d'un côté les résultats d'indépen dance de la section 1 du chapitre II (cf. théorème II.1.12) permettent d'affirmer que, sous les hypothèses d'abélianité énoncées, limage de $\varepsilon_{K}^{S}$ dans $u_{\ell} / \mu_{\ell}$ est un $\mathbb{Z}_{\ell}[\Delta]$-module de caractère $\left[\sum_{p \in S \cup\{\infty\}} x_{p}-1\right] \wedge x_{\text {rég }}$. Et, d'un autre côté la théorie du corps de classes local affirme l'existence d'un épimorphisme canonique $u_{\ell} \longrightarrow I_{l}=\underset{l \mid \ell}{\oplus} I_{I}(L / K)$ de $u_{\ell}$ sur la somme directe des groupes d'inertie attachés aux places au-dessus de $L$ dans l'extension procy clique $L / K$, épimorphisme ayant pour noyau le sous-groupe $U_{\ell}^{\prime}$ de $U_{\ell}$ formé des éléments de $u_{\ell}$ qui sont normes à chaque étage fini de la tour $\mathrm{L} / \mathrm{K}$. Maintenant, comme chaque caractère $\ell$-adique irréductible du groupe $\Delta$ est représenté une fois et une seule dans le caractère régulier, le quotient $\delta_{K}^{S} / \delta_{K}^{S} \cap \eta_{L / K} \simeq s\left(\varepsilon_{K}^{S}\right) / s\left(\varepsilon_{K}^{S}\right) \cap u_{l}^{\prime}$ est donc un $\mathbb{Z}_{\ell}[\Delta]$-module de caractère

$\nu_{L / K}^{S}=\left[\left(\sum_{p \in S \cup\{\infty\}} x_{p}-1\right) \wedge x_{\text {rég }}\right] \wedge x x_{l}=\left[\sum_{p \in S \cup\{\infty\}} x_{p}-1\right] \wedge x_{l}$;

ce qui établit dans ce cas le résultat annoncé. Plus précisément, en vertu de l'inégalité déjà connue $\nu_{L / K}^{S} \leq\left[\sum_{p \in S \cup\{\infty\}} x_{p}-1\right] \wedge x(x-1)$, nous obtenons, comme attendu :

$\nu_{L / K}^{S}=\left[\sum_{p \in S \cup\{\infty\}} x_{p}-1\right] \wedge x x_{l}=\left[\sum_{p \in S \cup\{\infty\}} x_{p}-1\right] \wedge x\left(x_{l}-1\right)$, et, en outre, la condition nécessaire: $\left[\sum_{p \in S \cup\{\infty\}} X_{p}-1\right] \wedge x=1$. Si $\chi$ n'est pas le caractère unité, cette dernière éga lité s'écrit encore:

$$
x_{p} \wedge x=0, \quad \forall p \in S \cup\{\infty\} .
$$

Elle exprime que la restriction à $\Delta_{p}$ du caractère $\chi n^{\prime}$ est jamais le caractère unité, dès que la place $p$ est complètement décomposée dans l'extension procyclique $\mathrm{L} / \mathrm{K}$. 
- Si $x$ est le caractère unité, il vient seulement :

$x_{p} \wedge 1=0, \quad \forall p \in S \backslash\{\infty\}$.

Iln'y a donc pas dans ce cas de place finie de $\mathbb{Q}$ qui soit complète ment décomposée dans $L / K$.

$\underline{2}^{\text {ème }}$ cas $:$ Si $S$ contient $\ell$, écrivons $S^{\prime}=S \backslash\{l\}$, et faisons choix de llune des places $\mathfrak{l}$ de $K$ au-dessus de $l$; fixons $m$ assez grand pour avoir simultanément $l^{l^{m}} \in P_{K}$ et $D_{l}^{l^{m}}(L / K) \subset l_{l}^{l^{m}}(L / K)$; puis, prenons $x$ dans le tensorisé $R_{K}=\mathbb{Z}_{\ell} \otimes_{\mathbb{Z}} K^{x}$ engendrant l'idéal $l^{\ell^{m}}$, et $x_{\mathfrak{l}}$ dans le complété $K_{\mathfrak{l}}^{x}$, norme locale dans la tour $L / K$, de même valuation que $x$. Cela posé, le sous-module $\tilde{\delta}_{K}$ de $R_{K}$ engen dré par $\varepsilon_{K}^{S^{\prime}}$ et par les conjugués de $x$, qui est d'indice fini dans $\delta_{K}^{S}$, s'envoie non canoniquement dans $U_{\ell}$ au moyen de l'homomorphisme $\tilde{s}$ défini par $\mathbb{Z}_{\ell}[\Delta]$-linéarité à partir des identités :

$\tilde{s}(\epsilon)=s(\varepsilon)$, pour $\epsilon \in \varepsilon_{K}^{S^{\prime}} ; \quad \tilde{s}(x)=\left(\frac{x}{x_{I}}, x, \ldots, x\right) \epsilon \underset{T \in \Delta / \Delta_{I} I^{T}}{u^{T}}=u_{\ell}$.

Le corollaire II.1.27 nous dit alors que limage $\tilde{s}\left(\tilde{\varepsilon}_{K}^{S}\right)$ est le produit de $\tilde{s}\left(\mu_{k}\right)$ et d'un $\mathbb{Z}_{\ell}[\Delta]$-module de caractère $\left[\left(\sum_{p \in S \cup\{\infty\}} x_{p}-1\right) \wedge x_{\text {rég }}\right]-\psi$,

où $\psi$ est soit le caractère nul, soit llun des facteurs irréductibles du caractère $x_{l}$. Maintenant, comme nous avons pris le soin de choisir $x_{l}$ comme norme locale, l'application $\tilde{s}$ factorise comme plus haut les symboles de Hasse, et nous obtenons, par le même argument, l'iden tíé :

$$
\nu_{L / K}^{S}=\left[\left[\left(\sum_{p \in S \cup\{\infty\}} x_{p}-1\right) \wedge x_{r e g}\right]-\psi\right] \wedge x x_{l} \cdot
$$

- Si $x_{\ell}$ et $x x_{\ell}$ sont étrangers, autrement dit si $x$ ne divise pas $x_{l}$, le caractère $\psi$, qui divise $x_{\ell}$, n'est pas représenté dans $x_{x_{\ell}}$, et nous obtenons, comme attendu :

$v_{L / K}^{S}=\left(\sum_{p \in S \cup\{\infty\}} x_{p}-1\right) \wedge x_{\ell}=\left(\sum_{p \in S \cup\{\infty\}} x_{p}-1\right) \wedge x\left(x_{l}-1\right)$,

puisque tous les $x_{p}$ sont alors étrangers à $x$.

- Sinon, $x$ divise $x_{l}$ et $x_{l}$ est stable par translation de $x$.

11 vient alors: 
$\nu_{L / K}^{S} \geq\left[\left(\sum_{p \in S \cup\{\infty\}} x_{p}-1\right) \wedge x_{l}\right] \wedge\left[x x_{l}-\psi\right]=\left[\sum_{p \in S \cup\{\infty\}} x_{p}-1\right] \wedge\left[x x_{l}-\psi\right]$. Et comme nous avons a priori $\nu_{L / K}^{S} \leq\left(\sum_{p \in S U\{\infty\}} x_{p}-1\right) \wedge\left(x_{x_{l}}-\chi x\right)$, le caractère $\psi$ ne peut être que $\chi$; d'où le résultat annoncé.

Au passage, nous avons démontré la réciproque du lemme 22 .

SCOLIE III.2.23. - Soient $L$ une $\mathbb{Z}_{\ell}$-extension d'un corps abélien $K$, métabélienne sur le corps des rationnels, et $X$ le caractère $l$-adique du groupe de Galois $\Delta=G a l(K / \mathbb{Q})$ qui définit le produit semi-direct Gal $(L / Q) \simeq I \times \Delta$. Le comportement dans la tour $L / K$ des places de $K$ au-dessus d'une même place $p$ de $\mathbb{Q}$ est déterminé comme suit :

(i) Si $p$ vaut $\ell$, les places au-dessus de $p$ sont presque totalement ramifiées dans $L / K$.

(ii) Si p est la place à l'infini, les places au-dessus de $p$ sont complètement décomposées dans $L / K$.

Dans tous les autres cas :

(iii) ou bien le groupe de décomposition $\Delta_{p}$ de $p$ dans l'extension abélienne $K / Q$ est contenu dans le noyau de $\mathcal{X}$, auquel cas les places au-dessus de $p$ sont presque totalement inertes dans $L / K$;

(iv) ou bien la restriction de $X$ à $\Delta_{p}$ n'est pas le caractère unité, auquel cas les places au-dessus de p sont complètement dé composées dans la tour $\mathrm{L} / \mathrm{K}$.

Remarques. - (i) Lorsque $\chi$ n'est pas le caractère unité (i.e. lorsque $L$ n'est pas abélienne sur $Q$ ), l'identité $x_{p} \wedge x=0$ vaut pour la place à l'infini : Le corps $K$ ne peut, en effet, posséder une $\mathbb{Z}_{\ell}$-exten sion associée au caractère $x$, si $x$ est représenté dans le $\mathbb{Z}_{\ell}[\Delta]$ module des unités $\delta_{K} / \mu_{K}$, i.e. si $x$ divise $\left(x_{\infty}-1\right)$.

(ii) En revanche, lorsque $\chi$ est le caractère unité, il est trivialement contenu dans le caractère induit $\chi_{\infty}$, et l'égalité $\chi_{p} \wedge x=0$ est donc en défaut pour la place à l'infini. Dans ce cas, $L$ est né cessairement la $\mathbb{Z}_{\ell}$-extension cyclotomique de $K$. Le scolie redonne alors le fait bien connu que les places ultramétriques de $K$ sont fini ment décomposées dans la tour cyclotomique. 
COROLLAIRE 111.2 .24 . - Sous les hypothèses du théorème 22 , le $\ell$ quotient des $S$-genres de l'extension procyclique $L / K$ est composé direct d'un $\mathbb{Z}_{\ell}[\Delta]$-module projectif dont le caractère $X_{L / K}^{S}$ est donné par la formule :

$$
x_{L / K}^{S}=x\left(x_{l}-1\right)-x\left(x_{l}-1\right) \wedge\left(\sum_{p \in S^{\prime}} x_{p}-1\right) \text {, }
$$

où $S^{\prime}$ est la réunion de la place à linfini et des places de $S$ qui ne sont pas presque totalement inertes dans la tour $L / K$.

Démonstration: Désignons par $S_{i}$ le sous-ensemble de $S$ formé des places $p$ qui sont presque totalement inertes dans $L / K$. Dlaprès le co rollaire 19 , le caractère $\chi_{L / K}^{S}$ est donné par la formule :

$$
\begin{aligned}
x_{L / K}^{S}=\sum_{p \in R \cup S} \delta_{p} x x_{p}-\nu_{L / K}^{S}-x & =\sum_{p \in S_{i}} x x_{p}+x x_{l}-\nu_{L / K}^{S}-x \\
& =\sum_{p \in S_{i}} x_{p}+x\left(x_{l}-1\right)-\nu_{L / K}^{S} .
\end{aligned}
$$

D'un autre côté, le théorème ci-dessus nous permet d'écrire dans tous les cas:

$$
\nu_{L / K}^{S}=\sum_{p \in S_{i}} x_{p}+\left(\sum_{p \in S^{\prime}} x_{p}-1\right) \wedge x\left(x_{l}-1\right) \text {. }
$$

D'où le résultat annoncé .

Applications. - (i) Si L est la $\mathbb{Z}_{\ell}$-extension cyclotomique de $K$, les places ultramétriques autres que $\ell$ sont presque totalement inertes dans $L / K$. Le caractère $X_{L / K}^{S}$ ne peut donc prendre que deux valeurs:

Lorsque $S$ ne contient pas $\ell$, il vient :

$$
x_{L / K}^{S}=x_{L / K}=\left(x_{\ell}-1\right) \wedge \bar{x}_{\infty}=x_{\ell} \wedge \bar{x}_{\infty}
$$

si $\bar{X}_{\infty}=X_{\text {rég }}-X_{\infty}$ désigne le supplémentaire du caractère $\chi_{\infty}$. L'égalité $X_{L / K}=0$ a lieu lorsque $K$ est totalement réel (auquel cas $\bar{X}_{\infty}$ est nul), ou bien lorsque $K$ est une extension quadratique imaginaire dlun sous corps $K$, totalement réel si les places au-dessus de $\ell$ ne se décomposent pas dans $K / K_{+}$, (auquel cas $x_{\ell}$ et $\bar{X}_{\infty}$ sont étrangers). Lorsque $S$ contient $\ell$, il vient tout simplement $x_{L / K}^{S}=0$, et le groupe $G_{L}^{S} / K$ est donc fini ; clest le résultat de semi-simplicité de Greenberg $\left[G b_{1}\right]$. 
(ii) Soit $L$ l'unique $\mathbb{Z}_{l}$-extension d'un corps quadratique imaginaire $K$, qui est prodiédrale sur $\mathbb{Q}$. Si $\ell$ est décomposée dans $K / \mathbb{Q}$, le caractère $\chi_{L / K}^{S}$ vaut 1 , lorsque $S$ est vide ou réduit à la place à llinfini, et nul dans tous les autres cas.

- Si $\ell$ n'est pas décomposée dans $K / Q$, le caractère $X_{L / K}^{S}$ est toujours nul. Clest le résultat de Gillard $\left[G i_{1}\right]$. 
$-237-$ 
CHAPITRE IV

STRUCTURE DES $\Lambda[\triangle]$-MODULES

REPRÉSENTATIONS $\ell$-ADIQUES ASSOCIÉES AUX INVARIANTS CYCLOTOMIQUES 


\section{1. - STRUCTURE DES $\Lambda[\Delta]$-MODULES .}

1.- Présentation de l'algèbre d'lwasawa généralisée.............. IV.2

a.- Position du problème............................. IV.2

b. - Définition de l'algèbre d'lwasawa généralisée............... IV.4

c.- Décomposition semi-locale de l'algèbre $\Sigma \ldots \ldots \ldots \ldots \ldots \ldots \ldots \ldots \ldots$...... IV 6

2.- Structure des $\Lambda[\Delta]$-modules dans le cas abélien............... IV.9

a.- Paramètres attachés à un $\Lambda[\Delta]$-module noethérien........... IV.9

b. - Etude de la filtration d'un $\Lambda[\Delta]$-module noethérien associée aux idéaux $\nabla_{n} \ldots \ldots \ldots \ldots \ldots \ldots \ldots \ldots \ldots \ldots \ldots \ldots \ldots \ldots \ldots \ldots . \ldots . \ldots . \ldots 11$

c. - Suites paramétrées de $\mathbb{Z}_{\ell}[\Delta]$-modules finis ............... IV. 15

3.- Structure des $\Lambda[\Delta]$-modules dans le cas métabélien............ IV.20

a.- Description des $\Sigma$-modules projectifs de type fini........... IV.21

b. - Classification des $\Sigma$-modules noethériens................ IV.25

c.- Paramètres attachés à un $\Sigma$-module noethérien............. IV. 30

2.- REPRÉSENTATIONS l-ADIQUES ASSOCIÉES AUX INVARIANTS CYCLOTOMIQUES.

1.- Etude du groupe de Galois de la $\ell$-extension abélienne non ramifiée $\ell$-décomposée maximale de $\mathrm{K}$

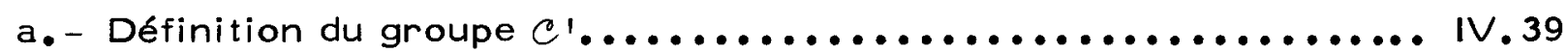

b. - Comparaison avec le groupe des classes au sens ordinaire....... IV.43

c.- Capitulation et structure des groupes de classes............. IV.50

2.- Etude du groupe de Galois de la $\ell$-extension abélienne

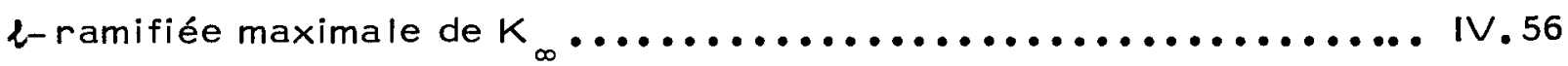

a.- Définition du groupe . ........................... IV.56

b. - Etude du sous-groupe de torsion $\tau$.................. IV.60

c. - Capitulation pour les groupes $\mathcal{U}_{\mathrm{n}}$, et application à la $K$-théorie...................................... IV 65 
3.- Etude du groupe de Galois de la $b$-extension hilbertienne maximale de $\mathrm{K}$

IV. 69

a.- Définition du groupe $\#$............................ IV.69

b. - Parallèle entre les conjectures de Leopoldt et de Gross....... . IV.73

c. - Inégalités du miroir ( $\ell$ impair ) ....................... IV.76

d. - Application à une conjecture de Coates.................... IV.79

\section{APPENDICE}

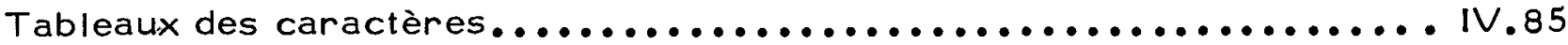

a. - Caractères associés aux suites paramétrées de $\mathbf{Z}_{\ell}[\Delta]$-modules finis.

IV.86

b. - Caractères associés aux $\Lambda[\Delta]$-modules noethériens (avec conjugaison complexe) 
$-242-$ 
L'introduction de l'algèbre formelle $\Lambda=Z_{\ell}[[\gamma-1]]$ dans la théorie des corps cyclotomiques est due à Jean-Pierre Serre. De fait, les théorèmes de structure sur les $\Lambda$-modules de type fini per mettent de clarifier substantiellement l'arithmétique des $\mathbb{Z}_{\ell}$-exten sions, et conduisent ainsi à des démonstrations simples des résul tats essentiels d'Iwasawa sur cette question.

Nous nous proposons ici d'étendre ces théorèmes de structure au cas plus général où l'anneau des scalaires est une algèbre de groupe $\Lambda[\Delta]$ à coefficients dans l'anneau d'Iwasawa $\Lambda=\mathbb{Z}_{\ell}[[\gamma-1]]$, le groupe $\Delta$ étant supposé abélien, d'ordre étranger à $\ell$, et muni d'une action par conjugaison sur le groupe procyclique $\Gamma=\gamma^{\mathbf{Z}_{\ell}}$. Les résultats proposés s'organisent en deux volets largement indépen dants : Le cas abélien, d'une part qui correspond à l'action triviale, et relève des méthodes de l'algèbre commutative, en particulier des résultats de Serre; le cas métabélien, d'autre part, qui correspond à une action non triviale, et s'obtient par passage à la limite à partir des techniques classiques des algèbres à produits croisés .

Auparavant, nous introduisons la notion de suite paramétrée de $\mathcal{Z}_{\ell}[\Lambda]$-modules finis, qui joue un rôle essentiel dans l'étude arithmétique développée plus loin. 
1.- PRÉSENTATION DE L'ALGËBRE DIIWASAWA GÉNÉRALISEE $\Lambda[\Delta]$. a.-Position du problème.

L'algèbre d'Iwasawa classique $\Lambda$ est l'algèbre des séries formelles à une indéterminée $\mathbb{Z}_{\ell}[[T]]$ sur l'anneau des entiers $\ell$-adiques. C'est un anneau local, d'idéal maximal $\mathbb{R}=\ell \Lambda+\Gamma \Lambda$, complet pour la topologie $\mathfrak{M}$-adique, et régulier de dimension 2 . Les idéaux premiers principaux d'un tel anneau sont de deux types (cf. [Sa $\left.{ }_{2}\right], p_{0} 60$ ) :

- lidéal $\ell \Lambda$, d'une part, engendré par $\ell$,

- les idéaux premiers $P(T) \Lambda$ engendrés par les polynô mes irréductibles distingués de l'anneau $\mathbb{Z}_{\ell}[T]$.

Enfin, les $\Lambda$-modules de type fini sont entièrement classifiés à pseudo-isomorphisme ${ }^{(\star)}$ près : chaque classe de pseudo-isomorphiedans la catégorie des $\Lambda$-modules noethériens contient un unique module qui s'écrit comme somme directe d'exemplaires de $\Lambda$ et de quotients $\Lambda / p^{n}$, où $p$ est un idéal premier principal de $\Lambda$; un tel module est dit élémentaire.

L'introduction de l'algèbre $\Lambda$ dans l'étude des corps cyclotomiques est dûe à Serre $\left.{ }^{(\star \star}\right)\left(c_{0}\left[S_{1}\right]\right)$ : Si $K_{\infty}$ est une $\mathbb{Z}_{l}$-exten sion diun corps de nombres $K, i . e$. une extension abélienne de $K$, dont le groupe de Galois $I=\operatorname{Gal}\left(K_{\infty} / K\right)$ est isomorphe à $\mathbb{Z}_{l}$, il existe, pour chaque naturel $n$, un unique sous-corps $K_{n}$ de $K{ }_{\infty}$ de degré $\ell^{n}$ sur $K$. Son groupe de Galois $\Gamma_{n}=\operatorname{Gal}\left(K_{n} / K\right)$ est isomor phe à $\mathbb{Z} / \ell^{n} \mathbb{Z}$, et la limite projective des algèbres de groupe

$$
\Lambda_{\Gamma}=\stackrel{\lim }{\longleftarrow} \mathbb{Z}_{\ell}\left[\Gamma_{n}\right]
$$

est, par définition, l'algèbre dilwasawa construite sur le groupe $I$. Le choix d'un progénérateur arbitraire $\gamma$ du groupe $\Gamma$ permet, en effet, de décrire $\Lambda_{\Gamma}$ comme algèbre de séries formelles en llindéterminée $(\gamma-1)$, l'anneau obtenu

$$
\Lambda_{\Gamma}=\mathbb{Z}_{\ell}[[\gamma-1]]
$$

étant en fait indépendant du choix de l'élément $\gamma$. Le théorème de structure rappelé plus haut permet alors de démontrer facilement le résultat essentiel d'lwasawa, qui peut s'énoncer comme suit :

(*) Un pseudo-isomorphisme est un morphisme à noyau et conoyau finis. $(\star \star)$ Dans les premiers articles sur la question $\left(\left[\mathrm{Iw} w_{3}\right],\left[\mathrm{Iw} w_{4}\right]\right)$, Iwasawa ne considère que des $\Gamma$-modules . 
Théorème fondamental. - Soient $X$ un $\Lambda$-module de type fini, noté additivement, et $x_{n}$ le quotient $x / \nu_{n} x$, avec $\nu_{n}=\sum_{k=0}^{\ell^{n-1}} \gamma^{k}$. Si tous les $X_{n}$ sont finis, il existe trois constantes entières $\lambda, \mu$ et $\nu$, avec $\lambda \geq 0$ et $\mu \geq 0$, telles que l'ordre $\ell^{x_{n}}$ de $x_{n}$ soit donné, pour $n$ assez grand, par la formule :

$$
x_{n}=\mu^{n}+\lambda n+\nu \text {. }
$$

Naturellement, Iwasawa s'intéressait avant tout au cas où $x_{n}$ est le $\ell$-groupe des classes d'idéaux du corps $K_{n}$, et clest dans ce cadre bien précis qu'il a formulé son théorème. Dans la forme abstraite donnée ci-dessus le résultat slapplique cependant à bien d'autres groupes, sous la condition quils soient représentables comme quotients finis $X / \nu_{n} \times$ associés à un $\Lambda$-module noethérien . En revanche, certains modules classiques ne sont pas de cette forme: Par exemple le quotient d'exposant $\ell^{n}$ du $\ell$-groupe des classes infinitésimales $a_{n}{ }^{(\star)}$ du corps $k_{n}$ est bien un groupe fini, mais il ne s'écrit pas comme quotient $X / \nu_{n} X$ d'un $\Lambda$-module noethérien $X$; malgré tout, son ordre est donné asymptotiquement par une formule analogue à celle présentée dans le théorème fondamental (mais faissant intervenir un invariant supplémentaire p). Nous donnerons plus loin une forme plus générale du théorème fondamental, permettant de rendre compte de ce type de situation.

Ensuite, il peut être intéressant d'étudier ce qui se produit lorsque la $\mathbb{Z}_{\ell}$-extension considérée $K_{\infty} / K$ est galoisienne sur un sous-corps $F$ de $K$, par exemple lorsque $K$ est abélien sur $F$, de degré relatif $d=[K: F]$ étranger avec $l$. Cette situation se rencontre naturellement lorsque, $\ell$ étant impair, le corps $F$ ne contient pas les racines $\ell$-ièmes de l'unité, et que l'on désire cependant faire appel à la théorie de Kummer : L'extension cyctotomique $K_{\infty}$, engendrée sur $K$ par les racines d'ordre $l$-primaire de l'unité, est une $\mathbb{Z}_{l}$-extension du corps $K=F\left[\zeta_{l}\right]$; elle est abélienne sur $F$; et le

$\left({ }^{\star}\right)$ D'après les résultats de la section 11.2 , le groupe $a_{n}$ s'identifie au groupe de Galois Gal $\left(M_{n} / K_{n}\right)$ de la $\ell$-extension abélienne $\ell$-ramifiée maximale de $K_{n}$. 
degré relatif $d=[K: F]$ divise $(\ell-1)=\left[\mathbb{Q}\left[\zeta_{\ell}\right]: \mathbb{Q}\right]$. Dans ce cas, on retrouve les invariants de $F$ à partir de ceux de $K$ en faisant appel à la décomposition semi-locale de l'algèbre de Galois Gal(K/F). Mais il peut arriver aussi que la $\mathbb{Z}_{\ell}$-extension considérée $K_{\infty} / K$ ne soit pas abélienne sur le corps de base $F$. Par exemple, si $F$ est le corps des rationnels, et $K$ une extension abélienne imaginaire de $\mathbb{Q}$, de degré d divisant $(\ell-1)$, l'exactitude de la conjecture de Leopoldt pour $K$ permet d'affirmer qu'il existe exactement $\left(\frac{d}{2}+1\right) \mathbb{Z}_{\ell}$-ex tensions de $K$ qui sont galoisiennes sur $\mathbb{Q}:$ la $\mathbb{Z}_{\ell}$-extension cyclo tomique, qui est associée au caractère unité, et les $\frac{d}{2} \mathbb{Z}_{\ell}$-exten sions galoisiennes mais non abéliennes sur $\mathbb{Q}$, qui sont associées aux caractères $\ell$-adiques imaginaires $\left.{ }^{(}\right)$du groupe $\Delta=\operatorname{Gal}(K / \mathbb{Q})$. Dans ce cas, les $\Lambda$-modules étudiés sont aussi des $\mathbb{Z}_{\ell}[\Delta]$-modules, et la rencontre de ces deux structures se traduit par des congruences significatives sur leurs invariants d'lwasawa ( $\mathrm{cf} .\left[\mathrm{Gi}_{1}\right],\left[\mathrm{CK}_{1}\right]$ ). Pour bien comprendre l'origine de ces congruences, il est alors nécessaire d'étudier les groupes concernés en tant que $\Lambda[\Delta]$-modules.

\section{b.- Définition de l'algèbre d'lwasawa généralisée.}

Dans tout ce qui suit, nous adoptons les conventions suivantes:

\section{Conventions - -}

(i) $l$ est un entier premier.

(ii) $\Gamma_{\chi} \Delta$ est un groupe profini, noté multiplicativement, produit semi-direct d'un sous-groupe procyclique $\Gamma$, isomorphe à $\mathbb{Z}_{\ell}$, et d'un groupe abélien $\Delta$, d'ordre d étranger à $\ell$.

(iii) L'homomorphisme de $\Delta$ dans Aut $\Gamma$, qui définit la loi de groupe sur le produit $\Gamma_{X} \Delta$, se factorise par un caractère $l$-adique $X$ du groupe $\Delta$, ce que traduit l'identité de conjugaison :

$$
\tau \gamma \tau^{-1}=\gamma^{X(\tau)}
$$

(*) Les caractères imaginaires absolument irréductibles sont ceux qui prennent la valeur $(-1)$ sur la conjugaison complexe. 
où $Y$ est un progénérateur arbitraire de $\Gamma$, et $\tau$ un élément quelconque de $\Delta$.

(iv) $H=\operatorname{Ker} \chi$ est le noyau du caractère $\chi$, et $m=(\Delta: H)$ son ordre, qui divise $d \wedge(\ell-1)$.

Cela étant, nous posons :

DEFINITIONIV.1.1.- Nous appelons algèbre d'Iwasawa du groupe profini $\Gamma_{\chi} \Delta$, et nous notons $\Sigma=\Lambda[\Delta]$, l'algèbre à produits croisés du groupe abélien $\Delta$ sur l'anneau d'lwasawa $\Lambda=\mathbb{Z}_{\ell}[[\gamma-1]]$, tordue par l'identité :

$$
\tau \gamma \tau^{-1}=\gamma^{\chi(\tau)}, \quad \forall \tau \in \Delta \text {. }
$$

Lorsque $X$ est le caractère unité du groupe $\Delta$, l'algèbre $\Lambda[\Delta]$ est l'algèbre abélienne de $\Delta$ à coefficients dans l'anneau $\Lambda$. Dans tous les autres cas , $\Lambda[\Delta]$ est une algèbre gauche, isomorphe au produit tensoriel $\mathbb{Z}_{\ell}[[\gamma-1]] \otimes_{\mathbb{Z}_{\ell}} \mathbb{Z}_{\ell}[\Delta]$ muni de la multiplication non commutative induite par l'identité :

$$
\left(\gamma^{\prime} \otimes \tau^{\prime}\right)(\gamma \otimes \tau)=\gamma^{\prime} \gamma^{X(\tau)} \otimes \tau^{\prime} \tau \text {. }
$$

Pour traduire commodément l'identité de conjugaison qui définit la multiplication dans l'algèbre $\Lambda[\Delta]$, il est naturel diintroduire l'élément résolvant :

$$
\theta=\frac{1}{\mathrm{~d}} \sum_{\tau \in \Delta} X\left(\tau^{-1}\right)\left[\gamma^{X(\tau)}-1\right]
$$

Il vient, en effet :

PROPOSITION IV.1.2. - La résolvante $\theta$ attachée à un progénérateur arbitraire du groupe $\Gamma$ satisfait la congruence :

$$
\text { (i) } \theta \equiv \gamma-1\left(\bmod (\gamma-1)^{2} \Lambda\right) \text {, }
$$

ainsi que la relation de commutation :

$$
\text { (ii) } \tau \theta \tau^{-1}=x(\tau) \theta, \quad \forall \tau \in \Delta \text {. }
$$

Elle est égale à $(\gamma-1)$, lorsque $\chi$ est le caractère unité; et, dans tous les cas, elle permet d'écrire l'algèbre d'Iwasawa $\mathbb{Z}_{\ell}[[\gamma-1]]$ sous la forme $\Lambda=\mathbf{Z}_{l}[[\theta]]$. 
Démonstration : Si $r$ est une racine de l'unité dans $\mathbb{Z}_{l}$, la formule du binôme nous donne l'identité :

$$
y^{r}-1=[1+(y-1)]^{r}-1=\sum_{k=1}^{\infty}\left(\begin{array}{c}
r \\
k
\end{array}\right)(y-1)^{k}
$$

II vient donc:

$$
\theta=\frac{1}{d} \sum_{\tau \in \Delta} X^{-1}(\tau)\left[\gamma^{X(\tau)}-1\right]=(\gamma-1)+\sum_{k \geq 2}\left[\frac{1}{d} \sum_{\tau \in \Delta} X\left(\tau^{-1}\right)\left(\begin{array}{c}
X(\tau) \\
k
\end{array}\right)\right](\gamma-1)^{k},
$$

ce qui établit la congruence annoncée.

Enfin, si $\tau$ est un élément quelconque de $\Delta$, nous avons:

$$
\begin{aligned}
\tau \theta \tau^{-1}=\frac{1}{\mathrm{~d}} \sum_{\sigma \in \Delta} X\left(\sigma^{-1}\right)\left[\tau \gamma^{X(\sigma)} \tau^{-1}-1\right] & =\frac{X(\tau)}{\mathrm{d}} \sum_{\sigma \in \Delta} X\left(\sigma^{-1} \tau^{-1}\right)\left[\gamma^{\chi(\sigma \tau)}-1\right] \\
& =\chi(\tau) \theta,
\end{aligned}
$$

ce qui achève la démonstration.

COROLLAIRE IV.1.3.- Le centre $C$ de l'algèbre $\sum$ est l'algèbre $\mathbb{Z}_{\ell}\left[\left[\theta^{m}\right]\right][H]$ du sous-groupe $H=\operatorname{Ker} \chi$ de $\Delta$, à coefficients dans l'anneau des séries formelles $\mathbb{Z}_{\ell}\left[\left[\theta^{m}\right]\right]$, où $m$ est l'ordre du caractère $X$.

L'algèbre tordue $\Sigma=\Lambda[\Delta]$ est un $C$-module libre de dimension $\mathrm{m}^{2}$; elle contient $\Lambda[H]$ comme sous-algèbre commutative ma ximale ; $\Lambda[H]$ est de dimension $m$ sur le centre $C$.

Démonstration: D'après la proposition 2 , tout élément $x$ de l'algèbre $\mathbb{Z}_{\ell}[[y-1]]$ s'écrit comme série formelle en la résolvante $\theta$, i.e. $x=\sum_{k \in N} x_{k} \theta^{k}$. La condition $x \in C$ se traduit donc par liidentité : $\sum x_{k} \theta^{k}=x=\tau x \tau^{-1}=\sum x_{k} \tau \theta^{k} \tau^{-1}=\sum x_{k} x^{k}(\tau) \theta^{k}$, pour tout $\tau$ de $\Delta$, c'est-à-dire $x_{k}\left(x^{k}(\tau)-1\right)=0, \forall T \in \Delta$, soit encore $x_{k}=0$, pour $k \not 0(\bmod m)$. D'où la caractérisation du centre.

\section{c.-Décomposition semi-simple de l'algèbre $\Sigma$.}

Désignons par $R_{\mathbb{Z}_{l}}^{+}(\Delta)$ le semi-groupe des caractères des $\mathbb{Z}_{\ell}[\Delta]$-modules noethériens et projectifs, par $R_{\mathbb{Z}_{\ell}}(\Delta)$ le groupe 
des caractères virtuels du groupe $\Delta$ sur l'anneau $\mathbb{Z}_{\ell}$. L'ordre $d$ du groupe abélien $\Delta$ étant supposé étranger à $\ell$, l'anneau $R_{\mathbb{Z}_{\ell}}(\Delta)$ est muni d'un produit scalaire à valeurs dans $\mathbb{Z}_{\ell}$

$$
\langle\varphi, \psi\rangle=\frac{1}{\mathrm{~d}} \sum_{\tau \in \Delta} \varphi\left(\tau^{-1}\right) \psi(\tau)
$$

et à chaque caractère $\ell$-adique irréductible $\varphi$ de $\Delta$ correspond un idempotent primitif de l'algèbre $\mathbb{Z}_{\ell}[\Delta]$ :

$$
e_{\varphi}=\frac{1}{d} \sum_{T \in \Delta} \varphi\left(T^{-1}\right)
$$

Dans la décomposition semi-locale $\mathbb{Z}_{\ell}[\Delta]=\underset{\varphi \in R_{\mathbb{Z}_{\ell}}^{\operatorname{irr}}(\Delta)}{\mathbb{Z}} \ell[\Delta]$ e $\varphi$ de l'algèbre de groupe $\mathbb{Z}_{\ell}[\Delta]$, chaque facteur isotypique $Z_{\varphi}=$

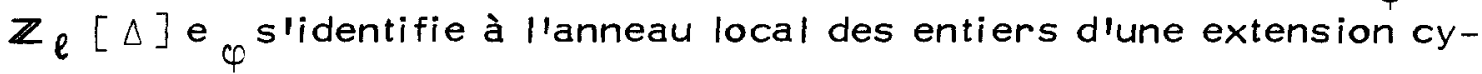
clotomique non ramifiée de $\mathbb{Q}_{\ell}$, qui a pour degré $\left[Z_{\varphi}: \mathbb{Z}_{\ell}\right]$ le de gré $\mathrm{d}_{\varphi}=\langle\varphi, \varphi\rangle$ du caractère $\varphi$. En particulier, tout $\mathbb{Z}_{\ell}[\Delta]-$ module noethérien sans $\mathbb{Z}_{\ell}$-torsion est somme directe d'exemplaires de $Z_{\varphi}$, donc $\mathbb{Z}_{\ell}[\Delta]$-projectif.

Malheureusement, cette décomposition semi-locale de l'algèbre $\mathbb{Z}_{\mathfrak{l}}[\Delta]$ ne se relève pas toujours en une décomposition d'algèbre de $\Lambda[\Delta]$ : la décomposition à droite par les idempotents primitifs $e_{\varphi}$ induit bien une décomposition de l'algèbre $\Sigma$ comme module à gauche sur elle-même :

$$
\Sigma={\underset{\varphi \in R}{\mathbb{Z}_{l}}}_{\operatorname{irr}_{l}}(\Delta)^{\Sigma} \varphi \text {, avec } \Sigma_{\varphi}=\Lambda[\Delta] \mathbf{e} \varphi,
$$

mais les relations de commutation ( $i i)$ montrent que cette décomposition n'est pas compatible avec la structure dlalgèbre dès que $X$ est non trivial. Plus précisément, lidentité (ii) se traduit ici par une trans lation des caractères :

PROPOSITION IV.1.4. - Les idempotents primitifs e $\varphi$ de l'algèbre $\mathbb{Z}_{\ell}[\Delta]$, associés aux caractères $\ell$-adiques irréductibles du groupe $\triangle$ vérifient lídentité de translation :

(iii) $\theta \mathrm{e}_{\varphi}=\mathrm{e}_{\varphi \mathrm{x}}{ }^{\theta}$.

En particulier, les idempotents centraux primitifs del'algèbre $\Sigma=\Lambda[\Delta]$ sont les idempotents associés auxinduits à $\Delta$ des caractères $\ell$-adiques irréductibles du noyau $H$ du caractère $X$. 
Démonstration : Nous avons immédiatement :

$\theta_{\varphi}=\frac{1}{d} \sum_{\tau \in \Delta} \varphi(\tau) \theta \tau^{-1}=\frac{1}{d} \sum_{\tau \in \Delta} \varphi(\tau) X(\tau) \tau^{-1} \theta=e_{\varphi \chi} \theta$, pour chaque caractère $\ell$-adique irréductible $\varphi$ de $\Delta$, et, plus généra lement :

$$
\theta \mathrm{e}_{\Phi}=\mathrm{e}_{\Phi X^{\theta}}
$$

si e $\Phi_{\Phi}=\frac{1}{\mathrm{~d}} \sum_{T \in \Delta} \Phi(\tau) \tau^{-1}$ est l'idempotent de l'algèbre $\mathbb{Z}_{\ell}[\Delta]$ associé à un caractère $\ell$-adique $\Phi$ de $R_{\mathbb{Z}}^{+}(\Delta)$, contenu dans le caractère régulier (i.e. à une somme finie disjointe de caractères irréductibles). Les idempotents centraux sont donc ceux qui vérifient l'identité $\mathrm{e}_{\Phi}=\mathrm{e}_{\Phi X}$, clest-à-dire les idempotents associés aux caractères $\Phi$, contenus dans le caractère régulier, et stables par translation de $X$. Les caractères $\Phi$ qui sont stables par translation de $X$ sont évidemment les caractères $\ell$-adiques nuls en-dehors de $H$; ce sont les " multiples " de l'induit à $\Delta$ du caractère unité du sous-groupe $H$. En effet, dans la décomposition absolument irréductible du caractère $\Phi$, la condition d'invariance $\Phi \chi=X$ exprime l'existence d'un caractère $\varphi$, à valeurs dans une extension de $\mathbb{Z}_{\ell}$, vérifiant liidentité : $\Phi=\varphi\left(1+x+\ldots+x^{m-1}\right)=\varphi \cdot 1 \stackrel{\Delta}{H}$. II vient en particulier res $\stackrel{\Delta}{\Delta} \Phi=$ $m$. res $\stackrel{H}{H}^{\Delta}$, et la restriction de $\varphi$ à $H$ est donc un caractère de $R \mathbb{Z}_{\ell}(H)$.

COROLLAIRE IV.1.5.- Dans la décomposition irréductible

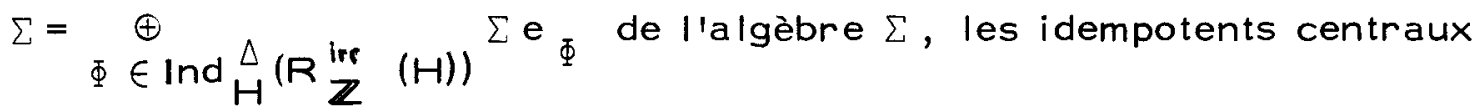
primitifs $e_{\Phi}$ sont associés aux caractères $\ell$-adiques $\Phi$ du groupe $\Delta$ qui sont induits par les caractères $\ell$-adiques irréductibles du noyau $H$. Les facteurs isotypiques $\Sigma_{\Phi}=\Sigma \mathrm{e}_{\Phi}$ s'identifient aux algèbres tordues $\Lambda_{\Phi} \otimes_{\mathbb{Z}_{\ell}[H]} \mathbb{Z}_{\ell}[\Delta]$, où $\Lambda_{\Phi}=\mathbb{Z}_{\Phi}[[\theta]]$ est l'algèbre des séries formelles sur l'extension non ramifiée $Z_{\Phi}$ de $\mathbb{Z}_{\ell}$ associée au caractère $\ell$-adique irréductible $\frac{1}{m} \operatorname{Res} \Delta_{H}^{\Delta} \Phi$. Ce sont des algèbres gauches de dimension $m^{2}$ sur leur centre $Z_{\Phi}\left[\left[\theta^{m}\right]\right]$, qui contiennent les $\Lambda_{\Phi}$ comme sous-algèbres commutatives maximales . 
Cela étant, le plan d'étude des $\Lambda[\Delta]$ - modules est clair :

- déterminer d'abord la structure et les propriétés des $\Lambda_{\Phi}$-modules ;

modules .

- étendre ensuite les résultats obtenus au cas des $\Sigma_{\Phi^{-}}$

Comme la somme directe des algèbres locales $\Lambda_{\Phi}$ (lorsque $\Phi$ parcourt l'ensemble des induits à $\Delta$ des caractères $\ell$-adiques irréductibles de $H$ ) est l'algèbre abélienne $\Lambda[H]$ du groupe $H$ à coefficients dans l'an neau d'lwasawa $\Lambda=\mathbb{Z}_{\ell}[[\theta]]$, la première étape n'est autre que l'étude du cas abélien $X=1$.

\section{2. - STRUCTURE DES $\Lambda[\Delta]$ - MODULES DANS LE CAS ABÉLIEN .}

Nous supposons dans cette section que $X$ est le caractère unité, c'est-à-dire que $\Lambda[\Delta]$ est l'algèbre abélienne du groupe $\Delta$ à coefficients dans l'anneau d'lwasawa $\mathbb{Z}_{\ell}[[\theta]]$, où $\theta=\gamma-1$ est la résol vante construite sur un progénérateur arbitraire du groupe $\Gamma \simeq \mathbb{Z}_{l}$.

a.-Paramètres attachés à un $\Lambda[\Delta]$ - module noethérien .

Lorsque l'algèbre $\Lambda[\Delta]$ est abélienne, la décomposition di recte $\mathbb{Z}_{\ell}[\Delta]=\underset{\varphi \in R}{\oplus}{\underset{\mathbb{Z}_{l}}{\text { irr }}(\Delta)}_{\mathbb{Z}_{\ell}(\Delta] \text { e }}^{\mathbb{Z}_{\varphi}}$ de l'algèbre du groupe $\mathbb{Z}_{\ell}[\Delta]$, comme somme directe dlanneaux locaux complets de valuation discrète $Z_{\varphi}$, se propage en une décomposition directe $\Lambda[\Delta]=\underset{\varphi \in R_{\mathbb{Z}}^{i r r}(\Delta)}{\oplus} \varphi[[\theta]]$

de l'algèbre $\Lambda[\Delta]$, où chaque facteur isotypique $\Lambda_{\varphi}=z_{\varphi}[[\theta]]$ est un anneau local complet régulier de dimension 2 , et un $\Lambda$-module libre de dimension $\mathrm{d}_{\varphi}=\left[\mathbf{z}_{\varphi}: \mathbb{Z}_{\ell}\right]=\langle\varphi, \varphi\rangle$.

Si donc $X$ est un $\Lambda[\Delta]$-module noethérien ( noté additive ment) les résultats de Serre ( $\mathrm{cf} .\left[\mathrm{Se}_{1}\right], \S 5$ ) permettent directement d'affirmer que chaque composante isotypique $X_{\varphi}=e_{\varphi} \times$ est pseudo-isomorphe, en tant que $\Lambda_{\varphi}$-module noethérien, à une somme 
directe finie de $\Lambda_{\varphi}$-modules élémentaires irréductibles ; ce qui s'écrit :

$$
x_{\varphi} \sim \Lambda_{\varphi}^{\rho} \varphi \oplus\left[\underset{\mathrm{i}=0}{\oplus} \varphi \Lambda_{\varphi} / f_{\varphi, i} \Lambda_{\varphi}\right] \oplus\left[\underset{\mathrm{j}=0}{\oplus} \Lambda_{\varphi}^{t} \Lambda / e^{m} \varphi, j \Lambda_{\varphi}\right]
$$

Dans cette formule, les $\left(f_{\varphi, i}\right)_{i=0, \ldots, s_{\varphi}}$ forment une famille décroissante de polynômes distingués de llanneau $Z_{\varphi}[\theta]$ (ordonnée par divisibilité), et les $\left(m_{\varphi, j}\right)_{j}=0, \ldots, t_{\varphi}$ sont des entiers naturels non nuls. La pseudo-décomposition est d'ailleurs essentiellement unique sous les conditions énoncées. Autrement dit :

THÉOREME IV.1.6.- Tout $\Lambda[\Delta]$-module noethérien $\times$ est pseudo-isomorphe à une somme directe finie de $\Lambda[\Delta]$-modules isotypiques élémentaires. Plus précisément, pour chaque caractère $\ell$-adique irréductible $\varphi$ du groupe $\Delta$, il existe un unique triplet $\left(\rho_{\varphi}, s_{\varphi}, t_{\varphi}\right)$ d'entiers naturels, une unique suite décroissante $\left(f_{\varphi, i}\right)_{i=0, \ldots, s_{\varphi}}$ de polynômes distingués de l'anneau $\mathrm{Z}_{\varphi}[\gamma-1]$, et une unique suite décroissante $\left(m_{\varphi, j}\right)_{j}=0, \ldots, t_{\varphi}$ d'entiers naturels non nuls, tels que la $\varphi$-composante $X_{\varphi}=e_{\varphi} \times$ du module $X$ soit $\Lambda[\Delta]$-pseudo-isomorphe à la somme directe :

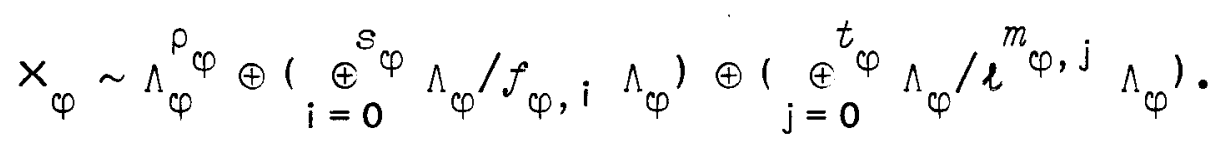

L'entier $\rho_{\varphi}$ est la dimension $\operatorname{dim}_{\Lambda_{\varphi}} \times_{\varphi}$ du $\Lambda_{\varphi}$-module $\times_{\varphi}$, et le polynôme $P_{\varphi}=\prod_{j=0}^{t} \varphi l^{m} \varphi, j \prod_{i=0}^{s} \varphi f_{\varphi, i}$ est le polynôme caractéristique de son sous-module de $\Lambda_{\varphi}$-torsion.

DÉFINITION IV.1.7.- Nous appelons paramètres d'un $\Lambda[\Delta]$-module noethérien $\times$ les caractères $\ell$-adiques du groupe $\Delta$ définis à partir des invariants d'Iwasawa des composantes isotypiques de $X$ par les formules

$$
\rho=\sum_{\varphi} \rho_{\varphi} \varphi, \quad \mu=\sum_{\varphi} \mu_{\varphi} \varphi, \quad \text { et } \lambda=\sum_{\varphi} \lambda_{\varphi} \varphi
$$

où, pour chaque caractère $l$-adique irréductible $\varphi$, les entiers $\rho_{\varphi}, \lambda_{\varphi}$, et $\mu_{\varphi}$ mesurent respectivement la dimension $\operatorname{dim}_{\Lambda_{\varphi}} \times_{\varphi}$ de la $\varphi^{\text {-composante }}$ 
de $\times$, ainsi que le degré $\sum_{i=0}^{s_{\varphi}}$ deg $f_{\varphi, i}$ et la $\ell$-valuation $\sum_{j=0}^{t} \varphi m_{\varphi, j}$ du polynôme caractéristique de son sous-module de $\Lambda_{\varphi}$-torsion.

L'algèbre $\Lambda_{\varphi}$ étant elle-même un $\Lambda$-module libre de dimension $d_{\varphi}=\langle\varphi, \varphi\rangle$, un calcul direct montre ainsi que les invariants classiques d'lwasawa d'un $\Lambda[\Lambda]$-module noethérien $X$, considéré comme $\Lambda$-module, ne sont autres que les degrés respectifs des paramètres de sa $\Lambda[\Delta]-$ structure. Ainsi,

$$
\operatorname{dim}_{\Lambda} X=\sum_{\varphi} \operatorname{dim}_{\Lambda} \Lambda_{\varphi}^{\rho} \varphi=\sum_{\varphi} \rho_{\varphi} \mathrm{d}_{\varphi}=\operatorname{deg} \rho
$$

et un résultat analogue vaut pour les paramètres $\lambda$ et $\mu$.

Cela dit, sous la forme générale donnée ci-dessus, les caractères $\rho, \mu$, et $\lambda$, associés à un $\Lambda[\Delta]$-module noethérien, sont caractérisés par le résultat fondamental suivant :

THÉORĖME IV.1.8.- Théorème des paramètres.- Soit $\times$ un $\Lambda[\Delta]-$ module noethérien de paramètres $\rho, \mu$, et $\lambda$. Si $\nabla_{n}=l^{n+1} \Lambda+w_{n} \Lambda$ désigne l'idéal de l'algèbre d'lwasawa $\Lambda=\mathbb{Z}_{\ell}[[\gamma-1]]$, engendré par l'élément $\ell^{n+1}$ et le polynôme $\omega_{n}=\left(\gamma^{\ell^{n}}-1\right)$, il existe un unique caractère $\ell$-adique virtuel $\nu$ du groupe $\Delta$, tel que l'ordre $e^{x_{n}^{\varphi}}$ de la $\varphi$-composante du quotient $\times / \nabla_{n} \times$ soit donné, pour chaque caractère $\ell$-adique irréductible $\varphi$, et tout $n$ assez grand, par la formule :

$$
x_{n}^{\varphi}=\left\langle\rho, \varphi>(n+1) l^{n}+\left\langle\mu, \varphi>l^{n}+\langle\lambda, \varphi>n+\langle\nu, \varphi>\text {. }\right.\right.
$$

La démonstration de ce résultat fait l'objet de la section qui vient :

b.-Etude de la filtration d'un $\Lambda[\Delta]$-module noethérien associée aux idéaux $\nabla_{n}$. PROPOSITION IV.1.9.- Pour chaque naturel $n$, désignons par $\nabla_{n}=e^{n+1} \Lambda+\omega_{n} \Lambda$ l'idéal de l'algèbre $\Lambda=\mathbb{Z}_{\ell}[[\gamma-1]]$ engendrée par l'élément $l^{n+1}$ et le polynôme $\omega_{n}=\gamma^{\ell^{n}}-1$. Alors : 
(i) Les idéaux $\left(\nabla_{n}\right) n \in \mathbb{N}$ forment une suite exhaustive strictement décroissante d'idéaux d'indice fini de l'algèbre d'lwasawa $\mathbb{Z}_{\ell}[[\gamma-1]]=\mathbb{Z}_{\ell}[\theta]$.

(ii) Un $\Lambda[\Delta]$-module compact $X$ est noethérien si et seulement s'il existe un $n$ pour lequel le quotient $X / \nabla_{n} \times$ est fini ; auquel cas les quotients $\times / \nabla_{n} \times$ sont tous des $\mathbb{Z}_{\ell}[\Delta]$-modules finis.

(iii) Un $\Lambda[\Delta]$-module noethérien $X$ est fini si et seulement si les sous-modules $\nabla_{n} \times$ sont nuls pour $n$ assez grand.

Démonstration : L'assertion (i) est sans malice : D'un côté, comme $\omega_{0}$ est égal à $y-1=\theta$, liidéal $\nabla_{0}$ est précisément liidéal maximal $\mathbb{m}$ de llalgèbre $\Lambda$; et l'identité $\left(\gamma^{l^{n}}-1\right)^{l}=\left(\gamma^{l^{n+1}}-1\right)+l\left(\gamma^{l^{n}}-1\right) P(\gamma)$, où $P$ est un polynôme convenable de l'anneau $\mathbb{Z}[\gamma]$ montre, par une récurrence évidente, que $\nabla_{n}$ est contenu dans la puissance $(n+1)$-ième de l'idéal $\mathfrak{M}$. D'un autre côté, un calcul immédiat donne

$$
\left(\Lambda: \nabla_{n}\right)=\left|\left(\mathbb{Z} / e^{n+1} \mathbb{Z}\right)\left[\Gamma_{n}\right]\right|=e^{(n+1) e^{n}},
$$

ce qui établit (i). Les assertions (ii) et (iii) résultent alors du fait que les idéaux $\left(\nabla_{n}\right) n \in \mathbb{N}$ forment une base de voisinages de 0 dans l'algèbre compacte $\mathbb{Z}_{\ell}[[\gamma-1]]$.

Cela étant, le théorème 8 résulte d'une suite de cinq lemmes et du calcul de l'indice $\left(Y: \nabla_{n} Y\right)$ lorsque $Y$ est élémentaire :

LEMME IV.1.10. - Soient $Z_{\varphi}$ une extension finie non ramifiée de $\mathbb{Z} l$, et $f$ un polynôme distingué de l'anneau $Z_{\varphi}[\gamma-1]$. Pour chaque naturel $n$ assez grand, il existe alors deux polynômes $a_{n}$ et $b_{n}$ dans $z_{\varphi}[\gamma-1]$ qui vérifient : $\sum_{k=0}^{l-1} y^{k l^{n}}=\frac{w_{n}+1}{w_{n}}=\ell\left(1+l a_{n}\right)+b_{n} f$. L'élément $\left(1+\ell a_{n}\right)$ est inversible dans $\Lambda_{\varphi}$.

Démonstration $(\star)$ : Raisonnons dans l'anneau quotient $z_{\varphi}[\gamma-1] / f z_{\varphi}[\gamma-1]$.

(*) Ce résultat peut être regardé comme une généralisation de $\left[\mathrm{Se}_{1}\right], \S 5$, th. 8 (iii); cf. aussi [Or], lemme p. 18. 
Si $e$ est le degré du polynôme $f$, nous avons $(\overline{\gamma-1})^{e} \equiv \overline{0}(\bmod \bar{l})$, et donc $\bar{\gamma} e^{n-1}-\overline{1} \equiv(\overline{\gamma-1}) e^{n-1} \equiv \overline{0}(\bmod \bar{l})$, dès que $n$ est assez grand.

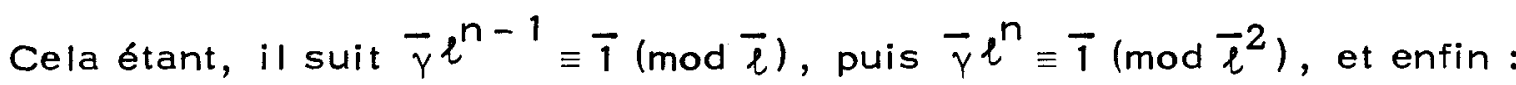

$$
\sum_{k=0}^{l-1} \bar{\gamma}^{k l^{n}} \equiv \bar{l}\left(\bmod \bar{l}^{2}\right) ; \text { ce qui est le résultat annoncé. }
$$

LEMME IV.1.11. - Soit $Y=T \oplus P$ un $\Lambda[\Delta]$-module élémentaire, somme directe de son sous-module de $\Lambda$-torsion $T$ et d'un sous-module projectif $P$. Pour chaque naturel $n$, posons $\partial^{n} Y=l^{n+1} Y \cap \omega_{n} Y$. Nous avons $\partial^{n} Y=\partial^{n_{1}} \oplus \partial^{n_{P}} P$, avec $\partial^{n} T=e^{n-n_{0}} \partial^{n_{0}} T$ et $\partial^{n_{P}}=\frac{\omega_{n}}{\omega_{n_{0}}} e^{n-n_{0}} \partial^{n_{0}} P$, pour $n_{0}$ assez grand, et tout $n$ plus grand que $n_{0}$.

Démonstration : Tout $\Lambda[\Delta]$-module noethérien et projectif $P$ étant facteur direct d'un module libre, nous avons immédiatement $\partial^{n} P=e^{n+1} P \cap \omega_{n} P=e^{n+1} \omega_{n} P$, par un argument de factorialité (les facteurs locaux $\Lambda_{\varphi}$ de l'algèbre $\Lambda[\Delta]$ sont des anneaux réguliers donc factoriels). En particulier, il suit $\partial^{n} P=\frac{\omega_{n}}{\omega_{n}} l^{n-n_{0}} \partial^{n_{0}} P$, comme an-
noncé.

Considérons maintenant le sous-module de torsion $T$. Ses facteurs indécomposables, en nombre fini, sont de deux types : des quotients de la forme $\Lambda_{\varphi} / e^{m} \Lambda_{\varphi}$, d'une part ; des quotients de la forme $\Lambda_{\varphi} / f \Lambda_{\varphi}$, avec $f$ distingué dans $Z_{\varphi}[\gamma-1]$, d'autre part. Désignons par $S$ la somme directe des seconds. Pour $n$ assez grand, nous avons $\ell^{n_{T}}=l^{n_{S}}$, et donc $\partial^{n_{T}}=\partial^{n} S$. Appliquons maintenant le lemme 10 à chacun des facteurs indécomposables de $S$. Pour $n$ assez grand, disons $n \geq n_{0}$, nous obtenons $\frac{w_{n}+1}{w_{n}} s=l s$, d'où :

$$
\begin{aligned}
& \partial^{n} s=e^{n+1} s \cap \omega_{n} s=e^{n-n_{0}} e^{n_{0}+1} s \cap \frac{w_{n}}{w_{n}} \omega_{n} s= \\
& =e^{n-n_{0}}\left[e^{n_{0}+1} s \cap \omega_{n_{0}} s\right]=e^{n-n_{0}} \partial^{n_{0}} s ;
\end{aligned}
$$

ce qui est le résultat attendu. 
LEMME IV.1.12. - Soient $Y$ un $\Lambda[\Delta]$-module élémentaire, et $X$ un sousmodule d'indice fini. Pour chaque caractère $\ell$-adique irréductible $\varphi$ du groupe $\Delta$, la suite $\left(\left(\partial^{n} Y_{\varphi}: \partial^{n} X_{\varphi}\right)\right)_{n \in \mathbb{N}}$ des $\varphi$-parties des indices $\left(\partial^{n} Y: \partial^{n} X\right)$ est stationnaire.

Démonstration : Désignons par $Y^{\text {tor }}$ le sous-module de torsion de $Y$, et notons pr la projection canonique $Y \rightarrow Y / Y^{\text {tor }}$. Nous avons :

$$
\left(\partial^{n} Y_{\varphi}: \partial^{n} X_{\varphi}\right)=\frac{\left(\partial^{n} Y_{\varphi}: \partial^{n} Y_{\varphi}^{\text {tor }}+\partial^{n} X_{\varphi}\right)}{\left(\partial^{n} Y_{\varphi}^{\text {tor }}+\partial^{n} X_{\varphi}: \partial^{n} X_{\varphi}\right)}=\frac{\left(p r\left(\partial^{n} Y_{\varphi}\right): \operatorname{pr}\left(\partial^{n} X_{\varphi}\right)\right)}{\left(\partial^{n} Y_{\varphi}^{\text {tor }}: \partial^{n} X_{\varphi}^{\text {tor }}\right)} \text {. }
$$

D'un côté, le lemme 11 nous donne $\operatorname{pr}\left(\partial^{n+1} Y_{\varphi}\right)=\ell \frac{\omega_{n+1}}{\omega_{n}} \operatorname{pr}\left(\partial^{n} Y_{\varphi}\right)$; et comme nous avons trivialement $\operatorname{pr}\left(\partial^{n+1} x_{\varphi}\right) \supset \ell \frac{\omega_{n}+1}{\omega_{n}} \operatorname{pr}\left(\partial^{n} x_{\varphi}\right)$, le numérateur, qui va donc décroissant pour $n$ assez grand, est stationnaire.

D'un autre côté, nous avons $\partial^{n+1} Y_{\varphi}^{\text {tor }}=l \partial^{n} Y_{\varphi}^{\text {tor }}$ et $\partial^{n+1} x_{\varphi}^{\text {tor }}=\ell \partial^{n} x_{\varphi}^{\text {tor }}$, pour $n \geq n_{0}$ par une extension facile du lemme 11 , puisque $l^{n_{0}} Y_{\varphi}^{\text {tor }}$ et $l^{n_{0}} X_{\varphi}^{\text {tor }}$ sont annulés par un même polynôme distingué $f \in Z{ }_{\varphi}[\gamma-1]$. Comme la multiplication par $\ell^{n_{0}}$ a tué le sous-module de $\mathbb{Z}_{\ell}$-torsion de $Y_{\varphi}^{\text {tor }}$, la multiplication par $\ell$ est injective sur $\ell^{n_{0}} Y_{\varphi}^{\text {tor }}$ et le dénominateur est stationnaire pour $n \geq n_{0}$ •

느MME IV.1.13.- Sous les hypothèses du lemme 12, la suite $\left(\left(\nabla_{n} Y_{\varphi}: \nabla_{n} X_{\varphi}\right)\right)_{n \in \mathbb{N}}$ des $\varphi$-parties des indices $\left(\nabla_{n} Y: \nabla_{n} X\right)$ est stationnaire.

Démonstration: Dans la suite exacte courte de $\Lambda[\Delta]$-modules finis $0 \rightarrow \partial^{n} Y_{\varphi} / \partial^{n} X_{\varphi} \rightarrow\left(e^{n+1} Y_{\varphi} / e^{n+1} X_{\varphi}\right) \oplus\left(\omega_{n} Y \varphi / \omega_{n} X_{\varphi}\right) \rightarrow \nabla_{n} Y_{\varphi} / \nabla_{n} X_{\varphi} \rightarrow 0$ les termes médians, qui vont décroissant, sont stationnaires. Le résultat annoncé résulte donc directement du lemme 12 précédent.

LEMME IV.1.14. - Soient $X$ un $\Lambda[\Delta]$-module noethérien, et $Y$ l'unique 
$\Lambda[\Delta]$-module élémentaire auquel il est pseudo-isomorphe (*). Pour chaque caractère $\ell$-adique irréductible $\varphi$ du groupe $\Delta$, la suite des quotients $\left(\left(X_{\varphi}: \nabla_{n} X_{\varphi}\right) /\left(Y_{\varphi}: \nabla_{n} Y_{\varphi}\right)\right)_{n \in N}$ est stationnaire.

Démonstration : Prenons un pseudo-isomorphisme $t$ de $X$ dans $Y$, et considérons la suite exacte courte :

$$
0 \rightarrow \mathrm{N} \longrightarrow \mathrm{X} \stackrel{t}{\rightarrow} \mathrm{Y} \rightarrow \mathrm{C} \longrightarrow 0 \text {. }
$$

Le noyau $N$ et le conoyau $C$ étant finis, choisissons $n_{0}$ assez grand pour avoir $N \cap \nabla_{n_{0}} X=0$, et $\nabla_{n_{0}} C=0$, i.e. $\nabla_{n} Y \subset t(X)$. Pour chaque $n \geq n_{0}$, nous obtenons alors la suite exacte

$0 \longrightarrow{ }^{-1} t\left(\nabla_{n} Y\right) / \nabla_{n} X \longrightarrow X / \nabla_{n} X \longrightarrow Y / \nabla_{n} Y \longrightarrow c \longrightarrow 0$; et pour chaque caractère $\ell$-adique irréductible $\varphi$ du groupe $\Delta$, la $\varphi$-partie de l'ordre du terme de gauche, qui est donnée par la formule $\left({ }^{-1} t\left(\nabla_{n} Y_{\varphi}\right): \nabla_{n} X_{\varphi}\right)=\left|N_{\varphi}\right|\left(\nabla_{n} Y_{\varphi}: \nabla_{n} t\left(X_{\varphi}\right)\right)$, est constante pour $n$ assez grand, en vertu du lemme 13 .

\section{c.-Suites paramétrées de $\mathbb{Z}_{\ell}[\Delta]$-modules finis $\cdot$}

DÉFINITION IV.1.15.- Nous disons qu'une suite $\left(x_{n}\right)_{n \in \mathbb{N}}$ de $\mathbb{Z}_{l}[\Delta]$ modules finis est paramétrée par les caractères $\ell$-adiques virtuels $\rho=\Sigma_{\varphi} \rho_{\varphi} \varphi, \quad \mu=\Sigma_{\varphi} \mu_{\varphi} \varphi, \quad \lambda=\Sigma_{\varphi} \lambda_{\varphi} \varphi$, et $\nu=\Sigma_{\varphi} \nu_{\varphi} \varphi$, lorsque, pour chaque caractère $l$-adique irréductible $\varphi$ du groupe $\Delta$, l'ordre $l^{x_{n}^{\varphi}}$ de la $\varphi$-composante $X_{n, \varphi}=e{ }_{\varphi} X_{n}$ du module $X_{n}$ est donnée asymptotiquement par la formule $(\star \star)$ :

$$
\begin{aligned}
& x_{n}^{\varphi}=\left\langle\rho, \varphi>(n+1) e^{n}\left\langle\mu, \varphi>e^{n}+\langle\lambda, \varphi>n+\langle\nu, \varphi\rangle=\right.\right. \\
& =d_{\varphi}\left(\rho \varphi^{n} \ell^{n+1}+\mu_{\varphi} e^{n}+\lambda_{\varphi} n+\nu_{\varphi}\right) .
\end{aligned}
$$

Remarque. - L'entier $x_{n}^{\varphi}$ étant toujours positif, le caractère $\rho$ interve-

$(\star)$ Si $\times$ n'est pas de torsion, il n'existe pas toujours de pseudo-isomorphisme de $Y$ dans $X$.

$(\star \star)$ Cette définition est plus restrictive que celle de $\left[\mathrm{Ja}_{\mathbf{g}}\right]$. 
nant dans la définition est toujours positif. II en est de même du caractère $\mu$ si $\rho$ est nul, et du caractère $\lambda$ si $\rho$ et $\mu$ sont tous deux nuls. En revanche, il peut arriver que les caractères $\lambda$ ou $\mu$ ne soient pas dans $R_{Z_{l}}^{+}(\Delta)$, dès lors que le caractère dominant y est. Des exemples sont donnés dans la section 2 .

Compte tenu du lemme 14 , le théorème 8 résulte de la proposition suivante :

PROPOSITION IV.1.16. - Soient $Y$ un $\Lambda[\Delta]$-module élémentaire, et $e^{y_{n}}$ l'ordre du quotient $Y / \nabla_{n} Y$.
(i) Pour $Y=\Lambda_{\varphi}$, il vient :
$y_{n}=d_{\varphi}(n+1) e^{n}$
(ii) Pour $Y=\Lambda_{\varphi} / e^{m} \Lambda_{\varphi}$, il vient : $y_{n}=d_{\varphi} m e^{n}=<m \varphi, \varphi>e^{n}$.
(iii) Pour $Y=\Lambda_{\varphi} / f \Lambda_{\varphi}$, il vient : $\quad y_{n}=d_{\varphi}\left(n-n_{0}\right) \operatorname{deg} f+y_{n_{0}}=$ $<\operatorname{deg} f \cdot \varphi, \varphi>n+<c^{\text {te }} \varphi, \varphi>$.

Démonstration : Distinguons les trois cas :

(i) Pour $Y=\Lambda_{\varphi}$, nous avons $Y / \nabla_{n} Y \simeq\left(Z_{\varphi} / e^{n+1} Z_{\varphi}\right)\left[I_{n}\right]$, avec $\Gamma_{n}=\Gamma / \Gamma^{l^{n}} \simeq \mathbb{Z} / e^{n} \mathbb{Z} ;$ d'où

$$
y_{n}=(n+1) d_{\varphi} l^{n}
$$

(ii) Pour $Y=\Lambda_{\varphi} / e^{m} \Lambda_{\varphi}$, et $n+1 \geq m$, nous avons : $Y / \nabla_{n} Y \simeq\left(Z_{\varphi} / l^{m} Z_{\varphi}\right)\left[\Gamma_{n}\right] ;$ d'où :

$$
y_{n}=m d_{\varphi} e^{n}
$$

(iii) Pour $Y=\Lambda_{\varphi} / f \Lambda_{\varphi}$, le lemme 10 nous montre que pour $n$ assez grand, disons $n \geq n_{0}$, nous avons $\nabla_{n} Y=e^{n-n_{0}} \nabla_{n_{0}} Y$; d'où :

$$
\left(Y: \nabla_{n} Y\right)=\left(Y: \nabla_{n_{0}} Y\right)\left(\nabla_{n_{0}} Y: l^{n-n_{0}} \nabla_{n_{0}} Y\right) ;
$$


et $\nabla_{n_{0}} Y$, qui est d'indice fini dans $Y$, est un $Z_{\varphi}$-module libre de dimension deg.f. Notons $l^{c_{0} d_{\varphi}}$, avec $c_{0} \in \mathbb{N}$, llordre du $Z_{\varphi}$-module fini $Y / \nabla_{n_{0}} Y$. Nous obtenons, comme attendu :

$y_{n}=y_{n_{0}}+d_{\varphi}\left(n-n_{0}\right) \operatorname{deg} f=d_{\varphi}\left[n \operatorname{deg} f+c^{\text {te }}\right], \quad$ avec $c^{\text {te }}=c_{0}-n \operatorname{deg} f$.

Dans le théorème 8 , le choix de la famille $\left(\nabla_{n}\right) n \in \mathbb{N}$ est suggéré par la théorie de Kummer. Néanmoins, d'autres choix sont possibles, comme le montre le résultat suivant :

THÉORÈME IV.1.17. - Soit $\times$ un $\Lambda[\Delta]$-module noethérien de paramètres $\rho, \mu$, et $\lambda$ dans $R_{Z_{\ell}}^{+}(\Delta)$. Pour chaque entier relatif $k$, et tout $n$ assez grand, désignons par $\nabla_{n, k}=e^{n+k} \Lambda+\omega_{n} \Lambda$ l'idéal de l'algèbre d'lwasawa $\Lambda=\mathbb{Z}_{\ell}[[\gamma-1]]$ engendré par l'élément $l^{n+k}$ et le polynôme $\omega_{n}=\gamma^{\ell^{n}}-1$. La suite des quotients $\left(X / \nabla_{n}, k^{X}\right)_{n}$ est alors une suite paramétrée de $\mathbb{Z}_{\ell}[\Delta]$-modules finis, de paramètres $\rho, \mu+(k-1) \rho, \lambda$, et $\psi_{k}$, pour un caractère $\ell$-adique virtuel convenable $\psi_{k}$ du groupe $\Delta$.

En particulier, les paramètres $\rho$ et $\lambda$ sont indépendants du choix de $k$; il en est de même du paramètre $\mu$, si $X$ est de torsion.

Démonstration : Ce résultat s'établit exactement comme le théorème 8 ; il suffit pour cela de reprendre les lemmes 11 à 14 , en remplaçant $\nabla_{n}$ par $\nabla_{n+k}$, i.e. $e^{n+1}$ par $e^{n+k}$. La différence provient de la proposition 16 (i). En effet, pour $Y=\Lambda_{\varphi}$, il vient :

$$
Y / \nabla_{n, k} Y \simeq\left(Z_{\varphi} / \ell^{n+k} Z_{\varphi}\right)\left[\Gamma_{n}\right] \text {, d'où : }\left(Y: \nabla_{n, K} Y\right)=e^{d} \varphi^{(n+k) l^{n}} \text {, }
$$

ce qui conduit à la formule :

$$
y_{n, k}=\langle\varphi, \varphi\rangle(n+1) e^{k}+\langle(k-1) \varphi, \varphi\rangle e^{k} \text {. }
$$

Lorsque le module $\times$ n'est pas de torsion, le choix de $k$ dans l'étude des quotients $\times / \nabla_{n, k} \times$ modifie donc le paramètre $\mu$.

Le lien entre ce dernier théorème et les résultats classiques de la théorie d'lwasawa est donné par les deux propositions suivantes. 
PROPOSITION IV.1.18. - Soit $X$ un $\Lambda[\Delta]$-module noethérien et de torsion, de paramètres $\mu$ et $\lambda$. Si les modules quotient $x_{n}=x / \omega_{n} \times$ sont finis, alors :

(i) II existe un caractère $l$-adique virtuel $\nu$, tel que la suite $\left(X_{n}\right)_{n \in N}$ soit paramétrée par les caractères $\rho=0, \mu, \lambda$, et $\nu$.

(ii) Pour chaque entier relatif $k$ fixé, les sous-modules $l^{n+k} x_{n}$ sont deux à deux isomorphes pour $n$ assez grand.

Démonstration : II suffit naturellement de faire la démonstration lorsque $X$ est isotypique.

(i) Supposons donc $X$ isotypique de caractère $\varphi$, et considérons les quotients finis $X / \omega_{n} \times$. Nous pouvons écrire:

$\left(X: \omega_{n} X\right)=\left(X: \nabla_{n} X\right)\left(\nabla_{n} X: w_{n} X\right)$. D'après le théorème 8 , le résultat annoncé sera donc établi si nous montrons que le groupe $\nabla_{n} \times / \omega_{n} \times$ est constant pour $n$ assez grand. Or nous avons $\nabla_{n} x / \omega_{n} x=\left(e^{n+1} x+\omega_{n} x\right) / \omega_{n} x=$ $e^{n+1} x /\left(e^{n+1} \times \cap \omega_{n} x\right)=e^{n+1} x / \partial^{n} x$; et, pour $n$ assez grand, la multiplication par $\ell^{n+1}$ tue le sous-module de $z_{\varphi}$-torsion. Nous ne restreignons donc pas la généralité en supposant $\times$ sans $Z_{\varphi}$-torsion, clest-à-dire (en vertu du théorème de structure rappelé en a) d'indice fini dans un $\Lambda_{\varphi}$-module élémentaire de polynôme caractéristique $f$ distingué dans $\mathrm{Z}_{\varphi}[\gamma-1]$. Cela étant, d'après le lemme 10 , nous obtenons

$$
\begin{aligned}
& \omega_{n} \times=e^{n-n_{0}} \omega_{n_{0}} \times \text {, pour } n \geq n_{0} \text {, d'où : } \\
& \quad \nabla_{n} \times / \omega_{n} \times \simeq e^{n+1} \times / l^{n-n_{0}} \partial^{n_{0}} \times \simeq e^{n_{0}+1} \times / \partial^{n} \times \simeq \nabla_{n_{0}} \times / \omega_{n_{0}} \times,
\end{aligned}
$$

comme annoncé.

(ii) Il vient de même : $e^{n+k} x_{n} \simeq e^{n+k} \times /\left(e^{n+k} \times \cap \omega_{n} x\right) \simeq$ $\simeq e^{n+k} \times / e^{n-n_{0}}\left(e^{n_{0}+k} \times n \omega_{n_{0}} x\right)$, d'où :

$$
e^{n+k} x_{n} \simeq e^{n_{0}+k} x /\left(e^{n_{0}+k} x \cap \omega_{n_{0}} x\right)=e^{n_{0}+k} x_{n_{0}},
$$

comme attendu.

PROPOSITION IV.1.19.- Soient $X$ un $\Lambda[\triangle]$-module noethérien et de torsion, de paramètres $\mu$ et $\lambda$, et $Y$ un sous-module diindice fini. Sup- 
posons choisi un entier naturel $m$, tel que les quotients $X_{n}=x / \frac{\omega_{n}}{\omega_{m}} Y$ soient finis, pour chaque $n \geq m$. Alors:

(i) II existe un caractère $\ell$-adique virtuel $\nu$ tel que la suite $\left(x_{n}\right)_{n \geq m}$ soit paramétrée par les caractères $\rho=0, \mu, \lambda$, et $\nu$.

(ii) Pour chaque entier relatif $k$ fixé, les sous-modules $\ell^{n+k} x_{n}$ sont deux à deux isomorphes pour tous les $\mathrm{n}$ assez grands.

Remarque : Ce dernier résultat peut être regardé comme une généralisation de la proposition $18 \mathrm{ci}$-dessus, qui correspond au cas $Y=\omega_{m} X .11$ a l'avantage de pouvoir s'appliquer, pour un choix arbitraire de $Y$, lors même que les quotients $X / \omega_{n} \times$ ne sont pas tous finis, ce qui a lieu lorsque certains des polynômes caractéristiques $f_{\varphi}$ des $\varphi$-composantes de $X$ admettent des facteurs cyclotomiques ( $i . e$. des diviseurs de la forme $\frac{\omega_{n}+1}{\omega_{n}}$, pour un $n$ dans $N$ ). En effet, pour $m$ assez grand et $n \geq m$, les polynômes $\frac{\omega_{n}}{\omega_{m}}$ sont étrangers à tous les $f_{\varphi}$, et les modules quotients $Y / \frac{\omega_{n}}{w_{m}} Y$ sont donc finis.

Démonstration : Ici encore, nous pouvons supposer $X$ isotypique dans la démonstration.

(i) L'égalité $\left(X: \frac{\omega_{n}}{\omega_{m}} Y\right)=(X: Y)\left(Y: \frac{\omega_{n}}{\omega_{m}} Y\right)$ permet de ne considérer que le cas $X=Y$. Cela étant, notons $K_{m}$ le noyau de $\omega_{n}$ dans $Y$, et $Z$ le quotient $Y / K_{m}$. La suite $\left(K_{n}\right)_{n \in N}$, suite croissante de sousmodules diun $\Lambda[\Delta]$-module noethérien, est stationnaire; nous avons donc $K_{n+1}=K_{n}$ pour $n$ assez grand, disons $n \geq n_{0}$. Dlaprès la suite exacte courte canonique

$$
0 \rightarrow K_{m} / \frac{\omega_{n}}{\omega_{m}} K_{n} \rightarrow Y / \frac{\omega_{n}}{\omega_{m}} Y \rightarrow \omega_{m} Y / \omega_{n} Y \rightarrow 0
$$

l'indice $\left(Y: \frac{w_{n}}{w_{m}} Y\right)$ se scinde en deux facteurs:

- le premier, $\left(K_{m}: \frac{w_{n}}{w_{m}} K_{n}\right)=\left(K_{m}: \frac{w_{n}}{w_{m}} K_{n}\right)=$ $=\left(K_{m}: \frac{\omega_{n}}{\omega_{m}} K_{n_{0}}\right)\left(\frac{\omega_{n}}{\omega_{m}} K_{n_{0}}: \frac{\omega_{n}}{\omega_{m}} K_{n_{0}}\right)$ s'écrit encore $\left(K_{m}: \frac{\omega_{n}}{\omega_{m}} K_{n}\right)=$ $=\left(K_{m}: \frac{\omega_{n}}{\omega_{m}} K_{n_{0}}\right)\left(\frac{\omega_{n}}{\omega_{m}} K_{n_{0}}: e^{n-n_{0}} \frac{\omega_{n_{0}}}{\omega_{m}} K_{n_{0}}\right)$, d'après le lemme 10 appliqué 
au module $K_{m}$ annulé par le polynôme distingué $\omega_{m}$. Dans la formule obtenue, le module $\frac{\omega_{n}}{\omega_{m}} K_{n_{0}}$, qui est dindice fini dans $K_{m}$, est somme directe d'un module fini et d'un $Z_{\varphi}$-module libre de dimension $\operatorname{deg} f_{m}$, si $f=f_{m} \cdot f_{m}^{\prime}$ est la décomposition du polynôme caractéristique de $Y$ comme produit d'un polynôme distingué $f_{\mathrm{m}}$ divisant une puissance de $\omega_{\mathrm{m}}$ et dlun polynôme $f_{\mathrm{m}}^{\prime}$ étranger à $\omega_{\mathrm{m}}$ •

- le second facteur $\left(\omega_{m} Y: \omega_{n} Y\right)=\left(\omega_{m} Z: \omega_{n} Z\right)$ s'écrit encore $\frac{\left(Z: w_{n} Z\right)}{\left(Z: w_{m} Z\right)}$. Comme il est fini, le polynôme caractéristique $f_{m}^{\prime} d u \Lambda_{\varphi}$-module $Z$ est étranger à tous les $\omega_{n}$; et l'indice $\left(Z: \omega_{n} Z\right)$ est donc donné par la proposition 18 .

Récapitulant le tout, nous obtenons le résultat attendu.

(ii) Tout comme pour la proposition 18, nous obtenons pour $n$ assez grand, disons $n \geq n_{0}: e^{n+k} X_{n} \simeq e^{n+k} \times /\left(e^{n+k} \times \cap \frac{\omega_{n}}{\omega_{m}} Y\right)=$ $=e^{n+k} \times / e^{n-n_{0}}\left(e^{n_{0}+k} \times \cap \frac{\omega_{n}}{\omega_{m}} y\right) \simeq e^{n_{0}+k} \times /\left(e^{n_{0}+k} \times \cap \frac{\omega_{n}}{\omega_{m}} y\right)=e^{n_{0}+k} \times x_{n}$, ce qui achève la démonstration.

\section{3. - STRUCTURE DES $\Lambda[\triangle]$-MODULES DANS LE CAS MÉTABÉLIEN .}

Nous supposons ici que $X$ n'est pas le caractère unité. Nous notons $H$ son noyau dans $\Delta$, et $m=(\Delta: H)$ son ordre, qui divise $d$ et $(l-1)$. Dans ce cas, l'algèbre $\Sigma=\Lambda[\Delta]$ est somme directe dlalgèbres gauches $\Sigma_{\Phi}=\Lambda_{\Phi} \otimes_{\mathbb{Z}_{\ell}[H]} \mathbb{Z}_{\ell}[\Delta]$, de dimension $\mathrm{m}^{2}$ sur leurs centres respectifs $C_{\Phi}=Z_{\Phi}\left[\left[\theta^{m}\right]\right]$, et qui contiennent les $\Lambda_{\Phi}$ comme sous-algèbres commutatives maximales. En particulier, si $F_{\Phi}=Q_{\Phi}\left(\left(\theta^{n}\right)\right)$ désigne le corps des fractions de l'algèbre $C_{\Phi}$, le tensorisé $F_{\Phi} \otimes_{C_{\Phi}} \Sigma_{\Phi}$ est une algèbre simple centrale sur $F_{\Phi}$, qui contient $Q_{\Phi}((\theta))$ comme sous-corps commutatif maximal. 


\section{a.-Description des $\Sigma$-modules projectifs de type fini .}

Dans l'étude préliminaire $(\S 1, c)$ nous avons distingué deux sortes didempotents dans l'algèbre $\Sigma$ :

- les idempotents primitifs $e_{\varphi}=\frac{1}{d} \sum_{\tau \in \Delta} \varphi\left(\tau^{-1}\right)_{T}$, qui sont associés aux caractères $\ell$-adiques irréductibles $\varphi$ du groupe $\Delta$.

- les idempotents centraux primitifs $\mathrm{e}_{\Phi}=\sum_{\varphi \mid \Phi} \mathrm{e}_{\varphi}$, qui sont associés aux induits à $\Delta$ des caractères $\ell$-adiques irréductibles du sousgroupe $H$.

Les premiers gouvernent la décomposition de $\Sigma$ comme module sur ellemême, les seconds sa décomposition d'algèbre.

PROPOSITION IV.1.20.- L'algèbre $\Sigma=\Lambda[\Delta]$ est somme directe d'un nombre fini d'algèbres gauches $\Sigma_{\Phi}$ de dimension $\mathrm{m}^{2}$ sur leurs centres respectifs $C_{\Phi}$; chacune des algèbres commutatives $C_{\Phi}$ est un anneau local complet régulier de dimension 2 . En particulier, tout $\Sigma$-module projectif de type fini est somme directe essentiellement unique de $\Sigma$ modules projectifs indécomposables de type fini.

(i) La décomposition de l'algèbre $\Sigma$, comme module sur ellemême, est donnée par :

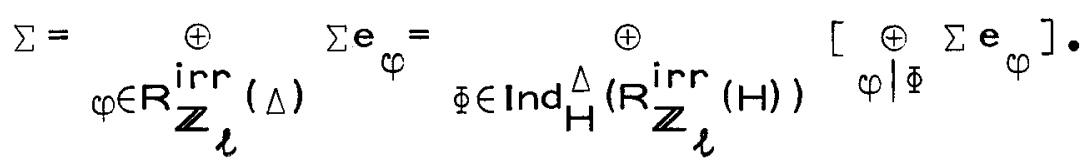

(ii) Pour chaque caractère $\Phi \in \operatorname{Ind}_{H}^{\Delta}\left(R_{\mathbb{Z}}^{i r r}(H)\right)$, il existe $m_{\Phi}$ classes d'isomorphie de $\Sigma_{\Phi}$-modules noethériens projectifs et indécomposables, si $m_{\Phi}$ désigne le nombre de caractères l-adiques irréductibles $\varphi$ intervenant dans la décomposition de $\Phi$; elles sont représentées par les $m_{\Phi}$-modules $\Sigma_{\varphi}$.

Démonstration : Le fait que tout $\Sigma$-module noethérien $\times$ s'écrive de façon essentiellement unique comme somme directe d'indécomposables résulte directement du théorème de Krull-Schmidt ${ }^{(\star)}$ appliqué à chacune des com-

( ) Pour plus de détails sur le théorème de Krull-Schmidt, on pourra consulter $[\mathrm{Re}]$, p. 88 . 
posantes locales $X_{\Phi}$ : l'algèbre $\Sigma_{\Phi}$ est un module de type fini sur son centre $C_{\Phi}$; et l'anneau $C_{\Phi}$ est local, complet et noethérien. Bien entendu, si $X$ est projectif, il en est de même de ses facteurs directs.

(i) La décomposition directe de $\Sigma$ est immédiate; il suffit donc de vérifier que les modules $\Sigma$ e sont indécomposables. Et cela résulte du fait que, pour chaque $\Sigma_{\Phi}$-module projectif noethérien et indécomposable $I$, le tensorisé $F_{\Phi}{ }^{\otimes} C_{\Phi} l$ est somme directe d'exemplaires de $Q_{\Phi}((\theta))$.

(ii) Dlaprès ce qui précède, il reste à montrer que deux modules $\Sigma \mathbf{e}_{\varphi}$ et $\Sigma \mathbf{e}_{\psi}$, associés à des caractères distincts, ne sont pas $\Sigma$-isomorphes. Ce problème ne se pose, dlailleurs, que lorsque $\varphi$ et $\psi$ sont deux facteurs irréductibles d'un même caractère $\Phi \in \operatorname{Ind}_{H}^{\Delta}\left(R_{\mathbb{Z}}^{i r r}(H)\right)$. Cela étant, supposons quili existe un tel isomorphisme $f$, et notons $\lambda e_{\psi}$, avec $\lambda=\sum_{i \in \mathbb{N}} \lambda_{i} \theta^{i} \in Z_{\Phi}[[\theta]]$, l'image de $e_{\varphi}$. D'un côté, nous aurions $\lambda \in Z_{\Phi}[[\theta]]^{*}$, i.e. $\lambda_{o} \in Z_{\Phi}^{*}$, puisque $f$ est un isomorphisme; et d'un autre $e_{\psi} \lambda e_{\psi}=$ $\mathrm{e}_{\psi} f\left(\mathrm{e}_{\varphi}\right)=f\left(\mathrm{e}_{\psi} \mathrm{e}_{\varphi}\right)=0$, d'où $\lambda_{0}=0$ : une contradiction.

SCOLIE IV.1.21.- Les $\Sigma$-modules noethériens qui sont $\Sigma$-projectifs sont exactement ceux qui sont $C$-projectifs (i.e. projectifs sur le centre $C=\mathbb{Z}_{\ell}\left[\left[\theta^{m}\right]\right][H]$ de l'algèbre $\Sigma$ ).

Démonstration : Le résultat sera acquis si nous montrons que tout $\Sigma-$ module $X$, noethérien et $C$-projectif, est un facteur direct de son tensorisé $X_{\Sigma}=\Sigma \otimes_{C} \times$. Or cela est clair, puisque la suite exacte canonique

$$
0 \rightarrow \operatorname{Ker} p \rightarrow x_{\Sigma} \underset{\Sigma}{\stackrel{p}{q}} \times \rightarrow 0
$$

définie par $p(\sigma \otimes x)=\sigma x$, est scindée par llapplication :

$$
q \mid \times \longmapsto \frac{1}{d} \sum_{\tau \in \Delta} \tau^{-1} \otimes(\tau x)
$$

PROPOSITION IV.1.22. - Tout idéal projectif de l'algèbre $\Sigma$ s'écrit comme somme directe indécomposable d'idéaux principaux

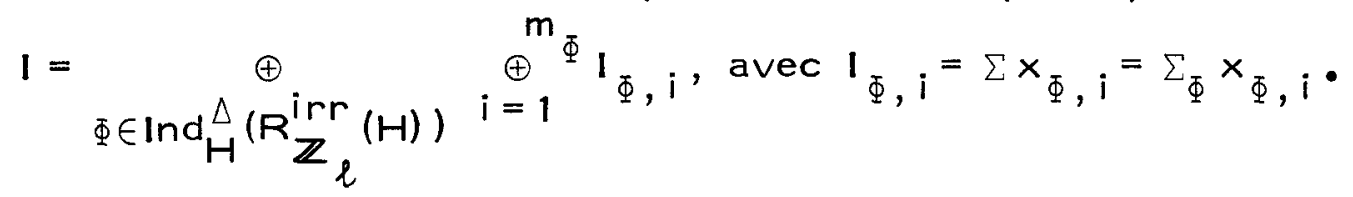


Dans cette décomposition, chaque idéal $I_{\Phi, i}$ est soit nul, auquel cas l'élément $x_{\Phi, i}$ est nul, soit isomorphe à l'un des $m_{\Phi}$ modules $\Sigma_{\varphi}$ pour $\varphi$ irréductible contenu dans $\Phi$, auquel cas l'élément $x_{\Phi, i}$ est invariant par multiplication à gauche par $e_{\varphi}$; nous disons alors que $x_{\Phi, i}$ est isotypique de caractère $\varphi$.

Démonstration : D'après la proposition 20 , tout idéal projectif 1 de l'algèbre $\Sigma$ s'écrit comme somme directe d'idéaux indécomposables principaux $1_{\varphi}=\Sigma x_{\varphi}=\Sigma_{\Phi} x_{\varphi} \simeq \Sigma_{\Phi} \mathbf{e}_{\varphi}=\Sigma \mathbf{e}_{\varphi}$. Cela étant, les relations d'or thogonalité des idempotents donnent immédiatement $e_{\psi} x_{\varphi}=0$, pour $\psi \neq \varphi$, i.e. $x_{\varphi}=e_{\varphi} x_{\varphi}$, comme annoncé. Enfin, pour chaque caractère $\Phi \in \operatorname{Ind}_{H}^{\Delta}\left(R_{\mathbb{Z}_{\ell}}(H)\right)$, le nombre de facteurs $I_{\Phi, i}$ intervenant effectivement dans la décomposition est majoré par l'entier $m_{\Phi}$, puisque chacun d'eux est un $\Lambda_{\Phi}$-module libre de dimension $\frac{m}{m_{\Phi}}(\star)$.

THÉORÈME IV.1.23.- Tout $\Sigma_{\Phi}$-module noethérien sans $\Lambda_{\Phi}$-torsion est contenu comme sous-module dindice fini dans un $\Sigma_{\Phi}$-module projectif. Autrement dit, tout $\Sigma$-module noethérien, dont les $\Phi$-composantes sont sans $\Lambda_{\Phi}{ }^{-}$ torsion, est pseudo-isomorphe à un $\Sigma$-module noethérien et projectif. Nous disons quiun tel module est quasi-projectif.

Démonstration : D'après le théorème de structure établi dans le cas abélien (th. 6), pour tout $\Lambda_{\Phi}$-module noethérien et sans torsion $X$, il existe un $\Lambda_{\Phi}$-module libre $L$ qui contient $\times$ comme sous-module dindice fini. Tout le problème consiste donc à montrer que si $X$ est un $\Sigma_{\Phi}$-module, il en est de même de $L$. Désignons par $C_{\Phi}=Z_{\Phi}\left[\left[\theta^{m}\right]\right]$ le centre de l'algèbre $\Sigma_{\Phi}$, et par $F_{\Phi}=Q_{\Phi}\left(\left(\theta^{m}\right)\right)$ son corps des fractions. Puisque $X$ est d'indice fini dans $L$, le produit tensoriel $F_{\Phi} \otimes_{C_{\Phi}} L$, qui contient $L$ de façon canonique, s'identifie au produit $F_{\Phi} \otimes_{C_{\Phi}} \times$; c'est donc un $\Sigma_{\Phi}$-module. Reste à vérifier que $L$ est stable pour l'action de $\Sigma_{\Phi}$. Pour chaque élément $\tau$ de $\Delta$, le conjugué $L^{\top}$ de $L$ contient $X^{\top}=X$ comme sous-module d'indice fini ; le

( $)$ On prendra garde au fait qu'un module indécomposable donné $\Sigma_{\varphi}$ peut ainsi être représenté plusieurs fois dans la décomposition d'un idéal 1 , ce qui ne peut arriver lorsque $\mathrm{l}$ est égal à $\Sigma$. 
sous-module $L^{\prime}=\sum_{T \in \Delta} L^{T}$ de $F_{\Phi} \otimes_{C} L$ engendré par $L$ est donc une extension de $L$ qui est d'indice fini ( $\left.L^{\prime}: L\right)$. A son tour $L^{\prime}$ est contenu comme sous-module d'indice fini dans un $\Lambda \widetilde{\Phi}^{- \text {module libre }} L^{\prime \prime}$, inclus dans $F_{\Phi} \otimes_{C} L$, et isomorphe à $L$. Cela étant, si $h$ est l'endomorphisme de $L$ " qui envoie $L$ " sur $L$, le déterminant det $h$ est nécessairement inversible puisque l'indice ( $L ": L$ ) est fini. Autrement dit, $h$ est inversible, et $L$ est égal à L", donc à L': Le module L est bien stable pour l'action de $\Sigma_{\Phi}$; comme il est $\Lambda_{\Phi}$-libre, il est $\Sigma_{\Phi}$-projectif en vertu du scolie 21 ; enfin, il contient $\times$ comme sous-module d'indice fini.

COROLLAIRE IV.1.24.- Tout $\Sigma$-module noethérien est pseudo-isomorphe à la somme directe d'un $\Sigma$-module noethérien et de torsion (i.e. d'un $\Sigma$ module noethérien dont les $\Phi$-composantes sont de $\Lambda_{\Phi}$-torsion) et d'un $\Sigma-$ module noethérien quasi-projectif.

Démonstration : II suffit naturellement de faire la démonstration lorsque $X$ est un $\Sigma_{\Phi}$-module noethérien. Dans ce cas, le sous-module de $C_{\Phi}$-torsion $T$ de $X$, qui est encore son sous-module de $\Lambda_{\Phi}$-torsion, est annulé par une puissance finie $P^{e}$ de son polynôme caractéristique $P \in Z_{\Phi}\left[\theta^{m}\right]$. Comme $P^{e}$ est central dans $\Sigma_{\Phi}$, son noyau $T=\operatorname{Ker} P^{e}$ dans $X$ est donc un $\Sigma_{\Phi}-$ module. En particulier dans la suite exacte courte canonique

$$
0 \longrightarrow T \longrightarrow X \longrightarrow Y=X / T \longrightarrow 0 \text {, }
$$

le terme de gauche $T$ est un $\Sigma_{\Phi}$-module noethérien et de torsion, et celui de droite $Y=X / T$ est un $\Sigma_{\Phi}$-module noethérien quasi-projectif. Cela étant, d'après le théorème $23 \mathrm{ci-dessus,} Y$ est contenu comme sous-module d'indice fini dans un $\Sigma_{\Phi}$-module projectif $L=\underset{i=1}{\oplus^{p}} \Sigma_{\Phi} y_{i}$. Faisons choix d'un polynôme distingué $f \in Z_{\Phi}\left[\theta^{m}\right]$, étranger au polynôme caractéristique $P$, annulant $L / Y$. Pour chaque naturel $n$, la multiplication par $f^{n}$ est alors un $\Sigma_{\Phi}$-endomorphisme de $X$, dont le noyau $N_{n}$ est un sous-module fini de $T$. Comme $X$ est noethérien, la suite $\left(N_{n}\right)_{n \in N}$ est stationnaire, et nous avons donc $N_{n}=N=\underset{k \in N}{U} N_{k}$ pour tout $n$ assez grand. Quittes à quotienter $X$ par le sous-module fini $N$, ce qui revient à faire un pseudo-isomorphisme, nous pouvons donc supposer désormais que la multiplication par $f$ est un monomorphisme de $X$. Dans ce cas, $X$ peut être regardé comme un 
sous-module de son tensorisé $f^{-1} X=f^{-1} C_{\Phi} \otimes_{C_{\Phi}} \times$; et son sous-module de torsion $T$ est alors contenu avec un indice fini dans celui $f^{-1} T$ de $f^{-1} \times$. Cela posé, pour chaque $i=1, \ldots, p$, nous avons $f y_{i} \in Y$, puisque $f$ annule le quotient $L / Y$. Faisons choix d'un $x_{i}$ isotypique de $X$ au-dessus de $f y_{i}$ : Nous définissons ainsi, par linéarité, un $\Sigma_{\Phi}$-morphisme de $f L$ dans $X$. Ce morphisme se prolonge de façon unique en un $\Sigma_{\Phi^{-}}$ morphisme $s$ de $Y$ dans $f^{-1} X$ (Si y appartient à $Y$, nous avons $f y \in f L$, et il suffit de poser $\left.s(y)=f^{-1} s(f y)\right)$. Cela étant, l'application $x \mapsto(x-s(x), \bar{x})$ est un $\Sigma_{\Phi}$-pseudo-isomorphisme de $x$ dans $f^{-1} T \oplus Y \sim T \oplus(X / T)$; ce qui achève la démonstration.

\section{b.- Classification des $\sum$-modules noethériens.}

Intéressons-nous maintenant aux $\Sigma_{\Phi}$-modules noethériens sans condition de projectivité. Un tel module $X$ est en particulier un $\Lambda_{\Phi}$-module de type fini, pseudo-isomorphe comme tel à une somme directe finie de quotients de $\Lambda_{\Phi}$ par des puissances didéaux premiers principaux. Pour rendre compte de la $\Sigma_{\Phi}$-structure du module $X$, il est naturel de remplacer les idéaux premiers principaux de l'algèbre $\Lambda_{\Phi}$ par des sousmodules projectifs convenables de l'algèbre $\Sigma_{\Phi}$.

LEMME IV.1.25.- Pour chaque caractère l-adique irréductible $\varphi$ du groupe $\Delta$, les sous-modules projectifs maximaux de llidéal $\Sigma_{\varphi}=\Sigma e_{\varphi}$ de l'algèbre $\Sigma$ sont de trois sortes:

(i) L'idéal $\ell \Sigma_{\varphi}$, isomorphe à $\Sigma_{\varphi}$, engendré par l'uniformisante $\ell$ de l'anneau $\mathrm{Z}_{\varphi}$. vante $\theta$.

(ii) L'idéal $\theta \Sigma_{\varphi}$, isomorphe à $\Sigma_{\varphi \chi}$, engendré par la résol-

(iii) Les idéaux $\Sigma e_{\varphi} f^{e} \varphi$, isomorphes à $\Sigma_{\varphi}$, associés aux polynômes distingués irréductibles de valuation nulle de l'algèbre gau-

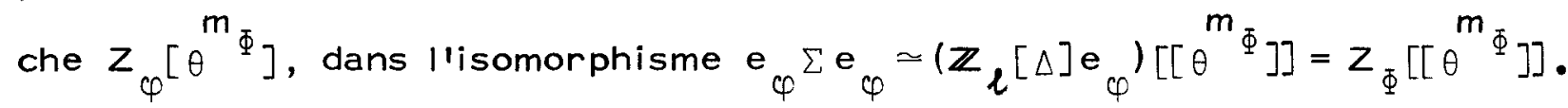

Démonstration : Considérons un sous-module $I_{\varphi}$ d'un idéal indécomposable $\Sigma_{\varphi}=\Sigma e_{\varphi}$ de l'algèbre $\Sigma_{\Phi}$. Si $I_{\varphi}$ est projectif, le théorème de structure 
établi plus haut (proposition 22) nous permet d'écrire $I_{\varphi}=\Sigma_{\Phi} e_{\psi} \times$, pour un caractère $\ell$-adique irréductible $\psi$ (représenté dans $\Phi$ ) et un $x$ dans $\Sigma$; et l'inclusion $\mathrm{I}_{\varphi} \subset \Sigma_{\varphi}$ nous donne alors immédiatement $\mathrm{I}_{\varphi}=\Sigma_{\Phi} \mathrm{e}_{\psi} \times \mathrm{e}_{\varphi}$. Ecrivons $x$ comme série formelle $x=\sum_{i \in N} x_{i} \theta^{i}$ à coefficients dans l'algèbre $\mathbb{Z}_{\ell}[\Delta]$. Les identités de commutation $e_{\psi} \theta^{i}=\theta^{i} e_{\psi x^{-i}}$ et les relations d'orthogonalité entre idempotents nous montrent que seuls interviennent dans les produits $e_{\psi} \times e_{\varphi}$ les termes $x_{i} \theta^{i}$ pour lesquels le caractère $\psi x^{-i}$ est égal à $\varphi$. Si donc $\psi$ s'écrit $\varphi x^{-\alpha}$, avec $\alpha \in\left\{0,1, \ldots,\left(m_{\Phi}-1\right)\right\}$, nous pouvons factoriser $\theta^{\alpha}$, et écrire $e_{\psi} \times e_{\varphi}=\theta^{\alpha} e_{\varphi}$ ye $e_{\varphi}$, avec $y \in\left(\mathbb{Z}_{\ell}[\Delta] e_{\varphi}\right)\left[\left[\theta^{m}\right]\right] \simeq z_{\varphi}\left[\left[\theta^{m}\right]\right]$. Utilisant alors le théorème de préparation de Weierstrass dans un cadre non commutatif, nous concluons que l'idéal $I_{\varphi}$ est engendré par un élément de la forme $\theta^{\alpha} l^{\mu} e_{\varphi} f$ e $\varphi$, où le produit $\mathrm{e}_{\varphi} f \mathrm{e}_{\varphi}$ correspond, dans liisomorphisme $\mathrm{e}_{\varphi} \mathrm{e}_{\varphi} \approx \mathrm{z}_{\varphi}\left[\left[\theta^{\mathrm{m}} \Phi\right]\right.$, à un polynôme distingué de valuation nulle de l'anneau $z_{\varphi}\left[\theta^{m}\right]$. Bien entendu, $I_{\varphi}$ est maximal, parmi les sous-modules projectifs de $\Sigma_{\varphi}$, si et seulement si l'élément obtenu $\theta^{\alpha} \ell^{\mu} e_{\varphi} f^{\mathrm{e}}{ }_{\varphi}$ est irréductible, ce qui conduit à la classification énoncée.

LEMME IV.1.26. - Tout idéal I de l'algèbre $\Sigma$, contenu dans l'idéal $\Sigma_{\varphi}$, est un sous-module d'indice fini d'un idéal projectif $P_{\varphi}=\Sigma l^{\mu} \theta^{\alpha} e_{\varphi} f e_{\varphi}$ de l'algèbre $\Sigma$.

Démonstration : L'idéal $I_{\varphi}$ est engendré par un nombre fini de générateurs $x_{1}, \ldots, x_{k}$, que le théorème de préparation de Weierstrass permet de choisir sous la forme $x_{i}=e^{\mu_{i}} \theta^{\alpha_{i}} f_{i} e_{\varphi}$, avec $f_{i}$ distingué de valuation nulle dans l'algèbre gauche $z_{\varphi}[\theta]$. Posons $\mu=\inf _{i=1}^{k} \mu_{i}, \alpha=\inf _{i=1}^{k} \alpha_{i}$; et prenons pour $f^{\prime}$ le plus grand diviseur commun des $f_{i}$, regardés comme polynômes formels en l'indéterminée $\theta$. Liidéal $I_{\varphi}^{\prime}=\Sigma \ell^{\mu} \theta^{\alpha} f^{\prime} e_{\varphi}$ contient $I_{\varphi}$ comme sous-module d'indice fini. S'il n'est pas projectif (i.e. si $f^{\prime}$ n'est pas dans $\left.z_{\varphi}\left[\theta^{m}\right]\right)$, posons $e_{\psi} l^{\mu} \theta^{\alpha} f^{\prime} e_{\varphi}=l^{\mu} \theta^{\alpha} f_{\psi}^{\prime} e_{\varphi}$, pour chaque caractère $\ell$-adique irréductible $\psi$ associé à $\varphi$ li.e. de la forme $\varphi x^{-k}$, pour $\left.k=0,1, \ldots,\left(m_{\Phi}-1\right)\right)$; et remplaçons $f^{\prime}$ par le plus grand diviseur commun $f^{\prime \prime}$ des $f_{\psi}^{\prime}$ : Nous obtenons un nouvel idéal $1_{\varphi}^{\prime \prime}=\Sigma \ell^{\mu} \theta^{\alpha} f^{\prime \prime} e{ }_{\varphi}$, qui 
contient $I_{\varphi}^{\prime}$ comme sous-module d'indice fini. Itérant le procédé, nous fabriquons ainsi une suite croissante d'idéaux $I_{\varphi}^{\prime} \subset I_{\varphi}^{\prime \prime} \subset I_{\varphi}^{\prime \prime \prime} \subset \ldots$ Cette suite est stationnaire, ce qui veut dire qu'au bout d'un nombre fini de transformations nous tombons sur un polynôme distingué $f$ qui est dans $z_{\varphi}\left[\theta^{m}\right]$. L'idéal $P_{\varphi}=\Sigma l^{\mu} \theta^{\alpha} e_{\varphi} f e_{\varphi}$ est l'idéal cherché.

DÉFINITION IV.1.27. - Pour chaque caractère $\ell$-adique $\Phi \in \operatorname{Ind}_{H}^{\Delta}\left(R_{\mathbb{Z}}^{i r r}(H)\right)$, nous appelons $\Sigma_{\Phi}$-module élémentaire toute somme directe finie d'exemplaires des modules suivants :

(i) Les $\Sigma_{\Phi}$-modules projectifs $\Sigma_{\Phi}$-modules projectifs $\Sigma_{\varphi}=\Sigma_{\Phi}$ e $\varphi^{\prime}$ associés aux $m_{\Phi}$-caractères $l$-adiques irréductibles $\varphi$ représentés dans $\Phi$.

(ii) Les quotients $\Sigma_{\varphi} / \theta^{\alpha} \Sigma_{\varphi}$ des modules précédents par leurs sous-modules respectifs engendrés par les puissances de la résolvante $\theta$.

(iii) Les quotients $\Sigma_{\varphi} / \ell^{\mu} \Sigma_{\varphi}$, correspondant aux puissances $\ell^{\mu}$ de l'uniformisante $l$ de l'anneau des scalaires $z_{\varphi}=\mathbb{Z}_{\ell}[\Delta] e_{\varphi}$.

(iv) Les quotients $\Sigma_{\varphi} / \Sigma e_{\varphi} f e_{\varphi}$, associés aux polynômes distingués de valuation nulle de l'algèbre gauche $z_{\varphi}\left[\theta^{\mathrm{m}}{ }^{\Phi}\right] \approx \mathbf{e}_{\varphi} \sum \mathbf{e}_{\varphi}$.

Cela posé, l'objet de cette section est d'établir le résultat fondamental suivant :

THEORËME IV.1.28.- Tout $\Sigma$-module noethérien est pseudo-isomorphe à un $\Sigma$-module élémentaire. De plus, ce dernier module est unique aux deux réserves suivantes près :

(i) Deux modules élémentaires $\Sigma_{\varphi} / \ell^{\mu} \Sigma_{\varphi}$ et $\Sigma_{\psi} / \ell^{\mu} \Sigma_{\psi}$, associés à deux caractères distincts $\varphi$ et $\psi$ représentés dans un même caractère $\Phi \in \operatorname{Ind}_{H}^{\Delta}\left(R_{\mathbb{Z}_{l}}^{\mathrm{irr}}(H)\right)$, sont pseudo-isomorphes.

(ii) Le même résultat vaut pour deux modules élémentaires $\Sigma_{\varphi} / \Sigma \mathbf{e}_{\varphi} f e_{\varphi}$ et $\Sigma_{\psi} / \Sigma e_{\psi} f e_{\psi}$, associés à deux caractères distincts $\varphi$ et $\psi$, représentés dans un même caractère $\Phi \in \operatorname{Ind}_{H}^{\Delta}\left(R_{\mathbb{Z}_{l}}^{i r r}(H)\right)$.

Démonstration de l'existence : D'après le corollaire 24 , nous ne restreignons pas la généralité en supposant que $X$ est un $\Sigma_{\Phi}$-module noethérien et de torsion. Cela étant, pour chaque idéal premier principal $p_{\Phi} d u$ centre $C_{\Phi}$ de l'algèbre $\Sigma_{\Phi}$, désignons par $X_{p_{\Phi}}$ le sous-module spectral de $X$ asso- 
cié à $\mathfrak{p}_{\Phi}$, c'est-à-dire l'ensemble des éléments $\times$ de $X$ qui sont annulés par une puissance de $p_{\Phi}$. Nous avons les résultats suivants :

(i) L'intersection $X_{\infty}$ des $X_{p_{\Phi}}$, lorsque $p_{\Phi}$ parcourt l'ensemble des idéaux premiers principaux de l'algèbre $C_{\Phi}$, est le plus grand $\mathrm{C}_{\Phi}$-sous-module fini de $X$; clest évidemment un $\Sigma_{\Phi}$-module.

(ii) La somme (non directe) des $X_{p_{\Phi}}$ est égale à tout $\times$, puisque celui-ci est de torsion.

(iii) Le groupe $X_{p_{\Phi}}$ se réduit à $X_{\infty}$ pour presque tout $\mathfrak{p}_{\Phi}$, en fait dès que $p_{\Phi}$ ne contient pas le polynôme caractéristique $P$ de $X$ (pour sa structure de $Z_{\Phi}\left[\left[\theta^{m}\right]\right]$-module).

En particulier, comme la surjection canonique $\underset{p_{\Phi} \ni P}{\oplus} \times p_{\Phi} \rightarrow X$ est clairement un $\Sigma_{\Phi}$-pseudo-isomorphisme, il nous suffit de faire la démonstration dans le cas où $X$ est annulé par une puissance finie $p_{\Phi}^{e}$ d'un idéal premier principal de l'algèbre $C_{\Phi}=Z_{\Phi}\left[\left[\theta^{m}\right]\right]$. Deux cas se présentent alors :

1. - L'idéal $p_{\Phi}$ est engendré par un polynôme distingué irréductible de valuation nulle $P_{\Phi}$ de l'anneau $Z_{\Phi}\left[\theta^{m}\right]$. Dans ce cas, la pseudodécomposition de $\times$ comme $\Lambda_{\Phi}$-module est de la forme :

$$
X \sim \underset{i=1}{\oplus^{n}} \Lambda_{\Phi} / P_{\Phi}^{e_{i}} \Lambda_{\Phi} \text {, avec } e=e_{1} \geq e_{2} \geq \ldots \geq e_{n}>0
$$

En effet, si $x \in X$ est un élément d'ordre maximal (pour la structure de $\Lambda_{\Phi}$-module de $\left.X\right)$, et $f \in Z_{\Phi}[\theta]$ le polynôme distingué qui engendre son annulateur, les conjugués $x^{\top}$ de $x$ (pour $\tau \in \Delta$ ) sont encore d'ordre maximal, et leurs annulateurs respectifs sont engendrés par les polynômes $\tau f \tau^{-1}=f^{\tau}$, donnés par $f^{\top}(\theta)=f(x(\sigma) \theta)$. Le polynôme $f$, qui est invariant par conjugaison, est donc dans le sous-anneau $Z_{\Phi}\left[\theta^{m}\right]$; c'est $P^{e}=P^{e} 1$. Plus généralement, si nous écrivons $\times \sim \underset{i=1}{\oplus}{ }^{n} \Lambda_{\Phi} / f_{\Phi, i} \Lambda_{\Phi}$ (avec $f_{\Phi, i} \in f_{\Phi, i-1} z_{\Phi}[\theta]$, pour $i=2, \ldots, n$ ) la pseudo-décomposition de $X$ comme $\Lambda_{\Phi}$-module, l'égalité $X=X^{\top}$ et l'unicité de cette décomposition nous prouvent que les polynômes $f_{\Phi, i}$ sont invariants par conjugaison, donc de la forme $f_{\Phi, i}=\mathrm{P}_{\Phi}^{e_{i}}$, pour un entier $e_{i}$, comme annoncé. Ce point 
acquis, faisons choix d'un sous-module $\Lambda_{\Phi}$-élémentaire $Y=\underset{i=1}{\oplus^{n}} \Lambda_{\Phi} X_{i} \simeq$ $\oplus_{i=1}^{n} \Lambda_{\Phi} / P{ }_{\Phi}^{e_{i}} \Lambda_{\Phi}$ de $X$. Quittes à remplacer $x_{1}$ par un de ses multiples $g x_{1}$ (pour un $g$ de $Z_{\Phi}\left[\theta^{m}\right]$ étranger à $P_{\Phi}$ ), nous pouvons supposer que $Y$ contient tous les conjugués $x^{\top}$ de $x$. L'un au moins des éléments isotypiques $e_{\varphi} x_{1}$ (pour $\varphi$ irréductible divisant $\Phi$ ) étant d'ordre maximal $P^{e}$ (sans quoi $x_{1}=\sum_{\varphi \mid \Phi} e_{\varphi} x_{1}$ serait annulé par $P^{e-1}$ ), choisissons-en un $y_{1}$ et considérons le $\Sigma$-module $\Sigma y_{1}=\Sigma_{\varphi} y_{1}$, qui est contenu dans $Y$. Le noyau $I_{\varphi}$ de la surjection canonique de $\Sigma_{\varphi}$ sur $\Sigma_{\varphi} y_{1}$ est un idéal projectif de $\Sigma_{\varphi}$. Dans le cas contraire, en effet, il serait contenu comme sous-module strict d'indice fini dans un tel idéal, et le quotient $\Sigma_{\varphi} / l_{\varphi}$, isomorphe à $\Sigma_{\varphi} y_{1}$, contiendrait un sous-module fini non nul, ce qui est exclu, puisque $Y$ est $Z_{\Phi}$-libre par construction. Ainsi $I_{\varphi}$ est de la forme $z_{\varphi}[\theta] f_{\varphi}$, pour un polynôme distingué $f$ de l'anneau $z_{\varphi}\left[\theta{ }^{m} \Phi\right]$, et nous avons d'après ce qui précède : $z_{\varphi}[\theta] f_{\varphi} \cap z_{\Phi}\left[\theta^{m}\right]=P_{\Phi}^{e_{1}} z_{\Phi}\left[\theta^{m}\right]$. En particulier le sous-module $\Sigma_{\varphi} y_{1}$ est somme directe d'un nombre fini d'exemplaires de $\Lambda_{\Phi} / P_{\Phi}^{e_{1}} \Lambda_{\Phi}$. L'existence de la pseudo-décomposition attendue s'obtient alors très simplement par récurrence sur l'entier $n$ :

Dans la suite exacte courte $0 \rightarrow \Sigma_{\varphi} \mathrm{y}_{1} \rightarrow x \rightarrow x^{\prime}=x / \Sigma_{\varphi} \mathrm{y}_{1} \rightarrow 0$, l'hypothèse de récurrence permet de faire apparaître un sous-module $\Sigma_{\Phi}$-élémentaire $\stackrel{n^{\prime}}{\oplus} \Sigma_{\Phi} \bar{y}_{j}$ d'indice fini dans le quotient $X^{\prime}$; et il suffit alors de relever les $\bar{y}_{i}$ dans $x$, en des éléments isotypiques de même ordre, ce qui est toujours possible en raison de la maximalité de $y_{1}$.

$$
\text { 2. - L'idéal } p_{\Phi} \text { est engendré soit par l'uniformisante } l \text {, soit }
$$
par la résolvante $\theta^{\mathrm{m}}$.

Dans ce cas encore, l'étude de $X$ comme $\Sigma_{\Phi}$-module se déduit de sa pseudo-décomposition comme $\Lambda_{\Phi}$-module, à partir des isomorphismes de $\Lambda_{\Phi^{-}}$ modules

$$
\Sigma_{\varphi} / e^{\mu} \Sigma_{\varphi} \simeq\left(\Lambda_{\Phi} / e^{\mu} \Lambda_{\Phi}\right)^{m / m} \quad, \quad \Sigma_{\varphi} / \theta^{\alpha} \Sigma_{\varphi} \simeq\left(\Lambda_{\Phi} / \theta^{\alpha} \Lambda_{\Phi}\right)^{m / m} \Phi
$$

et de la classification des sous-modules projectifs de $\Sigma_{\varphi}$ donnée plus haut.

Démonstration de l'unicité : Compte tenu du résultat d'unicité déjà connu pour la pseudo-décomposition d'un $\Lambda_{\Phi}$-module noethérien, les seuls points 
à vérifier sont les suivants :

(i) Deux modules projectifs $\Sigma_{\varphi}$ et $\Sigma_{\psi}$, associés à des caractères $\ell$-adiques distincts (mais contenus dans un même caractère $\Phi \in \operatorname{Ind}_{H}^{\Delta}\left(R_{\mathbf{Z}}^{i r r}(H)\right)$, ne sont pas pseudo-isomorphes : Nous savons déjà, par la proposition 20 (ii) que deux tels modules ne sont pas isomorphes. Comme tout $\Lambda_{\Phi}$-pseudo-isomorphisme d'un $\Lambda_{\Phi}$-module libre dans un autre est bijectif, notre assertion en résulte.

(ii) Deux quotients $\Sigma_{\varphi} / \theta^{\alpha} \Sigma_{\varphi}$ et $\Sigma_{\psi} / \theta^{\alpha} \Sigma_{\psi}$, associés à des caractères $\ell$-adiques distincts (mais contenus dans un même caractère $\Phi \in \operatorname{Ind}_{H}^{\Delta}\left(R_{\mathbb{Z}_{l}}^{i r r}(H)\right)$, ne sont pas pseudo-isomorphes : Quittes à quotienter si nécessaire, nous pouvons nous limiter à l'étude du cas $\alpha=1$. Cela étant, le premier module $\Sigma_{\varphi} / \theta \Sigma_{\varphi}$, isomorphe à $\mathbb{Z}_{\ell}[\Delta] \mathrm{e} \varphi \mathrm{Z}_{\varphi}$ est annulé par liidempotent $e_{\psi}$; tandis que le second $\Sigma_{\psi} / \theta \Sigma_{\psi}$, isomorphe à $\mathbb{Z}_{l}[\Delta] \mathrm{e}_{\psi} \approx Z_{\psi}$ est engendré par cet idempotent. Le seul $\Sigma$-morphisme de $\Sigma_{\varphi} / \theta \Sigma_{\varphi}$ dans $\Sigma_{\psi} / \theta \Sigma_{\psi}$ est donc le morphisme nul, qui n'est évidemment pas un pseudo-isomorphisme.

(iii) En revanche, deux quotients $\Sigma_{\varphi} / \ell^{\mu} \Sigma_{\varphi}$ et $\Sigma_{\psi} / \ell^{\mu} \Sigma_{\psi}$, ou encore deux quotients $\Sigma_{\varphi} / \Sigma_{\varphi} f$ et $\Sigma_{\psi} / \Sigma_{\psi} f$, associés à des caractères $\ell$-adiques $\varphi$ et $\psi$ contenus dans un même caractère $\Phi \in \operatorname{Ind}_{H^{\Delta}}\left(R_{\mathbf{Z}_{l}}^{\text {irr }}(H)\right)$, sont toujours pseudo-isomorphes. En effet, $\varphi$ s'écrit $\psi \chi^{\alpha}$, pour un $\alpha$ dans $\mathbf{N}$, et la multiplication à droite par $\theta^{\alpha}$, qui à l'idempotent $e_{\varphi}=e_{\psi \chi^{\alpha}}$ associe l'élément $e_{\varphi} \theta^{\alpha}=\theta^{\alpha} e_{\psi}$, induit, par passage au quotient, un $\Sigma$-pseudo-isomorphisme de $\Sigma_{\varphi} / e^{\mu} \Sigma_{\varphi}$ dans $\Sigma_{\psi} / e^{\mu} \Sigma_{\psi}$ (respectivement de $\Sigma_{\varphi} / \Sigma_{\varphi} f$ dans $\Sigma_{\psi} / \Sigma_{\psi} f$.

c.-Paramètres attachés à un $\Sigma$-module noethérien .

DÉFINITION IV.1.29.- Un E-module noethérien $X$ étant donné, introduisons sa pseudo-décomposition :

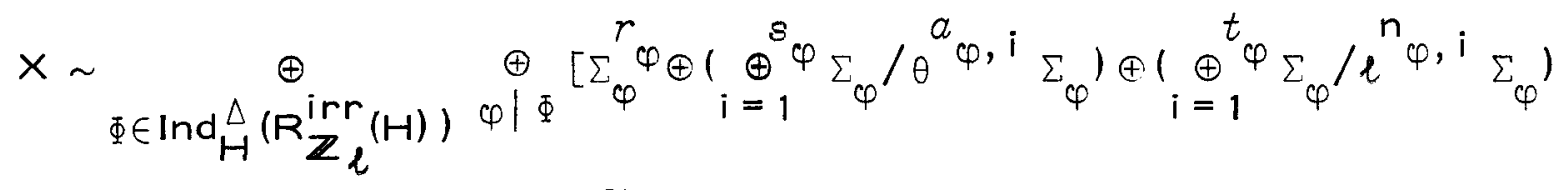

$$
\begin{aligned}
& \left.\oplus\left(\underset{i=1}{\oplus} \oplus_{\varphi} \Sigma_{\varphi} / \Sigma_{\varphi} f_{\varphi, i}\right)\right] ;
\end{aligned}
$$


les deux termes de gauche sont définis de façon canonique, et les deux termes de droite à translation près du caractère $\psi$ par une puissance de $X$; enfin, les $f_{\varphi, i}$ sont des polynômes en lindéterminée $\theta^{m}$.

Nous appelons paramètres du $\Sigma$-module $\times$ les caractères fractionnaires définis par les formules :

$$
\begin{aligned}
& \text { (i) } \rho=\sum_{\varphi} \rho_{\varphi} \varphi \text {, avec } \rho_{\varphi}=\frac{1}{m_{\Phi}} \sum_{k=0}^{m_{\Phi}-1} r X^{-k} \\
& \text { (ii) } \mu=\sum_{\varphi} \mu_{\varphi} \varphi \text {, avec } \mu_{\varphi}=\frac{1}{m_{\Phi}} \sum_{k=0}^{m_{\Phi}-1} \sum_{i=1}^{t} \varphi x^{-k} n x^{-k}, i \\
& \text { (iii) } \lambda=\sum_{\varphi} \lambda_{\varphi} \varphi \text {, avec } \lambda_{\varphi}=\frac{1}{m_{\Phi}} \sum_{k=0}^{m_{\Phi}-1} \sum_{i=1}^{u} \varphi x^{-k} \operatorname{deg} f x^{-k}, i+ \\
& +\sum_{k=0}^{m_{\Phi}-1} \sum_{i=1}^{s} \varphi x^{-k} \sup \left\{\left[\frac{\varphi x^{-k}, i-k}{m_{\Phi}}\right], 0\right\} \text {. }
\end{aligned}
$$

Les deux premiers sont des éléments du groupe $\frac{1}{m_{\Phi}} \operatorname{Ind}_{H}^{\Delta}\left(R_{\mathbb{Z}_{l}}(\Delta)\right) ;$ le troisième est de façon naturelle somme diun caractère fractionnaire induit (i.e. dans $\frac{1}{m_{\Phi}} \operatorname{lnd}_{H}^{\Delta}\left(R_{\mathbb{Z}_{\ell}}(H)\right)$ ) et d'un autre caractère de $\Delta$.

Conformément au théorème de paramétrage énoncé dans la section $2 \S \mathrm{a}$ (théorème 8 ), ces trois caractères mesurent la croissance avec $n$ des quotients $X / \nabla_{n} \times$ associés au $\Sigma$-module $X$. Leurs restrictions à $H$ sont, en effet, des caractères $\ell$-adiques de $H$; et il est facile de voir que ce sont les paramètres de $\times$, au sens de la définition 7 , pour sa structure de $\Lambda[H]$-module :

PROPOSITION IV.1.30.- Les restrictions au sous-groupe $H$ des trois paramètres $\rho, \mu$, et $\lambda$, attachés à un $\Sigma$-module noethérien $X$, sont les paramètres de $X$ dans $R_{\mathbb{Z}}(H)$, pour sa structure de $\Lambda[H]$-module.

Ainsi, pour chaque idempotent central primitif $e_{\Phi}$ de l'algèbre $\Sigma$, les résultats établis dans le cas abélien nous renseignent sur la croissance avec $n$ des $\Phi$-composantes respectives $X_{\Phi, n}=e_{\Phi} X_{n}$ des diverses suites $\left(X_{n}\right)_{n \geq n_{0}}$ de quotients finis de $X_{n}$ introduites dans la section 2 . 
Mais ils ne nous disent rien sur les $\varphi$-composantes de ses quotients, dès que plusieurs caractères $\ell$-adiques irréductibles $\varphi$ du groupe $\Delta$ sont représentés dans un même caractère induit $\Phi \in \operatorname{lnd}_{H}^{\Delta}\left(R_{\mathbb{Z}}^{i r r}(H)\right)$, puisque, dans ce cas, les $\varphi$-composantes $x_{\varphi}=e_{\varphi} \times$ ne sont pas des $\mathbb{Z}_{l}[\mathrm{H}]$-modules. En revanche, il est toujours possible de regarder les $X_{\varphi}$ comme modules sur les algèbres abéliennes $\widetilde{c}_{\Phi}=z_{\Phi}\left[\left[\theta^{m}\right]\right]$. II vient alors :

LEMME IV.1.31. - Soit $\times$ un $\Sigma$-module noethérien élémentaire et indécomposable.

(i) Si $X$ est projectif, i.e. $X \simeq \Sigma_{\psi}$ pour un $\psi \mid \Phi$, chacune de ses $\varphi$-composantes $X_{\varphi}=e_{\varphi} \times($ pour $\varphi \mid \Phi)$ est un $\tilde{c}_{\Phi}$-module libre de dimension $\frac{\mathrm{m}}{\mathrm{m}_{\Phi}}$ :

$$
x_{\varphi} \simeq \widetilde{\mathrm{C}}_{\Phi}^{\mathrm{m} / \mathrm{m}} \Phi
$$

(ii) Si $\times$ est un quotient $\Sigma_{\psi} / \mathcal{l}^{\mu} \Sigma_{\psi}$, pour un $\psi / \Phi$, ses $\varphi$-composantes (pour $\varphi \mid \Phi)$ sont toutes $\widetilde{C}_{\Phi}$-isomorphes au produit $\left(C_{\Phi} / \ell^{\mu} C_{\Phi}\right)^{m / m}$ :

$$
x_{\varphi} \simeq\left(\tilde{C}_{\Phi} / l^{\mu} \tilde{C}_{\Phi}\right)^{m / m} \Phi .
$$

(iii) Si $_{\mathrm{m}} \times$ est un quotient $\Sigma_{\psi} / \Sigma_{\psi} f$, pour un $\psi / \Phi$, avec $f$ distingué dans $Z_{\psi}\left[\theta^{m}\right]$, les $\varphi$-composantes $X_{\varphi}$ (pour $\varphi \mid \Phi$ ) sont sommes directes d'exemplaires du quotient $\tilde{\mathrm{C}}_{\Phi} / h \widetilde{\mathrm{C}}_{\Phi}$, où $h$ est le polynôme distingué de l'algèbre $z_{\Phi}\left[\theta^{m}\right]$ défini par $z_{\Phi}\left[\theta^{m}\right] \cap z_{\varphi}\left[\theta^{m}\right] f=h z_{\Phi}\left[\theta^{m}\right]$ :

$$
x_{\varphi} \simeq\left(\tilde{c}_{\Phi} / h \tilde{c}_{\Phi}\right)^{e} \text {. }
$$

(iv) Si $\times$ est un quotient $\Sigma_{\psi} / \theta^{\alpha} \Sigma_{\psi}$, pour un $\psi \mid \Phi$, ses $\varphi$-composantes pour $\varphi=\psi \chi^{\beta}$ (avec $\left.\beta=0,1, \ldots,\left(m_{\Phi}-1\right)\right)$ sont données par le $\tilde{\mathrm{C}}_{\Phi}$-isomorphisme :

$$
x_{\varphi} \simeq\left(\tilde{c}_{\Phi} / \theta^{m_{\Phi}\left[\frac{\alpha-\beta}{m_{\Phi}}\right]} \tilde{c}_{\Phi}\right)^{m / m_{\Phi}} .
$$

Démonstration : Lorsque l'entier $m_{\Phi}$ divise strictement l'ordre $m$ du caractère $x$, l'algèbre $\widetilde{C}_{\Phi}=z_{\Phi}\left[\left[\theta^{m}\right]\right]$ contient strictement la $\Phi$-composante 
$C_{\Phi}=Z_{\Phi}\left[\left[\theta^{m}\right]\right]$ du centre $C$ de $\Sigma$. Néanmoins, l'identité e $\varphi^{\theta^{m}}=\theta^{m} e_{\varphi}$, pour $\varphi$ divisant $\Phi$, montre que les $\varphi$-composantes d'un $\Sigma$-module $X$ sont encore des $\tilde{C}_{\Phi}$-modules. Pour $X=\Sigma_{\psi}=\sum_{\psi} \mathbf{e}_{\psi}$ et $\varphi=\psi x^{\beta}$, avec $\beta \in\left\{0,1, \ldots,\left(m_{\Phi}-1\right)\right\}$, il vient ainsi :

$$
x_{\varphi}=e_{\psi \chi^{\beta}} \mathbf{e}_{\psi}=\theta^{\beta} \mathbf{e}_{\psi} \mathbf{e}_{\psi} \text {, et la sous-algèbre } \mathbf{e}_{\psi} \sum \mathbf{e}_{\psi} \text {, iso- }
$$

morphe à l'algèbre gauche $z_{\psi}\left[\theta^{m}\right]$, est un $\widetilde{c}_{\Phi}$-module libre de dimension $\frac{m}{m_{\Phi}}$. Les quatre assertions du lemme en résultent.

LEMME IV.1.32. - Pour chaque naturel $n$, la résolvante

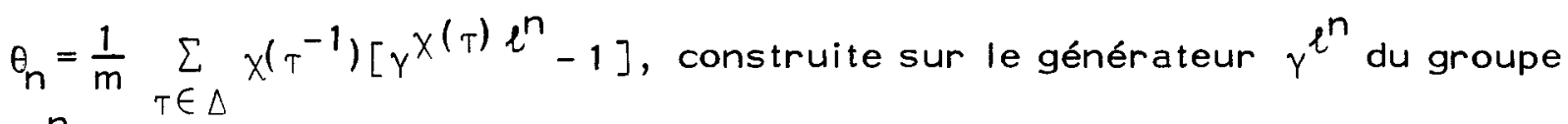
$\Gamma^{l^{n}}$, engendre l'idéal $\omega_{n} \Lambda$ de l'algèbre $\Lambda$, et vérifie les relations de conjugaison :

$$
\tau \theta_{n} \tau^{-1}=x(\tau) \theta_{n}, \quad \forall \tau \in \Delta
$$

En particulier, pour $n \geq n_{0}$, le quotient $\theta_{n} / \theta_{0}$ est contenu dans le centre $C$ de l'algèbre $\Sigma$.

Démonstration : 11 suffit de remplacer $\gamma$ par $\gamma^{l^{n}}$ dans la proposition 2 .

Nous pouvons maintenant énoncer l'analogue du théorème 8 pour le cas métabélien :

THEOREME IV.1.33. - Théorème des paramètres.- Soit $\times$ un module noethérien sur l'algèbre gauche $\Sigma=\Lambda[\Delta]$, de paramètres $\rho, \mu$, et $\lambda$. Si $\tilde{\nabla}_{n}=e^{n+1} \Lambda+\frac{\theta_{n}}{\theta_{0}} \Lambda$ désigne l'idéal de l'algèbre d'lwasawa $\Lambda=\mathbb{Z}_{\ell}[[\gamma-1]]$ engendré par l'élément $\ell^{n+1}$ et le quotient des résolvantes $\theta_{n} / \theta_{0}$, il existe un unique caractère $\ell$-adique virtuel $\nu$ du groupe $\Delta$, tel que l'ordre $e^{\tilde{x}_{n}^{\varphi}}$ de la $\varphi$-composante du quotient $\times / \widetilde{\nabla}_{n} \times$ soit donné, pour chaque caractère $\ell$-adique irréductible $\varphi$, et tout $n$ assez grand, par la formule :

$$
\tilde{x}_{n}^{\varphi}=\left\langle\rho, \varphi>(n+1) \frac{l^{n}-1}{m_{\Phi}}+\left\langle\mu, \varphi>\frac{l^{n}-1}{m_{\Phi}}+<\lambda, \varphi>n+\langle\nu, \varphi>.\right.\right.
$$


Démonstration: Le lemme 32 nous assure que le quotient $X / \widetilde{\nabla}_{n} \times$ est un $\Sigma$-module. Cela étant, la suite de lemmes établis dans la section 2 $\S \mathrm{b}$ nous permet de ramener la démonstration du théorème au cas où $x$ est un module élémentaire. D'après le lemme 31 , quatre cas se présentent donc :

(i) Pour $x=\Sigma_{\psi}$, et $\varphi=\psi x^{\beta}$, le quotient $x_{\varphi} / \tilde{\nabla}_{n} \times{ }_{\varphi}$ est un $z_{\varphi} / e^{n+1} z_{\varphi}$-module libre de dimension $\frac{e^{n}-1}{m_{\Phi}}$. Comme $z_{\varphi}$ est une extension non ramifiée de degré $d_{\varphi}=\langle\varphi, \varphi\rangle$ sur $\mathbb{Z}_{\ell}$, nous obtenons bien le résultat annoncé.

(ii) Pour $x=\Sigma_{\psi} / l^{k} \Sigma_{\psi}$, et $\varphi=\psi x^{\beta}$, le quotient $x_{\varphi} / \tilde{\nabla}_{n} \times$ est égal à $\times_{\varphi} / \frac{\theta_{n}}{\theta_{0}} \times{ }_{\varphi}$ pour $n$ assez grand; c'est un $z_{\varphi} / e^{k} z_{\varphi}$-module libre de dimension $\frac{\ell^{n}-1}{m_{\Phi}}$; d'où, ici encore, le résultat annoncé.

(iii) Pour $x=\Sigma_{\psi} / f \Sigma_{\psi}$, et $\varphi=\psi x^{\beta}$, la $\varphi$-composante $x_{\varphi}$ est un $z_{\varphi}$-module libre qui a pour dimension le degré deg $f$ du polynôme $f$ (regardé comme polynôme en l'indéterminée $\theta^{m}$ ) ; il en est de même pour chacun de ses sous-modules diindice fini $\tilde{\nabla}_{n} \times_{\varphi}$. Cela étant, le lemme 10 nous donne $\tilde{\nabla}_{n} x_{\varphi}=l^{n-n_{0}} \tilde{\nabla}_{n_{0}} \times$, pour $n \geq n_{0}$, d'où, comme attendu :

$$
\begin{aligned}
& \left(x_{\varphi}: \tilde{\nabla}_{n} x_{\varphi}\right)=\left(x_{\varphi}: \tilde{\nabla}_{n_{0}} x_{\varphi}\right)\left(\tilde{\nabla}_{n_{0}} x_{\varphi}: e^{n-n_{0}} \tilde{\nabla}_{n_{0}} x_{\varphi}\right)= \\
& =\left(x_{\varphi}: \tilde{\nabla}_{n_{0}} x_{\varphi}\right)\left(z_{\varphi}: e^{n-n_{0}} z_{\varphi}\right)^{\operatorname{deg} f} .
\end{aligned}
$$

(iv) Pour $x=\Sigma_{\psi} / \theta^{\alpha} \Sigma_{\psi}$, et $\varphi=\psi x^{\beta}$ avec $\beta \in\left\{0,1, \ldots,\left(m_{\Phi}-1\right)\right\}$, la décomposition de $X$ comme somme de ses $\varphi$-composantes fait intervenir la partie entière de la quantité $\frac{\alpha-\beta}{m_{\Phi}}$. Ceci explique la curieuse définition de l'invariant $\lambda$ dans ce cas.

SCOLIE IV.1.34. - Soient $\times$ un $\Sigma$-module noethérien et de torsion, de paramètres $\mu$ et $\lambda$, et $Y$ un sous-module dilindice fini. Supposons choisi un entier naturel $m$, tel que les quotients $X_{n}=X / \frac{\theta_{n}}{\theta_{m}} Y$ soient finis, pour
chaque entier naturel $n \geq m$. Alors: 
(i) Il existe un caractère $\ell$-adique virtuel $\nu$ du groupe $\Delta$ tel que l'ordre $l^{x_{n}^{\varphi}}$ de la $\varphi$-composante du groupe $x_{n}$ soit donné, pour chaque caractère $\ell$-adique irréductible $\varphi$, et tout $n$ assez grand, par la formule:

$$
x_{n}^{\varphi}=\left\langle\mu, \varphi>\frac{l^{n}-1}{m_{\Phi}}+<\lambda, \varphi>n+\langle\nu, \varphi>.\right.
$$

(ii) Pour chaque entier relatif $k$ fixé, les sous-modules $e^{n+k} x_{n}$ sont deux à deux $\Sigma$-isomorphes pour tous les $n$ assez grands. Démonstration : Identique à celle de la proposition 19 . 
$-278-$ 


\section{REPRÉSENTATIONS $\ell$-ADIQUES \\ ASSOCIÉES AUX INVARIANTS CYCLOTOMIQUES}

Nous explorons dans cette section les relations de dualité exposées dans le parallèle entre la théorie de Tate et celle de Kummer, mais en les regardant cette fois du point de vue de la théorie d'Iwasawa.

Notre propos est de montrer comment les paramètres associés aux $\Lambda[\Delta]$-modules classiques construits sur les groupes de classes, de radicaux ou de symboles qui expriment ces dualités se déduisent en fait les uns des autres par des formules complètement explicites ne faisant intervenir que des invariants galoisiens simples des extensions concernées. La notion-clé de cette étude est celle, introduite plus haut, de suite paramétrée, qui permet de ramener la démonstration des identités annoncées à l'étude asymptotique de certains modules finis définis à chaque étage fini de la tour cyclotomique. Le résultat essentiel, que résume le tableau publié en appendice, est que tous les paramètres rencontrés s'obtiennent à partir de deux seulement d'entre eux ${ }^{(*)}$ : Les invariants d'iwasawa $\mu_{c}$ et $\lambda_{c}$ associés au groupe de Galois $C^{\prime}$ de la $\ell$-extension abélienne non ramifiée $\ell$-décomposée maximale de la tour cyclotomique construite sur le corps considéré .

${ }^{\star}$ ) Encore l'invariant $\mu_{c}$ lui-même est-il conjecturalement nul . 


\section{0. - PRÉLIMINAIRES.}

Nous considérons, dans tout ce qui suit, le schéma de corps suivant : Un nombre premier $l$ étant fixé, nous notons $\zeta$ une racine primitive $\ell$-ième de l'unité, et $K / F$ une extension abélienne de corps de nombres, contenant $\zeta$, de degré d étranger à $\ell$, par exemple $K=F[\zeta]^{(\star)}$. Pour chaque naturel $n$, nous désignons par $F_{n}$ l'unique sous-extension cyclique de degré $l^{n}$ de la tour cyclotomique $F_{\infty} / F$; puis nous posons $K_{n}=K . F_{n}$. La réunion $K_{\infty}=K . F_{\infty}$ des corps $K_{n}$ est donc la tour cyclotomique sur $K$. D'un côté, son groupe de Galois $\Gamma=$ Gal $\left(K_{\infty} / K\right)$ est un groupe profini isomorphe à $\mathbb{Z}_{\ell}$, que nous écrivons $\Gamma=\gamma^{\mathbb{Z}} \ell$, en faisant choix d'un générateur topo logique arbitraire $\gamma$; d'un autre côté, le groupe $\Delta=\mathrm{Gal}\left(\mathrm{K}_{\infty} / F_{\infty}\right)$ est un groupe abélien, d'ordre $d$, isomorphe par restriction au groupe de Galois Gal(K/F); leur composé $G a l\left(K_{\infty} / F\right)$ est ainsi le produit direct de $\Gamma$ par $\triangle$.

L'ordre $d=[K: F]$ du groupe $\Delta$ étant inversible dans $\mathbb{Z} \ell$, nous savons qu'à chaque caractère $l$-adique irréductible $\varphi$ de $\Delta$ cor respond un idempotent primitif de l'algèbre $\mathbb{Z}_{\ell}[\Delta]$, donné par la formule :

$$
e_{\varphi}=\frac{1}{d} \sum_{T \in \Delta} \varphi\left(\tau^{-1}\right) \tau
$$

et que le facteur isotypique associé $Z_{\varphi}=\mathbb{Z}_{\ell}[\Delta]$ e $\varphi$ s'identifie à l'anneau local des entiers d'une extension cyclotomique non ramifiée de $\mathbb{Q}_{\ell}$, qui a pour degré $\left[Z_{\varphi}: \mathbb{Z}_{\ell}\right]$ le degré $d_{\varphi}$ du caractère $\varphi$. En particulier, tout $\mathbb{Z},[\Delta]$-module noethérien sans $\mathbb{Z} \ell^{\text {-torsion est }}$ somme directe d'exemplaires des $Z_{\varphi}$, donc $\mathbb{Z}_{\ell}[\Delta]$-projectif.

Le groupe $R \mathbb{Z}_{\ell}(\Delta)$ des caractères virtuels du groupe $\Delta$ sur l'anneau $\mathbb{Z}_{\ell}$ est la $\mathbb{Z}$-algèbre construite sur le semi-groupe $R_{\mathbb{Z}_{\ell}}^{+}(\Delta)$ des caractères des $\mathbb{Z}_{\ell}[\Delta]$-modules noethériens et projectifs. Clest un $\mathbb{Z}_{\ell}$-module libre, qui a pour base l'ensemble $R \mathbb{Z}_{\ell}^{(\Delta)}$ des carac tères $\ell$-adiques irréductibles du groupe $\Delta$. Il est muni canoniquement d'un produit scalaire :

${ }^{\star}$ ) Pour $\ell=2$, nous supposons en outre que $K$ ( et donc $F$ ) contient les racines quatrièmes de l'unité. 


$$
\langle\varphi, \psi\rangle=\frac{1}{d} \sum_{\tau \in \Delta} \varphi\left(\tau^{-1}\right) \psi(\tau)
$$

pour lequel les caractères irréductibles sont deux à deux or thogonaux, de carré scalaire $\langle\varphi, \varphi\rangle=\mathrm{d}_{\varphi}$, ainsi que d'une involution naturelle qui peut se définir comme suit : Pour chaque naturel $n$, désignons par $\mu_{n}$ le groupe des racines d'ordre $\ell$-primaire de l'unité contenues dans le corps $K_{n}$, et introduisons la réunion $\mu_{\ell}=\underline{\lim } \mu_{n}$ des $\mu_{n}$, ainsi que leur limite projective pour les applications normes :

$$
T_{\ell}=\stackrel{\lim }{\mu} \mu_{n} \text {. }
$$

Le groupe $T \mathbb{l}_{\ell}$ est, par définition, le module de Tate de l'extension;

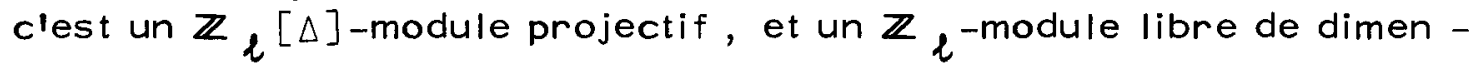
sion 1 . Si $w$ désigne le caractère qui lui correspond, l'application $x \longmapsto x^{*}$ définie par :

$$
x^{*}=w x^{-1}
$$

( avec la convention $\chi^{-1}(\tau)=\chi\left(\tau^{-1}\right)$, pour tout $\tau$ de $\Delta$ ) est une in volution du groupe $R_{\mathbb{Z}}(\Delta)$, appelée involution du miroir: Nous disons que $w$ est le caractère cyclotomique, et que $X^{*}$ est le reflet du caractère $x$.

\section{1. - ÉTUDE DU GROUPE DE GALOIS DE LA $\ell$-EXTENSION ABÉLIENNE} NON RAMIFIÉE $\ell$-DECOMPOSÉE MAXIMALE DU CORPS $K_{\infty} \cdot$

a.- Definition du groupe $C^{\prime}$.

Désignons par $C_{\infty}^{\prime}$ le $\ell$-corps des $\ell$-classes de Hilbert de la tour $K_{\infty}$, i.e. la $\ell$-extension abélienne maximale de $K_{\infty}$ qui est non ramifiée et où les places de $l$ se décomposent complètement . La notion d'extension non ramifiée, complètement décomposée en un en semble fini donné de places $S$, étant de caractère fini ( cf. [ $\left.\mathrm{Se}_{1}\right]$, $\$ 2$ ), le corps $C_{\infty}^{\prime}$ est la réunion croissante des $l$-corps des $l$ classes de Hilbert respectifs $C_{n}^{\prime}$ des corps $K_{n}$, et le groupe de Galois $C^{\prime}=$ Gal $\left(C_{\infty}^{\prime} / K_{\infty}\right)$ s'identifie ainsi, via l'isomorphisme $d u$ corps de classes, à la limite du système projectif ( pour les applications induites par la norme arithmétique ) :

$$
c^{\prime}=\left\lfloor\text { lim } a_{n}^{\prime}\right.
$$


des $\ell$-groupes de $\ell$-classes de diviseurs des corps $K_{n}$, i.e. des quotients $C_{n}^{\prime}$ des $l$-groupes de classes de diviseurs des corps $K_{n}$, par leurs sous-groupes respectifs $\mathcal{L}_{n}=C_{n}(l)$ engendrés par les places au-dessus de $l$.

DÉFINITIONIV.2.1.- Par $C^{\prime}$ nous entendons le groupe de Galois Gal ( $C_{\infty}^{\prime} / K_{\infty}$ ) de la $\ell$-extension abélienne non ramifiée $l$-décompo-

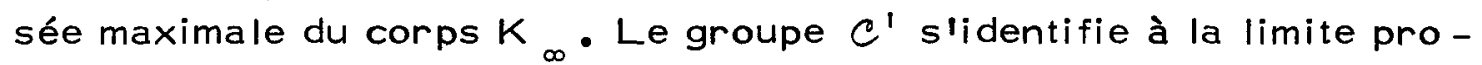
jective $\underline{\lim } C \ell_{\mathrm{n}}^{\prime}$ des $\ell$-groupes de classes de diviseurs des corps $K_{n}$; c'est un $\Lambda[\Delta]$-module noethérien et de torsion, dont nous écrivons $\rho_{c}=0, \mu_{c}$ et $\lambda_{c}$ les paramètres dans $R_{\mathbb{Z}_{l}}^{+}(\Delta)$.

Naturellement, le premier problème de la théorie d'lwasawa consiste à retrouver les groupes $C_{n}^{\prime}$ à partir de leur limite projec tive $C^{\prime}$. Rappelons ce résultat classique ( $\mathrm{cf}$. par exemple $\left[\mathrm{Se}_{1}\right]$ ) : Désignons par $K_{n}$ lintersection de $K_{\infty}$ avec le $l$-corps des $l$ classes de Hilbert $C_{0}^{\prime}$ de $K=K_{0}$ (de sorte que $K_{n}$ est la sous -ex tension maximale de $K_{\infty}$, qui est $\ell$-décomposée sur $K$ ), puis, pour chaque naturel $\mathrm{n} \geq \mathrm{n}_{0}$, introduisons le $\ell$-corps des $\ell$-genres $C_{\infty}^{\prime} / \mathrm{n}$ de l'extension procyclique $K_{\infty} / K_{n}$ (i.e. l'extension abélienne ma ximale de $K_{n}$, qui est non ramifiée et $\ell$-décomposée sur $K_{\infty}$ '. Le quotient des $l$-genres $g_{n}^{\prime}=\ell^{n}$ al $\left(C_{\infty}^{\prime} / n / K_{\infty}\right)=c^{\prime} / C^{\prime} \omega_{n}$, plus

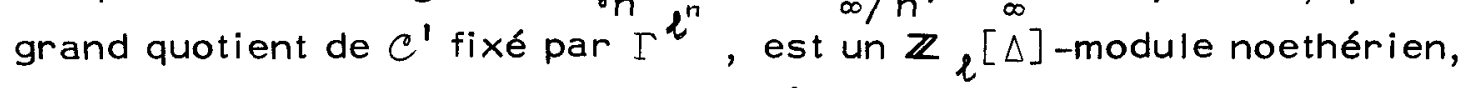
qui admet un quotient isomorphe à $C_{n}^{\prime}$ : Sous la condition $n \geq n_{0}$, en effet, l'extension $l$-décomposée $C_{n}^{\prime} / K_{n}$ est disjointe de la tour cyclotomique $K_{\infty} / K_{n}$, et le groupe des $l$-classes $\alpha_{n}^{\prime}$ s'identifie par conséquent au groupe de Galois Gal $\left(K_{\infty} C_{n}^{\prime} / K_{\infty}\right)$, qui est bien un quotient de $\mathcal{G}_{n}^{\prime}$.

Reste à évaluer le groupe Gal $\left(\mathrm{C}_{\infty}^{1} / \mathrm{K}_{\infty} \mathrm{C}_{n}^{\prime}\right)$, ou son quotient Gal $\left(C_{\infty}^{\prime} / n / K_{\infty} C_{n}^{\prime}\right)$. Un argument de projectivité ( cf. $\left[\mathrm{Iw}_{6}\right]$, th.8; [Or ], prop. $4.3 ;\left[\mathrm{Se}_{1}\right]$, th. 4 ) montre que, dès que $K_{n}$ contient le composé $K_{m}$ des sous-corps de décomposition des places de $K$ audessus de \& dans la tour $K_{\infty} / K$, le sous-groupe $C_{n}^{\prime}=G a l\left(C_{\infty}^{\prime} / K_{\infty} C_{n}^{\prime}\right)$ de $C^{\prime}$, qui fixe $K_{\infty} \cdot C_{n}^{\prime}$, est l'image par l'opérateur norme $\frac{\omega_{n}}{\omega_{m}}$ de celui $C_{m}^{\prime}$, qui fixe $K_{\infty} \cdot C_{m}^{\prime}$. Comme $C_{m}^{\prime}$ contient évidemment le sous groupe des $l$-genres $c^{\prime} \omega_{m}$ de l'extension $K_{\infty} / K_{m}$, il vient ainsi : 


$$
c l_{n}^{\prime}=c^{\prime} / c_{n}^{\prime}=c^{\prime} / c_{m}^{\prime}{ }^{w_{n} / w_{m}}=c^{\prime} /\left(c^{\prime w_{m}} c_{m}^{\prime}\right)^{w_{n} / w_{m}} ;
$$

ce qui est le résultat attendu.

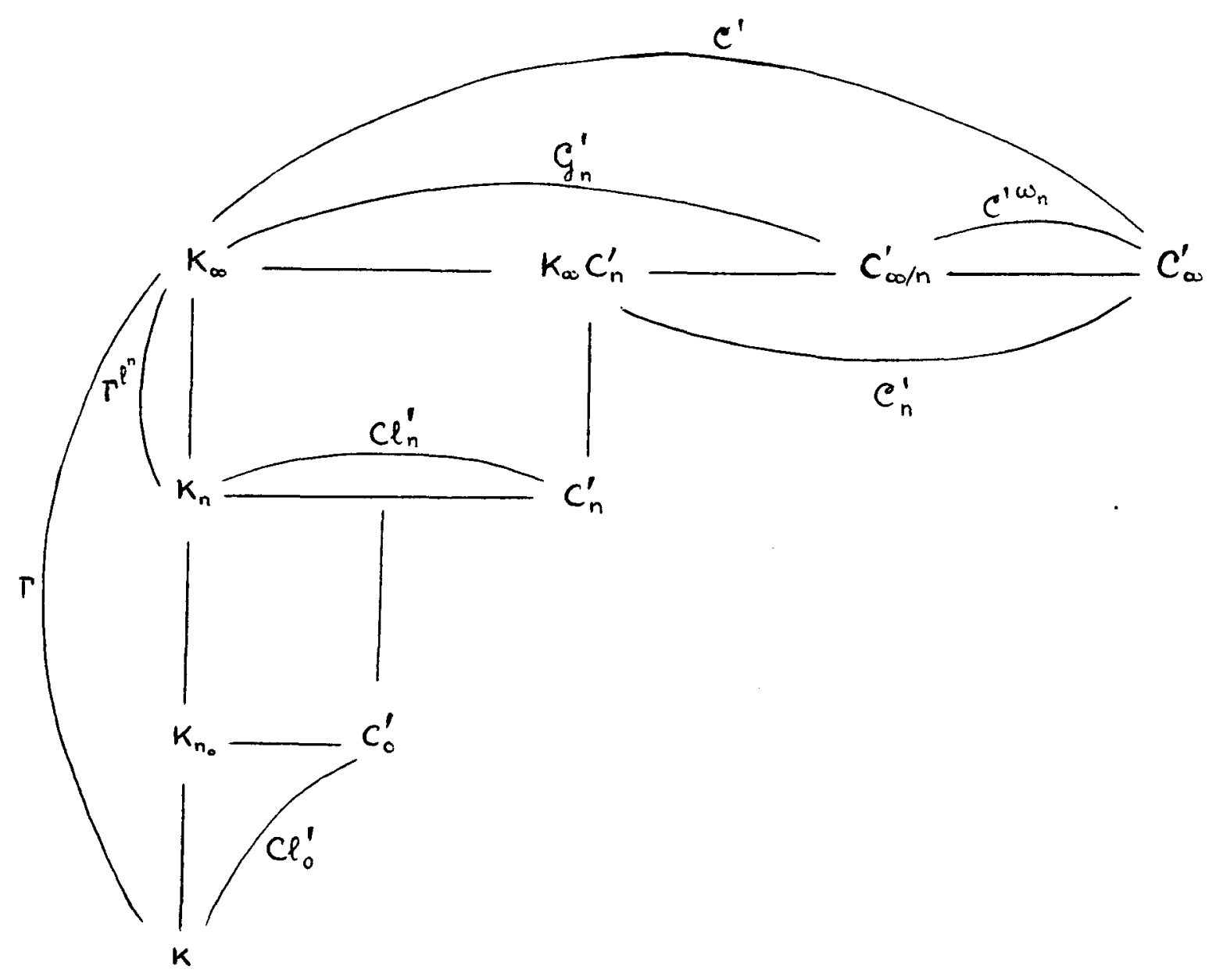

Le groupe $C$ vérifie, en effet, les hypothèses de la proposition IV.1.19; prenant pour r la $l$-valuation de l'ordre du $\ell$-Sylow $\mu_{0}$ du groupe des racines de l'unité dans $K$, nous pouvons donc énoncer ${ }^{(\star)}$ :

(*) Le théorème 2 qui suit est essentiellement bien connu, au moins dans le cas où $K$ est égal à $F[\zeta]$, pour lequel les représentations 1 -adiques irréductibles sont de degré 1 . L'introduction des quotients $\mathrm{Cl}_{n}^{\prime} / \mathrm{Cl}_{n}^{\prime} \ell^{n+r}$, indispensable à l'interprétationkummérienne des résultats obtenus, est due à $\left[\mathrm{Ja}_{9}\right]$. 
THÉORĖME IV.2.2. - Les caractères $\mu_{c}$ et $\lambda_{c}$ étant fixés par la définition 1 , il existe un unique caractère virtuel $\nu_{C}$ du groupe de Galois $\Delta=\operatorname{Gal}\left(\mathrm{K}_{\infty} / F_{\infty}\right)$, tel que pour chaque caractère $l$-adique ir réductible $\varphi$ de $\Delta$, et tout $n$ assez grand, l'ordre $\ell^{c^{\prime}} \varphi^{(n)}$ de la $\varphi$ composante du $\ell$-groupe $\mathrm{C}_{\mathrm{n}}^{\prime}$ des $\ell$-classes de diviseurs du corps $K_{\mathrm{n}}$ soit donné par la formule :

$$
c_{\varphi}^{\prime}(n)=\left\langle\mu_{c}, \varphi\right\rangle \ell^{n}+\left\langle\lambda_{c}, \varphi\right\rangle_{n}+\left\langle\nu_{c}, \varphi\right\rangle \text {. }
$$

Nous disons que la suite $\left(\mathrm{Cl}_{n}^{\prime}\right)_{n} \in \mathbb{N}$ est paramétrée par les caractères $\rho_{c}=0, \mu_{c}, \lambda_{c}$, et $\nu_{c}$.

SCOLIE IV.2.3. - 11 existe un unique caractère virtuel $\tilde{\nu}_{c} \leq \nu_{c} d u$ groupe $\Delta$, tel que pour chaque caractère $l$-adique ir réductible $\varphi$ de $\Delta$, et tout $n$ assez grand, l'ordre $e^{\tilde{c}_{\varphi}^{\prime}(n)}$ de la $\varphi$-composante du quotient $\mathrm{Cl}_{n}^{\prime} / \mathrm{Cl}_{\mathrm{n}}^{\prime} \mathrm{l}^{\mathrm{n}+\mathrm{r}}$ soit donné par la for mule:

$$
\tilde{c}_{\varphi}^{\prime}(n)=\left\langle\mu_{c}, \varphi\right\rangle \ell^{n}+\left\langle\lambda_{c}, \varphi\right\rangle n+\left\langle\tilde{\nu}_{c}, \varphi\right\rangle \text {. }
$$

Démonstration: D'après la proposition IV.1.19, les $\varphi$-composantes des sous-groupes $C_{n}^{\prime} \ell^{n+r}$ ont un ordre constant pour $n$ assez grand.

Dans la formule obtenue, la quantité $l^{\text {ntr }}$ mesure l'ordre du $\ell$-Sylow $\mu_{n}$ du groupe des racines de l'unité contenues dans le $n$ ième étage $K_{n}$ de la tour cyclotomique $K_{\infty} / K$. C'estaussi l'exposant de la $l$-extension abélienne kummérienne maximale du corps $K_{n}$. Convenons d'écrire $\Re_{n}=\left(e^{-n-r} \mathbb{Z} / \mathbb{Z}\right) \otimes_{\mathbb{Z}} K_{n}^{X}$ le radical kummérien associé. A chaque $\ell$-extension abélienne $L_{n}$ de $K_{n}$ correspond un sous-groupe de ce radical, défini par l'identité :

$$
\operatorname{Rad}\left(L_{n} / K_{n}\right)=\left\{e^{-n-r} \otimes x_{n} \in \Re_{n} \mid K_{n}\left[\sqrt[e]{x_{n}}\right] \subset L_{n}\right\},
$$

et caractérisé par l'isomorphisme du miroir :

$$
\operatorname{Rad}\left(L_{n} / K_{n}\right) \approx \operatorname{Hom}\left(\operatorname{Gal}\left(L_{n} / K_{n}\right), \mu_{n}\right) \text {. }
$$

En particulier, lor sque $L_{n}$ est la $\ell$-extension abélienne maximale non $r$ amifiée et $\ell$-décomposée $C_{n}^{\prime}$ de $K_{n}$, il vient :

$$
\operatorname{Rad}\left(c_{n}^{\prime} / K_{n}\right) \simeq \operatorname{Hom}\left(a_{n}^{\prime}, \mu_{n}\right) \simeq \operatorname{Hom}\left(a_{n}^{\prime} / a_{n}^{\prime} e^{n+r}, \mu_{n}\right) \text {. }
$$


COROLLAIRE IV.2.4. - La suite $\left(\Xi_{n}^{\prime}\right)_{n} \in \mathbb{N}$ des radicaux kummériens $\operatorname{Rad}\left(C_{n}^{\prime} / K_{n}\right)$ des $l$-extensions abéliennes maximales non ramifiées et $\ell$-décomposées des corps $K_{n}$ est par amétrée par les reflets res pectifs $\rho_{c}^{*}=0, \mu_{c}^{*}, \lambda_{c}^{*}$, et $\tilde{\nu}_{c}^{*}$ des paramètres attachés aux quo tients $\mathrm{Cl}_{n}^{\prime} / \mathrm{Cl}_{\mathrm{n}}^{\prime} \ell^{n+r}$ : Pour chaque caractère $\ell$-adique irréductible $\varphi$ du groupe $\Delta$, et tout $n$ assez grand, l'ordre $e^{\tilde{c}^{\prime}}{ }^{(n)}$ de la $\varphi-$ composante du groupe $\mathfrak{S}_{n}^{\prime}$ est donné par la formule:

$$
\tilde{c}_{\varphi}^{\prime}(n)=\left\langle w \mu_{c}^{-1}, \varphi\right\rangle l^{n}+\left\langle w \lambda_{c}^{-1}, \varphi\right\rangle n+\left\langle w \tilde{\nu}_{c}^{-1}, \varphi\right\rangle \text {. }
$$

Remarque : Supposons $\ell \neq 2$. Lorsque $\varphi$ est le caractère unité, la $\varphi$-composante du radical $\mathfrak{S}_{n}^{\prime}=\operatorname{Rad}\left(C_{n}^{\prime} / K_{n}\right)$ est le sous-groupe des radicaux de $\mathfrak{E}_{\mathrm{n}}^{\prime}$ construits sur les éléments de $F_{\mathrm{n}}$. La formule qui lui correspond s'écrit encore $\tilde{c}_{\varphi}^{\prime}(n)=\left\langle\omega, \mu_{c}\right\rangle \ell^{n}+\left\langle\omega, \lambda_{c}\right\rangle n+\left\langle\omega, \tilde{\nu}_{c}\right\rangle$. Si $w$ n'est pas le caractère unité (i.e. si $F$ ne contient pas les ra cines $\ell$-ièmes de ('unité), les quantités significatives $\left\langle w, \mu_{c}\right\rangle$ et $\left\langle\omega, \lambda_{c}\right\rangle$ ne sont pas définies dans $F_{\infty}$ : Dans ce cas, pour obtenir le radical de la plus gr ande extension kummérienne de $K_{n}$ qui provient par composition d'une $\ell$-extension abélienne non ramifiée $\ell$-décom posée de $F_{n}$, il convient de former non pas la composante de $\mathfrak{F}_{n}^{\prime}$ associée au caractère unité 1 , mais celle associée à son reflet $\omega=1^{*}$ (le caractère cyclotomique).

\section{b.- Comparaison avec le groupe des classes au sens ordinaire -}

Nous savons que, pour chaque naturel $n$, le $\ell$-groupe $C_{n}^{\prime}$ des $l$-classes de diviseurs du corps $K_{n}$ s'écrit comme quotient du $l-$ groupe des classes de diviseurs au sens ordinaire $C \ell_{n}$, par le sousgroupe, disons $\delta_{n}$, engendré par les classes dans $C_{n}$ des idéaux premiers de $K_{n}$ au-dessus de $l$. Les groupes $C_{n}$ donnent lieu naturellement à une description tout à fait semblable à celle des groupes $\alpha_{n}^{\prime}$. En particulier, leur limite projective $c=\underline{\lim } \alpha_{n}$ est un $\Lambda[\Delta]$-module noethérien et de torsion , qui admet $C^{\prime}$ comme quotient. Si d'ailleurs $m$ désigne l'indice du composé $K_{m}$ des sous-corps de décomposition des places de $K$ au-dessus de $\&$ dans la tour $K_{\infty} / K$, le

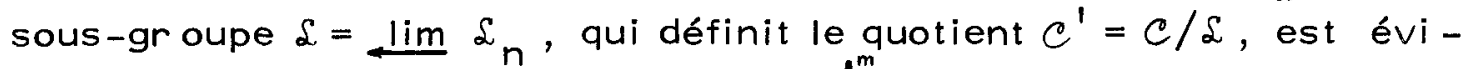
demment fixé par le groupe de Galois $\Gamma^{l^{m}}=\operatorname{Gal}\left(K_{\infty} / K_{m}\right)$, donc 
annulé par le polynôme $\omega_{m}=\gamma^{l^{m}}-1$. En particulier, pour chaque caractère $\ell$-adique irréductible $\varphi$ du groupe $\Delta$, les polynômes car actéristiques des $\varphi$-composantes respectives de $C$ et $C^{\prime}$ ne diffèrent donc que par des facteurs cyclotomiques (savoir les diviseurs de $\omega_{m}$ dans $Z_{\varphi}[\gamma-1]$, ou, ce qui est la même chose, dans $\left.\mathbb{Z}_{\ell}[\gamma-1]\right)$; ils sont, de ce fait, divisibles par les mêmes puissances de $\ell$.

PROPOSITION IV.2.5. - Le gr oupe de Galois $C=G a l\left(C_{\infty} / K_{\infty}\right)$ de la $\ell$-extension abélienne maximale non ramifiée $C_{\infty}$ de la tour $K_{\infty}$ est un $\Lambda[\Delta]$-module noethérien et de torsion, isomorphe à la limite projec tive $l$ im $l_{n}$ des $l$-groupes de classes de diviseurs des corps $K_{n}$. Ses paramètres dans $R_{\mathbb{Z}_{\ell}}^{+}(\Delta)$ sont donnés par les formules $\rho=0$; $\mu=\mu_{c} ; \lambda=\lambda_{c}+\lambda_{\ell}$, où $\lambda_{l}$ est l'unique paramètre non nul du sous module $\delta=\left\lfloor\right.$ lim $\delta_{n}$, qui définit le quotient $c^{\prime}=c / \delta$.

La détermination du paramètre $\lambda_{\ell}$ pose le problème de l'in terprétation des facteurs cyclotomiques dans la décomposition irré ductible des polynômes caractéristiques respectifs $\mathrm{PC}_{\varphi}$ et $\mathrm{Pc}_{\varphi}^{\prime}$ des $\varphi$-composantes respectives des $\Lambda[\Delta]$-modules $c$ et $C^{\prime}$. Pour chaque caractère $\ell$-adique $\varphi$ de $\Delta$, écrivons donc:

$$
P c_{\varphi}^{\prime}(\gamma-1)=\ell^{\mu} \varphi \Omega c_{\varphi}^{\prime}(\gamma-1) R c_{\varphi}(\gamma-1)
$$

la décomposition dans $Z_{\varphi}[\gamma-1]$ du polynôme caractéristique de la $\varphi$ composante du gr oupe $C^{\varphi^{\varphi}}$, comme produit d'une puissance de $l$, d'un polynôme cyclotomique $\Omega c^{\prime} \varphi$, et d'un polynôme distingué $R c$ étran ger à tous les $\omega_{i}$ : Le polynôme $\Omega c^{\prime}$ a mêmes facteurs irréductibles que le polynôme caractéristique $\tilde{\Omega}_{\varphi}^{\prime}{ }_{\varphi}^{\varphi}$ du quotient $c_{\varphi}^{\prime} / c_{\varphi}^{\prime}{ }^{(\star)}$. Ecrivons de même :

$$
P c_{\varphi}=e^{\mu} \varphi \Omega c_{\varphi}(\gamma-1) R c_{\varphi}(\gamma-1)
$$

la décomposition du polynôme caractéristique $P_{c_{\varphi}}$. Ici encore, le facteur cyclotomique $\Omega_{\sim} c_{\varphi}$ a mêmes facteurs irréductibles que le polynôme car actéristique $\tilde{\Omega}_{\varphi}{ }_{\varphi}$ du quotient $c_{\varphi} / c_{\varphi}^{\omega_{m}}$. Nous avons :

$(\star)$ Le quotient $C^{\prime} / C^{\prime w_{m} / w_{m}}$ étant fini ( cf. démonstration du théo rème 2$), P c^{\prime}$ est étranger à tous les $\frac{\omega_{n}}{\omega_{m}}$ (pour $\left.n \geq m\right)$. 
THÉORÈME IV.2.6. - Pour chaque naturel $n$ assez grand, la quantité $\mathrm{d}_{\varphi}$. deg $\widetilde{\Omega} c_{\varphi}^{\prime}$ mesure la $\varphi$-par tie du groupe de défaut de la conjecture de Gross dans le corps $K_{n}$. Par suite, les polynômes caractér istiques $P C_{\varphi}^{\prime}$ associés aux $\varphi$-composantes du $\Lambda[\Delta]$-module $C^{\prime}$ sont étrangers à chacun des polynômes cyclotomiques $\omega_{i}$ si et seulement si cette conjecture est vérifiée à chaque étage fini assez grand de la tour cyclotomique, ce qui a lieu en particulier dès que le corps $K$ est absolument abélien.

Démonstration: Fixons $n \geq m$ (autrement dit, supposons les places au-dessus de $\ell$ sans décomposition dans la tour $K_{\infty} / K_{n}$ ', et consi dérons le quotient des genres $g_{n}^{\prime}=c^{\prime} / c^{\prime w_{n}}$. D'un côté, le théorème de structure des $\Lambda[\Delta]$-modules nous montre que pour chaque caractère $\ell$-adique irréductible $\varphi$, la $\varphi$-composante du groupe $\zeta_{n}^{\prime}$

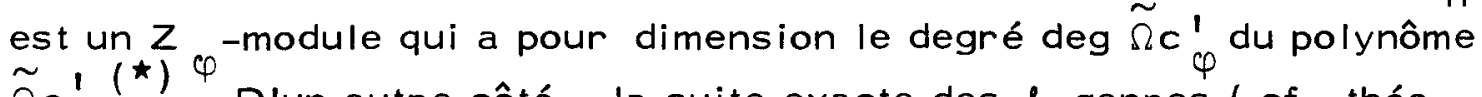

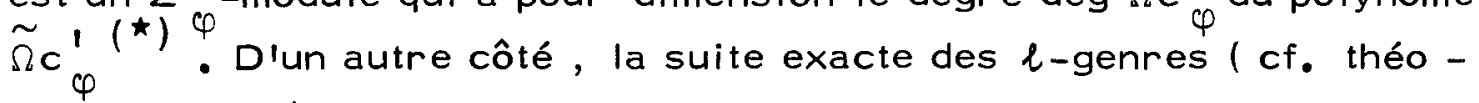
rème III.2. J, qui s'écr it ici :

$1 \longrightarrow \varepsilon_{n}^{\prime} / \varepsilon_{n}^{\prime} \cap \eta_{n} \longrightarrow \underset{I_{n} \mid l}{\widetilde{e}} D_{I_{n}}\left(C_{\infty}^{\prime} / n / K_{n}\right) \longrightarrow q_{n}^{\prime} \longrightarrow a_{n}^{\prime} \longrightarrow 1$,

nous prouve, puisque le groupe $C_{n}^{\prime}$ est fini, que la dimension sur $Z_{\varphi}$ de la $\varphi$-composante de $G_{n}^{\prime}$ est encore celle de la $\varphi$-composante du quotient :

$$
\left[\underset{I_{n} \mid l}{\tilde{e}_{1}} D_{I_{n}}\left(C_{\infty}^{\prime} / n / K_{n}\right)\right] / b_{\infty}^{\prime} C_{\infty}^{\prime} / K_{n}\left(\varepsilon_{n}^{\prime}\right)
$$

où le numérateur est la somme directe des groupes de décomposition des places de $K_{n}$ au-dessus de \& dans l'extension abélienne $C_{\infty}^{\prime} /{ }_{n} / K_{n}$, somme restreinte aux familles $\left(\sigma_{l}\right)_{l}$ qui vérifient la formule du produit $\prod_{\mathfrak{n}}\left|\ell \sigma{ }_{\mathfrak{n}}\right| K_{\infty}=1$; et le dénominateur est l'image, par les sym boles de Hasse $\left(\frac{, c_{\infty} / n / K_{n}}{I_{n}}\right)$, du tensorisé $\delta_{n}^{\prime}=\mathbb{Z}_{l} \otimes_{\mathbb{Z}} E_{n}^{\prime}$ du groupe des $\ell$-unités de $K_{n}$. Et comme nous ne nous intéressons

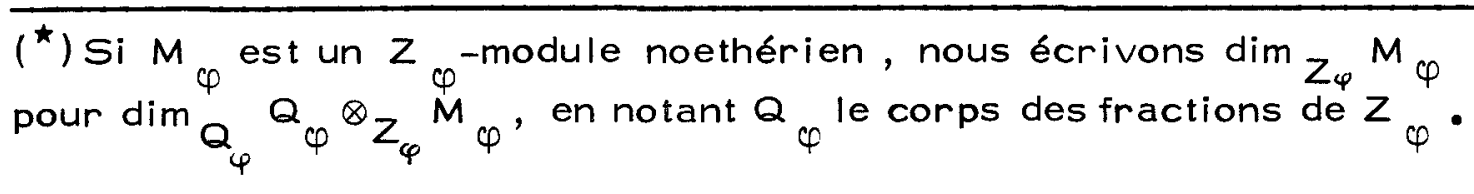


qu'aux dimensions, nous pouvons reécrire tout aussi bien cette identité sous la forme:

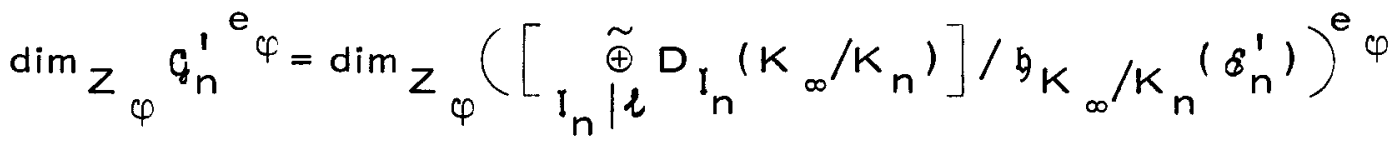

$$
\begin{aligned}
& =\operatorname{dim}_{Q_{\varphi}}\left(\left[\underset{I_{n} \mid l}{\tilde{\oplus}} \mathbb{Q}_{l} \otimes_{\mathbb{Z}_{l}} D_{I_{n}}\left(K_{\infty} / K_{n}\right)\right] / \zeta_{K_{\infty}} / K_{n}\left(Q_{l} \otimes_{\mathbb{Z}} E_{n}^{\prime}\right)\right)^{e} \varphi
\end{aligned}
$$

Il reste alors à reconnaître dans cette dernière expression le groupe de défaut de la conjecture de Gross. Pour cela, remarquons que, pour chaque place $I_{n}$ de $K_{n}$ au-dessus de $\ell$, le groupe de décompo sition $D_{1}\left(K_{\infty} / K_{n}\right)$ s'identifie canoniquement au groupe de Galois de l'extension cyclotomique locale au-dessus du complété $K_{n, l}$, c'està-dire, via liisomorphisme du corps de classes local, à un quotient de $K_{n, l}^{X}$. Pour transformer ce quotient, prenons la norme $N_{K}, I / Q_{l}$, puis le logarithme $\ell$-adique $\log _{\ell} \cdot$ Composant le tout, nous obtenons un isomorphisme naturel :

$$
\mathbb{Q}_{l} \otimes_{\mathbb{Z}_{l}} D_{I_{n}}\left(K_{\infty} / K_{n}\right) \simeq \mathbb{Q}_{l} \text {. }
$$

Dans la description obtenue, la formule du produit $I_{n}|l| \mathbb{Q}_{l} \otimes_{\mathbb{Z}} D_{I_{n}}\left(K_{\infty} / K_{n}\right) \longrightarrow \mathbb{Q}_{l} \otimes_{\mathbb{Z}}$ Gal $\left(K_{\infty} / K_{n}\right)$ devient llapplication diagonale $\underset{l_{n} \mid \ell}{\oplus} \mathbb{Q}_{\ell} \longrightarrow \mathbb{Q}_{\ell}$; et l'image d'une $\ell$-unité $\epsilon$ dans chaque facteur $\mathbb{Q}_{\ell}$ est le nombre $\log _{\ell} \circ N_{K},_{I_{n}} / \mathbb{Q}_{\ell}(\epsilon)=$ $\operatorname{Tr}_{K_{n, I_{n}} / Q_{l}}{ }^{\log _{l}} \epsilon$. Nous retrouvons bien la description du groupe de défaut de la conjecture de Gross donnée au chapitre 1.2; $2 \S c$.

DÉFINITIONIV.2.7.- Nous disons que le car actère $\delta_{\text {Gross }}=\sum_{\varphi}\left(\operatorname{deg} \widetilde{\Omega} c_{\varphi}^{\prime}\right)_{\varphi}$ est le caractère de défaut de la conjecture de Gross dans la tour cyclotomique $\mathrm{K}_{\infty} / \mathrm{K}$. Son degré deg $\delta_{\text {Gross }}=$ $\left\langle\delta_{\text {Gross }},{ }^{\delta}\right.$ Gross $\rangle$ est le défaut de la conjecture de Gross à chaque étage fini assez grand de la tour cyclotomique. 
COROLLAIRE IV.2.8.-Dans une tour cyclotomique, le défaut de la conjecture de Gross est majoré par l'invariant lambda d'lwasawa associé aux groupes de $l$-classes $\mathrm{Cl}_{\mathrm{n}}^{\prime}$. Plus précisément, on a l'iné galité entre caractèr es

$$
(\star):
$$

$$
\delta_{\text {Gross }} \leq \lambda
$$

Démonstration : Cela résulte directement des inégalités deg $\widetilde{\Omega}_{\varphi}^{\prime} \leq$ $\operatorname{deg} P c_{\varphi}^{\prime}$, pour chaque caractère $\varphi$.

Nous sommes maintenant en mesure de déterminer le paramètre $\lambda_{l}$ défini dans la proposition 5 . Pour chaque caractère $l$-a dique irréductible $\varphi$ du groupe $\Delta$, la participation de $\varphi$ dans $\Delta$ (i.e. la quantité $\frac{\left\langle\lambda_{\ell}, \varphi\right\rangle}{\langle\varphi, \varphi\rangle}=\frac{1}{d_{\varphi}}\left\langle\lambda_{\ell}, \varphi\right\rangle$ ) est égale au degré du quotient $\frac{\tilde{\Omega}_{\varphi}}{\tilde{\Omega}_{\varphi}^{\prime}}$. Sous la conjecture de Gross, elle se réduit donc au degré du numérateur $\tilde{\Omega}_{c}{ }^{(\star \star)}$, qui mesure la dimension sur $z_{\varphi}$ de la $\varphi$-partie du quotient des genres $c_{n}=c / c^{\omega_{n}}$ ( pour $\left.n \geq m\right)$. Par le même argument que plus haut, cette quantité s'écrit encore :

$$
\begin{aligned}
& \operatorname{dim}_{Z_{\varphi}} \dot{\varphi}_{n}^{e} \varphi=\operatorname{dim}_{Z}\left(\left[I_{n} \mid l I_{n}\left(K_{\infty} K_{n}\right)\right] / h^{b_{\infty}} K_{\infty} / K_{n}\left(\varepsilon_{n}\right)\right)^{e} \varphi
\end{aligned}
$$

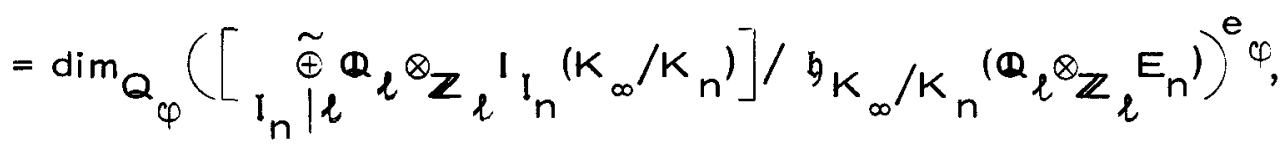

si $I_{\mathfrak{l}}\left(K_{\infty} / K_{n}\right)$ désigne le sous-groupe d'inertie de la place $I_{n}$ dans l'extension procyclique $K_{\infty} / K_{n}$, et $\varepsilon_{n}=\mathbb{Z}_{\ell} \otimes_{\mathbb{Z}} E_{n}$ le tensorisé du gr oupe des unités de $K_{n}$. Et, comme $I_{\mathfrak{l}}\left(K_{\infty} / K_{n}\right)$ est diindice fini

(*) Ce résultat est le pendant du théorème de Gillard sur le défaut de la conjecture de Leopoldt cité plus loin ( cf. $2 \S b$ ).

$(\star \star)$ Dans ce cas, on a même $\tilde{\Omega}_{\varphi}=\Omega_{c} c_{\varphi}$, puisque le module $\Sigma$ est annulé par le polynôme cyclotomique $\omega_{m}$. C'est le théorème de semisimplicité de Greenberg. 
dans $D_{1}\left(K_{\infty} / K_{n}\right)$ ( puisque les places au-dessus de $\ell$ sont presque totalement ramifiées dans $\left.K_{\infty} / K_{n}\right)$, le $\mathbb{Q}_{\ell}$-espace vectoriel $\mathbb{Q}_{l} \otimes_{\mathbb{Z}} I_{I}\left(K_{\infty} / K_{n}\right)$ n'est autre que $\mathbb{Q}_{l} \otimes_{\mathbb{Z}} D_{I}\left(K_{\infty} / K_{n}\right)$. Prenant à

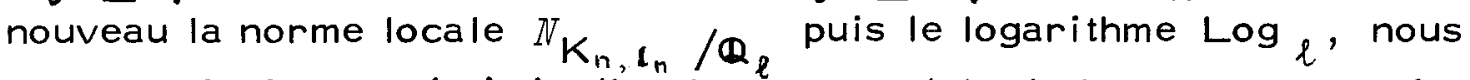
sommes ainsi ramenés à étudier le sous-module de la somme restreinte $\tilde{I}_{n}|l| \mathbb{Q}_{l}$ engendré par les images $\left(\operatorname{Tr}_{K_{n, I}} / \mathbb{Q}_{l}{ }^{\left.\circ \log _{l} \in\right)_{l} \mid l \text { des uni- }}\right.$ tés de $K_{n}$.

THÉORÈME IV.2.9.-- Supposons réunies les trois conditions suivantes:

(i) La conjecture de Leopoldt est vérifiée dans la tour cyclotomique $\mathrm{K}_{\infty} / \mathrm{K}$.

(ii) La conjecture de Gross est vérifiée dans la tour cyclotomique $\mathrm{K}_{\infty} / \mathrm{K}$.

(iii) L'extension abélienne $\mathrm{K} / \mathrm{F}$ admet une conjugaison com plexe (i.e. $K$ est une extension quadratique totalement imaginaire d'un sous-corps totalement réel de F).

Convenons alors de dire qu'un caractère $\ell$-adique du groupe de Galois $\Delta=G a l(F / K)$ est réel lorsque ses facteurs absolument irréductibles prennent la valeur +1 sur la conjugaison complexe; quil est imaginaire lorsquils prennent la valeur -1 .

Cela étant, le caractère $\lambda_{\ell}$ du groupe $\Delta$, associé à la suite $\left(\mathcal{L}_{n}\right)_{n} \in \mathbb{N}$ des sous-groupes respectifs des $\ell$-groupes de classes des corps $K_{n}$, engendrés par les images des places au-dessus de $l$, est égal à la partie imaginaire du caractère $\psi_{l}=\sum_{l \mid l} g_{l} X_{l}$, somme des induits à $\triangle$ des caractères des représentations unités des sous-groupes de décomposition dans $K / F$ des places de $l$ de $F$ au-dessus de $l$, comptés avec une multiplicité $g_{\mathfrak{l}}$ égale à leur indice de décomposition dans l'extension cyclotomique $K_{\infty} / K$ :

$$
\lambda_{l}=\psi_{l}^{-}=\sum_{I \mid l} g_{I} x_{I}^{-} \text {. }
$$

Et les paramètres du $\Lambda[\Delta]$-module $c=\left\lfloor\right.$ im $\alpha_{n}$ sont donnés par les formules :

$$
\rho=0 ; \quad \mu=\mu_{c} ; \quad \lambda=\lambda_{c}+\psi_{l}
$$


Remarque : Les conditions (i) et (ii) sont essentielles pour la démonstration du théorème 9 . La condition ( $i i i)$, en revanche, est purement technique et destinée à faciliter le calcul du paramètr e $\lambda_{\ell}$. Elle peut être ainsi remplacée par une condition de même nature, mais por tant cette fois sur les places au-dessus de $\ell$ (cf. scolie ci-dessous). On notera qu'elle suppose que $\ell$ soit impair.

Démonstration : Les trois conditions énoncées se propageant le long de la tour cyclotomique, ce n'est pas $r$ estreindre la généralité que de supposer les places au-dessus de $l$ sans décomposition dans $\mathrm{K}_{\infty} / \mathrm{K}$. La somme restreinte $\underset{\mathfrak{l} \mid \ell}{\oplus} Q_{\ell}$ est alors un $\mathbb{Q}_{\ell}[\Delta]$-module de caractère $\psi_{\ell}-1$, image par la trace semi-locale $\operatorname{Tr}_{\ell}=\left(\operatorname{Tr}_{K_{l}} / Q_{\ell}\right)^{\prime} \mid \ell d u$ noyau $\tilde{K}_{\ell}$ de la trace globale $\operatorname{Tr} K_{\ell} / Q_{\ell}$ dans l'algèbre semi-locale $K_{\ell}=\mathbb{Q}_{\ell} \otimes_{\mathbb{Q}} K$. Maintenant, par la conjecture de Leopoldt, les logarithmes $l$-adiques des unités de $K$ engendrent la composante réelle du noyau $\tilde{\mathrm{K}}_{\ell}$, et le sous-module de $\underset{\mathrm{I} / \ell}{\oplus} \mathbb{Q}_{\ell}$ engendré par les images des logarithmes des unités de $K$ est donc un $Q_{\ell}[\Delta]$-module de caractère $\psi_{l}^{+}-1$. II vient ainsi :

$$
\lambda_{l}=\left(\psi_{l}-1\right)-\left(\psi_{l}^{+}-1\right)=\psi_{l}^{-} \text {, }
$$

ce qui, compte-tenu de la proposition 5 , conduit au résultat annoncé.

SCOLIE IV.2.10. - Supposons réunies les conditions (i) et (ii) du théorème 9 , ainsi que la condition :

(iii)' Les places de F au-dessus de $\ell$ ont même décomposition dans l'extension abélienne $K_{\infty} / F$. Dans ces conditions, le paramètre $\lambda_{\ell}$ du groupe $\Sigma=\frac{\lim }{n} \mathcal{L}_{n}$ est donné par la formule :

$$
\lambda_{l}=\psi_{\ell} \wedge \bar{x}_{\infty}=g_{l}\left(x_{l} \wedge \bar{x}_{\infty}\right)
$$

où $x_{l}$ (respectivement $x_{\infty}$ ) est la somme $\sum_{I / l} x_{I}$ (respectivement $\left.\sum_{p \mid \infty} x_{p}\right)$ des induits à $\Delta$ des caractères des représentations unités des sous-groupes de décomposition dans $K / F$ des places de $F$ au-dessus de $l$ (respectivement à l'infini), et $\bar{x}_{\infty}=\sum_{p \mid \infty} \bar{x}_{p}=\sum_{p \mid \infty}\left(x-x_{p}\right)$, la somme des supplémentaires des $X_{p}$. 
Démonstration: Identique à celle du théorème 9 , l'hypothèse $\chi_{\infty} \wedge \bar{x}_{\infty}=0$ est remplacée par $x_{l} \wedge \bar{x}_{l}=0$.

COROLLAIRE IV.2.11. - Si K est un corps abélien imaginaire, et $F$ un sous-corps de $K$, le paramètre $\lambda_{\ell}$ est donné par la formule :

$$
\lambda_{\ell}=\psi_{l} \wedge \bar{x}_{\infty}=\psi_{l} \text {. }
$$

En particulier, l'égalité $\lambda_{\ell}=0$ a lieu si et seulement si les places au-dessus de $\ell$ dans le sous-corps réel maximal $K_{+}$de $K$ ne se décomposent pas dans l'extension quadratique $\mathrm{K} / \mathrm{K}_{+}$.

\section{c.- Capitulation et structure des groupes de classes -}

Les théorèmes de paramétrage appliqués au groupe $c=\left\lfloor\right.$ im $C_{n}$ montrent que, pour chaque entier relatif $k$ et chaque caractère $\ell$-adique irréductible $\varphi$ du groupe $\Delta$, les $\varphi$-composantes des sous-groupes $C_{n}^{e^{n+k}}$ ont un ordre constant, pour tout $n$ assez grand. Ce ré sultat peut, en fait, être sensiblement précisé :

PROPOSITION IV.2.12.- Soit $q \geq$ deg $\mu$ un majorant de l'invariant mu d'lwasawa du groupe $C$. Pour chaque naturel $n$ assez grand, l'homomorphisme d'extension $j_{n+1} / n$ de $C_{n}$ dans $C l_{n+1}$ induit un $\mathbb{Z}_{l}[\Delta]-$ isomorphisme canonique du sous-groupe $\mathrm{Cl}_{\mathrm{n}}^{l^{q}}$ sur le sous-groupe $C \ell_{n+1}^{q+1}$. En particulier, pour chaque entier relatif $k$ fixé, les grou pes $C l_{n}^{l^{n+k}}$ sont $\mathbb{Z}_{\ell}[\Delta]$-isomorphes dès que $n$ est assez grand.

Démonstration : Considérons le $\Lambda[\Delta]$-module $x=c^{l^{9}}$. Pour chaque caractère $\ell$-adique irréductible $\varphi$ du groupe $\Delta$, le polynôme caractéristique de la $\varphi$-composante de $X$ est un polynôme distingué $p_{\varphi}$ de l'anneau $Z_{\varphi}[\gamma-1]$, car il est étranger à $l$ d'après le choix de $q$. En particulier, le sous-module de $z_{\varphi}$-torsion, disons $T_{\varphi}$, du module $x_{\varphi}$ est donc fini . Si $x_{\varphi}$ ne se réduit pas à $T_{\varphi}$, le polynôme $p_{\varphi}$ est non trivial, et l'une de ses puissances annule $\top_{\varphi}$, de sorte que la puissance immédiatement supérieure, disons $f_{\varphi}$, annule $x_{\varphi}$; sinon, prenons pour $f_{\varphi}$ n'importe quel polynôme distingué annulant $T_{\varphi}$. Dans les deux cas, $f_{\varphi}$ annule $x_{\varphi}$. Cela étant, pour chaque $n$ 
assez grand, la $\varphi$-composante du groupe $c l_{n}^{l^{q}}$, qui est l'image de $\times$ par l'application norme $N_{\mathrm{n}}$, est un $Z_{\varphi}[\gamma-1]$-module annulé par $f_{\varphi}$, et le lemme 1.10 appliqué à chaque $\varphi$-composante du groupe $\mathrm{Cl}_{\mathrm{n}+1}$ nous prouve que l'image de ce groupe par la norme algébrique $\nu_{n+1 / n}=\frac{w_{n+1}}{w_{n}}=\sum_{k=0}^{l-1} \gamma^{k l}$ est le sous-groupe $a_{n+1}^{l}$. II vient donc :

$$
j_{n+1 / n}\left(a_{n}^{l^{q}}\right)=j_{n+1 / n} \circ N_{n+1 / n}\left(c_{n+1}^{l^{q}}\right)=\nu_{n+1 / n}\left(c l_{n+1}^{l^{q}}\right)=c l_{n+1}^{l^{q+1}}
$$

ce qui est le résultat annoncé.

THÉORĖME IV.2.13 ${ }^{(*)}$. - Lorsque le paramètre $\mu_{c}$ attaché au $\Lambda[\Delta]-$ module noethérien et de torsion $C=\left\lfloor\right.$ im $C \ell_{n}$ est nul, les assertions suivantes sont vérifiées pour $n$ assez grand :

(i) L'application naturelle $N_{\mathrm{n}}$ du groupe Csur le $\ell$-groupe des classes $\mathrm{Cl}_{n}$ induit un $\mathbb{Z}_{\ell}[\Delta]$ - isomorphisme du sous-groupe de $\mathbb{Z}_{\ell}$-torsion $T$ de $C$ sur le sous-groupe $\mathrm{Cap}_{\mathrm{n}}$ de $\mathrm{Cl}_{\mathrm{n}}$ for mé des classes de diviseurs de $K_{n}$ qui capitulent dans $K_{\infty}$.

(ii) Le sous-module Cap ${ }_{n}$ est unfacteur direct du $\ell$-groupe des classes $\mathrm{Cl}_{\mathrm{n}}$.

(iii) Pour chaque caractère $\ell$-adique irréductible $\varphi$ du groupe $\Delta$, il existe une famille $\left(\alpha_{1}, \ldots, \alpha_{\lambda_{\varphi}}\right)$ d'entiers relatifs, et une famille $\left(\alpha_{\lambda_{\varphi}+1}, \ldots, \alpha_{n_{\varphi}}\right)$ d'entiers naturels, tels que la décomposition élémentaire du $Z \varphi_{\varphi}^{- \text {module } C l_{n}^{e_{\varphi}}}$ s'écrive :

$$
\operatorname{cl}_{n}^{e} \varphi\left(\oplus_{i=1}^{\lambda} \varphi z_{\varphi} / e^{n+\alpha_{i}} z_{\varphi}\right) \oplus\left(\underset{i=\lambda \varphi_{\varphi}+1}{\oplus^{\prime} \varphi} z_{\varphi} / e^{\alpha i} z_{\varphi}\right) \text {. }
$$

Dans la décomposition obtenue, le terme de droite mesure la capitulation $\operatorname{Cap}_{n}^{e_{\varphi}}$, et le terme de gauche l'image $\widetilde{C}_{n}^{e_{\varphi}}$ de $\mathrm{Cl}_{n}^{e_{\varphi}}$ dans $\mathrm{Cl}_{\infty}^{e_{\varphi}}$; l'indice $\lambda_{\varphi}$ est la dimension sur $Z_{\varphi}$ de la $\varphi$-composante du groupe $C$, et l'indice $x_{\varphi}$ la dimension sur $F_{\varphi}{ }_{\varphi}=z_{\varphi} / l z_{\varphi}$ de la $\varphi$-composante du quotient $c / C l$.

(*) Ce résultat précise dans le cas cyclotomique considér é ici, celui plus général, obtenu en collaboration avec Grandet ( cf. [GJ]) . L'hypothèse $\mu_{c}=0$, est vérifiée dès que $K$ est absolument abélien, d'après un théorème de Ferrero et Washington ( cf. [FW ]) . 
SCOLIE IV.2.14.- Sous les hypothèses du théorème, la limite pro jective des groupes $\mathrm{Cl}_{n}^{e_{\varphi}}$ est un $Z_{\varphi}$-module noethérien isomorphe à la somme directe :

$$
c^{e} \varphi \simeq z_{\varphi}^{\lambda} \varphi \oplus(\underbrace{\oplus}_{i=\lambda}{ }_{\varphi}^{+1} z_{\varphi} / e^{\alpha_{i}} z_{\varphi})
$$

leur limite inductive est un $\mathrm{Z}_{\varphi}$-module divisible de corang $\lambda_{\varphi}$ :

$$
\mathrm{cl}_{\infty}^{\mathrm{e} \varphi} \simeq\left(\mathrm{Q}_{\varphi} / \mathrm{z}_{\varphi}\right)^{\lambda} \varphi
$$

Démonstration : Le paramètre $\mu$ étant supposé nul, pour chaque caractère $\ell$-adique irréductible $\varphi$ la $\varphi$-composante $c_{\varphi}=c^{e_{\varphi}} \mathrm{du}$ groupe profini $C$ est un $Z_{\varphi}$-module noethérien de la forme :

$$
c_{\varphi} \approx z_{\varphi}^{\lambda} \oplus \oplus T_{\varphi}
$$

où $T_{\varphi}$ est un $z_{\varphi}$-module fini. Désignons par $F_{\varphi}=z_{\varphi} / l z_{\varphi}$ le corps résiduel de l'anneau $Z_{\varphi}$ : notons $x_{\varphi}=\operatorname{dim}_{F_{\varphi}} c_{\varphi} / \ell c_{\varphi}$; faisons choix d'une pseudo-base $\left(x_{1}, \ldots, x_{x_{\varphi}}\right)$ de $c_{\varphi}$, et écrivons :

$$
c_{\varphi}=\left(\oplus_{i=1}^{\lambda} \varphi z_{\varphi} x_{i}\right) \oplus\left(\oplus_{i=\lambda}^{\oplus} \varphi+1 z_{\varphi} / e^{\alpha} z_{\varphi} x_{i}\right)
$$

avec $\quad \alpha_{\lambda_{\varphi}+1} \geq \ldots \geq \alpha_{\chi_{\varphi}}>0$

la décomposition élémentaire du $\mathrm{Z}_{\varphi}$-module $C$.

Fixons $m$ assez grand pour avoir $T_{\varphi}^{\ell^{m}}=1$, puis considér ons le quotient (d'ordre $e^{m \lambda} \varphi+\sum_{i=1}^{\lambda} \varphi \alpha_{i}$ ):

$$
c_{\varphi} / c_{\varphi}^{l^{m}}=\left(\oplus_{i=1}^{\lambda} \varphi z_{\varphi} / l^{m} z_{\varphi} \bar{x}_{i}\right) \oplus\left(\oplus_{i=\lambda}^{\oplus} \varphi z_{\varphi} / z^{\alpha_{i}} z_{\varphi} \bar{x}_{i}\right)
$$

Puisquil est fini, les quotients $C l_{n} / \mathrm{Cl}_{n}^{l^{n}}$, dont il est la limite projective, lui sont tous isomorphes pour $n$ assez grand. Ecrivons donc: 


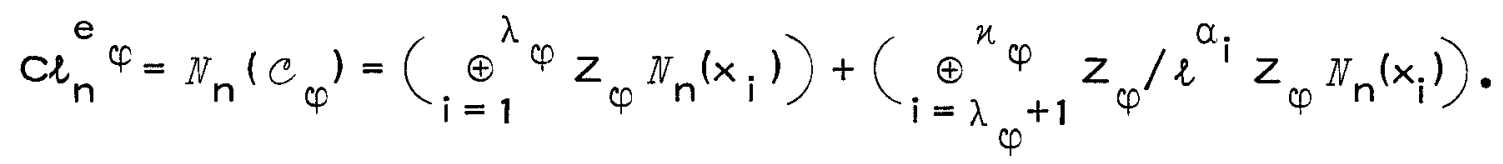

Nous obtenons :

$\left(c l_{n} / c_{n}^{l^{m}}\right)^{e} \varphi=\left(\sum_{i=1}^{\lambda} \varphi z_{\varphi} / e^{m} z_{\varphi} N_{n}\left(\bar{x}_{i}\right)\right)+\left(\sum_{i=\lambda}^{x} \varphi z_{\varphi} / z^{\alpha} / z_{\varphi} N_{n}\left(\bar{x}_{i}\right)\right)$

et l'égalité des indices $\left(\mathrm{Cl}_{n}^{e_{\varphi}}: \mathrm{Cl}_{n}^{l^{m}} \mathrm{e}_{\varphi}\right)=\left(c_{\varphi}: c_{\varphi}^{l^{m}}\right)$ nous prouve que toutes les sommes sont directes:

$$
\begin{aligned}
\left(c_{n} / c_{n}^{l^{m}}\right)^{e} \varphi & =\left(\underset{i=1}{\oplus \varphi} z_{\varphi} / e^{m} z_{\varphi} N_{n}\left(\bar{x}_{i}\right)\right) \oplus\left(\underset{i=\lambda}{\oplus}{ }^{x} \varphi z_{\varphi} / l^{\alpha_{i}} z_{\varphi} N_{n}\left(\bar{x}_{i}\right)\right) \\
& \simeq c_{\varphi} / c_{\varphi}^{l^{m}} .
\end{aligned}
$$

En particulier, le sous-groupe $C_{p_{\varphi, n}}={\underset{i=\lambda}{\oplus} \lambda_{\varphi}+1}_{\varphi} z_{\varphi} / e^{\alpha_{i}} z_{\varphi} / N_{n}\left(x_{i}\right)=$

$N_{\mathrm{n}}\left(\mathrm{T}_{\varphi}\right)$ est donc un sous-module pur, c'est-à-dire un facteur direct du $l$-groupe des classes $C_{n}^{e_{\varphi}}$. Nous allons voir que $C_{p}, n$ est exactement la $\varphi$-partie de la capitulation $\mathrm{Cap}_{n}$. Pour cela, fixons $n$ assez grand, faisons choix d'une pseudo-base $\left(x_{n, 1}, \ldots, x_{n, x_{\varphi}}\right)$ du $Z_{\varphi}$-module $\mathrm{Cl}_{n}^{e_{\varphi}}$, et écrivons :

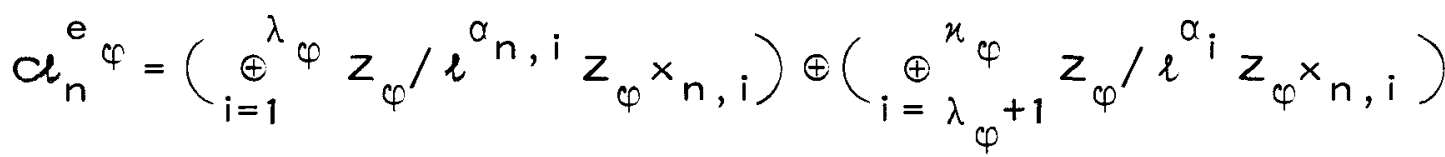

avec $\left\{\begin{array}{l}\alpha_{n, 1} \geq \ldots \geq \alpha_{n, \lambda} \geq m \geq \alpha_{n, \lambda_{\varphi}+1} \geq \ldots \geq \alpha_{n_{\varphi}} \\ x_{n, i}=N_{n}\left(x_{i}\right), \text { pour } i=\lambda_{\varphi}+1, \ldots, x_{\varphi},\end{array}\right.$

la décomposition élémentaire correspondante, les conditions sur les $\alpha_{n, i}$ étant imposées par la décomposition du quotient $\left(\alpha_{n} / \alpha_{n}^{\ell^{m}}\right) e_{\varphi}$. Par surjectivité de la norme, et compte-tenu de l'isomorphisme ca nonique $\mathrm{Cl}_{n} / \mathrm{Cl}_{n}^{l} \simeq \mathrm{Cl}_{n+1} / \mathrm{Cl}_{n+1}^{l} \simeq \mathrm{c} / \mathrm{c}^{l}$, nous pouvons relever les $x_{n, i}$ en un système de générateurs de $\mathrm{Cl}_{n+1}^{e_{\varphi}}$, et écrire :

$a_{n+1}^{e} \varphi\left(\oplus_{i=1}^{\lambda} \varphi z_{\varphi} / e^{\alpha+1, i} z_{\varphi} x_{n+1, i}\right) \oplus\left(\underset{i=\lambda_{\varphi}+1}{\oplus^{x} \varphi} z_{\varphi} / e^{\alpha} z_{\varphi} \times{ }_{n+1, i}\right)$ 
avec $\left\{\begin{array}{l}N_{n+1 / n}\left(x_{n+1, i}\right)=x_{n, i} \text { pour } i \leq \lambda_{\varphi} \\ x_{n+1, i}=N_{n+1}\left(x_{i}\right), \text { pour } i>\lambda_{\varphi},\end{array}\right.$ en notant $l^{\alpha+1, i}$ l'ordre de $x_{n+1, i}$ dans $C l_{n+1}^{e_{\varphi}}$.

Cela posé, faisons choix d'un multiple distingué $f_{\varphi}$ du polynôme caractéristique $\mathrm{PC}_{\varphi}$, annulant $c_{\varphi}$, et appliquons le lemme 1. 10. L'égalité entre opérateurs $\nu_{n+1 / n}=l\left(1+l a_{n+1 / n}\right)$, où $\nu_{n+1 / n}=\frac{\omega_{n+1}}{\omega_{n}}$ est la norme algébrique, nous prouve que les $x_{n+1, i}$ sont, à l'action près d'un inversible de l'algèbre de Galois, les étendus des $x_{n, i}$. En particulier, nous avons donc $\alpha_{n+1, i} \leq 1+\alpha_{n, i}$, pour $\mathrm{i}=1, \ldots, \lambda_{\varphi}$. Mais ce résultat n'est compatible avec l'identité $\left|c l_{n+1}^{e_{\varphi}}\right| /\left|C l_{n}^{e_{\varphi}}\right|=\ell^{\langle\lambda, \varphi\rangle}=\ell^{d_{\varphi} \lambda_{\varphi}}$, que si nous avons effective ment l'égalité $\alpha_{n+1, i}=1+\alpha_{n, i}$, pour $i=1, \ldots, \lambda_{\varphi}$, ainsi qu'une somme directe :

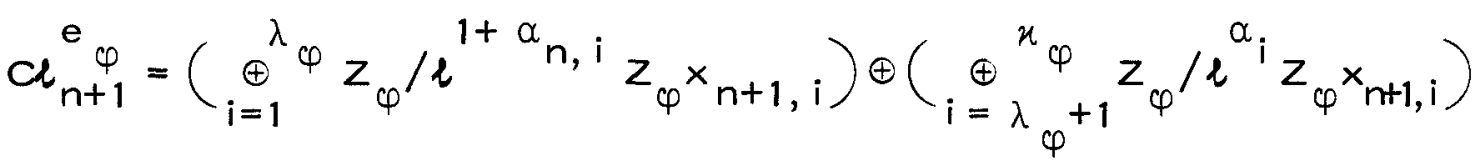

Procédant alors par récurrence, nous pouvons fabriquer, pour chaque $i=1, \ldots, x_{\varphi}$, une famille projective $\left(x_{n, i}\right)_{n} \geq n_{0}$ de classes de diviseurs, possédant les propriétés suivantes :

1) Les éléments $x_{i}^{\prime}=\frac{\varliminf i m}{n} \times_{n, i}$ forment une pseudo-base du groupe profini $C_{\varphi}$ :

$$
c_{\varphi}=\left(\underset{i=1}{\oplus} \varphi z_{\varphi} \times{ }_{i}^{\prime}\right) \oplus\left(\underset{i=\lambda \varphi^{+1}}{\oplus^{\lambda} \varphi} z_{\varphi} / l^{\alpha} z_{\varphi} \times{ }_{i}^{\prime}\right)
$$

Les $\lambda_{\varphi}$ premiers sont libres, les autres de torsion, d'ordres respectifs $e^{\alpha}$.

2) Pour chaque $n \geq n_{o}$, les éléments $x_{n, i}=N_{n}\left(x_{i}^{\prime}\right)$ for ment une pseudo-base du $Z_{\varphi}$-module $\mathrm{Cl}_{n}^{\mathrm{e}_{\varphi}}$. Les $\lambda_{\varphi}$ premiers sont d'ordres respectifs $l^{n+\alpha_{i}}$, pour un $\alpha_{i}$ dans $\mathbb{Z}$; les autres d'ordres respectifs $\ell^{\alpha_{i}}$, indépendant de $n$. 
Cela étant, l'homomorphisme d'extension $j_{n+1 / n}$ envoie le groupe $\mathrm{Cl}_{n}^{e_{\varphi}}$ sur le sous-groupe $\mathrm{Cl}_{n+1}^{l e_{4}}$ de $\mathrm{Cl}_{n+1}^{e_{\varphi}}$, et le sous-groupe $C_{p}, \mathrm{n}=N_{\mathrm{n}}\left(T_{\varphi}\right)$ de $C_{\mathrm{n}}^{\mathrm{e}}$ sur le sous-groupe $C_{p}, \mathrm{n+1}=N_{\mathrm{n}+1}\left(T_{\varphi}\right)$ de $\mathrm{Cl}_{n+1}^{e_{\varphi}}$. En particulier, les éléments de $\mathrm{Cl}_{n}^{e_{\varphi}}$ qui capitulent dans $\mathrm{Cl} e_{n+1}^{e_{\varphi}}$ forment un sous-groupe d'ordre $\left|l_{n}^{e_{\varphi}}\right| /\left|l_{n+1}^{l e_{\varphi}}\right|=\ell^{d_{\varphi}}\left(x_{\varphi}-\lambda_{\varphi}\right)$; ce sont les éléments d'ordre $l$ de la somme $C_{p}{ }_{\varphi, n}=\underset{i=\lambda_{\varphi}+1}{x_{\varphi}} z_{\varphi} / l^{\alpha_{i}} z_{\varphi} \times{ }_{n}$. Plus généralement, ceux qui capitulent dans $C_{n+k}^{e_{\varphi}}$ sont les éléments de $l^{k}$-torsion du sous-groupe $C_{P}, n$. Et les éléments de $\mathrm{Cl}_{n}^{e_{\varphi}}$ qui capitulent dans $\mathrm{Cl}_{\infty}^{e_{\varphi}}$ sont donc exactement les éléments de $C_{p} \varphi, n$. Enfin, comme la restriction au sous-groupe $\widetilde{C}_{l_{n}}^{e_{\varphi}}=$ $\oplus_{i=1}^{\lambda} \varphi z_{\varphi} / e^{n+\alpha_{i}} z_{\varphi} x_{n, i}$ de l'homomorphisme d'extension $j_{n}$ est injective, le groupe $\mathrm{Cl}_{\infty}^{\mathrm{e}_{\varphi}}=\underline{\lim } \mathrm{Cl}_{n}^{\mathrm{e}_{\varphi}}$ s'identifie par conséquent à la réunion croissante des groupes $\underset{\mathrm{C} l_{n}}{e_{\varphi}}$; c'est un $z_{\varphi}$-module divi sible de corang $\lambda_{\varphi}$.

PROPOSITIONIV.2.15. - Pour chaque ensemble fini $S$ de places de $\mathbb{Q}$, désignons par $C l_{n}^{S}$ le $\ell$-groupe des $S$-classes de diviseurs du corps $K_{n}(i . e$. le quotient du $\ell$-groupe des classes de diviseurs de $K_{n}$ par le sous-groupe $C_{n}(s)$, engendré par les classes des divi seurs construits sur les places au-dessus de $S$ ).

(i) Si $S$ ne contient pas $l$, le groupe $c^{S}=\left\lfloor\right.$ im $C_{n}^{S}$ est un $\Lambda[\Delta]$-module noethérien et de torsion, qui a mêmes paramètres que le groupe $c=\lfloor$ lim $\mathrm{Cl}$.

(ii) Si $S$ contient $l$, le groupe $c^{S}=\lim C l_{n}^{S}$ est un $\Lambda[\Delta]$ - module noethérien et de torsion, qui a mêmes paramètres que le groupe $C^{\prime}=\left\lfloor\right.$ im $C l_{n}^{\prime}$.

Dans tous les cas, le paramètre $\mu$ est celui $\mu_{c}$ du groupe $C^{\prime}$. En particulier, sous la condition $\mu_{c}=0$, tous les groupes $C e_{n}^{S}$ vérifient les assertions du théorème 13 et du scolie 14 .

Démonstration : Si $S$ ne contient pas $l$, la loi de décomposition dans une tour cyclotomique montre que les places de $K$ au-dessus de $\ell$ sont 
finiment décomposées dans $K_{\infty} / K$. En particulier, les groupes $C \ell_{n}(S)$ (respectivement $C_{n}^{\prime}(S)$ ) ont un ordre borné indépendamment de $n$. II vient donc:

$$
c^{S} \sim c \text { et } c^{\prime S}=c^{S \cup\{l\}} \sim c^{\prime} \text {, comme annoncé. }
$$

En fait, il est facile de voir qu'il n'y a qu'un nombre fini de groupes $c^{S}$ distincts pour une $\mathbb{Z}_{\mathfrak{l}}$-extension donnée : En effet, la famille des sous modules ( $C(S)$ ) possède un plus grand élément dans l'ensemble noethérien des sous-modules de $C$; ce qui revient à dire qu'il existe un ensemble fini de places, disons $T$, tel qu'on ait $C^{S}=C^{\top}$ dès que $S$ contient $T$. Dans le cas, considéré ici, de la $\mathbb{Z}_{\ell}$-extension cyclotomique diun corps de nombres $T$ est simplement l'ensemble des places au-dessus de $l$. D'après le corps de classes local, en effet, les places de $K_{\infty}$ étrangères à $\ell$ sont complètement décomposées dans toute $\ell$-extension de $K_{\infty}$ où elles ne se ramifient pas, de sorte que nous avons identiquement :

$$
c^{S}=c^{\prime} \text {, lorsque } S \text { contient } 2, \quad C^{S}=c, \text { sinon . }
$$

\section{2. - ÉTUDE DU GROUPE DE GALOIS DE LA $\ell$-EXTENSION ABÉLIENNE $\ell$-RAMIFIÉE MAXIMALE DU CORPS $K_{\infty} \cdot$}

a.-Définition du groupe $a$.

Désignons par $M_{\infty}$ la $\ell$-extension abélienne $\ell$-ramifiée maximale du corps $K_{\infty}$, et notons $a=\operatorname{Gal}\left(M_{\infty} / K_{\infty}\right)$ son groupe de Galois . L'extension $M_{\infty}$ est évidemment la réunion des $\ell$-extensions abéliennes $\ell$-ramifiées maximales $M_{n}$ des sous-corps de degré fini $K_{n}$ de $K_{\infty}$, et nous disons que $M_{n}$ est le $l$-corps des $l$-classes in finitésimales de $K_{n}$, en accord avec les résultats du chapitre II, 2 , qui interpr ètent le groupe de Galois $a_{n}=$ Gal $\left(M_{n} / K_{n}\right)$ comme quotient du tensorisé $\delta_{n}^{\prime}=\mathbb{Z}_{\ell} \mathbb{Z}_{\mathbb{Z}} D_{n}^{\prime}$ du groupe $D_{n}^{\prime}$ des diviseurs de $K_{n}$ étrangers à $l$, par le sous-module $p_{n}^{\infty}$ des diviseurs principaux-in finitésimaux, image dans le sous-module $P_{n}^{\prime}=\mathbb{Z} \ell^{\otimes} P_{n}^{\prime}$ des divi seurs principaux étrangers à $l$, du sous-groupe infinitésimal $k_{n}^{\infty} d u$ tensorisé $K_{n}^{X}=\mathbb{Z}_{l} \otimes_{\mathbb{Z}} K_{n}^{X}$. Autrement dit :

DÉFINITIONIV.2.16. - Par a nous entendons le groupe de Galois Gal $\left(M_{\infty} / K_{\infty}\right)$ de la $\ell$-extension abélienne $\ell$-ramifiée maximale $d u$ 
corps $K_{\infty}$, qui slidentifie à la limite projective $\varliminf_{\mathrm{m}} a_{\mathrm{n}}$ des $\ell$-groupes de $l$-classes infinitésimales $D_{n}^{\prime} / p_{n}^{\infty}$ des corps $K_{n}$. Le groupe $a$ est un $\Lambda[\Delta]$-module noethérien, dont nous notons $\rho_{a}, \mu_{a}$, et $\lambda_{a}$ les paramètres dans $R_{\mathbb{Z}_{\ell}}^{+}(\Delta)$.

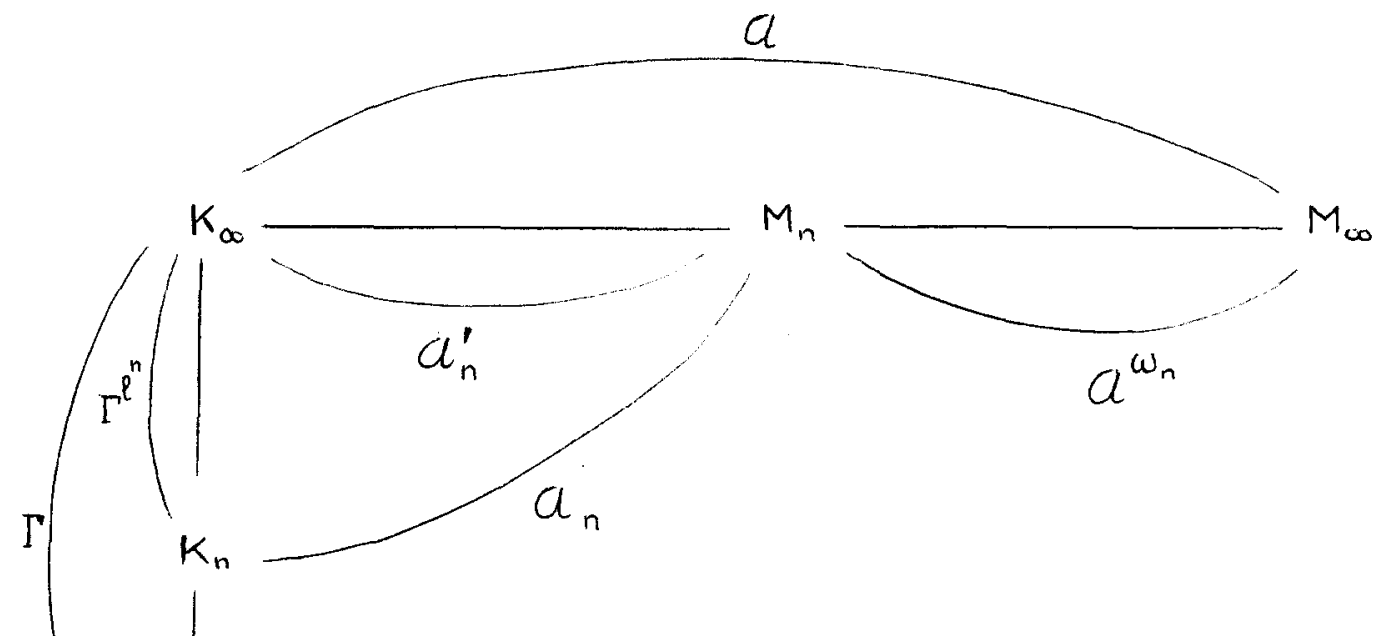

La tour cyclotomique $\mathrm{K}_{\infty} / \mathrm{K}$ étant $\ell$-ramifiée, pour chaque naturel $n$ le corps des $\ell$-classes infinitésimales $M_{n}$ est la sous-extension maximale de $M_{\infty}$ qui est abélienne sur $K_{n}$. Le groupe de Galois $a_{n}^{\prime}=\operatorname{Gal}\left(M_{n} / K_{\infty}\right)$, plus grand quotient de $a$ fixé par $I_{n}$, est ainsi le quotient des $l$ genres infinitésimaux $a / a^{\omega}$ de l'extension procyclique $K_{\infty} / K$; et le groupe $a_{n}=\operatorname{Gal}\left(M_{n} / K_{n}\right)$ est donc isomorphe, comme $\mathbb{Z}_{l}[\Delta]$ module, à la somme directe :

$$
a_{\mathrm{n}} \simeq \Gamma_{\mathrm{n}} \oplus a_{\mathrm{n}}^{\prime}=\Gamma^{l^{n}} \oplus a / a^{\omega_{n}},
$$

puisque $I_{n}$ se relève dans $a_{n}$, par un argument de projectivité. En particulier, si $r$ désigne, comme plus haut, la $\ell$-valuation de l'ordre du groupe des racines de l'unité dans $K$, il vient :

$$
a / a^{l^{n+r}} \simeq \Gamma_{n} / \Gamma_{n+r} \oplus a / a^{\nabla n, r} ;
$$

d'où, d'après le théorème 1.17: 
PROPOSITION IV.2.17. - La suite $\left(a_{n} / a_{n}^{l^{n+r}}\right)_{n \in \mathbb{N}}$ des quotients d'exposant $\ell^{n+r}$ des groupes de $l$-classes infinitésimales des corps $K_{n}$ est paramétrée par les caractères $\rho_{a}, \mu_{a}+(r-1) \rho_{a}, \lambda_{a}+1$, et $\tilde{\nu}_{a}$, pour un caractère $\ell$-adique virtuel convenable $\tilde{\nu}_{a}$ du groupe $\Delta$.

Autrement dit, pour chaque caractère $\ell$-adique irréductible $\varphi$ de $\Delta$, l'ordre $l^{\tilde{a}}(n)$ de la $\varphi$-composante du quotient $a_{n} / a_{n}^{l^{n+r}}$ est donné asymptotiquement par la formule :

$\tilde{a}_{\varphi}(n)=\left\langle\rho_{a}, \varphi\right\rangle(n+1) l^{n}+\left\langle\mu_{a}+(r-1) \rho_{a}, \varphi\right\rangle \ell^{n}+\left\langle\lambda_{a}+1, \varphi\right\rangle n+\left\langle\tilde{\nu}_{a}, \varphi\right\rangle$.

COROLLAIRE IV.2,18. - La suite $\left(\Re_{n}\right)_{n} \in \mathbb{N}$ des radicaux kummé riens $\operatorname{Rad}\left(M_{n} / K_{n}\right)$, associés aux $\ell$-extensions abéliennes $\ell$-ramifiées maximales $M_{n}$ des corps $K_{n}$, est paramétrée par les caractères $\rho_{a}^{*}, \mu_{a}^{*}+(r-1) \rho_{a}^{*}, \lambda_{a}^{*}+\omega$, et $\tilde{\nu}_{a}^{*}$, reflets des précédents dans linvolution du miroir.

Nous sommes maintenant en mesure d'exprimer les para mètres du groupe $a$ à partir de ceux du groupe $c^{\prime}$. Convenons de désigner :

- par $X_{\infty}=\sum_{p \mid \infty} X_{p}$, la somme des induits à $\Delta$ des caractères des représentations unités des sous-groupes de décomposition des places à l'infini du corps $F$ dans l'extension abélienne $\mathrm{K} / \mathrm{F}$;

- par $\psi_{\ell}=\sum_{l \mid l} g_{\mathfrak{l}} x_{\mathfrak{l}}$, la somme des induits à $\Delta$ des caractères des représentations unités des sous-groupes de décomposition des places I de $F$ au-dessus de $l$ dans $K / F$, comptés avec une multiplicité glégale au nombre de places de $K_{\infty}$ au-dessus de chacune d'elles. Cela posé, nous avons :

THÉORĖME IV.2.19. - Les paramètres $\rho_{a}, \mu_{a}$, et $\lambda_{a} d u$ groupe de Galois $a$ de la $\ell$-extension abélienne $\ell$-ramifiée maximale $M_{\infty} d u$ corps $K_{\infty}$ sont donnés, en fonction de ceux $\mu_{c}$ et $\lambda_{c}$ du groupe de Galois $C^{\prime}$ de sa sous-extension non ramifiée $l$-décomposée maximale $C_{\infty}^{\prime}$, par les formules suivantes:

(i) $\rho_{a}=x_{\infty}^{*}=w x_{\infty}$

(ii) $\mu_{\mathrm{a}}=\mu_{\mathrm{c}}^{*}=w \mu_{\mathrm{c}}^{-1}$

(iii) $\lambda_{a}=\lambda_{c}^{*}+\left(\psi_{l}-1\right)^{*}=\omega\left[\lambda_{c}^{-1}+\left(\psi_{\ell}-1\right)\right]$. 
Pour établir ce résultat, nous nous appuyerons sur le lemme :

\section{LEMME IV.2.20.-}

(i) La suite $\left(\mu_{n}\right)_{n} \in \mathbb{N}$ des $\ell$-groupes de racines de l'unité des corps $K_{n}$ est paramétrée par les caractères $p=0 ; \mu=0$; $\lambda=\omega ; \nu=r \omega$.

(ii) La suite $\left(E_{n} / E_{n}^{l^{n+r}}\right)_{n \in \mathbb{N}}$, où $E_{n}$ est le groupe des unités de $K_{n}$, est paramétrée par les caractères $\rho=X_{\infty} ; \mu=(r-1) \chi_{\infty}$; $\lambda=\omega-1 ; \nu=r(\omega-1)$

(iii) La suite $\left(E_{n}^{\prime} / E_{n}^{\prime} e^{n+r}\right)_{n} \in N$, où $E_{n}^{\prime}$ est le groupe des $\ell$-unités de $K_{n}$, est paramétrée par les caractères $\rho=X_{\infty}$; $\mu=(r-1) x_{\infty} ; \lambda=\omega+\left(\psi_{\ell}-1\right) ; \nu=r\left[\omega+\left(\psi_{\ell}-1\right)\right]$.

Démonstration du lemme : L'assertion (i) résulte directement de la définition du caractère cyclotomique $\omega$, le groupe $\mu_{n}$ étant un $Z_{\omega}-$ module d'ordre $\ell^{n+r}$. L'assertion (ii) provient du théorème de représentation de Herbrand, qui nous dit que le produit $Q \otimes_{\mathbb{Z}} E_{n}$ est un $\mathbb{Q}[\Delta]$-module de caractère $\ell^{n} x_{\infty}-1$. L'assertion (iji) s'obtient de même, le produit $\mathbb{Q} \otimes \mathbb{Z} E_{n}^{\prime}$ étant un $\mathbb{Q}[\Delta]$-module de caractère $\ell^{n} x_{\infty}+\psi_{\ell}-1$, dès que $K_{n}$ contient le composé $K_{m}$ des sous-corps de décomposition des places de $K$ au-dessus de $\ell$ dans l'extension $K_{\infty} / K$.

Démonstration du théorème : La suite exacte de dualité (i) du théorème 11.2 .13 s'écrit :

$1 \longrightarrow E_{n}^{\prime}=E_{n}^{\prime} / E_{n}^{\prime} e^{n+r} \longrightarrow \Re_{n}=\operatorname{Rad}\left(M_{n} / K_{n}\right) \longrightarrow l_{l^{n+r}} C l_{n}^{\prime}-1$. Le terme de gauche est paramétré par le lemme 20 (iii) : le terme médian, par le corollaire 18 ; et le terme de droite par le scolie 3. Ecrivant que la somme alternée des paramètres est nulle, nous obtenons :

$\left.{ }^{\star}{ }^{\star}\right)$ On notera que le paramètre $\lambda=\omega-1$ n'est pas dans $R_{\mathbf{Z}}^{+}(\Delta)$, lorsque $F$ ne contient pas $\zeta$. 


$$
\left.\begin{array}{llc}
\rho_{a}^{*} & x_{\infty}+0 \\
\mu_{a}^{*}+(r-1) \rho_{a}^{*} & =(r-1) x_{\infty}+\mu_{c} \\
\lambda_{a}^{*}+\omega & =\omega+\left(\psi_{l}-1\right)+\lambda_{c} \\
\tilde{\nu}_{a}^{*} & =r\left[\omega+\left(\psi_{l}-1\right)\right]+\tilde{\nu}_{c}
\end{array}\right\} \text { d'où }\left\{\begin{array}{l}
\rho_{a}^{*}=x_{\infty} \\
\mu_{a}^{*}=\mu_{c} \\
\lambda_{a}^{*}=\lambda_{c}+\left(\psi_{l}-1\right) \\
\tilde{\nu}_{a}^{*}=\tilde{\nu}_{c}+r\left[\omega+\left(\psi_{l}-1\right)\right]
\end{array}\right.
$$

ce qui conduit bien au résultat annoncé, les caractères $\chi_{\infty}$ et $\psi_{l}$, sommes d'induits de caractères unités, étant égaux à leurs pseudoinverses respectifs $x_{\infty}^{-1}$ et $\psi_{l}^{-1}$.

\section{b.- Etude du sous-groupe de torsion $\ell$.}

DÉFINITION IV.2.21. - Par $\&$ nous entendons le sous-module de $\Lambda$ torsion du groupe de Galois $a=\mathrm{Gal}\left(\mathrm{M}_{\infty} / \mathrm{K}_{\infty}\right)$ de la $l$-extension abélienne $\ell$-ramifiée maximale du corps $K_{\infty}$. Clest un $\Lambda[\Delta]$-module noethérien de paramètres $0, \mu_{a}$, et $\lambda_{a}$. Nous notons $Z_{\infty}$ son corps des invariants.

Le sous-module de $\Lambda$-torsion $\sigma$ du groupe $a$ permet de décrire, pour chaque naturel $n$, le sous-groupe de $\mathbb{Z} \ell^{\text {-torsion }} d u$ groupe des $\ell$-classes infinitésimales du corps $K_{n}$. En effet, dans la décomposition directe :

$$
a_{n} \simeq \Gamma^{l^{n}} \oplus a / a^{w_{n}}
$$

le sous-groupe de torsion $\sigma_{n}$ de $a_{n}$ s'identifie à celui du sous-groupe $a_{n}^{\prime}=a / a^{\omega_{n}}$, donc, finalement, au sous-groupe de torsion du quotient $\sigma / \zeta^{\omega_{n}}$. Ainsi, tout comme pour la suite $\left(C \ell_{n}^{\prime}\right)_{n} \in \mathbb{N}$, la détermination des paramètres associés à la suite $\left(\tau_{n}\right)_{n} \in \mathbb{N}$ pose le problème de l'interprétation des facteurs cyclotomiques dans la fac torisation irréductible des polynômes caractéristiques respectifs $\mathrm{Pa}_{\varphi}$ des $\varphi$-composantes du module $G$. Ecrivons donc:

$$
\operatorname{Pa}_{\varphi}(\gamma-1)=e^{\mu_{\varphi}^{*}} \Omega \mathrm{a}_{\varphi}(\gamma-1) \mathrm{Ra}_{\varphi}(\gamma-1)
$$

la décomposition dans $Z_{\varphi}[\gamma-1]$ du polynôme $\mathrm{Pa}_{\varphi}$ comme produit d'une puissance de $\ell$, d'un polynôme cyclotomique $\Omega a_{\varphi}$, et d'un polynôme 
distingué $\mathrm{Ra}_{\varphi}$ étranger à tous les $\omega_{i}$. Fixons $\mathrm{m}^{\prime}$ assez grand pour

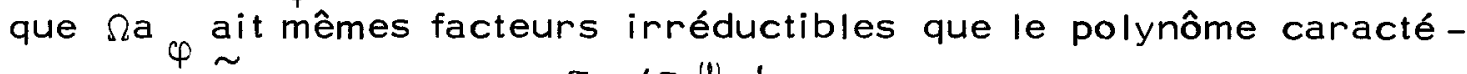
ristique $\widetilde{\Omega a}_{\varphi}$ du quotient $\sigma_{\varphi} / \sigma_{\varphi}^{\omega_{m}^{\prime}}$. Cela posé, nous avons $(\star) \stackrel{\varphi}{:}$

THÉORÈME IV.2.22. - Pour chaque naturel n assez grand, la quantité d $\varphi^{\text {. deg } \widetilde{\Omega} a} \varphi$ mesure la $\varphi$-partie du groupe de défaut de la conjecture de Leopoldt dans le corps $K_{n}$. Par suite, les polynômes caractéristiques $\mathrm{Pa} \varphi$ associés aux $\varphi$-composantes du $\Lambda[\Delta]$-module $\tau$ sont étrangers à chacun des polynômes cyclotomiques $\omega_{i}$ si et seulement si cette conjecture est vérifiée à chaque étage fini assez grand de la tour cyclotomique, ce qui a lieu en particulier dès que le corps $K$ est absolument abélien.

Démonstration : Le groupe de défaut de la conjecture de Leopoldt dans le corps $K_{n}$ est le noyau $\delta_{n}^{\infty}$, dans le tensorisé $\delta_{n}=\mathbb{Z}_{l} \otimes_{\mathbb{Z}} E_{n}$ du groupe des unités, de llapplication de semi-localisation $\delta_{n} \stackrel{s}{\longrightarrow} u_{n}=$ $I_{n} \mid l^{\oplus} U_{I_{n}}$ à valeurs dans le groupe des unités principales du complété semi-local de $K_{n}$. C'est évidemment un $\mathbb{Z}_{\ell}[\Delta]$-module projectif . Pour déterminer son caractère, disons $\delta_{n}$, nous allons évaluer de deux façons celui du groupe de Galois $a_{n} / \sigma_{n}$.

- D'un côté, puisque la définition des polynômes $\widetilde{\Omega}_{\varphi}$ est indépendante du choix de l'entier $m^{\prime}$, pour chaque $n \geq m^{\prime}$ le quotient $a_{n}^{\prime} / \sigma_{n}$ du groupe $a_{n}^{\prime}=a / a^{\omega_{n}}$ par son sous-module de torsion $\sigma_{n}$ est un $\mathbb{Z}_{\ell}[\Delta]$-module projectif de caractère $\ell^{n} \rho_{a}+\sum_{\varphi} \operatorname{deg} \tilde{\Omega}_{a} \varphi \cdot \varphi \cdot$

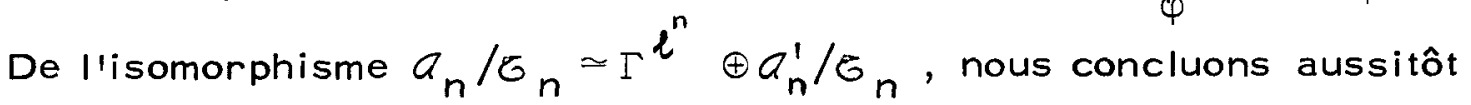
que le groupe $a_{n} / \delta_{n}$ est lui-même un $\mathbb{Z}_{\ell}[\Delta]$-module de caractère $e^{n} \rho_{a}+1+\sum_{\varphi} \operatorname{deg} \tilde{\Omega}_{a} \varphi \varphi=e^{n} x_{\infty}^{*}+1+\sum_{\varphi} \operatorname{deg} \Omega a \varphi^{\cdot} \varphi \cdot$

( $\left.{ }^{\star}\right)$ Ce résultat est dû essentiellement à Gillard ( cf. $\left[G i_{2}\right]$, th. 1 ), qui l'énonce en termes de rangs. Sous la forme proposée ici, il est le pendant exact du théorème 6 . 
- D'un autre côté, nous avons directement $a_{n} \sim u_{n} / s\left(\varepsilon_{n}\right)$ par la théorie du corps de classes (cf. Ch. $11.2,1 \S \mathrm{d}$ ) donc $Q_{l} \otimes_{\mathbb{Z}} a_{n}=Q_{l} \otimes_{\mathbb{Z}} u_{n} / Q_{l} \otimes_{\mathbb{Z}} s\left(\delta_{n}\right)$. Dans cet isomorphisme, le numérateur s'envoie isomorphiquement sur le groupe additif $\mathbb{Q}_{\ell} \otimes_{\mathbb{Q}} \mathrm{K}_{\mathrm{n}}$ par action du logarithme: clest un $\mathbb{Q}_{\ell}[\Delta]$-module de caractère $\ell^{n}[F: \mathbb{Q}] x_{r e ́ y}$. Quant au dénominateur, son caractère vaut exacte ment $\left(l^{n} x_{\infty}-1\right)-\delta_{n}$, puisque le produit $\mathbb{Q}_{l} \otimes_{\mathbb{Z}} E_{n}$ est un $\mathbb{Q}_{\ell}[\Delta]-$ module de caractère $\ell^{n} x_{\infty}-1$.

Egalant les deux expressions obtenues, nous en déduisons :

$$
\delta_{n}=e^{n}\left[x_{\infty}+x_{\infty}^{*}-[F: \mathbb{Q}] x_{r} \dot{e j g}\right]+\sum_{\varphi} \operatorname{deg} \Omega_{\varphi} \varphi ;
$$

ce qui établit le théorème, en vertu du lemme:

LEMME IV.2.23. - La somme $\chi_{\infty}+\chi_{\infty}^{*}$ est égale à $[F: Q] X$.

Démonstration : Désignons par $r_{F}$ (respectivement $c_{F}$ ) le nombre de places réelles (respectivement complexes) du corps $F$. Si $p$ est complexe, nous avons $x_{p}=x_{\text {rég }}$ donc $\chi_{p}+x_{p}^{*}=2 x_{\text {réy }}$. Si $p$ est réelle, il y a ramification, donc $x_{p}+\chi_{p}^{*}=\chi_{p}+\omega \chi_{p}=\chi_{\text {rég }}$, puisque wagit non trivialement sur le sous-groupe de décomposition $\Delta_{p}$. 11 vient donc $x_{\infty}+x_{\infty}^{*}=\sum_{p \mid \infty}\left(x_{p}+x_{p}^{*}\right)=\left(r_{F}+2 c_{F}\right) x_{r}$ ég $=[F: \mathbb{Q}] x_{r}$ ég , comme attendu.

DÉFINITION IV.2.24. - Nous disons que le caractère $\delta$ Leopoldt $=$ $\sum\left(\right.$ deg $\left.\widetilde{\Omega} a_{\varphi}\right) \varphi$ est le caractère de défaut de la conjecture de Leopoldt $\varphi$

dans la tour cyclotomique $K_{\infty} / K$. Son degré $\left\langle\delta_{\text {Leopoldt }}\right.$, $\left.\delta_{\text {Leopoldt }}\right\rangle$ mesure le défaut de la conjecture de Leopoldt à chaque étage fini assez grand de la tour cyclotomique.

COROLLAIRE IV.2.25. - Dans une tour cyclotomique, le défaut de la conjecture de Leopoldt est majoré par l'invariant $\lambda$ d'lwasawa associé aux groupes de $l$-classes $C l_{n}^{\prime}{ }^{(*)}$. Plus précisément, nous avons l'inégalité entre caractères :

$$
\delta_{\text {Leopoldt }} \leq \lambda_{c}^{*}
$$

( $\left.{ }^{\star}\right)$ Enoncé en termes de rangs, ce résultat est dû à Gillard (cf. [Gi 2$]$, th. 2 ). 
Démonstration: La majoration triviale $\operatorname{deg} \widetilde{\Omega a}_{\varphi} \leq \operatorname{deg} \mathrm{Pa}_{\varphi}$ donnant seulement l'inégalité :

$$
\delta_{\text {Leopoldt }} \leq \lambda_{a}=\lambda_{c}^{*}+\omega\left(\psi_{l}-1\right),
$$

il est nécessaire de procéder autrement: Nous savons par le théo rème 22 que, pour $n$ assez grand, le groupe $\varepsilon_{n}^{\infty}$ des unités infinité simales du corps $K_{n}$ est un $\mathbb{Z}_{\ell}[\Delta]$-module projectif de caractère $\delta_{n}=\delta_{\text {Leopoldt }}$. Considérons donc l'extension abélienne $K_{\infty}\left[\sqrt[\ell]{\delta}_{n}^{\infty}\right] / K_{\infty}^{\left({ }^{\star}\right)}$ : Elle est non ramifiée aux places étrangères à $\ell$ ( puisque son radical est représenté par des unités) et complète ment décomposée aux places divisant $\ell$ (puisqu'il est représentépar des infinitésimaux). Son groupe de Galois Gal $\left(K_{\infty}\left(\sqrt[\ell]{\delta}_{n}^{\infty}\right) / K_{\infty}\right)$ est donc un quotient du groupe $C^{\prime}=\mathrm{Gal}\left(\mathrm{C}_{\infty}^{\prime} / \mathrm{K}_{\infty}\right)$. Mais c'est aussi un $\mathbb{Z}_{\ell}[\Delta]$-module projectif de caractère $\delta_{n}^{*}=\delta_{\text {Leopoldt }}^{*}$. II vient donc $\delta_{\text {Leopoldt }}^{*} \leq \lambda_{c}$, i.e. $\delta_{\text {Leopoldt }} \leq \lambda_{c}^{*}$, comme annoncé .

Remarque : Le fait que le défaut de la conjecture de Leopoldt soitborné dans la tour $K_{\infty} / K$ prouve que le groupe de défaut $\varepsilon_{n}^{\infty}$ est constant pour $n$ assez grand. En effet, $\varepsilon_{n}^{\infty}$ estalors d'indice fini dans $\varepsilon_{n+1}^{\infty}$, en vertu de l'égalité des rangs; et c'est aussi un sous-module pur de $\varepsilon_{n+1}^{\infty}$, sans quoi l'extension $k_{n+1} / K_{n}$ serait engendrée par la racine $\ell$-ième diune unité infinitésimale de $\mathrm{K}_{\mathrm{n}}$, donc $\ell$-décomposée ; ce qui n'est pas.

COROLLAIRE IV.2.26. - La suite $\left(8_{n}\right)_{n} \in \mathbb{N}$ des radicaux kummé riens $\operatorname{Rad}\left(Z_{n} / K_{n}\right)$ des composées $Z_{n}$ des $\mathbb{Z}_{\ell}$-extensions des corps $K_{n}$ est paramétrée par les caractères $x_{\infty},(r-1) x_{\infty}, \omega+\delta_{\text {Leopoldt }}^{*}$ et $r\left(w+\delta_{\text {Leopoldt }}^{*}\right)$.

Démonstration : Pour chaque caractère $\ell$-adique irr éductible $\varphi$ de $\Delta$, écrivons $l^{\tilde{z}_{\varphi}(n)}$ llordre de la $\varphi$-composante du radical $8_{n}$. Celaposé, pour $n \geq m$, nous savons déjà que le groupe de Galois $a_{n} / \sigma_{n}=$ Gal $\left(Z_{n} / K_{n}\right)$ est un $\mathbb{Z}_{\ell}[\Delta]$-module projectif de caractère

${ }^{\star}$ ) clest-à-dire la plus grande $l$-extension kummér ienne de $\mathrm{K}_{\infty}$ dont le radical est représenté par des unités infinitésimales. 
$\ell^{n} x_{\infty}^{*}+1+\delta_{\text {Leopoldt }}$. De l'isomorphisme canonique :

$\operatorname{Rad}\left(z_{n} / K_{n}\right) \simeq \operatorname{Hom}\left(\operatorname{Gal}\left(z_{n} / K_{n}\right), \mu_{n}\right)$,

donné par la théorie de Kummer, nous déduisons donc la formule:

$$
\tilde{z}_{\varphi}(n)=\left\langle e^{n} x_{\infty}+\omega+\delta_{\text {Leopoldt }}^{*}, \varphi\right\rangle(n+1) ;
$$

ce qui est précisément l'assertion du corollaire.

COROLLAIRE IV.2.27. - Supposons vérifiée la conjecture de Leopoldt dans la tour cyclotomique $K{ }_{\infty} / K$. Alors la suite $\left(\sigma_{n} / \sigma_{n}^{l^{n+r}} I_{n} \in \mathbb{N}\right.$ des quotients d'exposant $\ell^{n+r}$ des sous-groupes de torsion $\sigma_{n}$ est paramétrée par les caractères $0, \mu_{c}^{*}, \lambda_{c}^{*}+\omega\left(\psi_{l}^{-1)}\right.$, et $\tilde{\nu}_{c}^{*}+\omega\left(\psi_{l}^{-1}\right)$.

Nous allons donner deux démonstrations de ce résultat : la première en évaluant directement l'ordre $\ell^{\tilde{t}(n)}$ de la $\varphi$-compo sante du quotient $\sigma_{n} / \sigma_{n}^{l^{n+r}}$, pour un caractère $\ell$-adique irréductible arbitraire $\varphi$; la seconde en écrivant que $\ell^{\tilde{t}(n)}$ est encore l'ordre de la $\varphi$-composante du sous-groupe de $\ell^{n+r}$-torsion $\ell^{n+r} \sigma_{n}=$ $\ell^{n+r} a_{n}$ du groupe $a_{n}$.

Première démonstration: L'isomorphisme de dualité $\left.B_{n}=\operatorname{Hom}\left(a_{n} / a_{n}^{l^{n+r}}\right) /\left(\sigma_{n} / \sigma_{n}^{e^{n+r}}\right), \mu_{n}\right)$ nous conduit immédiatement à l'identité $\tilde{a}_{\varphi}(n)-\tilde{t}_{\varphi}(n)=\tilde{z}_{\varphi^{*}}(n)$, où $\varphi^{*}$ est le reflet du carac tère $\varphi$. Cela étant, l'indice $\tilde{a}_{\varphi}(n)$ est donné par la proposition 17 , et llindice $\tilde{z}_{\varphi^{*}}(n)$ par le corollaire 26 ; d'où le résultat annoncé : $\tilde{t}_{\varphi}(n)=\left\langle\mu_{c}^{*}, \varphi\right\rangle \ell^{n}+\left\langle\lambda_{c}^{*}+\omega\left(\psi_{l}-1\right), \varphi\right\rangle n+\left\langle\tilde{\nu}_{c}^{*}+r \omega\left(\psi_{l}-1\right), \varphi\right\rangle$.

Deuxième démonstration : D'après la proposition 11.2 .6 , la suite exacte de dualité (ii) du théorème 11.2 .13 s'écrit, pour $n$ assez grand :

$1 \longrightarrow\left({ }_{I_{n} \mid l}^{\oplus} \mu_{I_{n}}\right) / \mu_{n} \longrightarrow l^{n+r} a_{n} \longrightarrow \mathbb{c}_{n}^{\prime}=\operatorname{Rad}\left(c_{n}^{\prime} / K_{n}\right) \longrightarrow 1$

11 vient donc:

$\tilde{t}_{\varphi}(n)=\left\langle\omega\left(\psi_{l}-1\right), \varphi\right\rangle+\tilde{c}_{\varphi}^{\prime}(n)$, et l'indice $\tilde{c}_{\varphi^{*}}^{\prime}(n)$ est donné par le corollaire 4 ; d'où, à nouveau, le résultat . 
c.- Capitulation pour les gr oupes $\sigma_{n}$, et application à la $K$-théorie.

Le corollaire 27 montrant que la suite $\left(\sigma_{n} / \sigma_{n}^{e^{n+r}}\right)_{n} \in \mathbb{N}$ des quotients de $\ell^{n+r}$ - torsion des groupes $G_{n}$ est paramétrée, il est naturel d'attendre un résultat analogue pour la suite $\left(\zeta_{n}{ }_{n} \in \mathbb{N}\right.$ elle-même. Pour l'obtenir, nous allons comparer les groupes $G_{n}$ avec les quotients des genres $\sigma / \sigma^{\omega_{n}}$ associés au sous-module de $\Lambda$ torsion $\sigma$ du groupe de Galois $a=\operatorname{Gal}\left(M_{\infty} / K_{\infty}\right)$. Sous la conjecture de Leopoldt, il est facile de voir, en effet, que le groupe $\zeta$ est la limite projective des groupes $\zeta_{n}$ : D'un côté, les groupes $\sigma_{n}$ étant finis, leur limite projective $\lim _{n} \sigma_{n}$ est bien un sous-module de tor sion de $a$, donc un sous-module de $\sigma$. D'un autre côté, sous la conjecture de Leopoldt, les quotients $\sigma / \sigma^{\omega_{n}}$ sont finis, et contenus dans $a_{n}^{\prime}=a / a^{W_{n}}$, donc dans le sous-module de $\mathbb{Z}_{l} l^{\text {tor } \operatorname{sion}} \sigma_{n}$; ce qui prouve en retour que $G$ est contenu dans la limite projective des $G_{n}$. L'ident ité ainsi obtenue :

$$
\sigma=\varliminf_{n}
$$

n'entraîne pas cependant l'égalité :

$$
\sigma_{n}=\sigma / \sigma^{\omega_{n}} \text {, pour chaque naturel } n \text {, }
$$

puisque rien n'assure que l'application norme $N_{\mathrm{n}}$ de $\sigma$ dans $\sigma_{\mathrm{n}}$ soit une surjection. De fait, la théorie du corps de classes, qui inter prète le quotient $G_{\mathrm{n}} / N_{\mathrm{n}}(\sigma)$ comme groupe de Galois de l'extension abélienne $\left(M_{n} \cap Z_{\infty}\right) / Z_{n}$, montre que ce défaut de surjectivité me sure la partie de la torsion de $a_{n}$ qui est absorbée par les $\mathbb{Z} l^{- \text {ex }}$ tensions lorsquion monte la tour cyclotomique. Par la théorie de Kummer, le groupe dual $\left(\sigma_{n} / N_{n}(\sigma)\right)^{\vee}=\operatorname{Hom}\left(\sigma_{n} / N_{n}(\sigma), Q_{\ell} / \mathbb{Z}_{\ell}\right)$ s'identifie au noyau de l'application naturelle:

$$
\bar{R}_{2}\left(\mathrm{~K}_{\mathrm{n}}\right) \longrightarrow \bar{R}_{2}\left(\mathrm{~K}_{\infty}\right) \text {, }
$$

où $\bar{R}_{2}\left(K_{\mathrm{n}}\right)=\check{\zeta}_{\mathrm{n}}$ est le dual de Pontr jagin du groupe $\sigma_{\mathrm{n}}$, et $\left.{ }^{\star}\right) \bar{R}_{2}\left(\mathrm{~K}_{\infty}\right)$ celui du groupe $\zeta$; il correspond donc à une capitulation

Pour l'évaluer, partons de la description du groupe $C / \sigma$ donnée par la théorie d'lwasawa. Les théorèmes de structure de la

(*) Les groupes $\bar{R}_{2}\left(\mathrm{~K}_{\mathrm{n}}\right)$ sont définis au chapitre $\mathrm{I} .1,2 \S \mathrm{b}$. 
section 1 nous prouvent que le quotient $a / \sigma$, qui est sans $\mathbb{Z} \ell^{\text {-tor - }}$ sion, est contenu avec un indice fini dans un $\Lambda[\Delta]$-module projec tif $p$, ce que nous pouvons écrire :

$1 \longrightarrow \mathrm{a} / \mathrm{G} \longrightarrow \mathrm{P} \longrightarrow \mathrm{F} \longrightarrow 1$, pour un module fini $\mathfrak{F}$.

Comparant cette suite exacte à elle-même, par action de l'opérateur $w_{n}=\gamma^{l^{n}}-1$, puis formant le diagramme du serpent, nous obtenons la suite exacte :

$$
1 \longrightarrow \xi^{\Gamma_{n}} \longrightarrow a / \sigma a^{\omega_{n}} \longrightarrow p / p^{\omega_{n}} \longrightarrow \xi^{\omega_{n}} \longrightarrow 1
$$

qui nous prouve, puisque le quotient $p / p^{\omega_{n}}$ est $\mathbb{Z}_{\ell}[\Delta]$-projectif , que le groupe $\mathfrak{F}^{\Gamma_{n}}$ s'identifie canoniquement au sous-groupe de $\mathbb{Z}_{l}$ torsion du quotient $a / \delta a^{\omega_{n}}$. Naturellement, pour $n$ assez grand, $\omega_{n}$ annule ${ }^{\prime}$, et $\Gamma_{n}$ est égal à .

Cela étant, dans la suite exacte courte canonique :

$$
1 \longrightarrow 6 a^{\omega_{n}} / a^{\omega_{n}} \longrightarrow a / a^{\omega_{n}} \longrightarrow a / b a^{\omega_{n}} \longrightarrow 1
$$

le terme de gauche $\tau a^{\omega_{n}} / a^{\omega_{n}} \simeq \sigma / \sigma^{\omega_{n}}$ étant fini sous la conjec ture de Leopoldt, le sous-groupe de torsion du terme de droite, qui est isomorphe à $F$, est limage dans le quotient $a / \varepsilon a^{w_{n}}$ du sous groupe de torsion $\sigma_{n}$ de $a / a^{\omega_{n}}$. Par restriction aux sous-groupes de torsion, nous obtenons donc la suite courte :

$$
1 \longrightarrow \sigma / \varepsilon^{w_{n}} \stackrel{N_{n}}{\longrightarrow} \varepsilon_{n} \longrightarrow 1
$$

qui nous prouve que le groupe de défaut cherché est précisément . II vient donc:

THÉORĖME IV,2.28.- Sous la conjecture de Leopoldt, la limite pro-jective des sous-modules de $\mathbb{Z} \ell^{\text {torsion }} G_{n}$ des $\ell$-groupes de clas ses infinitésimales $a_{n}$ des corps $K_{n}$ est le sous-module de $\Lambda$-torsion $\sigma$ de la limite projective des $a_{n}$; c'est un $\Lambda[\Delta]$-module noethérien et de torsion, de paramètres $\mu_{c}^{*}$, et $\lambda_{c}^{*}+\omega\left(\psi_{l}-1\right)$. De plus, il existe un paramètre $\ell$-adique virtuel $\nu_{a}$ du groupe $\Delta$, tel que, pour chaque caractère $\ell$-adique irréductible $\varphi$ de $\Delta$, et tout $n$ assez grand, l'or dre $l^{t(n)}$ de la $\varphi$-composante du groupe $G_{n}$ soit donné par la formule : $t_{\varphi}(n)=\left\langle\omega \mu_{c}^{-1}, \varphi\right\rangle \ell^{n}+\left\langle\omega \lambda_{c}^{-1}+\omega\left(\psi l^{-1}\right), \varphi\right\rangle n+\left\langle\nu_{a}, \varphi\right\rangle$. 
SCOLIE IV.2.29. - Supposons la conjecture de Leopoldt vérifiée dans la tour $K_{\infty} / K$, et $n$ assez grand. Alors :

(i) Le quotient $\sigma_{n} / N_{n}(\sigma)$ est isomorphe au groupe ź́fini ci-dessus 。

(ii) Le noyau de l'homomorphisme naturel $\bar{R}_{2}\left(\mathrm{~K}_{\mathrm{n}}\right) \longrightarrow \bar{R}_{2}\left(\mathrm{~K}_{\infty}\right)_{\ell}$ est isomorphe au dual de ${ }^{*}$.

Cette dernière assertion est l'analogue pour les groupes $\bar{R}_{2}\left(\mathrm{~K}_{\mathrm{n}}\right)_{l}$ du résultat de Coates sur les noyaux réguliers $R_{2}\left(\mathrm{~K}_{\mathrm{n}}\right)_{l}$ de la $K$-théorie ( $\mathrm{cf} .\left[\mathrm{Co}_{1}\right], \S 4$, th. 7 ). Considérons, en effet, le tensorisé $\overline{\bar{a}}=\overline{\mathrm{U}}_{l}^{2} \otimes_{\mathbb{Z}} a$ du groupe $a$ par le carré tensoriel $\overline{\mathrm{T}}_{l}^{2}$ de l'opposé du module de Tate $\pi,=\lim \mu_{n}$. Les résultats du chapitre $1^{(*)}$ montrent que le dual de Pontrjagin, disons $\overline{\bar{\sigma}}_{n}$, du groupe $\bar{R}_{2}\left(\mathrm{~K}_{n}\right)_{\ell}$ est le sous-groupe de $\mathbb{Z}_{\ell}$-torsion du quotient des genres $\Gamma_{n} a=a / a^{\omega_{n}}$ associé à $a$. Bien entendu, comme $a$ est un $\Lambda[\Delta]$-module noethérien de paramètres $\chi_{\infty}, \omega \mu_{c}^{-1}$, et $\omega\left(\lambda_{c}^{-1}+\left(\psi_{l}-1\right)\right)$, le groupe $\overline{\bar{a}}$ est, lui , un $\Lambda[\Delta]$-module noe thérien de paramètres $\omega^{-2} x_{\infty}, \omega^{-1} \mu_{c}^{-1}$ et $\omega^{-1}\left(\lambda_{c}^{-1}+\left(\psi_{l}-1\right)\right)$. Son

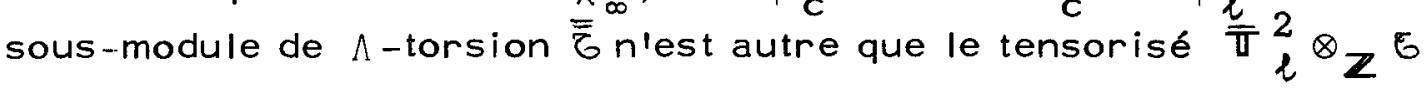
de celui de $a$, et, comme expliqué dans le chapitre 1 , le résultat fondamental de la théorie du $K_{2}$ montre que son polynôme caractéris tique est étranger aux $\omega$. Si donc $\overline{\bar{z}}$ désigne le tensorisé $\bar{u}_{l}^{2} \otimes_{\mathbb{Z}} \bar{z}$ du groupe $\Im$, les mêmes arguments que plus haut conduisent à la suite exacte :

$$
1 \longrightarrow \overline{\bar{\sigma}} / \overline{\bar{\sigma}}^{\omega_{n}} \longrightarrow \overline{\bar{\sigma}}_{n} \longrightarrow \overline{\bar{z}} \longrightarrow 1 \text {, }
$$

qui redonne, par passage aux duaux, la suite exacte de Coates:

$$
1 \longrightarrow \stackrel{\check{\bar{F}}}{\longrightarrow} \bar{R}_{2}\left(\mathrm{~K}_{\mathrm{n}}\right)_{l} \longrightarrow R_{2}\left(\mathrm{~K}_{\infty}\right)_{l}^{\Gamma_{\mathrm{n}}} \longrightarrow 1 \text {. }
$$

En particulier, le théorème 9 de $\left[\mathrm{Co}_{1}\right]$ peut être précisé comme suit :

(*) section $2, \$ 2$. 
THEOREME IV.2.30.- La limite projective des duaux de Pontrjagin $\sigma_{\mathrm{n}}$ des noyaux réguliers $R_{2}\left(\mathrm{~K}_{\mathrm{n}_{2}}{ }^{\prime}\right.$ des corps $\mathrm{K}_{\mathrm{n}}$ est le sous-module de $\Lambda$-torsion $\overline{\bar{\sigma}}$ du tensorisé $\overline{\bar{a}}=\overline{\bar{U}}^{2}{ }^{\otimes^{\otimes}} Z_{l} a$ du groupe $a=\operatorname{Gal}\left(\mathrm{M}_{\infty} / \mathrm{K}_{\infty}\right)$ par le carré de l'opposé du module de Tate. Le groupe $\overline{\bar{\sigma}}$ est un $\Lambda[\Delta]$ module noethérien et de torsion, de paramètres $\omega^{-1} \mu_{c}^{-1}$ et $\omega^{-1}\left(\lambda_{c}^{-1}+\left(\psi_{\ell}-1\right)\right)$, et la suite $\left(R_{2}\left(K_{n}\right)_{\ell}\right)_{n} \in \mathbb{N}$ est paramétrée par les caractères $\omega \mu_{c}, \omega\left(\lambda_{c}+\left(\psi_{\ell}-1\right)\right)$, et $\nu_{r}$, pour un caractère $\ell$-adique virtuel $\nu_{r}$ du groupe $\Delta$. Autrement dit, pour chaque caractère $\ell$-adique irréductible $\varphi$ du groupe $\Delta$, et tout $n$ assez grand, l'ordre $l^{r(n)}$ de la $\varphi$-composante du module $R_{2}\left(\mathrm{~K}_{\mathrm{n}}\right){ }_{l}$ est donné par la formule:

$r_{\varphi}(n)=\left\langle\omega \mu_{c}, \varphi\right\rangle \ell^{n}+\left\langle\omega \lambda_{c}+w\left(\psi_{\ell}-1\right), \varphi\right\rangle n+\left\langle\nu_{r}, \varphi\right\rangle$.

SCOLIE IV.2.31. - Soit $n$ un entier naturel assez grand. Alors :

$$
\begin{aligned}
& \text { (i) Le quotient } \overline{\bar{\sigma}}_{n} / N_{n}(\overline{\bar{\sigma}}) \text { est isomorphe au tensorisé } \\
& \overline{\bar{F}}=\bar{\tau}_{l}^{2} \otimes_{Z} \text { du groupe } \text {. } \\
& \text { (ii) Le noyau de } 1 \text { homomorphisme naturel } \\
& R_{2}\left(\mathrm{~K}_{\mathrm{n}}\right)_{\ell} \longrightarrow R_{2}\left(\mathrm{~K}_{\infty}\right)_{\ell} \text { est isomorphe au dual de } \overline{\overline{\mathrm{z}}} \text {. }
\end{aligned}
$$

Enfin, un résultat de paramétrage vaut encore pour les quotients $R_{2}\left(\mathrm{~K}_{\mathrm{n}}\right)_{\ell} / R_{2}\left(\mathrm{~K}_{\mathrm{n}}\right)^{\ell^{\mathrm{n}+\mathrm{r}}}$ :

PROPOSITION IV.2.32. - La suite $\left(R_{2}\left(\mathrm{~K}_{n}\right)_{l} / R_{2}\left(\mathrm{~K}_{\mathrm{n}}\right)^{l^{\mathrm{n}+r}}\right)_{\mathrm{n} \in \mathbb{N}}$ des quotients d'exposant $\ell^{n+r}$ des noyaux réguliers est paramétrée par les caractères $0, \omega \mu_{c}, \omega \lambda_{c}+\omega\left(\psi_{l}-1\right)$, et $\omega \tilde{\nu}_{c}+r \omega\left(\psi l^{-1)}\right.$.

Démonstration: Les théorèmes de dualité du chapitre 1 conduisent à l'isomorphisme canonique ${ }^{(\star)}$ :

$$
\mu_{n} \otimes_{Z_{l}} \bar{R}_{2}\left(\mathrm{~K}_{\mathrm{n}}\right)_{l} \simeq \bar{\mu}_{\mathrm{n}} \otimes_{\mathbb{Z}_{l}} R_{2}\left(\mathrm{~K}_{\mathrm{n}}\right)_{l}
$$

où $\bar{R}_{2}\left(\mathrm{~K}_{\mathrm{n}}\right)_{l}$ est le dual de Pontrjagin du groupe $\sigma_{\mathrm{n}}$, et $\bar{\mu}_{n}$ l'opposé de $\mu_{n}$. Cela étant, d'après la proposition 27 , la suite $\left(l^{n+r}{ }^{6}{ }^{\prime} n \in \mathbb{N}\right.$

(*) of. Section 2, 4§a: corcllatire 1.2.31. 
des sous-groupes de $\ell^{n+r}$-torsion des groupes $\sigma_{n}$ est paramétrée par les caractères $\omega \mu_{c}^{-1}, \omega \lambda_{c}^{-1}+\omega\left(\psi_{l}-1\right)$, et $\omega \tilde{\nu}_{c}^{-1}+r \omega\left(\psi_{l}-1\right)$. Les paramètres associés à la suite $\left(\mu_{n} \otimes_{\mathbb{Z}_{\ell}} \bar{R}_{2}\left(\mathrm{~K}_{n}\right)_{\ell}\right)_{n} \in \mathbb{N}=$ $\left(\bar{\mu}_{n} \otimes_{Z_{l}} R_{2}\left(\mathrm{~K}_{n}\right)_{l}\right)_{n} \in \mathbf{N}$ sont donc exactement $\mu_{c}, \lambda_{c}+\left(\psi_{l}-1\right)$, et $\tilde{\nu}_{c}+r\left(\psi_{\ell}-1\right)$; ce qui conduit au résultat annoncé .

\section{3. - ÉTUDE DU GROUPE DE GALOIS DE LA \&-EXTENSION HILBERTIENNE} MAXIMALE DU CORPS $K_{\infty}$.

\section{a.-Définition du groupe $\boldsymbol{*}$.}

Pour chaque naturel $n$, désignons par $H_{n}$ la $l$-extension hilbertienne maximale du corps $K_{n}$, $i . e$. la composée des $\ell$-extensions cycliques de $K_{n}$, qui se plongent dans une $\ell$-extension cycli que de degré arbitrairement grand. Le corps $H_{n}$, qui contient évi demment la composée $Z_{n}$ des $\mathbb{Z}_{\ell}$-extensions de $K_{n}$, est contenu, en revanche, dans la $l$-extension abélienne $l$-ramifiée maximale $M_{n}$ ' puisque la condition de plongement qui définit $H_{n}$ exclut toute ramifi cation en-dehors de $l$ (Le degré d'une $\ell$-extension cyclique totale ment ramifiée en une place $p$ étrangère à $l$ est borné, en vertu du corps de classes local, par sa $\ell$-partie du nombre de racines de lunité dans le complété $p$-adique du corps de basel. Plus précisé ment, la théorie du corps de classes global ${ }^{(*)}$ montre que $H_{n}$ est la sous-extension de $M_{n}$ fixée par l'image $\operatorname{Gal}\left(M_{n} / H_{n}\right)$ dans $\operatorname{Gal}\left(M_{n} / K_{n}\right)$ de la somme directe $\hat{\mu}_{\mathrm{n}}=\stackrel{I}{\mathrm{n}} \mid \ell_{\ell}^{\oplus} \mu_{\mathrm{l}}$ des $l$-groupes de racines de llunité des complétés de $K_{n}$ pour les places au-dessus de $l$. Sous la conjecture de Leopoldt, le théorème 1.1 .18 conduitainsi à l'isomor phisme :

$$
\operatorname{Gal}\left(M_{n} / H_{n}\right) \simeq \hat{\mu}_{n} / \mu_{n} \text {, }
$$

c'est-à-dire à la suite exacte de $\mathbb{Z}_{\ell}[\Delta]$-modules finis :

(*) cf.ch. $1.1,23 b$ 


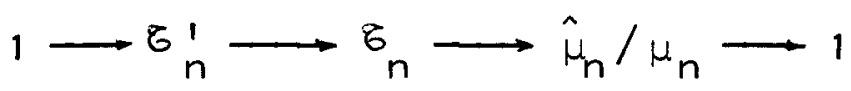

où $\sigma_{n}^{\prime}=\operatorname{Gal}\left(H_{n} / Z_{n}\right)$ désigne le sous-groupe de torsion de $A_{n}=$ $\operatorname{Gal}\left(H_{n} / K_{n}\right)$, et $G_{n}=\operatorname{Gal}\left(M_{n} / Z_{n}\right)$ celui du groupe $a_{n}=$ $\operatorname{Gal}\left(M_{n} / K_{n}\right)$.

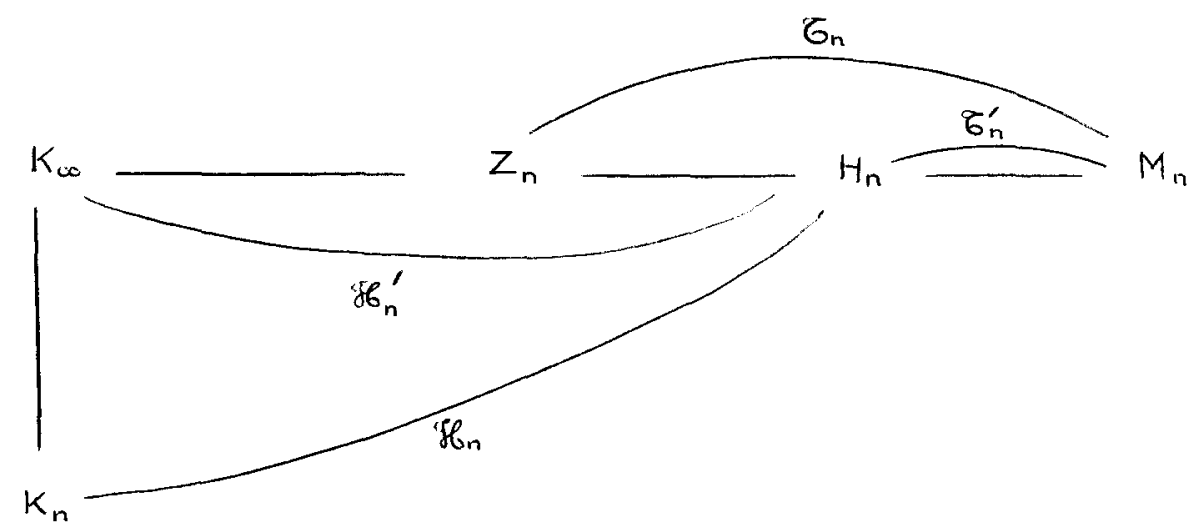

Maintenant, l'ordre du groupe $\sigma_{n}$ étant donné par le théorème 28 ,

nous obtenons :

THÉORËME IV.2.33. - Sous la conjecture de Leopoldt, la limite projective des sous-modules de $\mathbb{Z} \ell^{\text {torsion }} \mathcal{G}_{n}^{\prime}$ des groupes de Galois respectifs $\sharp_{n}=G a l\left(H_{n} / K_{n}\right)$ des $l$-extensions hilbertiennes maxi males $H_{n}$ des corps $K_{n}$ est le sous-module de $\Lambda$-torsion $\sigma^{\prime}$ de la li mite projective $H_{\text {des groupes }} H_{n}$; c'est un $\Lambda[\Delta]$-module noethérien et de torsion, dont les paramètres $\mu_{c}^{*}$ et $\lambda_{c}^{*}$ sont les reflets de ceux du groupe $C^{\prime}$ dans linvolution du miroir.

En particulier, pour chaque caractère $\ell$-adique irréduc tible $\varphi$ du groupe $\Delta$, et tout $n$ assez grand, l'ordre $l^{t^{\prime}(n)}$ de la $\varphi-$ composante $\sigma_{n}^{\prime}$ est donné par la formule :

$t_{\varphi}^{\prime}(n)=\left\langle\omega \mu_{c}^{-1}, \varphi\right\rangle \ell^{n}+\left\langle\omega \lambda_{c}^{-1}, \varphi\right\rangle n+\left\langle\nu_{a}-(r-1) \omega\left(\psi_{l}-1\right), \varphi\right\rangle$.

Démonstration : La formule finale résulte directement du théorème 28 , puisque le quotient $\hat{\mu}_{n} / \mu_{n}=\underset{I_{n}|l| \mu_{I_{n}} / \mu_{n}}{\oplus}$ est un $\left(\mathbb{Z} / \ell^{n+r} \mathbb{Z}\right)[\Delta]-$ module projectif de caractère $\omega\left(\psi_{\ell}-1\right)$, dès que $n$ est assez grand 
( pour $n \geq m$, l'extension cyclotomique $K_{n} / K_{m}$ est non décomposée aux places au-dessus de $l$, et tous les groupes locaux $\mu_{l}$ ont bien même ordre $e^{n+r}$ que le groupe global $\mu_{n}$ ). Reste à vérifier que la limite projective des $\sigma_{n}^{\prime}$ slidentifie au sous-groupe de $\Lambda$-torsion du groupe de Galois $\boldsymbol{H}=\mathrm{Gal}\left(\mathrm{H}_{\infty} / \mathrm{K}_{\infty}\right)$ associé à la réunion $H_{\infty}$ des corps $H_{n}$. Pour cela, il suffit de remarquer que $H_{n}$ est le corps des genres associé à $H_{\infty}$ dans l'extension procyclique $K_{\infty} / K_{n}$ : En effet, pour chaque $k$ dans $\mathbb{N}$, la norme semi-locale $N_{n+k} / n$ de $\hat{\mu}_{n+k}$ dans $\hat{\mu}_{n}$ est une surjection; et $H_{n}$, qui est la sous-extension maximale de $M_{n}$ fixée par l'image de $\hat{\mu}_{n}$ dans $G a l\left(M_{n} / K_{n}\right)$, est aussi la sous extension maximale de $H_{n+k}$ qui est abélienne sur $K_{n}(\star)$, ce que nous pouvons traduire par lidentité:

$$
H_{n}^{\prime}=x / H^{w_{n}} \text {, }
$$

en désignant par $H_{n}^{\prime}$ le groupe de Galois Gal $\left(H_{n} / K_{\infty}\right)$. Cela étant, comme d'un côté les groupes $G_{n}$ sont finis, leur limite projective

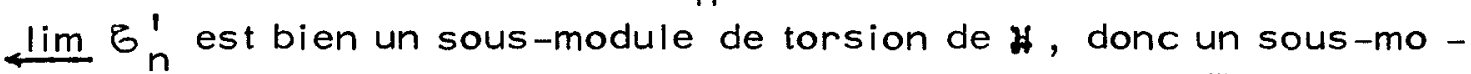
dule de $\sigma^{\prime}$; et, d'un autre côté, les quotients $\sigma^{\prime} / \sigma^{\prime} \omega_{n}$, qui sont finis sous la conjecture de Leopoldt ( puisque le polynôme caractéristique du sous-module $\sigma^{\prime}$ de $G$ est alors étranger à tous les $\omega_{i}$ ), étant contenus dans $H_{n}^{\prime}=\not f / \|^{W_{n}}$, ils sont bien dans son sous-module de torsion $\sigma_{n}^{\prime}$, ce qui prouve en retour que $\sigma^{\prime}$ est contenu dans la li mite projective des $\sigma_{n}^{\prime}$.

Le comportement des groupes $\sigma_{n}^{\prime}$ est donc exactement parallèle à celui des groupes $b_{n}$. Comme celui-ci, il admet une inter prétation simple en termes de $K$-théorie, les noyaux hilbertiens $\mathrm{H}_{2}\left(\mathrm{~K}_{\mathrm{n}}\right)_{l}$ venant, ici remplacer les noyaux réguliers $R_{2}\left(\mathrm{~K}_{\mathrm{n}}\right)_{l}$. Enonçons sans démonstration l'analogue du théorème 30 :

THÉOREMME IV.2.34.- La limite projective des duaux de Pontrjagin $\overline{\bar{\sigma}}_{n}^{\prime}$ des noyaux hilbertiens $H_{2}\left(K_{n}\right)$, des corps $K_{n}$ est le sous-mo dule de $\Lambda$-torsion $\overline{\bar{\varepsilon}}^{\prime}$ du tensorisé $\overline{\mathcal{H}}^{l}=\overline{\bar{T}}_{l}^{2} \otimes_{\mathbf{Z}}$ \# du groupe de Galois

(*) Pour plus de détails, voir le chapitre $1.2,1 \S$ b où sont exposés les éléments du corps de classes utilisés ici. 
H $=\mathrm{Gal}\left(\mathrm{H}_{\infty} / \mathrm{K}_{\infty}\right)$ par le carré de l'opposé du module de Tate. Le groupe $\bar{E}$ ' est un $\Lambda[\Delta]$-module noethérien et de torsion, de paramètres $\omega^{-1} \mu_{c}^{-1}$ et $\omega^{-1} \lambda_{c}^{-1}$, et la suite $\left(H_{2}\left(K_{n}\right)_{\ell}\right)_{n} \in \mathbb{N}$ est paramétrée par les caractères $\omega \mu_{c}, w \lambda_{c}$, et $\nu_{h}$, pour un caractère $\ell-$ adique virtuel convenable $\nu_{h}$ du groupe $\Delta$.

Autrement dit, pour chaque caractère $\ell$-adique irréductible $\varphi$ du groupe $\Delta$, et tout $n$ assez grand, l'ordre $l^{h \text { (n) }}$ de la $\varphi$-com posante du module $\mathrm{H}_{2}\left(\mathrm{~K}_{\mathrm{n}}\right)_{\ell}$ est donné par la formule :

$$
h_{\varphi}(n)=\left\langle\omega \mu_{c}, \varphi\right\rangle \ell^{n}+\left\langle\omega \lambda_{c}, \varphi\right\rangle n+\left\langle\nu_{h}, \varphi\right\rangle \text {. }
$$

SCOLIEIV.2.35.- Sous la conjecture de Leopoldt, la suite $\left(\sigma_{n}^{\prime} / \sigma_{n}^{\prime} l^{n+r}\right)$ des quotients d'exposant $l^{n+r}$ des sous-groupes de torsion des groupes $\dot{A}_{n}$ est paramétrée par les quatre caractères 0 , $\mu_{c}^{*}, \lambda_{c}^{*}$, et $\tilde{\nu}_{h}$, où $\tilde{\nu}_{h}$ est un caractère $\ell$-adique virtuel du groupe $\Delta$.

Démonstration: Nous savons, par le théorème 33 , que le groupe $H=\operatorname{Gal}\left(H_{\infty} / K_{\infty}\right)$ est un $\Lambda[\Delta]$-module noethérien de paramètres $X_{\infty}^{*}$, $\mu_{c}^{*}$, et $\lambda_{c}^{*}$. Dlaprès les théorèmes de paramétrage établis dans la section 1 (cf. th. IV.1.17), la suite des quotients $\left(H_{n}^{\prime} / H_{n}^{\prime} e^{n+r}\right)_{n \in N}$ est donc paramétrée par les caractères $x_{\infty}^{*}, \mu_{c}^{*}+(r-1) \chi_{\infty}^{*}, \lambda_{c}^{*}$, et $\tilde{\nu}_{h}$, pour un caractère virtuel convenable $\tilde{\nu}_{h}$ du groupe $\Delta$. En par ticulier, la suite $\left(\not_{n} / A_{n}^{l^{n+r}}\right)_{n \in \mathbb{N}}$ est, elle, paramétrée par les caractères $x_{\infty}^{*}, \mu_{c}^{*}+(r-1) x_{\infty}^{*}, \omega+\lambda_{c}^{*}$, et $\tilde{\nu}_{h}$. Parmi ceux-ci, $x_{\infty}^{*}$, $(r-1) x_{\infty}^{*}$, $\omega$, et 0 mesurent, en vertu du lemme 26 , et sous la conjecture de Leopoldt, la part de $\not_{n} / \sharp_{n}^{l^{n+r}}$ qui provient des $\mathbb{Z}_{\ell}$ extensions de $K_{n}$. Restent donc $0, \mu_{c}^{*}, \lambda_{c}^{*}$, et $\tilde{\nu}_{h}$, qui corres pondent aux quotients $\sigma_{n}^{1} / \sigma_{n}^{\prime} e^{n+r}$; ce qui est le résultatannoncé.

COROLLAIRE IV.2.36.- Sous la conjecture de Leopoldt, la suite de terme général $\mu_{n} \otimes_{\mathbb{Z}_{l}} \bar{H}_{2}\left(K_{n}\right)_{l} \approx \bar{\mu}_{n} \otimes_{\mathbb{Z}_{l}} H_{2}\left(K_{n}\right)_{l}$ est paramétrée par les caractères $0, \mu_{c}, \lambda_{c}$, et $\tilde{\nu}_{h}^{*}$.

Démonstration : Rappelons que nous avons défini par $\bar{H}_{2}\left(\mathrm{~K}_{\mathrm{n}}\right)$ le dual de Pontrjagin du groupe $\sigma_{n}^{\prime}{ }^{(\star)}$, et que llisomorphisme canonique

$\left(^{\star}\right)$ cf. Chap. $1.2,3 \S b$. 
(sous la conjecture de Leopoldt) $\mu_{n} \otimes_{\mathbb{Z}_{l}} \bar{H}_{2}\left(\mathrm{~K}_{\mathrm{n}}\right)_{\ell} \simeq \bar{\mu}_{\mathrm{n}} \otimes_{\mathbb{Z}_{l}} H_{2}\left(\mathrm{~K}_{\mathrm{n}}{ }^{\prime} \ell\right.$ est établi dans le chapitre 1.2 (théorème 1.2.52). Cela dit, les quo tients $\bar{H}_{2}\left(K_{n}\right)_{l} / \bar{H}_{2}\left(K_{n}\right)_{l}^{l n+r}$ sont les duaux respectifs des groupes $\ell^{n+r} \sigma_{n}^{\prime}$, et le corollaire résulte immédiatement du scolie 35 .

\section{b.-Parallèle entre les conjectures de Leopoldt et de Gross .}

Nous avons vu que le défaut de la conjecture de Gross dans la tour cyclotomique $K_{\infty} / K$ est lié à la présence de facteurs cyclo tomiques dans la décomposition irréductible des polynômes caracté ristiques respectifs des composantes isotypiques du groupe de Galois $c^{\prime}=\left\lfloor\right.$ lim $C_{n}^{\prime}$ de la $\ell$-extension abélienne non ramifiée $l$-décomposée maximale $C_{\infty}^{\prime}$ du corps $K_{\infty}$; et que, de façon semblable, le défaut de la conjecture de Leopoldt dans $K_{\infty} / K$ est lié à la présence de facteurs cyclotomiques dans la décomposition irréductible des polynômes caractéristiques respectifs des composantes isotypiques du sous-module de $\Lambda$-torsion $\zeta$ du groupe de Galois $a=\underline{\lim } a_{n}$ de la $l$-extension abélienne $\ell$-ramifiée maximale $M_{\infty}$ du corps $K_{\infty}$. Ainsi présenté, le parallèle entre les conjectures de Gross et de Leopoldt reste cependant imparfait, puisque les groupes $\sigma$ et $C^{\prime}$ n'ont pas la même taille, l'invariant lambda du groupe $\sigma$ étant, en général, strictement plus gros que celui du groupe $C^{\prime}$. Pour ajuster la comparaison, il convient de remplacer le groupe $\sigma$ par son quotient $\sigma^{\prime}$, étudié plus haut. II vient, en effet :

LEMME IV.2.37.- En toute hypothèse, il existe une suite exacte courte de $\Lambda[\Delta]$-modules :

$$
1 \longrightarrow\left({ }_{l_{\infty} \mid l}^{\oplus} \sigma_{l}\right) / \pi_{l} \longrightarrow \sigma \longrightarrow \sigma^{\prime} \longrightarrow 1,
$$

où $\pi_{l}$ est le module de Tate, $I_{\infty}$ parcourt les places de $K_{\infty}$ audessus de $l$, et le terme de gauche est un $\mathbb{Z}_{\ell}[\Delta]$ - module projectif de caractère $\omega\left(\psi_{l}-1\right)$. 
Démonstration: II est facile de voir que le lemme est vér ifié sous la conjecture de Leopoldt. Dans ce cas, en effet, la suite exacte courte qui définit le groupe $\tau_{n}^{\prime}$ ( cf. théorème 1.2.25) :

$$
1 \longrightarrow\left({ }_{I_{n} \mid l}^{\oplus} \mu_{I_{n}}\right) / \mu \longrightarrow \sigma_{n} \longrightarrow \sigma_{n}^{\prime} \longrightarrow 1
$$

s'écrit, à la limite :

$$
1 \longrightarrow\left({ }_{I_{\infty} \mid l}^{\oplus} \pi_{l}\right) / \pi_{l} \longrightarrow \sigma \longrightarrow \sigma_{1} \longrightarrow 1 ;
$$

ce qui est précisément le résultat annoncé .

Les choses sont un peu moins simples lorsque la conjecture de Leopoldt est en défaut :

l'application naturelle $\left(\underset{l_{n} \mid \ell}{\oplus} \mu_{l}\right) / \mu_{n} \rightarrow_{n}$, donnée par la théorie du corps de classes, n'est pas, en général, injective; et nous avons seulement une suite exacte longue :

$1 \longrightarrow \sqrt{\delta_{n}^{\infty}} / \mu_{n} \delta_{n}^{\infty} \longrightarrow\left(I_{n} \mid \ell \mu^{\mu_{n}}\right) / \mu_{n} \longrightarrow \sigma_{n} \longrightarrow \sigma_{n}^{\prime} \longrightarrow 1$, où $\varepsilon_{n}^{\infty}$ désigne le groupe des unités infinitésimales du corps $K_{n}$, et $\sqrt{\delta_{n}^{\infty}}$ son radical dans le tensorisé $k_{n}^{x}=\mathbb{Z}_{l} \otimes_{\mathbb{Z}} K_{n}^{x}$. Mais, comme les seuls éléments divisibles du groupe $k{ }_{\infty}^{x}=Z_{l} \otimes_{\mathbb{Z}} K_{\infty}^{X}$ sont les racines de l'unité, le seul fait que le défaut de la conjecture de Leopoldt (i.e. le rang du $\mathbb{Z} \ell_{\ell}$-module $\varepsilon_{n}^{\infty}$ ) soitborné dans la tour cyclotomique $K_{\infty} / K$ montre que les quotients $\sqrt{\delta}_{n}^{\infty} / \mu_{n} \varepsilon_{n}^{\infty}$ restent bornés lorsque $n$ croit; et nous obtenons encore, par passage à la limite projective, la suite exacte courte :

$1 \longrightarrow\left({ }_{1}^{\oplus \mid \ell} \pi_{\ell}\right) / \pi_{\ell} \longrightarrow \lim _{n}{ }_{n} \longrightarrow \lim _{n} \longrightarrow 1$

Maintenant, les deux quotients $\sigma / \lim \sigma_{n}$ d'une part, et $\sigma^{\prime} / \lim _{n}^{\prime}$ d'autre part, mesurent l'un et l'autre le groupe de Galois relatif des

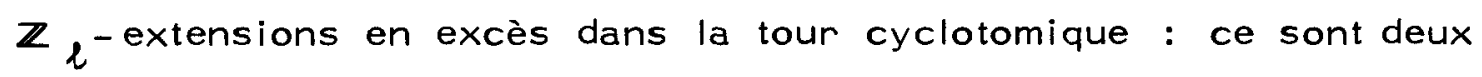
$\mathbb{Z}_{\ell}[\Delta]$-modules projectifs de même caractère : $\delta_{\text {Leopoldt }}$. II vient donc, comme plus haut : 


$$
1 \longrightarrow\left({ }_{l_{\infty} \mid l}^{\oplus} \pi_{l}\right) / \pi_{l} \longrightarrow \sigma \longrightarrow \delta^{\prime} \longrightarrow 1 ;
$$

ce qui établit le lemme.

Cela étant, nous pouvons résumer les diverses interpréta tions des conjectures de Gross et de Leopoldt développées plus haut (section $1, \S b$, et section $2, \S b$ ) de la manière synoptique suivante :

Interprétation cyclotomique de la conjecture de Gross
Interprétation cyclotomique de la conjecture de Leopoldt
Soit $C^{\prime}=\operatorname{Gal}\left(C_{\infty}^{\prime} / K_{\infty}\right)$ le groupe de Galois de la Lextension abélienne non ramifiée L-décomposée maximale $C_{\infty}^{\prime}$ du corps $K_{\infty}$; c'est un $\Lambda[\Delta]$-module noethe rien et de torsion de paramètres :

$$
\mu_{c} \text { et } \lambda_{c}
$$

Pour chaque caractère $\ell$-adique irréductible $\emptyset$ du groupe $\Delta$, écrivons :

$$
P c_{\varphi}^{\prime}(\gamma-1)=e^{H_{\varphi}} \Omega c_{\varphi}^{\prime}(\gamma-1) R c_{\varphi}(\gamma-1)
$$

la décomposition dans $Z_{\varphi}[\gamma-1]$ du polynôme caracté ristique de la $\varphi$-composante de $c^{\prime}$ comme produit d'une puissance de $l$, diun polynôme cyclotomique ${ }^{(*)}$, et d'un polynôme distingué étranger à tous les $\omega_{i}$; le polynôme $\Omega c_{\phi}^{\prime}$ a les mêmes facteurs irréductibles que le polynôme caractéristique $\tilde{\Omega} c_{\phi}^{\prime}$ commun aux $\varphi$-composantes des quotients $C^{\prime} / C^{\prime \omega_{n}}$ pour chaque $n$ assez grand. Le caractère :

$$
{ }^{\delta} \text { Gross }=\sum_{\varphi}\left(\operatorname{deg} \tilde{\Omega} c_{\varphi}^{\prime}\right)
$$

est le caractère de défaut de la conjecture de Gross dans la tour cyclotomique $K_{\infty} / K$. Il vérifie trivia lement la majoration :

$$
{ }^{8} \text { Gross } \leqslant \lambda_{c}
$$

En particulier, la conjecture de Gross est vérifiée dans la tour cyclotomique $K{ }_{\infty} / K$ dès que l'invariant lambda du groupe $C^{\prime}$ est nul .

(*) i.e. d'un polynôme dont les facteurs irr éductibles sont cyclotomiques. 
c.- Inégalités du miroir ( $l$ impair) .

Nous supposons, dans cette section, que le corps F est totalement réel, et que ses places à l'infini ont même sous-corps de décomposition, disons $\mathrm{K}^{+}$, dans l'extension abélienne $\mathrm{K} / F$. Dans ce cas, il est naturel d'écrire chaque caractère $\ell$-adique $\chi$ du groupe $\Delta$ comme somme $x^{+}+\chi^{-}$d'un caractère réel et d'un caractère imaginaire, en posant :

DÉFINITION IV.2.38. - Lorsque le corps $K$ est une extension quadratique totalement imaginaire d'un sur-corps $\mathrm{K}^{+}$de $\mathrm{F}$ totalement réel, le groupe de décomposition commun $\Delta_{\infty}$ des places à liinfini de $F$ dans $K / F$ est le groupe d'ordre 2 , engendré dans $\Delta$ par la conjugaison complexe. Nous disons alors qu'un caractère $l$-adique irréductible $\varphi$ du groupe $\Delta$ est réel lorsquili est représenté dans le caractère $x_{\infty}$, qu'il est imaginaire dans le cas contraire ; et qu'un caractère virtuel quelconque $\varphi$ est réel lorsque ses facteurs irréductibles sont réels, quil est imaginaire lorsqu'ils sont imaginaires. Les sous-groupes de $R \mathbb{Z}_{\ell}(\Delta)$, engendrés, le premier par les caractères irréductibles réels, le second par ceux imaginaires, sont deux sous-groupes supplémentaires, images l'un de l'autre dans l'involution du miroir.

Cela posé, fixons $n$ assez grand, et considérons le schéma de corps où sont représentées la $\ell$-extension hilbertienne maximale $H_{n}$ du corps $K_{n}$, la composée $Z_{n}$ des $\mathbb{Z}_{\ell}$-extensions de $K_{n}$, et la $\ell$-extension abélienne non ramifiée $\ell$-décomposée maximale $C_{n}^{\prime}$ de $K_{n}$ :

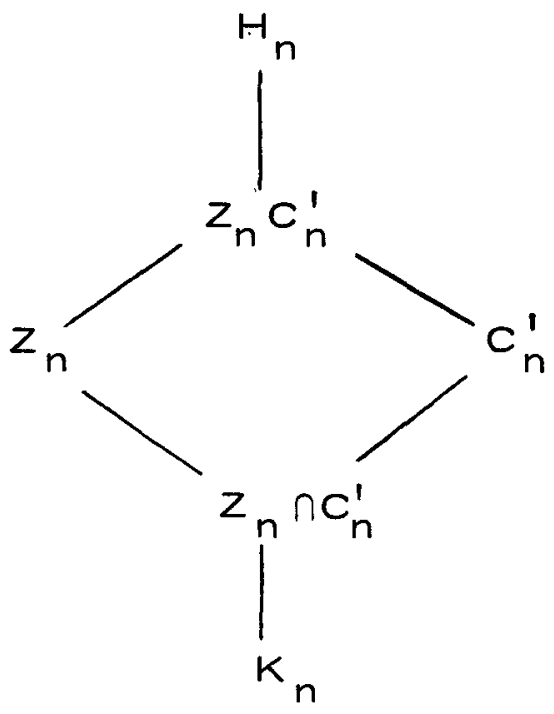


Nous avons :

LEMME IV.2.39.-Supposons que l'extension K/F possède une conju gaison complexe (i.e. que $K$ soit une extension quadratique totalement imaginaire d'un sur-corps totalement réel de F). Alors:

(i) Sous la conjecture de Leopoldt, le groupe de Galois Gal ( $\left.Z_{n} \cap C_{n}^{\prime} / K_{n}\right)$ est totalement imaginaire.

(ii) Sous la conjecture de Gross, le groupe de Galois Gal $\left(H_{n} / Z_{n} C_{n}^{\prime}\right)$ est totalement réel.

Démonstration : Lorsque l'extension abélienne $\mathrm{K} / \mathrm{F}$ possède une conjugaison complexe $F$, les idempotents orthogonaux $e_{+}=\frac{1}{2}(1+\bar{\tau})$ et $e_{-}=\frac{1}{2}(1-\vec{\tau})$ permettent d'écrire chaque $\mathbb{Z}_{\ell}[\Delta]$-module comme somme directe dlun module totalement réel et d'un module totalement imaginaire. Cela étant :

(i) La conjecture de Leopoldt dans le corps $K_{n}$ affirme que la partie réelle du groupe de Galois Gal $\left(Z_{n} / K_{n}\right)$ se réduit au seul groupe Gal $\left(K_{\infty} / K_{n}\right)$. Comme la tour cyclotomique $K_{\infty} / K_{n}$ est totalement ramifiée aux places au-dessus de $l$ dès que $n$ est assez grand, il vient par ailleurs : $C_{n}^{\prime} \cap K_{\infty}=K_{n}$; et donc $\operatorname{Gal}\left(\left(Z_{n} \cap C_{n}^{\prime}\right) / K_{n}\right)^{+}=\operatorname{Gal}\left(\left(K_{\infty} \cap C_{n}^{\prime}\right) / K_{n}\right)=1$, comme annoncé . (ii) D'après la théorie du corps de classes (cf. Ch. I.1, $2 \S b)$ le groupe de Galois Gal $\left(H_{n} / C_{n}^{\prime} Z_{n}\right)$ s'identifie au quotient ${\sqrt{\mu_{n}, l}} / \mu_{n, l}$, où $\mu_{n, l}=\mathfrak{l}_{n}|l| \mu_{l}$ est le groupe des racines de l'unité dans le complété semi-local $K_{n, l}^{x}$ associé à $K_{n}$, et $\sqrt{\mu_{n}}, \ell$ son radical dans le produit $\mu_{n, l} \delta_{n, l}^{\prime}$, où $\delta_{n, l}^{\prime}$ est l'image dans $K_{n, l}^{x}$ du tensorisé $l$-adique $\delta_{n}^{\prime}=\mathbb{Z}_{\ell} \otimes_{\mathbb{Z}} E_{n}^{\prime}$ du groupe des $l$ - unités de $K_{n}$. La conjecture de Gross dans le corps $K_{n}$ affirme que la restriction de llapplication de semi-localisation aux composantes imaginaires détermine une injection de $\xi_{n}^{\prime}-$ dans $k_{n, \bar{l}}^{x} \cdot 11$ vient alors $\sqrt{\mu}_{n, l^{-}}=\mu_{n, l^{\prime}}$, i.e. Gal $\left(H_{n} / C_{n}^{\prime} z_{n}\right)^{-}=1$, ce qui est le résul tat annoncé.

Passons maintenant à la limite, et considér ons le schéma de corps : 


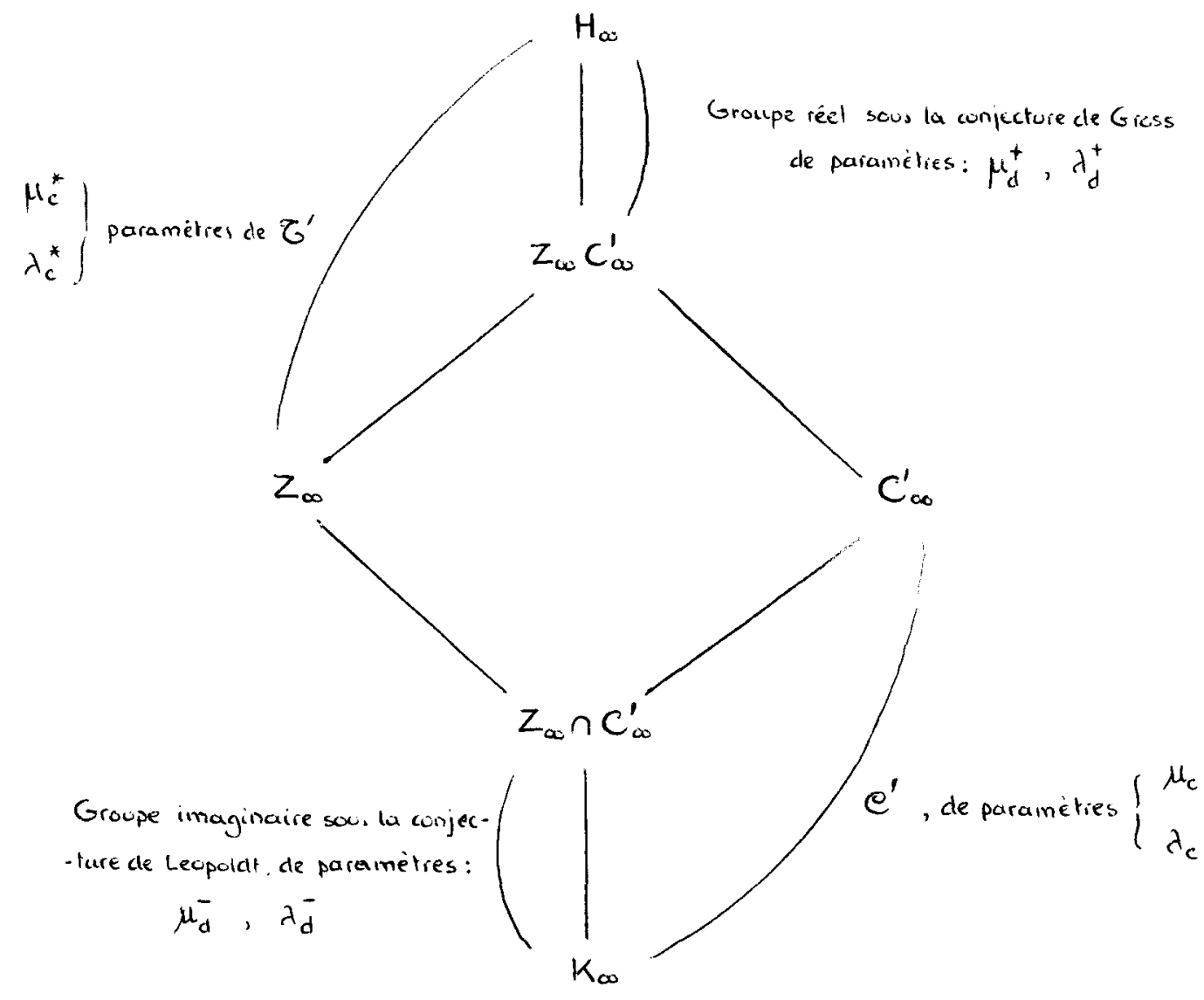

Dans celui-ci, les groupes $C^{\prime}=\mathrm{Gal}\left(\mathrm{C}_{\infty}^{\prime} / \mathrm{K}_{\infty}\right)$ et $G^{\prime}=$ Gal $\left(H_{\infty} / Z_{\infty}\right)$ sont les images l'un de l'autre dans l'involution du miroir : le premier est un $\Lambda[\Delta]$-module noethérien et de torsion de pa ramètres $\mu_{c}$ et $\lambda_{c}$; le second, de paramètres $\mu_{c}^{*}$ et $\lambda_{c}^{*}$. Enfin, les deux groupes $\mathrm{Gal}\left(\mathrm{H}_{\infty} / Z_{\infty} \mathrm{C}_{\infty}^{\prime}\right)$ et $\mathrm{Gal}\left(\left(Z_{\infty} \cap \mathrm{C}_{\infty}^{\prime}\right) / \mathrm{K}_{\infty}\right)$ sont respectivement réels et imaginaires, l'un en vertu de la conjecture de Gross, l'autre en vertu de celle de Leopoldt. Ecrivant donc $\mu_{d}^{+}$et $\lambda_{d}^{+}$les paramètres ( réels) du premier, puis $\mu_{\mathrm{d}}^{-}$et $\lambda_{\mathrm{d}}^{-}$ceux (imaginaires) du second, évaluant ensuite de deux façons différentes les paramètres du groupe Gal $\left(\left(z_{\infty} C_{\infty}^{\prime}\right) / z_{\infty}\right) \simeq \operatorname{Gal}\left(C_{\infty}^{\prime} /\left(z_{\infty} \cap C_{\infty}^{\prime}\right)\right)$, nous obte nons sans peine les équations :

$$
\left\{\begin{array} { l } 
{ \mu _ { c } ^ { - } - \mu _ { d } ^ { - } = \mu _ { c } ^ { + * } } \\
{ \lambda _ { c } ^ { - } - \lambda _ { d } ^ { - } = \lambda _ { c } ^ { + * } }
\end{array} \quad \text { et } \quad \left\{\begin{array}{l}
\mu_{c}^{+}=\mu_{c}^{-*}-\mu_{d}^{+} \\
\lambda_{c}^{+}=\lambda_{c}^{*}-\lambda_{d}^{+}
\end{array}\right.\right.
$$

ce que nous pouvons résumer sous la forme : 


$$
\mu_{c}^{-}-\mu_{c}^{+*}=\mu_{d}^{-}=\mu_{d}^{+*} \geq 0 \quad \lambda_{c}^{-}-\lambda_{c}^{+*}=\lambda_{d}^{-}=\lambda_{d}^{+*}=0 .
$$

THÉORĖME IV.2.39. (Inégalités du miroir) - Lorsque l'extension $K / F$ possède une conjugaison complexe $(i . e$. quand $K$ est une extension quadratique totalement imaginaire d'un sur-corps totalement réel de $F$ ), si les conjectures de Gross et de Leopoldt sont vérifiées dans la tour cyclotomique $K_{\infty} / K$, les paramètres $\mu_{c}$ et $\lambda_{c}$ du groupe de Galois $C^{\prime}=G a l\left(C_{\infty}^{\prime} / K_{\infty}\right)$ de la $\ell$-extension abélienne maximale $C_{\infty}^{\prime}$ non ramifiée et $\ell$-décomposée du corps $K_{\infty}$ vérifient les inégalités du miroir :

$$
\mu_{c}^{-} \geq \mu_{c}^{+*} \quad \text { et } \quad \lambda_{c}^{-} \geq \lambda_{c}^{+*}
$$

Remarque 1.- Comme nous l'avons vu, les conjectures de Leopoldt et de Gross ne concernent en fait que les invariants lambda . L'iné galité $\mu_{c}^{-} \geq \mu_{c}^{+}$vaut donc encore en l'absence de ces deux conjectures. De fait, l'invariant mu du groupe $C^{\prime}$ coïncide avec celui du groupe $C$ dont $C^{\prime}$ est le quotient ( cf. $1 \S \mathrm{b}$ ), et l'inégalité obtenue $\mu_{c}^{-} \geq \mu_{c}^{+*}$ n'est autre que la traduction en termes de caractères de celle bien connue deg $\mu_{c}^{-} \geq \operatorname{deg} \mu_{c}^{+}$entre les invariants mu d'Iwasawa du groupe $C$.

Remarque 2. - En revanche, les groupes $C$ et $C^{\prime}$ n'ont pas même pa ramètre lambda. D'après le théorème 9 , en effet, sous les hypo thèses précédentes, le paramètre lambda du groupe $c$ est donné par la formule: $\quad \lambda(c)=\lambda_{c}+\lambda_{l}=\lambda_{c}+\psi_{l}$ ( où $\psi_{l}$ est le caractère défini dans la section $2 \S$ a l. L'inégalité obtenue $\lambda_{c}^{-} \geq \lambda_{c}^{+*}$ est donc plus forte que celle bien connue $\operatorname{deg}\left(\lambda_{c}^{-}+\psi_{l}^{-}\right) \geq \operatorname{deg} \lambda_{c}^{+}$entre les invariants lambda d'Iwasawa du groupe $c$.

\section{d.- Application à une conjecture de Coates.}

Supposons, comme plus haut, que $K$ soit une extension qua dratique totalement imaginaire d'un sur-corps totalement réel $K_{+}$de $F$, et que les conjectures de Leopoldt et de Gross soient en outre vérifiées dans la tour cyclotomique $\mathrm{K}_{\infty} / \mathrm{K}$. Dans ce cas, les calculs qui 
précèdent permettent de résoudre complètement une conjecture avan cée par Coates sur la description kummérienne du corps $K_{\infty}$.

Considérons les trois extensions abéliennes $\ell$-ramifiées suivantes du corps $\mathrm{K}_{\infty}$ :

$K_{\infty}\left[\sqrt[8]{E}^{\prime}\right]$, engendrée par les racines d'ordre $\ell$-primaire des $\ell$-unités de $K_{\infty}$;

$K_{\infty}[\sqrt[l]{E}]$, engendrée par les racines d'ordre $l$-primaire des unités de $K_{\infty}$;

$Z_{\infty}$, fixée par le sous-module de $\Lambda$-torsion $G$ du groupe de Galois $a=\operatorname{Gal}\left(M_{\infty} / K_{\infty}\right)$ $(\star)$

Les inclusions suivantes sont bien connues :

$$
Z_{\infty} \subset K_{\infty}[\sqrt[l]{E}] \subset K_{\infty}\left[\sqrt[l]{\frac{\infty}{E}} i^{\prime}\right] \subset M_{\infty} .
$$

Rappelons brièvement comment les obtenir : Comme $K_{\infty}\left[\sqrt[l^{\infty}]{E^{\prime}}\right]$ contient évidemment $K_{\infty}[\sqrt[\ell]{E}]$, et que l'extension $K_{\infty}[\sqrt[\ell]{E}] / K_{\infty}$ est $\ell-r a-$ mifiée, il suffit de vérifier que $Z_{\infty}$ est contenue dans $K_{\infty}[\sqrt[\ell]{E}]$; autrement dit, que Gal $\left(M_{\infty} / K_{\infty}[\sqrt[\ell]{E}]\right)$ est un $\Lambda$-module de torsion. Pour voir cela, introduisons les radicaux kummériens :

$\Re=\left\{l^{-m} \otimes x \in\left(\mathbb{Q}_{l} / \mathbb{Z}_{l}\right) \otimes_{\mathbb{Z}} K_{\infty}^{x} \mid v_{p}(x) \equiv 0\left(\bmod l^{m}\right), \forall p \in \mathrm{Pl}_{K_{\infty}}\right.$ étrangère à $\left.l\right\}$, puis :

$\mathbb{E}=\left\{e^{-m} \otimes x \in\left(Q_{l} / \mathbb{Z}_{l}\right) \otimes_{\mathbb{L}} K_{\omega}^{x} \mid x \in E\right\}=\left(\mathbb{Q}_{l} / \mathbb{Z}_{l}\right) \otimes_{\mathbb{L}} E ;$

et considér ons le groupe $\alpha$, défini par la suite exacte courte canonique de $\Lambda[\Delta]$-modules:

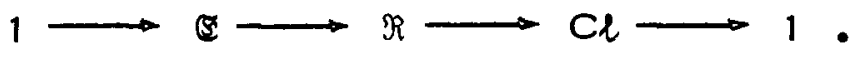

Une vérification immédiate montre que l'application qui, à l'élément $\ell^{-m} \otimes x$ de $\Re$, associe la classe $c l\left(\sqrt[\ell^{n}]{(x)}\right)$ de l'idéal $\sqrt[\ell^{m}]{(x)}$, induit un isomorphisme naturel du groupe $C l$ sur le $\ell$-groupe des classes d'i déaux du corps $K_{\infty}$ (On notera que l'appartenance à $\Re$ assure que liidéal principal est une puissance $l^{m}$-ième en-dehors de $l$, et que

$\left.{ }^{\star}\right)$ Sous la conjecture de Leopoldt dans $K_{\infty}$, l'extension $Z_{\infty}$ est la réunion $U_{n} \in \mathbb{N} Z_{n}$ des composées des $\mathbb{Z} \ell^{\text {-extensions respectives des }}$ corps $K_{n}$. 
les idéaux de $K_{\infty}$ construits sur les places au-dessus de $l$ sont des puissances $l^{k}$-ièmes pour tout $k$ ). Revenons maintenant aux groupes de Galois . Dlaprès l'isomorphisme fondamental de la théorie de Kummer, nous obtenons donc:

$$
\begin{aligned}
\operatorname{Gal}\left(M_{\infty} / K_{\infty}[\sqrt[l]{E}]\right)=\operatorname{Hom}\left(C l, \mu_{l}\right) & =\operatorname{Hom}\left(\frac{\lim }{n} c l_{n}, \mu_{l}\right) \\
& \sim \frac{\lim }{n} \operatorname{Hom}\left(C l_{n}, \mu_{l}\right),
\end{aligned}
$$

par un argument classique de la théorie d'lwasawa ( cf. $\left[\mathrm{Iw}_{6}\right], \S 5$, th. 11$)$. Désignons par $c l_{n}^{*}$ le reflet Hom $\left(C l_{n}, \mu_{l}\right)$ du groupe $C l_{n}$, puis par $C^{*}=\frac{\lim }{n} l_{n}^{*}$ la limite projective des groupes ainsi définis. Le groupe obtenu $C^{*}$ est alors un $\Lambda[\Delta]$-module noethérien, qui est de $\Lambda$-torsion, et dont les paramètres dans $R_{\mathbb{Z}_{\ell}}^{+}(\Delta)$ sont les reflets de ceux de $c=\frac{\lim }{n} \alpha_{n}$ dans linvolution du miroir. Nous disons que $C^{*}$ est le reflet du groupe $c=\mathrm{Ga} /\left(\mathrm{C}_{\infty} / \mathrm{K}_{\infty}\right)$ étudié plus haut Le groupe de Galois $\operatorname{Gal}\left(M_{\infty} / K_{\infty}[\sqrt[i]{E}]\right)$ s'évalue de la même façon : La suite exacte courte canonique loù $\mathbb{E}^{\prime}=\left(\mathbb{Q}_{l} / \mathbb{Z}_{l}\right) \otimes_{\mathbb{Z}} E^{\prime}$ est le radical kummérien de l'extension $K_{\infty}\left[\sqrt[2]{E^{\prime}}\right] / K_{\infty}$ ) :

$$
1 \longrightarrow \mathbb{F}^{\prime} \longrightarrow \mathrm{Cl}^{\prime} \longrightarrow 1
$$

montre que le quotient $\mathrm{Cl}=\Re / \mathbb{E}^{\prime}$ s'identifie au $l$-groupe des $l$ classes d'idéaux de $K_{\infty}$, et, par suite, que le groupe de Galois :

$$
\operatorname{Gal}\left(M_{\infty} / K_{\infty}[\sqrt[l]{E}]\right) \simeq \operatorname{Hom}\left(\mathrm{C} \ell, \mu_{\ell}\right)
$$

est pseudo-isomorphe au reflet $c^{\prime *}=\frac{\lim }{n} C_{n}^{\prime *}=\frac{\lim }{n} \operatorname{Hom}\left(\mathrm{Cl}_{n}^{\prime *}, \mu_{l^{\prime}}\right.$ du groupe $C^{\prime}=\mathrm{Ga}\left(\mathrm{C}_{\infty}^{\prime} / \mathrm{K}_{\infty}\right)$.

Enonçons ces résultats qui précisent un théorème classique dilwasawa ( $\mathrm{cf} \cdot\left[\mathrm{Iw}_{6}\right], \S 8$, th。 16 ) :

THÉORĖME IV.2.40.- Soient $K_{\infty}=K\left[\mu_{\ell}\right]$ la $\mathbb{Z}_{\ell}$-extension cyclotomique construite sur un corps $K$ contenant les racines $2 \ell$-ièmes de l'unité, et $M_{\infty}$ la $\ell$-extension abélienne $\ell$-ramifiée maximale de $K_{\infty}$ • 
(i) II existe un pseudo-isomorphisme canonique du groupe de Galois $\operatorname{Gal}\left(M_{\infty} / K_{\infty}[\sqrt[l]{E}]\right) \simeq \operatorname{Hom}\left(C l, \mu_{l}\right)$ dans le reflet $c^{*}=$ $\frac{\lim }{\mathrm{n}} \mathrm{Cl}_{\mathrm{n}}^{*}$ du groupe de Galois $C=\operatorname{Gal}\left(\mathrm{C}_{\infty} / \mathrm{K}_{\infty}\right)$ de la $\ell$-extensionabélienne non ramifiée maximale de $K_{\infty}$.

(ii) II existe un pseudo-isomorphisme canonique du groupe de Galois $\operatorname{Gal}\left(M_{\infty} / K_{\infty}\left[\sqrt[l]{E_{\infty}^{\prime}}\right]\right) \simeq \operatorname{Hom}\left(C l^{\prime}, \mathbb{H}_{\ell}\right)$ dans le reflet $C^{\prime *}=\frac{\lim }{n} C l_{n}^{\prime *}$ du groupe de Galois $C^{\prime}=\operatorname{Gal}\left(C_{\infty}^{1} / K_{\infty}\right)$ de la $\ell$-ex tension abélienne non ramifiée $\ell$-décomposée maximale du corps $K_{\infty}$. En particulier, lorsque $K$ est abélien sur un sous-corps $F$ de degré relatif d étranger à $l$, de groupe de Galois $\Delta$, les paramè tres associés à $\operatorname{Gal}\left(\mathrm{M}_{\infty} / \mathrm{K}_{\infty}[\sqrt[\ell]{E}]\right.$ ) (respectivement à $\left.\operatorname{Gal}\left(M_{\infty} / K_{\infty}[\sqrt[l]{E}]\right)\right)$ dans $R_{\mathbb{Z}}(\Delta)$ sont les reflets dans linvo lution du miroir de ceux associés à $\mathrm{Gal}\left(\mathrm{C}_{\infty} / \mathrm{K}_{\infty}\right)$ (respectivement à $\left.\operatorname{Gal}\left(\mathrm{C}_{\infty}^{\prime} / \mathrm{K}_{\infty}\right)\right)$.

COROLLAIRE IV.2.41.- Supposons que l'extension abélienne $\mathrm{K} / \mathrm{F}$ admette une conjugaison complexe (i.e. que $K$ soit une extension qua dratique totalement imaginaire d'un sur-corps $K$, totalement réel de $F$ ), et que les conjectures de Leopoldt et de Gross soient vérifiées dans la tour cyclotomique $K_{\infty} / K$. Le groupe de Galois $\operatorname{Gal}\left(K_{\infty}[\sqrt[l]{E}] / Z_{\infty}\right)$ est alors un $\mathbb{Z}_{\ell}[\Delta]$-module projectif et un $\Lambda[\Delta]$-module noethérien paramétré par le seul caractère :

$$
\lambda_{c}=\omega\left(\psi_{l}^{+}-1\right) \text {. }
$$

En particulier, l'égalité $Z_{\infty}=K_{\infty}[\sqrt[2]{E}]$ a lieu si et seulement si $\psi_{\ell}^{+}$est le caractère unité, c'est-à-dire lorsquil n'existe qu'un seul idéal premier au-dessus de $\ell$ dans le sous-corps réel maximal de $K_{\infty}$.

Démonstration: Un argument classique de la théorie d'lwasawa (cf. $\left[\mathrm{Iw}_{6}\right], \S 6.3$ ) montre que le groupe de Galois Gal ( $\mathrm{K}_{\infty}[\sqrt[i]{E}] / \mathrm{K}_{\infty}$ ) ne possède aucun sous- $\Lambda$-module fini non nul . L'égalité $K_{\infty}[\sqrt[l]{E}]=Z_{\infty}$ a donc lieu sous la seule condition que les paramètres 
du groupe $\mathrm{Gal}\left(\mathrm{K}_{\infty}[\sqrt[i]{E}] / Z_{\infty}\right)$ soient nuls. Or ceux-ci sont connus par le théorème 40 . En effet, les paramètres du groupe $6=$ $\mathrm{GaI}\left(\mathrm{M}_{\infty} / \mathrm{Z}_{\infty}\right)$ sont donnés par le théorème 19 ; et ceux du groupe $C=$ Gal ( $C_{\infty} / K_{\infty}$ ) par la proposition 5 et le corollaire 11 , sous les conjectures de Leopoldt et de Gross. II vient donc:

$$
\begin{aligned}
& \text { (i) Pour } E\left\{\begin{array}{l}
\mu=\mu_{c}^{*} \\
\lambda=\lambda_{c}^{*}+\omega\left(\psi_{l}-1\right)
\end{array}\right. \\
& \text { (ii) Pour } c^{*}\left\{\begin{array}{l}
\mu=\mu^{*} \\
\lambda=\left(\lambda+\psi_{l}^{-}\right)^{*}=\lambda^{*}+\omega_{l}^{-}
\end{array}\right.
\end{aligned}
$$

D'où le résultat annoncé.

L'identité $Z_{\infty}=K_{\infty}[\sqrt[i]{E}]$ constitue la conjecture de Coates, qui l'a établie dans le cas où il n'existe qu'une seule place au-dessus de $l$ dans le corps $K_{\infty}\left(c f .\left[\mathrm{Co}_{1}\right]\right)$. Un premier contre-exemple a été donné par Iwasawa. Le fait que l'égalité $Z_{\infty}=K_{\infty}[\sqrt[2]{E}]$ ait lieu, lorsque $K$ est absolument abélien, sous la condition nécessaire et suffisante qu'iln'y ait qu'une seule place au-dessus de $\ell$ dans le souscorps réel maximal de $K_{\infty}$ a été prouvé dans $\left[\mathrm{Ja}_{9}\right](\S 5$, th. 15$)$. Une démonstration de ce résultat a également été donnée indépendamment par Wingberg ( $\mathrm{cf} .[\mathrm{Wi}]$ ) à l'aide des théorèmes de dualité globale de Poitou-Tate. 
$-326-$ 
Nous donnons ci-dessous les paramètres des principaux modules rencontrés dans la section 2 .

Les notations sont donc celles de la section précédente. En particulier $l$ est un nombre premier, $K$ une extension abélienne d'un corps de nombres $F$, contenant les racines $\ell$-ièmes de llunité, de groupe de Galois $\Delta$, de degré d étranger à $\ell$; et $K K_{\infty}$ est la $\mathbb{Z} l^{-}$ extension cyclotomique de $K$. Le groupe $\Delta$ est identifié à son relèvement canonique dans $\mathrm{Gal}\left(\mathrm{K}_{\infty} / F\right)$.

Les paramètres dont il est question ici sont tous des caractères du groupe $\Delta$ à valeurs dans l'anneau $\mathbb{Z} l$ des entiers $l$-adiques. Tous s'expriment en fonction de cinq caractères de base définis comme suit :

(i) Le caractère cycl otomique w est le caractère de l'action de $\Delta$ sur les racines de l'unité : c'est le caractère du module de Tate $\sigma_{l}=\frac{\lim }{n} \mu_{n}$, qui est la limite projective des $l$-Sylow des groupes de racines de l'unité attachés aux sous-corps $K_{n}$ de degrés finis sur $K$.

(ii) Le caractère à I'infini $x_{\infty}=\sum_{p / \infty} x_{p}$ est la somme des induits à $\triangle$ des caractères des représentations unités des sous groupes de décomposition des places à linfinini de $F$ dans l'extension $\mathrm{K} / \mathrm{F}$.

(iii) Le caractère de Ia $l$-decomposition $\psi_{\ell}=\sum_{I \mid l} g_{I} X_{I}$ est la somme des induits à $\triangle$ des caractères des représentations unités des sous-groupes de décomposition des places de F au-dessus de $f$, chacun compté avec une multiplicité égale à l'indice de décomposition de la place en question dans la tour $K_{\infty} / K$. 
(iv) Enfin, les deux paramètres $\lambda$ et $\mu$ sont les paramètres construits sur les invariants d'Iwasawa des $\ell$-groupes de $\ell$-classes de diviseurs des corps $K_{n}\left(i_{\text {. e. }}\right.$ des quotients respectifs $\mathrm{Cl}_{n}^{\prime}$ des $l-$ groupes $\mathrm{Cl}_{\mathrm{n}}$ de classes de diviseurs des corps $\mathrm{K}_{n}$, par les sousgroupes correspondants engendrés par les images des places au-dessus de $\ell$ ). Ces paramètres sont notés $\lambda_{c}$ et $\mu_{c}$ dans la section 2 .

\section{a.- Caractères associés aux suites paramétrées de $\mathbf{Z}_{\ell}[\Delta]$-modules finis.}

Conformément à la définition IV.1.15, nous disons qu'une

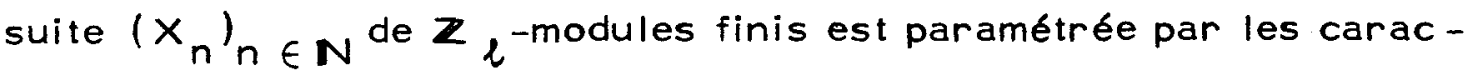
tères $\ell$-adiques virtuels $\rho=\sum_{\varphi} \rho_{\varphi} \varphi, \mu=\sum_{\varphi} \mu_{\varphi} \varphi, \lambda=\Sigma_{\varphi} \lambda_{\varphi} \varphi$, et $\nu=\sum_{\varphi} \nu_{\varphi} \varphi$, lorsque, pour chaque caractère $l$-adique irréduc tible $\varphi$ du groupe $\Delta$, llordre $e^{\varphi}$ de la $\varphi$-composante $x_{n, \varphi}=e_{\varphi} X_{n}$ du module $X_{n}$ est donné asymptotiquement par la formule :

$$
x_{n}^{\varphi}=\langle p, \varphi\rangle(n+1) \ell^{n}+\langle\mu, \varphi\rangle \ell^{n}+\langle\lambda, \varphi\rangle n+\langle\nu, \varphi\rangle .
$$

\begin{tabular}{|c|c|c|c|c|c|}
\hline \multicolumn{6}{|c|}{ TABLEAU I } \\
\hline \multirow{2}{*}{\multicolumn{2}{|c|}{$\begin{array}{l}\text { Modules } \\
\text { paramétrés }\end{array}$}} & \multirow{2}{*}{ Definition } & \multicolumn{3}{|c|}{ Paramètres } \\
\hline & & & $c$ & $\lambda$ & $\mu$ \\
\hline $\begin{array}{l}E_{n} / E_{n}^{n+1} \\
E_{n}^{\prime} / E_{n}^{\prime n+1} \\
\quad d_{n}^{\prime}\end{array}$ & & $\begin{array}{l}H_{n} \text { est le } \ell \text {-groupe des racines de l'unité dans } K_{n} \\
E_{n} \text { est le groupe des unités du corps } K_{n} \\
E_{n}^{\prime} \text { est le groupe des } \ell \text {-unités du corps } K_{n} \\
C_{n}^{\prime} \text { est le } \ell \text {-groupe des } \ell \text {-classes de } K_{n}\end{array}$ & $\begin{array}{c}0 \\
x_{\infty} \\
x_{\infty} \\
0\end{array}$ & $\begin{array}{l}w-1 \\
k+\left(l^{-1}\right)\end{array}$ & $\begin{array}{l}0 \\
0 \\
0 \\
1\end{array}$ \\
\hline $\begin{array}{l}\operatorname{Gal}\left(C_{n}^{\prime} / K_{n}\right) \\
\operatorname{Rad}\left(C_{n}^{\prime} / K_{n}\right)\end{array}$ & & $\begin{array}{l}C_{n}^{\prime} \text { est la } \ell \text {-extension abélienne maximale d'exposant } \ell^{n+1} \\
\text { de } K_{n} \text { qui est non ramifiée el } \ell \text {-décomposée }\end{array}$ & $\begin{array}{l}0 \\
0\end{array}$ & $\stackrel{\lambda}{\omega_{\lambda}^{-1}}$ & $\omega_{\mu}^{-1}$ \\
\hline $\begin{array}{l}\operatorname{Gal}\left(M_{n} / K_{n}\right) \\
\operatorname{Rad}\left(M_{n} / K_{n}\right)\end{array}$ & & $\begin{array}{c}M_{n} \text { est la } \ell \text {-extension abélienne maximaled'exposant } \ell^{n+1} \\
\text { de } K_{n} \text { qui est } l \text {-ramifiée }\end{array}$ & $x_{\infty}^{\infty}$ & $\begin{array}{c}w \lambda^{-1}+1+w\left(2^{-1}\right) \\
\lambda+w+\left(l^{-1}\right)\end{array}$ & $\omega_{\mu}^{-1}$ \\
\hline $\begin{array}{l}\operatorname{Gal}\left(H_{n} / K_{n}\right) \\
\operatorname{Rad}\left(H_{n} / K_{n}\right)\end{array}$ & & $\begin{array}{l}H_{n} \text { est la composée des } \ell \text {-extensions cycliques d'expo- } \\
\text { sant } \ell^{n+1} \text { de } K_{n} \text { qui sont cycliquement plongeables }\end{array}$ & $x_{\infty}^{\infty}$ & $\begin{array}{l}w \lambda^{-1}+1 \\
\lambda+w\end{array}$ & $\omega_{\mu}^{-1}$ \\
\hline $\begin{array}{c}\operatorname{Gal}\left(Z_{n} / K_{n}\right) \\
\operatorname{Rad}\left(Z_{n} / K_{n}\right) \\
u_{n}\end{array}$ & $\star$ & 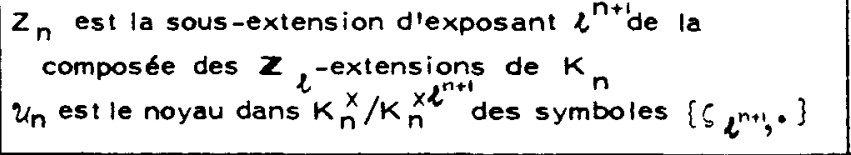 & $\begin{array}{l}w x_{\infty} \\
x_{\infty} \\
x_{\infty}\end{array}$ & $\begin{array}{l}1 \\
w\end{array}$ & $\begin{array}{l}0 \\
0 \\
0\end{array}$ \\
\hline $\begin{array}{l}\operatorname{Ga}\left(M_{n} / Z_{n}\right) \\
\operatorname{Rad}\left(M_{n} / Z_{n}\right) \\
R^{n+1} R_{2}\left(K_{n}\right)\end{array}$ & * & $\begin{array}{l}e^{n+1} R_{2}\left(K_{n}\right) \text { est le sous-groupe de } e^{n+1} \text {-torsion du noyau } \\
\text { dans } K_{2}\left(K_{n}\right) \\
\text { des symboles réguliers attachés aux places de } K_{n}\end{array}$ & $\begin{array}{l}0 \\
0 \\
0\end{array}$ & $\begin{array}{c}\omega \lambda^{-1}+\omega\left(\psi_{2}-1\right) \\
\lambda+\left(2_{2}-1\right) \\
\omega \lambda+w\left(l^{-1}\right)\end{array}$ & $w_{\mu}^{-1}$ \\
\hline $\mid \begin{array}{l}\operatorname{Gal}\left(H_{n} / Z_{n}\right) \\
\operatorname{Rad}\left(H_{n} / Z_{n}\right) \\
{ }^{n+1} H_{2}\left(K_{n}\right)\end{array}$ & * & $\begin{array}{l}e^{n+1} H_{2}\left(K_{n}\right) \text { est le sous-groupe de } l^{n+1} \text {-torsion du noyau } \\
\text { dans } K_{2}\left(K_{n}\right) \\
\text { des symboles de Hi lbert attachés aux places de } K_{n}\end{array}$ & $\begin{array}{l}0 \\
0 \\
0\end{array}$ & $\begin{array}{l}\omega \lambda^{-1} \\
\lambda \\
\omega \lambda\end{array}$ & $\omega_{\mu}^{\omega \mu^{-1}}$ \\
\hline
\end{tabular}




\section{b. - Caractères associés aux $\Lambda[\Delta]$-modules noethériens en présence d'une conjugaison complexe.}

Nous supposons ici que $\ell$ est impair et que l'extension abélienne $K / F$ admet une conjugaison complexe $(i$.e. que $K$ est une extension quadratique totalement imaginaire d'un sur-corps totalement réel de $k$ ). Nous écrivons $x=x^{+}+x^{-}$la décomposition canonique d'un caractère $l$-adique du groupe $\Delta$ en ses composantes réelles et imaginaires.

En accord avec la définition $\mathrm{V} .1 .7$, nous notons $\Lambda[\Delta]=$ $\oplus_{\varphi} \Lambda_{\varphi}$ l'algèbre semi-locale du groupe $\Delta$ à coefficients dans l'anneau d'lwasawa $\Lambda=Z_{\ell}[[\gamma-1]]$, et nous appelons paramètres d'un $\Lambda[\Delta]$ modules noethérien les trois caractères $\ell$-adiques :

$$
\rho=\sum_{\varphi} \rho_{\varphi} \varphi, \quad \mu=\sum_{\varphi} \mu_{\varphi} \varphi, \quad \text { et } \lambda=\sum_{\varphi} \lambda_{\varphi} \varphi \text {, }
$$

où, pour chaque caractère $\ell$-adique irréductible $\varphi$ de $\Delta$, les entiers naturels $\rho_{\varphi}$, $\mu_{\varphi}$, et $\lambda_{\varphi}$ sont les invariants d'lwasawa de la $\varphi$-composante de $X$ regardée comme $\Lambda_{\varphi}$-module : l'entier $\rho_{\varphi}$ est la dimension sur $\Lambda_{\varphi}$ du module $X_{\varphi}$; les entiers $\lambda_{\varphi}$ et $\mu_{\varphi}$ sont respectivement le degré et la $\ell$-valuation du polynôme caractéristique $P_{\varphi} \in Z_{\varphi}[Y-1]$ de son sous-module de $\Lambda_{\varphi}$-torsion.

\begin{tabular}{|c|c|c|c|c|c|}
\hline \multicolumn{6}{|c|}{ TABLEAU $\|$} \\
\hline \multirow{2}{*}{\multicolumn{2}{|c|}{$\Lambda[\Delta]$-modules }} & \multirow{2}{*}{ Definition } & \multicolumn{2}{|r|}{ Paramètres } & \\
\hline & & & 0 & $\lambda$ & $u$ \\
\hline $\begin{array}{l}\operatorname{Gal}\left(M_{\infty} / K_{\infty}\right) \\
\operatorname{Gal}\left(H_{\infty} / K_{\infty}\right)\end{array}$ & & $\begin{array}{l}M_{\infty}=\underset{n \in \mathbb{N}}{U} M_{n} \text { est abelienne } \ell \text {-ramifice maximale sur } K_{\infty} \\
H_{\infty}=\underset{n \in \mathbb{N}}{U} H_{n} \text { est hilbertienne maximale sur } K_{\infty}\end{array}$ & $w x_{\infty}$ & $\begin{array}{c}w \lambda^{-1}+w\left(2^{-1}\right) \\
w \lambda^{-1}\end{array}$ & $\operatorname{lin}^{-1}$ \\
\hline $\begin{array}{l}\operatorname{Gal}\left(z_{\infty} / K_{\infty}\right) \\
\operatorname{Gal}\left(k_{\infty} \sqrt[2]{E_{\infty}} / K_{\infty}\right)\end{array}$ & $\star$ & $\begin{array}{l}Z_{\infty}=U_{n \in N} Z_{n} \text { est composée des } Z_{\ell} \text {-extensions des corps } K_{n} \\
E_{\infty} \text { est le groupe des unités des corps } K_{\infty}\end{array}$ & $\begin{array}{l}x_{\infty} \\
x x_{\infty}\end{array}$ & $w\left(+\frac{i}{i}\right)$ & 0 \\
\hline $\begin{array}{l}\left.\text { Gall } C_{\infty} / K_{\infty}\right) \\
\operatorname{Gal}\left(M_{\infty} / K_{\infty} \sqrt[i]{E}\right)\end{array}$ & 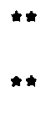 & $\begin{array}{l}C_{\infty}=U_{n \in N} C_{n} \text { est abélienne non ramifiée maximale sur } K_{\infty} \\
E_{\infty} \text { ess le groupe des unités de } K{ }_{\infty}\end{array}$ & 0 & $\lambda+\bar{l}$ & $\omega \mu^{-1}$ \\
\hline $\begin{array}{l}\text { Gall } C_{\infty}^{\prime} / K K_{\infty}^{\prime} \\
\operatorname{Gal}\left(M_{\infty} / K i_{\infty}^{i} E_{\infty}^{\prime}\right)\end{array}$ & & $\begin{array}{l}C^{\prime} \text { esi la sous-extension } \ell \text {-decomposée maximale de } C_{\infty} \\
E_{\infty}^{\prime} \text { est le groupe des } \ell \text {-unités de } K_{\infty}\end{array}$ & 0 & $\begin{array}{l}\lambda \\
\lambda^{-1}\end{array}$ & $\omega_{N^{-1}}^{\mu}$ \\
\hline
\end{tabular}

(*) L'astérisque repare les modules pour lesquels le calcul de l'invariant lambda fait intervenir la conjecture de Leopoldt.

(*) La double astérisque repère ceux pour lesquels le même calcul fait appel en outre a la conjecture de Gross.

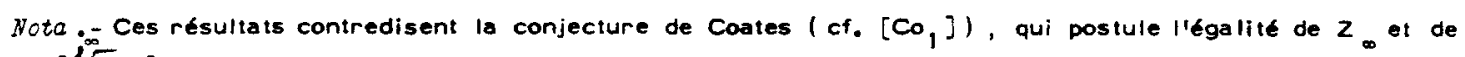
$K_{\infty}[\sqrt[i]{E}]$ 


\begin{tabular}{|c|c|c|c|c|}
\hline \multicolumn{5}{|c|}{ TABLEAU 1} \\
\hline \multirow{2}{*}{\multicolumn{2}{|c|}{$\begin{array}{l}\text { Modules } \\
\text { paramétrés }\end{array}$}} & \multirow{2}{*}{ Définition } & \multicolumn{2}{|r|}{$P$} \\
\hline & & & $p$ & \\
\hline $\begin{array}{l}E_{n} / E_{n}^{\ell^{n+1}} \\
E_{n}^{\prime} / E_{n}^{1 \ell^{n+1}} \\
C_{n}\end{array}$ & & $\begin{array}{l}\mu_{n} \text { est le } \ell \text {-groupe des racines de l'unité dans } K_{n} \\
E_{n} \text { est le groupe des unités du corps } K_{n} \\
E_{n}^{\prime} \text { est le groupe des } \ell \text {-unités du corps } K_{n} \\
C \ell_{n}^{\prime} \text { est le } \ell \text {-groupe des } \ell \text {-classes de } K_{n}\end{array}$ & $\begin{array}{c}0 \\
x_{\infty} \\
x_{\infty} \\
0\end{array}$ & $\omega+$ \\
\hline $\begin{array}{l}\operatorname{Gal}\left(C_{n}^{\prime} / K_{n}\right) \\
\operatorname{Rad}\left(C_{n}^{\prime} / K_{n}\right)\end{array}$ & & $\begin{array}{l}C_{n}^{\prime} \text { est la } \ell \text {-extension abélienne maximale d'exposant } \ell^{n+1} \\
\text { de } K_{n} \text { qui est non ramifiée et } l \text {-décomposée }\end{array}$ & $\begin{array}{l}0 \\
0\end{array}$ & \\
\hline $\begin{array}{l}\operatorname{Gal}\left(M_{n} / K_{n}\right) \\
\operatorname{Rad}\left(M_{n} / K_{n}\right)\end{array}$ & & $\begin{array}{c}M_{n} \text { est la } l \text {-extension abélienne maximale d'exposant } l^{n+1} \\
\qquad \text { de } K_{n} \text { qui est } l \text {-ramifiée }\end{array}$ & $\begin{array}{l}\omega x_{\infty} \\
x_{\infty}\end{array}$ & $\begin{array}{c}\omega \lambda^{-1} \\
\lambda+\end{array}$ \\
\hline $\begin{array}{l}\operatorname{Gal}\left(H_{n} / K_{n}\right) \\
\operatorname{Rad}\left(H_{n} / K_{n}\right)\end{array}$ & & $\begin{array}{l}H_{n} \text { est la composée des } \ell \text {-extensions cycliques d'expo- } \\
\text { sant } \ell^{n+1} \text { de } K_{n} \text { qui sont cycliquement plongeables }\end{array}$ & $\begin{array}{l}w x_{\infty} \\
x_{\infty}\end{array}$ & \\
\hline$\left|\begin{array}{c}\operatorname{Gal}\left(Z_{n} / K_{n}\right) \\
\operatorname{Rad}\left(Z_{n} / K_{n}\right) \\
u_{n}\end{array}\right|$ & $\begin{array}{l}\star \\
\star\end{array}$ & $\begin{array}{l}Z_{n} \text { est la sous-extension d'exposant } \ell^{n+1} \text { de la } \\
\text { composée des } \mathbb{Z} \ell^{-e x t e n s i o n s} \text { de } K_{n} \\
u_{n} \text { est le noyau dans } K_{n}^{x} / K_{n}^{x l^{n+1}} \text { des symboles }\left\{\zeta_{l^{n+1}, \cdot}\right\}\end{array}$ & $\begin{array}{l}\omega x_{\infty} \\
x_{\infty} \\
x_{\infty}\end{array}$ & \\
\hline $\begin{array}{l}\operatorname{Gal}\left(M_{n} / Z_{n}\right) \\
\operatorname{Rad}\left(M_{n} / Z_{n}\right) \\
e^{n+1} R_{2}\left(K_{n}\right)\end{array} \mid$ & ネ & $\begin{array}{l}l^{n+1} R_{2}\left(K_{n}\right) \text { est le sous-groupe de } l^{n+1} \text {-torsion du noyau } \\
\text { dans } K_{2}\left(K_{n}\right) \\
\text { des symboles réguliers attachés aux places de } K_{n}\end{array}$ & $\begin{array}{l}0 \\
0 \\
0\end{array}$ & $\begin{array}{l}\omega \lambda^{-1} \\
\lambda+ \\
\omega \lambda+\end{array}$ \\
\hline $\begin{array}{l}\operatorname{Gal}\left(H_{n} / Z_{n}\right) \\
\operatorname{Rad}\left(H_{n} / Z_{n}\right) \\
e^{n+1} H_{2}\left(K_{n}\right)\end{array}$ & ネ & $\begin{array}{l}l^{n+1} H_{2}\left(K_{n}\right) \text { est le sous-groupe de } l^{n+1} \text {-torsion du noyau } \\
\text { dans } K_{2}\left(K_{n}\right) \\
\text { des symboles de Hilbert attachés aux places de } K_{n}\end{array}$ & $\begin{array}{l}0 \\
0 \\
0\end{array}$ & \\
\hline
\end{tabular}




\begin{tabular}{|c|c|c|}
\hline & & TABLEAU $\|$ \\
\hline \multicolumn{2}{|l|}{$\Lambda[\Delta]$-modules } & Définition \\
\hline $\begin{array}{l}\operatorname{Gal}\left(M_{\infty} / K_{\infty}\right) \\
\operatorname{Gal}\left(H_{\infty} / K_{\infty}\right)\end{array}$ & & $\begin{array}{l}M_{\infty}=\underset{n \in \mathbb{N}}{U} M_{n} \text { est abélienne } l \text {-ramifiée maximale sur } K_{\infty} \\
H_{\infty}=\underset{n \in \mathbb{N}}{U} H_{n} \text { est hilbertienne maximale sur } K_{\infty}\end{array}$ \\
\hline $\begin{array}{l}\operatorname{Gal}\left(Z_{\infty} / K_{\infty}\right) \\
\operatorname{Gal}\left(K_{\infty} \sqrt[\ell]{E}_{\infty} / K_{\infty}\right)\end{array}$ & $\star$ & $\begin{array}{l}Z_{\infty}=U_{n \in \mathbb{N}} Z_{n} \text { est composée des } \mathbb{Z}_{\ell} \text {-extensions des corps } K_{n} \\
E_{\infty} \text { est le groupe des unités des corps } K_{\infty}\end{array}$ \\
\hline $\begin{array}{l}\operatorname{Gal}\left(C_{\infty} / K_{\infty}\right) \\
\operatorname{Gal}\left(M_{\infty} / K_{\infty} \sqrt[i c]{E}\right)\end{array}$ & $\star \star$ & $\begin{array}{l}C_{\infty}=U_{n \in \mathbb{N}} C_{n} \text { est abélienne non ramifiée maximale sur } K_{\infty} \\
E_{\infty} \text { est le groupe des unités de } K_{\infty}\end{array}$ \\
\hline $\begin{array}{l}\left.\text { Gall } C_{\infty}^{\prime} / K_{\infty}^{\prime}\right) \\
\operatorname{Gal}\left(M_{\infty} / K_{\infty} \sqrt[\ell^{\prime}]{E_{\infty}^{\prime}}\right)\end{array}$ & & $\begin{array}{l}C_{\infty}^{\prime} \text { est la sous-extension } \ell \text {-décomposée maximale de } C_{\infty} \\
E_{\infty}^{\prime} \text { est le groupe des } \ell \text {-unités de } K_{\infty}\end{array}$ \\
\hline
\end{tabular}

(*) L'astérisque repère les modules pour lesquels le calcul de l'invariant lambc conjecture de Leopoldt.

( $\star \star)$ La double astérisque repère ceux pour lesquels le même calcul fait appel en out

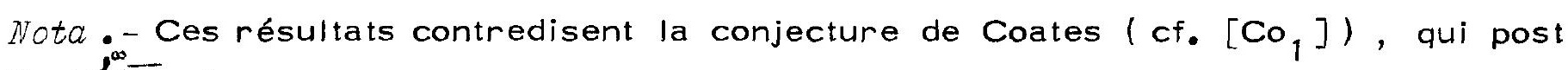
$K_{\infty}\left[\sqrt[l^{\infty}]{E_{\infty}}\right]$. 
$-332-$ 
[AF ] J.V. ARMITAGE \& A. FRÖHLICH .- Class numbers and unit signatures - Mathematika 14 (1967), 54-58.

[Ar ] M. ARNAUDON.- Etude des normes dans les extensions galoisiennes de corps de nombres -

Thèse de $3^{\text {ème }}$ cycle , Bordeaux (1976).

[AT ] E. ARTIN \& J. TATE . - Class field theory - Benjamin , New-York Amsterdam (1967).

[AX] J. AX.- On the units of an algebraic number field III. J. Math. 9 (1965), 584-589 .

[Ba] A. BAKER . - Linear forms in the logarithms of algebraic numbers Mathematika 13 (1966), 204-216.

[Bb] N. BOURBAKI .- Eléments de Mathématique, Livre II, Ch.7 (Algèbre: Modules sur les anneaux principaux) - Hermann, Paris (1964).

$\left[B h_{1}\right]$ M. BHASKARAN .- Construction of genus field and some applica tions - J. Numb. Th. 11 (1979), $488-497$; J. Numb. Th. 19 (1984), 449-451.

$\left[\mathrm{Bh}_{2}\right]$ M. BHASKARAN .- Some fur ther remarks on genus field - J. Numb. Th. 21 (1985), 256-259 .

[Bo] R.J. BOND .- Some results on the capitulation problem J. Numb. Th. 13 (1981), 246-254 . 
[BP ] F. BERTRANDIAS \& J.-J. PAYAN .- T-extensions et invariants cyclotomiques - Ann. Sci. Ec. Norm. Sup. 5 ( 1972), $517-548$.

[Br] A. BRUMER . - On the units of algebraic number fields Mathematika 14 (1967), 121-124.

[BR] A. BRUMER \& M. ROSEN .- Class number and ramification in number fields - Nagoya Math. Jo 23 (1963), 97-101.

[BS] J. BROWKIN \& A. SCHINZEL - - On Sylow 2 -subgroups of $K_{2} \mathrm{O}_{\mathrm{F}}$ for quadratic number field - J. reine angew. Math. 331 (1981), 104-113.

[Bw] J. BROWKIN .- The functor $K_{2}$ for the ring of integers of a number field - Prépublication.

$\left[\mathrm{Ca}_{1}\right]$ J. E. CARROLL - - On the 2 -primary part of $K_{2} \theta$ and on $\mathbb{Z}_{2}$-extensions for imaginary quadratic fields - Ph. D. Thesis, Cambridge Mass. (1973).

$\left[\mathrm{Ca}_{2}\right]$ J.E. CARROLL .-On determining the quadratic subfields of $\mathbb{Z}_{2}$-extensions of complex quadratic fields Compositio Math. 30 (1975), 259-271 .

$\left[\mathrm{Ca}_{3}\right]$ J.E. CARROLL . - On a relationship between the 2 -primary part of the tame kernel and $\mathbb{Z}_{2}$-extensions for complex qua dratic fields -

[Cd] A. CANDIOTTI .- Computation of Iwasawa invariants and $K_{2}$ Compositio Math. 29 (1974), 89-111.

[CF ] J.W.S. CASSELS \& A. FRÖHLICH .- Algebraic Number Theory Academic Press, London - New-York (1967). 
$\left[\mathrm{Ch}_{1}\right]$ C. CHEVALLEY - - Sur la theorie du corps de classes dans les corps locaux finis et les corps locaux - J. Fac. Sci. Tokyo 2 (1933), 365-476.

$\left[\mathrm{Ch}_{2}\right]$ C. CHEVALLEY - - La théorie du corps de classes - Ann. of Math. 2 (1940), 394-419.

[CK $\left.{ }_{1}\right]$ J.E. CARROLL \& H. KISILEVSKY. - On Iwasawa's $\lambda$ invariant for

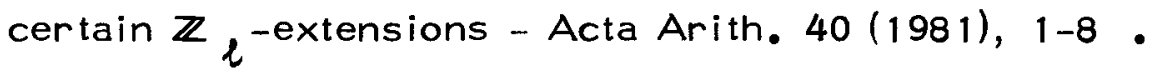

$\left[\mathrm{CK}_{2}\right]$ J.E. CARROLL \& H. KISILEVSKY.- On the Iwasawa invariants of certain $\mathbb{Z}_{\mathrm{p}}$-extensions - Compositio Math. 49 (1983), 217-229.

$\left[\mathrm{CK}_{3}\right]$ J.E. CARROLL $\&$ H. KISILEVSKY.- Initial layers of $\mathbb{Z} l^{\text {exten }}$ sions of complex quadratic fields - Comp. Math. 32 (1976), 157-168 。

[Co ${ }_{1}$ ] J. COATES.- On $K_{2}$ and some classical conjectures in algebraic number theory - Ann. of Math. 95 (1972), 99-116 .

$\left[\mathrm{Co}_{2}\right]$ J. COATES - Research problems : arithmetic questions in K-theory Algebraic $K$-theory 11 , Lecture Notes in Math. 341 (1973), $521-523$.

$\left[\mathrm{Co}_{3}\right]$ J. COATES.-p-adic L-functions and Iwasawa's theory - Algebraic number fields, Academic Press, London - New-York San Francisco (1977).

[CR] G. CORNELL \& M. ROSEN .- Group - theoretic constraints on the structure of the class group - J. Numb. Th. 13 (1981), $1-11$.

[CW ] S.U. CHASE \& W.C. WATERHOUSE .- Moore's theorem on unique ness of reciprocity laws - Inv. Math. 16 (1972), 267-270。 
[Du ] D.S. DUMMIT .- An extension of Iwasawa's theorem on finitely ge nerated modules over power series rings Manusc. Math. 43 (1983), 229-259 .

[EKW] M. EMSALEM , H. KISILEVSKY \& D.B. WALES .- Indépendance Iinéaire sur $\overline{\mathbf{Q}}$ de logarithmes $\mathrm{p}$-adiques de nombres algébriques et rang $p$-adique du groupe des unités d'un corps de nombres - J. Numb. Th. 19 (1984), 384-391.

[Em] M. EMSALEM .- Rang $p$-adique de groupes de $S$-unités d'un corps de nombres - C.R. Acad. Sci. Paris 297 (1983), 225-227 .

[Fd $\left.{ }_{1}\right]$ L.J. FEDERER .- p-adic L-Functions, Regulators, and Iwasawa modules - Ph. D. Thesis, Princeton (1982).

$\left[\mathrm{Fd}_{2}\right]$ L.J. FEDERER - - Regulators, Iwasawa modules, and the main conjecture for $p=2$ - Modern trends in number theory related to Fermat's last theorem, Birkhauser

$\left[\mathrm{Fd}_{3}\right]$ L.J. FEDERER .- Noetherian $\mathbb{Z}_{\mathrm{p}}[[\gamma-1]]$-modules, adjoints, and Iwasawa theory - Prépublication .

$\left[\mathrm{Fd}_{4}\right]$ L.J. FEDERER .- Genera theory for S-class groups Prépublication.

$\left[\mathrm{Fd}_{5}\right]$ L.J. FEDERER •- R-generalized $\mathrm{S}$-class groups, Kummer theory, and characteristic power series - Prépublication .

[FG] L.J. FEDERER \& B. GROSS .- Regulators and Iwasawa Modules Inv. Math. 62 (1981), 443-457.

$\left[\mathrm{Fr}_{1}\right]$ A. FRÖHLICH.- The genus field and genus number in algebraic number fields - Mathematika 6 (1959), 40-46 \& 142-146.

$\left[\mathrm{Fr}_{2}\right]$ A. FRÖHLICH.- On fields of class two - Proc. London Math. Soc. 4 (1954), 235-256. 
$\left[\mathrm{Fr}_{3}\right]$ A. FRÖHLICH .- Central extensions, Galois groups, and ideal class groups of number fields - Contemporary mathematics 24 (1983).

$\left[F u_{1}\right]$ Y. FURUTA . - The genus field and genus number in algebraic num ber fields - Nagoya Math. J. 29 (1967), 281-285.

$\left[\mathrm{Fu}_{2}\right]$ Y. FURUTA.- Über das Geschlecht und die Klassenzahl eines rela tiv-Galoissches Zahlkörpers von PrimzahlpotenzgradeNagoya Math. J. 37 (1970), 197-200.

$\left[\mathrm{Fu}_{3}\right]$ Y. FURUTA.- On nilpotent factors of congruent ideal class groups of Galois extensions - Nagoya Math. J. 62 (1976), $13-28$.

$\left[\mathrm{Fu}_{4}\right]$ Y. FURUTA. - Note on class number factors and prime decomposi tion - Nagoya Math. J. 66 (1977), 167-182.

$\left[\mathrm{Fu}_{5}\right]$ Y. FURUTA.- A norm residue map for central extensions of an al gebraic number field - Nagoya Math. J. 93 (1984), 61-69.

[FW] P. FURTW ÄNGLER . Beweis des Hauptidealsatzes für den Klassenkörper algebraische Zahlkörper - Abh. Math. Sem. Hamburg 7 (1930), 14-36.

[FW ] B. FERRERO \& L. WASHINGTON . - The Iwasawa invariant $u_{p}$ vanishes for abelian number fields - Ann. of Math. 109 (1979), 377-395.

$\left[\mathrm{Ga}_{1}\right]$ D. A. GARBANATI .- Units signatures, and even class numbers, and relative class numbers - J. reine angew. Math. 275 $(1975), 376-384$.

$\left[\mathrm{Ga}_{2}\right]$ D. A. GARBANATI .- Units with norm -1 and signature of units J. reine angew. Math. 283 (1976), 164-175 . 
$\left[\mathrm{Ga}_{3}\right]$ D. A. GARBANATI. - The Hasse norm theorem for non cyclic exten sions of the rationals - Proc. London Math. Soc. 37 (1978), 143-164.

$\left[\mathrm{Ga}_{4}\right]$ D. A. GARBANATI . - Invariants of the ideal class groups and the Hasse norm theorem - J. reine angew. Math. 297 (1978), $159-171$.

$\left[G b_{1}\right]$ R. GREENBERG - On a certain $\ell$-adic representation Inv. Math. 21 (1973), 117-124.

$\left[\mathrm{Gb}_{2}\right]$ R. GREENBERG . - On the Iwasawa invariants of totally real number fields - Am. J. Math. 98 (1976), 263-284.

$\left[\mathrm{Gb}_{3}\right]$ R. GREENBERG .- A note on $K_{2}$ and the theory of $\mathbb{Z}_{\mathrm{p}}$-extensions Am. J. Math. 100 (1978), 1235-1245 .

$\left[\mathrm{Gb}_{4}\right]$ R. GREENBERG .- On the structure of certain Galois groups Inv. Math. 47 (1978), 85-89 .

[Gd] L.J. GOLDSTEIN .- On prime discriminants - Nagoya Math. J. 45 (1971), 119-127.

[GG] G. GRAS \& M.N. GRAS . - Signature des unités cyclotomiques et pa rité du nombre de classes des extensions cyclotomiques de $\mathbb{Q}$ de degré premier impair Ann. Sci. Inst. Fourier 35 (1975), 1-22.

[Gi $\left.{ }_{1}\right]$ R. GILLARD - - Remarques sur certaines extensions prodiédrales de corps de nombres - C.R. Acad. Sci. Paris 282 (1976), 13-15.

[Gi $\left.{ }_{2}\right]$ R. GILLARD . - Formulations de la conjecture de Leopoldt, et étude d'une condition suffisante - Abh. Math. Sem. Hamburg 48 (1979), $125-138$. 
$\left[\mathrm{Gi}_{3}\right] \mathrm{R}$. GILLARD.$-\mathbb{Z}_{\ell}$-extensions, fonctions $L \ell$-adiques, et unités cyclotomiques - Sém. Th. Nombres, Bordeaux $(1976 / 77)$, exp. $n^{\circ} 4$.

[Gi $\left.{ }_{4}\right]$ R. GILLARD .- Fonctions L p-adiques des corps quadratiques ima ginaires et de leurs extensions abéliennes J. reine angew. Math. (à parâitre)

[GI] H. GARLAND.- Afiniteness theorem for $K_{2}$ of number fields Ann. of Math. 94 (1971), 534-548.

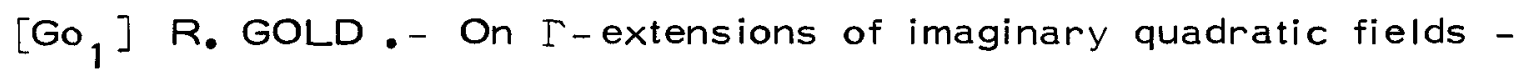
Pacific J. Math. 40 (1972),

$\left[\mathrm{Go}_{2}\right]$ R. GOLD.- The non triviality of certain $\mathbb{Z}_{\ell}$-extensions J. Numb. Th. 6 (1974), 369-373.

$\left[\mathrm{Go}_{3}\right]$ R. GOLD . - Examples of Iwasawa invariants - Acta Arith.26 (1975), 21-32.

$\left[G r_{1}\right]$ G. GRAS .- Sur les $\ell$-classes d'idéaux dans les extensions cycliques de degré premier $\ell$-Ann. Sci. Inst. Fourier 23,3 (1973), 1-48.

$\left[\mathrm{Gr}_{2}\right]$ G. GRAS - Nombre de $\varphi$-classes invariantes. Application aux clas ses des corps abéliens - Bull. Soc. Math. France 106 (1978), 337-364.

$\left[\mathrm{Gr}_{3}\right]$ G. GRAS .- Sur les invariants lambda d'Iwasawa des corps abéliens Pub. Math. Fac. Sci. Besançon (1978/79) .

$\left[\mathrm{Gr}_{4}\right]$ G. GRAS .-Groupe de Galois de la p-extension abélienne p-ramifiée maximale d'un corps de nombres - J. reine angew. Math. 333 (1982), 86-132.

[Gr 5 ] G. GRAS - Logarithme p-adique et groupes de Galois J. reine angew. Math. 343 (1983), 64_80. 
$\left[G r_{6}\right]$ G. GRAS - Logarithme p-adique, p-ramification abélienne et $K_{2}$ Sém. Th. Nombres, Bordeaux (1982/83), exp. n 12 .

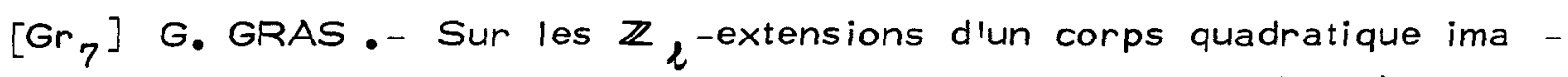
ginaire - Ann. Sci. Inst. Fourier 33,4 (1983), 1-18.

$\left[G r_{8}\right]$ G. GRAS - Plongements kummériens dans les $\mathbb{Z}_{\mathrm{p}}$-extensions Compositio Math. 55, (1985), 383-396.

[Gr $\left.{ }_{9}\right]$ G. GRAS . - Decomposition and inertia groups in $\mathbb{Z}_{p}$-extensions (A paraître dans JoFac. Sci. Tokyo IA, 1986).

[Gs] B. GROSS . - p-adic L-series at $s=0$ - J. Fac. Sci. Tokyo 1 A 28 (1981), 979-994.

[Gu $\left.{ }_{1}\right]$ S.J. GURAK . - The Hasse norm principle - J. reine angew. Math. 299 (1978), 16-27.

$\left[\mathrm{Gu}_{2}\right]$ S.J. GURAK .- The Hasse norm principle in non abelian extensions J. reine angew. Math. 299 (1978),

$\left[\mathrm{Gu}_{3}\right]$ S.J. GURAK . - Ideal -theoretic caracterization of the relative genus field - J. reine angew. Math. 303/304 (1978), 314-318.

$\left[\mathrm{Ha}_{1}\right]$ H. HASSE . - Neue Begründung und Verallgemeinerung der Theorie des Normenrestsymbols - J. reine angew. Math. 162 (1931), 134-144.

$\left[\mathrm{Ha}_{2}\right] \mathrm{H}$. HASSE - Bericht über neuere Untersuchungen und Probleme aus der Theorie der algebraischen Zahlkörper 11 Physica Verlag, Würzburg (1965).

$\left[\mathrm{Ha}_{3}\right]$ H. HASSE .- Zur Geschlechtertheorie in quadratischen ZahlkörpernJ. Math. Soc. Japan 3 (1951), 45-51.

[Ha $\left.{ }_{4}\right]$ H. HASSE - A supplement on Leopoldt's theory of genera in abelian number fields - J. Numb. Th. 1 (1969), 4-7 . 
$\left[\mathrm{Hd}_{1}\right]$ F.P. HEIDER .- Zur Theorie der zahlentheoretischen Knoten Dissertation, Köln (1978).

$\left[\mathrm{Hd}_{2}\right]$ F.P. HEIDER - - Strahlknoten und Geschlechterkörper mod $\mathfrak{M}$ J. reine angew. Math. 320 (1980), 52-67.

$\left[\mathrm{Hd}_{3}\right]$ F.P. HEIDER - - Kapitulationsproblem und Knotentheorie Manuscripta Math. 46 (1984), 229-272 .

[He $\left.{ }_{1}\right]$ J. HERBRAND . - Nouvelle démonstration et généralisation d'un théorème de Minkowski-C.R. Acad. Sci. Paris (1930), 1282 .

$\left[\mathrm{He}_{2}\right]$ J. HERBRAND .- Sur les unités d'un corps algébrique C.R. Acad. Sci. Paris 192 (1931), 24-27 \& 188 .

[Hi ] D. HILBERT .- Théorie des corps de nombres algébriques Ann. Fac. Toulouse (1909/10); Hermann, Paris (1913).

[Ho ] T. HONDA.- On absolute class fields of certain algebraic number fields - J. reine angew. $203(19)$, 80-89.

[Is] M. ISHIDA.- The genus field of algebraic number fields Lecture Notes in Math. 555, Springer Verlag, Berlin, Heidelberg, New York (1976).

[IT ] S. IYANAGA \& T. TAMAGAWA.- Sur la théorie du corps de classes sur le corps des nombres rationnels - J. Math. Soc. Japan 3 (1951), 220-227.

$\left[\mathrm{IW}_{1}\right] \mathrm{K}$. IWASAWA .- A note on the group of units of an algebraic number field - J. Math. pures appl. 35 (1956), 189-192 .

$\left[\mathrm{IW}_{2}\right] \mathrm{K}$. IWASAWA.- A note on class numbers of algebraic number fieldsAbh. Math. Sem. Hamburg 20 (1956), 257-258. 
$\left[\mathrm{IW}_{3}\right] \mathrm{K}$. IWASAWA.- On $\Gamma$-extensions of algebraic number fields Bull. Am. Math. Soc. 65 (1958), 183-226.

$\left[\mathrm{IW}_{4}\right] \mathrm{K}$. IWASAWA.- On some invariants of cyclotomic fields Am. J. Math. 80 (1958), 773-783.

$\left[\mathrm{IW}_{5}\right] \mathrm{K}$. IWASAWA.- On the $\mu$ invariants of $\mathbb{Z}_{\ell}$-extensions - Number theory, algebraic geometry, and commutative algebra, Kinokuniya, Tokyo (1973), 1-11.

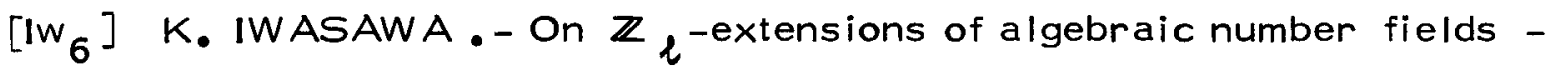
Ann. of Math. 98 (1973), 243-326 .

$\left[\mathrm{IW}_{7}\right] \mathrm{K}$. IWASAWA.-Riemann Hurwitz formula and $\mathrm{p}$-adic Galois repre sentations for number fields - Tôhoku Math. J. 33,2 (1981), 263-288.

$\left[\mathrm{IW}_{8}\right] \mathrm{K}$. IWASAWA.- On cohomology groups of units for $\mathbb{Z}_{\mathrm{p}}$-extensions Amer. J. Math. 105 (1983), 189-200.

$\left[\mathrm{Ja}_{1}\right] \quad J_{\bullet}-\mathrm{F}$. JAULENT •-Sur la théorie des genres dans une extension cyclique de degré $\ell^{m}$, métabélienne sur un sous-corpsPub. Math. Fac. Sci. Besançon (1980/81).

$\left[\mathrm{Ja}_{2}\right]$ J.-F. JAULENT .- Théorie d'lwasawa des tours métabéliennes Sém. Th. Nombres, Bordeaux (1980/81), exp. n²1.

$\left[\mathrm{Ja}_{3}\right]$ J.-F. JAULENT .- Sur la théorie des genres dans les tours méta béliennes - Sém. Th. Nombres, Bordeaux (1981/82), exp. $n^{\circ} 24$.

[ $\left.\mathrm{Ja}_{4}\right]$ J.-F. JAULENT .- Sous-groupe ambige, quotient des genres et théorie d'lwasawa - Sém. DPP (1981/82); Birkhäuser (1983).

[ $\left.\mathrm{Ja}_{5}\right]$ J.-F. JAULENT •- $\&$-classes infinitésimales d'un corps de nombres algébriques - Ann. Sci. Inst. Fourier 34, 2 (1984). 
[ $\left.\mathrm{Ja}_{6}\right]$ J.-F. JAULENT .- Sur l'indépendance $\ell$-adique de nombres algébriques - J. Numb. Th. 20, (1985), 149-158 .

$\left[\mathrm{Ja}_{7}\right]$ J.-F. JAULENT .-Sur quelques représentations $\ell$-adiques liées aux symboles et à la $\ell$-ramification - Sém. Th. Nombres, Bordeaux (1983/84), exp. $n^{\circ}$

$\left[\mathrm{Ja}_{8}\right]$ J.-F. JAULENT .- Sur la formule du produit pour le symbole de reste normique généralisé - Acta Arithmetica 45, 3 (1985), 67-72 .

[ $\left.\mathrm{Ja}_{9}\right]$ J.-F. JAULENT 。- Représentations $\ell$-adiques et invariants cyclotomiques - Pub. Math. Fac. Sci. Besançon (1983/84).

[GJ ] M. GRANDET \& J.-F. JAULENT .- Sur la capitulation dans une $\mathbb{Z} \mathfrak{l}^{-}$ extension - J. reine angew. 362 (1985), 213-217.

[Je] W. JEHNE .- On knots in algebraic number theory - Prépublication.

[Ka] S. KATAYAMA.- On the Galois cohomology groups of $C_{K} / D_{K}$ Japan J. Math. 8 (1982), 407-415 .

[Kd] Y. KIDA.- -extensions of C.M. fields and cyclotomic invariants J. Numb. Th. 12 (1980), 519-528.

[Ki $\left.{ }_{1}\right]$ H. KISILEVSKY. - Some results related to Hilbert's theorem 94 J. Numb. Th. 2 (1970), 199-206 .

$\left[\mathrm{Ki}_{2}\right]$ H. KISILEVSKY.- Some non-semi-simple Iwasawa module Compositio Math. 49 (1983), 399-404.

[Ko] M. KOBAYASHI .- The connected component of the idele class group of an algebraic number field - Pac. J. Math. 106 (1983), 129-134.

[Kb] N. KOBLITZ . - p-adic analysis: a short course on recent work London Math. Soc. Lecture Notes 46 , Cambridge University Press, Cambridge (1980). 
[Ku ] T. KUBOTA.- Galois group of the maximal abelian extension of an algebraic number field - Nagoya Math. J. 12 (1957), 177-189.

$\left[\mathrm{Kz}_{1}\right]$ L.V. KUZ'MIN .- The Tate module for algebraic number fields IzV. Akad. Nauk. SSR. Math. 36 (1972), 267-327 ; Math. USSR IzV. 6 (1972).

$\left[\mathrm{Kz}_{2}\right]$ L.V. KUZ'MIN .- Some duality theorems for cyclotomic $\Gamma$-extensions of algebraic number fields of C.M. type Math. USSR IzV. 14 (1980), 441-498。

$\left[\mathrm{Kz}_{3}\right]$ L.V. KUZ'MIN .- Some remarks on the $\ell$-adic Dirichlet theorem and the $\ell$-adic regulator - Math. USSR IzV. 19 (1982), $445-478$.

[KC] A. KRAMER \& A. CANDIOTTI .- On $K_{2}$ and $\mathbb{Z} l^{\text {-extensions of num- }}$ ber fields - Am。J. Math. 100 ( 1978), 177-196.

[La] S. LANG .- Algebraic Number Theory - Addison Wesley (1970).

[Le $\left.{ }_{1}\right]$ H.W. LEOPOLDT - - Zur Geschlechtertheorie in abelschen Zahl körper - J. Math. Soc. Japan 3 (1951), 45-51.

[Le ${ }_{2}$ ] H.W. LEOPOLDT .- Zur Arithmetik in abelschen Zahlkörpern J. reine angew. Math. 209 (1962), 54-71.

[Ma] K. MASUDA.- An application to the general norm residue symbol Proc. Am. Math. Soc. 10 (1959), 245-252.

$\left[\mathrm{MK}_{1}\right]$ K. MIYAKE . - On the units of an algebraic number field J. Math. Soc. Japan 34 (1982), 515-525.

$\left[\mathrm{Mk}_{2}\right]$ K. MIYAKE - Central extensions and Schur's multiplicators of Galois groups - Nagoya Math. J. 90 (1983), 134-144. 
[MK 3 ] K. MIYAKE .- On central extensions of a Galois extension of algebraic number fields - Nagoya Math. J. 93 (1984), 133-148.

$\left[\mathrm{Mi}_{1}\right] \mathrm{H}_{\text {. MIKI }}$ - On the maximal abelian $\ell$-extension of a finite algebraic number field with given ramification Nagoya Math. J. 70 (1978), 183-202.

$\left[\mathrm{Mi}_{2}\right] \mathrm{H}$. MIKI . - On the Leopoldt conjecture on the $\mathrm{p}$-adic regulators J. Numb. Th。 ( à paraître)

[MS] H. MIKI \& H. SATO .- Leopoldt's conjecture and Reiner 's theoremJ. Math. Soc. Japan 36, 1 (1985), 47-52 .

[Ne] O. NEUMANN .- On p-closed number fields and an analogue of Riemann's existence theorem - in Algebraic number fields, Academic Press, London (1977).

$\left[\mathrm{Ng}_{1}\right]$ T. NGUYEN QUANG DO . - Sur la structure galoisienne des corps locaux et la théorie d'lwasawa - J. reine angew. Math. 333 (1982), 133-143; Comp. Math. 46 (1982), 85-119.

$\left[\mathrm{Ng}_{2}\right]$ T. NGUYEN QUANG DO.- Valeurs en $s=1$ de certaines fonctions d'Iwasawa - Groupe d'Etude d'Analyse Ultramétrique (1984) .

$\left[\mathrm{Ng}_{3}\right]$ T. NGUYEN QUANG DO . - Formations de classes et modules d'Iwa sawa - Proceedings Number Theory, Noor dwykerkout (1983), Lecture Note in Math. 168 .

$\left[\mathrm{Ng}_{4}\right]$ T. NGUYEN QUANG DO.- Sur la $\mathbb{Z}_{\mathrm{p}}$-torsion de certains modules galoisiens - Prépublication.

[NK] J. NEUKIRCH . - Class Field Theory - Springer-Verlag , Berlin , Heidelberg, New-York, Tokyo (1985).

[NT ] D. BERTRAND ,..., M. WALDSCHMIDT .- Les Nombres Tr anscendants - Mém. Soc. Math. de France, 112 , fasc. 1 (1984). 
[Oe] J. OESTERLE .- Travaux de Ferrero et Washington sur le nombre de classes d'idéaux des corps cyclotomiques Sém. Bourbaki (1978), exp. n 535 .

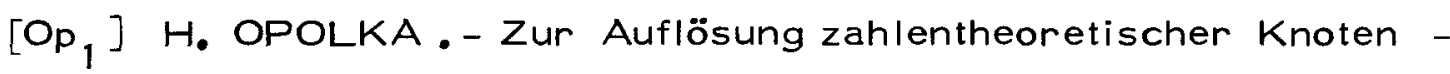
Math. Z. 173 (1980), 95-103.

$\left[\mathrm{Op}_{2}\right]$ H. OPOLKA.- Extensions of number fields defined by cohomology groups - Nagoya Math. J. 92 (1983), 179-186.

[Or $\left.{ }_{1}\right]$ B. ORIAT - Essai de généralisation du Spiegelungssatz Thèse, Besançon (1980).

[Or $\left.{ }_{2}\right]$ B. ORIAT - Introduction à la théorie d'lwasawa - Pub. Math. Fac. Sci. Besançon (1980/81).

[Re ] I. REINER .- Maximal orders - Academic Press, London (1975).

[Rn] G. RENAULT .- Algèbre non commutative - Gauthier-Villars, Paris (1975).

[Rz] M. RAZAR . - Central and genus class fields and the Hasse norm theorem - Compositio Math. 35 (1977), 281-298.

[Sa, ${ }_{1}$ P. SAMUEL - Algèbre locale - Gauthier-Villars, Paris (1953).

$\left[\mathrm{Sa}_{2}\right]$ P. SAMUEL - - Théorie algébrique des nombres - Hermann, Paris (1971).

[Sc] R. SCHIPPER .- On the behavior of ideal classes in cyclic unramified extensions of prime degree -

[Se $\left.{ }_{1}\right]$ J.-P. SERRE .- Classes des corps cyclotomiques (d'après Iwasawa)Sém. Bourbaki (1958), exp. 174 .

$\left[\mathrm{Se}_{2}\right]$ J. -P. SERRE - Corps locaux - Hermann, Paris (1968) • 
$\left[\mathrm{Se}_{3}\right]$ J.-P. SERRE .- Représentations linéaires des groupes finis Hermann, Paris (1971).

$\left[\mathrm{Se}_{4}\right]$ J.-P. SERRE - - Sur le résidu de la fonction zêta p-adique d'un corps de nombres - C.R. Acad. Sci. Paris 287 (1978), $183-188$.

[Sh] S. SHIRAl . - On the central class field mod $\mathfrak{M}$ of Galois extensions of an algebraic number field - Nagoya Math. J. 71 (1978), 61-85.

[Si] W.M. SINNOT .- On p-adic L-functions and the Riemann-Hurwitz genus formula - Compositio Math. 53 (1984), 3-17.

[St] H.M. STARK .- The genus theory of number fields Com. pur a appl. Math. 29 (1976), 805-811 .

[Sz] A. SCHOLZ .- Totale Normenreste, die keine Normen sind, als Euzenger nicht abelscher Körper erweiterungen J. reine angew. Math. 172 (1936), 100-107; 182 (1940), 217-234 。

$\left[\mathrm{Ta}_{1}\right]$ J. TATE - Symbols in arithmetic - Actes Congrès International Math. 1 (1970), 201-211。

$\left[\mathrm{Ta}_{2}\right]$ J. TATE . - Letter from Tate to Iwasawa on a relation between $K_{2}$ and Galois cohomology - Algebraic K-theory II , Lecture Notes in Math. 342 (1973).

$\left[\mathrm{Ta}_{3}\right]$ J. TATE .- Relations between $K_{2}$ and Galois cohomology Inv. Math. 36 (1976), 257-274 .

[TBS] J. TATE . - Les conjectures de Stark sur les fonctions L d'Artin en $s=0$ - Birkhäuser, Boston, Basel, Stuttgart (1984).

[TK] T. TAKAGI - - Ueber eine Theorie des relativ abelschen ZahlkörpersJ. Coll. Sci. Tokyo (1920). Voir aussi : Collected papers, Iwanami, Tokyo (1973). 
[Ts] O. TAUSSKY . - A remark conceming Hilbert's theorem 94 J. reine angew. Math. 240 (1970), 435-438 .

[Wa $\left.{ }_{1}\right]$ M. WALDSCHMIDT .- Transcendance et exponentielles en plusieurs variables - Inv. Math. 63 (1981), 97-127.

$\left[\mathrm{Wa}_{2}\right] \mathrm{M}$. WALDSCHMIDT - - A lower bound for the p-adic rank of the units of an algebraic number field-Actes Congrès Budapest (1981).

[We ] A. WEIL - - Sur la théorie du corps de classes - J. Math. Soc. Japan 3 (1951), 1-35.

[Wi] K. WINGBERG .- Duality theorems for $\Gamma$-extensions of algebraic number fields - Compositio Math. 55 (1985), 333-381 .

[Yo] H. YOKOI - On the class number of a relatively cyclic number fieldNagoya Math. J. (1947), 31-44.

[Hr] M. HORIE .- On the genus field in algebraic number fields Tokyo J. Math. 6 (1983), 363-380. 